\title{
Measurements of the W Boson Mass with the D0 Detector
}

\author{
A Dissertation Presented \\ by \\ Rafael Coelho Lopes de Sá \\ to \\ The Graduate School \\ in Partial Fulfillment of the
}

Requirements

for the Degree of

Doctor in Philosophy

in

Physics

Stony Brook University

August/2013 
Copyright by

Rafael Coelho Lopes de Sá

Creative Commons BY-NC Bir. (\$)

2013 


\section{Stony Brook University \\ The Graduate School \\ Rafael Coelho Lopes de Sá}

We, the dissertation committee for the above candidate for the

Doctor of Philosophy degree, hereby recommend

acceptance of this dissertation

John Hobbs - Dissertation Advisor

Professor, Physics and Astronomy

Paul Grannis - Chairperson of Defense

Professor, Physics and Astronomy

Patrick Meade - Internal Member

Assistant Professor, Physics and Astronomy

Jan Stark - External Member
Staff Scientist, French National Centre for Scientific Research

Robert Hirosky - External Member

Professor, Department of Physics, University of Virginia

This dissertation is accepted by the Graduate School

Charles Taber

Interim Dean of the Graduate School 
Abstract of the Dissertation

\title{
Measurements of the $W$ Boson Mass with the D0 Detector
}

\author{
by \\ Rafael Coelho Lopes de Sá \\ Doctor of Philosophy \\ in \\ Physics \\ Stony Brook University
}

2013

\section{Thesis est omnis divisa in partes tres.}

In the first part, we describe what is the $W$ boson mass in the context of the Standard Model. We discuss the prominent role this physical observable plays in the determination of the internal self consistency of the Electroweak Sector. We review measurements and calculation of the $W$ boson mass done in past and argue about the importance and feasibility of improving the experimental determination. We give a description of the Fermilab Tevatron Collider and the D0 detector, highlighting the relevant parts for the measurement described in this Dissertation.

In the second part, we give a detailed description of a measurement of the $W$ boson mass using the D0 Central Calorimeter. The measurement uses $1.68 \times 10^{6}$ candidates from $W \rightarrow e v$ decays, corresponding to $4.3 \mathrm{fb}^{-1}$ of integrated luminosity collected from 2006 to 2009 . We measure the mass using the transverse mass, electron transverse momentum, and missing transverse energy distributions. The transverse mass and electron transverse momentum measurements are the most precise and are combined to give $M_{W}=80.367 \pm 0.013$ (stat) \pm 0.023 (syst) $\mathrm{GeV}=80.367 \pm 0.026 \mathrm{GeV}$. This is combined with an earlier D0 result determined using an independent $1 \mathrm{fb}^{-1}$ data sample, also with central electrons only, to give $M_{W}=80.375 \pm 0.023 \mathrm{GeV}$. The uncertainty in the measurement is dominated by the determination of the calorimeter electron energy scale, the $W$ sample size, the knowledge of the parton distribution function.

In the third part, we discuss methods of reducing the dominant uncertainties in the $W$ boson mass measurements. We show that introducing electrons detected in the End Calorimeters greatly reduce the measurement systematic uncertainty, especially the one 
related to the parton distribution functions. We describe a precise calibration of the End Calorimeter using $Z \rightarrow e e$ events corresponding to $4.3 \mathrm{fb}^{-1}$ of integrated luminosity. The calibration is an important milestone in a measurement that explores a larger part of the D0 Calorimeter. We present parametrized models that describe the response of the End Calorimeters to electron showers and soft hadronic particles, giving special attention to the specific challenges of a measurement in the forward region: the inhomogeneity of the uninstrumented materials, the large hadronic energy flow in the calorimeter and the jet misidentification probability. 
I dedicate this Dissertation to Luciana, to whom I owe the strength to have made it. 


\section{Contents}

\begin{tabular}{ll}
\hline I Preliminaries & 1
\end{tabular}

1 The W Boson and its Mass in the Standard Model 2

1.1 Electroweak Precision Observables . . . . . . . . . . . . . . . . . . . . . 3

$1.2 \quad W$ Boson Production at the Tevatron . . . . . . . . . . . . . . . . . . . 4

1.3 Previous $W$ Boson Mass Calculations and Measurements . . . . . . . . . . 7

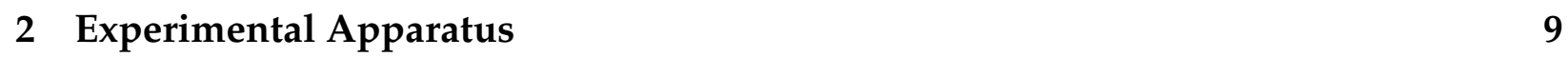

2.1 The Fermilab Tevatron Collider . . . . . . . . . . . . . . . . . . . . . . . . 9

2.2 The Tevatron Bunch Structure and Luminosity . . . . . . . . . . . . . . . . 11

2.3 The Run II D0 Detector $\ldots \ldots \ldots \ldots$

$2.3 .1 \quad$ Silicon Microstrip Tracker . . . . . . . . . . . . . . . . . . . 13

2.3 .2 Central Fiber Tracker . . . . . . . . . . . . . . . . . . . . . . . . . . . . 15

2.3 .3 Solenoid and Toroid . . . . . . . . . . . . . . . . . . . . . . . . . . 16

2.3 .4 Muon System . . . . . . . . . . . . . . . . . . . . . . . . . . . 17

2.3 .5 Luminosity Monitor . . . . . . . . . . . . . . . . . . . . . . . . . 18

2.4 The D0 Calorimetry System $\ldots \ldots \ldots \ldots$

$2.4 .1 \quad$ Liquid Argon Calorimeter . . . . . . . . . . . . . . . . . . 18

2.4 .2 Inter-Cryostat Detector . . . . . . . . . . . . . . . . . . . . . 21

2.4 .3 The Central and Forward Preshowers . . . . . . . . . . . . . 21

$2.4 .4 \quad$ Calorimeter Readout System . . . . . . . . . . . . . . . . . . . . . 23

2.4 .5 Anomalous Currents . . . . . . . . . . . . . . . . . . . . . . . 26

2.5 The D0 Single Electron Trigger $\ldots \ldots \ldots \ldots$

II W Boson Mass Measurement with Central Electrons 34

3 Measurement Strategy 35

3.1 Conventions $\ldots \ldots \ldots \ldots \ldots \ldots \ldots$

3.2 Event Characteristics . . . . . . . . . . . . . . . . . . . . . . . . . 35

3.3 Mass Measurement Strategy . . . . . . . . . . . . . . . . . . . . . 36

3.4 Additional Kinematic Variables . . . . . . . . . . . . . . . . . . . . . . . . . . 38 
4 Data Reconstruction 40

4.1 Electron Reconstruction . . . . . . . . . . . . . . . . . . . . . . 41

4.2 Vertex Reconstruction . . . . . . . . . . . . . . . . . . . . . . . . 44

4.3 Raw Missing $E_{T}$ and Recoil Reconstruction . . . . . . . . . . . . . . . . 44

4.4 SET Reconstruction $\ldots \ldots \ldots \ldots \ldots \ldots$

4.5 Corrected Missing $E_{T}$ Reconstruction . . . . . . . . . . . . . . . . . . . . . . . . . . . . . .

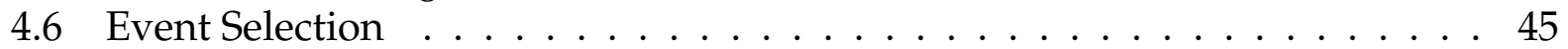

5 Dead Material Correction to the Electron Response 47

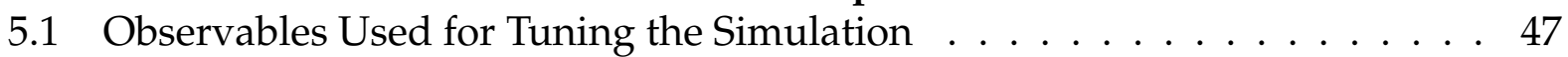

5.2 Improvements in the Shower Simulation of Electromagnetic Showers and in the D0 Material Model . . . . . . . . . . . . . . 50

5.3 Energy-Loss Corrections . . . . . . . . . . . . . . . . . . . . 53

$5.4 \quad 4.3 \mathrm{fb}^{-1}$ Analysis Validation $\ldots \ldots \ldots \ldots \ldots$. . . . . . . . . . 54

6 Generators for Full and Fast Simulation 56

6.1 QCD Corrections-Boson $q_{T} \ldots \ldots \ldots \ldots$. . . . . . . . . . . . . . . . . . . . . . . . . 57

6.2 Electroweak Corrections $\ldots \ldots \ldots \ldots \ldots$

6.3 Parton Distribution Functions $\ldots \ldots \ldots$. . . . . . . . . . . . . 58

7 Determination of the Fast Simulation Parameters 59

7.1 Primary Vertex Parametrization . . . . . . . . . . . . . . . . . . 60

7.2 Electron Efficiency Parametrization . . . . . . . . . . . . . . . . 60

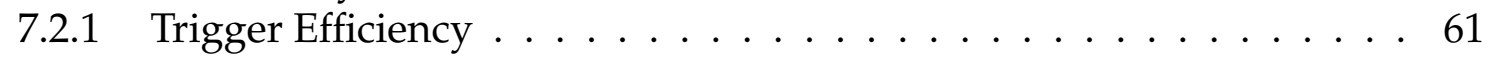

$7.2 .2 \quad$ FSR Efficiency $\ldots \ldots \ldots \ldots \ldots$. . . . . . . . . . . . . . . . . . . . . . . . . . . . . . 62

7.2 .3 Track-Matching Efficiency . . . . . . . . . . . . . . . . 63

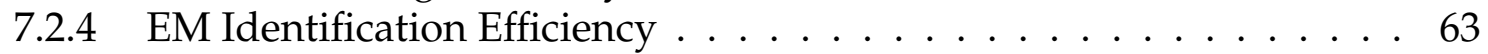

$7.2 .5 \phi_{\bmod }$ Efficiency $\ldots \ldots \ldots \ldots \ldots \ldots$. . . . . . . . . . . . . . . . . . . . . . . . 65

7.2 .6 Electron $\phi$ Efficiency $\ldots \ldots \ldots$. . . . . . . . . . . . . 66

7.2 .7 Hadronic Energy Dependent Efficiency . . . . . . . . . . . . . . . . . 66

7.2 .8 Monte Carlo Validation $\ldots \ldots \ldots$. . . . . . . . . . . . . . . . . . . . . . . . 68

7.2 .9 Residual Efficiency Corrections . . . . . . . . . . . . . . . . . . 68

7.2 .10 Systematic Uncertainty due to Efficiencies . . . . . . . . . . . . . . . 70

7.3 Electron Response Parametrization . . . . . . . . . . . . . . . . . . . 70

7.3 .1 Photon Radiation Effects . . . . . . . . . . . . . . . . . . . . 71

$7.3 .2 \quad$ Luminosity Dependence of the Calibration . . . . . . . . . . . . 71

$7.3 .3 \quad \eta$-Dependence of the Calibration . . . . . . . . . . . . . . . . . . . . . . . . . . . . . .

7.3 .4 Energy Response and Resolution . . . . . . . . . . . . . . . . . . 73

7.3 .5 Determination of the Constant Term in Resolution . . . . . . . . . . . 76

7.3 .6 Electron-Window Effects . . . . . . . . . . . . . . . . . . . 77

7.4 Hadronic Recoil Parametrization . . . . . . . . . . . . . . . . . . . . 79

$7.4 .1 \quad$ Recoil Model . . . . . . . . . . . . . . . . . 81

7.4 .2 Fit Results . . . . . . . . . . . . . . . . . . . . 83 
$7.4 .3 \quad$ Recoil Modeling Systematic Uncertainties . . . . . . . . . . . . . . . 84

8 Backgrounds 86

$8.1 \quad Z \rightarrow e e$ Background . . . . . . . . . . . . . . . . . . . . 86

8.2 Multijet Background . . . . . . . . . . . . . . . . . . . . . . . . 87

$8.3 \quad W \rightarrow \tau v$ Background $\ldots \ldots \ldots \ldots$. . . . . . . . . . 87

\begin{tabular}{llr}
\hline & Results & 89
\end{tabular}

\begin{tabular}{ll}
\hline 10 Combination & 93
\end{tabular}

\begin{tabular}{lr}
\hline 11 Consistency Checks & 96
\end{tabular}

III W Boson Mass Measurement with Forward Electrons 101

12 Understanding the PDF uncertainty in the $W$ mass measurement 102

12.1 Partons and PDF uncertainty $\ldots \ldots \ldots 2 \ldots \ldots \ldots$

12.2 Detectors and PDF uncertainties $\ldots \ldots$. . . . . . . . . . . 105

13 Calibration of the D0 End Calorimeters for the W Mass Measurement 110

13.1 General Idea of the Calorimeter Calibration . . . . . . . . . . . . . . 110

13.2 A Review of the $W$ Mass Group Modifications to GEANT . . . . . . . . . 112

13.3 The D0 Geometry Model in the Forward Region $\ldots$. . . . . . . . . . . . . 116

13.4 Improvements in the Material Model . . . . . . . . . . . . . . . . . . . 118

13.4 .1 Uncertainties . . . . . . . . . . . . . . . . . . . . . . . . 123

13.4.2 Closure Test of the Parametrized Model . . . . . . . . . . . . . . . . . 124

13.4 .3 Consistency Checks . . . . . . . . . . . . . . . . . . . . . 124

13.5 The Energy Loss Correction Function . . . . . . . . . . . . . . . . . 126

13.6 Absolute Calibration with Improved Dead Material Model . . . . . . . . . 128

13.7 Systematic Uncertainties $\ldots \ldots \ldots 131$

14 Parametrized Models for a Forward Electrons fast MC 136

14.1 Final State Radiation Efficiency and Response for Forward Electrons . . . 136

14.1.1 Closure test of the FSR model . . . . . . . . . . . . . . . 137

14.2 Leakage Model for Forward Electrons . . . . . . . . . . . . . . . . . . . . 141

14.3 Underlying Energy Model for Forward Electrons . . . . . . . . . . . . . . . 142

14.3 .1 Rotated clusters and $\Delta u_{T}$ library $\ldots \ldots$. . . . . . . . . . . . . . . 143

14.3.2 $\Delta u_{T} \rightarrow \Delta E_{T}$ Mapping and Closure Test of the Underlying Event

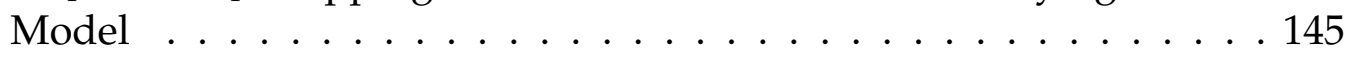

14.4 Single Photon Model for Forward Electrons . . . . . . . . . . . . . . . . . 146

14.4.1 Closure Test for Out-of-Cone FSR Photons . . . . . . . . . . . . . . 148

14.5 The Hard Recoil Model . . . . . . . . . . . . . . . . . . . . . . . . . . . . . 148

14.6 Resolution Model for Forward Electrons . . . . . . . . . . . . . . . . . . . 149 
14.6 .1 Closure Test for the Resolution Model . . . . . . . . . . . . . . . . . . 150

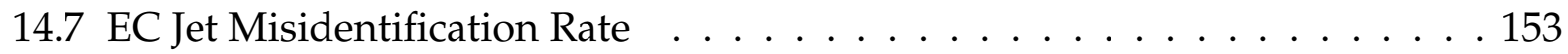

14.8 Full MC Efficiency Model for Forward Electrons . . . . . . . . . . . . . 156

14.8 .1 EMID Efficiency $\ldots \ldots \ldots \ldots \ldots \ldots$

14.8 .2 Track Matching Efficiencies . . . . . . . . . . . . . . . . . 158

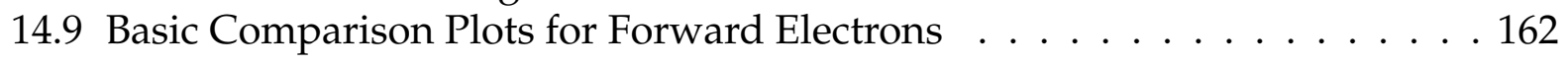

\begin{tabular}{lr}
\hline IV Conclusions & 164
\end{tabular}

$\begin{array}{ll}15 \text { Conclusions } & 165\end{array}$

\begin{tabular}{ll}
\hline Appendices & 176
\end{tabular}

\begin{tabular}{|l|l|l|l|}
\hline A Renormalization of the Electroweak Sector of the Standard Model & 177
\end{tabular}

A.1 The Weak Angle. . . . . . . . . . . . . . . . . . . . . . . . . . . 178

A.2 $\quad$ W Boson Mass . . . . . . . . . . . . . . . . . . . . . . . . . . . . . . . . . 179

A.3 Renormalization of the Gauge Boson Propagator . . . . . . . . . . . . . . 179

A.4 Photon-Z Mixing $\ldots \ldots \ldots 181$

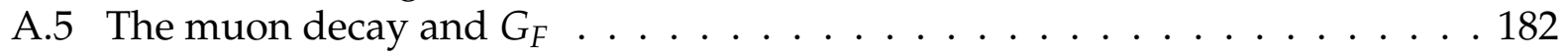

\begin{tabular}{ll}
\hline B Central Calorimeter fast MC Comparison Plots & 186
\end{tabular}

B.1 $\quad Z \rightarrow e e$ Comparison Plots $\ldots \ldots \ldots$. . . . . . . . . . . . . 186

B.2 $\quad W \rightarrow e v$ Comparison Plots $\ldots \ldots \ldots$. . . . . . . . . . . . . . . . . . . . 189

\begin{tabular}{|l|l|}
\hline C Parametrized Model for the End-Cap Calibration & 191
\end{tabular}

C.1 Ad hoc Geometries . . . . . . . . . . . . . . . . . . . . . . . . . . . . . . . 191

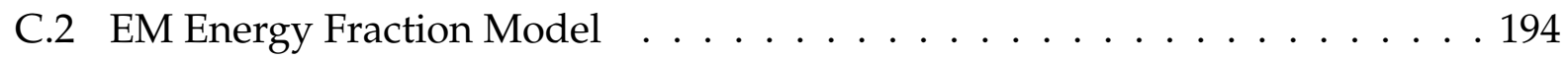

C.3 Geometry Eigensets and Energy Loss Function . . . . . . . . . . . . . . . 203 


\section{List of Figures}

1.1 The $p_{T}^{e}$ (left) and $m_{T}$ (right) spectra for simulated $\mathrm{W}$ bosons without detector resolution effects and $q_{T}=0$ (solid line), with the natural $q_{T}$ spectrum at the Tevatron (shaded area), and with the full $q_{T}$ distribution and all detector resolution effects included (points). All curves are normalized to unit area and they all include acceptance effects. . . . . . . . . . . . . . 6

2.1 Scheme of the complete Run II acceleration chain that led to the interactions

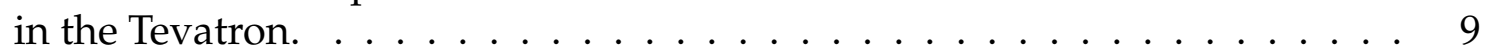

2.2 Integrated luminosity delivered by the Tevatron (green line) and recorded by the D0 experiment (blue line). The long horizontal lines are periods of extended Tevatron shutdown. In particular, the shutdown between April and June 2006 divides Run IIa and Run IIb. The overall recording efficiency at D0 was 90\% and basically limited by the Trigger Framework (see Sec. 2.5) dead time and by temporary problems with the detector. $\ldots . . . . . .11$

2.3 Tevatron bunch structure during Run I (top) and Run II (bottom). The first bunch of each super-bunch was different since there were no interactions right before it. The interactions involving first bunches would be naturally less prone to out-of-time pileup and associated detector effects...... .12

2.4 Cross section of the D0 detector showing the many sub-detectors that com-

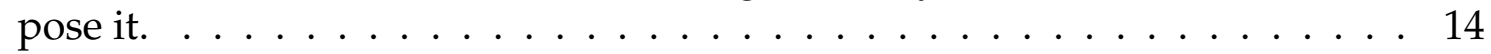

2.5 A drawing of the D0 Silicon Microstrip Tracker. There are 6 barrels interspersed by F-disks and additional four H-disks that provided tracking in

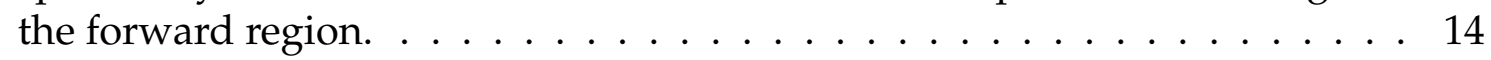

2.6 Technical drawing of the D0 central tracker system. . . . . . . . . . . 16

2.7 Momentum resolution for muon tracks with normal incidence $(\eta=0)$ with and without SMT hits. The yellow band corresponds to muons with incident angle $\eta=1.6$ and, hence, outside the CFT coverage. Those central tracks relied only on SMT hits. $\ldots \ldots \ldots \ldots$. . . . . . . . . . . . . . . . .

2.8 Flattened projective towers of the D0 calorimeter. . . . . . . . . . . . . . 19

2.9 Unit cell of the D0 calorimeter with the two liquid argon gaps on both sides of the copper readout pad. . . . . . . . . . . . . . . 21

2.10 The MIP and shower active layers of the Forward Preshower with the lead absorber in between. . . . . . . . . . . . . . . 22 
2.11 Assembly of the Forward Preshower. The "chimney" on the bottom right part of the detector is only notched on the south Preshower to allow space for the cryogenic services. . . . . . . . . . . . . . 23

2.12 Central calorimeter sampling resolution. The left plot shows the strong angular dependence of the sampling resolution due to the dead material introduced before the calorimeter. The dependence is stronger than the naïve estimation $\propto 1 / \sqrt{\sin \theta}$ [1]. The plot on the right shows the energy dependence of the sampling resolution also due to the large amount of dead material. The sampling resolution behavior of the D0 calorimeter violates the naïve $1 / \sqrt{E}$ behavior. $\ldots \ldots \ldots \ldots 24$

2.13 Waveforms in the output of the pre-amplifier and shaper circuits. The output form the charge sensitive amplifier had a long time constant (out of scale) while the signal from the shaper had a maximum after $320 \mathrm{~ns}$ and a return to zero after $1.2 \mu \mathrm{s} . \mid \ldots \ldots \ldots \ldots \ldots$

2.14 The BLS system always introduces a measurement error due to the decay of the previous waveform during the $396 \mathrm{~ns}$. (left) Two minimum bias signals when the error does not create negative energies. (right) A minimum bias signal after a "physics" signal, creating negative energy. . . . . . . . . . . . 25

2.15 Long-term behavior of the current drawn from a HV supply that fed one of the CC EM4 modules. The hatched areas represent extended shutdowns of the Tevatron collider. . . . . . . . . . . . . . . . 27

2.16 Scheme of the accumulation of ions responsible for the Malter process. The ion layer extracts electrons from the metal increasing the current until eventually the oxide breaks down and quenches the ions. . . . . . . . . . . . 27

2.17 Slow turn-on curve of the current from the HV supply after turning it on in the absence of beam in the Tevatron. Left: Current from the 32 supplies that fed the Central Calorimeter modules. Right: North End Calorimeter, where no Malter process is observed. Although not shown, the south End Calorimeter supplies behave like the north ones. Note especially the difference in the vertical axis scale, in units of $\mu \mathrm{A}$ : currents in the CC are many times larger than the one in the EC in the absence of beam. . . . . . . . 28

2.18 Behavior of the current from the HV supplies in the Central (left) and north End-Cap (right) Calorimeters during a period with many Tevatron stores. The plots clearly show the currents following the variation of the instantaneous luminosity and, in the Central Calorimeter, the Malter build-up of change between the stores. . . . . . . . . . . . . . . . . 29

2.19 Malter breakdown. When enough ions accumulate on the surface of the uranium oxide, the insulation is broken and the argon ions quenched. The build up process begins anew and the cycle repeats. . . . . . . . . . . . 29 
2.20 First principle calculation of the HV drop due to twice the normal current expected at $300 \times 10^{30} \mathrm{~cm}^{-2} \mathrm{~s}^{-1}$. The voltage variation can be as large as $20 \%$ at the center of the module. The HV is fed from two corners clearly seen on the figure. . . . . . . . . . . . . . . . . 29

2.21 Apparent position of the $Z \rightarrow e e$ mass peak in collider data CC-CC events before and after a long shutdown in Run IIb (red and blue points, respectively). The green points are from full MC simulations with unsuppresed zero-bias overlay that takes into account the energy flow from pileup and the negative energy from baseline subtraction, but not the HV sag due to the large currents in the central calorimeter. . . . . . . . . . . . . . 31

2.22 Resistive circuit that models the HV distribution in the calorimeter modules. This equivalent circuit is used to translate the current estimates into the HV available at the gaps of each cell with which the change in drift velocity can be calculated. . . . . . . . . . . . . . . 32

2.23 Typical Tevatron store. The black line shows the rate of the L1 trigger output, the blue line the rate of the L2 trigger output and, finally, the red line shows the rate of output of L3 that was being written on tape. The dashed magenta line shows the decrease of instantaneous luminosity during the store. The discontinuities in the triggers rates indicate the start of new D0 runs when the trigger rates were adjusted to accept more events. $\ldots . . . .33$

3.1 Deposit of energy in each calorimeter cell for a $W$ event candidate (run number 223477, event number 45476599). The blue bars indicate the deposit in the electromagnetic calorimeter while the red bars indicate the energy in the hadronic calorimeter. The green box at $\eta=0$ represents the event missing transverse energy. There are two large and clustered deposits of energy in the event, but only one is predominantly electromagnetic and identified as the electron. . . . . . . . . . . . . 36

3.2 a) Definition of $\eta$ and $\xi$ axis for $Z \rightarrow e e$ events. b) Definition of $u_{\|}$and $u_{\perp}$. As can be seen in this figure, $u_{\|}$is negative when opposite to the electron

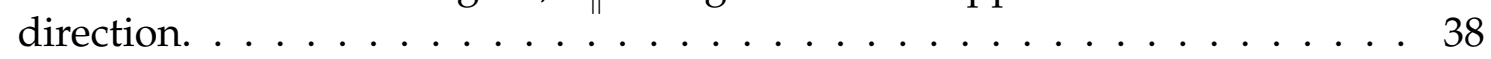

4.1 Instantaneous luminosity profiles for Run IIa and Run IIb. The Run IIb W candidate events have an average of two additional reconstructed primary vertices while this number was below one during Run IIa. $\quad$. . . . . . . . . . 40

4.2 The 13 calorimeter towers defined as the electron reconstruction window. The window is centered on the tower with the highest transverse energy. A circle of radius $\Delta R=0.2$ is shown for comparison. $\ldots$. . . . . . . . . . 41

4.3 Face size, in $\mathrm{cm}$, of an EM cluster as a function of the cluster detector $\eta$. The left plot shows the maximum diameter, in $\mathrm{cm}$, of an EM cluster in the $\Delta \phi$ direction, while the right plot shows the maximum diameter, also in $\mathrm{cm}$, of an EM cluster in the $\Delta \eta$ direction. . . . . . . . . . . . . . . . . . 42 
5.1 Overview of the material in front of the Central Calorimeter. This drawing shows a cross-sectional view of the central tracking system in the $x-$ $z$ plane. Also shown are the locations of the solenoid, the Preshower detectors, Luminosity Monitors and the Calorimeters. . . . . . . . . . . . . . 48

5.2 The average longitudinal shower profile (along the shower axis) of electrons with $\mathrm{E}=45 \mathrm{GeV}$ simulated using the GFlash parametrization. The depth of each different readout section of the central calorimeter is indicated for an electron with normal incidence $\eta=0$ (left) and with nonnormal incidence $\eta=1$ (right). $\ldots \ldots \ldots \ldots$. . . . . . . . . 48

5.3 The ratio of the data to simulation for the means of the EM fraction distributions in $Z \rightarrow e e$ events for each of the first three EM layers and each of the $15 \eta$ categories shown before the correction described here has been applied. Each of the three horizontal lines indicates the result of a fit of a common constant to the 15 data points from a given EM layer. The fit was performed for EM1, EM2 and EM3. . . . . . . . . . . . . . . . 50

5.4 Fit for $\mathrm{nX}_{0}$, the amount of uninstrumented material (in radiation lengths) added to the nominal material in the improved simulation of the D0 detector. The solid and dotted vertical lines show respectively the best fit and one standard deviation uncertainties for $\mathrm{nX}_{0}$. This fit is performed with the $Z \rightarrow$ ee data sample from our $1 \mathrm{fb}^{-1}$ measurement (see Sec. 5.4 )

5.5 Stability check: results of the fit for $\mathrm{n} \mathrm{X}_{0}$, performed separately for each of the three layers (EM1, EM2 and EM3). The result of the combined fit is also shown for comparison. . . . . . . . . . . . . . . 52

5.6 The ratio of the data to simulation for the means of the EM fraction distributions in $Z \rightarrow e e$ events for each of the first three EM layers and each of the $15 \eta$ categories shown after the correction described here has been applied. Each of the three horizontal lines indicates the result of a fit of a common constant to the 15 data points from a given EM layer. The fit was performed for EM1, EM2 and EM3. . . . . . . . . . . . . . . . . . 52

5.7 The data/simulation ratios for the means of the EM fraction distributions in $W \rightarrow$ ev events for each of the first three EM layers and each of the five $\eta$ bins after the correction. The dashed horizontal lines indicate the variations in the EM fractions from the uncertainty in the material tune due to the limited size of the $\mathrm{Z}$ sample. $\ldots \ldots \ldots \ldots$

5.8 The true energy spectrum for electrons in simulated $W$ events which pass the full selection (upper panel), and the ratio of measured minus true energy to the true energy as a function of true energy in $Z$ and $W$ electrons

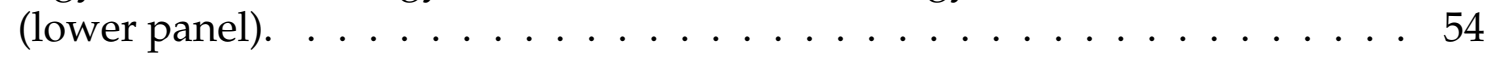


5.9 (left) Each line represents the ratio of the mean EM fractions (EMF) simulated with ZB overlay to the same sample simulated without overlay. It represents the contribution from extra $p \bar{p}$ interactions and noise to the mean EM fractions, which is determined separately for the Run IIa sample (dotted lines) and the Run IIb sample (continuous lines). The ratio between the continuous to the dashed line is used as a correction factor to the EMF fractions measured in RunIIa when comparing them to the Run IIb fractions. (right) Ratio of the means of the EM fraction distributions in $\mathrm{Z} \rightarrow$ ee events between the Run IIa analysis and the present Run IIb analysis, separately for each of the four EM layers and each of the 15 standard $\eta$ categories. . . . 55

7.1 Trigger efficiency as a function of $p_{T}^{e}$ for the three triggers used. The triggers are explained in section $2.5 \mid \ldots \ldots \ldots \ldots$. . . . . . . . . . . 62

7.2 Electron identification efficiency for electrons accompained by FSR determined from full MC as a function of the fraction of the energy carried by the photon. Each pane corresponds to a different $\Delta R$ region, and the distributions over $\eta$ and $E^{e}$ have been integrated for clarity. . . . . . . . . . . . . . 64

7.3 Track-matching efficiency as a function of $z_{V}$ and $\eta$ in data. The efficiency is proportional to the area of the boxes. $\ldots \ldots \ldots 65$

7.4 Track-matching efficiency as a function of $p_{T}^{e}$ in 13 bins of $\eta$ in full MC. The shapes reflect the fact that tracks from electrons with higher energy are more easily matched to the calorimeter cluster. This relative efficiency is arbitrarily normalized to 0.98 at $p_{T}^{e}=45 \mathrm{GeV}$. The total track-matching efficiency is the product of the relative efficiencies shown in Fig.|7.3 and Fig.|7.4. 66

7.5 (left) Mean difference between $\phi^{\mathrm{EM}}$ and $\phi^{\text {trk }}$ in module units as a function of $\phi_{\text {mod }}^{\text {trk }}$. (right) $\phi_{\text {mod }}$ electron reconstruction efficiency as a function of the

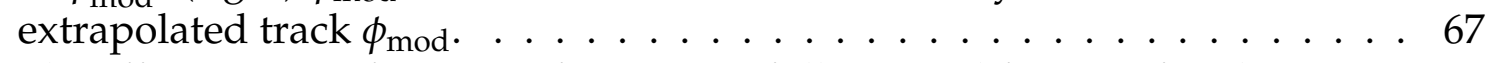

7.6 The efficiency as a function of true $p_{T}^{e}$ in full MC and fast MC for electrons in $Z \rightarrow e e$ (left) and $W \rightarrow e v$ (right) events. $\ldots \ldots \ldots \ldots$

7.7 The efficiency as a function of $\eta_{\text {det }}$ in full MC and fast MC for electrons in $Z \rightarrow e e$ (left) and $W \rightarrow e v$ (right) events. . . . . . . . . . . . . . 69

7.8 Electron efficiency ratio (data/full MC) as a function of SET (left) and instantaneous luminosity (right) for HMatrix, loose-track-match, tight-trackmatch, and the overall (HMatrix $\times$ loose-track-match $\times$ tight-track-match) criteria. Only the "All" requirements correction is used in analysis. The others are shown to illustrate where the major improvements arise. $\quad$. . . . . 70

7.9 Electron energy correction determined from full MC as a function of fraction of the energy carried out by the photon. $\ldots . . . . . . . .72$

7.10 (left) Result of the $Z$ mass fit per $\eta_{\text {det }}$ category prior to applying $\eta$-dependent corrections (Table 5.2). (right) Result of the translation into one relative gain

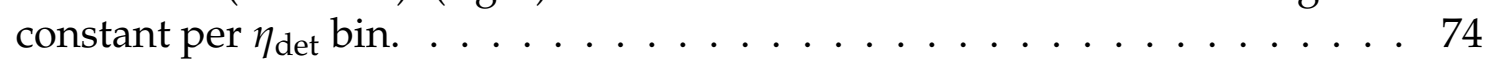

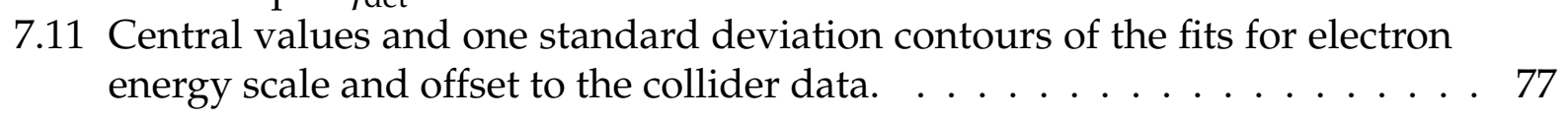


7.12 Mean $\Delta E$ as a function of SET and instantaneous luminosity comparing full MC and fast MC. . . . . . . . . . . . . . . . . 79

7.13 Mean $\Delta E$ as a function of true $p_{T}^{e}$ and $\eta_{\text {det }}$ comparing full MC and fast MC. $\quad 79$

7.14 Mean $\Delta E$ as a function of $u_{\|}$comparing full MC and fast MC. $\quad \ldots$. . . . . 80

$7.15\left\langle\Delta u_{\|}\right\rangle$as a function of instantaneous luminosity separately for sub-samples with different SET (left) and as a function of SET instantaneous luminosity for sub-samples with different instantaneous luminosity (right). Instanta-

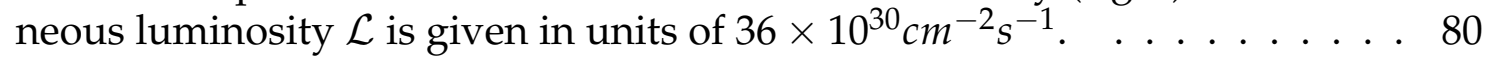

$7.16\left\langle\Delta u_{\|}\right\rangle$as a function of $u_{\|}$separately for various bins of SET. . . . . . . . . . 81

7.17 The distribution of the recoil relative $p_{T}$ and $\phi$ resolutions for full MC (boxes) and fit (contours) for $q_{T}=[4.5,5] \mathrm{GeV}$ (left) and $q_{T}=[18,20] \mathrm{GeV}$

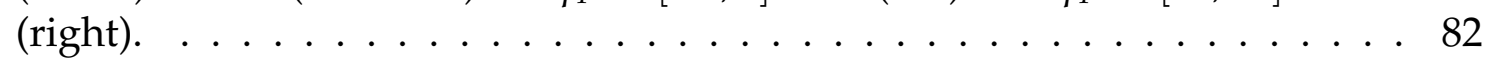

7.18 Data and fast MC comparison of the mean and RMS of the $\eta_{i m b}$ for the ten different bins in $p_{T}^{L} \ldots \ldots \ldots \ldots \ldots \ldots$

8.1 (left) Tight track match efficiency as a function of the electron $p_{T}^{e}$ measured relative to the loose track match requirement. (right) Probability of a jet object that passes the loose track match requirement to pass the tight track

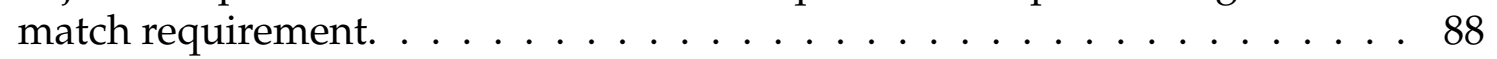

8.2 The $m_{T}, p_{T}^{e}$ and $Z_{T}$ distributions for the three backgrounds $Z$ (red), MJ
(black) and $W \rightarrow \tau v$ (blue) with absolute normalization. $\ldots \ldots 8$

9.1 The $Z$ mass distribution in data and from the fast MC (top) and the $\chi$ values

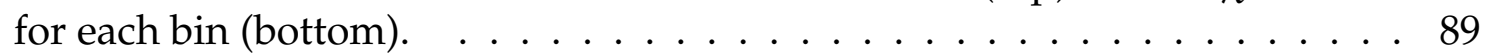

9.2 The $m_{T}$ distribution for data and fast MC with backgrounds added (top), and the $\chi$ value for each bin (bottom). . . . . . . . . . . . . . 90

9.3 The $p_{T}^{e}$ distribution for data and fast MC with backgrounds added (top) and the $\chi$ value for each bin (bottom). . . . . . . . . . . . 90

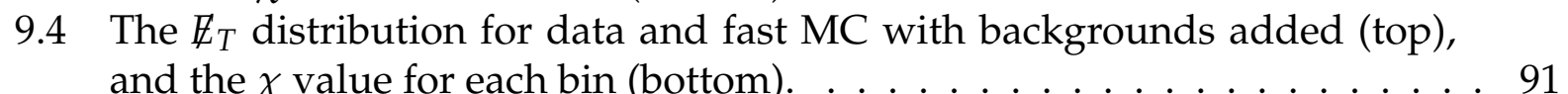

10.1 The D0 Run II measurement of $M_{W}$ plotted with the world-average mass of the top quark $M_{t}$ at $68 \%$ CL. by area (red ellipse). The new world-average for $M_{W}$ is also plotted (black ellipse). The thin blue band is the prediction of $M_{W}$ in the Standard Model given by equation $\mid 1.4$, chapter $\mid 1$, assuming $M_{H}=125.7 \pm 0.4 \mathrm{GeV} \ldots \ldots \ldots \ldots \ldots$

11.1 Impact of the fitting range on the fitted value of the observables. . . . . . . . 97

11.2 Consistency checks by splitting the $W$ and $Z$ samples in subsets with different instantaneous luminosity and time. . . . . . . . . . . . . . 98

11.3 Consistency checks by splitting the $W$ and $Z$ samples in subsets with different detector $\eta$ and $u_{\|} \cdot \ldots \ldots \ldots \ldots$. . . . . . . . . . . . . . . . . . 11.4 Consistency checks by splitting the $W$ and $Z$ samples in subsets with dif-
ferent electron $\phi_{\bmod }$ and recoil $\phi . \ldots \ldots \ldots$ 
12.1 (top left) Propagation of the variation of each member of the CTEQ6.6 error eigenset to the fitted value of the $W$ boson mass using the transverse mass distribution of pseudo-experiments simulated with RESBOS. (top right) PDF-induced correlation between the $W$ mass and the $u / d$ PDF as a function of the parton momentum fraction for three different mass scales. (bottom) Variation of the $u / d$ PDF in each member of the CTEQ6.6 error

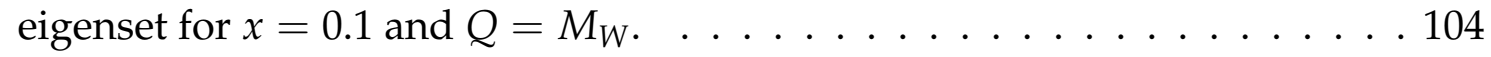

12.2 (top left) Propagation of the variation of each member of the CT10 error eigenset to the fitted value of the $W$ boson mass using the transverse mass distribution of pseudo-experiments simulated with RESBOS. (top right) PDF-induced correlation between the $W$ mass and the $u / d$ PDF as a function of the parton momentum fraction for three different mass scales. (bottom) Variation of the $u / d$ PDF in each member of the CT10 error eigenset for $x=0.1$ and $Q=M_{W} \ldots \ldots \ldots \ldots \ldots \ldots$

12.3 (top left) Propagation of the variation of each member of the CT10w error eigenset to the fitted value of the $W$ boson mass using the transverse mass distribution of pseudo-experiments simulated with RESBOS. (top right) PDF-induced correlation between the $W$ mass and the $u / d$ PDF as a function of the parton momentum fraction for three different mass scales. (bottom) Variation of the $u / d$ PDF in each member of the CT10w error eigenset for $x=0.1$ and $Q=M_{W} . \ldots \ldots \ldots \ldots$. . . . . . . . . . . . . . . . . . . . .

12.4 Propagation of the variation in each member of the CTEQ6.6 error eigenset to the fitted value of the $W$ boson mass using the transverse mass observable. On the left, the propagation with central electrons $\left(\left|\eta_{\text {det }}\right|<1.05\right)$ only. On the right, the same propagation but including a restricted region of the forward calorimeter $\left(1.5<\left|\eta_{\text {det }}\right|<2.5\right)$. The propagation was done with events generated by RESBOS and simulated with the $W$ mass fast MC. The total uncertainty is the sum in quadrature of each variation.. . . . . . . . . 107

12.5 Propagation of the variation in each member of the CTEQ6.6 error eigenset to the fitted value of the $W$ boson mass using the electron transverse momentum observable. On the left, the propagation with central electrons $\left(\left|\eta_{\text {det }}\right|<1.05\right)$ only. On the right, the same propagation but including a restricted region of the forward calorimeter $\left(1.5<\left|\eta_{\text {det }}\right|<2.5\right)$. The propagation was done with events generated by RESBOS and simulated with the $W$ mass fast MC. The total uncertainty is the sum in quadrature of each

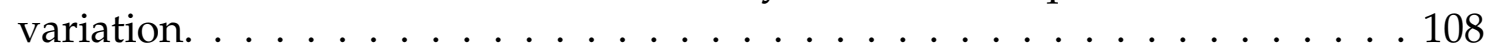


12.6 Propagation of the variation in each member of the CTEQ6.6 error eigenset to the fitted value of the $W$ boson mass using the missing transverse energy observable. On the left, the propagation with central electrons $\left(\left|\eta_{\text {det }}\right|<\right.$ 1.05) only. On the right, the same propagation but including a restricted region of the forward calorimeter $\left(1.5<\left|\eta_{\text {det }}\right|<2.5\right)$. The propagation was done with events generated by RESBOS and simulated with the $W$ mass fast MC. The total uncertainty is the sum in quadrature of each variation. . . . . . 108

12.7 Dependence of the PDF uncertainty on the hadronic recoil relative response and resolution. The PDF uncertainty is propagated using $m_{T}$ fits of RESBOS pseudo-experiments simulated with the fast MC and the CTEQ6.6 error set. The recoil transverse momentum vector is simulated as $\vec{u}_{T}=-\alpha \vec{p}_{T}^{W}+$ $\beta^{\prime} \hat{x}+\beta^{\prime \prime} \hat{y}$ where $\beta^{\prime}$ and $\beta^{\prime \prime}$ are independent random variables normally distributed with common width $\beta$. . . . . . . . . . . . . . 109

13.1 (top) Average shower for a $45 \mathrm{GeV}$ electron in the $\mathrm{CC}$ with $\eta=0$ and $\eta=1$. (bottom) Average shower for a $100 \mathrm{GeV}$ electron in the EC with $\eta=2$. The simulations use the GFlash parametrization and represent the average over 1000 simulated EM showers. . . . . . . . . . . . . . . . . . 114

13.2 The mean electron energy (thick) versus $\eta$ for electrons from $Z$ (red dashes) and $W$ (black solid line) events in data. The thin lines indicate the standard deviations of the energy distributions versus $\eta$. . . . . . . . . . . 115

13.3 Comparison between the parametrization of the bremsstrahlung cross section present in GEANT (blue points) and the first principle calculation by Seltzer and Berger used for this calibration (green points). . . . . . . . . 116

13.4 Geometry of the D0 GEANT model around the central and End Calorimeter cryostats. The red hatched areas are logical mother volumes. The blue solid areas are the cryostat walls and the internal support structure of the rohacell excluder. The pink hatched areas represent a series of other dead materials that, although important for the overall material budget, will have smaller importance to the strategy of this calibration (solenoid, CPS and FPS absorbers). The green solid area represents the cylinder that was added in the CC calibration. Finally, the cyan hatched areas are the sensitive elements of the forward calorimeter. . . . . . . . . . . . . . 117

13.5 Ratio of the mean of the EM energy fraction distribution for data and full MC. The full MC is simulated with the settings described in Sec. 13.2. The average value and $\chi^{2}$ around the mean are calculated in the range $1.5<$

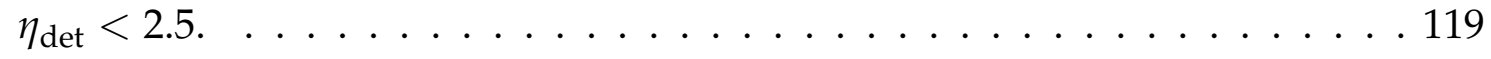


13.6 Best fit for north (top panels) and south (bottom panels) cryostats that minimize the test statistic 13.10 . The dash-dotted green lines represent the average $\eta_{\text {det }}$ from $Z \rightarrow$ ee data events in each of the $12 \eta_{\text {det }}$ bins with boundaries $(1.5,1.55,1.6,1.65,1.7,1.8,1.9,2.0,2.1,2.2,2.3,2.4,2.5)$ used to calculate the value of $\chi^{2}$. The vertical and horizontal axis in the left panels are absolute coordinates of the volume in the D0 geometry model, ie, relative to the center of the tracking system. The central panels show the energy fraction distribution after the minimization for EM1 (red), EM2(green), EM3 (blue) and EM4 (yellow) layers. The right panels show the amount of dead material (in radiation lengths of stainless steel) added or subtracted for each $\eta_{\text {det }}$ bin. The shaded areas depict the uncertainty due to the dead material, whose procedure is explained in 13.4.1 . . . . . . . . . . . . . 121

13.7 EM energy fractions for layers EM1, EM2 and EM3 after adding a disk with constant projective thickness in front of the south calorimeter. The values $\chi^{2}$ in the vertical axis are with respect to unity. The bottom right pad shows a summary of how much extra material would be needed to bring the EMLF ratio to one for each layer. In this panel, the uncertainties have been multiplied by 10 , for easier visualization. Similar results are observed for the north calorimeter. . . . . . . . . . . . . . . . 123

13.8 Comparison between the full d0gstar simulation and the parametrized model prediction in the south EC for EM1 (top left), EM2 (top right), EM3 (bottom left) and EM4 (bottom right) layers. Both simulations are done with the same 750k ResBos CC-EC events at the generator level. $\quad$. . . . . . 125

13.9 Comparison between the full d0gstar simulation and the parametrized model prediction in the south EC for EM1 (top left), EM2 (top right), EM3 (bottom left) and EM4 (bottom right) layers. Both simulations are done with the same 750k RESBOS CC-EC events at the generator level. $\quad$. . . . . . 126

13.10Determination of the correction to the material model in different $\phi$ bins for the north (left) and south (right) End Calorimeters. . . . . . . . . . . . . . 127

13.11Dependence of the layer weights correction with the instantaneous luminosity. The consistency among luminosity bins shows that the model for the luminosity dependence of the gains in d0s im works sufficiently well for this calibration. . . . . . . . . . . . . . . . . . 127

13.12Absolute calibration for Run Ilb1. The red thin error bars are statistical and the this blue errors bars are from the propagation of the material uncertainty through the energy loss function and through the layer weights

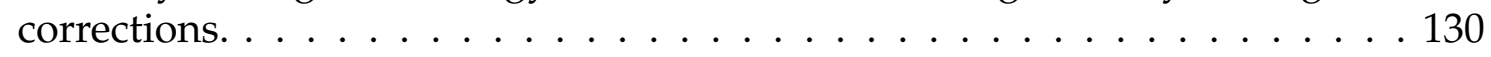

13.13Absolute calibration for Run IIb2. The red thin error bars are statistical and the this blue errors bars are from the propagation of the material uncertainty through the energy loss function and through the layer weights

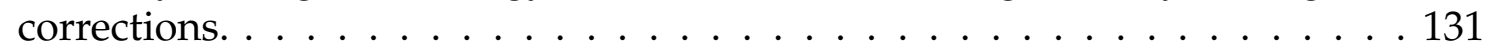


13.14Invariant mass distribution of $Z \rightarrow e e$ events before and after the determination of the in calibration constants. The left (right) panel shows the distribution for Run IIb1 (Run IIb2) candidates. . . . . . . . . . . . . . . . . . 134

13.15Propagation of uncertainty from the dead material determination using $Z \rightarrow$ ee events to the fitted $W$ boson mass using the transverse mass distribution. The left panel considers a measurement with forward electrons $1.5<\left|\eta_{\text {det }}\right|<2.5$ only, while the right panel includes central electrons

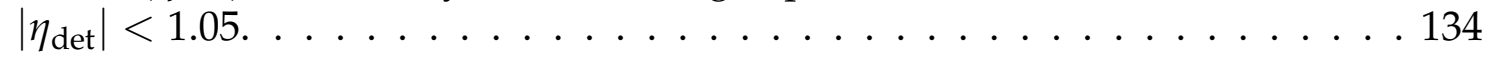

13.16Propagation of uncertainty from the dead material determination using $Z \rightarrow$ ee events to the fitted $W$ boson mass using the electron transverse momentum distribution. The left panel considers a measurement with forward electrons $1.5<\left|\eta_{\text {det }}\right|<2.5$ only, while the right panel includes cen-

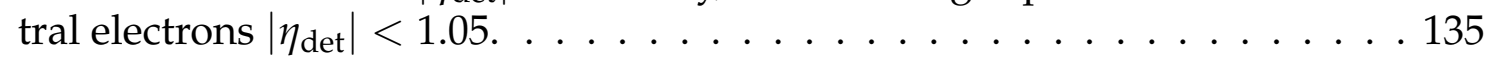

13.17Propagation of uncertainty from the dead material determination using $Z \rightarrow e e$ events to the fitted $W$ boson mass using the missing transverse energy distribution. The left panel considers a measurement with forward electrons $1.5<\left|\eta_{\text {det }}\right|<2.5$ only, while the right panel includes central

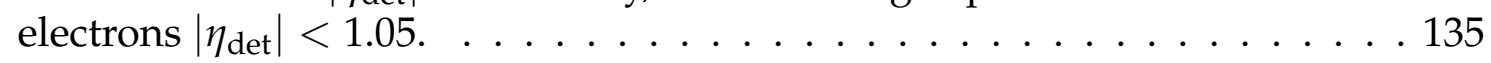

14.1 Efficiency of detection of an electron in the presence of a showered photon as a function of the fraction of the momentum carried by the photon in bins of $\Delta R$ between the electron and the photon. . . . . . . . . . . . 138

14.2 Fraction of the photon energy that does not contribute to the electron cluster reconstructed energy as a function of the fraction of the momentum carried by the photon in bins of $\Delta R$ between the electron and the photon. $\quad .139$

14.3 Comparison between the photon energy fraction (left) and $\Delta R$ (right) between the electron and photon for reconstructed electrons in full MC and fast MC, where the fast MC uses the parametrized model described here. . . 140

14.4 Comparison between the photon energy loss in reconstructed electrons with FSR in fast and full MC. . . . . . . . . . . . . . . . . . . 140

14.5 Example of a reconstructed full MC electron shower in the EC, after ZB subtraction. The blue bars represent the energy inside the electron cluster. The red is the leaked electron energy into the recoil system. We call attention to how irregular a EC cluster can be. . . . . . . . . . . . . . . . 141

14.6 Leakage model for both central electrons, dashed line on the left and open makers on the right, and forward electrons, full line on the left and full markers on the right. (left) The probability of an electron produced at angle $\eta$ to leak part of its transverse momentum. In the EC, pratically all EM clusters have some leakage. (right) Average transverse momentum fraction leaked when it happens. The model is shown separately for the case with no showered electron and for the case with one showered electron closer than $\Delta R=0.3$, and integrated over SET. . . . . . . . . . . . . . . . 142 
14.7 Average energy deposited in rotated cluster, in bins of $\left|\eta_{\text {det }}\right|$, measured in a $W \rightarrow e v$ full MC sample. The large tower of energy around $\Delta(i \phi)=0$ is the electron cluster and the small deposit of energy opposite to it is the hard recoil of longitudinally boosted $W$ bosons, which are kinematically correlated to the electron. The region $10<|\Delta(i \phi)|<20$ is free of both contamination and used for the average. . . . . . . . . . . . . . 143

14.8 Average transverse hadronic energy in rotated clusters as a function of $\left|\eta_{\text {det }}\right|$, SET, instantaneous luminosity $\mathcal{L}$ and $u_{\|}$. We use $W \rightarrow e v$ events, and the average is done on rotated clusters around the same $i \eta$ ring the electron cluster is centered on. We remove, from the average, clusters with electron and hard recoil contamination. The strong SET dependence shows the importance of the soft recoil for the underlying energy flow. We consider both SET and $\mathcal{L}$ dependence simultaneously to describe effects related to the calorimeter readout, such as the BLS system 2.4 .4 . The complete model is a function of the four variables shown here. . . . . . . . . . . . . . . . . 144

14.9 Probability distribution for the transverse hadronic energy in rotated clusters as a function of SET, showing the dependence of the noise fluctuations on this variable. . . . . . . . . . . . . . . . . . 145

14.10The electron cluster $\Delta E_{T}$, in $\mathrm{GeV}$, as a function of detector $\eta$ (left), SET (center) and instantaneous luminosity (right). The red markers shows the closure test with a toy fast MC which uses the parametrized model described in this section. . . . . . . . . . . . . . . . . 146

14.11Probability of an out-of-cluster FSR photon to reach the calorimeter and leave a visible deposit of energy as a function of the photon energy (left), $\eta$ (center) and event SET (right). . . . . . . . . . . . . . . . . . 147

14.12The EC calorimeter response to soft photons as a function of the photon pseudo-rapidity $\eta$ and event ZB SET. . . . . . . . . . . . . . . . . . . . . . 147

14.13Comparision between the fraction of the photon energy deposited in the recoil system as measured in full MC (red dots) and in the toy fast MC (blue dots) using the model described in this Section. . . . . . . . . . . . 148

14.14 (left) Projected relative response $\left(u_{T} \cdot q_{T}\right) /\left(q_{T} \cdot q_{T}\right)$ of the recoil transverse vector $u_{T}$ with respect to the boson generator transverse momentum $q_{T}$ as a function of $q_{T}$. (right) SET distribution from the hard recoil only. The blue histograms were generated from full MC samples without overlay while the red histograms are generated form histograms with ZB overlay in which the underlying energy was removed event by event. Once again, the importance of the underlying energy in bringing the cells closer to the noise suppression threshold is clearly seen in the amount of visible soft energy in the detector. . . . . . . . . . . . . . . . . 149 
14.15Hard recoil parametrization with new $Z \rightarrow v v$ full MC. The left panel shows the relative response and direction for relatively low $Z q_{T}$ when the recoil is dominated by soft gluon emission. In this case the angular correlation is very soft. $\ldots \ldots \ldots$. . . . . . . . . . . . . . 150

14.16Distribution of the reconstructed energy, after energy loss correction, for monochromatic electrons with true energy $55 \mathrm{GeV}$ (left) and $85 \mathrm{GeV}$ (right). The model used to describe the electron sampling resolution is the convolution of a Landau distribution with a gaussian. The Landau describes the fluctuations in the radiative energy loss, while the gaussian describes the sampling fluctuations. $\ldots \ldots \ldots \ldots 151$

14.17(left) Gaussian width in the energy distribution for single monochromatic electrons with energy $55 \mathrm{GeV}$ (red markers) and $85 \mathrm{GeV}$ (blue markers). As expected, the large amount of dead material introduces strong angular dependence. Due to the non-uniformity of the material budget in the forward region, the distribution is not smooth as for the CC. (right) Energy dependence for electrons reconstructed with $2.0<\eta_{\text {det }}<2.1$. The dependence is fitted to a polynomial in $1 / \sqrt{E}$ with an important contribution from the

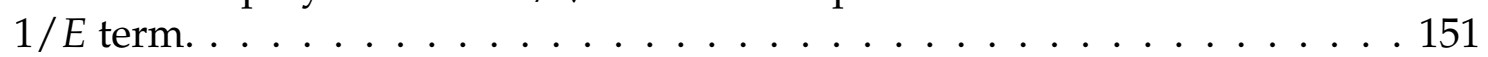

14.18Distribution of the electron fractional energy fluctuation for both full MC (blue markers) and fast MC (red markers). This comparison uses a sample of single electrons in which all the energy fluctuation is due to the sampling resolution and, therefore, represents a good test for our model. . . . . . . . . 152

14.19We compare the RMS of the electron fractional energy sampling fluctuation in both full MC (blue markers) and fast MC (red markers) for events with different $\eta$ (left) and energy (right) regimes. . . . . . . . . . . . . . . . . . 152

14.20Probability of a highly electromagnetic jet in the EC to fake an electron with a associated track as a function of the spatial track matching probability

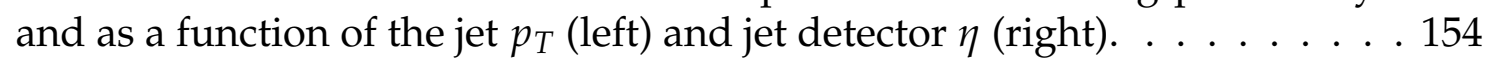

14.21 Relative probability of a highly electromagnetic jet in the EC to fake an electron with a associated track as a function of the minimum track $p_{T}$ criterion and as a function of the jet $p_{T}$ (left) and jet detector $\eta$ (right). . . . . . . . 155

14.22Relative probability of a highly electromagnetic jet in the north (left) and south (right) ECs to fake an electron with the same track criteria as in the central calorimeter analysis, as a function of the jet $p_{T}$ and jet detector $\eta . .155$

14.23Parametrization of the fake rate probability in the north EC as a function of the jet $p_{T}$ and $\eta_{\text {det }}$. The left plot shows a graph of the parametrized function. The right plot shows the signed $\chi$ distribution with respect to the parent distribution. . . . . . . . . . . . . . . . 156

14.24Parametrization of the fake rate probability in the south EC as a function of the jet $p_{T}$ and $\eta_{\text {det }}$. The left plot shows a graph of the parametrized function. The right plot shows the signed $\chi$ distribution with respect to the parent distribution. . . . . . . . . . . . . . . . 157 
14.25EMID efficiency as a function of the electron $p_{T}$ and $\eta_{\text {det }}$. The efficiency is derived using the tag and probe method (left) and the truth method (right). 158

14.26Projection of the EMID efficiency over electron $p_{T}$ (left) and $\eta_{\text {det }}$ (right). The bottom plots show the ratio between the efficiency derived by the truth method to the tag and probe method. . . . . . . . . . . . . . . 159

14.27Loose track match efficiency as a function of the electron $p_{T}$ and $\eta_{\text {det }}$. The efficiency is derived using the tag and probe method (left) and the truth

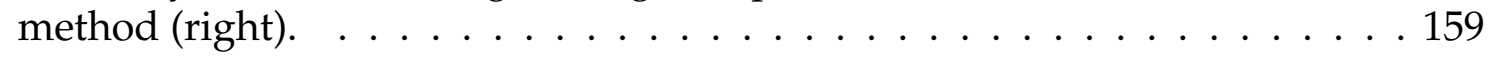

14.28Projection of the loose efficiency on electron $\eta$ (left) and $z_{V}$ (right). The bottom plots show the ratio between the efficiency derived by the truth method to the tag and probe method. . . . . . . . . . . . . . 160

14.29Loose track match efficiency as a function of the electron $\eta$ and $z_{V}$. The efficiency is derived using the tag and probe method (left) and the truth method (right). The EC electrons are required to satisfy the fiducial criterion $z_{V} \times \eta<0$ when $\left|z_{V}\right|>30 \mathrm{~cm} . \ldots \ldots \ldots 16 \ldots \ldots \ldots$. . . . . . . . . . .

14.30Projection of the loose efficiency on electron $\eta$ (left) and $z_{V}$ (right). The bottom plots show the ratio between the efficiency derived by the truth method to the tag and probe method. The EC electrons are required to satisfy the fiducial criterion $z_{V} \times \eta<0$ when $\left|z_{V}\right|>30 \mathrm{~cm} . \ldots 161$

14.31 Tight track match efficiency as a function of the electron $\eta$ and $z_{V}$. The efficiency is derived using the tag and probe method (left) and the truth method (right). The EC electrons are required to satisfy the fiducial criterion $z_{V} \times \eta<0$ when $\left|z_{V}\right|>30 \mathrm{~cm} . \ldots \ldots \ldots 2$

14.32Comparison between fast and full MC transverse mass $m_{T}$ (top left), electron transverse momentum $p_{T}^{e}$ (top right), and missing transverse energy $\mathbb{E}_{T}$ (bottom) distributions from $W \rightarrow$ ev events. The fast MC has all the models described in this section but lacks any tune of the electron response and resolution, electron efficiency and hadronic response and resolution using $Z \rightarrow e e$ events. The efficiency model is incomplete, as can be clearly seen in low energy part of the histograms. . . . . . . . . . . . . . 163

15.1 Cover of the Physical Review Letters issue in which the latest D0 and CDF measurements of the $W$ boson mass have been published. . . . . . . . . . 166

A.1 Feynman diagram for the muon decay in the Standard Model. We consider only loop corrections to the propagator, ignoring sub-leading corrections from the vertex renormalization as well as box-like diagrams. On the last diagram, $B$ represents either a vector boson $Z, \gamma$ or the Higgs boson $H . \mid$. . . 183

B.1 (left) Comparison between data and fast MC for the $p_{T}^{e}$ distribution of the electrons from $Z \rightarrow e e$. (right) the corresponding $\chi$ value per bin. . . . . . . 186

B.2 (left) Comparison between data and fast MC for the $p_{T}^{Z}$ distribution of $Z \rightarrow$ ee events. (right) the corresponding $\chi$ value per bin. $\ldots . . . . . .187$ 
B.3 (left) Comparison between data and fast MC for the $u_{T}$ distribution of $Z \rightarrow$ ee events. (right) the corresponding $\chi$ value per bin. $\ldots$. . . . . . . . 187

B.4 (left) Comparison between data and fast MC for the $u_{\|}$distribution of electrons in $Z \rightarrow e e$ events. (right) the corresponding $\chi$ value per bin. . . . . . . 187

B.5 (left) Comparison between data and fast MC for the $u_{\perp}$ distribution of electrons in $Z \rightarrow e e$ events. (right) the corresponding $\chi$ value per bin... .188

B.6 (left) Comparison between data and fast MC for the $\mathbb{E}_{T}$ distribution of in $Z \rightarrow e e$ events. (right) the corresponding $\chi$ value per bin. $\ldots . . . . .188$

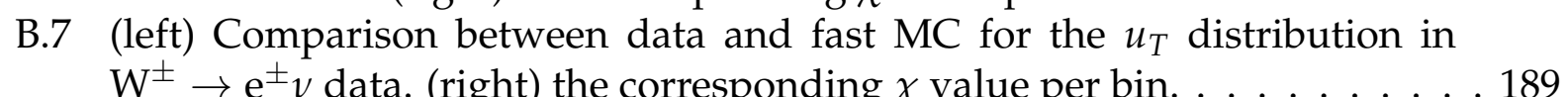

B.8 (left) Comparison between data and fast MC for the $u_{\|}$distribution in $\mathrm{W}^{ \pm} \rightarrow \mathrm{e}^{ \pm} v$ data. (right) the corresponding $\chi$ value per bin. $\ldots$. . . . . . . 189

B.9 (left) Comparison between data and fast MC for the $u_{\perp}$ distribution in $\mathrm{W}^{ \pm} \rightarrow \mathrm{e}^{ \pm} v$ data. (right) the corresponding $\chi$ value per bin. . . . . . . . . . . 190

B.10 (left) Comparison between data and fast MC for the physics $\eta$ distribution of electrons from $\mathrm{W}^{ \pm} \rightarrow \mathrm{e}^{ \pm} v$. (right) Comparison between data and fast MC for the detector $\eta$ distribution of electrons from $\left.W^{ \pm} \rightarrow \mathrm{e}^{ \pm} v.\right]$. . . . . . 190

B.11 (left) Comparison between data and fast MC for the instantaneous luminosity distribution of $\mathrm{W}^{ \pm} \rightarrow \mathrm{e}^{ \pm} v$ events. (right) Comparison between data and fast MC for the SET distribution of $\mathrm{W}^{ \pm} \rightarrow \mathrm{e}^{ \pm} v$ events. . . . . . . . . . . 190

C.1 Data and fullMC mean of the EMF distributions in bins of $\eta_{\text {det }}$ using the

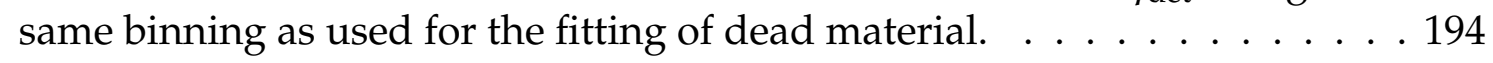

C.2 Parametrized model for the variation of the mean EM1 energy fraction as a function of the variation of the dead material. Since the model is built with independent full MC samples, the bins are statistically independent. . . . . 195

C.3 Parametrized model for the variation of the mean EM2 energy fraction as a function of the variation of the dead material. Since the model is built with independent full MC samples, the bins are statistically independent. . . . . 196

C.4 Parametrized model for the variation of the mean EM3 energy fraction as a function of the variation of the dead material. Since the model is built with independent full MC samples, the bins are statistically independent. . . . . . 197

C.5 Parametrized model for the variation of the mean EM4 energy fraction as a function of the variation of the dead material. Since the model is built with independent full MC samples, the bins are statistically independent. . . . . 198

C.6 Parametrized model for the variation of the mean EM1 energy fraction as a function of the variation of the dead material. Since the model is built with independent full MC samples, the bins are statistically independent. . . . . . 199

C.7 Parametrized model for the variation of the mean EM2 energy fraction as a function of the variation of the dead material. Since the model is built with independent full MC samples, the bins are statistically independent. . . . . 200 
C.8 Parametrized model for the variation of the mean EM3 energy fraction as a function of the variation of the dead material. Since the model is built with independent full MC samples, the bins are statistically independent. . . . . 201

C.9 Parametrized model for the variation of the mean EM4 energy fraction as a function of the variation of the dead material. Since the model is built with independent full MC samples, the bins are statistically independent. . . . . 202

C.10 South end-cap, eigenset 1 (two upper panels) and 2 (two bottom panels) with variations corresponding to $\pm 1 \sigma$ where $\sigma$ is the square root of the corresponding eigenvalue. The eigenvectors are ordered by decreasing eigen-

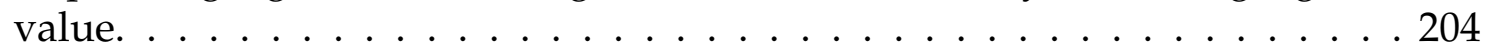

C.11 South end-cap, eigenset 3 (two upper panels) and 4 (two bottom panels) with variations corresponding to $\pm 1 \sigma$ where $\sigma$ is the square root of the corresponding eigenvalue. The eigenvectors are ordered by decreasing eigen-

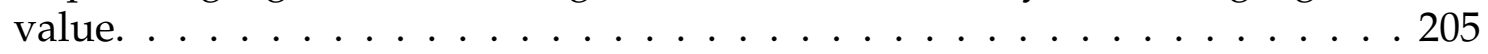

C.12 South end-cap, eigenset 5 (two upper panels) and 6 (two bottom panels) with variations corresponding to $\pm 1 \sigma$ where $\sigma$ is the square root of the corresponding eigenvalue. The eigenvectors are ordered by decreasing eigen-

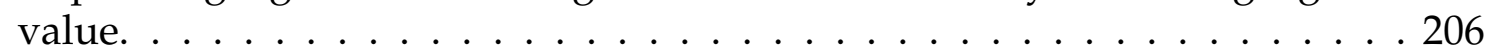

C.13 South end-cap, eigenset 7 (two upper panels) and 8 (two bottom panels) with variations corresponding to $\pm 1 \sigma$ where $\sigma$ is the square root of the corresponding eigenvalue. The eigenvectors are ordered by decreasing eigenvalue. . . . . . . . . . . . . . . . . . . 207

C.14 South end-cap, eigenset 9 (two upper panels) and 10 (two bottom panels) with variations corresponding to $\pm 1 \sigma$ where $\sigma$ is the square root of the corresponding eigenvalue. The eigenvectors are ordered by decreasing eigen-

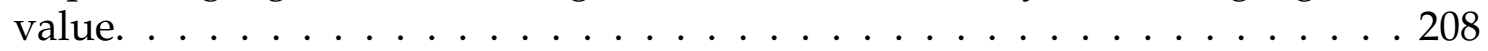

C.15 North end-cap, eigenset 1 (two upper panels) and 2 (two bottom panels) with variations corresponding to $\pm 1 \sigma$ where $\sigma$ is the square root of the corresponding eigenvalue. The eigenvectors are ordered by decreasing eigen-

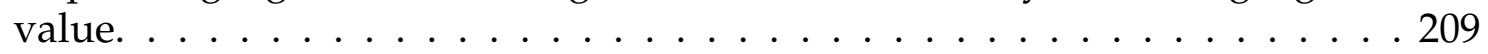

C.16 North end-cap, eigenset 3 (two upper panels) and 4 (two bottom panels) with variations corresponding to $\pm 1 \sigma$ where $\sigma$ is the square root of the corresponding eigenvalue. The eigenvectors are ordered by decreasing eigen-

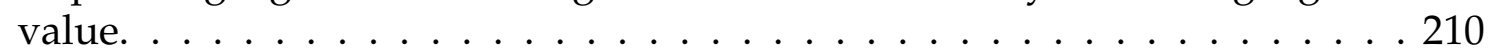

C.17 North end-cap, eigenset 5 (two upper panels) and 6 (two bottom panels) with variations corresponding to $\pm 1 \sigma$ where $\sigma$ is the square root of the corresponding eigenvalue. The eigenvectors are ordered by decreasing eigen-

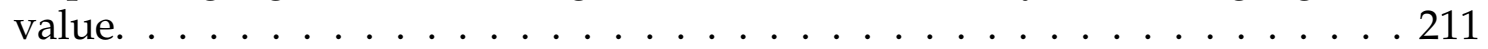

C.18 North end-cap, eigenset 7 (two upper panels) and 8 (two bottom panels) with variations corresponding to $\pm 1 \sigma$ where $\sigma$ is the square root of the corresponding eigenvalue. The eigenvectors are ordered by decreasing eigen-

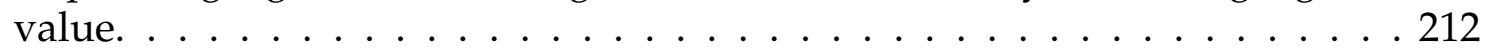


C.19 North end-cap, eigenset 9 (two upper panels) and 10 (two bottom panels) with variations corresponding to $\pm 1 \sigma$ where $\sigma$ is the square root of the corresponding eigenvalue. The eigenvectors are ordered by decreasing

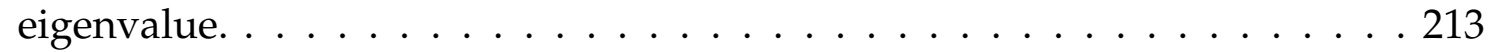

C.20 North End Calorimeter energy loss function fit for using the D0 nominal geometry model. . . . . . . . . . . . . . . . . 214

C.21 South End Calorimeter energy loss function fit for using the D0 nominal geometry model. $\ldots \ldots \ldots \ldots . \ldots \ldots . \ldots \ldots \ldots$

C.22 North End Calorimeter energy loss function fit for using the ad hoc ECdevelB1

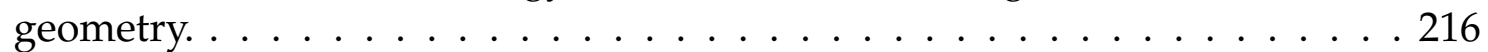

C.23 South End Calorimeter energy loss function fit for using the ad hoc ECdevelB1

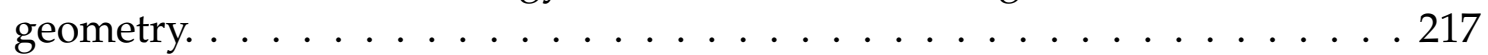

C.24 North End Calorimeter energy loss function fit for using the ad hoc ECdevelB2

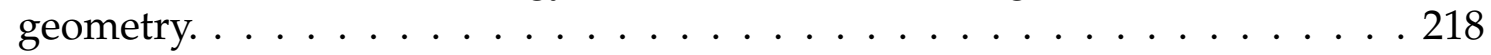

C.25 South End Calorimeter energy loss function fit for using the ad hoc ECdevelB2

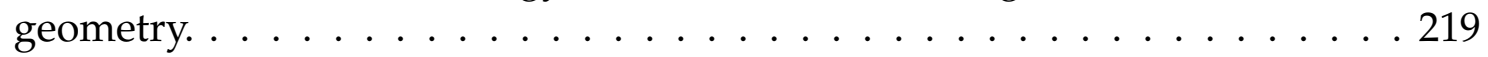

C.26 North End Calorimeter energy loss function fit for using the ad hoc ECdevelB3

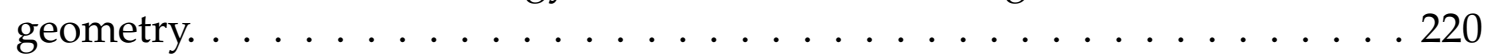

C.27 South End Calorimeter energy loss function fit for using the ad hoc ECdevelB3

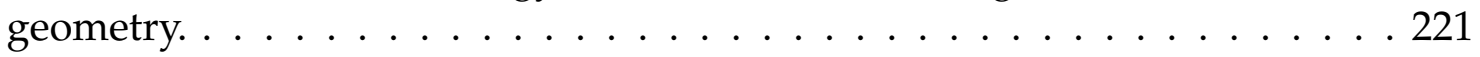

C.28 North end-cap, energy loss functions for the first six $\eta_{\text {det }}$ bins $\left(1.5<\eta_{\text {det }}<\right.$ 1.9). The shaded area represents the 3 standard deviations propagated from the material tune. . . . . . . . . . . . . . . . . . 222

C.29 North end-cap, energy loss functions for the last six $\eta_{\text {det }}$ bins $\left(1.9<\eta_{\text {det }}<\right.$ 2.5). The shaded area represents the 3 standard deviations propagated from the material tune. . . . . . . . . . . . . . . . 223

C.30 South end-cap, energy loss functions for the last six $\eta_{\text {det }}$ bins $\left(1.9<\eta_{\text {det }}<\right.$ 2.5). The shaded area represents the 3 standard deviations propagated from the material tune. . . . . . . . . . . . . . . . . 224

C.31 South end-cap, energy loss functions for the last six $\eta_{\text {det }}$ bins $\left(1.9<\eta_{\text {det }}<\right.$ 2.5). The shaded area represents the 3 standard deviations propagated from the material tune. . . . . . . . . . . . . . . . 225 


\section{List of Tables}

2.1 Pitch, inner and outer radii of the barrel and disks in the D0 Silicon Microstrip Detector. . . . . . . . . . . . . . . . . 15

2.2 Thickness of the different layers and sections of the D0 liquid argon calorime-

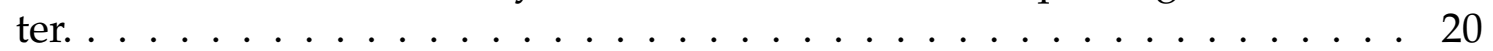

2.3 Typical pedestal RMS values for some layers of the D0 calorimeter. . . . . . 26

$5.1 \quad$ Definition of bins in electron $|\eta| . \ldots \ldots$. . . . . . . . . . . . . 49

5.2 Definition of $\eta$ categories for $Z \rightarrow e e$ events. . . . . . . . . . . . . . . . 49

6.1 Event generators for $W$ boson and $Z$ boson processes used in this analysis. PYTHIA is used for the full MC closure test and for estimating PDF uncertainties. WGRAD and ZGRAD are used only for estimation of QED theory

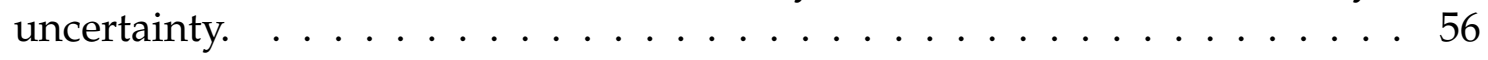

$7.1 \quad$ Results of the fits for electron energy scale and offset to the collider data. . 76

9.1 Results from the fits to data. The uncertainty is only that from the data candidate $W$ boson sample statistics. The $\chi^{2} /$ d.o.f. values are computed

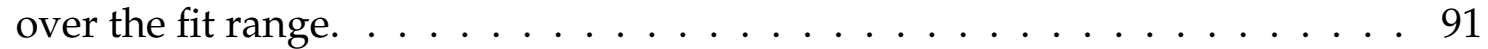

9.2 Systematic uncertainties on the $W$ mass results. The dominant systematic uncertainty comes from the electron energy scale, and this is determined by the statistical power of the $Z$ event sample. . . . . . . . . . . . . . 92

10.1 Correlation matrices for the $W$ boson statistical, recoil scale and resolution and the PDF uncertainties. The correlation matrix for each of the other uncertainties is assumed to have a $100 \%$ correlation among the $m_{T}, p_{T}^{e}$ and $E_{T}$ results. $\ldots \ldots \ldots \ldots \ldots \ldots$

12.1 Comparison between the PDF uncertainty propagated to the fitted $W$ boson mass using the transverse mass distribution from the fast MC tuned for the measurement with $4.3 \mathrm{fb}^{-1} \ldots \ldots \ldots$. . . . . . . . . . . . 103

12.2 PDF uncertainty in the D0 $W$ boson mass measurement. The uncertainty is propagated from the CTEQ6.6 error set using pseudo-experiments generated with ResBos and simulated with the $W$ mass fast MC. . . . . . . . . . . 107 
13.1 Material in front of the first calorimeter layer as seen by an electron traveling from the interaction point to the CC at normal incidence. . . . . . . . . 112

13.2 Layer weights applied during data reconstruction in the D0 calorimeter. The values are further multiplied by a global ADC to GeV conversion factor of $3.086 \times 10^{-4} \mathrm{GeV} /$ count. . . . . . . . . . . . . . 113

13.3 Best modification of the internal cryostat wall (CC+1 volume in d0gstar). These modifications are implemented in geometry p20_ECdevelB6 in package d0AltGeom. The coordinates are given with respect to the center of the calorimeter coordinate system and additional shifts are necessary to translate them to the center of the tracking system which is used as reference for the D0 global coordinate system. The additional shifts used in this study are those present in the D0 GEANT geomtry model. . . . . . . . . . . . . . 122

13.4 Multiplicative correction to the reconstruction default layer weights. They are determined as the inverse of the mean energy fraction across the region $1.5<\eta_{\text {det }}<2.5$ after the improvement in the material model.. . . . . . . . 124

13.5 Energy loss functions using the best fit for the material tune. The function used is $f_{\mathrm{EL}}\left(E_{\text {reco }}\right)=p_{0}+p_{1} \log \left(E_{\text {reco }}\right)+p_{2} \log ^{2}\left(E_{\text {reco }}\right)+p_{3} \log ^{3}\left(E_{\text {reco }}\right)$ with $E_{\text {reco }}$ in $\mathrm{GeV}$. The fit uncertainties are negligible compared to the propagated uncertainties from the dead material tune. . . . . . . . . . . . . 129

13.6 Calibration constants for the north in ring using the improved energy loss functions and layer weights corrections. The first uncertainty is statistical, the second due to the uncertainty on the material tune in the forward direction. The determination is done separately for Run IIb1 and Run IIb2. . 132

13.7 Calibration constants for south in ring using the improved energy loss functions and layer weights corrections. The first uncertainty is statistical, the second due to the uncertainty on the material tune in the forward direction. The determination is done separately for Run IIb1 and Run IIlb2. . 133

14.1 Parametrization of the fake rate probability in the north and south ECs. . . . 156

C.1 Polycone model for the D0 end-cap internal cryostat wall (volume CC+1 in d0gstar). The number of points was increased by simple interpolation to provide additional flexibility in the fit. The coordinates are given with respect to the center of the calorimetry system and, in d0gstar, additional shifts are applied to translate them to the center of tracker system, which is

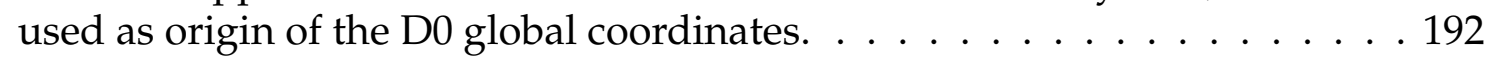

C.2 The three ad hoc variations built by varying $z$ and $r_{\max }$ in the polycone model of the internal cryostat wall. . . . . . . . . . . . . . 193 


\section{Acknowledgments}

It was a long journey that was not travelled through the easiest path and that was not travelled alone. I owe a lot to those who taught me, who supported me, who believed in my dreams and who, ultimately, made this Dissertation possible.

Firstly, I would like to thank my advisor and friend, Prof. John Hobbs. You were able to see my weaknesses and made your best to make me rise above them. You taught me how to do research and how to focus on doing things that no one has done before. Thanks for continously supporting me through easy and hard times. I must also thank the experimental high-energy group at Stony Brook University and at the D0 experiment. In particular, I would like to thank Dean Schamberger and Jan Stark for teaching me so much and for always believing in me. Secondly, I would like to thank my friends Jan Gutt and Alexander Stoffers. You were more than friends, you were brothers to me. Finally, my most heartfelt thanks to my mother Celeste and my sister Cristina, for having never doubted my craziest dreams. And to Luciana, my love, who has always been my side when I needed and who is the bravest and kindest person I have ever met.

The $W$ mass measurements presented here are not the work of a single person. My main individual contributions to the measurement with Central Electron were the determination of the Hadronic Recoil Response, as described in Sec. 7.4 the study and determination of the impact of the Parton Distribution Functions in the $W$ mass measurement, as described in Secs. 6.3 and 12; the calculation and propagation of all uncertainties that were reevaluated for the measurement with $4.3 \mathrm{fb}^{-1}$, but the one associated to the efficiency model, as briefly described throughtout Part II of this Dissertation; and the final combination of measurements, as described in Sec. 10. I had also individual contributions in the combination procedure with other $W$ boson mass measurements to determine the new world average value and uncertainty, as it is very briefly described in Sec. 10. All the work in Part III, that describes the initial efforts for a future measurement with Forward Electrons is my individual contribution. I acknowledge the incommensurable help of all the Run IIb 12 D0 W mass working group, whose list of names is too long to write here, without whom I would not be able to complete my individual contributions and who were also the main authors of the other parts of the measurement described here. I must thank the Run IIa D0 W mass working group for having paved an important track that now allows us to understand the D0 calorimeter in fine details and perform the type of precision measurement described here. I would also like to show my appreciation to the renewed Run IIb 34 D0 W mass working group, who will take the knowledge described here and perform the final D0 W measurements. Last, but not least, I would like to acknowledge the D0 Editorial Board that reviewed this work and whose careful evaluation of our methodology forced me to learn more about the detector and about the choices we made.

My work during the year prior to my arrival at Stony Brook University was supported by FAPESP, process number 06/591885. 


\section{Part I}

\section{Preliminaries}




\section{Chapter 1}

\section{The W Boson and its Mass in the Standard Model}

Since the observation of the Higgs boson by the ATLAS [2] and CMS [3] collaborations and measurement of its mass, the electroweak section of the Standard Model has become over-constrained and we can test its internal consistency without any unknown degree of freedom. This type of statistical analysis has been performed by many groups, including the GFitter collaboration [4], whose results we use here to underscore the importance of the measurement of the $W$ boson mass in understanding the electroweak sector of the Standard Model.

The electroweak sector of the Standard Model is a $S U(2) \times U(1)$ gauge theory with spontaneous symmetry breaking generated by the Higgs scalar field. Such theory has two gauge coupling constants, one for each gauge group, as well as the quadratic and quartic couplings in the Higgs lagrangian. The physical spectrum contains three massive eigenstates $W_{\mu}^{ \pm}, Z_{\mu}$ and one massless $A_{\mu}$ that can be expressed as function of the $S U(2)$ and $U(1)$ gauge fields $W_{\mu}^{a}, B_{\mu}$ :

$$
\begin{aligned}
W_{\mu}^{ \pm} & =\frac{1}{\sqrt{2}}\left(W_{\mu}^{1} \mp i W_{\mu}^{2}\right), \\
\left(\begin{array}{c}
Z_{\mu} \\
A_{\mu}
\end{array}\right) & =\left(\begin{array}{cc}
\cos \theta_{W} & \sin \theta_{W} \\
-\sin \theta_{W} & \cos \theta
\end{array}\right)\left(\begin{array}{c}
W_{\mu}^{3} \\
B_{\mu}
\end{array}\right),
\end{aligned}
$$

where $\theta_{W}$ is the weak angle defined as:

$$
\cos \theta_{W}=\frac{M_{W}}{M_{Z}},
$$

and the masses are defined as the real part of the poles of the propagators of each field. The mass eigenstates of the Higgs doublet are the physical Higgs field with mass $M_{H}$, the neutral and charged Goldstone bosons $\chi, \phi^{ \pm}$. The three physical masses together with the two coupling constants can be expressed as a function of the four original parameters in the electroweak lagrangian, which introduces a well defined relationship between them in the renormalization procedure. 
For global fits and consistency checks, it is convenient to use the Fermi constant $G_{F}$ in place of the $S U(2)$ gauge coupling constant. The Fermi constant is the coupling constant of the old Fermi four-fermion theory and in the Standard Model is defined through the expression for the muon decay time:

$$
\tau_{\mu}^{-1}=\frac{G_{F}^{2} m_{\mu}^{5}}{192 \pi^{3}} F\left(\frac{m_{e}^{2}}{m_{\mu}^{2}}\right)\left(1+\frac{3 m_{\mu}^{2}}{5 M_{W}^{2}}\right)\left(1+\Delta q_{\mathrm{QED}}\right)
$$

where $F(x)=1-8 x-12 x^{2} \ln x+8 x^{3}-x^{4}$ and $\Delta q_{\mathrm{QED}}$ encodes the pure QED corrections. The term $3 m_{\mu}^{2} / 5 M_{W}^{2}$ is not strictly from the Fermi theory since it comes from the $W$ propagator, but its numerical effect is negligible even considering the current precision with which the Fermi constant is determined [5].

The relationship between the electroweak parameters can then be derived by calculating the equivalent expression for the muon decay time in the Standard Model and comparing to Eq. 1.3. The result is usually cast in the following form:

$$
M_{W}^{2}\left(1-\frac{M_{W}^{2}}{M_{Z}^{2}}\right)=\frac{\pi \alpha}{\sqrt{2} G_{F}}(1+\Delta r)
$$

where $\alpha=e^{2} / 4 \pi$ is the fine structure constant. The higher-order corrections $\Delta q$ have been calculated to full two-loop order [6, 7].

\subsection{Electroweak Precision Observables}

Besides the five natural observables defined above, others less directly related to the constants in the lagrangian can be defined. Theoretically, those observables are no less fundamental than the physical masses and, experimentally, they can be determined with similar precision. There are an infinite number of such observables. In practice, only a few have been measured with enough precision to be relevant in a global fit. In Ref. [4] there is a comprehensive list of the observables usually used. Most of them are $Z$ pole observables in Drell-Yan events, such as the total cross-section into hadrons or forward-backward asymmetries in specific $Z$ decay channels. These observables have been measured by the LEP and SLD experiments [8,9] with a precision that cannot be reached in hadron collides experiments. There are four precision observables for which hadron collider experiments currently have the best determination: the $W$ boson mass $M_{W}$ and width $\Gamma_{W}$, the top quark mass $m_{T}$ and the Higgs boson mass $M_{H}$. We will focus on these.

From these four observables, the $W$ boson width and the Higgs boson mass have limited interest. The SM prediction for the $W$ boson width is so much more precise than the measurement that any additional measurement will hardly change the status of the fit. The Higgs mass, on the contrary, is extremely relevant for the fit but is already so much better measured than its prediction $\bigsqcup^{1}$ that it could just be fixed to the measured value.

\footnotetext{
${ }^{1}$ In electroweak global fits, the prediction for $M_{H}$ is obtained as the value that maximizes likelihood
} 
The $W$ boson mass and the quark top mass are the two observable for which slight improvements in the precision with which they are measured can significantly change our knowledge about the consistency of the SM and the possibility of New Physics (NP).

In the GFitter fit, the observables that are left floating are the Higgs mass, the $Z$ boson mass, the contribution from the five lighter quarks to the evolution of the fine structure constant $\Delta \alpha^{(5)}$, the strong $S U(3)$ coupling constant $\alpha_{s}$ and the quark masses. Those, together with $G_{F}$, can be thought as their choice of independent variables. For the rest, their predictions are obtained from individual profile likelihood scans. After the precise measurement of the Higgs boson mass, for the first time in history, it is possible to determine the value of the $W$ boson mass with better precision than it has been directly measured. The GFitter prediction [4] is:

$$
\begin{aligned}
M_{W}^{\text {pred }} & =80.3593 \pm 0.0056_{m_{T}} \pm 0.0026_{M_{Z}} \pm 0.0018_{\Delta \alpha^{(5)}} \pm 0.0017_{\alpha_{s}} \pm 0.0002_{M_{H}} \pm 0.0040_{\text {theo }} \\
& =80.359 \pm 0.011 \mathrm{GeV} .
\end{aligned}
$$

where the subscripts indicate the source of the uncertainty in the likelihood scans. This has to be compared to $15 \mathrm{MeV}$ of uncertainty in the direct measurement after the most recent D0 measurement described in this Dissertation and the most recent CDF [10] measurement. It is expected that the prediction will become even more precise in the near future with improved determinations of the top quark mass from the Tevatron and LHC experiments. It is also conceivable to imagine improved determinations of $\Delta \alpha^{(5)}$ from high-intensity low-energy colliders [11] and from lattice calculations [12]. Therefore, for the $W$ mass to continue to be relevant, it is necessary to improve its measurement to the $10 \mathrm{MeV}$ level and this dissertation describes the efforts made at the D0 experiment toward this goal.

\subsection{W Boson Production at the Tevatron}

W bosons at the Tevatron are mainly produced via the partonic interaction (and its CPconjugated process):

$$
d\left(p_{d}\right)+\bar{u}\left(p_{u}\right) \rightarrow e^{-}\left(p_{e}\right)+\bar{v}\left(p_{v}\right) .
$$

The spin averaged invariant amplitude for this process is [13]:

$$
\left|\mathcal{M}\left(d \bar{u} \rightarrow e^{-} v\right)\right|^{2}=16\left(2 \sqrt{2} G_{F} M_{W}^{2}\right)^{2}\left|V_{u d}\right|^{2} \frac{\left(p_{u} \cdot p_{e}\right)^{2}}{\left(\left(p_{u}+p_{e}\right)^{2}-M_{W}^{2}\right)^{2}+M_{W}^{2} \Gamma_{W}^{2}},
$$

function without including the direct measurements performed at the LHC experiments. The value quoted from [4] is $94_{-22}^{+25} \mathrm{GeV}$ while the naïve combination, diregarding correlations, between the ATLAS [2] and CMS [3] direct measurements is $125.7 \pm 0.4 \mathrm{GeV}$. 
where $V_{u d}$ is the CKM matrix entry. If we define $\theta^{*}$ to be the $e^{+}\left(e^{-}\right)$polar angle of emission in the $W^{+}\left(W^{-}\right)$rest frame, measured with respect to the direction of the incident $\bar{p}$ $(p)$, then,

$$
\left(p_{u} \cdot p_{e}\right)^{2}=\frac{M_{W}^{4}}{16}\left(1+\cos \theta^{*}\right)^{2} \Rightarrow \cos \theta^{*}=\left(1-\frac{4 p_{T}^{e 2}}{M_{W}^{2}}\right)^{1 / 2}
$$

Thus, the angular distribution of the electron, when averaged between $W^{+}$and $W^{-}$, is:

$$
\frac{1}{\sigma} \frac{d \sigma}{d \cos \theta^{*}}=\frac{3}{8}\left(1+\cos ^{2} \theta^{*}\right)
$$

If we now use equation 1.8 , the electron transverse momentum distribution is given by:

$$
\frac{1}{\sigma} \frac{d \sigma}{d p_{T}^{e 2}}=\frac{3}{M_{W}^{2}}\left(1-\frac{4 p_{T}^{e 2}}{M_{W}^{2}}\right)^{-1 / 2}\left(1-\frac{2 p_{T}^{e 2}}{M_{W}^{2}}\right),
$$

which is strongly peaked at $p_{T}^{e}=M_{W} / 2$. This peak is known as Jacobian Peak and the basic idea of a $W$ boson mass measurement in a hadron collider is to accurately determine its position. The same peak is observed in the neutrino transverse mass distribution, which can be experimentally measured with the missing transverse energy, and in the transverse mass distribution, in which the transverse mass is defined as:

$$
m_{T}^{2}=2 p_{T}^{e} p_{T}^{v}\left(1-\cos \Delta \phi_{e v}\right)
$$

The transverse momentum distribution is smeared by the $W$ finite width, by final state QED radiation, by detector effects but, more importantly, by the $W$ boson transverse momentum, which is dominated by the emission of initial state QCD radiation. Figure 1.1 shows the effect of these smearing in the electron transverse momentum and transverse mass distributions

At the Tevatron, most $W$ bosons are produced with relatively little transverse momentum compared to its invariant mass, ie. $q_{T} \ll Q$. This simple observation complicates considerably the calculation of the $W$ transverse momentum distribution. Schematically, the NLO real cross section can be written as [14]:

$$
\frac{d \sigma^{R}}{d q_{T}^{2}}=\alpha_{S}\left(A \frac{\ln \left(Q^{2} / q_{T}^{2}\right)}{q_{T}^{2}}+B \frac{1}{q_{T}^{2}}+C\left(q_{T}^{2}\right)\right)
$$

where $A$ and $B$ are perturbatively calculable factors. The two terms that diverge as $q_{T} \rightarrow 0$ come from the singular behavior of the invariant amplitude when a soft gluon is emitted. Order by order, the virtual corrections regularize the integral, but the first factor is large when:

$$
\alpha_{s} \ln \frac{Q^{2}}{q_{T}^{2}} \gtrsim 1 \Rightarrow q_{T} \lesssim 10 \mathrm{GeV}
$$



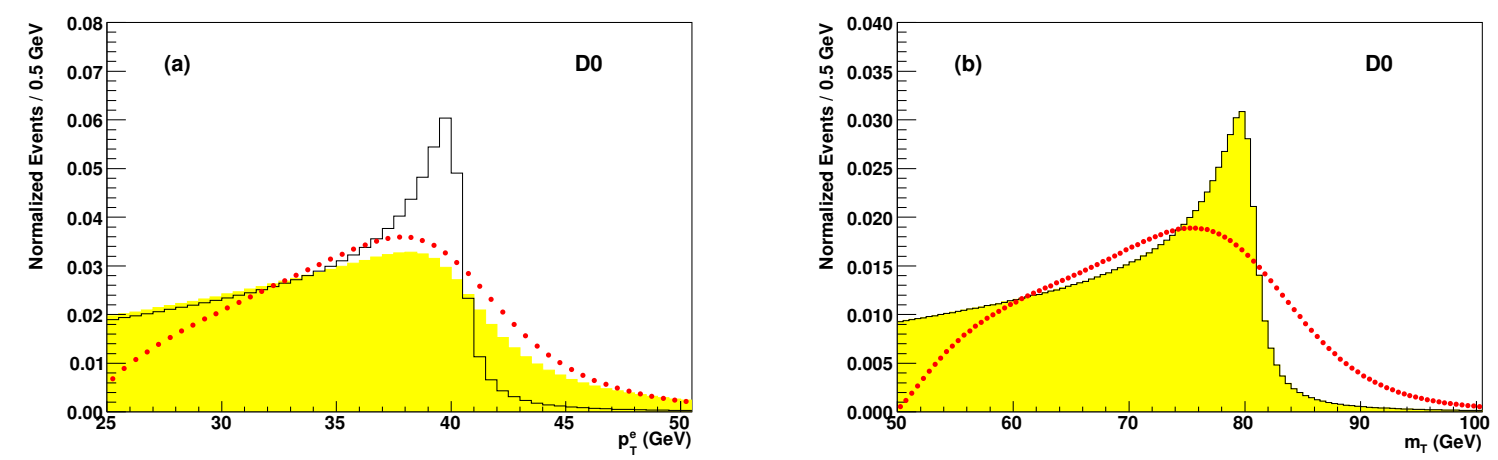

Figure 1.1: The $p_{T}^{e}$ (left) and $m_{T}$ (right) spectra for simulated $\mathrm{W}$ bosons without detector resolution effects and $q_{T}=0$ (solid line), with the natural $q_{T}$ spectrum at the Tevatron (shaded area), and with the full $q_{T}$ distribution and all detector resolution effects included (points). All curves are normalized to unit area and they all include acceptance effects.

which is exactly the situation at the Tevatron. This same divergence exists at every perturbation order:

$$
\frac{1}{\sigma} \frac{d \sigma}{d q_{T}^{2}} \simeq \frac{1}{q_{T}^{2}}\left[A_{1} \alpha_{S} \ln \frac{Q^{2}}{q_{T}^{2}}+A_{2} \alpha_{S}^{2} \ln ^{3} \frac{Q^{2}}{q_{T}^{2}}+\cdots+A_{n} \alpha_{S}^{n} \ln 2 n-1 \frac{Q^{2}}{q_{T}^{2}}+\cdots\right]
$$

Thus, for low boson $q_{T}$, the convergence at fixed order in perturbation theory is bad. Fortunately, this series can be resummed. The resummation is done in the impact parameter space as was first worked out by Collins, Soper and Sterman [15]. The so called CSS formalism is used in RESBOS where the cross section for $W$ production is written as [16]:

$$
\begin{aligned}
& \left(\frac{d \sigma\left(h_{1} h_{2} \rightarrow V\left(\rightarrow \ell_{1} \bar{\ell}_{2}\right) X\right)}{d Q^{2} d y d q_{T}^{2} d \phi_{V} d \cos \theta d \phi}\right)_{r e s}=\frac{1}{96 \pi^{2} S} \frac{Q^{2}}{\left(Q^{2}-M_{V}^{2}\right)^{2}+Q^{4} \Gamma_{V}^{2} / M_{V}^{2}} \\
& \quad \times\left\{\frac{1}{(2 \pi)^{2}} \int d^{2} b e^{i \vec{q}_{T} \cdot \vec{b}} \sum_{j, k} \widetilde{W}_{j \bar{k}}\left(b_{*}, Q, x_{1}, x_{2}, \theta, \phi, C_{1}, C_{2}, C_{3}\right) \widetilde{W}_{j \bar{k}}^{N P}\left(b, Q, x_{1}, x_{2}\right)\right. \\
& \left.\quad+Y\left(q_{T}, Q, x_{1}, x_{2}, \theta, \phi, C_{4}\right)\right\} .
\end{aligned}
$$

The same formula can be used for $W$ and $Z$ production, which are collectively referred as $V$. In Eq. 1.15, the parton momentum fractions are defined as $x_{1}=e^{y} Q / \sqrt{s}$ and $x_{2}=e^{-y} Q / \sqrt{s}$, where $\sqrt{s}$ is the center-of-mass energy of the hadrons $h_{1}$ and $h_{2}$. The renormalization group invariant quantity $\widetilde{W}_{j \bar{k}}(b)$ sums to all orders in $\alpha_{S}$ the singular terms that behave as $q_{T}^{-2} \times\left[1\right.$ or $\left.\ln \left(q_{T}^{2} / Q^{2}\right)\right]$ for $q_{T} \rightarrow 0$. To avoid the Landau pole, the 
impact parameter in $\widetilde{W}_{j \bar{k}}(b)$ is replaced by:

$$
b_{*}=\frac{b}{\sqrt{1+\left(b / b_{\max }\right)^{2}}}
$$

which introduces a cutoff at large values of $b$. This region cannot be calculated perturbatively, and a phenomenological non-perturbative form factor $\widetilde{W}_{j \bar{k}}^{N P}\left(b, Q, x_{1}, x_{2}\right)$ is introduced. This measurement uses the BNLY parametrization as form factor, Eq. 1.16, fitted to the Tevatron Run I data [17]

$$
\widetilde{W}_{j \bar{k}}(b)=\exp \left[\left(-g_{1}-g_{2} \ln \left(Q / Q_{0}\right)-g_{1} g_{3} \ln \left(100 x_{1} x_{2}\right)\right) b^{2}\right] .
$$

The values used in the simulations for the measurements described in this Dissertation are:

$$
g_{1}=0.21_{-0.01}^{+0.01}, \quad q_{2}=0.68_{-0.02}^{+0.01}, \quad g_{3}=-0.6_{-0.04}^{+0.05}
$$

\subsection{Previous $W$ Boson Mass Calculations and Measurements}

The first calculations of the radiative corrections $\Delta q$ in Eq.1.4 were done Marciano and Sirlin [19] and have been recently reviewed in [20]. The most up to date calculation includes, as discussed above, the full two-loop order calculation, but the fermionic corrections in three and four loops are also known [6]. When Eq. 1.4 is interpreted as a prediction of the value of the $W$ boson mass, the yet not calculated higher-order corrections result in $4 \mathrm{MeV}$ of uncertainty. The calculation is usually done in the so called on-shell renormalization scheme [21] where the renormalized mass is the physical mass. In this scheme all renormalized equations have direct physical meaning. The relationship between the pole (on-shell) mass and the modified minimium subtraction (MS) mass is also known to two-loop precision [22, 23].

In the past, when the Higgs boson mass was not measured, the relationship 1.4 was of great interest, since $\Delta r$ is dependent on the value of $m_{T}$ and $M_{H}$ and could be reinterpreted as an indirect determination of the Higgs boson mass. Due to the large mass splitting between the top and bottom quarks and breaking of the $\mathrm{SU}(2)$ custodial symmetry, the quark top mass has a strong contribution to the renormalization of the $\rho$ parameter:

$$
\rho=\frac{1+\widehat{\Pi}_{W}\left(q^{2}\right)}{1+\widehat{\Pi}_{Z}\left(q^{2}\right)}
$$

where $\Pi_{W, Z}$ are the $W, Z$ self-energy functions (see definition 4 in App. A). Today, equation 1.4 is seen as a powerful test of the electroweak theory by providing a direct and testable relationship between $M_{W}$ and $m_{t}$.

\footnotetext{
${ }^{2}$ Recently, the necessity for this non-perturbative parametrization to describe the Tevatron data has been challenged [18], but their calculation is not publicly available as a Monte Carlo generator yet.
} 
Experimentally, the $W$ boson mass was first measured by the UA2 [24] and UA1 [25] and collaborations using essentially the same methods that will be described in this dissertation. The H1 [26] and ZEUS [27] collaborations at HERA also performed early measurements of the $W$ boson mass, but it was at LEP and at the Fermilab Tevatron Collider that the first precision measurements were performed. At LEP, the ALEPH [28], DELPHI [29], L3 [30] and OPAL [31] collaborations measured the $W$ boson mass by direct reconstruction of $e^{+} e^{-} \rightarrow W^{+} W^{-} \rightarrow q \bar{q} q \bar{q}$ and $e^{+} e^{-} \rightarrow W^{+} W^{-} \rightarrow q \bar{q} \ell v$ invariant mass, reaching a combined precision of $31 \mathrm{MeV}$ when combined with their previous measurements from threshold scans.

At the Tevatron, after early measurements by both D0 and CDF, the early Run II measurements (see Sec. 2.1) became competitive with the LEP results. CDF [10] published a measurement of the $W$ boson mass using $2.2 \mathrm{fb}^{-1}$ of integrated luminosity in the $W \rightarrow e v$ and $W \rightarrow \mu v$ channels with $19 \mathrm{MeV}$ of uncertainty. Their measurement using the $m_{T}$ distribution in the muon channel is the single most precise measurement of the $W$ boson mass. In Run II, D0 published a measurement of the $W$ mass with $1 \mathrm{fb}^{-1}$ from Run IIa data [32] and the measurement described in this dissertation with $4.3 \mathrm{fb}^{-1}$ from Run IIb data [33]. Combined, the two D0 Run II measurements published so far have a precision of $23 \mathrm{MeV}$. Both D0 and CDF use similar measurement strategies but very different calibration procedures. The world average value of all direct $W$ boson mass measurement is, today, $80.385 \pm 0.015 \mathrm{GeV}$ [34], dominated by the Tevatron Run II and by the LEP measurements. 


\section{Chapter 2}

\section{Experimental Apparatus}

\subsection{The Fermilab Tevatron Collider}

The Fermilab Tevatron Collider was the last accelerator in a long chain that produced interactions between protons and anti-protons with a center-of-mass energy of $1.96 \mathrm{TeV}$. It was operated between 1987 and September 2011 (but only between 2002 and 2011 with $1.96 \mathrm{TeV})$, when it was decommissioned. The complete chain, from the proton extraction to the interaction points can be seen on Fig. 2.1.

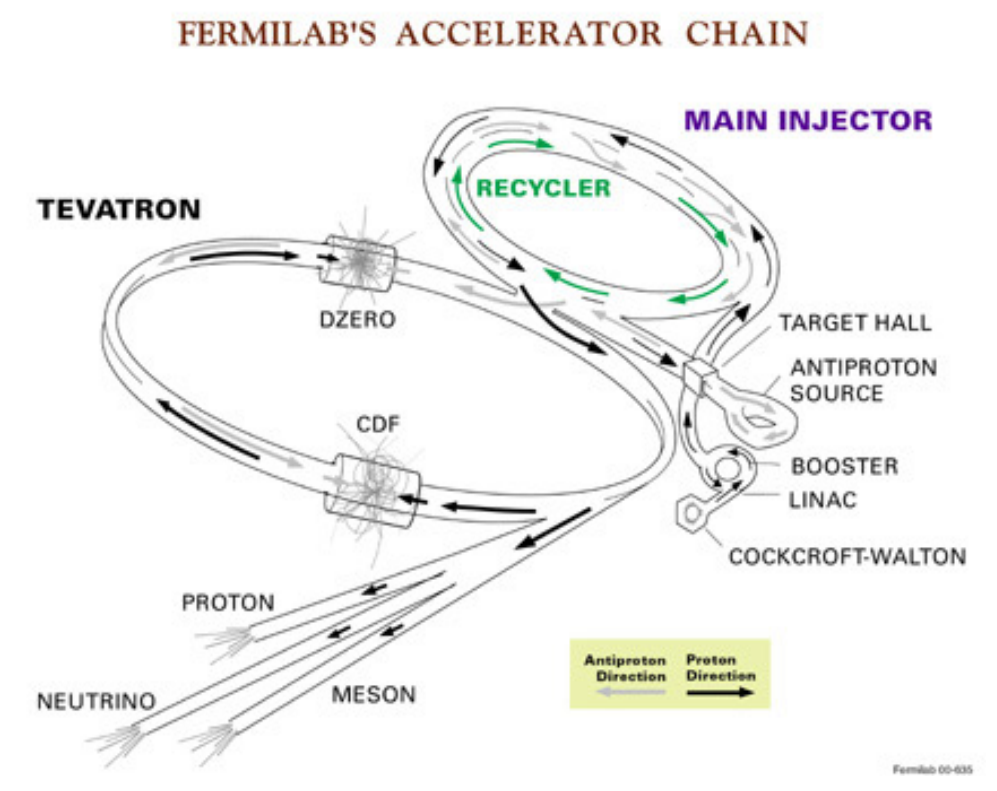

Figure 2.1: Scheme of the complete Run II acceleration chain that led to the interactions in the Tevatron.

The beginning of the process consisted in producing free protons by ionizing hydro- 
gen gas. After the ionization, the protons were accelerated by an electrostatic CockcroftWalton accelerator to an energy of $750 \mathrm{keV}$. Then, accelerated by a RF linear accelerator (LINAC) to $400 \mathrm{MeV}$ and by the Booster circular accelerator to $8 \mathrm{GeV}$. When approximately $5 \times 10^{12}$ protons were available, they were transferred to the Main Injector circular accelerator where they were accelerated to $120 \mathrm{GeV}$.

To produce anti-protons, a fraction of the protons from the Main Injector was directed toward a Ni-Cu target, producing a particle shower, out of which anti-protons were selected and collimated by lithium lenses. The anti-proton efficiency production was very low, approximately 1 to every $10^{5}$ protons in the nickel target, which has been the limiting factor for the Tevatron luminosity during its operations. The anti-protons were produced with a large momentum distribution and were cooled down by the Debuncher and accumulated in a small storage ring. They would be transferred and further accumulated in the Recycler storage ring until approximately $10^{12}$ anti-protons were available, when they were then transferred to the Main Injector.

The Main Injector accelerated protons and anti-protons to $150 \mathrm{GeV}$ and then transferred them to the Tevatron collider where they were further accelerated to $980 \mathrm{GeV}$ per beam. After acceleration, the beams were scrubbed and focused to interact in two points $\mathrm{B} 0$ and D0, where the CDF and D0 detectors, respectively, recorded the result of the interactions. The typical instantaneous luminosity during the last years of operations was $\mathcal{L} \approx 200 \times 10^{30} \mathrm{~cm}^{-2} \mathrm{~s}^{-1}$ with an average of 6 simultaneous interactions per crossing (the record instantaneous luminosity was $\mathcal{L} \approx 430 \times 10^{30} \mathrm{~cm}^{-2} \mathrm{~s}^{-1}$ ). The luminosity varied during operations due to the protons lost on the interactions or due to vacuum imperfections. Tipically after 16 hours of operations, the luminosity was low enough to justify disposing the leftover protons in the Beam Dump area. The anti-protons were transferred back to the Recycler and re-used. Each new filling of the Tevatron collider was referred to as a store. Contrary to proton-proton colliders like the LHC, the two beams in the Tevatron traveled inside the same vacuum pipe, in opposite directions. This was possible by the use of electrostatic separators that directed the proton and antiproton beams into different helical orbits, keeping them apart except at the B0 and D0 interaction points, where the CDF and D0 detectores were located. One advantage of this setup is that the crossing angle between the beams is effectively zero. The disadvantage is the strong de-focusing after the interactions that accounted for $40 \%$ of the luminosity loss during a store.

The Tevatron operation was divided in epochs, or Runs. The Run I began in 1992 and ended in 1996 after both D0 and CDF were able to, independently, observe the quark top for the first time in history. After a long shutdown when both detectors and accelerator were upgraded, there were the Run IIa between 2001 and April 2006, and then the Run $\mathrm{IIb}$ between June 2006 and September 2011. The Tevatron collider delivered $11.9 \mathrm{fb}^{-1}$ of integrated luminosity during Run II, out of which D0 recorded $10.7 \mathrm{fb}^{-1}$ (see Fig. 2.2). 


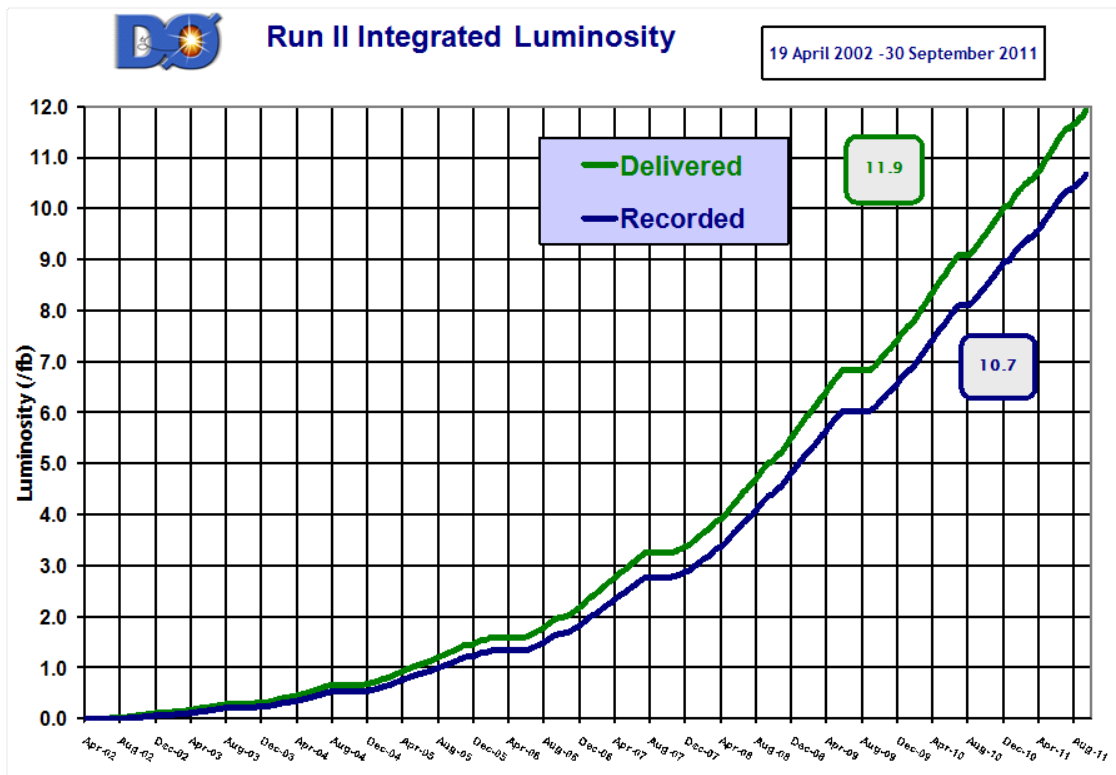

Figure 2.2: Integrated luminosity delivered by the Tevatron (green line) and recorded by the D0 experiment (blue line). The long horizontal lines are periods of extended Tevatron shutdown. In particular, the shutdown between April and June 2006 divides Run IIa and Run IIb. The overall recording efficiency at D0 was 90\% and basically limited by the Trigger Framework (see Sec. 2.5) dead time and by temporary problems with the detector.

\subsection{The Tevatron Bunch Structure and Luminosity}

The protons and anti-protons revolving around the Tevatron were grouped into bunches. One revolution around the Tevatron took $21 \mu \mathrm{s}$, corresponding to a revolution frequency of $47.713 \mathrm{kHz}$. The acceleration radio-frquency(RF) at the Tevatron worked at $53.104 \mathrm{MHz}$, yielding 1113 RF buckets in one turn, separated by $18.85 \mathrm{~ns}$. A set of seven consecutive buckets defined a tick, and the first bucket of every tick could be filled with protons or anti-protons. There were 159 ticks per turn. Not every tick was filled, though. During Run I, there were 6 bunches evenly spaced. During Run II, there were 36 bunches. They were grouped in 3 super-bunches of 12 bunches each. Inside each super-bunch, the bunches were spaced with 2 empty ticks. Therefore, an interaction occurred every $396 \mathrm{~ns}$. Between each super-bunch, there was a space, called cosmic gap, of 20 ticks or approximately $2.64 \mu \mathrm{s}$. One consequence of the bunch structure at Run II is that D0 and CDF did not see the same collisions and could, in principle, have different delivered luminosities 1 . The Tevatron accelerator division, however, tried its best to deliver equivalent amount of interactions to both experiments.

\footnotetext{
${ }^{1}$ The optics lattice was also slightly different at the B0 and D0 interaction points[35].
} 


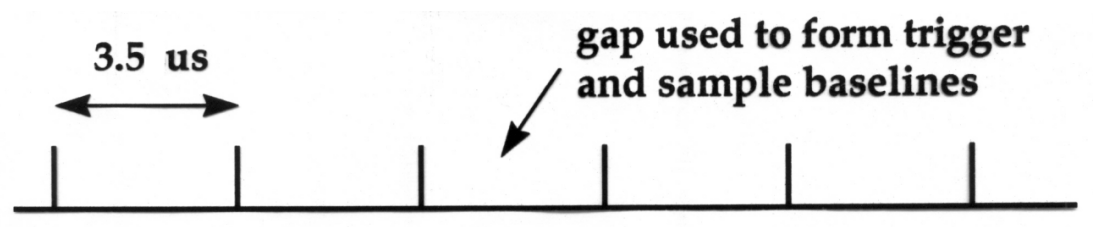

Run I $6 \times 6$

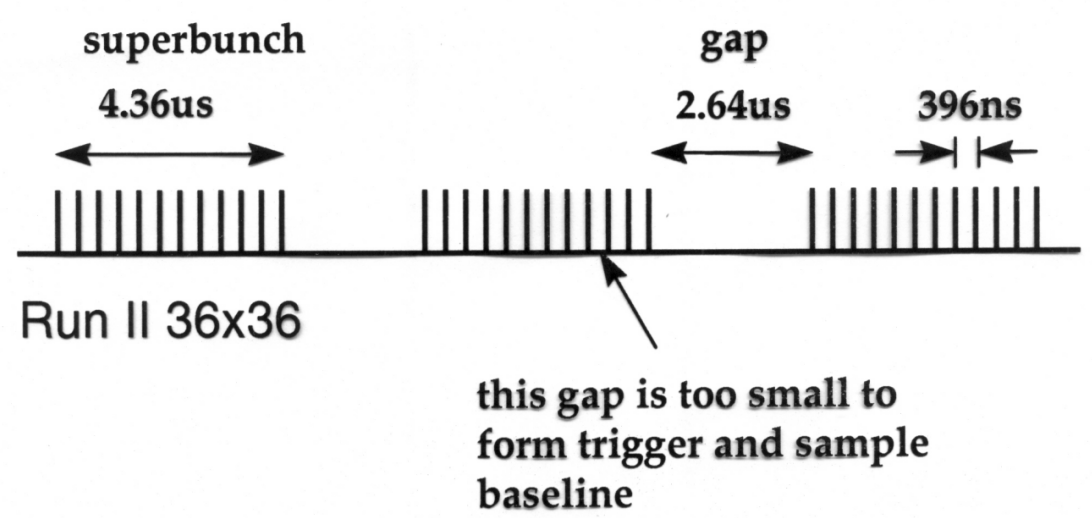

Figure 2.3: Tevatron bunch structure during Run I (top) and Run II (bottom). The first bunch of each super-bunch was different since there were no interactions right before it. The interactions involving first bunches would be naturally less prone to out-of-time pileup and associated detector effects.

In the absence of a crossing angle, the instantaneous luminosity is given by [36]:

$$
\mathcal{L}=\frac{f B N_{p} N_{\bar{p}}}{2 \pi\left(\sigma_{p}^{2}+\sigma_{\bar{p}}^{2}\right)} F\left(\sigma_{l} / \beta^{*}\right),
$$

where $f$ is the revolution frequency, $B$ is the number of bunches in each beam, $N_{p}\left(N_{\bar{p}}\right)$ is the number of protons (anti-protons) in a bunch, $\sigma_{p}\left(\sigma_{\bar{p}}\right)$ is the RMS size of the proton (anti-proton) beam and $F$ is a form-factor that depends on the ratio of the bunch length $\sigma_{l}$ to the beta function at the interaction point $\beta^{*}$. In this Dissertation, we express the instantaneous luminosity in units of $10^{30} \mathrm{~cm}^{-2} \mathrm{~s}^{-1}=1 \mu \mathrm{b}^{-1} \mathrm{~s}^{-1}$.

The D0 detector had two Luminosity Monitors (see Sec.2.3.5) close to the beam pipe on the face of the north and south End Calorimeters (see Sec.2.4.1), to measure the number of interactions. An interaction at D0 was defined as a coincidence signal in both Luminosity Monitors. The number of interactions $n$ per crossing follows a Poisson distribution with mean $\mu=\mathcal{L} \sigma_{\mathrm{LM}} / f$ where $\sigma_{\mathrm{LM}}$ is the inelastic luminosity cross section corrected by the Luminosity Monitors' acceptance and efficiency [37], which allow the luminosity to be 
measured as:

$$
\mathcal{L}=\frac{f}{\sigma_{\mathrm{LM}}} \mu=-\frac{f}{\sigma_{\mathrm{LM}}} \ln (1-P(n>0)),
$$

Unfortunately, $\mu$ is not constant in every tick, and the luminosity was measured independently 159 times per turn:

$$
\mathcal{L}=-\frac{f / 159}{\sigma_{\mathrm{LM}}} \sum_{i=1}^{159} \ln \left(1-\frac{\Delta \mathrm{LM}_{i}}{\Delta \mathrm{ticks} / 159}\right)
$$

where $\Delta \mathrm{LM}_{i}$ and $\Delta$ ticks are, respectively, the number of coincidences in the luminosity monitors and the number of ticks in a pre-determined period of time called the Luminosity Block.

\subsection{The Run II D0 Detector}

Figure 2.4 shows a representation of all sub-detectors that composed the D0 detector. It was a typical high-energy, general purpose, detector [38]. Tracking was provided by two sub-detectors: the Silicon Microstrip Tracker and the Central Fiber Tracker, that were immersed in a solenoidal magnetic field of 1.9T. Those were new detection systems installed during the Run II upgrade. Calorimetry was provided by three Liquid Argon (LAr) calorimeters, scintillator fibers Central and Forward Preshowers detectors, and the scintillator pads Inter-Cryostat Detector (ICD). The Preshowers Detectors were also new in Run II, but the rest of the system had been maintained from Run I. The calorimeter readout electronic system was re-designed and improved to cope with the increased rate of interactions and instantaneous luminosity. Muon detectors were located outside the calorimeter which provided both precision position measurement and fast signal for triggering. Here, we give a brief description of each element and a thorough description of the calorimeter system that is the most relevant to the measurement described in this Dissertation 2

\subsubsection{Silicon Microstrip Tracker}

The D0 Silicon Microstrip Tracker (SMT) provided tracking and vertex information throughout most of the luminous region. Since the interaction region at D0 was quite long, the SMT was designed with barrels interspersed by disks, as well as additional disks in the

\footnotetext{
${ }^{2}$ During part of the Run II, a set of roman pots (FPD - forward proton detectors) took data in special Tevatron runs with very large $\beta^{*}$. The FPD was comprised of six castles, four of which as quadrupoles and two as dipole detectors. Each pole of the castles contained scitillating fiber spectrometers in six stereo layers that provided angle and momentum measurement of protons from elastic and diffraction interactions. Since the measurement described in this Dissertation does not use any data from the period in which FPD were installed, we do not describe it further[39].
} 


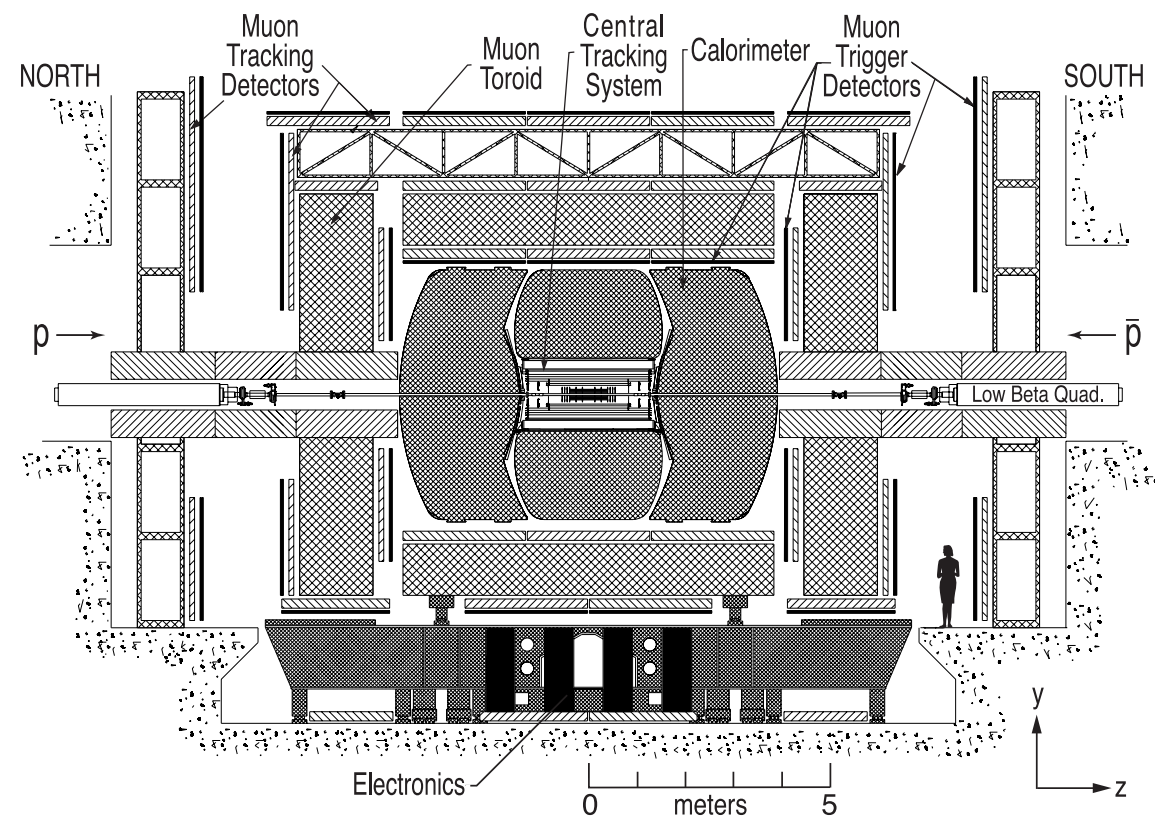

Figure 2.4: Cross section of the D0 detector showing the many sub-detectors that compose it.

forward region. The detectors in the barrel measured a detector hit $r-\phi$ coordinates 3 , while the disks were able to measure the full $r-\phi$ and $r-z$ coordinates.

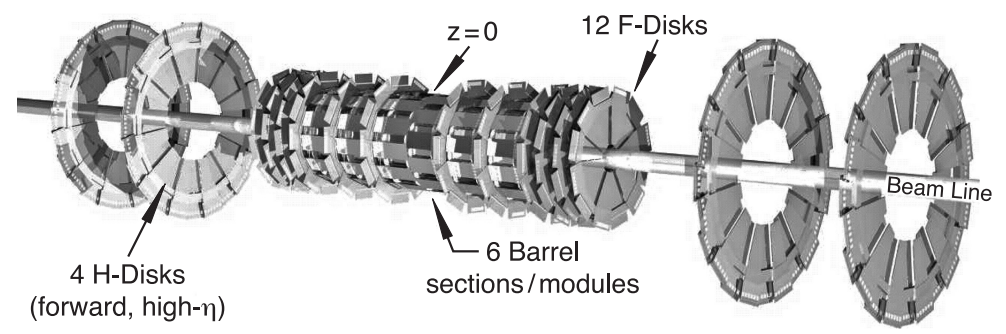

Figure 2.5: A drawing of the D0 Silicon Microstrip Tracker. There are 6 barrels interspersed by F-disks and additional four H-disks that provided tracking in the forward region.

The barrels were built with four layers and each layer was composed of intercalated ladders. Each barrel had a total of 432 ladders. The disks that capped the barrels, or Fdisks, had 12 double-sided wedge detectors. In the forward regions, four additional largediameter disks, the H-disks, provided additional tracking with 24 wedges each. The full system consisted of 792,576 channels readout with 128-channel SVXIIe ASICs. Table 2.1

\footnotetext{
${ }^{3}$ Here we define "hit" simply as a signal in a silicon strip above noise threshold.
} 
has a summary of the geometry of each individual module of the SMT, including the pitch which determines the spatial resolution.

\begin{tabular}{|c|c|c|c|}
\hline \hline Module & Layer & Pitch $(\mu \mathrm{m})$ & Radius $(\mathrm{cm})$ \\
\hline F-disk & - & $50 / 62.5$ & 2.57 (inner) -9.96 (outer) \\
\hline H-disk & - & 40 & 9.5(inner) - 26 (outer) \\
\hline & 1 & 50 & 2.715 \\
Central Barrel(4) & 2 & 50 & 4.55 \\
& 3 & 153.5 & 7.582 \\
& 4 & 62.5 & 10.51 \\
\hline & 1 & 50 & 2.715 \\
Outer Barrel (2) & 2 & 50 & 4.55 \\
& 3 & 50 & 7.582 \\
& 4 & 62.5 & 10.51 \\
\hline \hline
\end{tabular}

Table 2.1: Pitch, inner and outer radii of the barrel and disks in the D0 Silicon Microstrip Detector.

During the shutdown between Run IIa and Run IIb, the two outer H-disks were disconnected and an additional barrel layer (L0) was installed. The L0 had sensors convering the the region between $z=-38 \mathrm{~cm}$ and $z=38 \mathrm{~cm}$ in two different radii, $16.1 \mathrm{~mm}$ and $17.6 \mathrm{~mm}$, with sensor pitches of 71 and $81 \mu \mathrm{m}$, respectively. The L0 improved the impact parameter resolution for low $p_{T}$ particles and the identification of bottom quarks, which was important for Higgs physics and B physics measurements [40].

\subsubsection{Central Fiber Tracker}

The D0 Central Fiber Tracker was a scintillating fiber tracker located just outside the SMT. It was composed of eight coaxial cylinders with radius from 20 to $52 \mathrm{~cm}$, and each cylinder supported two doublets of scintillating fibers $835 \mu \mathrm{m}$ thick. The six outermost cylinders were $2.52 \mathrm{~m}$ long, while the two innermost cylinders were only $1.66 \mathrm{~m}$ long to accommodate the SMT H-disks. In each cylinder, the fibers of one doublet layer were always parallel to the axis (axial layer) while the other doublet was at a relative angle of $+3^{\circ}$ or $-3^{o}$ (stereo layers).

The scintillating fibers were coupled to clear fiber waveguides that carried the scintillation light to visible light photon counters (VLPC), which are cryogenic photo-detectors with excellent quantum efficiency $(>75 \%)$, high gain $(22,000$ to 65,000$)$ and low gain dispersion. The fibers in each doublet were mounted a half-fiber shifted between the two layers so that the resolution was better than the individual fiber thickness. When the position of the fibers was known better than $50 \mu \mathrm{m}$, the spatial resolution of a doublet was $100 \mu \mathrm{m}$. The signal from the VLPCs was read by analog front-end boards that also provided information for the level 1 central tracker trigger system (L1CTT). The L1CTT used 


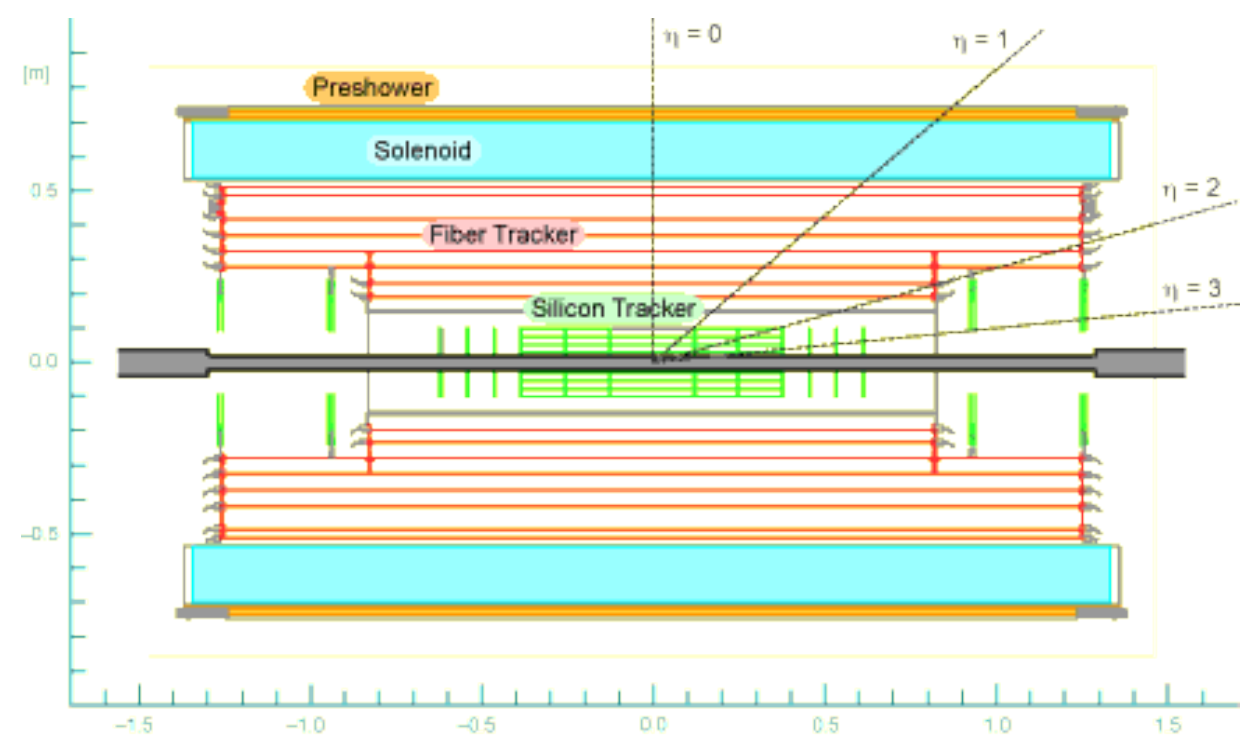

Figure 2.6: Technical drawing of the D0 central tracker system.

only the information from the axial layers and was able to trigger on very low $p_{T}$ tracks $(>1.5 \mathrm{GeV})$. The overall momentum resolution of the tracking system (SMT + CFT) can be determined using low $p_{T}$ muons, for which the momentum measurement in the Muon System 2.3.4 had small weight. Figure 2.7 shows the momentum resolution determination for muons with normal incidence and with $\eta=1.6$. The latter is outside the CFT coverage and, thus, relies only on the SMT hits.

\subsubsection{Solenoid and Toroid}

One of the most challenging upgrades of the D0 detector for Run II was the introduction of a superconducting solenoid magnet in the space available between the central calorimeter cryostat and the then new central tracker. The D0 superconducting solenoid had $2.73 \mathrm{~m}$ in length and only $1.42 \mathrm{~m}$ in diameter. It added, by itself, 1 radiation length $\left(X_{0}\right)$ to the detector material budget. It provided a solenoidal field of $1.9 \mathrm{~T}$ of intensity was important to permit the momentum measurement of charged tracks and tracking pattern recognition.

Outside the calorimeter, a system of central and forward toroids with $1.8 \mathrm{~T}$ and 1.9 $\mathrm{T}$, respectively, completed the D0 magnetic field allowing independent momentum measurements in the Muon System. The toroids were already present in the Run I but had their current reduced by $6 \%$, since, in Run II, the momentum could also be measured in the central tracker. Both solenoid and toroid were designed in such way that their polarity could be switched during operations. That has been shown to be very important to

\footnotetext{
${ }^{4}$ The design intensity was $2 \mathrm{~T}$, but a cracking noise was heard when the solenoid was first turned on at full current in reverse configuration and it was then decided to operate it with a smaller current.
} 


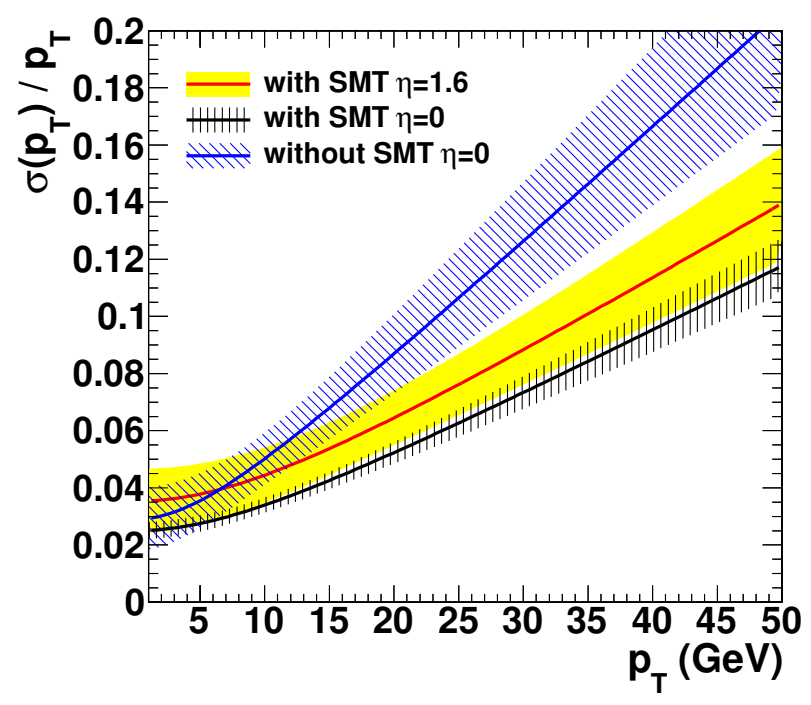

Figure 2.7: Momentum resolution for muon tracks with normal incidence $(\eta=0)$ with and without SMT hits. The yellow band corresponds to muons with incident angle $\eta=$ 1.6 and, hence, outside the CFT coverage. Those central tracks relied only on SMT hits.

cancel systematic uncertainties and allowed D0 to perform delicate asymmetry measurements [41, 42] $]^{5}$

\subsubsection{Muon System}

The D0 Muon System consisted of three layers, one before and two after the toroidal magnets, named A, B, and C, respectively. The Muon System was divided into the central muon system, that covered the region $|\eta| \lesssim 1.0$ and forward muon system in the region $1.0 \lesssim|\eta| \lesssim 2.0$. For triggering, the A-layer and C-layer of the central muon system were equipped with 630 scintillation counters named $A \phi$ Counters and all the three layers in the forward region were equipped with 4214 scintillation counters named Pixel Counters. They provided a fast signal with good time resolution for muon identification.

The muon momentum measurement was made with proportional drift tubes (PDT) installed in the three layers of the central muon system and mini-drift tubes (MDT) installed in the three layers of the forward region. They provided accurate coordinate information with resolution better than $1 \mathrm{~mm}$ in the direction perpendicular to the wires which were arranged perpendicular to the toroidal magnetic field. Due to the large amount of material in the liquid argon calorimeters, only muons with momentum larger than $2.5 \mathrm{GeV}$ reached the A-layer and, with the additional energy loss in the toroid, only muons with

\footnotetext{
${ }^{5}$ Also important to enable the change of polarity in the solenoidal magnetic field was the use of a lightbased scintillating fibers central tracker instead of one based on charged carrier drifts, which would introduce extra uncertainties from $\mathbf{E} \times \mathbf{B}$ effects.
} 
momentum over $3.5 \mathrm{GeV}$ left signal in all three layers throughout the whole covarage of the muon system.

\subsubsection{Luminosity Monitor}

The Luminosity Monitor (LM) consisted of two arrays of scintillation counters attached to the D0 End Calorimeters. Each array consisted of 24 wedges that provided coverage of the region $2.7<|\eta|<4.4$ and were read by photo-multiplier tubes (PMT). The Luminosity Monitor readout identified hits that were in a window $6.4 \mathrm{~ns}$ wide around the nominal time of flight from the interaction point. This time window corresponded to three times the time of flight through the typical luminous region. Beam halo particles were easily identifiable since they would arrive approximately $9 \mathrm{~ns}$ early in one of the sides. A valid luminosity signal was triggered when there were at least one in-time hit in both north and south LMs. A crossing with more than six early hits was considered too noisy to allow an accurate luminosity measurement and halo-vetoed. All other crossings were called live crossings and accepted for triggering. Because of the halo-veto, the Luminosity Monitor was considered to be the Level 0 of the D0 trigger system (see Sec. 2.5).

\subsection{The D0 Calorimetry System}

The main part of the D0 calorimetric system was composed of three sampling UraniumLiquid Argon (U-LAr) calorimeters. They were in three cryostats: the Central Calorimeter (CC), covering the region $|\eta| \lesssim 1.1$, and the north and south End Calorimeters (EC) covering the region $1.5<|\eta|<4.0$. To avoid large regions of uninstrumented material along the shower devolpment and the resulting lateral leakage it would cause, which is particularly harmful to missing $E_{T}$ resolution [1], the energy flow in the region between the cryostats was measured with a sampling scintillator tile calorimeter, the InterCryostat Detector (ICD), covering the region $1.1<|\eta|<1.4$. Within the central and forward cryostats, there were further single cells called the massless gaps covering the region $0.8<|\eta|<1.3$ and improving the hermiticity of the calorimeter. The system was completed by the Preshowers Detectors which were at the same time calorimetric and tracking detectors. They provided enhanced spatial matching between calorimeter clusters and central tracks and could, in principle, be used to improve the knowledge of the energy lost by showers in the solenoid and upstream material. The Central Preshower (CPS) covered the region $|\eta|<1.3$ while the two Forward Preshowers (FPS) sampled showers in the region $1.5<|\eta|<2.5$.

\subsubsection{Liquid Argon Calorimeter}

The three liquid argon calorimeters were segmented longitudinally, parallel to the calorimeter cylindrial axis, and transversally. The longitudinal segmentation defined different sections of the calorimeter which had different design choices and goals. The first four layers 
composed the electromagnetic (EM) section of the calorimeter. It used thin depleted uranium absorbers 3 and $4 \mathrm{~mm}$ thick in the CC and EC, respectively. The next three layers of the $\mathrm{CC}$ and four layers of the $\mathrm{EC}$ formed the fine hadronic $(\mathrm{FH})$ section of the calorimeter. It used uranium-niobium absorbers $6 \mathrm{~mm}$ thick. The last layer of the CC is the central calorimeter coarse hadronic $(\mathrm{CCCH})$ section that used $46.5 \mathrm{~mm}$ plates of copper as absorber. In the EC, the coarse hadronic layer section used stainless steel absorbers $46.5 \mathrm{~mm}$ thick. Transversally, the calorimeter was segmented in pseudo-projective towers $0.1 \times 0.1$ wide in $\eta \times \phi$ space. The third EM layer was more finely segmented into $0.05 \times 0.05$ cells. The segmentation was coarser after $\eta=3.2$, since, at these large angles, the face size of a $0.1 \times 0.1$ tower would be comparable to one Molière radius in uranium. Figure 2.8 has a flattened representation of the pseudo-projective towers and layers just described. The high $\eta$ region of the EC hadronic calorimeter was referred to as inner section, followed by the middle section, and, only in the coarse hadronic layer, an outer section.

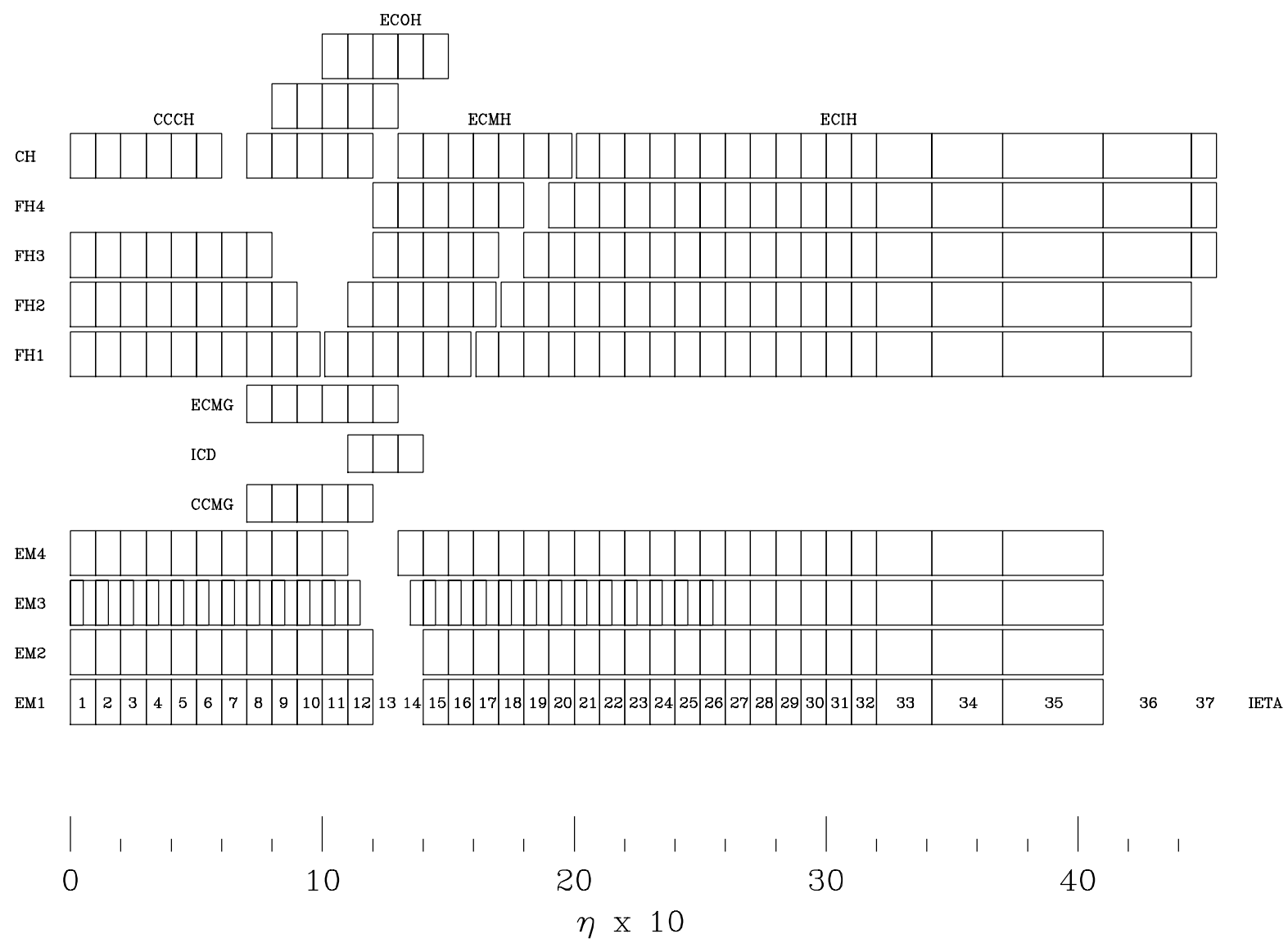

Figure 2.8: Flattened projective towers of the D0 calorimeter.

The thickness of each layer varied by section and layer. Table 2.2 has a summary of the number of radiation lengths $X_{0}$ for the EM layers and nuclear absorption lengths $\lambda_{A}$ for 
the hadronic ones. The liquid argon unit cell is shown in Fig. 2.9. The absorbers were kept at ground and the high-voltage (HV) was delivered through the resistive coat. The typical operation HV was $2 \mathrm{kV}$, which yielded an average electron drift time of $450 \mathrm{~ns}$ through the $2.3 \mathrm{~mm}$ argon gaps. The long drift time was clearly a challenge for a crossing time of $396 \mathrm{~ns}$ and the ionization charge was necessarily under-sampled by the Run II readout. The resistive coat is isolated from the copper readout pads by a layer of G10 epoxy. The image charges in the pads were read by $30 \Omega$ coxial cables. Cells in different regions of the calorimeter had different sizes and, therefore, different capacitance.

\begin{tabular}{|c|c|c|}
\hline \hline Section & Layer & Thickness \\
\hline & 1 & $1.4 X_{0}$ \\
CCEM & 2 & $2.0 X_{0}$ \\
& 3 & $6.8 X_{0}$ \\
& 4 & $9.8 X_{0}$ \\
\hline & 1 & $1.6 X_{0}$ \\
ECEM & 2 & $2.6 X_{0}$ \\
& 3 & $7.9 X_{0}$ \\
& 4 & $9.3 X_{0}$ \\
\hline & 1 & $1.3 \lambda_{A}$ \\
CCFH & 2 & $1.0 \lambda_{A}$ \\
& 3 & $0.75 \lambda_{A}$ \\
\hline CCCH & - & $3.2 \lambda_{A}$ \\
\hline ECFH (inner) & $1-4$ & $1.1 \lambda_{A}$ \\
ECFH (middle) & $1-4$ & $0.9 \lambda_{A}$ \\
\hline ECFH (inner) & - & $4.1 \lambda_{A}$ \\
ECFH (middle) & - & $4.4 \lambda_{A}$ \\
ECFH (outer) & - & $6.0 \lambda_{A}$ \\
\hline \hline
\end{tabular}

Table 2.2: Thickness of the different layers and sections of the D0 liquid argon calorimeter.

The assembly of the calorimeter was done in modules. In the CC EM calorimeter, there were $32 \phi$-modules extending through the whole length of the calorimeter. Between these modules there were small cracks where the shower detection efficiency and energy responses were reduced. The CC hadronic sections were built from only $16 \phi$-modules. In the EC, the EM and inner hadronic calorimeter were built on a monolithic ring and there were no cracks. The middle and outer hadronic were built from 16 wedge-shaped modules. The typical resistance of an CC EM module was measured in situ, at $83 \mathrm{~K}$, to be $1.5 \pm 0.4 \mathrm{G} \Omega$ which means a typical sheet resistivity of $170 \pm 40 \mathrm{M} \Omega / \square^{6}$

\footnotetext{
${ }^{6}$ In an ohmic resistive sheet, the resitance between two points depend only on the ratio $l / w$, where $l$ and $w$ are the dimensions parallel and perpendicular to the direction of the current density, what we represent by the number of squares that can be fitted between the two points.
} 


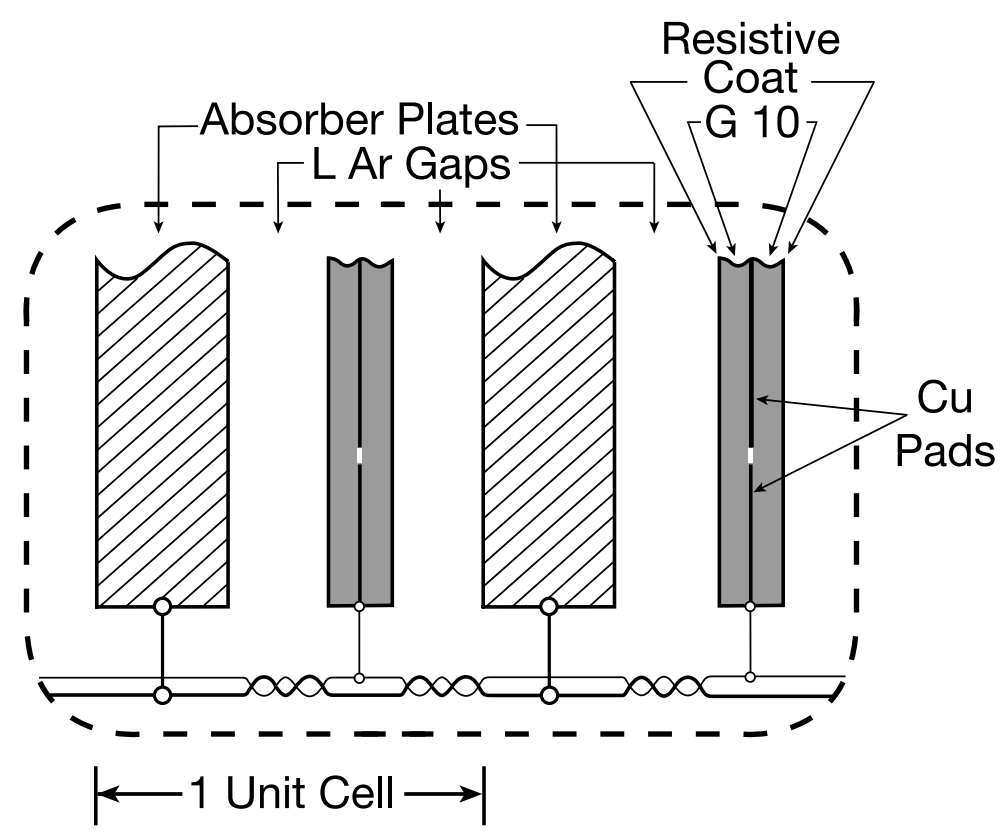

Figure 2.9: Unit cell of the D0 calorimeter with the two liquid argon gaps on both sides of the copper readout pad.

\subsubsection{Inter-Cryostat Detector}

The Inter-Cryostat Detector (ICD) was a series of 0.5 inch thick scintillating tiles enclosed in light-tight aluminum boxes. Each tile covered an area of $0.3 \times 0.4$ in $\eta \times \phi$ space and was divided in 12 sub-tiles $0.1 \times 0.1$ wide by a reflective epoxy layer. The scintillation light from each sub-tile was read by wavelength shifting fibers (WLS), then transported with clear fibers to PMTs. The signal was pre-amplified in such way to be compatible with the calorimeter readout BLS and ADC systems (see section 2.4.4 for the definition). The PMT responses were monitored by a LED calibration system, but they were never stable enough to be used as a precision measurement, nor was the EM sampling sufficient to yield comparable resolution.

\subsubsection{The Central and Forward Preshowers}

The Central and Forward Preshowers (CPS and FPS, respectively) were made of triangular strips of scintillators. The triangles were mounted interleaved with no dead-space between them, improving the position determination. The distance between the center of fibers in different layers was $3.05 \mathrm{~mm}$ and $3.91 \mathrm{~mm}$ in the CPS and FPS, respectively. In the CPS, the strips were mounted on three coaxial cylinders located between the solenoid 
and the Central Calorimeter outer cryostat wall 7 . The strips on the three cylinders were arranged in an axial-u-v geometry with stereo angles of $23.774^{\circ}$ and $-24.016^{\circ}$. Since each cylinder was composed of eight modules, and given the length of the cylinder, this particular choice of angle made the end of axial and stereo fibers to align perfectly. Between the solenoid and the first CPS cylinder, an additional lead radiator $1 X_{0}$ thick was introduced.

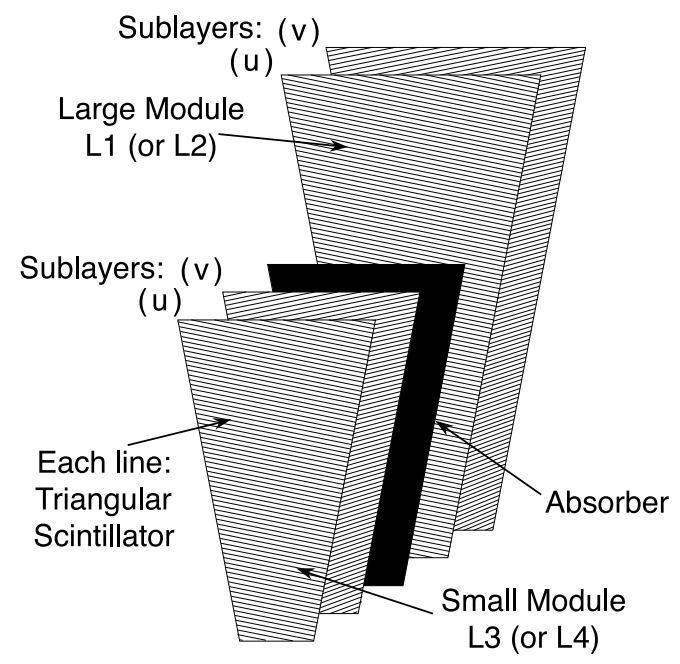

Figure 2.10: The MIP and shower active layers of the Forward Preshower with the lead absorber in between.

The north and south FPS were mounted on the End Calorimeter cryostats, between the Luminosity Monitor and inner edge of the Inter-Cryostat Detectors. Each detector was made with two layers and a thick $2 X_{0}$ lead radiator between them. The upstream layers were known as MIP layers while the downstream layers, after the radiator, were known as shower layers. The shower layer covered the region $1.5<|\eta|<2.5$, while the MIP layers and radiator covered only $1.65<|\eta|<2.5$ as shown on Fig. 2.10. The region between $1.5<|\eta|<1.65$ was in the shadow of the solenoid, which added $3 X_{0}$ by itself. The MIP and shower layers were composed of two u-v sub-layers at stereo angles of $\pm 22.5^{\circ}$. They were mounted on $22.5^{\circ}$ wide wedges making the fibers perpendicular to the $\phi$-boundary. Each MIP sub-layer had 206 scintillator strips and each shower sub-layer had 288. In the south FPS, there were four special wedges that only covered the region $1.8<|\eta|<2.5$. They were notched to accommodate the solenoid cryogenic services, sometimes called the "chimney". Successive FPS layers were offset to avoid cracks in $\phi$. Figure 2.11 summarizes the description given here.

The signal from each fiber was read using wavelength shifting fibers that carried the signal to the periphery of the detector where it was coupled to clear fibers and converted to electric pulses by the same VLPCs used for the Central Fiber Tracker. The Preshow-

\footnotetext{
${ }^{7}$ In this Dissertation, we define the outer and inner walls with respect to the inside of the cryostats and not with respect to the center of the detector, what is the more commonly adopted convention.
} 


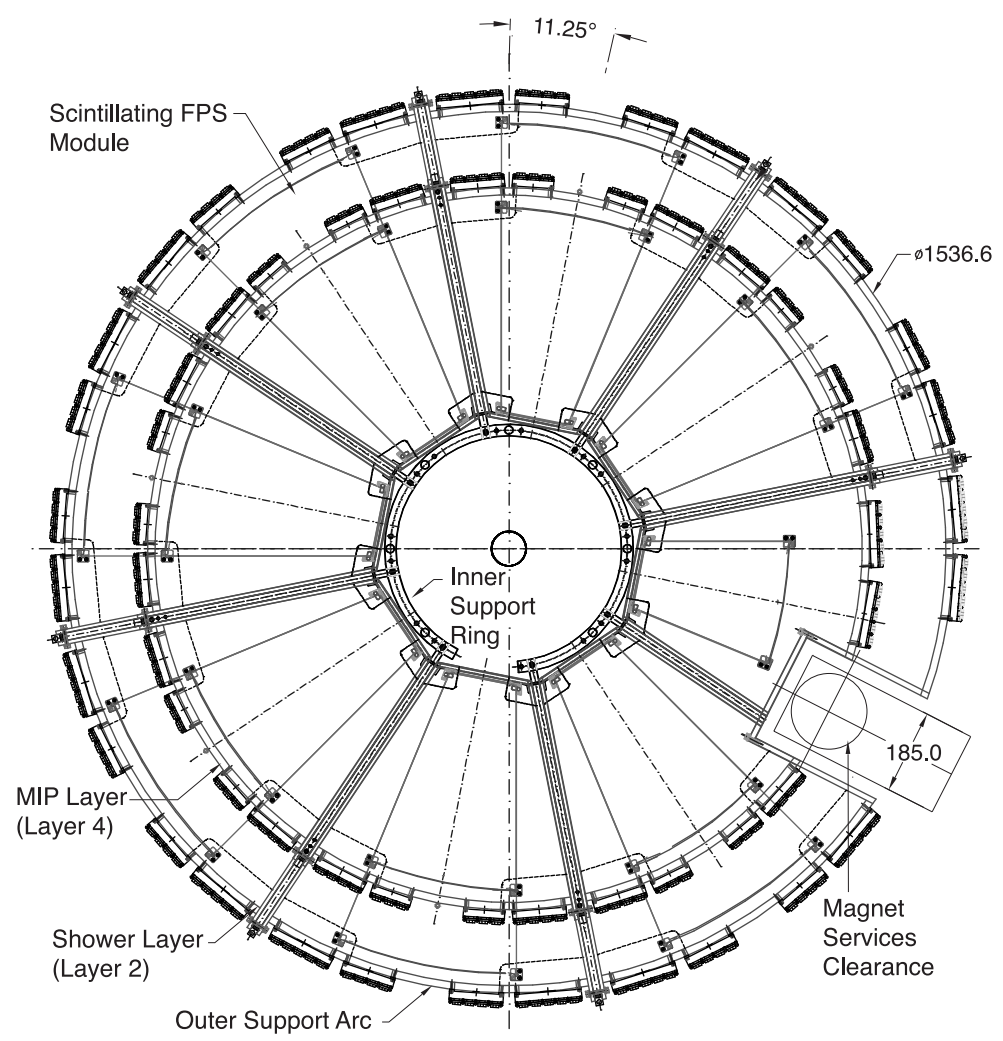

Figure 2.11: Assembly of the Forward Preshower. The "chimney" on the bottom right part of the detector is only notched on the south Preshower to allow space for the cryogenic services.

ers electronics, unfortunately, had a small readout dynamical range and were quickly saturated, rendering them useless for the analysis in this Dissertation. The addition of the dead material in the Preshower absorbers had profound destructive impact in the D0 calorimeter resolution. The effect on the central calorimeter resolution was thoroughly studied for the Run IIa $W$ boson mass measurement [43]. Figure 2.12] shows how the dead material introduced a strong energy and angular dependence on the sampling resolution of the D0 calorimeter.

\subsubsection{Calorimeter Readout System}

This section describes the readout system of the LAr and ICD calorimeters. The signal was pre-amplified by charge-sensitive amplifiers. These amplifiers had dual JFET inputs that maintained a low noise level. The charge integrated signal in the pre-amplifiers output had a rise time of $450 \mathrm{~ns}$ but a very long decay time of $15 \mu \mathrm{s}$. The signal was differentiated and shaped into a unipolar waveform with maximum at $320 \mathrm{~ns}$ and return to zero after 1.2 $\mu$ s (see Fig. 2.13). Because of the short time between crossings, only $2 / 3$ of the signal was 

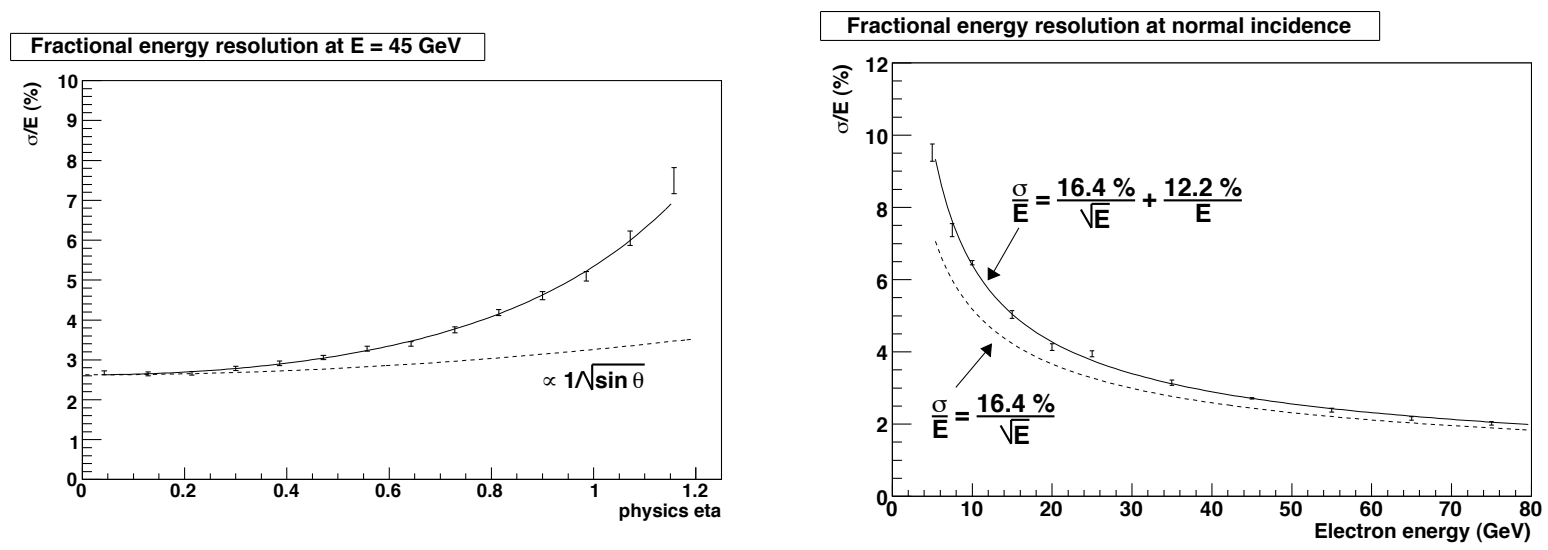

Figure 2.12: Central calorimeter sampling resolution. The left plot shows the strong angular dependence of the sampling resolution due to the dead material introduced before the calorimeter. The dependence is stronger than the naïve estimation $\propto 1 / \sqrt{\sin \theta}[1]$. The plot on the right shows the energy dependence of the sampling resolution also due to the large amount of dead material. The sampling resolution behavior of the D0 calorimeter violates the naïve $1 / \sqrt{E}$ behavior.

actually sampled. The pre-amplifier circuit compensated for the different capacitances of different calorimeter cells to assure that every output had a similar waveform.

The analog signal was sampled every $132 \mathrm{~ns}$, including a sample at the peak response for a given crossing, and held in switched capacitor arrays (SCA) until a trigger decision was made. There were SCAs for both L1 and L2 triggers (see Sec. 2.5). The baseline subtraction (BLS) system subtracted the signal at the peak from the one 3 samples (396 ns) before. This corresponds to an estimate of the baseline voltage above which the new signal rose. In practice, this systems always introduced a small error corresponding to the decay of the previous waveform during these $396 \mathrm{~ns}$, as can been seen on Fig. 2.14.

The BLS error was particularly relevant when reading a minimum bias signal after a "physics" signal in the previous crossing. When that happened, it was possible to have large negative energies read, since the decay of the "physics" signal would be larger than the minimum bias amplitude. During Run I, when the Tevatron operated at low luminosities, this situation was very rare. By the end of Run II, this error introduced an explicit luminosity dependence in many calorimeter-based measurements. The baseline subtracted signal was digitized by a 12-bit successive approximation ADC. To increase the dynamical range, two pre-amplifier gain paths (x1 and $x 8)$ were available.

The pedestal signals of the calorimeter cells were routinely measured between the stores. The mean values were determined by the readout electronics while the RMS $(\sigma)$ was a function of the thermal noise, and therefore capacitance of each cell. Table 2.3 shows typical values of the pedestal RMS for different sections of the D0 calorimeter. 


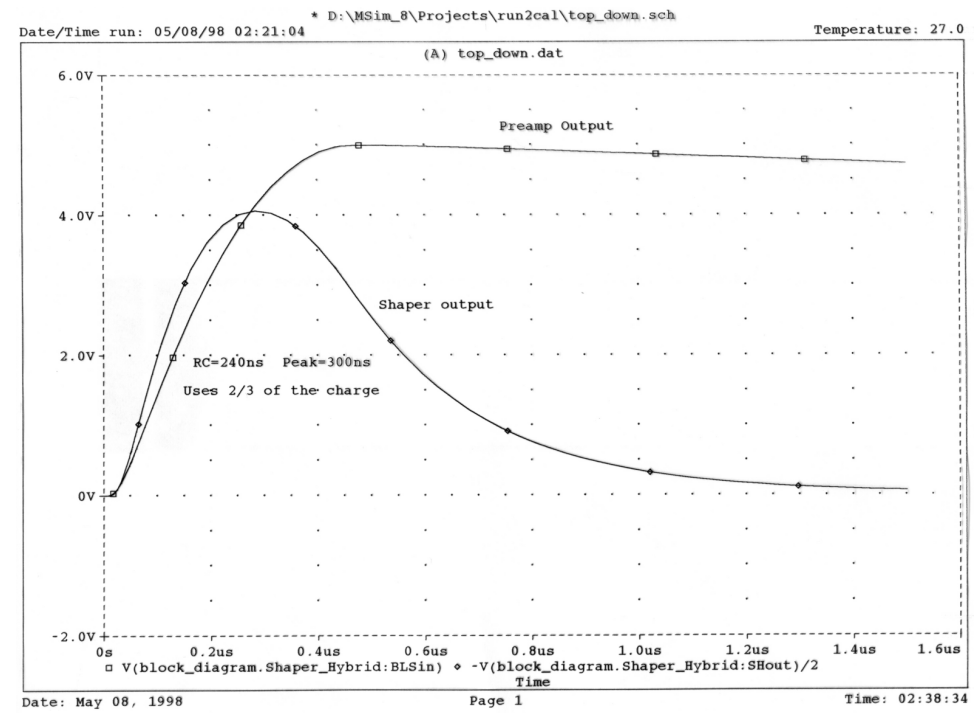

Figure 2.13: Waveforms in the output of the pre-amplifier and shaper circuits. The output form the charge sensitive amplifier had a long time constant (out of scale) while the signal from the shaper had a maximum after $320 \mathrm{~ns}$ and a return to zero after $1.2 \mu \mathrm{s}$.
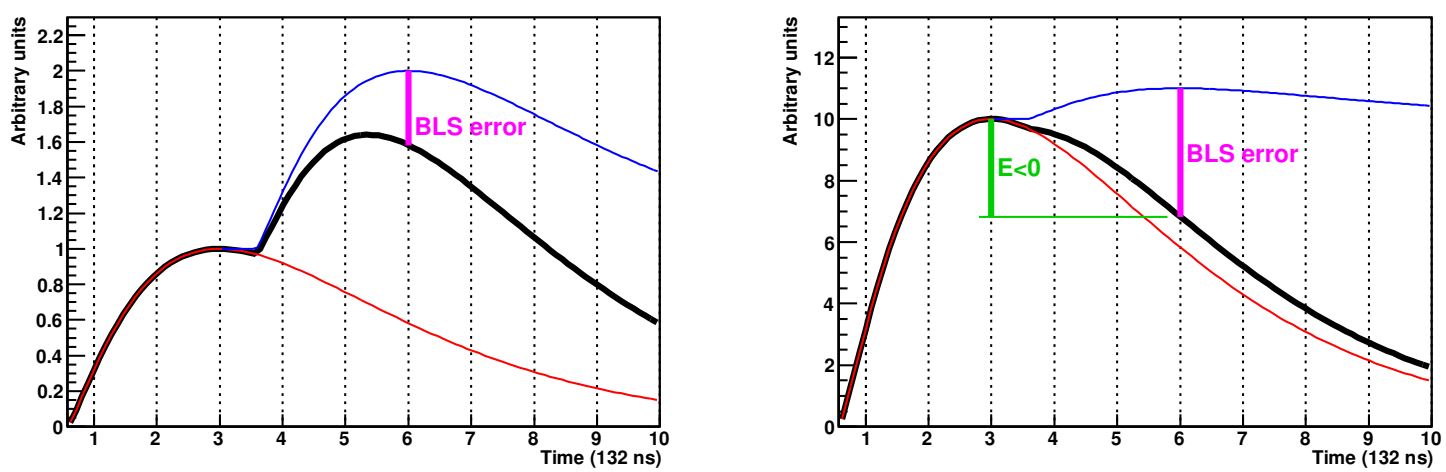

Figure 2.14: The BLS system always introduces a measurement error due to the decay of the previous waveform during the $396 \mathrm{~ns}$. (left) Two minimum bias signals when the error does not create negative energies. (right) A minimum bias signal after a "physics" signal, creating negative energy. 
The readout system always subtracted the mean value from the readout signal. For most events, there was a hardware-based $1.5 \sigma$ zero-suppression applied to all cells. During the Run II operations, D0 collected events with a zero-bias (ZB) trigger without any zerosuppression. This was a special trigger, based solely on the accelerator clock, that had constant low exposure and sampled the calorimeter sequentially in each tick with bunch crossing.

\begin{tabular}{|c|c|c|}
\hline \hline Layer & Pedestal RMS (ADC) & Pedestal RMS (MeV) \\
\hline CC (EM1) & 3.1 & 48 \\
EC (EM1) & 3.2 & 50 \\
CC (EM3) & 2.0 & 25 \\
CC (FH1) & 6.6 & 80 \\
CC (CH) & 6.4 & 297 \\
\hline \hline
\end{tabular}

Table 2.3: Typical pedestal RMS values for some layers of the D0 calorimeter.

During the reconstruction, the $1.5 \sigma$ zero suppressed signals were further suppressed keeping only positive energies above $2.5 \sigma$. In a later stage, a high-level T42.5 algorithm [44, 45] would suppress even further cells below $4 \sigma$ unless the cell was a 3D-neighbor of another cell already above $4 \sigma$.

\subsubsection{Anomalous Currents}

During the operations of the Central Calorimeter, a steady increase of the current drawn from the high-voltage (HV) sources was observed, as shown in Fig. 2.15. They caused sizable changes in the response of the D0 calorimeter. The goal of this section is to describe the cause, the effects and the modeling of the calorimeter readout system to large currents.

Large currents were present even in the absence of beam and were sometimes called dark currents. The effect is reminiscent of a phenomenon first observed by Günterschulze in the early 1930s [46] and by Malter in the late 1940s [47, 48]. In what today is known as the Malter process, a layer of oxide is formed over an anode which accumulates ions from the medium on its surface. Figure 2.16 has a scheme of the process. The field from the ions, when sufficiently intense, extracts electrons from the metal and increases the current. The oxide eventually breaks down and discharges the surface.

The D0 calorimeter is similar to the original Malter setup. A layer of uranium oxide $\left(\mathrm{UO}_{2}\right)$, if formed over the absorber surface, will collect argon ions in the gap. The field will build up, increasing the number of electrons tunneling through the oxide into the gap and contributing to the current in the resistive coats. The resistivity of uranium oxide at $83 \mathrm{~K}$ $\left(1.4 \times 10^{16} \Omega \mathrm{cm}\right)$ is very close to the resistivity of aluminum oxide at room temperature $\left(1.0 \times 10^{14} \Omega \mathrm{cm}\right)$ [49], the original insulator studied by Malter.

The anomalous current in the Central Calorimeter was known since right after the calorimeter assembly, from test beam and Run I measurements [50]. Unfortunately, both 


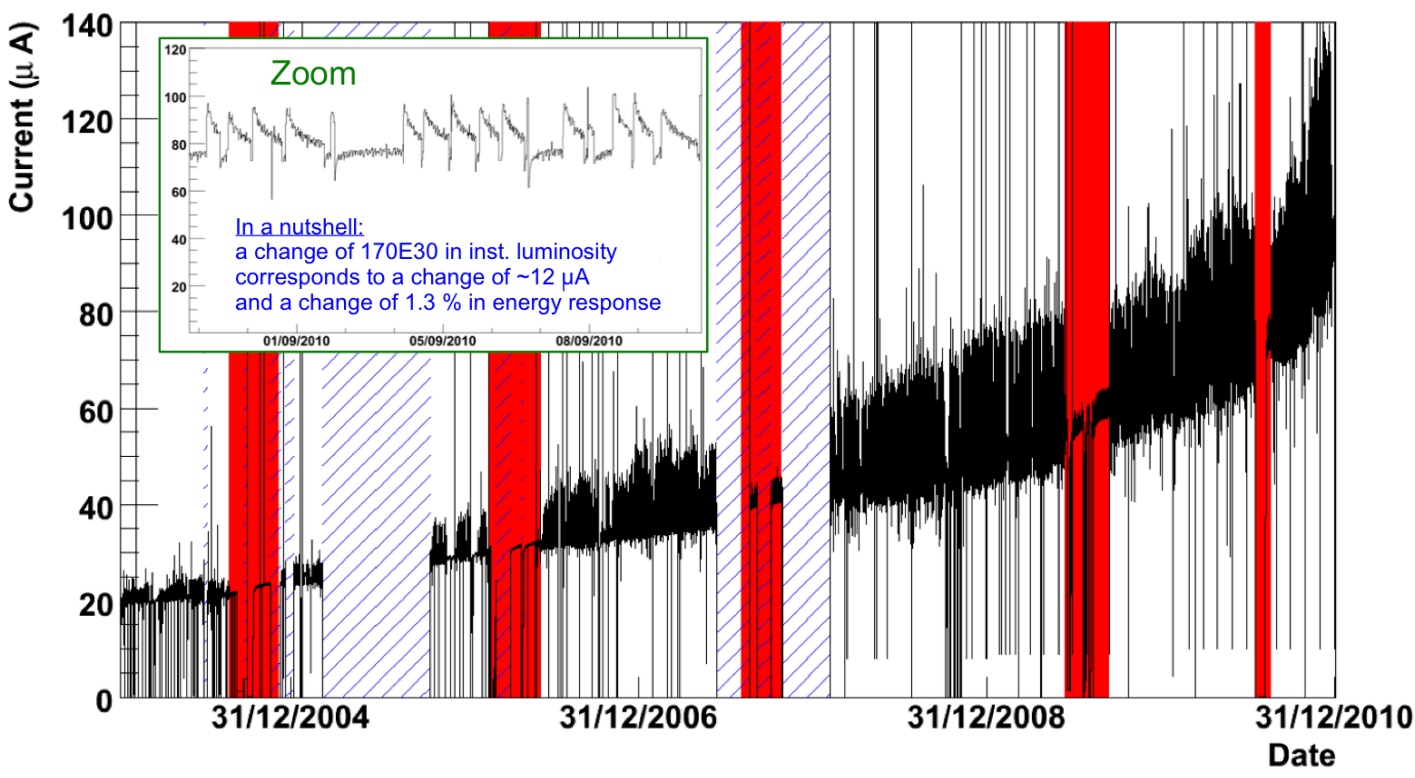

Figure 2.15: Long-term behavior of the current drawn from a HV supply that fed one of the CC EM4 modules. The hatched areas represent extended shutdowns of the Tevatron collider.

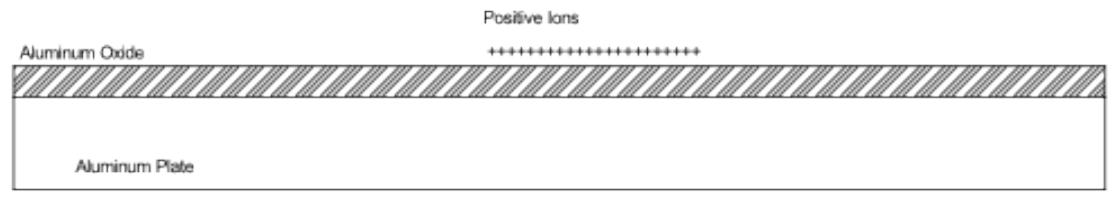

Figure 2.16: Scheme of the accumulation of ions responsible for the Malter process. The ion layer extracts electrons from the metal increasing the current until eventually the oxide breaks down and quenches the ions. 
data are no longer available. However, from 2002 to 2012, an online archive system recorded the HV current in each of the 32 central calorimeter HV supplies every 5 minutes and this data can be used to study the phenomenon. Occasionally, during 2010 to 2012, the monitoring rate was increased to $1 \mathrm{~Hz}$ to better understand the current behavior.

Figure 2.17 shows the current from the HV supplies right after they were turned on, without any beam at the Tevatron. The build-up curve is exactly the same as described by Malter in Fig. 12 of his paper [48]. An equivalent turn-on phenomenon was never observed in the End Calorimeters, since special care was taken in the construction against the accumulation of large and thick $\mathrm{UO}_{2}$ layers on the surface of the absorbers.
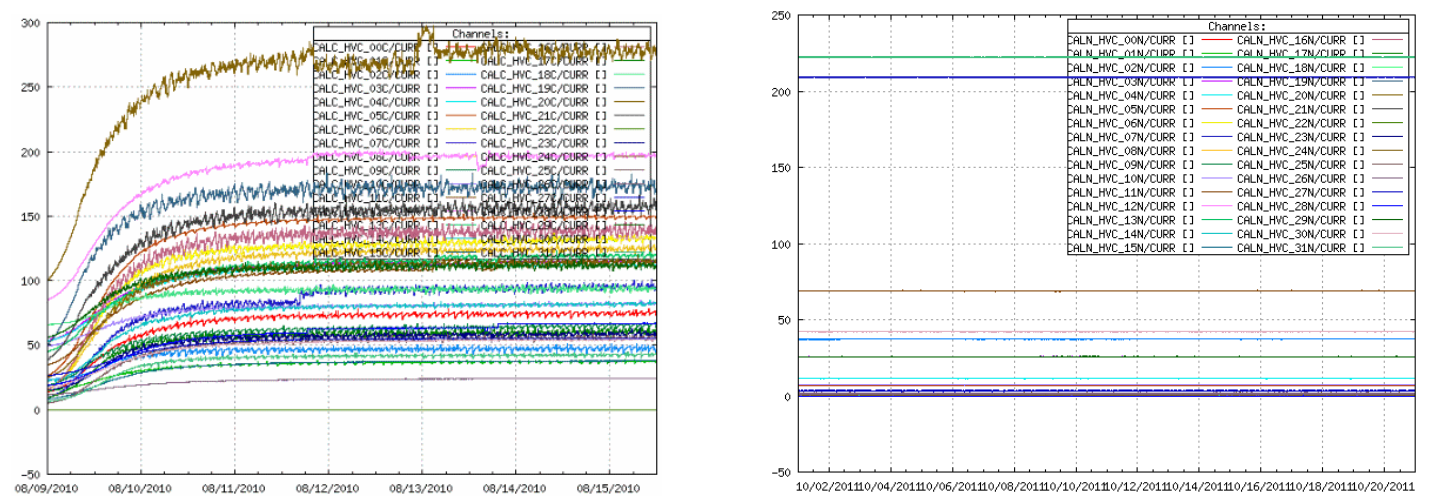

Figure 2.17: Slow turn-on curve of the current from the HV supply after turning it on in the absence of beam in the Tevatron. Left: Current from the 32 supplies that fed the Central Calorimeter modules. Right: North End Calorimeter, where no Malter process is observed. Although not shown, the south End Calorimeter supplies behave like the north ones. Note especially the difference in the vertical axis scale, in units of $\mu \mathrm{A}$ : currents in the $\mathrm{CC}$ are many times larger than the one in the $\mathrm{EC}$ in the absence of beam.

With interacting beams in the Tevatron, the current drawn from the CC HV supplies can be very large, since the charge from the energy flowing in the calorimeters adds to the already large baseline current. Figure 2.18 shows an interval of time with five Tevatron stores. It is easy to see the effect of the varying instantaneous luminosity as well as the build-up of charge between the stores 8 . It is also possible to see the quenching of the argon ions when the oxide breakdown by looking at a single store, as shown on Fig. 2.19.

The large dark currents together with the currents drawn in high luminosity runs of the Tevatron creates a visible sag in the HV across the high resistivity coating. With reasonable assumptions for the boundary conditions, a finite element calculation of the voltage drop can be seen on Fig. 2.20. The voltage drop creates a luminosity dependence of the otherwise stable gains of the liquid argon calorimeter. Since the HV is fed from the corners of the modules, it also generates a $\eta$ dependence of the gains.

\footnotetext{
${ }^{8}$ The observed current increase was slower with beams present due to the radiation damage in the $\mathrm{UO}_{2}$ structure that generate acceptor sites that trap electrons, increasing the effective resistivity [51].
} 

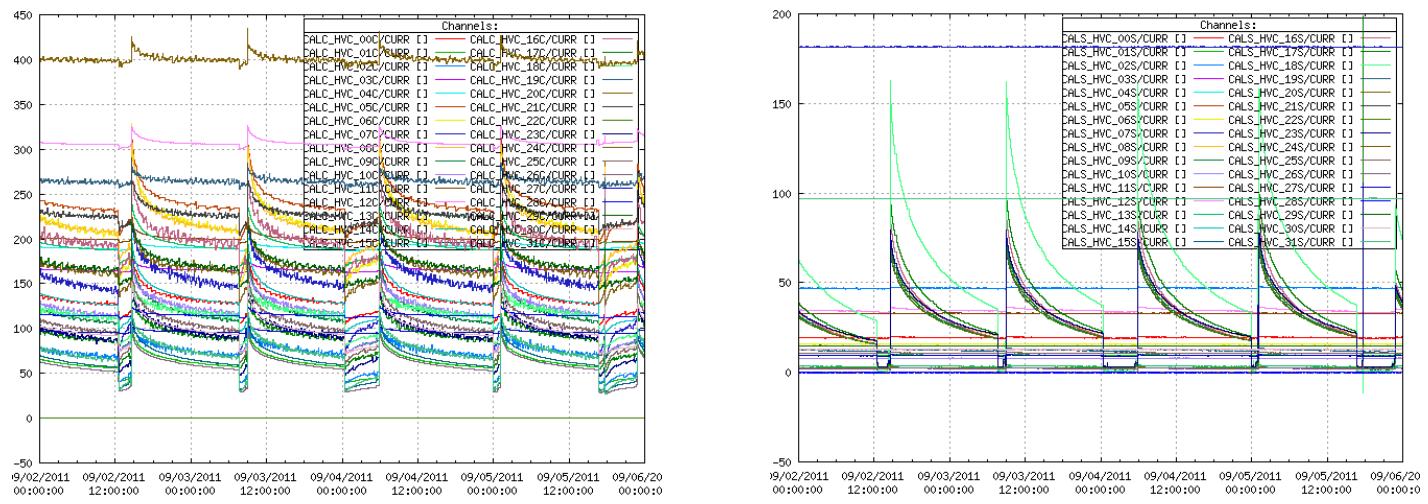

Figure 2.18: Behavior of the current from the HV supplies in the Central (left) and north End-Cap (right) Calorimeters during a period with many Tevatron stores. The plots clearly show the currents following the variation of the instantaneous luminosity and, in the Central Calorimeter, the Malter build-up of change between the stores.
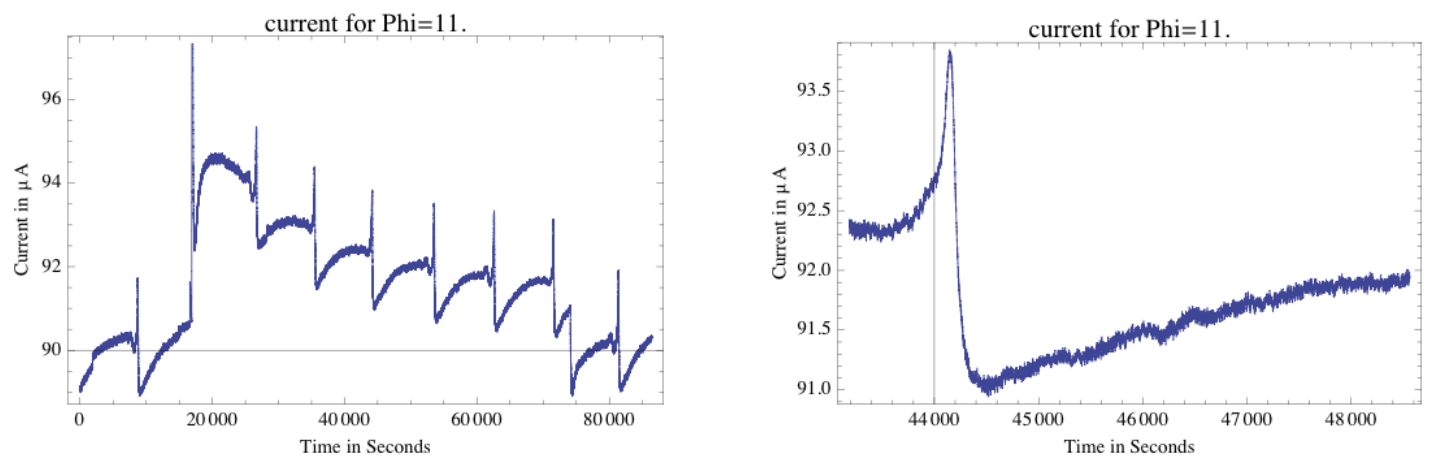

Figure 2.19: Malter breakdown. When enough ions accumulate on the surface of the uranium oxide, the insulation is broken and the argon ions quenched. The build up process begins anew and the cycle repeats.

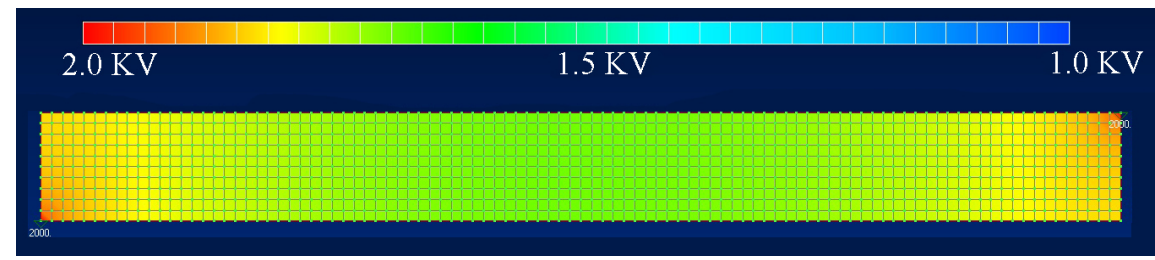

Figure 2.20: First principle calculation of the HV drop due to twice the normal current expected at $300 \times 10^{30} \mathrm{~cm}^{-2} \mathrm{~s}^{-1}$. The voltage variation can be as large as $20 \%$ at the center of the module. The HV is fed from two corners clearly seen on the figure. 
The importance of the HV drop was first observed by studying the luminosity dependence of the reconstructed position of the $Z \rightarrow e e$ invariant mass peak during high luminosity runs (see Fig. 2.21). Several phenomena were identified that could potentially generate the observed variation:

1. Energy flow from additional $p \bar{p}$ interactions in the same bunch crossing (pileup).

2. Negative energy from baseline subtraction.

3. Space-charge effects related to the accumulation of positive argon ions in the readout gaps [52].

4. Recombination of electrons and argon ions.

5. Reduction of HV due to losses across resistive material at high ionization currents.

Additional $p \bar{p}$ interactions increase the underlying energy, moving the apparent $Z$ mass up, while the other four effects generate a non optimal readout of the ionization charge and reduce the apparent $Z$ mass. The two first effects are properly taken into account in the simulation by using zero-bias overlay [53], while the other three are not usually considered. Space-charge effects and electron-ion recombinations can be shown to have very limited impact in the D0 calorimeter. Therefore, the only effect not modeled in the simulation is exactly due to the HV drop described here.

To model the effect of the HV drop we use a very simple resistive circuit equivalent of the distribution in the calorimeter modules as depicted in Fig. 2.22. As an input to the model, we need an estimate of the current drawn from each cell, which is not directly accessible from the HV current measurement, since a single supply feeds many liquid argon di-gaps. We use the ionization energy from unsuppresed zero-bias events to estimate this current. There are many shortcomings in this choice. First, the ionization energy in zerobias events is very close to one ADC count, which introduces large quantization errors. Even if the ionization energy was measured exactly, the readout system only samples a small fraction of the total ionization charge. Thus, late ionization charges, like those from thermal neutrons, are not taken into account.

The measured average ionization energy $\bar{E}$ is translated into an incomplete $I_{\text {vis }}$ estimate of the current using the relation:

$$
I_{\mathrm{vis}}=\frac{f w \bar{E} s}{n_{g}} \times \frac{2 e}{E_{\mathrm{ion}}}
$$

where $f$ is the bunch crossing frequency, $w$ is the inverse of the weight factor applied in the energy reconstruction (see Sec. 13.2 and, in particular, table 13.2), $s$ is the sampling fraction, $n_{g}$ is the number of LAr gaps ganged together in the cell, $e$ is the electron charge and $E_{\text {ion }}=15.8 \mathrm{eV}$ is the ionization energy of the liquid argon. With this initial estimate, we sum the currents from the cells of a common HV supply and compare to the measured current to derive a global correction factor $I=\alpha I_{\mathrm{vis}}$ of $\alpha=2.5$. The equivalent resistive 


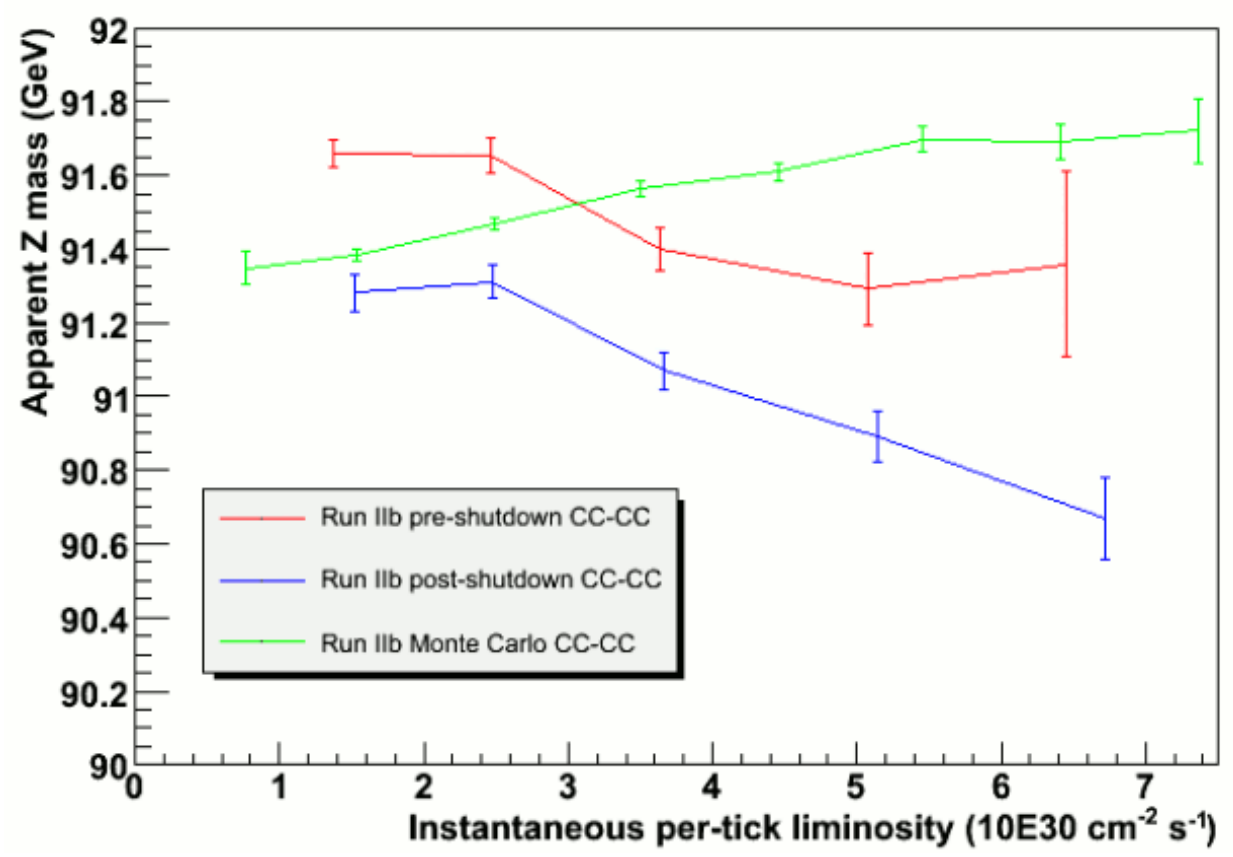

Figure 2.21: Apparent position of the $Z \rightarrow e e$ mass peak in collider data CC-CC events before and after a long shutdown in Run IIb (red and blue points, respectively). The green points are from full MC simulations with unsuppresed zero-bias overlay that takes into account the energy flow from pileup and the negative energy from baseline subtraction, but not the HV sag due to the large currents in the central calorimeter.

circuit is used to translate the currents from the cells in a module to the voltage drop before each gap. Finally, since the calorimeter readout is essentially an "initial current readout", we use the measured relation between the HV and electron drift velocity [54] to translate the voltage drop into a drop of the initial current:

$$
i_{e}=q \frac{v_{e}(V)}{\Delta X}
$$

where $\Delta X$ if the gap width and $v_{e}(V)$ the drift velocity as a function of the voltage $V$. This model was introduced in the D0 simulation and is used in some parts of this Dissertation.

\subsection{The D0 Single Electron Trigger}

The D0 trigger system was composed of three levels L1, L2 and L3. The L1 and L2 triggers were programmed in dedicated hardware that performed fast calculations of low-level physical observables, while the L3 was a computer farm that performed a fast reconstruction of the events and the decision was made on high-level physical objects reconstructed 


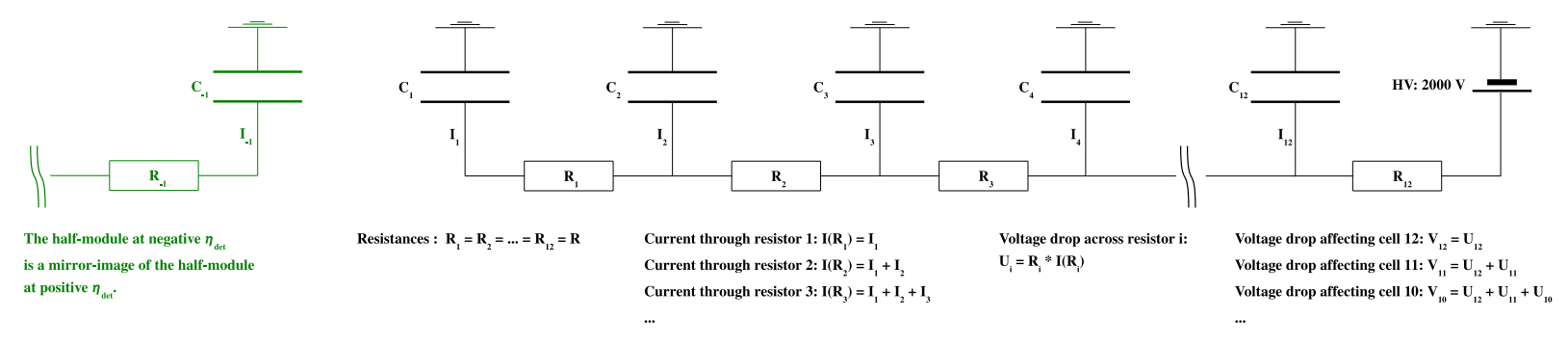

Figure 2.22: Resistive circuit that models the HV distribution in the calorimeter modules. This equivalent circuit is used to translate the current estimates into the HV available at the gaps of each cell with which the change in drift velocity can be calculated.

from the full read out. Figure 2.23 shows the rates of these triggers in a typical store. The L1 trigger reduced the rate of events from $1.7 \mathrm{MHz}$ down to a range of 0.9 to $1.8 \mathrm{kHz}$. The L2 further reduced this rate to a range between 500 and $900 \mathrm{~Hz}$. Finally, the L3 output was reduced to a rate between 100 and $200 \mathrm{~Hz}$, and those events were written to the tape system. The typical variation of these rates during a Tevatron store can be seen on Fig. 2.23.

Here we concentrate on the trigger used for the $W$ boson mass measurement. We used single electron, calorimeter-only based triggers [55]. This allowed unbiased tagand-probe efficiency studies.

The level 1 calorimeter (L1CAL) trigger received an analog signal from the BLS system with the sum of each EM and HAD trigger tower. This signal was digitized at 4 times the frequency corresponding to a bunch crossing of $132 \mathrm{~ns}$ and passed through a 8-tap 10-bit finite impulse response (FIR) filter and subsequent peak detector. After the frequency has been reduced to the bunch crossing, the peak signal was then used with a look up table to assess the transverse energy for that trigger tower.

The same processing was applied to all 2560 trigger towers. The trigger towers did not use the full granularity of the D0 calorimeter, but were $0.2 \times 0.2$ wide in $\eta \times \phi$ space. Even so, a single calorimeter trigger tower contained only a small fraction of the energy of a shower. This was especially true for jets. Thus, after digitization, the calorimeter L1 trigger used even coarser regions, composed of $2 \times 2$ trigger towers, to look for local depositions of energy. It used a sliding window algorithm, studied extensively by the ATLAS collaboration [56], to look for the $2 \times 2$ local maximum (window) of transverse energy $E_{T}$ in regions of interest (ROI) $5 \times 5$ wide. The $2 \times 2$ window selected, together with the neighbor towers, were used to estimate the $E_{T}$ of the shower.

The triggers used in this measurement, E1_SHT25 and E1_SHT27, required a trigger $E_{T}$ higher than $19 \mathrm{GeV}$ at L1 in any region of interest with $\left|\eta_{\text {det }}\right|<3.2$. For L2, these triggers had two pathways. If the trigger $E_{T}$ was larger than $25 \mathrm{GeV}$, it was automatically accepted. If it was between 19 and $25 \mathrm{GeV}$, a likelihood for the energy deposition to be an 


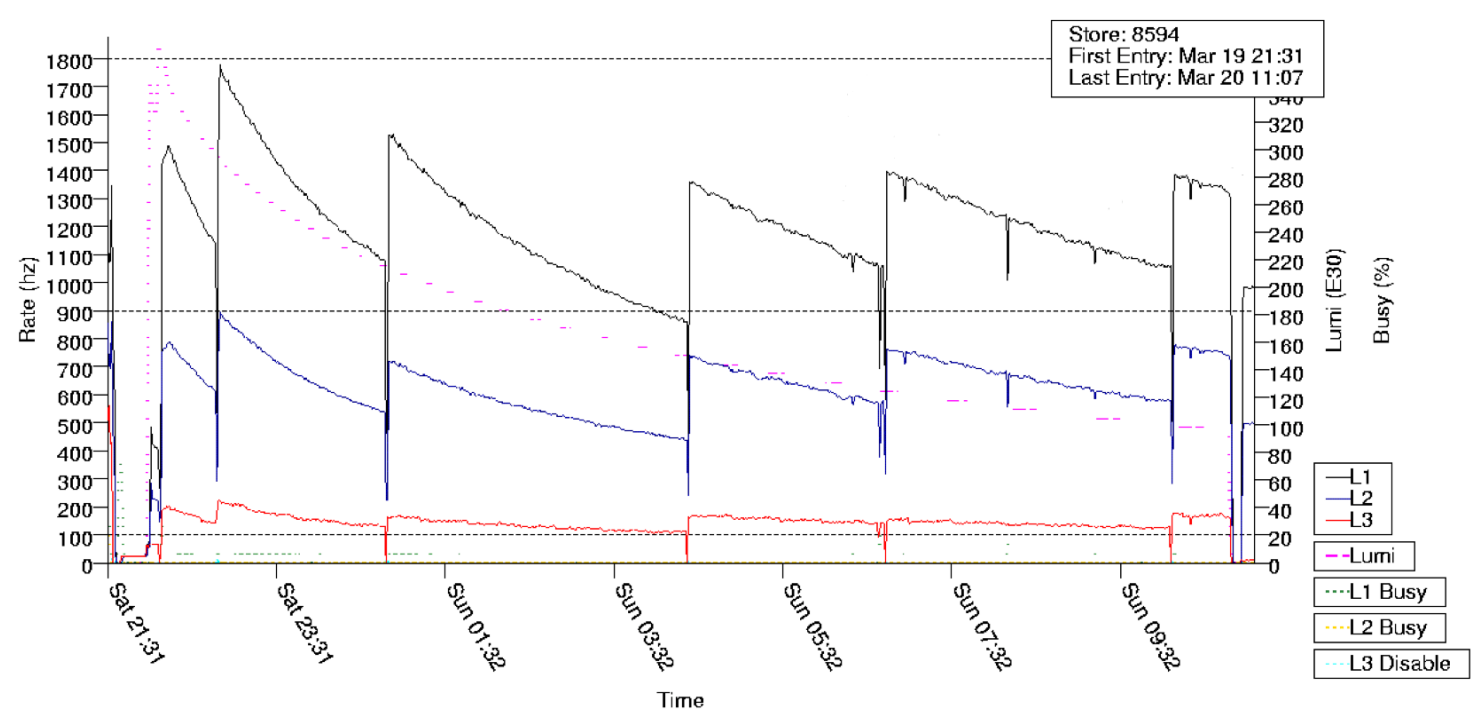

Figure 2.23: Typical Tevatron store. The black line shows the rate of the L1 trigger output, the blue line the rate of the L2 trigger output and, finally, the red line shows the rate of output of L3 that was being written on tape. The dashed magenta line shows the decrease of instantaneous luminosity during the store. The discontinuities in the triggers rates indicate the start of new D0 runs when the trigger rates were adjusted to accept more events.

electromagnetic shower was calculated 9 The likelihood was calculated using the 9 trigger towers around the seed tower with largest $E_{T}$ in the sliding window. The likelihood included the following observables: EM fraction of the seed trigger tower, EM fraction of $3 \times 3$ region around seed trigger tower, isolation of the seed trigger tower with respect to the $3 \times 3$ region around it, and ratio of EM fraction of the seed to the EM fraction of the 3 $\times 3$ region around it. Finally, the L3 triggers require a reconstructed electron with transverse energy larger that 25 (27) GeV for the E1_SHT25 (E1_SHT27) trigger and calorimeter $|\eta|<3.6$. The calorimeter $\eta$ was calculated with an energy weighted average using the full granularity of EM3 $(0.5 \times 0.5$ in $\eta \times \phi$ space $)$. The weights were different depending on the region of the calorimeter the cluster was reconstructed.

\footnotetext{
${ }^{9} \mathrm{~A}$ previous version of this trigger, referred in this Dissertation as v15, used to consider only isolation between 19 and $22 \mathrm{GeV}$ and accept clusters above that. The version described in more details in the text will be referred as v16. These versions are not the trigger versions, but the version of the trigger menu used by the D0 DAQ.
} 


\section{Part II}

\section{W Boson Mass Measurement with Central Electrons}




\section{Chapter 3}

\section{Measurement Strategy}

\subsection{Conventions}

A momentum vector $\vec{p}$, in the D0 standard coordinate system, is represented by its cartesian coordinates, $p_{x}, p_{y}, p_{z}$ where $\hat{z}$ is the direction of the proton beam and $\hat{y}$ points upward. It is convenient to use a cylindrical coordinate system in which the same vector is given by the magnitude of its components perpendicular to the beam direction called transverse momentum, $p_{T}$, its azimuthal angle $\phi$, and $p_{z}$. In some situations, spherical coordinates are used, in which $p_{z}$ is replaced by either the polar angle $\theta$ or the pseudorapidity $\eta=-\ln \tan (\theta / 2)$. When referring to instrumental effects, sometimes it is convenient to define $\eta_{\text {det }}$ as the pseudorapidity the particle would have had it been produced at $z_{V}=0$ and reached the calorimeter at the same distance from the axis of the detector. We use units in which $\hbar=c=1$ throughout this dissertation.

\subsection{Event Characteristics}

We measure the decays of the $W$ boson in the electron channel $W \rightarrow e v$ and at the same time measure $Z \rightarrow$ ee decays which provide an important calibration sample. In this dissertation, by electron we mean electron or positron unless specifically noted. The electrons typically have transverse momenta of about half the mass of the decaying boson and are well isolated in the calorimeter. Isolated high $p_{T}$ electrons are dominantly produced by $W$ and $Z$ decays and allow us to select a clean sample of $W$ and $Z$ boson events. The D0 calorimeter is well-suited for a precise measurement of electron energies, providing a single electron energy resolution $4.5 \%$ averaged over the electron acceptance in this analysis with the angular and energy spectrum of electrons from $W$ boson decay.

In the process $p \bar{p} \rightarrow(W \rightarrow e v)+X$ we select the electron by requiring $\left|\eta_{\text {det }}\right|<1.05$ and use all other particles detected up to $\left|\eta_{\text {det }}\right|<4.2$ for the hadronic recoil measurement. We cannot detect recoil particles with $\left|\eta_{\text {det }}\right|>4.2$, but their transverse momenta are small and can be neglected in the recoil system transverse momentum, $\vec{u}_{T}$. 

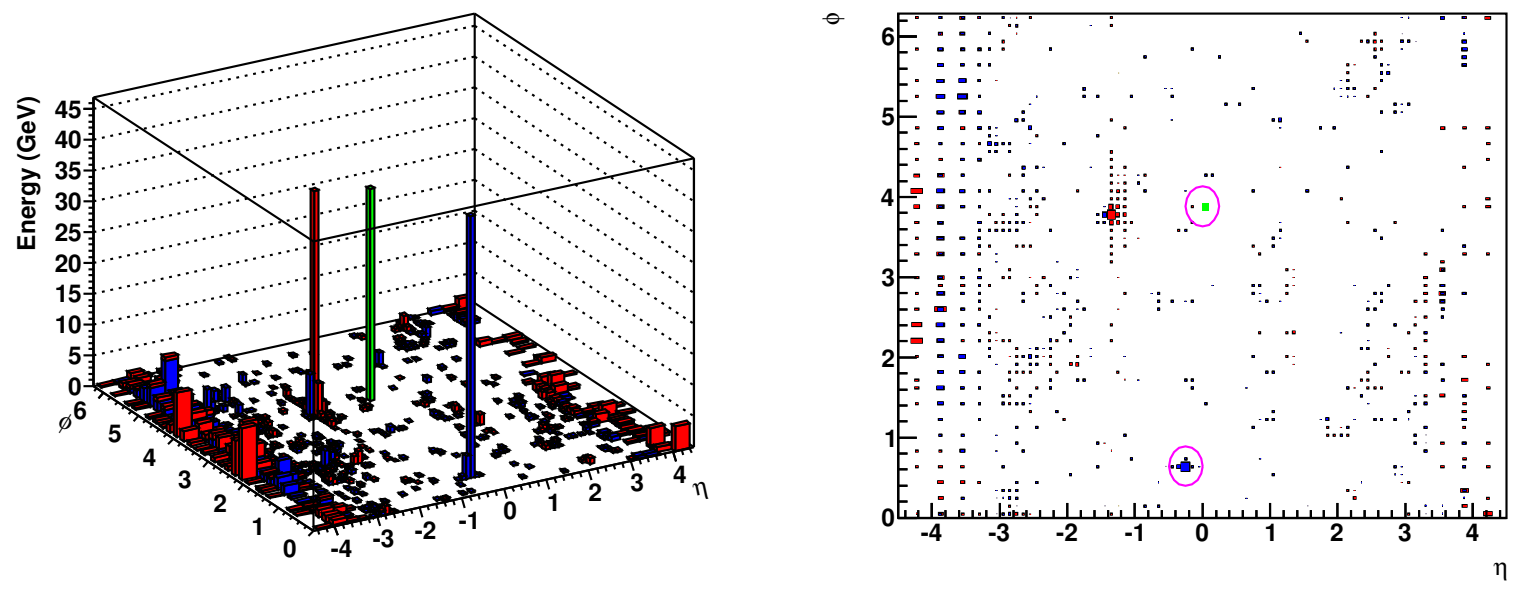

Figure 3.1: Deposit of energy in each calorimeter cell for a $W$ event candidate (run number 223477, event number 45476599). The blue bars indicate the deposit in the electromagnetic calorimeter while the red bars indicate the energy in the hadronic calorimeter. The green box at $\eta=0$ represents the event missing transverse energy. There are two large and clustered deposits of energy in the event, but only one is predominantly electromagnetic and identified as the electron.

A candidate $W$ boson event is characterized by a measurement of the electron momentum $\vec{p}^{e}$ and $\vec{u}_{T}$. The neutrino escapes undetected but the magnitude and direction of its transverse momentum are inferred from the event missing transverse energy, $\vec{E}_{T} \equiv-\left(\vec{p}_{T}^{e}+\vec{u}_{T}\right)$. The signature of a $W \rightarrow e v$ decay is therefore an isolated high- $p_{T}$ electron and large missing transverse momentum. An example of a $W$ candidate is shown in Fig. 3.1.

The signature of $Z \rightarrow$ ee decay consists of two isolated high- $p_{T}$ electrons. In a manner similar to candidate $W$ boson events, a candidate $Z$ boson event is characterized by a measurement of the two electron momenta and $\vec{u}_{T}$.

\subsection{Mass Measurement Strategy}

Since we cannot reconstruct the longitudinal component of the neutrino momentum, we must resort to variables different from the invariant mass. To measure the $W$ mass we use the following three kinematic variables: the $W$ transverse mass $m_{T}$, the electron transverse momentum $p_{T}^{e}$, and the neutrino transverse momentum $p_{T}^{v}\left(\mathbb{E}_{T}\right)$. In principle, the electron longitudinal momentum could also be used [57], but it not explored further in the work described in this Dissertation.

The $m_{T}$ and $p_{T}^{e}$ measurements provide a powerful cross-check because of their complementary systematic uncertainties. The shape of the $m_{T}$ distribution is dominated by the 
detector resolution (mainly the resolution due to the recoil system energy measurement), while the $p_{T}^{e}$ spectrum is affected by the transverse momentum of the $W$ boson, and hence the recoil system and initial-state radiation.

The $p_{T}^{v}$ measurement is sensitive to the same systematic uncertainties as both $m_{T}$ and $p_{T}^{e}$ and has significantly poorer experimental resolution, but this measurement is still useful for a cross-check. Moreover, since the correlations among these three measurements are not $100 \%$, we can combine these results.

Because of the various detector acceptance and resolution effects, the shapes of the distributions of these variables cannot be calculated analytically. The measurement of $M_{W}$ is obtained by a comparison of the spectra of the three different measurement variables with templates generated from Monte Carlo simulation with varied $W$ masses. This requires templates with very high statistics ( $\sim 10^{9}$ events) to characterize the different systematic uncertainties while ensuring that statistical fluctuations from the Monte Carlo simulation are negligible. The detailed D0 detector simulation (full $\mathrm{MC}$ ) is too slow to generate many samples of this size, and it also does not reproduce the detector performance in data with sufficient precision to measure the $W$ boson mass. To generate appropriate templates, a parametrized Monte Carlo simulation (fast MC) has been developed to generate large samples on a reasonable time scale and to provide a detailed description of the detector performance. $Z \rightarrow e e$ events are used to determine the parametrization. This allows a determination of the fast MC parameters, including details of the hadronic recoil system, from the data itself. Since the $Z$ boson mass is known with a high precision, its value can be used to calibrate the energy scale of the electromagnetic (EM) part of the calorimeter. Care must be taken to ensure that the calibrations using the $Z$ boson are valid at the somewhat lower average energy of the electrons from $W$ boson decay. Once this has been established, the $W$ boson mass measurement is, to a good approximation, a measurement of the ratio of $W$ boson and $Z$ boson masses.

A binned likelihood comparing collider data and simulated events (a template) is computed for each of the $m_{T}, p_{T}^{e}$ and $\mathbb{Z}_{T}$ distributions. Since the templates have many more events than the data, the likelihoods are calculated using the Poisson probability for bin $i$ with $m_{i}$ expected events from the template to have $n_{i}$ observed events from the data distribution with the total likelihood formed from the product over all bins in a distribution:

$$
\mathcal{L}=\prod_{i=1}^{N} \frac{e^{-m_{i}} m_{i}^{n_{i}}}{n_{i} !}
$$

We take the logarithm of the likelihood and find

$$
-\ln \mathcal{L}=\sum_{i=1}^{N}\left(-n_{i} \ln m_{i}+m_{i}+\ln \left(n_{i} !\right)\right) .
$$

Templates are generated for different hypothetical $W$ boson mass values with $10 \mathrm{MeV}$ steps between values. This procedure effectively gives a mass-dependent likelihood for each of the $m_{T}, p_{T}^{e}$ and $\mathbb{E}_{T}$ distributions. We then measure $M_{W}$ using MINUIT [58] to 
find the mass value which maximizes the mass-dependent likelihood. The minimization is performed separately for each of the three observables, and during the minimization, interpolation is performed between templates produced at the discrete input mass hypotheses.

The precision needed for this analysis merits special care that the $M_{W}$ measurement not be biased toward or away from the current world average value. To this end a blinded analysis procedure has been developed. The code that provides the template fits uses an unknown but recoverable offset in an interval of $[-2,2] \mathrm{GeV}$ around the $M_{W}$ value with which the templates have been generated. It therefore reports true differences between different mass fits, allowing systematic studies, while keeping the measured $M_{W}$ value unknown. The same offset is applied to the result of the fit to $m_{T}, p_{T}^{e}$ and $\mathbb{E}_{T}$ so that the relative agreement between the three observables is visible.

\subsection{Additional Kinematic Variables}

In $Z \rightarrow e e$ decays the di-electron momentum is given by $\vec{p}_{e e}=\vec{p}^{e_{1}}+\vec{p}^{e_{2}}$ and the dielectron invariant mass is: $m_{e e}=\sqrt{2 E^{e_{1}} E^{e_{2}}(1-\cos \omega)}$ where $\omega$ is the opening angle between the two electrons. When tuning the simulation and making comparisons with $Z \rightarrow e^{+} e^{-}$data, it is useful to define a coordinate system, first introduced by UA2 [24], in the plane transverse to the beams that depends only on the electron directions, but not on electron energies. We call the axis along the inner bisector of the two electrons the $\eta$ axis and the axis perpendicular to that the $\xi$ axis. Figure 3.2(a) illustrates these definitions.

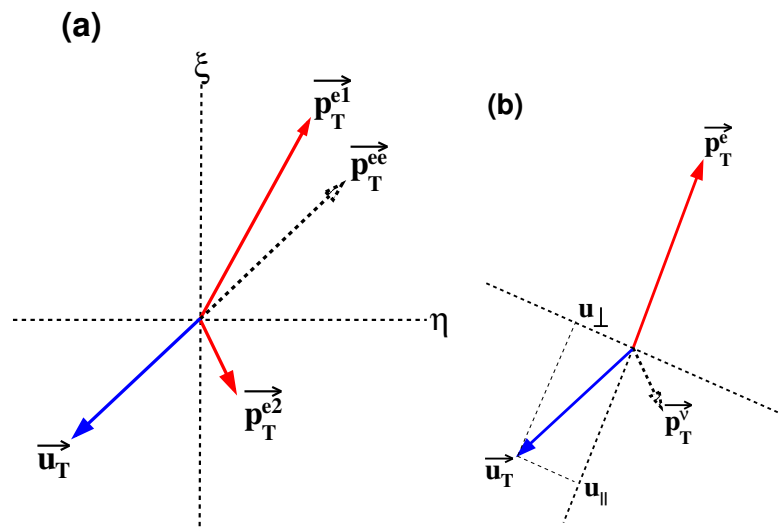

Figure 3.2: a) Definition of $\eta$ and $\xi$ axis for $Z \rightarrow e e$ events. b) Definition of $u_{\|}$and $u_{\perp}$. As can be seen in this figure, $u_{\|}$is negative when opposite to the electron direction.

For $W \rightarrow e v$ decays, useful quantities are the projection of the recoil system transverse momentum on the electron direction:

$$
u_{\|}=\vec{u}_{T} \cdot \hat{p}_{T}^{e}
$$


and the projection on the direction perpendicular to the electron:

$$
u_{\perp}=\vec{u}_{T} \cdot\left(\hat{p}_{T}^{e} \times \hat{z}\right)
$$

Figure 3.2(b) illustrates these definitions for $W$ events but it is also useful for each electron from $Z \rightarrow e e$ events.

The two variables $u_{\|}$and $u_{\perp}$ are useful to study the correlation between the recoil system and the electron direction. Another variable, the scalar sum of all transverse energies (Scalar $E_{T}$ or SET) measured by the calorimeter except those energies associated with electrons, reflects the total hadronic activity in the calorimeter. 


\section{Chapter 4}

\section{Data Reconstruction}

The data sample for this measurement (Run $\mathrm{IIb}$ ) includes data with a total integrated luminosity of $4.3 \mathrm{fb}^{-1}$ taken between June 2006 and June 2009. Figure 4.1 compares the instantaneous luminosity profile of this Run IIb measurement with the profile of our previous [32] measurement using data recorded from 2002-2006 (Run IIa). The Run IIb instantaneous luminosity is much higher and much of the effort for this measurement is spent in dealing with the multiple interactions (pileup) and calorimeter gain variation resulting from high beam intensity in Run IIb.

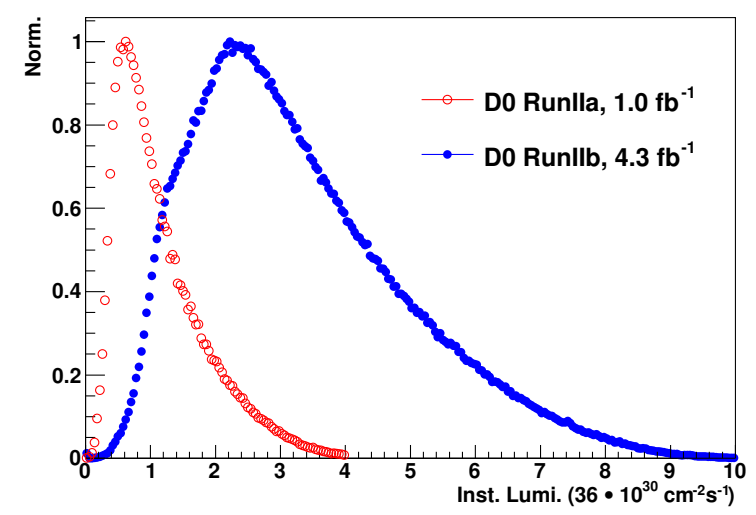

Figure 4.1: Instantaneous luminosity profiles for Run IIa and Run IIb. The Run IIb $W$ candidate events have an average of two additional reconstructed primary vertices while this number was below one during Run IIa.

This high beam instantaneous luminosity results in extra $p \bar{p}$ interactions in the same beam crossing as the event of interest. We measure the effect of this pileup by collecting $p \bar{p}$ interactions in random beam crossings which are labeled zero-bias (ZB) events. There are also extra interactions not due to the hard parton-parton collision of interest coming from the reactions of spectator partons in the same $p \bar{p}$ collision as the hard col- 
lision. These extra interactions are studied using minimum-bias (MB) events, which are selected by requiring a coincidence between Luminosity Monitor scintillation counters. They are selected from MB events by requiring zero or one reconstructed primary (hard collision) vertex. The number of multiple interactions accompanying an event of interest scales with luminosity, while the contribution of spectator partons is independent of it 1 .

\subsection{Electron Reconstruction}

The measured EM energy associated with an electron $\left(E_{\mathrm{EM}}^{\mathrm{raw}}\right)$ in the central calorimeter is the sum of the energies in all EM cells whose centers lie in a cone of radius $\Delta R=0.2^{2}$ centered on the tower with the highest transverse energy. The precise definition of the electron energy reconstruction window is shown in Fig. 4.2 . The total energy $E_{\mathrm{Tot}}^{\mathrm{raw}}(\Delta R)$ is the sum of the energies in all nearest-neighbors cells for a given distance $\Delta \bar{R}=2$ centered on the highest $p_{T}$ tower, where $\bar{R}$ counts the distance in number of cells, over all layers of the calorimeter.

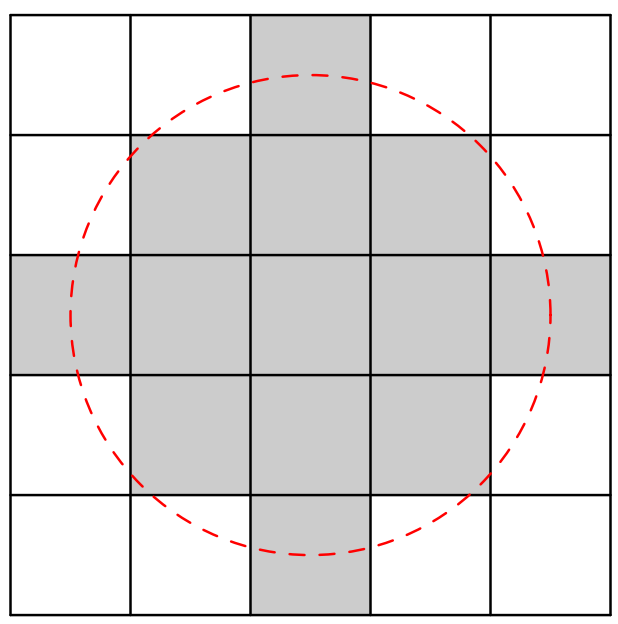

Figure 4.2: The 13 calorimeter towers defined as the electron reconstruction window. The window is centered on the tower with the highest transverse energy. A circle of radius $\Delta R=0.2$ is shown for comparison.

In the EC, the definition of the electron cluster does not have a fixed window size, but

\footnotetext{
${ }^{1}$ The model described here is called "ZB plus one scatter" model, where the one scatter is described by the MB events. It describes very well the underlying event for events with large number of reconstructed primary vertices but fails at low primary vertice multiplicity. Therefore, the MB library is reweighted using the SET of $Z \rightarrow e e$ events with low $Z$ transverse momentum and collected at low luminosity[59]

${ }^{2}$ In this Dissertation we define $\Delta R=\sqrt{(\Delta \eta)^{2}+(\Delta \phi)^{2}}$
} 
is determined by the formula:

$$
\Delta \bar{R}=\left\lfloor 1+\frac{64}{2 \pi} \times \frac{r_{\mathrm{EC}}}{r_{\mathrm{est}}}\right\rfloor
$$

where $r_{\mathrm{EC}}=10 \mathrm{~cm}, r_{\mathrm{est}}$ is the distance from the hottest cell in the cluster to the calorimeter cylindrical axis $r_{\text {est }}=\sqrt{x_{\text {hot }}^{2}+y_{\text {hot }}^{2}}$ and $\Delta \bar{R}$ is the maximal distance that a nearestneighbor may lie and still be a candidate to be included in the cluster (for instance, for the CC, we would define $\Delta \bar{R}=2$ ). In EC clusters, there is also a depth $(z)$ limitation with respect to the hottest cell in the cluster 3 . A cell whose coordinate $\left(x_{\text {cell }}, y_{\text {cell }}\right)$ does not satisfy

$$
\sqrt{\left(x_{\text {hot }}-x_{\text {proj }}\right)^{2}+\left(y_{\text {hot }}-y_{\text {proj }}\right)^{2}}<r_{\mathrm{EC}} ; \quad x, y_{\text {proj }}=x, y_{\text {cell }} \times \frac{z_{\text {hot }}}{z_{\text {cell }}}
$$

is not included in the cluster, even if it is within the window determined by Eq. 4.1. Owning to the variable size of the window, the face size ${ }^{4}$ of an EM cluster is strongly dependent on its position, as shown in Fig. 4.3.
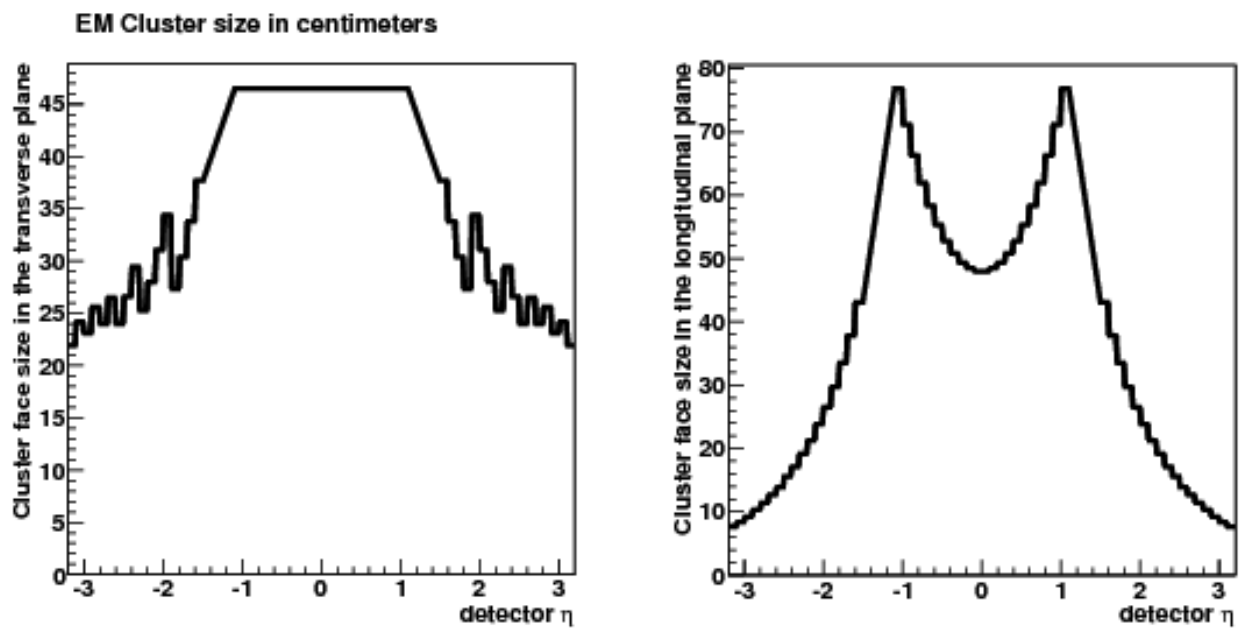

Figure 4.3: Face size, in $\mathrm{cm}$, of an EM cluster as a function of the cluster detector $\eta$. The left plot shows the maximum diameter, in $\mathrm{cm}$, of an EM cluster in the $\Delta \phi$ direction, while the right plot shows the maximum diameter, also in $\mathrm{cm}$, of an EM cluster in the $\Delta \eta$ direction.

To identify this cluster of EM energy as resulting from the detection of a true electron, four parameters are used as defined below:

\footnotetext{
${ }^{3}$ The reconstruction algorithm prefers cells in EM3 as seed cell for an EC cluster and will only choose an EM1, EM2 or EM4 cell if its energy is larger than ten times the energy of the hottest cell in EM3.

${ }^{4}$ We define the face size as the maximum diameter, in EM3, of an EM cluster.
} 
- EM fraction: A true electron will deposit nearly all of its energy in the EM layers of the calorimeter. Therefore the EM fraction

$$
f_{E M} \equiv \frac{E_{\mathrm{EM}}^{\mathrm{raw}}(\Delta R<0.2)}{E_{\mathrm{Tot}}^{\mathrm{raw}}(\Delta R<0.2)}
$$

is expected to be close to 1 .

- Isolation: In an electron shower most of the energy is deposited in a tight cone with little energy around it. Therefore

$$
f_{\text {iso }} \equiv \frac{E_{\mathrm{Tot}}^{\mathrm{raw}}(\Delta R<0.4)-E_{\mathrm{EM}}^{\mathrm{raw}}(\Delta R<0.2)}{E_{\mathrm{EM}}^{\mathrm{raw}}(\Delta R<0.2)}
$$

is expected to be close to 0 . Since jets tend to generate a wider shower, the isolation criterion is a strong criterion for jet rejection.

- HMatrix: The transverse and longitudinal shapes of an electron shower are well modeled by Monte Carlo simulations. Therefore it is possible to compare the shape of the EM cluster to that of an electron in Monte Carlo to create a covariance matrix. The inverse of this matrix is used to determine a $\chi_{H M}^{2}$ value, which should be small if the cluster is likely to be an electron. [60]

HMatrix7 (used in the CC) is built from the following variables: EM fractions in layers $1,2,3,4$, shower energy weighted RMS in the $\phi$ direction, $\log \left(E_{\mathrm{Tot}}^{\mathrm{raw}}\right)$, and $z_{V} / \sigma_{z}$ (the vertex $z$ position, $\sigma_{z}$ is defined below).

HMatrix8 (used in the EC) is built from the same variables as HMatrix7 plus the shower energy weighted RMS in $z$.

- Track Match: A track is reconstructed from SMT and CFT hits and is required to have $p_{T}>10 \mathrm{GeV}$. It is considered to be matched with an EM cluster if it is within 0.05 in $\Delta \eta$ and 0.05 in $\Delta \phi$ of the cluster centroid. The quality of the match is determined by

$$
\chi_{\mathrm{TM}}^{2} \equiv\left(\frac{\Delta \phi}{\sigma_{\phi}}\right)^{2}+\left(\frac{\Delta z}{\sigma_{z}}\right)^{2}
$$

where $\phi$ and $z$ are found by extrapolating the track to the third EM layer of the calorimeter. Here $\sigma_{\phi}$ and $\sigma_{z}$ are the measured RMS resolutions of $\Delta \phi$ and $\Delta z$.

In the initial reconstruction, electromagnetic clusters are required to have transverse energy $E_{T}^{\text {raw }}>1.5 \mathrm{GeV}$ and $\mathrm{EM}$ fraction $f_{\mathrm{EM}}>0.9$. If the cluster has a track loosely $(\Delta R<0.2)$ matched to it, it is considered a candidate electron. Photons are reconstructed when similar criteria are satisfied, but without an associated track.

After electron identification is completed for $W \rightarrow e v$ and $Z \rightarrow e e$ events (Sec. 4.6), each electron window is defined and the energy of an electron $E^{e, \text { raw }}$ is redefined as the sum of the energies in all four electromagnetic calorimeter (EM1 to EM4) and first fine 
hadronic layer (FH1) cells in the 13 towers of the electron window (Fig. 4.2) centered on the tower with the highest transverse energy:

$$
E^{e, \text { raw }}=\sum_{i} E_{i}^{\text {raw }}
$$

The FH1 layer is included to more fully contain the electromagnetic shower. The corrected electron energy $E^{e}$ is defined by applying the dead material correction (Sec. 5).

In this analysis, the direction of the electron is always taken as the direction of the matched track.

$$
\begin{aligned}
& \theta^{e}=\theta_{\text {track }} \\
& \phi^{e}=\phi_{\text {track }}
\end{aligned}
$$

The momentum of the electron, ignoring its mass, is given by

$$
\vec{p}^{e}=E^{e}\left(\begin{array}{l}
\sin \theta^{e} \cdot \cos \phi^{e} \\
\sin \theta^{e} \cdot \sin \phi^{e} \\
\cos \theta^{e}
\end{array}\right)
$$

and the transverse energy of the electron is defined as $p_{T}^{e}=E^{e} \sin \theta^{e}$. Corresponding to this definition, the raw transverse energy of the electron is given by $p_{T}^{e, \text { raw }}=E^{e, \text { raw }} \sin \theta^{e}$, without the dead material correction.

\subsection{Vertex Reconstruction}

The $W$ boson production point along the beam line $z_{V}$ is determined either using the standard D0 primary vertex algorithm (which uses a Kalman filter algorithm [61]), or is taken as the point of closest approach of the electron track to the beam line if this electron track vertex position differs by more than $2 \mathrm{~cm}$ from the point selected by the primary vertex algorithm. For $Z$ boson events the $z_{V}$ is taken to be the average of the two points of closest approach of the electron tracks. The production point must satisfy the condition $\left|z_{V}\right|<60 \mathrm{~cm}$.

\subsection{Raw Missing $E_{T}$ and Recoil Reconstruction}

The raw missing energy vector in the transverse plane is calculated by taking the vector sum

$$
{\overrightarrow{\mathbb{E}_{T}}}^{\mathrm{raw}}=-\sum_{i} E_{i}^{\mathrm{raw}} \sin \theta_{i}\left(\begin{array}{c}
\cos \phi_{i} \\
\sin \phi_{i}
\end{array}\right)=-\sum_{i} \vec{E}_{T}^{i \mathrm{raw}}
$$

where the sum runs over all calorimeter cells that were readout except cells in the coarse hadronic calorimeter and ICD (since they would introduce undesirable noise). Here the $E_{i}^{\text {raw }}$ are cell energies, and $\phi_{i}$ and $\theta_{i}$ are the azimuth and polar angle of center of cell $i$ with respect to the primary vertex. 


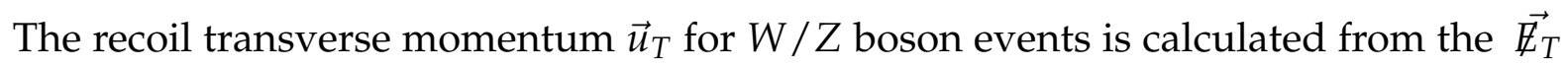
and the electron(s) transverse momentum:

$$
\vec{u}_{T}^{\mathrm{raw}}=-{\overrightarrow{\mathbb{E}_{T}}}^{\mathrm{raw}}-\sum_{e} \vec{p}_{T}^{e \text { raw }}
$$

We do not use a hadronic energy scale correction for the recoil $p_{T}$, and thus:

$$
\vec{u}_{T} \equiv \vec{u}_{T}^{\mathrm{raw}}
$$

\subsection{SET Reconstruction}

The SET is defined as the scalar sum of the transverse energies of all calorimeter cells

$$
\mathrm{SET}=\sum_{i} E_{i}^{\mathrm{raw}} \sin \theta_{i}
$$

excluding cells inside the electron reconstruction window, the coarse hadronic and cells from the ICD detector.

\subsection{Corrected Missing $E_{T}$ Reconstruction}

The corrected $\overrightarrow{\mathbb{E}_{T}}$ is calculated from $\vec{u}_{T}$ and corrected $\vec{p}_{T}^{e}$. For $W \rightarrow e v$ events,

$$
\overrightarrow{\mathbb{E}}_{T}=-\vec{u}_{T}-\vec{p}_{T}^{e}
$$

For $Z \rightarrow e e$ events,

$$
{\overrightarrow{\mathbb{E}_{T}}}=-\vec{u}_{T}-\vec{p}_{T}^{e_{1}}-\vec{p}_{T}^{e_{2}}
$$

\subsection{Event Selection}

Vector boson events are selected with the following requirements. In the $W \rightarrow$ ev selection requirements, electron refers to the reconstructed electron candidate with highest transverse energy and, for the $Z \rightarrow e e$ selection requirements, the two highest ones.

Vertex requirement:

- $\left|z_{V}\right|<60 \mathrm{~cm}$

Electron requirements:

- $f_{E M}>0.9, f_{\text {iso }}<0.15$

- HMatrix7 $<12$ in CC and HMatrix8 $<20$ in EC (the EC electrons are used for tag and probe studies) 
- in calorimeter fiducial region in $\eta$ and $\phi$ (we only use the region $0.1<\phi_{\bmod }<0.9$ to avoid cracks between CC modules, see Sec.7.2.5)

- $p_{T}>25 \mathrm{GeV}$

- The associated track must have at least one SMT hit, $p_{T}>10 \mathrm{GeV}$ and a good track match (track match probability $P\left(\chi_{\mathrm{TM}}^{2}\right)>0.01$ based on $\chi_{\mathrm{TM}}^{2}-$ Sec. 4.1).

$\mathrm{Z} \rightarrow e e$ candidate selection requirements:

- At least one electron passes trigger requirements of all three trigger levels.

- Electron $\left|\eta_{\text {det }}\right|<1.05$, except for studies of electron efficiency which allow one electron to be in the EC region $1.5<\left|\eta_{\text {det }}\right|<2.3$.

- $u_{T}<15 \mathrm{GeV}$

- $70<m_{e e}<110 \mathrm{GeV}$

$W \rightarrow e v$ candidate selection requirements:

- The electron must pass trigger requirements of all three trigger levels.

- $\mathbb{E}_{T}>25 \mathrm{GeV}$

- Electron $\left|\eta_{\text {det }}\right|<1.05$

- $u_{T}<15 \mathrm{GeV}$

- $50<m_{T}<200 \mathrm{GeV}$

After the above selections, 54,512 candidate $Z \rightarrow e e$ events remain with both electrons in the CC, which we use to determine the EM calibration, and 1,677,489 candidate $W \rightarrow e v$ events remain which are used to determine $M_{W}$. 


\section{Chapter 5}

\section{Dead Material Correction to the Electron Re- sponse}

Figure 5.1 shows an overview of the material in front of the CC cryostat. An electron traveling from the interaction point to the CC at normal incidence encounters about 3.7 radiation lengths $\left(\mathrm{X}_{0}\right)$ of material before reaching the first active layer of liquid argon: $0.2 \mathrm{X}_{0}$ in the inner detector, $0.9 \mathrm{X}_{0}$ in the solenoid, $0.3 \mathrm{X}_{0}$ in the Preshower detector plus $1.0 \mathrm{X}_{0}$ in the associated lead, and $1.3 \mathrm{X}_{0}$ in the cryostat walls plus related support structures. As a consequence of dead material in front of the $\mathrm{CC}$, the measured response energy has significant non-linear dependence on the true energy and the angle of impact. We describe here the derivation of the corrections to the electron response which are applied to data because of dead material. This correction is derived from a simulation of the detector response to electrons in which the shower description has been improved relative to the default GEANT description and the amount of dead material has been tuned. The dead material tuning was derived with the $Z \rightarrow e e$ data sample of the $1 \mathrm{fb}^{-1}$ (Run IIa) analysis [32] and re-validated for this analysis (Sec. [5.4. A comprehensive account of the calibration method can be found in [62].

\subsection{Observables Used for Tuning the Simulation}

To estimate the contribution of dead material we exploit the longitudinal segmentation of the calorimeter readout. This means looking at the EM fractions, i.e. at the fraction of the measured electron energy deposited in each one of the longitudinal layers EM1, EM2, EM3, EM4, and FH1. The depositions in EM4 and FH1 give contributions which are negligible in the tuning procedure.

Electrons produced at different angles cross different amounts of dead material and the fraction of energy deposited in each layer will therefore be different, as shown in figure 5.2 that depicts the average of 1000 showers simulated with the GFlash [63] parametrization. We split the $Z$ event sample into categories based on electron physics pseudo- 


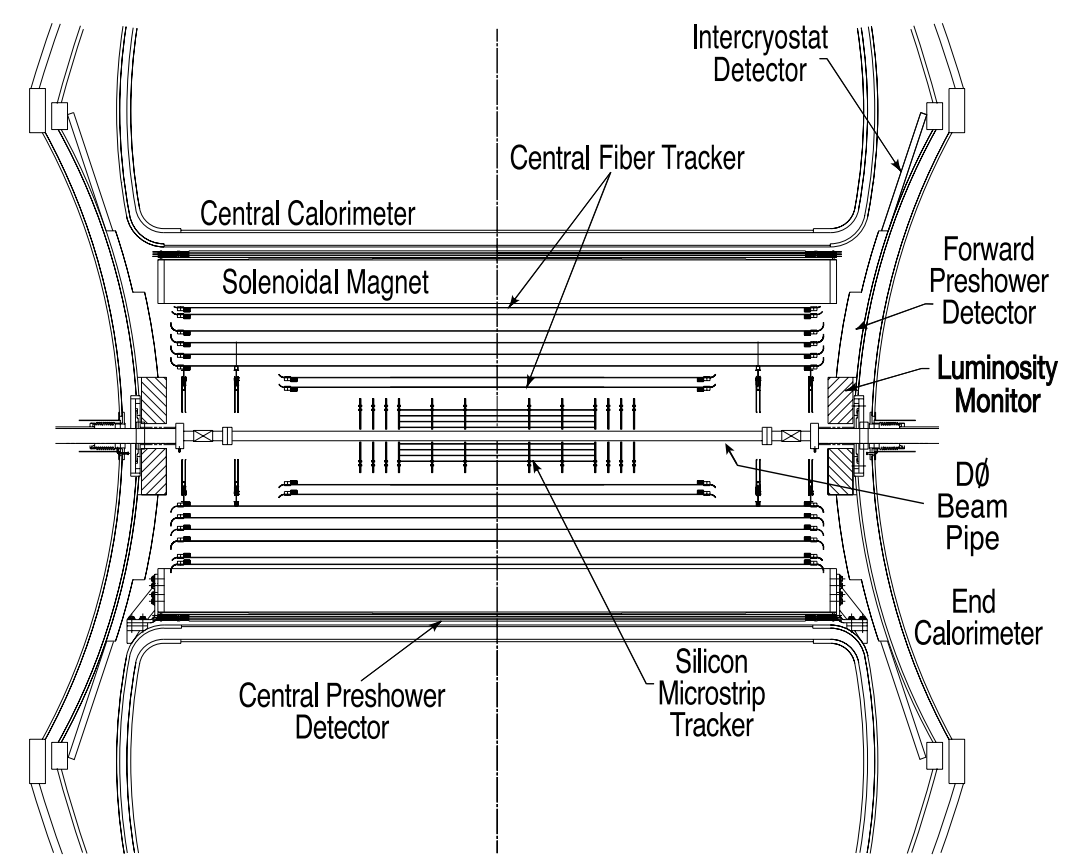

Figure 5.1: Overview of the material in front of the Central Calorimeter. This drawing shows a cross-sectional view of the central tracking system in the $x-z$ plane. Also shown are the locations of the solenoid, the Preshower detectors, Luminosity Monitors and the Calorimeters.
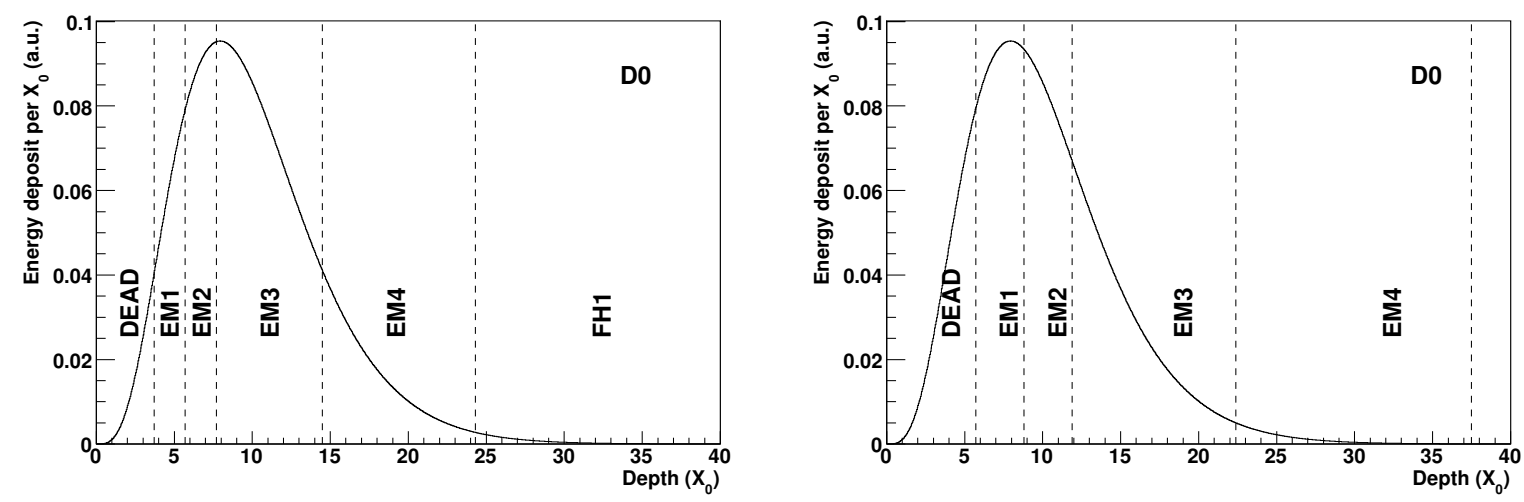

Figure 5.2: The average longitudinal shower profile (along the shower axis) of electrons with $\mathrm{E}=45 \mathrm{GeV}$ simulated using the GFlash parametrization. The depth of each different readout section of the central calorimeter is indicated for an electron with normal incidence $\eta=0$ (left) and with non-normal incidence $\eta=1$ (right). 


\begin{tabular}{|c|l|}
\hline \hline bin 0: & $|\eta|<0.2$ \\
bin 1: & $0.2 \leq|\eta|<0.4$ \\
bin 2: & $0.4 \leq|\eta|<0.6$ \\
bin 3: & $0.6 \leq|\eta|<0.8$ \\
bin 4: & $0.8 \leq|\eta|$ \\
\hline
\end{tabular}

Table 5.1: Definition of bins in electron $|\eta|$.

\begin{tabular}{|c|c|}
\hline \hline Category & Combination of $\eta$ bins \\
\hline \hline 10 & $0-0$ \\
11 & $0-1$ \\
12 & $0-2$ \\
13 & $0-3$ \\
14 & $0-4$ \\
15 & $1-1$ \\
16 & $1-2$ \\
17 & $1-3$ \\
18 & $1-4$ \\
19 & $2-2$ \\
20 & $2-3$ \\
21 & $2-4$ \\
22 & $3-3$ \\
23 & $3-4$ \\
24 & $4-4$ \\
\hline \hline
\end{tabular}

Table 5.2: Definition of $\eta$ categories for $Z \rightarrow e e$ events.

rapidity $(\eta)$. We define five bins of $|\eta|$ used as a measure of the angle of incidence on the dead material. The definition of the bins is given in table 5.1. We classify a $Z$ event into one of 15 distinct categories shown in Table 5.2 according to the $|\eta|$ bins of the two electrons. We do not distinguish the leading and the subleading electron to avoid consideration of the calorimeter energy corrections which we are trying to determine.

We compare the mean of the EM fraction distribution for each layer in each category between the $Z \rightarrow e e$ data and simulation. As can be seen in Fig. 5.3 which is made prior to applying the correction procedure described below, a large disagreement between data and simulation is present. The difference arises from both imprecision in the simulation of electromagnetic showers and inadequacies of the D0 material model included in the full MC. 


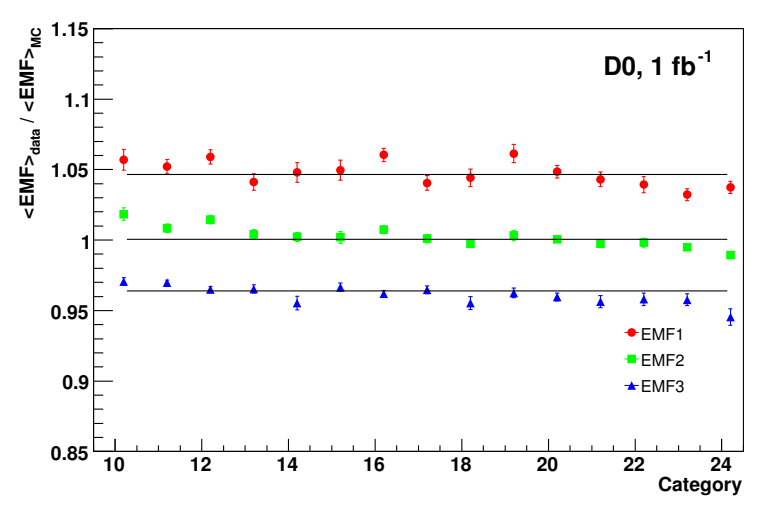

Figure 5.3: The ratio of the data to simulation for the means of the EM fraction distributions in $Z \rightarrow e e$ events for each of the first three EM layers and each of the $15 \eta$ categories shown before the correction described here has been applied. Each of the three horizontal lines indicates the result of a fit of a common constant to the 15 data points from a given EM layer. The fit was performed for EM1, EM2 and EM3.

\subsection{Improvements in the Shower Simulation of Electromagnetic Show- ers and in the D0 Material Model}

Because of the large amount of material in front of and forming the calorimeter, a precise simulation of the electromagnetic shower is needed to ensure acceptable understanding of the electron energy reconstruction as a function of true energy and angle of incidence. Several improvements were needed to the standard GEANT [64] simulation to have a good description of the energy deposition and depth of the shower. The improvements will be discussed in details in section 13.2. They consisted in a careful evaluation of the parameters in the transport algorithms and interaction cross-sections. We call the simulation with these improvements a ultra-detailed simulation of the D0 detector. Although these modifications allow a much better description of the showering before and in the calorimeter, it also represents a factor of eighty increase in the processing time for GEANT event simulations. We use the ultra-detailed simulation only for studies of the calorimeter response to electron showers and the impact of the dead material on them.

As shown in Fig. 5.3 the data has a higher deposition in EM1 than the MC, so additional uninstrumented material must be added in front of the calorimeter to the detector model in the full MC. We choose a relatively low atomic number material, copper, and place it inside the solenoid. The shape of the copper is a cylindrical shell with the same axis as the solenoid. Along the $z$ direction, it extends over the length of the solenoid. The shape of the missing material is driven by the observation that the materials in front of the central calorimeter have a geometry that is close to cylindrical. The thickness of the cylinder was chosen to be constant since there was no visible $\eta$ category dependency in 


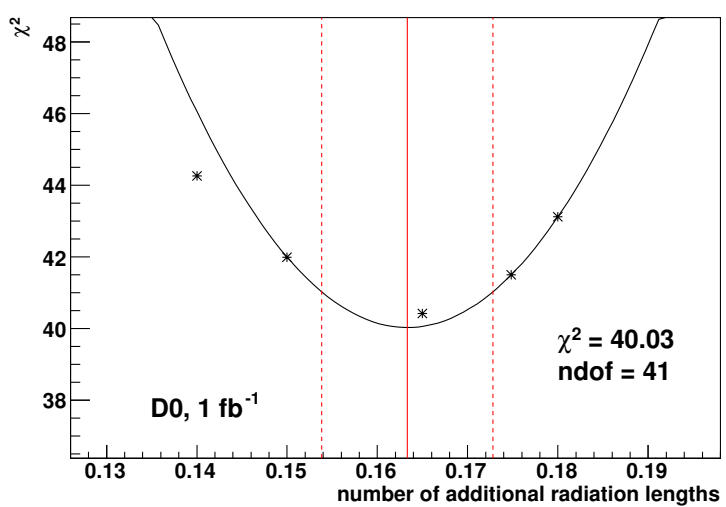

Figure 5.4: Fit for $n X_{0}$, the amount of uninstrumented material (in radiation lengths) added to the nominal material in the improved simulation of the D0 detector. The solid and dotted vertical lines show respectively the best fit and one standard deviation uncertainties for $\mathrm{nX}_{0}$. This fit is performed with the $\mathrm{Z} \rightarrow$ ee data sample from our $1 \mathrm{fb}^{-1}$ measurement (see Sec. 5.4)

the correction needed to the dead material model, as is shown in Fig. 5.3 .

We use the improved GEANT model to simulate the electrons from $Z \rightarrow e e$ events. For these events, the thickness of the additional copper material is varied. We then build a parametrized model of the mean EM fractions and the fluctuations around the average as a function of the copper thickness $\left(\mathrm{nX}_{0}\right)$. As shown in Fig. 5.3. we fit the ratio of the mean EM fraction in data to that in $\mathrm{MC}$ as a function of the $Z$ event category to a constant for each of the first through third EM layers. We then form a total $\chi^{2}$ from the sum of the individual $\chi^{2}$ values from the three layer fits. This is shown as a function of the thickness of the additional copper material in Fig. 5.4. This figure also shows the parabolic fit giving the $\chi^{2}$ minimum corresponding to the final thickness used in our tuned simulation, $\mathrm{nX}_{0}=$ $0.1633 \pm 0.0095$. Because of the small energy deposit in EM4, we do not include it in our fits.

As a cross-check, we repeat the fit for $n \mathrm{X}_{0}$ separately for each of the three layers. The results are summarized in Fig. 5.5. Good agreement is found between the overall fit and the results of the individual layers. The ratio of mean EM fraction in data to that in full MC after adding the missing material is shown in Fig. 5.6. We interpret the small residual deviations from unity as layer weight correction factors that must be applied during data reconstruction.

Figure 5.7 shows the EM fraction for electrons from $W$ boson decay, using the same binning as in Table 5.1 after adding to the simulation the additional copper material corresponding to the value derived above. The precision with which we have measured the material in front of the detector contributes directly to the energy measurement of the electron and therefore to the $W$ boson mass. Our measurement of $M_{W}$ depends critically on the assumption that the calibration done at the $Z$ mass pole is valid down to the $W$ 


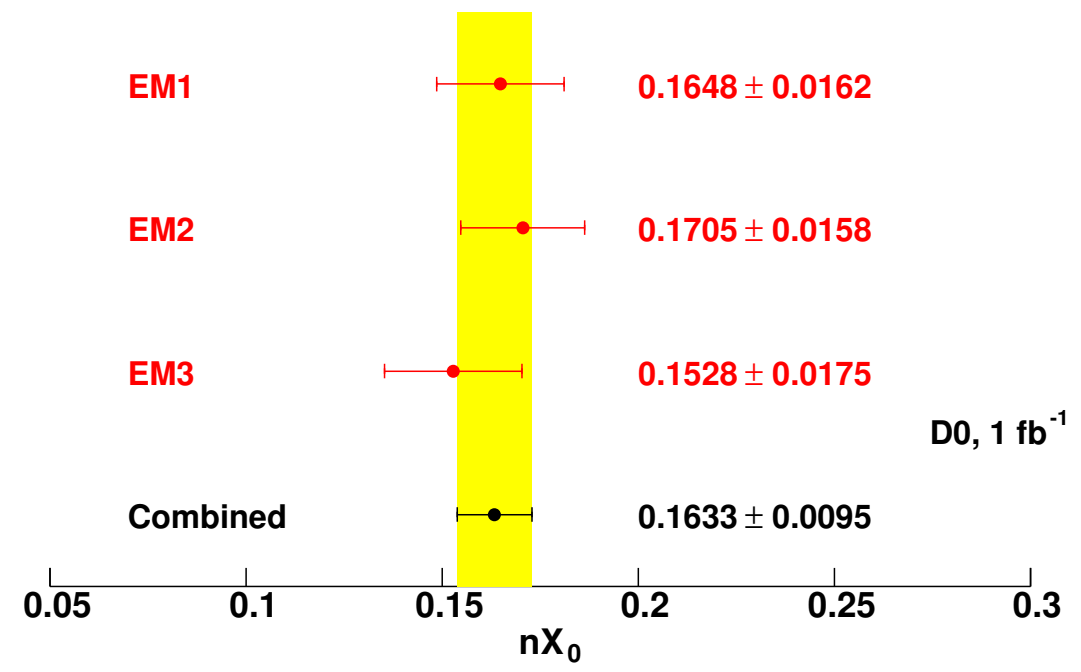

Figure 5.5: Stability check: results of the fit for $n \mathrm{X}_{0}$, performed separately for each of the three layers (EM1, EM2 and EM3). The result of the combined fit is also shown for comparison.

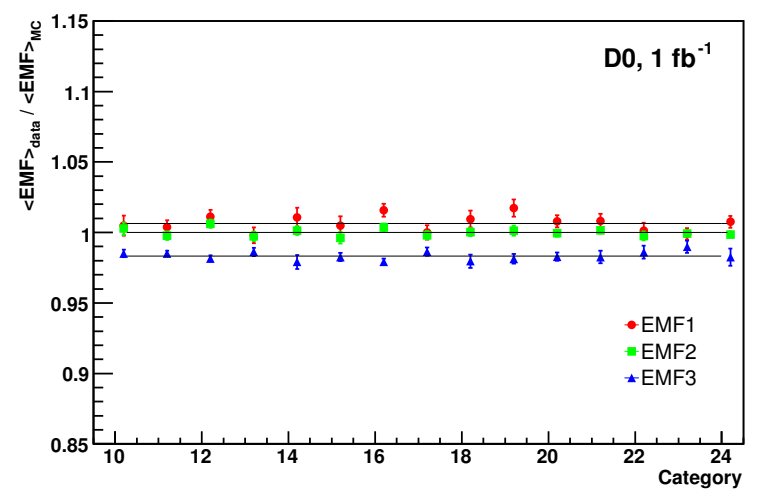

Figure 5.6: The ratio of the data to simulation for the means of the EM fraction distributions in $Z \rightarrow e e$ events for each of the first three EM layers and each of the $15 \eta$ categories shown after the correction described here has been applied. Each of the three horizontal lines indicates the result of a fit of a common constant to the 15 data points from a given EM layer. The fit was performed for EM1, EM2 and EM3. 
boson mass scale. A mismeasured material distribution would be the primary source of a non-linearity in this scaling.

The uncertainty on the $W$ boson mass arising from the material tune is derived by varying the additional material by plus and minus one standard deviation shown in Fig. 5.4. However, because the overall EM calibration is performed with a specific amount of material assumed, some variation arising from the material thickness uncertainty would be absorbed into the calibration and reduce the impact of the material variation. We build fast MC models of the response considering the combination of the material variation and the impact of calibration procedure.

The fast MC models resulting from plus and minus one sigma variations in the additional material are used to generate $W$ boson events. The $m_{T}, p_{T}^{e}$ and $\mathbb{E}_{T}$ distributions from these events are fit to templates generated with the default parametrization and the resulting value is compared to the known input mass used. We find shifts of $4 \mathrm{MeV}$ using the $m_{T}$ distribution for the fit, $6 \mathrm{MeV}$ using the electron $p_{T}^{e}$ distribution and $7 \mathrm{MeV}$ for the $\mathbb{E}_{T}$ distribution.

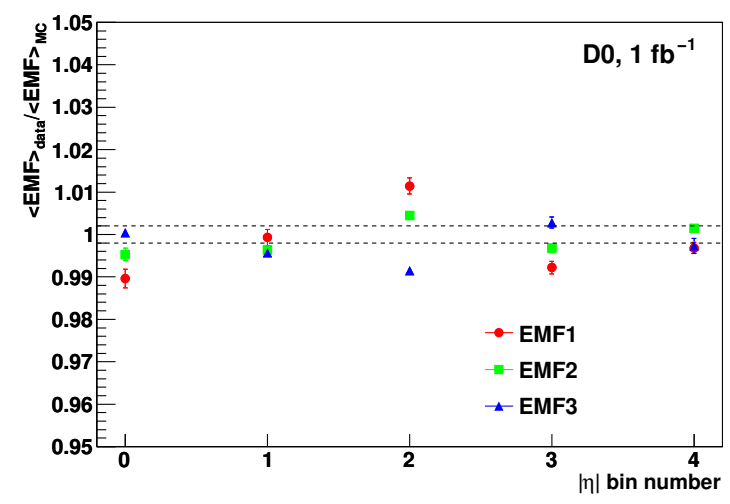

Figure 5.7: The data/simulation ratios for the means of the EM fraction distributions in $W \rightarrow e v$ events for each of the first three EM layers and each of the five $\eta$ bins after the correction. The dashed horizontal lines indicate the variations in the EM fractions from the uncertainty in the material tune due to the limited size of the $Z$ sample.

\subsection{Energy-Loss Corrections}

The average electron energy loss is recovered with correction functions determined using single-energy electron full MC samples with incident energies from $1 \mathrm{GeV}$ to $135 \mathrm{GeV}$ and the improvements described above. The precision of the corrections is therefore limited by the statistical precision of the full MC sample. As will be discussed in Sec. 7.3.4 final tuning of the electron energy response using $Z \rightarrow e e$ events from the data would partially 
fix some imperfections in the energy-loss parametrization, for example, a global scale mistake in the energy-loss function.

The electrons from $Z \rightarrow e e$ populate one band in $E^{e}$ versus $\eta$ space and electrons from $W \rightarrow e v$ populate another band. If the energy dependence of the energy-loss correction is not correctly derived, the energy scale tuned on $Z \rightarrow e e$ events will be slightly incorrect when applied to $W \rightarrow e v$ events. To estimate the effect from the different $E^{e}$ versus $\eta$ distributions of $Z \rightarrow e e$ electrons and $W \rightarrow e v$ electrons, we calculate the difference between the reconstructed and the true electron energy in $\mathrm{MC}$ and divide it by the true energy and plot it as a function of the true electron energy. This fractional energy difference reflects the imperfection of the energy-loss corrections owning to statistical fluctuations in the determination of its energy dependency. The result is shown in Fig. 5.8. Any difference in the corrections between $Z \rightarrow e e$ and $W \rightarrow e v$ events will be translated into the reconstructed electron energy and then into the measured $W$ mass. After propagating the correction difference to the $W$ mass, we assign a systematic uncertainty of $4 \mathrm{MeV}$, limited by the statistics of the full MC Z sample used to derive the energy-loss functions, because of the different $E^{e}$ versus $\eta$ distributions of electrons from $Z \rightarrow e e$ and $W \rightarrow e v$ for the variables $m_{T}, p_{T}^{e}$ and $\mathbb{E}_{T}$.

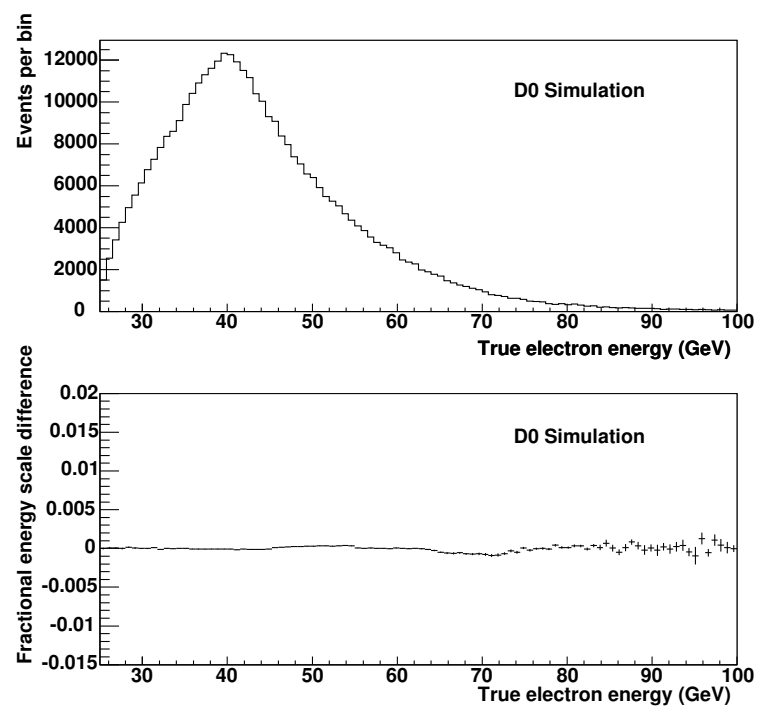

Figure 5.8: The true energy spectrum for electrons in simulated $W$ events which pass the full selection (upper panel), and the ratio of measured minus true energy to the true energy as a function of true energy in $Z$ and $W$ electrons (lower panel).

\section{$5.44 .3 \mathrm{fb}^{-1}$ Analysis Validation}

The dead material correction presented here was derived with the $Z \rightarrow e e$ data sample of our $1 \mathrm{fb}^{-1}$ (Run IIa) analysis. It is used again here, for the $4.3 \mathrm{fb}^{-1}$ (Run IIb) analysis, 
because the distribution of EM fractions is essentially identical to the distribution of EM fractions in the Run IIa measurement. There are two differences between this measurement and the Run IIa measurement relevant to EM showers:

- increased pileup in Run IIb

- insertion of an inner silicon tracking layer (L0) before Run IIb $\left(\sim 0.003 \mathrm{X}_{0}\right)$

The inclusion of L0 is a very small contribution to the total amount of dead material when compared to the CFT, solenoid, CPS and cryostat, that remained unchanged throughout Run II. Figure 5.9 shows the contribution from extra $p \bar{p}$ interactions and noise to the mean EM fractions in $Z \rightarrow e e$ events, estimated separately for Run IIa and Run IIb. Figure 5.9 also shows the EM fraction distributions in $Z \rightarrow e e$ data for Run IIa and Run IIb, after correcting the Run IIb data by the Run IIa/Run IIb ratio. The Run Ilb EM fractions are not significantly different from the Run IIa EM fractions.
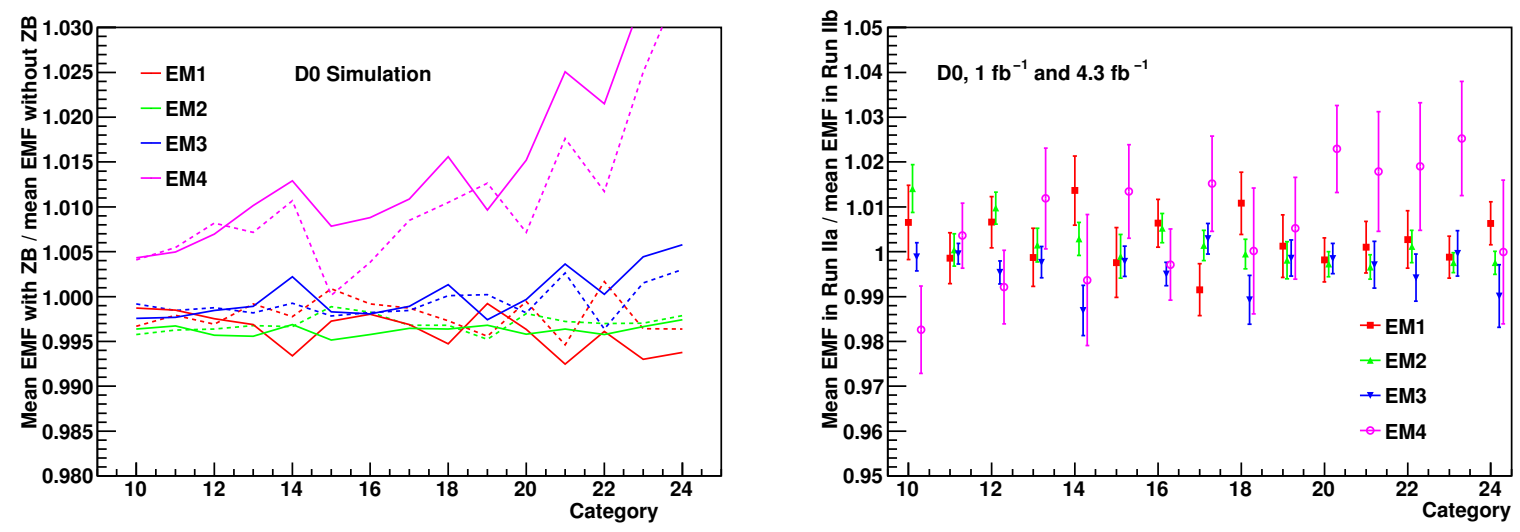

Figure 5.9: (left) Each line represents the ratio of the mean EM fractions (EMF) simulated with $\mathrm{ZB}$ overlay to the same sample simulated without overlay. It represents the contribution from extra $p \bar{p}$ interactions and noise to the mean EM fractions, which is determined separately for the Run Ila sample (dotted lines) and the Run IIb sample (continuous lines). The ratio between the continuous to the dashed line is used as a correction factor to the EMF fractions measured in RunIIa when comparing them to the Run Ilb fractions. (right) Ratio of the means of the EM fraction distributions in $Z \rightarrow e e$ events between the Run IIa analysis and the present Run IIb analysis, separately for each of the four EM layers and each of the 15 standard $\eta$ categories. 


\section{Chapter 6}

\section{Generators for Full and Fast Simulation}

The initial step in constructing templates for extracting the $W$ boson mass is simulation of vector boson production and decay kinematics. The list of event generators used in this analysis are listed in Table 6.1. We use the RESBOS [65, 16, 17] program coupled with the CTEQ6.6 NLO parton distribution functions [66] as the default event generator. RESBOS provides a good description of the dominant QCD effects, namely the emission of multiple gluons, that drives the shape of the boson $q_{T}$ distribution at low boson $q_{T}$. The $W$ boson $q_{T}$ spectrum has a significant impact on the simulated $p_{T}^{e}$ and $p_{T}^{v}$ spectra so an accurate description of this is an important ingredient of the $W$ boson mass measurement.

The dominant effect from EW corrections on the $W$ boson mass measurement arises from radiation of a single photon from the final state charged lepton. This process is simulated by combining RESBOS with PHOTOS [67]. We use PHOTOS in a configuration where it generates up to two photons in the final state. The effect of additional photons is, to a very good approximation, absorbed into the calibration of the electron energy scale based on $Z \rightarrow e e$ events.

\begin{tabular}{c|ccc}
\hline \hline Tool & Process & QCD & EW \\
\hline RESBOS & $W, Z$ & NLO & - \\
PYTHIA & $W, Z$ & LO & QED FSR \\
\hline WGRAD & $W$ & LO & complete $\mathcal{O}(\alpha)$ \\
ZGRAD & $Z$ & LO & complete $\mathcal{O}(\alpha)$ \\
PHOTOS & & & QED FSR,$\leq 2$ photons \\
\hline \hline
\end{tabular}

Table 6.1: Event generators for $W$ boson and $Z$ boson processes used in this analysis. PYTHIA is used for the full MC closure test and for estimating PDF uncertainties. WGRAD and ZGRAD are used only for estimation of QED theory uncertainty. 


\subsection{QCD Corrections-Boson $q_{T}$}

RESBOS uses the triple differential cross section $d^{3} \sigma / d q_{T} d y d Q$ for $Z / \gamma^{*}$ and $W$ boson processes, where $q_{T}$ is the boson transverse momentum, $y=\frac{1}{2} \ln \left[\left(E+p_{z}\right) /\left(E-p_{z}\right)\right]$ is the boson rapidity and $Q$ is the boson mass, available as grid files to generate events. These differential cross sections are calculated using the CSS $q_{T}$ resummation technique for low boson $q_{T}$ matched to a fixed order calculation at high $q_{T}$. The resummation is performed in impact parameter space with Sudakov exponents calculated to NNLL precision and Wilson coefficients calculated to NLO precision. At large impact parameters, the perturbative calculation is frozen out and a phenomenological non-perturbative parametrization is introduced. The grid files used in this measurement use the BNLY [17] parametrization which is a function of three variables, $g_{1}, g_{2}$ and $g_{3}$.

The observed boson $q_{T}$ spectrum in this measurement is mostly sensitive to $g_{2}$ and has very limited sensitivity to the other non-perturbative parameters and scales in the cross section. Therefore, we take the uncertainty in $g_{2}$ as representative of the boson production model uncertainty. We use the world average for $g_{2}$ [17], and the uncertainty is propagated using pseudo-experiments generated by varying $g_{2}$ within its quoted uncertainty. We find uncertainties of 2,5 and $2 \mathrm{MeV}$ for the $m_{T}, p_{T}^{e}$ and $\mathbb{E}_{T}$ fits, respectively.

\subsection{Electroweak Corrections}

In the fast MC care was taken to model the EW corrections to $W$ boson production and decay as well as the detector response to the emitted photon(s). The most important correction is the real emission of final state photons, since it takes away some of the energy of the electron and the invariant mass of the electron-neutrino will be smaller than the $W$ boson invariant mass, biasing the measurement.

As discussed above, we use PHOTOS to simulate the leading effects of real photon emission. To estimate the uncertainties from this modeling, we explore the difference from the shower simulation done by PНОтОS and the complete EW NLO calculation available in WGRAD [68] and ZGRAD [69]. WGRAD and ZGRAD cannot be used to measure the $W$ boson mass, since they do not include higher order QCD corrections, but are adequate to estimate the purely EW uncertainties.

WGRAD allows both shower and complete EW NLO calculations. We generate pseudoexperiments using both options and fit them against templates prepared with PHOTOS. The difference of the fitted $W$ boson mass is taken as a measure of the uncertainty and is found to be $5 \mathrm{MeV}$ for the $m_{T}$, $p_{T}^{e}$ and $\mathbb{E}_{T}$ fits.

To estimate the uncertainty in the EW NLO calculation itself, we study the dependence of the measured $W$ boson mass with the soft and collinear cutoffs that are introduced to avoid the infrared divergence in the cross-section. We find shifts of $2 \mathrm{MeV}, 1 \mathrm{MeV}$, and $3 \mathrm{MeV}$ for the $m_{T}, p_{T}^{e}$, and $\mathbb{E}_{T}$ fits respectively.

Finally, an experimental scale is also present in the FSR simulation: the radius of the cone used as boundary between photons whose energy is detected as part of the electron 
cluster or as part of the unclustered recoil. The simulation uses the value $R=0.3$ as standard, and we vary it by the size of a cell of the D0 calorimeter, between 0.2 and 0.4 , to estimate the uncertainty coming from this experimentally introduced scale. We find uncertainties of $(1,1,5) \mathrm{MeV}$ for the $\left(m_{T}, p_{T}^{e}, \mathbb{E}_{T}\right)$ fits respectively.

\subsection{Parton Distribution Functions}

Parton distribution functions (PDF) enter into the $W$ mass measurement through their effects on the detector acceptance and kinematics of the decay electron. In the ideal case of full pseudorapidity acceptance and no kinematic cuts, the lack of knowledge of the PDFs would introduce no uncertainty on $M_{W}$.

We determine the systematic uncertainty arising from the PDFs using PYTHIA and the CTEQ6.1 PDF set [70] which is available at LO. We generate pseudo-experiments using the 40 CTEQ6.1 error eigenfunction sets each of which corresponds to a one-sided uncorrelated variation of the PDF parameters at 90\% C.L. The pseudo-experiments from each of the 40 uncertainty sets are compared to mass templates generated using the nominal set. Following the CTEQ prescription, we take the average of the two-sided variation $\left|M^{+}-M^{-}\right| / 2$ as the estimate of the uncertainty for each uncorrelated combination of the PDF parameters and determine the total uncertainty by summing the 20 variations in quadrature. To obtain the uncertainty with $68 \% \mathrm{CL}$ we introduce an extra factor of 1.6 in the estimate:

$$
\Delta M_{W}=\frac{1}{1.6} \sqrt{\sum_{i=1}^{20}\left(\frac{M_{i}^{+}-M_{i}^{-}}{2}\right)^{2}}
$$

The final uncertainty is found to be $11 \mathrm{MeV}, 11 \mathrm{MeV}$, and $14 \mathrm{MeV}$ for $m_{T}, p_{T}^{e}, \mathbb{E}_{T}$ methods. These values are slightly larger than those in the Run IIa measurement [32] due to the deterioration of the hadronic recoil resolution at higher luminosity. Further details can be found in Sec. 12 . 


\section{Chapter 7}

\section{Determination of the Fast Simulation Parameters}

As described in Sec. 3.2. $W(Z)$ events are characterized by the measurements of the electron(s) and hadronic recoil in the event. Our fast simulation is designed to reproduce these measurements and their correlations starting from the truth-level four-vector generated by the event genertors described in Sec. 6 . The simulation consists of four parts: (1) simulation of the vertex $z$ position, (2) simulation of the electron reconstruction and identification efficiency, (3) simulation of the electron energy measurement and (4) simulation of the hadronic recoil energy measurement. The first of these is needed to predict the detector region(s) with which the electron(s) interact when computing efficiencies and reconstructed energy. The last three items are directly affected by the presence of FSR, hence, in our fast simulation, photons within the electron energy reconstruction window (Fig. 4.2) of a parent electron are merged back into the electron, treating the resulting electron plus photon(s) system as the reconstructed electron. This procedure takes into account the reconstruction inefficiency induced by the photons as well as the probability of low energy photons to reach the calorimeter. Photons far from electrons are reconstructed as part of the recoil system and so are described in our fast simulation. Descriptions of these effects are included in the corresponding sections below.

We describe here the models used in the fast MC to simulate data and full MC. Separate tunes are required for data and for full $\mathrm{MC}$ because our full $\mathrm{MC}$ does not describe our data with an accuracy sufficient to measure the $W$ boson mass. We perform the full measurement of the $W$ boson mass twice: once with full $\mathrm{MC}$ and once with data. By treating the full MC events as data and using the same parametrized detector model, but with different parameters, we validate our experimental procedure. In our full MC measurement we obtain a difference of our measured $Z$ mass from the input mass of $-3 \pm 4 \mathrm{MeV}$ and a difference of our measured $W$ mass from the input mass of $-2 \pm 5 \mathrm{MeV}$ from the fit to the $m_{T}$ distribution, $-2 \pm 5 \mathrm{MeV}$ from the fit to the fit to the $p_{T}^{e}$ distribution, and $+5 \pm 6$ $\mathrm{MeV}$ from the fit to the $\mathbb{E}_{T}$ distribution. These uncertainties are statistical, reflecting the size of the full MC sample. 


\subsection{Primary Vertex Parametrization}

We select only events with primary vertex position $\left|z_{V}\right|<60 \mathrm{~cm}$ and electrons with $\left|\eta_{\text {det }}\right|<1.05$ for the final analysis. Since the electron $\eta_{\text {det }}$ depends on the electron $\eta$ and the primary vertex position, we need a model that can be used to predict the primary vertex distribution in the fast MC. The beam shape is modeled as a convolution of a Gaussian bunch length with a Lorentzian shape set by the accelerator $\beta^{*}$ functions in both transverse directions. The shape parameters are determined from fits to the vertex distribution for randomly triggered beam crossings.

\subsection{Electron Efficiency Parametrization}

The $m_{T}, p_{T}^{e}$ and $\mathbb{E}_{T}$ distributions are sculpted by (in)efficiencies in electron identification which depend on event kinematics and the hadronic environment. This sculpting introduces bias in the measured $W$ boson mass which must be accounted for. We accomplish this by having an efficiency model in the fast $\mathrm{MC}$ which results in the simulation reproducing the sculpting effects. In this section we discuss the components of the fast MC model used to predict the combined electron reconstruction and identification efficiencies. We begin by giving an overview of the model, discuss each of the components, and end with a discussion of the model validation.

The efficiency model begins by determining the calorimeter related identification efficiencies using the full MC simulation. Then data control samples are used to model dependence not included in the full MC including the trigger, whose effect is not included at all in the full simulation, and imperfections in the tracking efficiency description. In the last steps data control samples are used again to provide final corrections to the full MC model used as the starting point. The final corrections are rather modest in scale largely because in our full MC processing ZB events from collider data are added to simulated events at the (simulated) raw data processing level. This allows the use of control data to model the effects from changing instantaneous luminosity, which is the leading source of inefficiency.

The electron identification efficiency model must be multi-dimensional and depend on all quantities that introduce bias in the reconstructed mass. In the ideal case, a single multi-dimensional efficiency would be derived which would depend on all necessary variables and automatically include all correlations. However, the $Z \rightarrow$ ee control data sample is not large enough to establish a model by binning the efficiency in all relevant variables to derive a single function.

Many of the dependencies are, however, largely uncorrelated with each other and our full MC program can describe parts of the efficiency reasonably well. Because of this we build an efficiency model which assumes partial factorization and is derived using a mix of full MC, data and data versus fast MC comparisons. The overall efficiency $\epsilon$ can be 
written as a product of several terms

$$
\begin{aligned}
\epsilon= & \epsilon_{\text {trig }}\left(p_{T}^{e}\right) \times \epsilon_{\mathrm{FSR}}\left(X, \Delta R, \eta, E^{e}\right) \times \epsilon_{\mathrm{EMID}}\left(\eta_{\mathrm{det}}, p_{T}^{e}\right) \times \epsilon_{\text {trk }}\left(z_{V}, \eta, p_{T}^{e}\right) \times \epsilon_{\phi_{\text {mod }}}\left(\phi_{\text {mod }}\right) \\
& \times \epsilon_{\phi}\left(\phi^{e}\right) \times \epsilon_{\mathrm{had}}\left(S E T, p_{T}^{e}, \eta_{\mathrm{det}}, \mathcal{L}, u_{\|}\right) \times R_{1}(\mathrm{SET}, \mathcal{L}) \times R_{2}\left(u_{\|}\right)
\end{aligned}
$$

in which $\epsilon_{\text {trig }}$ measures the trigger efficiency for recording events in the sample, $\epsilon_{\mathrm{FSR}}$ measures the efficiency effects arising from radiated photons, $\epsilon_{\text {trk }}$ measures the efficiency of the track selection requirement, $\epsilon_{\mathrm{EMID}}$ measures the efficiency of the calorimetric requirements used in the electron selection, and $\epsilon_{\phi_{\text {mod }}}$ measures the efficiency depending on how near the electron is to a calorimeter module boundary. The efficiency $\epsilon_{\phi}$ models electron $\phi$ dependent efficiency and $\epsilon_{\text {had }}$ quantifies the effect on electron finding arising from hadronic activity in the event. The term $\epsilon_{\text {had }}$ is essential for describing the effect of multiple $p \bar{p}$ interactions in the electron identification. Finally, $R_{1}(\mathcal{L}, \mathrm{SET})$ is introduced to account for the poor quality of the efficiency description in full MC at high instantaneous luminosity, especially the one related to tracking matching, while $R_{2}\left(u_{\|}\right)$is introduced to describe the fine details of the hard recoil (see Sec. 7.4.1 for a definition of the term) in the electron identification and reconstruction efficiency that were not totally described by the rather coarse binning of the Hadronic Energy Dependent efficiency in the $u_{\|}$variable. The first five of these, $\epsilon_{\text {trig }}$ through $\epsilon_{\phi_{\bmod }}$ are derived directly from either full MC or data. The correction $R_{1}$ is derived from a comparison of the efficiency in data and full MC, while $R_{2}$ is derived from a comparison of the efficiency between data and fast MC in which all previously determined efficiencies are applied to the fast MC. We describe each of these efficiencies in the following sections, and also define the variables upon which they depend. We note in passing that the overall normalization of the total efficiency does not enter this analysis because the fast MC yields are always normalized to the data or full MC yield.

\subsubsection{Trigger Efficiency}

Events used in this analysis must satisfy at least one of three triggers requiring the EM object at trigger Level 3 to have $p_{T}^{L 3}>25 \mathrm{GeV}$ or $p_{T}^{L 3}>27 \mathrm{GeV}$. For this analysis, a oneto-one correspondence between a run period and a specific trigger is enforced. To make the correspondence, we choose the lowest $p_{T}$ threshold unprescaled trigger available for the period. The efficiency for any of these three triggers will be less than unity near the threshold because of energy measurement differences between the trigger system and the offline reconstruction program. The efficiency modeling these effects, $\epsilon_{\text {trig, }}$, is thus a function of electron $p_{T}$.

A tag-and-probe method is used with collider $Z \rightarrow e e$ candidate events to measure the trigger efficiency as a function of $p_{T}^{e}$. We require one electron (the tag) in a $Z \rightarrow e e$ event to pass all selection requirements including the trigger. The other electron (the probe) is initially required to pass the full selection except no requirement is made regarding the trigger. The efficiency is then determined from the fraction of times the probe electron passes the trigger whose efficiency is being measured. For this efficiency determination, we allow the tag electron to be in the EC. 
The resulting measured efficiency is shown in Fig. 7.1 for each of the three triggers. When simulating the trigger in the fast MC a mix of the three efficiencies is used such that each occurs at the same relative frequency as in the data determined from the integrated luminosity exposure for each trigger. This efficiency is only used when using the fast MC to simulate events for comparison to collider data. It does not apply to full MC analysis.

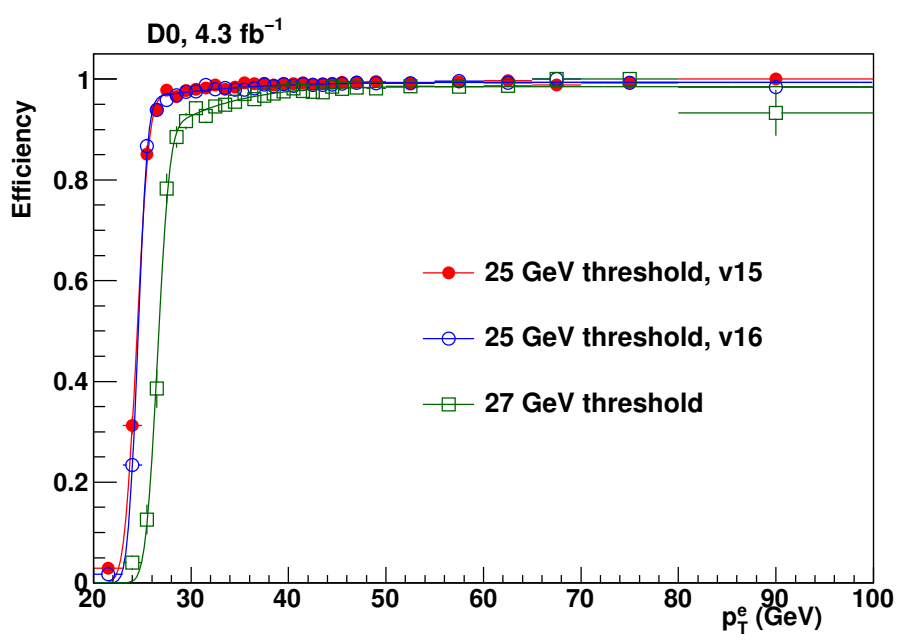

Figure 7.1: Trigger efficiency as a function of $p_{T}^{e}$ for the three triggers used. The triggers are explained in section 2.5 .

\subsubsection{FSR Efficiency}

Radiated photons (FSR) close to or inside the electron reconstruction cone will affect the electron identification efficiency because of isolation, shower shape and track matching requirements. To account for these effects we introduce an electron efficiency $\epsilon_{\mathrm{FSR}}\left(X, \Delta R, \eta, E^{e}\right)$. Here $X$ is the fraction of the electron energy carried by the photon and:

$$
\Delta R=\sqrt{[\phi(e)-\phi(\gamma)]^{2}+[\eta(e)-\eta(\gamma)]^{2}}
$$

with $\phi(e)-\phi(\gamma)$ taken modulo $2 \pi$, measures the separation between the electron and photon.

The parametrization is derived by studying electron reconstruction efficiency using two full MC samples: one with single electrons having the kinematics of those from $W \rightarrow e v$ decay which are accompanied by normal FSR photons, and a second sample that includes exactly the same events as the first one, except that the electron has not been showered. Both samples have ZB event overlay, and the same ZB event is overlaid on a given $W$ event in each of the two samples. The ratio of the electron yields in the first sample to that in the second sample defines this efficiency. The efficiency is determined in bins of the four variables, $X, \Delta R, \eta$, and $E^{e}$. 
Figure 7.2 shows examples of the electron reconstruction efficiency versus $X$ in each of twelve $\Delta R$ bins. The first bin covers $0.0<\Delta R<0.05$ and the last bin covers $0.55<\Delta R<$ 0.6 . The shapes of these efficiencies as functions of $X$ and $\Delta R$ are primarily a combination of effects of the photon distorting the cluster shower shape and cluster centroid position causing either the EM or track match requirements to fail and of the photon carrying sufficient energy that the electron fails either the track or cluster $p_{T}$ requirement.

The efficiency in the first three $\Delta R$ bins is mainly driven by the track matching requirement and, to a lesser extent, by the shower shape requirement: while the photon is still close enough to the electron for most of its energy to be deposited in the same reconstruction cone, the shower shape at large values of $X$ becomes too different from that expected for a single electron, and more importantly, the calorimeter based estimate of the cluster position deviates significantly from the track based expectation. In the last three $\Delta R$ bins the photon is far away from the electron cone and does not directly interfere with the electron reconstruction. Besides these obvious limiting cases, there other interesting features. At large $\Delta R$ and large $X$, the efficiency drops because the cluster begins to be reconstructed around the photon, which will not have an associated track. At intermediate $\Delta R$ and intermediate $X$ there is a bump in the efficiency reconstruction when the photon is sufficiently far from the electron to not spoil the shower shape and track match requirements but it still not energetic enough to have EM cluster reconstructed around it.

\subsubsection{Track-Matching Efficiency}

The track-matching efficiency $\epsilon_{\text {trk }}\left(z_{V}, \eta, p_{T}\right)$ is described as a product of two efficiencies, one expressed as a function of $z_{V}$ and $\eta$ and the second expressed as a function of $p_{T}$ and $\eta$. The first of these is derived using the tag-and-probe method applied to $Z \rightarrow e e$ candidate events. The probe electron is initially required to pass all selections except the tracking requirements. The resulting efficiency is shown in Fig. 7.3. Because this is derived for both variables simultaneously, the correlations are automatically included. The second function describes the $p_{T}$ dependence and correlation with $\eta$ of the track requirements. Due to the limited size of $Z$ boson sample, it is derived from full MC and arbitrarily normalized to 0.98 at $p_{T}=45 \mathrm{GeV}$. The result is shown Fig.7.4.

\subsubsection{EM Identification Efficiency}

The efficiency accounting for the EM cluster finding, HMatrix, isolation, and EM fraction requirements is derived from $Z \rightarrow e e$ data, again the using tag-and-probe method. For this determination, the probe object is a track which passes our tracking requirements, and the invariant mass of the track and tag electron is required to be consistent with a $Z$ boson. This efficiency is a function of $\eta_{\text {det }}$ and electron $p_{T}$. 

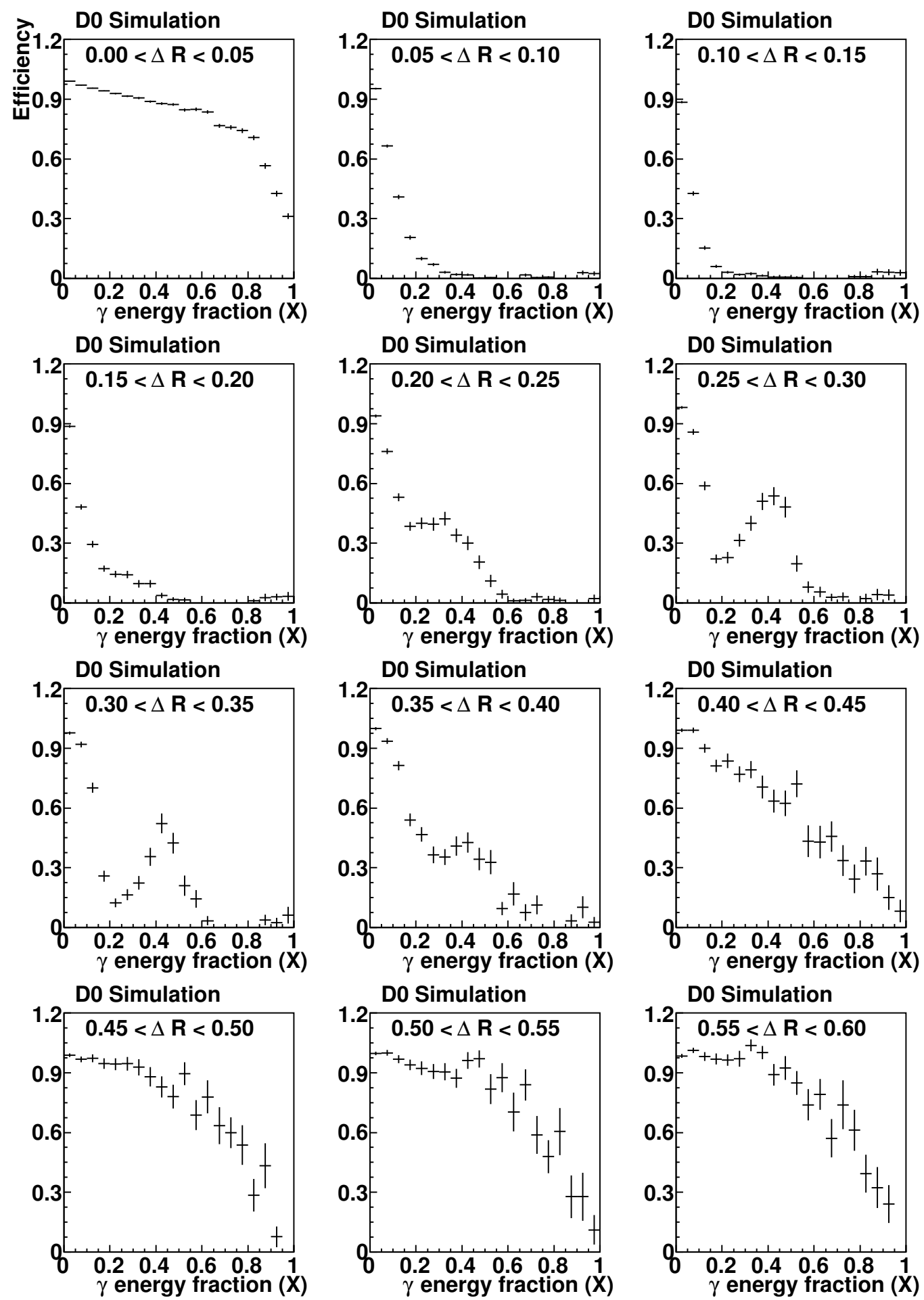

Figure 7.2: Electron identification efficiency for electrons accompained by FSR determined from full MC as a function of the fraction of the energy carried by the photon. Each pane corresponds to a different $\Delta R$ region, and the distributions over $\eta$ and $E^{e}$ have been integrated for clarity. 


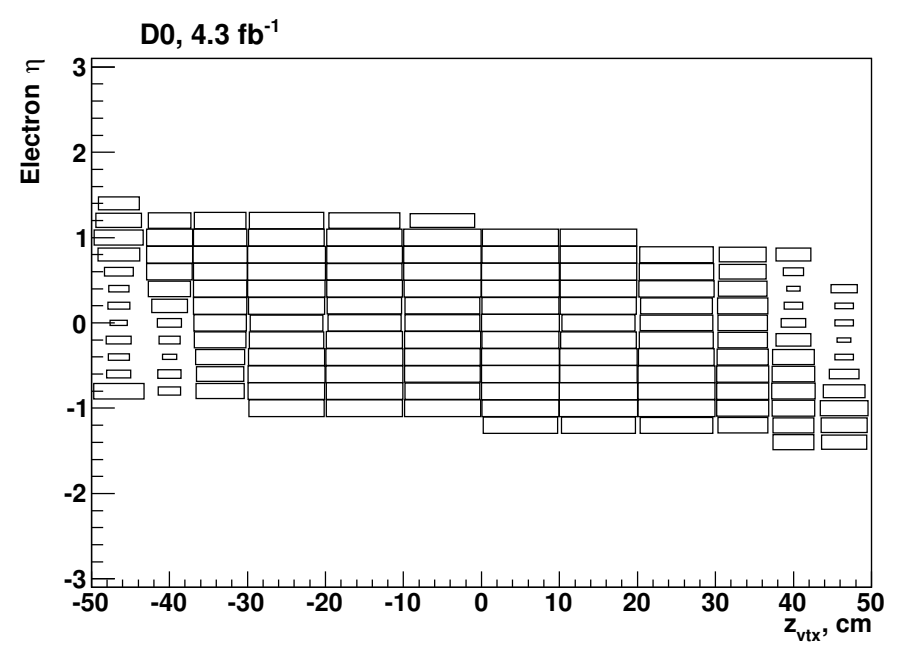

Figure 7.3: Track-matching efficiency as a function of $z_{V}$ and $\eta$ in data. The efficiency is proportional to the area of the boxes.

\subsection{5 $\phi_{\text {mod }}$ Efficiency}

The D0 calorimeter has $32 \mathrm{EM}$ modules in the CC region. Each module has a width of $2 \pi / 32 \sim 0.2$ radians in $\phi$. Between any two adjacent modules there is an uninstrumented region (crack) of the width of $\sim 0.02$ radians in $\phi$. An intra-module $\phi$ variable, $\phi_{\text {mod }}$ is defined as the fractional part of $32 \phi / 2 \pi$. This variable measures the angular position within any module as a fraction of module width (with $0 \leq \phi_{\bmod } \leq 1$ ). Each of the EM1, EM2 and EM4 layers in an EM module consists of two readout cells. The central value $\phi_{\text {mod }}=0.5$ corresponds to the inter-cell boundary. The values close to 0 and 1 are the module edges. The EM3 layer is segmented twice as finely in both $\eta$ and $\phi(0.05$ radians wide). The $\phi_{\text {mod }}$ values at $0.25,0.5$, and 0.75 correspond to the inter-cell boundaries of EM3.

Because of dead regions inside and outside an EM module, the reconstructed electron cluster barycenter $\phi$ value $\left(\phi^{\mathrm{EM}}\right)$ is biased away from these dead regions. The left panel on figure 7.5 shows the $\phi_{\text {mod }}^{\mathrm{EM}}$ bias, namely $\phi_{\text {mod }}^{\mathrm{EM}}-\phi_{\text {mod }}^{\text {trk }}$ as a function of $\phi_{\text {mod }}^{\text {trk }}$, which is calculated from the extrapolated track $\phi$. Since $\phi^{\text {trk }}$ is unbiased, we can see a strong tendency for the $\phi^{\mathrm{EM}}$ to move away from these dead region. The $\phi_{\text {mod }}$ efficiency is derived using the tag-and-probe method applied to $Z$ candidate events and is shown in Fig. 7.5 . The efficiency variation with $\phi_{\text {mod }}^{\text {trk }}$ is small except near the edges. We therefore apply a fiducial requirement, $0.1 \leq \phi_{\text {mod }}^{\text {trk }} \leq 0.9$, restricting our analysis to the region of stable $\phi_{\bmod }^{\text {trk }}$ efficiency. 


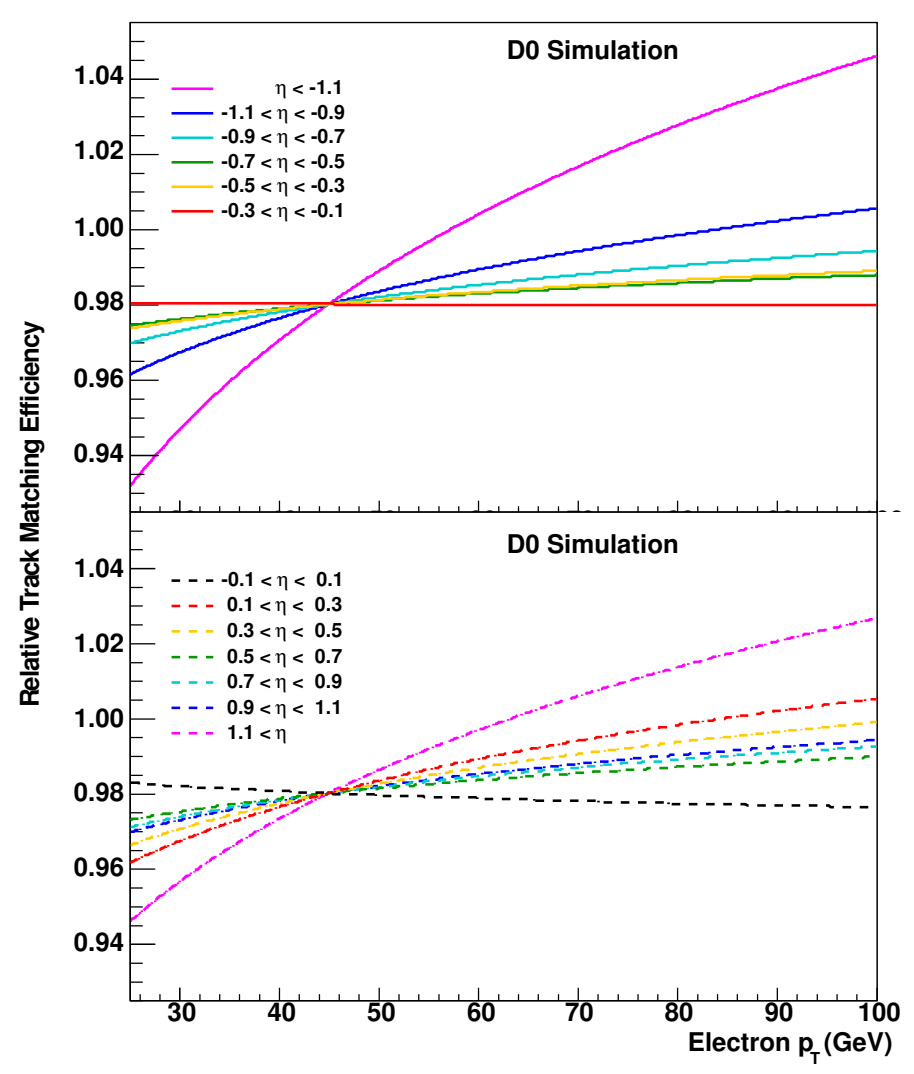

Figure 7.4: Track-matching efficiency as a function of $p_{T}^{e}$ in 13 bins of $\eta$ in full MC. The shapes reflect the fact that tracks from electrons with higher energy are more easily matched to the calorimeter cluster. This relative efficiency is arbitrarily normalized to 0.98 at $p_{T}^{e}=45 \mathrm{GeV}$. The total track-matching efficiency is the product of the relative efficiencies shown in Fig.7.3 and Fig.7.4.

\subsubsection{Electron $\phi$ Efficiency}

The reconstructed electron $\phi$ distribution from $W \rightarrow e v$ events is not uniform. Once the $\phi_{\text {mod }}$ induced effects are incorporated, we attribute the remaining overall $\phi$ dependence to small-scale imperfections in the detector, primarily dead tracker regions and calorimeter cells. Since the left-over imperfections do not interfere with the energy response of the calorimeter, this efficiency is determined by dividing the $\phi$ distribution in data or full MC by that from the corresponding fast $\mathrm{MC}$ after including all previous fast $\mathrm{MC}$ efficiencies.

\subsubsection{Hadronic Energy Dependent Efficiency}

The efficiencies described thus far are directly related to kinematic properties of the electron and radiated photons. Effects arising from the presence of hadrons in the same events 

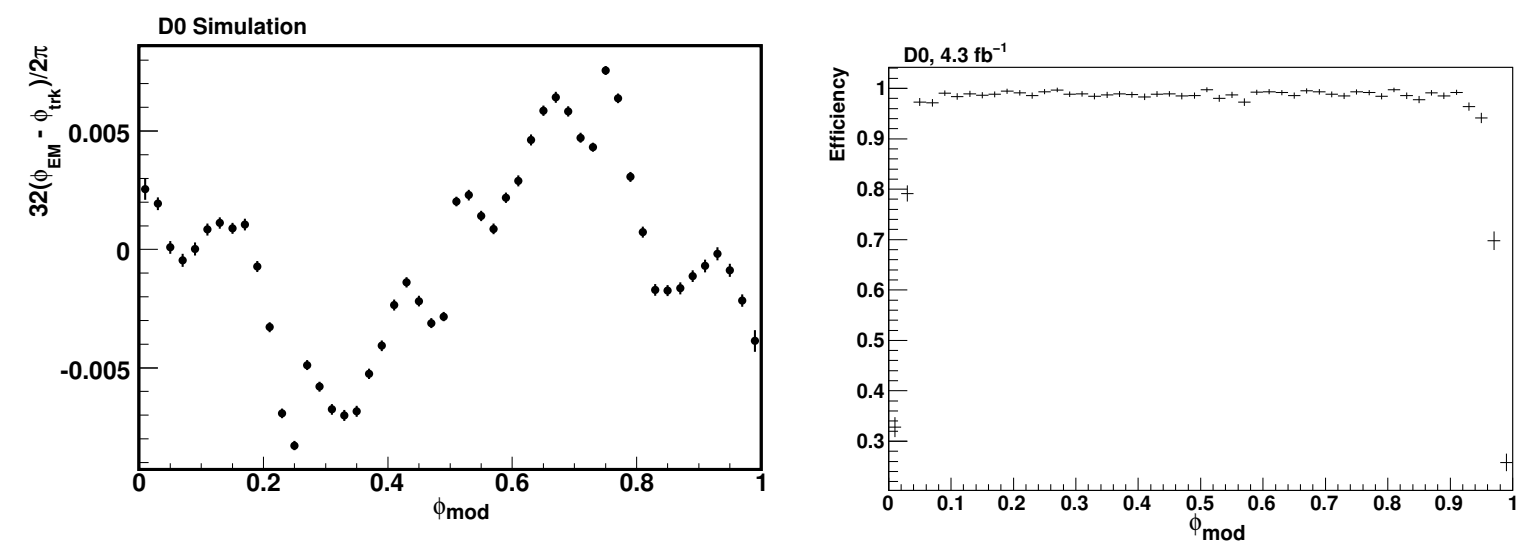

Figure 7.5: (left) Mean difference between $\phi^{\mathrm{EM}}$ and $\phi^{\text {trk }}$ in module units as a function of $\phi_{\mathrm{mod}}^{\text {trk }}$. (right) $\phi_{\text {mod }}$ electron reconstruction efficiency as a function of the extrapolated track $\phi_{\text {mod }}$.

have been accounted for indirectly through the presence of the recoil and additional $p \bar{p}$ interactions in events used to derive the efficiencies, but the independent effects of the hadronic underlying energy are not specifically studied. The hadronic efficiency model accounts for the EM cluster reconstruction efficiency which is strongly affected by the presence of hadronic energy in the calorimeter cells. It also collective describes any residual $p_{T}^{e}$ and SET dependency of the electron reconstruction and identification efficiency.

The hadronic efficiency $\epsilon_{\text {had }}\left(\mathrm{SET}, \mathcal{L}, u_{\|}, p_{T}^{e}, \eta_{\text {det }}\right)$ depends on five variables, the first three being direct measures of the hadronic energy. The use of SET accounts for the impact of energy from additional interactions, and the use of $u_{\|}$accounts for the orientation of the hard recoil and the electron. The $p_{T}^{e}$ dependence arises because higher energy electrons are less affected by a fixed amount of nearby hadronic energy than lower energy electrons. Finally, the use of instantaneous luminosity $\mathcal{L}$ accounts for the different behaviour of the calorimeter read out at different instantaneous luminosity regimes.

The efficiency is derived in a multi-step process and uses as observable the ZB-event SET and true MC $p_{T}^{e}$ in both full MC and fast MC. These variables are chosen because they are not modified during the fast simulation and, thus, provide a robust observables to describe the cluster reconstruction efficiency as well as the impact of the hadronic energy on it, especially in high luminosity environments. The first step is then to create a version of the fast MC which has the $\mathrm{SET}_{Z B} \times p_{T}^{e}$, true distribution reweighted to agree with the full MC distribution. This provides a high statistics target to the fast MC model.

In the next step, we compare the number of events in the original and reweighted fast $\mathrm{MC}$ in bins of $\left(u_{\|}, p_{T}^{e}, \eta_{\mathrm{det}}, \mathcal{L}\right)$. This ratio between the number of events in each bin is taken as the initial estimate of the efficiency. Although the overall efficiency normalization is arbitrary in our model, it is important to respect the relative normalizations and thus the need for this initial estimate. In each bin, we compare the distribution of the ratio $\mathrm{SET} / p_{T}^{e}$ between the original and the reweighted fast MC. The ratio between the two is 
smoothed using a polynomial function and the average value is shifted to one so that it can be interpreted as a perturbation over the initial estimate. The hadronic efficiency is then the product of the initial estimate and the SET / $p_{T}^{e}$ perturbation in each bin.

This model is derived separately for $W \rightarrow e v$ and $Z \rightarrow e e$ events, since, in the case of $Z \rightarrow e e$ events, the correlated kinematics introduces extra dependencies which would not be described by the application of the efficiency derived from $W \rightarrow e v$ full MC twice.

\subsubsection{Monte Carlo Validation}

As part of the full MC measurement, we validate our efficiency model using the MC generator level information to check the efficiency modeling as a function of the many variables that were used to parametrize the model. Figure 7.6 shows the comparison of the total electron efficiency (except for the trigger-which is determined in data for use in the fast MC) as a function of true $p_{T}^{e}$ in full MC and fast MC. Figure 7.7 shows the comparison of total electron efficiency as a function of $\eta_{\text {det }}$ in full MC and fast MC. For electrons in $Z \rightarrow e e$ events and in $W \rightarrow e v$ events, we observe our efficiency model in fast $\mathrm{MC}$ accurately describes the efficiency in full MC.
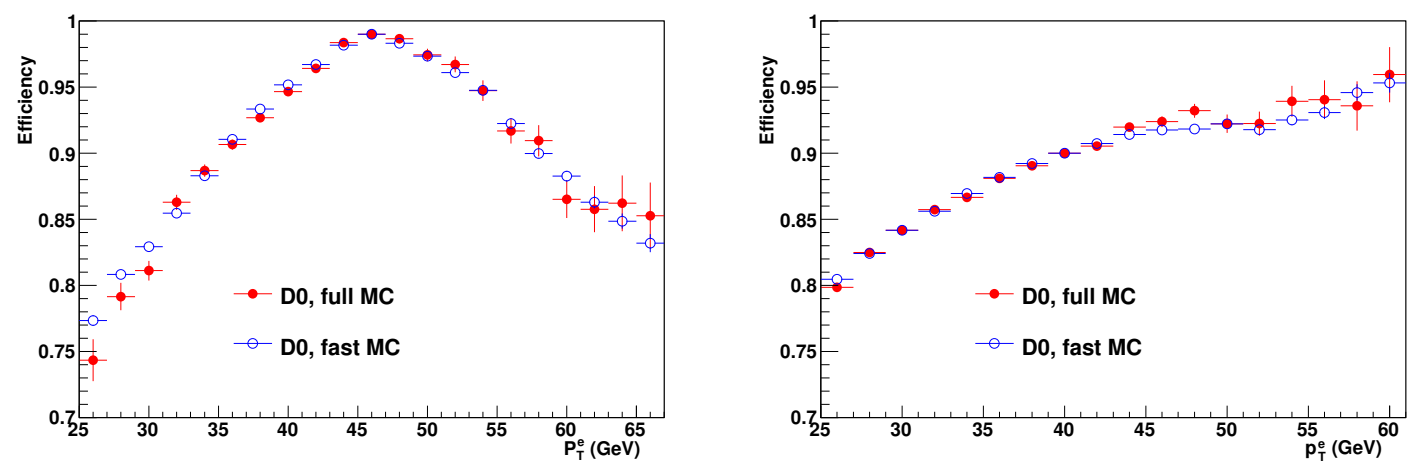

Figure 7.6: The efficiency as a function of true $p_{T}^{e}$ in full MC and fast MC for electrons in $Z \rightarrow e e$ (left) and $W \rightarrow e v$ (right) events.

We conclude that the electron reconstruction and identification efficiency in full MC is well described by the fast $\mathrm{MC}$ model, and minimal adjustments are needed to describe the data. This success of the detailed simulation is in large part due to the fact that the main source of inefficiency (pile-up) is modeled by the ZB overlay, ie. by the superposition of collider data events during randomly selected bunch crossings.

\subsubsection{Residual Efficiency Corrections}

The efficiencies discussed thus far assumes that the full MC, when used, accurately describes the dependencies of the efficiencies and that the biases arising from the tag and 

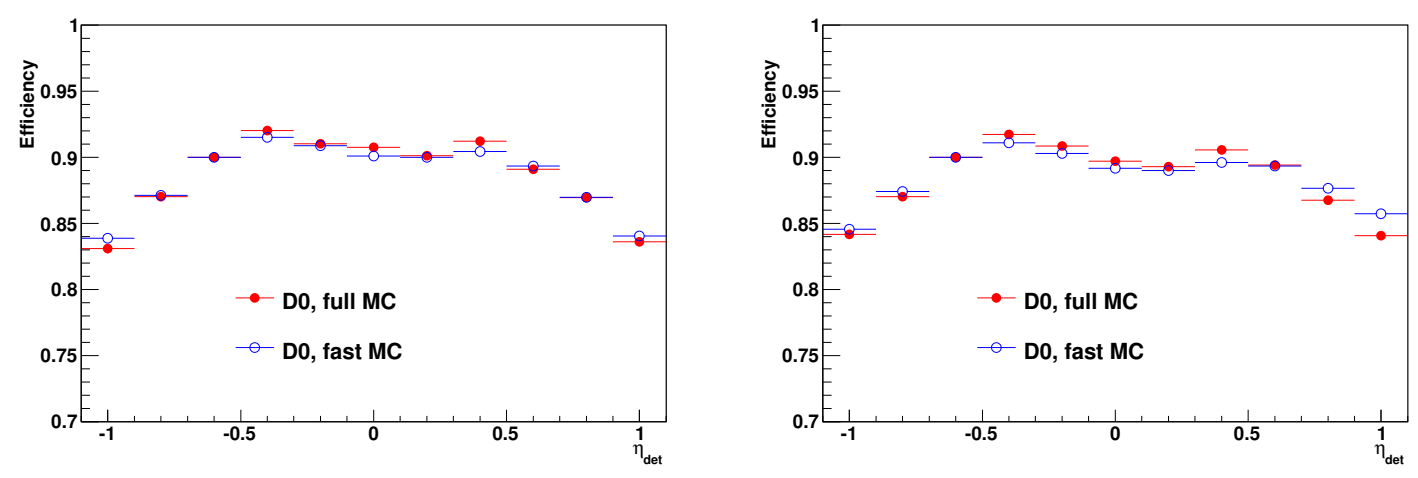

Figure 7.7: The efficiency as a function of $\eta_{\text {det }}$ in full MC and fast MC for electrons in $Z \rightarrow e e$ (left) and $W \rightarrow e v$ (right) events.

probe method are small. After applying the above efficiencies to the fast $\mathrm{MC}$, comparisons are made between the full $\mathrm{MC}$ and data or fast $\mathrm{MC}$ and data. Based on these comparisons, two independent residual efficiency corrections $R_{1}(\mathrm{SET}, \mathcal{L})$ and $R_{2}\left(u_{\|}\right)$are derived.

The first of these $R_{1}(\mathrm{SET}, \mathcal{L})$ is derived by measuring the electron identification efficiency as a function of SET and $\mathcal{L}$ in both $Z \rightarrow e e$ data and full MC. The ratio of the efficiency from data to that from the full MC defines this relative efficiency correction. The ratios are shown in Fig. 7.8 for projections on the SET and $\mathcal{L}$ axes respectively. This efficiency is a correction needed for data analysis to the $\epsilon_{\text {had }}$ efficiency derived previously by comparing full MC to fast MC.

When measuring the data efficiency to use in this data to full $\mathrm{MC}$ ratio, rather than directly counting the number of probe electron, dielectron invariant mass distributions are produced in bins of the variable(s) used to parametrize the correction. Two distributions are made, one from a loose sample in which the probe electron is not required to satisfy the selection under study, and a tight sample in which the probe electron is required to satisfy all the selection requirements. The $Z \rightarrow e e$ contribution in each distribution is determined by fitting the distribution to $Z \rightarrow e e$ signal and background components, giving a pure $Z \rightarrow e e$ yield.

The second residual efficiency correction $R_{2}\left(u_{\|}\right)$addresses imperfections in the $u_{\|}$dependency of the efficiency model. This is derived using the same technique of measuring the dielectron invariant mass distribution in bins of $u_{\|}$, but taking the ratio of the efficiencies calculated in data to those derived in fast MC $Z \rightarrow e e$ events. The correction used is:

$$
R_{2}\left(u_{\|}\right)= \begin{cases}1.0 & \text { for } u_{\|}<0 \\ 1.0-0.0025 \times u_{\|} & \text {for } u_{\|}>0\end{cases}
$$



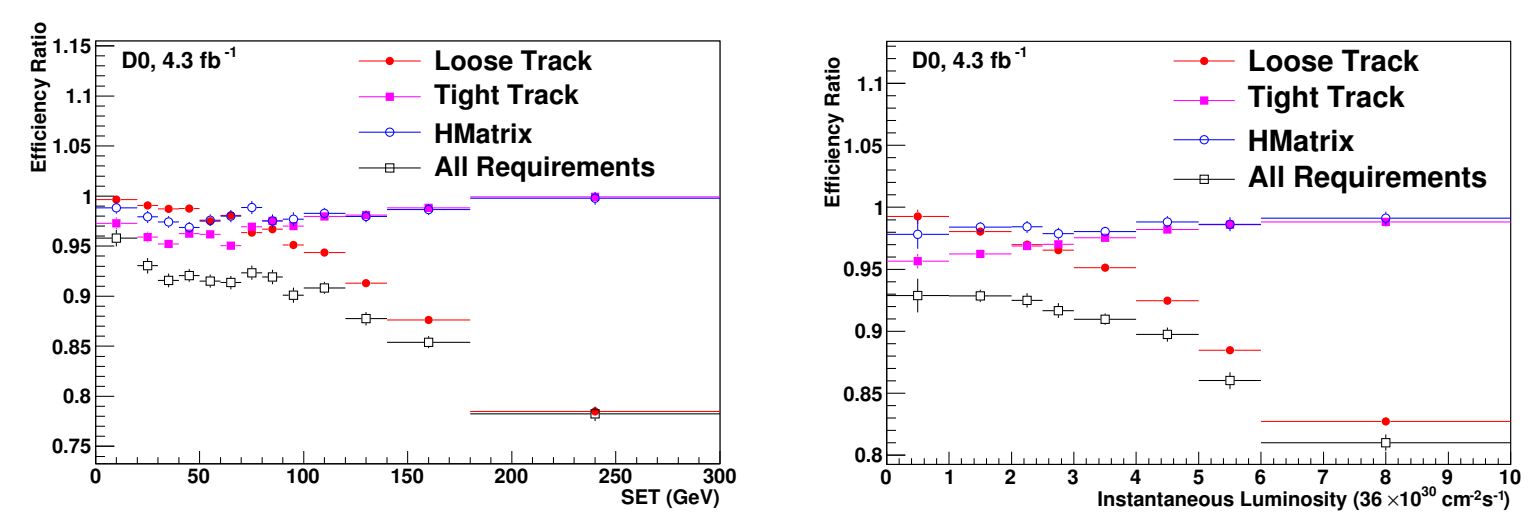

Figure 7.8: Electron efficiency ratio (data/full MC) as a function of SET (left) and instantaneous luminosity (right) for HMatrix, loose-track-match, tight-track-match, and the overall (HMatrix $\times$ loose-track-match $\times$ tight-track-match) criteria. Only the "All" requirements correction is used in analysis. The others are shown to illustrate where the major improvements arise.

\subsubsection{Systematic Uncertainty due to Efficiencies}

The most significant efficiency-related uncertainty results from the adjustment of the final residual uncertainty correction $R_{2}$. The resulting uncertainties on $M_{W}$ are 1,2 , and $5 \mathrm{MeV}$ respectively for the $m_{T}, p_{T}^{e}$, and $\mathbb{E}_{T}$ methods.

\subsection{Electron Response Parametrization}

The electron response model is composed of three parts: the response model, the resolution model and the underlying energy model. A short introduction to the model is provided, and detailed descriptions are then given in each of the sections.

The response model describes the average reconstructed electron energy for a given electron true energy. We begin by building a parametrized model for the contribution of radiated photons to the reconstructed electron cluster energy, since the energy from these photons is not distinguishable from the electron energy itself after reconstruction. We then correct residual luminosity and $\eta$ dependencies of the response which are not described by the data calibration. Finally, we use the $Z \rightarrow e e$ data and the well known value of the $Z$ boson mass to calibrate the absolute energy scale.

The resolution model describes the fluctuations in the reconstructed electron energy. The EM calorimeter sampling resolution is modeled from a full MC sample which includes the improvements described in Sec. 5.2. This allows a detailed description of the dependence of the sampling term with the amount of dead material upstream of the calorimeter as well as the energy and angular dependencies that it creates. We use $Z \rightarrow e e$ data and the well known value of the $Z$ boson width to calibrate the resolution constant 
term. The resolution model does not describe the noise fluctuations by itself, since most of this contribution comes from the underlying hadronic energy inside the electron reconstruction cone.

The underlying energy model describes both the average contribution of the hadrons to the electron reconstructed energy and the fluctuations around this value. The model is based on measurements in $W \rightarrow e v$ data of the energy flow in calorimeter sections with the same geometry as the electron reconstruction cone, but away from it. The effect of zero suppression on the recoil energy transported into the electron reconstructed cluster is described using full MC.

\subsubsection{Photon Radiation Effects}

Photon radiation from the $W$ boson decay electron can bias the mass measurement when the energy from radiated photons is not included in the reconstructed electron energy. This happens if the radiated photon is far enough away from the electron that its energy is not counted in the electron energy, or if the photon energy is absorbed totally or partially by dead material in front of the calorimeter.

The radiated photons arise either from final state radiation (FSR) at the electron generation stage or during the interaction of the electron with dead material in front of the calorimeter (bremsstrahlung). The bremsstrahung energy loss is corrected in full MC by the electron energy loss correction (see Sec. 5). The FSR energy loss is modeled in fast $\mathrm{MC}$.

The average energy response difference between no FSR and FSR (for the same $\eta$ and energy bin as Fig. 7.2) is given in Fig. 7.9. Again 12 plots are shown for the same $\Delta R$ bins. The horizontal axis represents the fraction of energy carried out by the photon $(X)$. The vertical axis is defined as a ratio $\kappa$ : the difference between the reconstructed electron energy with and without FSR divided by the difference between the true electron energy with and without FSR, which is interpreted as the photon energy.

$$
\kappa=\frac{E_{\text {reco }}-E_{\text {reco }}[\text { not showered }]}{E_{\text {true }}[\text { not showered }]-E_{\text {true }}} \text {. }
$$

At high $\Delta R$ we expect $\mathcal{K}=-1$ because the photon is far away from the electron and does not contribute to the reconstructed electron energy. At low $\Delta R$, we expect large negative values of $\kappa$ due to losses in the dead material, and we expect these losses to be of lesser importance as $X$ increases. At intermediate $\Delta R$ and large values of $X, \kappa \simeq 1$ since now the EM cluster is reconstructed around the photon. The final FSR energy loss parametrization is done as a function of the same variables as the FSR efficiency, namely: $\Delta R, X, \eta_{\text {det }}$ and $E^{e}$.

\subsubsection{Luminosity Dependence of the Calibration}

The current $M_{W}$ measurement explores a much higher luminosity regime than our previous measurement (Fig. 4.1) and we observe a significant dependence of the energy re- 

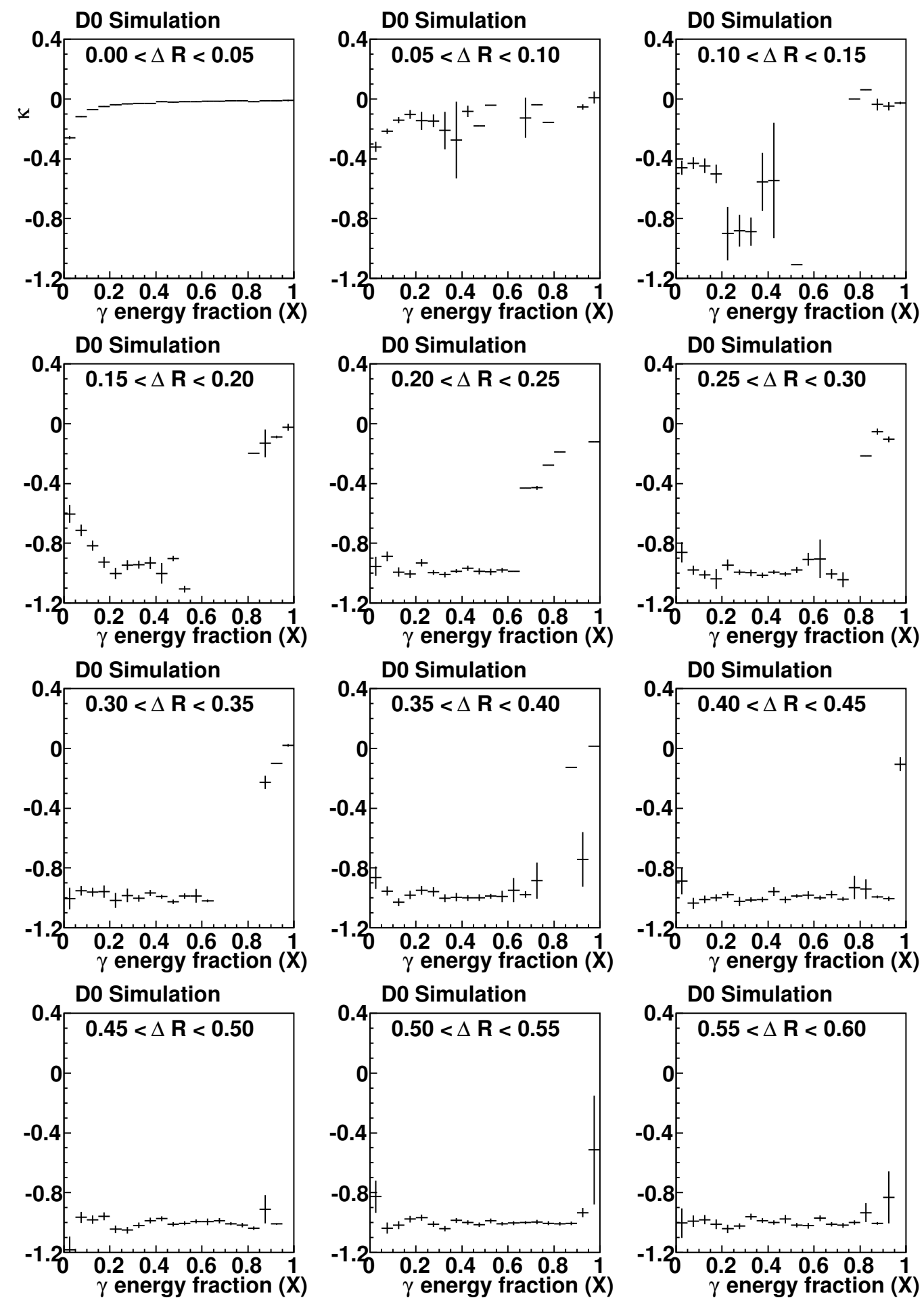

Figure 7.9: Electron energy correction determined from full MC as a function of fraction of the energy carried out by the photon. 
sponse with the luminosity due to anomalous currents described in Sec. 2.4.5. The dependence was studied with the resistive circuit model for the HV distribution in the CC modules. An additional global scale factor of 0.88 is derived from the luminosity dependence of the $Z$ peak position measured in data. We simulate single electrons at different energies, angles and luminosities, both with and without the tuned model of luminosity dependence, to parametrize the response change for electrons as a function of instantaneous luminosity and $\eta_{\text {det }}$. For electrons at normal incidence, where the effect is maximal, the fractional change in response at a luminosity of $L=120 \times 10^{30} \mathrm{~cm}^{-2} \mathrm{~s}^{-1}$ is $0.42 \%$. A possible dependence on electron energy has been considered and found to be negligible.

\subsection{3 $\eta$-Dependence of the Calibration}

The procedure used to calibrate the EM calorimeter explicitly includes an equalization of the energy response of towers at different $\eta$ values. This procedure is relatively simple in that it just adjusts the gains in such a way that the position of the $Z$ mass peak in data is the same for any combination of $\eta$ values of the two electrons in a $Z \rightarrow e e$ event. This procedure does not account for the $\eta$-dependence of the underlying energy flow which implies that reconstructed $Z$ boson mass should have a small $\eta$ dependence. This is a small effect that is not relevant for most purposes, but we do take it into account in the measurement of the $W$ boson mass by simulating this dependence in fast MC.

To derive an $\eta_{\text {det }}$-dependent correction to the electron energy scale, we split our sample of CC-CC Z $\rightarrow e e$ events into 15 categories as defined in Table 5.2 (Sec. 5.1). We use our standard procedures to fit for the $Z$ boson mass, separately for each category. Our standard procedures use $m_{e e}$ templates produced using fast $\mathrm{MC}$, in which the effect of the underlying energy is included. The results of these mass fits are summarized in Fig.7.10. We define one relative gain constant for each $\left|\eta_{\text {det }}\right|$ bin (Table 5.1), and we translate the 15 mass values from Fig. 7.10 into the values of the 5 relative gain constants. The world average value [71] of the $Z$ mass is used to translate energies into dimensionless perelectron relative gains. The results of the translation are shown in Fig.7.10. They are used in fast $\mathrm{MC}$ for the simulation of the $\eta_{\text {det }}$ non-uniformity in the calorimeter gains.

\subsubsection{Energy Response and Resolution}

Starting from the FSR-photon-merged true electron energy, the reconstructed electron energy is simulated as

$$
E=R_{\mathrm{EM}}\left(E_{0}\right) \otimes \sigma_{\mathrm{EM}}\left(E_{0}\right)+\Delta E,
$$

where $E_{0}$ is the FSR-photon-merged true electron energy, $E$ is the reconstructed energy, $R_{\mathrm{EM}}\left(E_{0}\right)$ is the response, $\sigma_{\mathrm{EM}}$ is the energy resolution for electromagnetic objects, and $\Delta E$ is the correction term arising from electron reconstruction window effects. The operation $f_{1}\left(E_{0}\right) \otimes f_{2}\left(E_{0}\right)$ represents the convolution of a $\delta\left(E-E_{0}\right)$ function centered in $E_{0}$ with a gaussian distribution of width $f_{2}\left(E_{0}\right)$. 

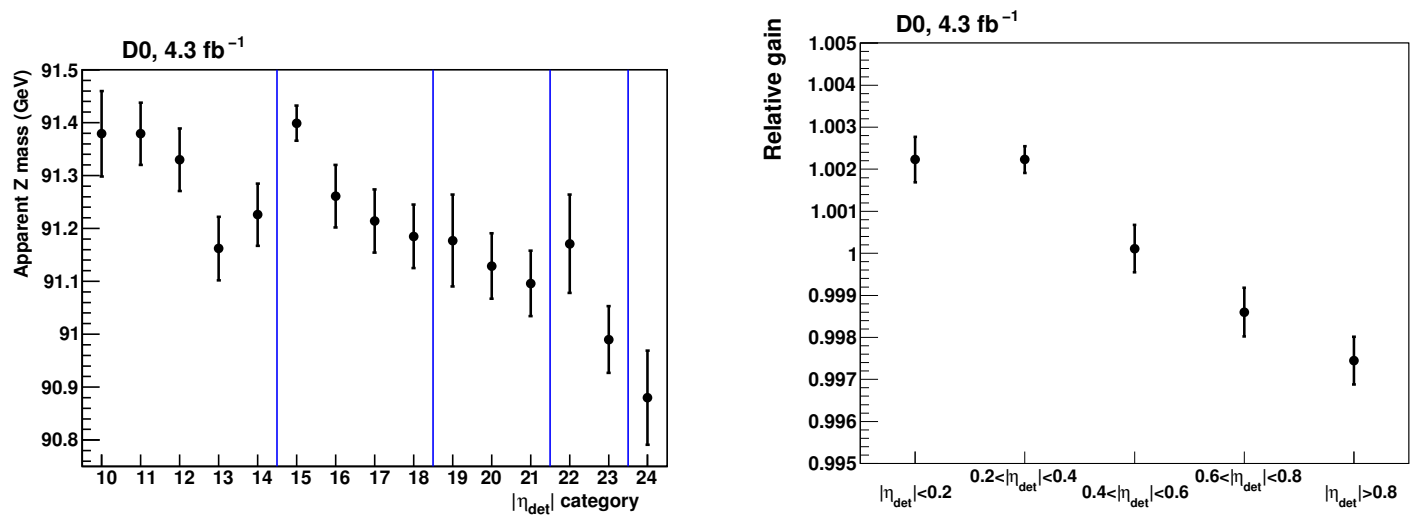

Figure 7.10: (left) Result of the $Z$ mass fit per $\eta_{\text {det }}$ category prior to applying $\eta$-dependent corrections (Table 5.2). (right) Result of the translation into one relative gain constant per $\eta_{\text {det }}$ bin.

The resolution of the EM calorimeter $\sigma_{\mathrm{EM}}$ is modeled as

$$
\frac{\sigma_{\mathrm{EM}}\left(E_{0}\right)}{E_{0}}=\sqrt{C_{\mathrm{EM}}^{2}+\frac{S_{\mathrm{EM}}^{2}}{E_{0}}+\frac{N_{\mathrm{EM}}^{2}}{E_{0}^{2}}} .
$$

in which $C_{\mathrm{EM}}, S_{\mathrm{EM}}$ and $N_{\mathrm{EM}}$ are constants used in the constant, sampling and noise terms respectively. Due to the material in front of the calorimeter, the sampling term parameter $S_{\mathrm{EM}}$ depends on electron energy and incident angle, and is parametrized as

$$
S_{\mathrm{EM}}\left(E_{0}, \eta\right)=S_{0} \exp \left[S_{1}\left(\frac{1}{\sin \theta}-1\right)\right]+\frac{\left(S_{2} \eta+S_{3}\right)}{\sqrt{E_{0}}}
$$

where,

$$
\begin{array}{lc}
S_{0}= & 0.15294 \pm 0.00005 \mathrm{GeV}^{1 / 2} \\
S_{1}= & 1.543 \pm 0.007 \\
S_{2}= & -0.025 \pm 0.001 \mathrm{GeV} \\
S_{3}= & 0.172 \pm 0.002 \mathrm{GeV}
\end{array}
$$

The values of the smearing parameters $S_{0}$ to $S_{3}$ are determined from the improved simulation of the D0 detector that has been tuned using $Z \rightarrow e e$ data, as discussed in Sec. 5 . and the uncertainties in the parameters are dominated by the limited precision of the material tune.

The value of $N_{\mathrm{EM}}$ is set to zero since most of the noise is already included in the fluctuations in the model of the energy flow from the underlying event into the electron energy cone. The extraction of $C_{\mathrm{EM}}$ from the width of the $Z$ mass peak is discussed in Sec.7.3.5. 
The energy response for electrons in (7.4) is modeled as

$$
R_{\mathrm{EM}}\left(E_{0}\right)=F_{\eta-e q}\left(\eta_{\mathrm{det}}\right) \times F_{\mathrm{HV}-\mathrm{loss}}\left(L, \eta_{\text {det }}\right) \times\left(\alpha \times\left(E_{0}-\overline{E_{0}}\right)+\beta+\overline{E_{0}}\right)
$$

where $F_{\mathrm{HV}-\text { loss }}\left(L, \eta_{\mathrm{det}}\right)$ implements the model of the luminosity-dependence of the calorimeter gains due to the HV loss that is discussed in Sec. 7.3.2, and $F_{\eta-e q}\left(\eta_{\text {det }}\right)$ describes the $\eta$-non-uniformity discussed in Sec.7.3.3. The parameters $\alpha$ and $\beta$ are referred to as scale and offset, and $\overline{E_{0}}=43 \mathrm{GeV}$ is a reference value for the energy of electrons in $Z \rightarrow e e$ events. The values of $\alpha$ and $\beta$ are determined from $Z \rightarrow e e$ events in collider data. The constant $\overline{E_{0}}$ is introduced to reduce the correlation between the parameters $\alpha$ and $\beta$ to improve the stability of the numerical evaluation of the covariance matrix of the simultaneous fit for $\alpha$ and $\beta$.

When the electron $\phi$ position within the CC EM module is such that $\phi_{\bmod }<0.2$ or $\phi_{\text {mod }}>0.8$, the modeling of the electron energy response and resolution in fast MC are modified compared to the description above to account for the uninstrumented regions. We model the loss in response by a simple linear function with respect to the boundary distance:

$$
R_{\text {crack }}\left(E_{0}\right)=R_{\mathrm{EM}}\left(E_{0}\right) \times\left[1-\alpha_{\text {crack }}-\beta_{\text {crack }} \times\left(\left|\phi_{\text {mod }}-0.5\right|-0.3\right)\right],
$$

where the values of $\alpha_{\text {crack }}=0.0062 \pm 0.0003$ and $\beta_{\text {crack }}=0.0035 \pm 0.002$ are determined by studying the $Z \rightarrow e e$ invariant mass distributions as a function of $\phi_{\bmod }$ of both electrons. Similarly, for electrons near module boundaries, we derive a correction to a gaussian resolution model by introducing a power-law tail, as in the Crystal Ball function [72]. The decay power is fixed to 10 and the power law is used for fluctuations below the mean energy larger than:

$$
\sigma_{\text {crack }}\left(E_{0}\right)=\sigma_{\mathrm{EM}}\left(E_{0}\right) \times\left[\alpha_{\mathrm{CB}}-\beta_{\mathrm{CB}} \times\left(\left|\phi_{\text {mod }}-0.5\right|-0.3\right)\right]
$$

where $\alpha_{\mathrm{CB}}=1.71 \pm 0.02$ and $\beta_{\mathrm{CB}}=6.75 \pm 0.11$.

The determination of the values of the global parameters $\alpha$ and $\beta$ is one of the most important steps in the measurement of the $W$ boson mass. The scale and offset cannot be distinguished from one another to the precision required using only the $M_{Z}$ distribution. However, the fact that electrons from $Z$ decays are not monochromatic can be exploited to constrain the energy dependence of the energy response. The measured $Z$ mass $\left(M_{Z}\right)$ is calculated from:

$$
M_{Z}=\sqrt{2 E^{e_{1}} E^{e_{2}}(1-\cos (\omega))}
$$

where $\omega$ is the opening angle between the two electrons.

Substituting and Taylor expanding with $\beta \ll E^{e_{1}}+E^{e_{2}}$ gives (ignoring $\overline{E_{0}}$ )

$$
M_{Z} \approx \alpha \times M_{Z}^{0}+f_{Z}^{0} \times \beta+O\left(\beta^{2}\right)
$$

where $f_{Z}$ is a kinematic variable defined as:

$$
f_{Z}=\frac{\left(E_{0}^{e_{1}}+E_{0}^{e_{2}}\right)(1-\cos (\omega))}{M_{Z}^{0}}
$$


in which quantities denoted by a zero sub- or superscript are calculated with all corrections except the $\alpha$ and $\beta$ correction.

This equation relates the observed mass to the scale, offset and the true energies of the electrons. From the $M_{Z}$ vs. $f_{Z}$ distribution it is then possible to extract the scale and offset. By varying both the scale and offset, templates of the two dimensional distribution $M_{Z}$ versus $f_{Z}$ in the fast MC are compared to the equivalent distribution in data. The final values of $\alpha$ and $\beta$ used are those which minimize the likelihood formed during the comparison.

The scale and offset are determined separately in different bins of instantaneous luminosity. The luminosity $\mathcal{L}$ is expressed in units of $36 \times 10^{30} \mathrm{~cm}^{-2} \mathrm{~s}^{-1}$. Table 7.1 summarizes the scale and offset parameters, along with the correlation coefficients from the fits. A graphical representation of the fit results is shown in Fig. 7.11. As can be seen from Fig. 7.11, the results from the fits for each of the bins in instantaneous luminosity agree well with each other. This shows that our model of the underlying energy flow into the electron window (Sec. 7.3.6) and the model of the luminosity-dependence of the calorimeter gains (Sec. 7.3.2) are correctly accounting for the luminosity dependence of the detector response to electrons. We could simply use one luminosity-averaged set of parameters for the scale and offset. We actually use the different values per bin in luminosity because one does not lose statistical power (ie. one does not increase the systematic uncertainty in the $W$ boson mass due to the electron energy scale by splitting into luminosity bins.

\begin{tabular}{c|c|c|c|c}
\hline \hline & $0<L<2$ & $2<L<4$ & $4<L<6$ & $L>6$ \\
\hline$\alpha$ & $1.0237 \pm 0.0043$ & $1.0164 \pm 0.0030$ & $1.0181 \pm 0.0047$ & $1.0300 \pm 0.0074$ \\
$\beta(\mathrm{GeV})$ & $0.129 \pm 0.032$ & $0.188 \pm 0.022$ & $0.208 \pm 0.034$ & $0.158 \pm 0.053$ \\
Correlation & -0.796 & -0.786 & -0.783 & -0.764 \\
\hline \hline
\end{tabular}

Table 7.1: Results of the fits for electron energy scale and offset to the collider data.

The dominant systematic uncertainty on our measurement of $M_{W}$ is the precision with which we measure the mean electron energy response. The uncertainties in the energy scale and offset are individually large but they are highly correlated. We propagate the correlated uncertainties in the scale and offset parameters to our measurement of $M_{W}$ and obtain uncertainties of $16 \mathrm{MeV}$ using the $m_{T}$ and $\mathbb{Z}_{T}$ distributions and $17 \mathrm{MeV}$ for the $p_{T}^{e}$ distribution.

\subsubsection{Determination of the Constant Term in Resolution}

For data, unlike the full $\mathrm{MC}$, the constant term $C_{\mathrm{EM}}$ is also important. It arises primarily from residual channel-to-channel calibration differences and describes an energyindependent contribution to the fractional energy resolution. Thus, its main impact is felt at high electron energies where the sampling term is suppressed by its approximate $1 / \sqrt{E}$ 


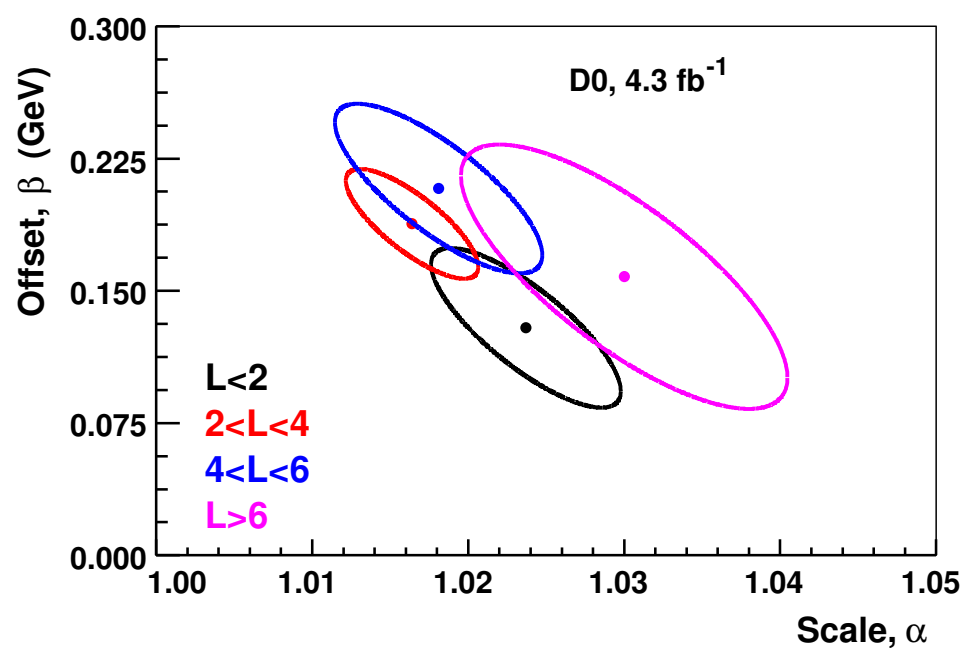

Figure 7.11: Central values and one standard deviation contours of the fits for electron energy scale and offset to the collider data.

behavior. The value of $C_{\mathrm{EM}}$ is extracted from the width of the $Z$ boson mass peak with the sampling term modeled as described above. The noise term is modeled in fast MC via the fluctuations in the model of the underlying event energy leaking into the electron reconstruction window. It does not represent an important contribution to the energy resolution for electrons from decays of $W$ and $Z$ bosons. The value of $C_{\mathrm{EM}}$ is determined using template fitting to the dielectron invariant mass distribution. The best fit value for $C_{\mathrm{EM}}$ is

$$
C_{\mathrm{EM}}=(1.997 \pm 0.073) \%
$$

which is in good agreement with our determination in Run IIa and with the Run II design goal of $2 \%$.

The propagation of the uncertainty due to electron energy resolution to $M_{W}$ requires the remeasurement of the constant term in pseudo-experiments (using the same procedure described in Sec. 5.2). The sampling term of the calorimeter is parametrized as a function of the number of radiation lengths $n X_{0}$ added upstream of the calorimeter. The measured constant term is correlated with the sampling term, so part of the of the uncertainty introduced by the sampling term will be compensated by the determination of the constant term. The contribution is found to be small, and is estimated to be $2 \mathrm{MeV}$ for the $M_{W}$ measurement using $m_{T}$ and $p_{T}^{e}$ and $3 \mathrm{MeV}$ for $\mathbb{B}_{T}$

\subsubsection{Electron-Window Effects}

To reconstruct an electron, we must define an electron-energy-reconstruction window (Fig.4.2). However, the energy in an electron-reconstruction window arises not only from the electron, but also from hadronic recoil, spectator parton interactions, and additional 
$p \bar{p}$ collisions. There are also effects from the suppression of electronic noise. These bias both the reconstructed electron energy and the reconstructed recoil energy. Extra energy is given to the electron from the recoil, and it is excluded from the reconstruction of the $u_{T}$. The additional energy added to the electron window is called $\Delta E$ (Eqn. 7.4, Sec.7.3.4), while the additional transverse energy subtracted from the recoil in the electron window is called $\Delta u_{\|}$(Eqn. 7.14) in Sec.7.4.

Contrary to what one might expect $\Delta u_{\|}$is not equal to $\Delta E \sin \theta^{e}$ for two reasons:

- The energy loss due to dead material in front of the calorimeter is corrected for the electron, but not for the recoil.

- Zero-suppression has different effects near a large concentrated energy $(\Delta E)$ compared to a small diffuse background energy $\left(\Delta u_{\|}\right)$.

To study electron window effects we construct a $\Delta u_{\|}$library by recording the energy deposition in random windows from $W \rightarrow e v$ events in collider data and full MC. These random windows are electron-reconstruction windows (Fig. 4.2), but selected to avoid any electron energy contribution. Events in this library sample the same luminosity profile as collider data used to measure the $W$ mass.

To derive the electron identification efficiency dependence on $\Delta u_{\|}$and to convert the $\Delta u_{\|}$to $\Delta E$, we need to know the energy difference of a given electron with and without the $\Delta u_{\|}$overlay including zero suppression.

- Electron only: contains only the electron and FSR photons.

- No electron: contains everything except the electron and FSR photons, ie. the hard recoil, spectator parton interactions, and additional $p \bar{p}$ interactions.

- Full sample: contains the complete event.

All three versions of a given event are passed through the standard reconstruction chain, including zero suppression. In the end, for a given event and a given electron reconstruction window, we can obtain the pure electron energy deposition $\left(E_{1}^{e}\right)$ from the electron only sample, the $\Delta u_{\|}$from the no electron sample, and the overlaid electron energy $\left(E_{2}^{e}\right)$ from the full sample. Therefore, $\Delta E=E_{2}^{e}-E_{1}^{e}$. Results of the dependence of $\Delta E$ on SET, $\mathcal{L}, p_{T}^{e}$, $\eta_{\text {det }}$, and $u_{\|}$are shown in Figs. 7.12 to 7.14 comparing the full MC and fast MC.

The studies discussed here leading to the $\Delta E$ determination also give information on the $\Delta u_{\|}$for the recoil system discussed in Sec. 7.4. We show the dependence of the mean $\Delta u_{\|} \equiv\left\langle\Delta u_{\|}\right\rangle$on $\mathcal{L}$ in Fig. 7.15 for various bins of SET. As we should expect, for the full SET range, $\left\langle\Delta u_{\|}\right\rangle$shows a strong dependence on $\mathcal{L}$. But in a fixed bin of SET there is almost no dependence on $\mathcal{L}$. In Fig. 7.15 we also study the dependence of $\left\langle\Delta u_{\|}\right\rangle$on SET for various bins of $\mathcal{L}$. We can see that $\left\langle\Delta u_{\|}\right\rangle$varies linearly with SET for any $\mathcal{L}$ (except for anomalies at very low $\mathcal{L}$ ). This implies that SET is a good measure of the dominant contribution to $\left\langle\Delta u_{\|}\right\rangle$arising from the $\mathrm{ZB}$ events from additional $p \bar{p}$ collisions. 

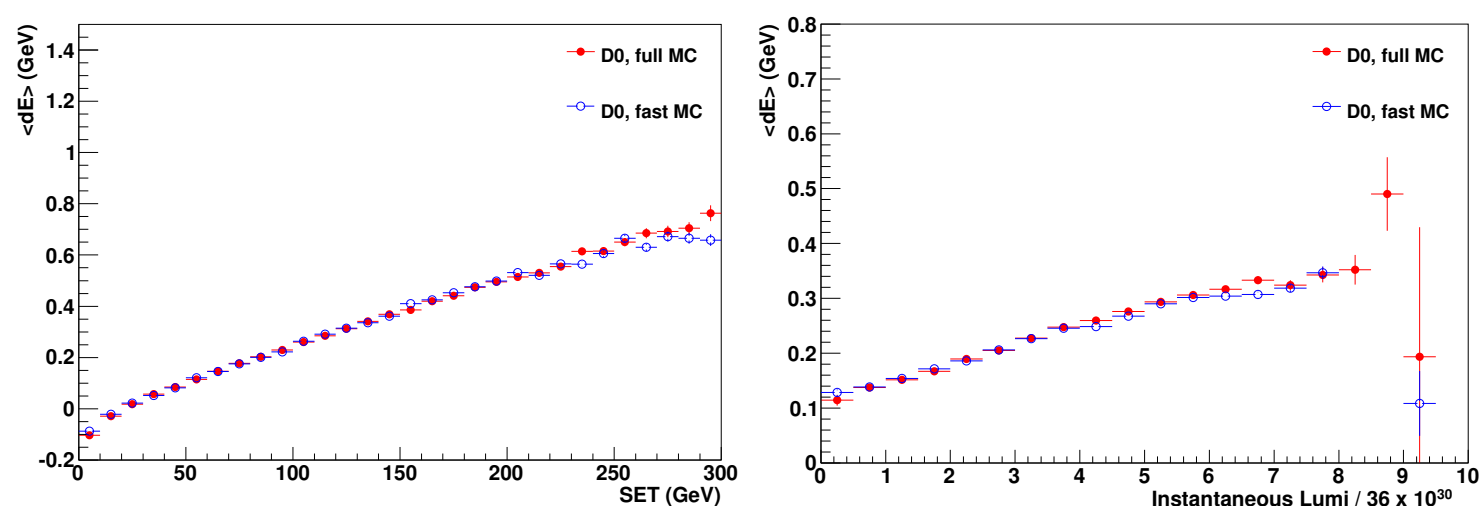

Figure 7.12: Mean $\Delta E$ as a function of SET and instantaneous luminosity comparing full $\mathrm{MC}$ and fast MC.
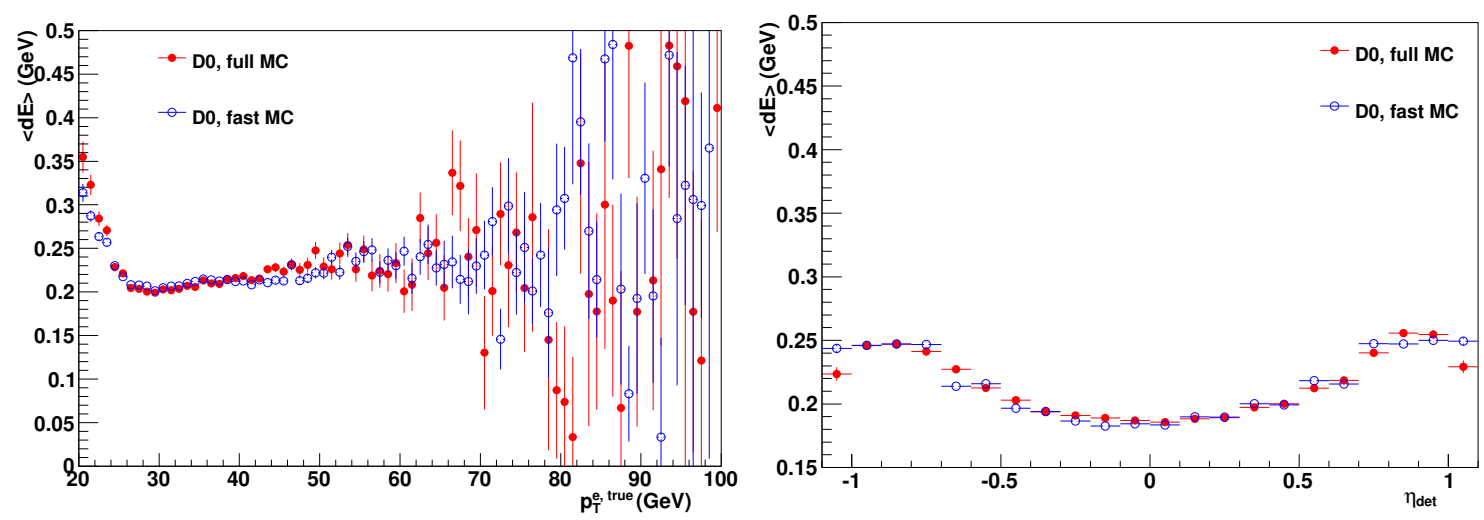

Figure 7.13: Mean $\Delta E$ as a function of true $p_{T}^{e}$ and $\eta_{\text {det }}$ comparing full MC and fast MC.

We show the dependence of mean $\left\langle\Delta u_{\|}\right\rangle$on $u_{\|}$in Fig. 7.16 for various bins of SET. For all SET the mean decreases as $u_{\|}$increases from negative values toward zero. But in a fixed bin of SET the $\left\langle\Delta u_{\|}\right\rangle$always increases with increasing $u_{\|}$as the recoil gets closer to the electron window. Our interpretation is that at fixed SET, the soft recoil component (ZB events) is fixed and we can study the hard recoil which is controlled by $u_{\|}$.

\subsection{Hadronic Recoil Parametrization}

The hadronic recoil simulation in the fast Monte Carlo uses a multi-component model which can be logically divided as:

$$
\vec{u}_{T}=\vec{u}_{T}^{\text {HARD }}+\vec{u}_{T}^{\text {SOFT }}+\vec{u}_{T}^{\text {ELEC }}+\vec{u}_{T}^{\text {FSR }}
$$




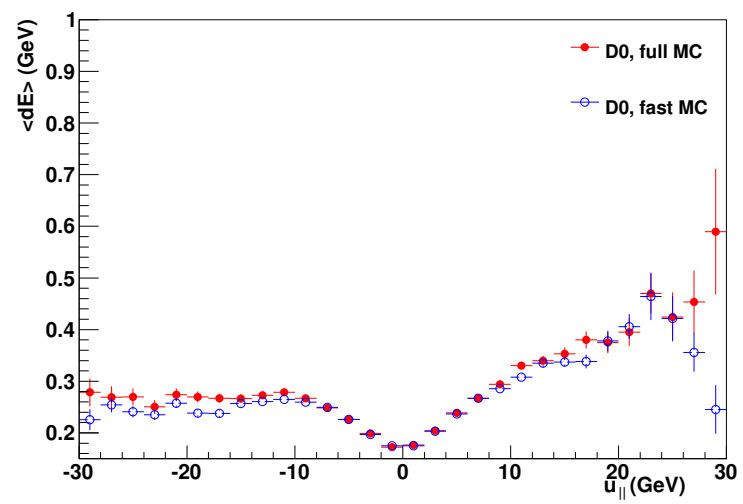

Figure 7.14: Mean $\Delta E$ as a function of $u_{\|}$comparing full MC and fast MC.
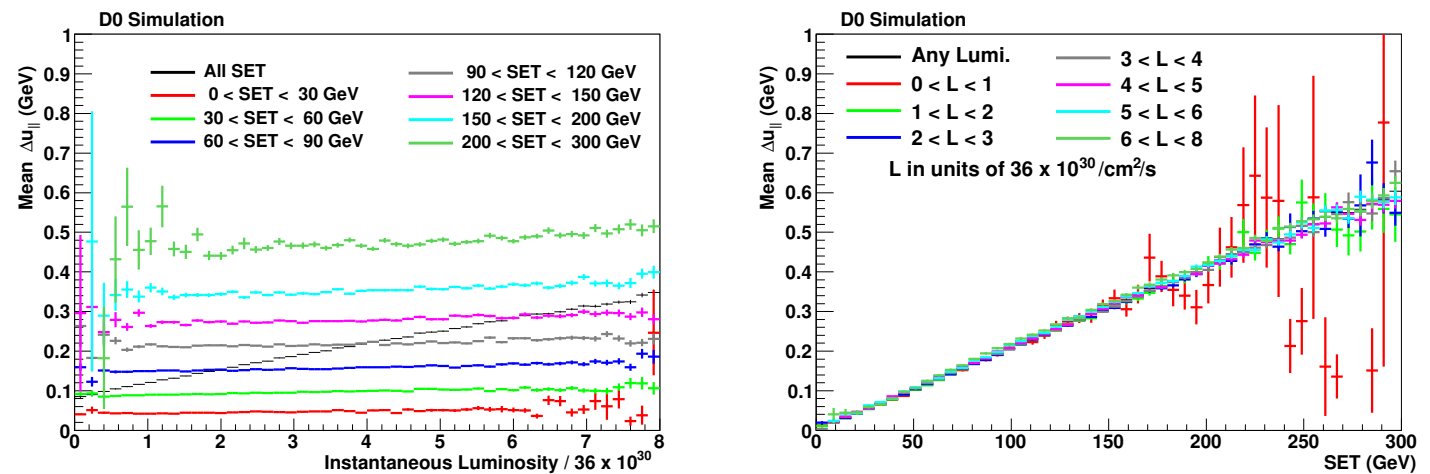

Figure 7.15: $\left\langle\Delta u_{\|}\right\rangle$as a function of instantaneous luminosity separately for sub-samples with different SET (left) and as a function of SET instantaneous luminosity for subsamples with different instantaneous luminosity (right). Instantaneous luminosity $\mathcal{L}$ is given in units of $36 \times 10^{30} \mathrm{~cm}^{-2} \mathrm{~s}^{-1}$. 


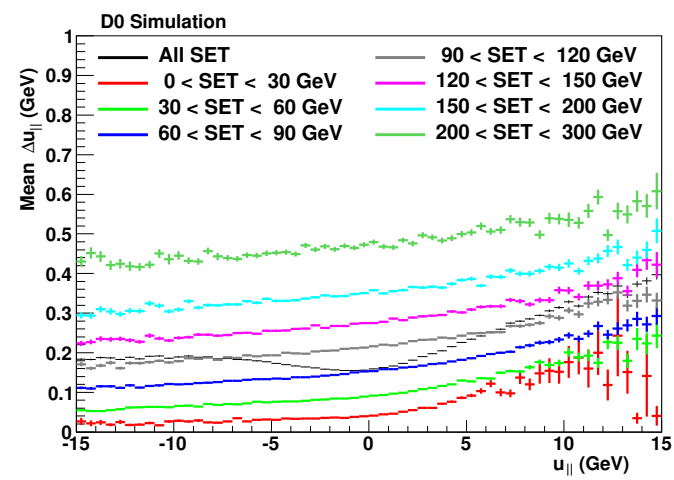

Figure 7.16: $\left\langle\Delta u_{\|}\right\rangle$as a function of $u_{\|}$separately for various bins of SET.

where $\vec{u}_{T}^{\text {HARD }}$ is the dominant part of the recoil balancing the vector boson, $\vec{u}_{T}^{\text {SOFT }}$ describes the zero-bias and minimum-bias contribution, $\vec{u}_{T}^{\text {ELEC }}$ models the hadronic energy in the electron window and electron energy leakage out of the window and $\vec{u}_{T}^{\mathrm{FSR}}$ is the out-of-cone electron FSR contribution ${ }^{1}$. The third of these, $\vec{u}_{T}^{\text {ELEC }}$, is defined as

$$
\vec{u}_{T}^{\mathrm{ELEC}}=-\Delta u_{\|} \widehat{p_{T}^{e}}+\vec{p}_{T}^{\mathrm{LEAK}},
$$

where $\Delta u_{\|}$was discussed in Sec. 7.3.6. The leakage model is determined using single electron full MC samples and parametrized as a function of $E^{e}$ and $\eta_{\mathrm{det}}^{e}$ separately for the cases with and without FSR photons.

\subsubsection{Recoil Model}

An initial hard recoil model is derived from a special sample of $Z \rightarrow v v$ full MC events generated with PYTHIA without simulation of multiple parton interactions and without overlay of zero-bias events. The generated events were processed through the full chain of the D0 detector simulation and reconstruction software. Since the neutrinos escape undetected, all the energy measured in the detector can be attributed to the recoil alone. This full MC sample contains 600,000 $Z \rightarrow v v$ events. To obtain kinematics similar to $Z \rightarrow e e$ events, both neutrinos from a $Z$ boson decay are required to have $|\eta|<1.3$.

The initial model simulates the magnitude $\left(u_{T}^{\text {hard }}\right)$ and direction $(\phi)$ of the reconstructed hard recoil as a function of the negative of the generator-level transverse momentum of the vector boson $\vec{q}_{T}$. The model is parametrized using two variables, the relative $p_{T}$

$$
R=\frac{u_{T}^{\text {hard }}-q_{T}}{q_{T}}
$$

\footnotetext{
${ }^{1}$ The model for the out-of-cone electron FSR response is described in detail in Sec. 14.4.1.
} 
and the angular resolution

$$
\Delta \phi=\phi\left(\vec{u}_{T}^{\text {hard }}\right)-\phi\left(\vec{q}_{T}\right) \quad(|\Delta \phi|<\pi)
$$

The $Z \rightarrow v v$ sample is divided into 32 bins of $q_{T}$. For each bin the distribution of $R$ and $\Delta \phi$ distribution is smoothed to a continuous probability density $P(R, \Delta \phi)$. The smoothing function is a product of a $\log$-normal distribution in $R$ with a normal distribution in $\Delta \phi$. Two examples of such event probability density functions are shown in Figs. 7.17 for the $q_{T}=[4.5,5] \mathrm{GeV}$ and $q_{T}=[18,20] \mathrm{GeV}$ bins. For low values of $q_{T}$ there is a correlation between $R$ and $\Delta \phi$ which is properly described by assuming that the mean of the lognormal has a linear dependence on $\Delta \phi$. The smoothing fits are shown in Fig. 7.17 as colored contours. From these, the simulated $R$ and $\Delta \phi$ values for a fast MC event were chosen by randomly sampling the probability density corresponding to the input $q_{T}$.
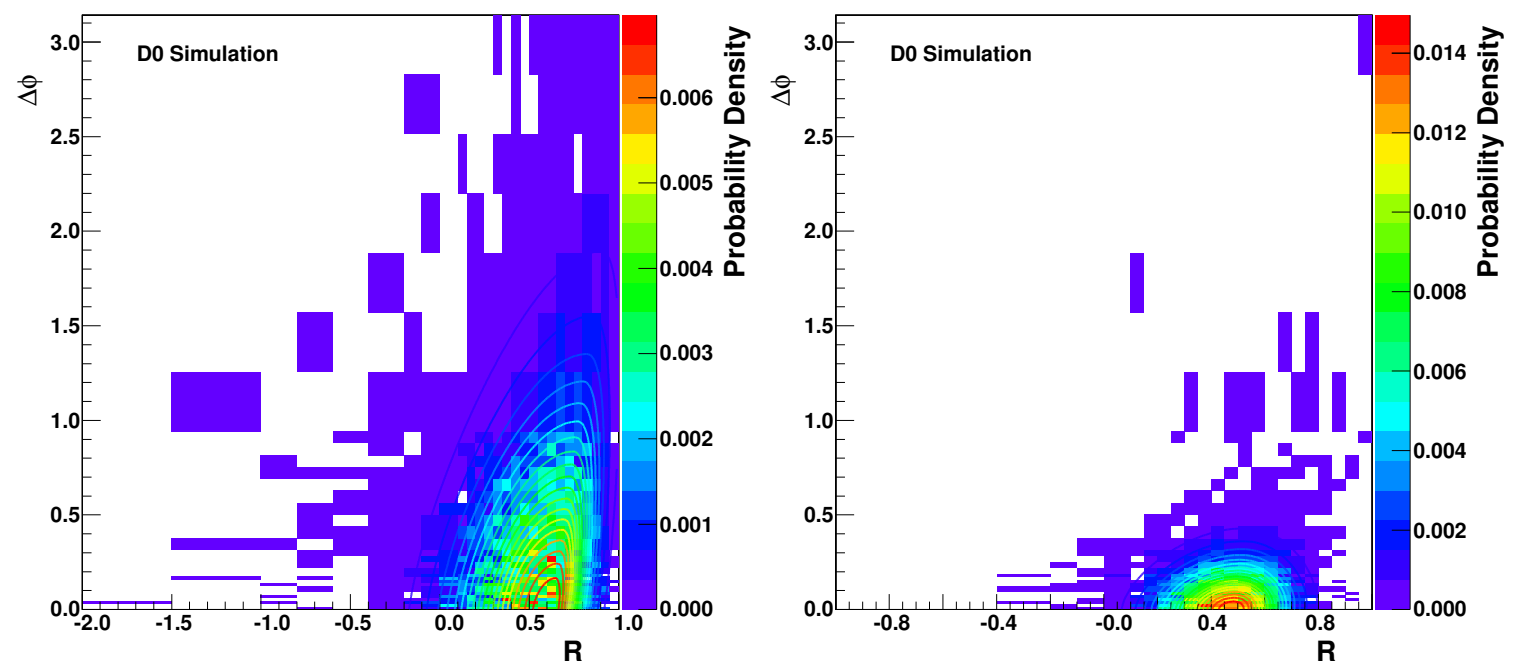

Figure 7.17: The distribution of the recoil relative $p_{T}$ and $\phi$ resolutions for full MC (boxes) and fit (contours) for $q_{T}=[4.5,5] \mathrm{GeV}$ (left) and $q_{T}=[18,20] \mathrm{GeV}$ (right).

The initial hard recoil model described thus far applies to full MC $Z \rightarrow v v$ events. To correct for imperfections in the simulation, additional parameters are introduced and applied to the component $u_{\|}^{\text {hard }}=u_{T}^{\text {hard }} \cos \left(\Delta \phi^{\text {hard }}\right)$ in the direction of $q_{T}$, to give the corrected recoil denoted by $u_{\|}^{\text {HARD: }}$

$$
\begin{aligned}
u_{\|}^{\mathrm{HARD}} / q_{T}=( & \left.r_{0}+r_{1} e^{-q_{T} / \tau_{\mathrm{HAD}}}\right)\left(\bar{R}\left(q_{T}\right)+1\right) \\
& +\sigma_{0}\left(u_{\|}^{\text {hard }} / q_{T}-\bar{R}\left(q_{T}\right)-1\right) .
\end{aligned}
$$

The perpendicular component $u_{\perp}^{\mathrm{HARD}}=u_{T}^{\text {hard }} \sin \left(\Delta \phi^{\text {hard }}\right)$ remains unmodified. The mean values $\bar{R}\left(q_{T}\right)=\left\langle\left(u_{\|}^{\text {hard }}-q_{T}\right) / q_{T}\right\rangle$ are determined from the smoothed distributions for 
$\left(R, \Delta \phi^{\text {hard }}\right)$. The smearing parameters $r_{0}, r_{1}, \tau_{\mathrm{HAD}}$, and $\sigma_{0}$ are determined as described below.

The soft recoil is modeled from the measured recoils in collider data $\mathrm{MB}$ and $\mathrm{ZB}$ events. In addition to being selected by the $M B$ trigger, the $M B$ events are required to have zero or one reconstructed primary vertex. The $\mathrm{ZB}$ events are sampled to give the instantaneous luminosity distribution observed in the data. We created lists of the magnitude and direction of recoil in the $\mathrm{MB}$ and $\mathrm{ZB}$ events, and for a given fast $\mathrm{MC}$ event, the simulated soft recoil is created by taking one $\overrightarrow{\mathrm{u}}_{T}$ value from each of the MB and ZB lists and combining them to give the soft recoil

$$
\vec{u}_{T}^{\mathrm{SOFT}}=\sqrt{\alpha_{\mathrm{MB}}} \vec{u}_{T}^{\mathrm{MB}}+\vec{u}_{T}^{\mathrm{ZB}}
$$

where $\alpha_{\mathrm{MB}}$ is a new parameter.

We determine values for the five phenomenological parameters $r_{0}, r_{1}, \tau_{\mathrm{HAD}}, \sigma_{0}$ and $\alpha_{\mathrm{MB}}$ by fits comparing data (or full MC) to the fast MC simulation using the momentum imbalance between the $p_{T}$ of the dielectron system and the recoil $u_{T}$ in $Z \rightarrow e e$ events is projected on the bisector $\hat{\eta}$ of the electron and positron directions

$$
\eta_{i m b} \equiv\left(\vec{p}_{T}^{e e}+\vec{u}_{T}\right) \cdot \hat{\eta}
$$

as shown in Fig. 3.2. The bisector is chosen to reduce coupling between the electron energy scale and the hadronic recoil. This occurs because the bisector is independent of fluctuations in the measured electron energies.

The $\eta_{i m b}$ distributions are created in bins of reconstructed $p_{T}^{e e}$ for both data (or full MC) and fast MC. The five parameter values are determined by constructing separate fast MC samples with varying values of the parameters and finding the parameter values which minimize the $\chi^{2}$ difference between the $\eta_{i m b}$ means (as functions of $r_{0}, r_{1}$ and $\tau_{\mathrm{HAD}}$ ) and RMS values (as functions of $\sigma_{0}$ and $\alpha_{\mathrm{MB}}$ ) of the data and fast MC distributions. The fits using the mean and the RMS are performed independently.

\subsubsection{Fit Results}

The results from the minimization of the $\eta_{i m b}$ mean as a function of $p_{T}^{e e}$ for collider data are

$$
\begin{aligned}
r_{0} & =1.0471 \pm 0.0077 \\
r_{1} & =2.07 \pm 0.39 \\
\tau_{\mathrm{HAD}} & =2.51 \pm 0.32 \mathrm{GeV}
\end{aligned}
$$

and the results from the minimization of the RMS are

$$
\begin{aligned}
\sigma_{0} & =1.238 \pm 0.040 \\
\alpha_{\mathrm{MB}} & =0.633 \pm 0.064 .
\end{aligned}
$$


The corresponding two correlation matrices are:

$$
\begin{aligned}
& r_{0} \\
& r_{0} \\
& r_{1} \\
& \tau_{\mathrm{HAD}}
\end{aligned}\left(\begin{array}{ccc}
1.0 & 0.30 & -0.49 \\
0.30 & 1.0 & -0.90 \\
-0.49 & -0.90 & 1.0
\end{array}\right)
$$

and

$$
\left.\sigma_{0} \begin{array}{cc}
\sigma_{0} & \alpha_{\mathrm{MB}} \\
1.0 & -0.675 \\
-0.675 & 1.0
\end{array}\right)
$$

Because the mean and RMS fits are performed separately, possible correlations between the $r_{0}, r_{1}$ and $\tau_{\mathrm{HAD}}$ parameter set and the $\sigma_{0}$ and $\alpha_{\mathrm{MB}}$ parameter set are not considered. There is a slight correlation, and the two fits are repeated until stability in both is reached. Figs. 7.18 show the comparison of the mean and the width of the $\eta_{i m b}$ between data and fast MC for the ten different $p_{T}^{Z}$ bins. The quantity $\chi$ is defined as the ratio of the difference between data and fast MC divided by the uncertainty in the data for each bin.

\subsubsection{Recoil Modeling Systematic Uncertainties}

The $Z$ sample statistical precision determines the precision of the five parameters, and these are then propagated to the $W$ mass to determine the recoil modeling systematic uncertainty. To do the propagation, we diagonalize the covariance matrices above to get uncorrelated variations. These are then used to determine uncertainties in the uncorrelated basis. We find uncertainties of $5.4 \mathrm{MeV}, 6.1 \mathrm{MeV}$ and $13.6 \mathrm{MeV}$ for the $m_{T}$, $p_{T}^{e}$ and $\mathbb{E}_{T}$ results. The correlation matrix for the final fits is

$\begin{array}{ccc}m_{T} & p_{T}^{e} & \mathbb{E}_{T} \\ m_{T} & p_{T}^{e} \\ \mathbb{Z}_{T} & \left(\begin{array}{ccc}1.000 & 0.754 & 0.571 \\ 0.754 & 1.000 & 0.128 \\ 0.571 & 0.128 & 1.000\end{array}\right)\end{array}$



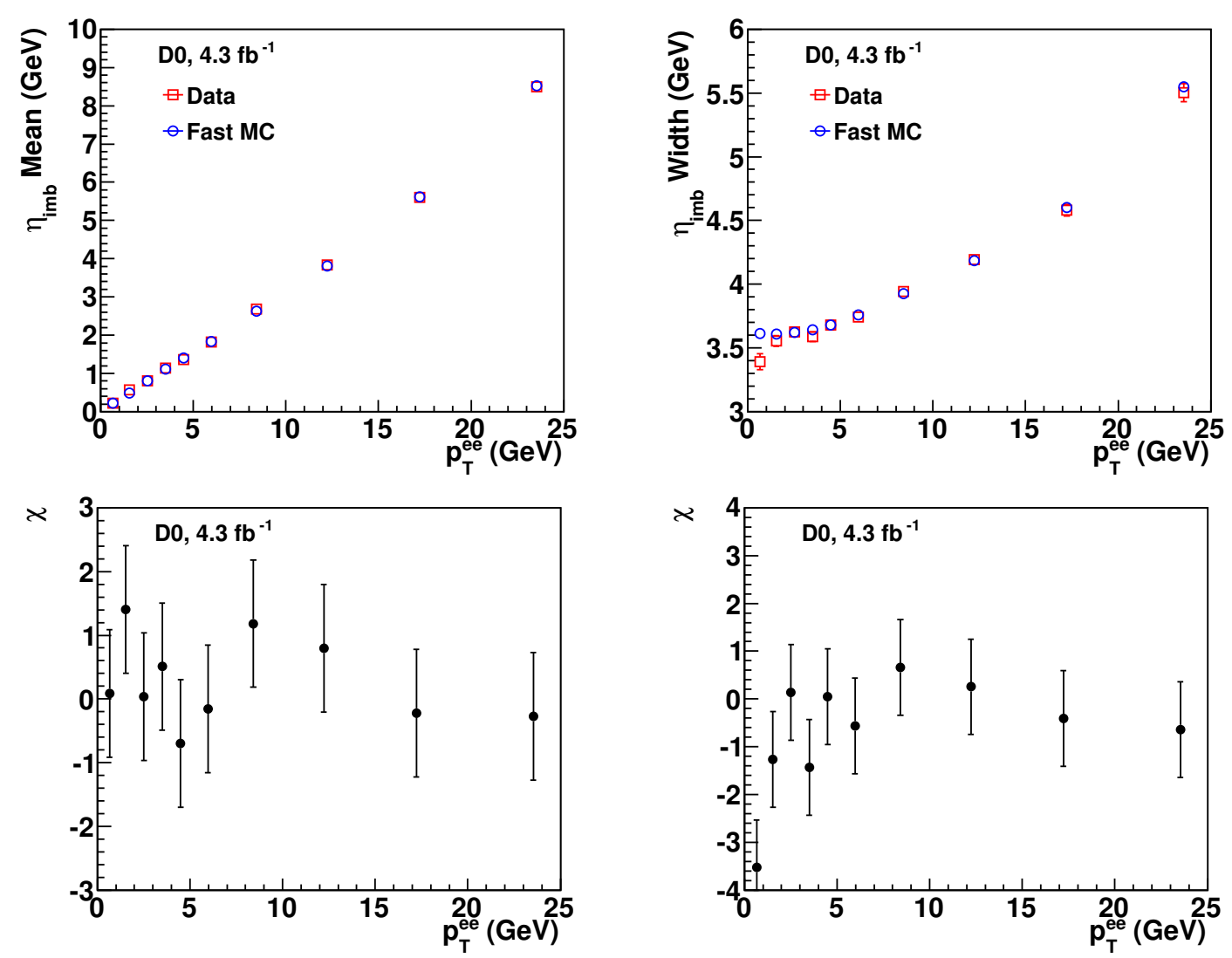

Figure 7.18: Data and fast MC comparison of the mean and RMS of the $\eta_{i m b}$ for the ten different bins in $p_{T}^{Z}$. 


\section{Chapter 8}

\section{Backgrounds}

There are three significant backgrounds in the $W$ sample:

- $Z \rightarrow e e$ events in which one electron escapes in a poorly instrumented region of the detector,

- Multijet events (MJ) in which a jet is misidentified as an electron and $\mathbb{Z}_{T}$ arises from misreconstruction

- $W \rightarrow \tau v \rightarrow e v v v$ events.

The first two components are measured using data control samples, and the third is estimated using simulation.

\subsection{Z $\rightarrow$ ee Background}

$Z \rightarrow e e$ events are present in the $W \rightarrow e v$ sample when there is substantial missing transverse momentum from mismeasurement. We directly estimate the $Z \rightarrow e e$ contamination from the $W \rightarrow e v$ sample, selecting events which pass the full $W$ sample analysis selection, modified to include selection of an additional reconstructed object chosen to indicate that the selected event is likely a $Z$ boson decay. Most often the second object is in the inter-cryostat region, which is outside the electron acceptance in this analysis and has poor sampling of hadronic energy flow since the Inter-Cryostat Detector (ICD) is not included in MET reconstruction. The $Z \rightarrow e e$ background from events in which neither electron is in the ICD region is negligible.

Because we cannot directly identify electrons in this region, we estimate the number of $Z \rightarrow e e$ using jets and tracks. The jet must have a matched track such that the invariant mass of this track and the electron is consistent the $Z$ boson mass. To estimate the absolute 
number of $Z \rightarrow e e$ events in the $W \rightarrow$ ev sample we measure the number of $Z \rightarrow e e$ candidates and use Equation (8.1):

$$
N(Z \rightarrow e e \text { background })=\frac{N^{Z \rightarrow e e}(e, j e t)}{\epsilon_{j e t}^{\prime} \times A(e, t r k)},
$$

where $N^{Z \rightarrow e e}(e, j e t)$ is the number of selected events passing the $W$ plus additional object selection, $\epsilon_{j e t}^{\prime}=\epsilon_{j e t} \times A(e, j e t) / A(e, t r k)$ is the relative efficiency to find a jet given the presence of a track and $A(e, t r k)$ is the track acceptance in the invariant mass window considered, both measured in data control samples. The fraction of $Z \rightarrow e e$ background events in the $W \rightarrow$ ev candidate sample is found to be $1.08 \pm 0.02 \%$.

\subsection{Multijet Background}

The MJ background is determined using a loose sample obtained by replacing the standard tight track-matching requirement with a less restrictive requirement depending only on the directions of the track and an EM cluster which uses a wider region for the track and cluster match than the standard requirement. This sample contains all events selected by the standard selection requirements, but has a significantly higher contamination from MJ background than the standard sample. The probabilities for electron candidates in $W$ events $\left(\epsilon_{e}\right)$ and in multijet events $\left(\epsilon_{f}\right)$ to pass the full matching requirements given that they already satisfy the direction-based match are determined in control samples. The probability for real electrons is determined from $Z$ data, and the probability for electron candidates in multijet events is determined from dijet events. These probabilities are parametrized as a function of electron $p_{T}$ and can be seen in Fig. 8.1. The loose sample event yield $N_{L}$, the standard sample event yield $N$, and the two probabilities are then used to determine the multijet background yield in each bin $i$ of a distribution by solving the system of equations

$$
\begin{aligned}
& N_{L}^{(i)}=N_{W}^{(i)}+N_{\mathrm{MJ}}^{(i)} \\
& N^{(i)}=\epsilon_{e}^{(i)} N_{W}^{(i)}+\epsilon_{f}^{(i)} N_{\mathrm{MJ}}^{(i)}
\end{aligned}
$$

for the MJ background, $\epsilon_{f} N_{\mathrm{MJ}}$. The $\epsilon_{e}^{(i)}$ and $\epsilon_{f}^{(i)}$ values for a given event are determined using the $p_{T}$ dependent forms. The contribution from MJ events is found to be $1.02 \pm$ $0.06 \%$ of the selected $W \rightarrow$ ev candidate sample.

\subsection{W $\rightarrow \tau v$ Background}

The $W \rightarrow \tau v \rightarrow e v v v$ contribution is determined from simulation of the process using RESBOS for event generation, TAUOLA [73, 74] for $\tau$ decay and fast MC for detector simulation. Because the electron arises from a secondary decay, the momenta are lower than 

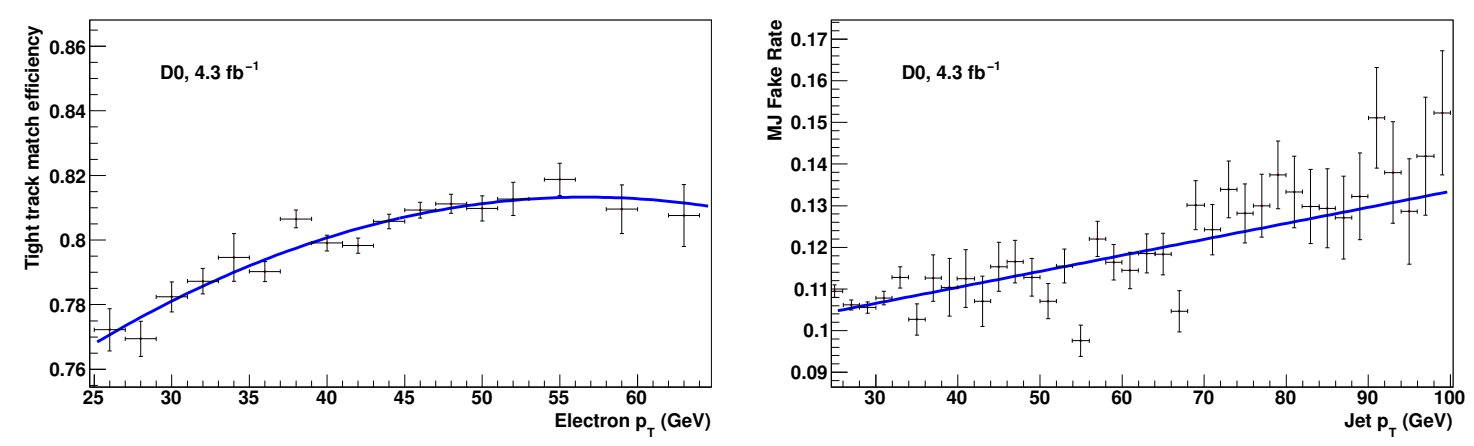

Figure 8.1: (left) Tight track match efficiency as a function of the electron $p_{T}^{e}$ measured relative to the loose track match requirement. (right) Probability of a jet object that passes the loose track match requirement to pass the tight track match requirement.

that from direct $W \rightarrow e v$ and the distribution is broader. The background contribution from $W \rightarrow \tau v$ is $(1.668 \pm 0.004) \%$, with the uncertainty dominated by the uncertainty in the $\tau \rightarrow e v v$ branching ratio [71]. The uncertainty in the $M_{W}$ measurement arising from incorporating the $W \rightarrow \tau v \rightarrow e v v v$ events as background instead of a (W mass dependent) signal is small.

Propagated $M_{W}$ uncertainties are at most $1 \mathrm{MeV}$ for both QCD and $W \rightarrow \tau v$ backgrounds for all three observables, and $1 \mathrm{MeV}, 2 \mathrm{MeV}$, and $1 \mathrm{MeV}$ for the $m_{T}, p_{T}^{e}$, and $\mathbb{E}_{T}$ observables for the $Z \rightarrow e e$ background. Distributions of the three background contributions are shown in Fig. 8.2 .
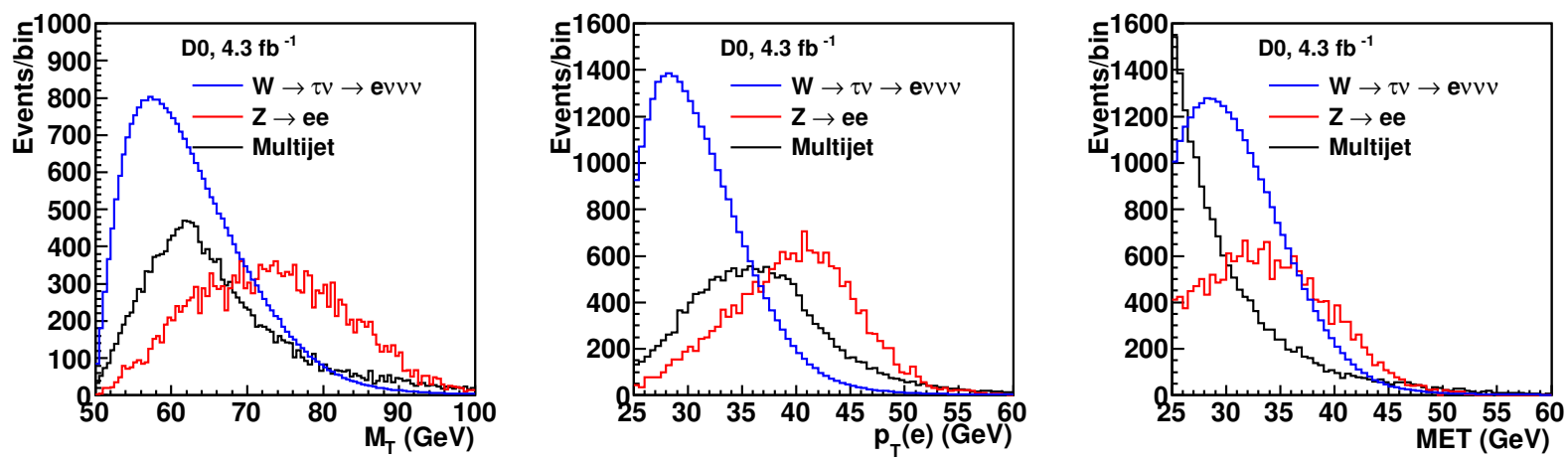

Figure 8.2: The $m_{T}, p_{T}^{e}$ and $\not_{T}$ distributions for the three backgrounds $Z$ (red), MJ (black) and $W \rightarrow \tau v$ (blue) with absolute normalization. 


\section{Chapter 9}

\section{Results}

The $M_{W}$ results are determined by fits between the data and fast $\mathrm{MC}$ templates. Figure 9.1 shows the agreement between data and fast $\mathrm{MC}$ in fitting the invariant mass distribution of $Z \rightarrow e e$ events. Figures $9.2-9.4$ show comparisons of the data to the fast MC for the distributions we use to measure the $W$ boson mass including the fitting range used. The fitting ranges were determined by minimizing the sum in quadrature of the PDF and statistical uncertainties, which are the most sensitive uncertainties to this choice [75].
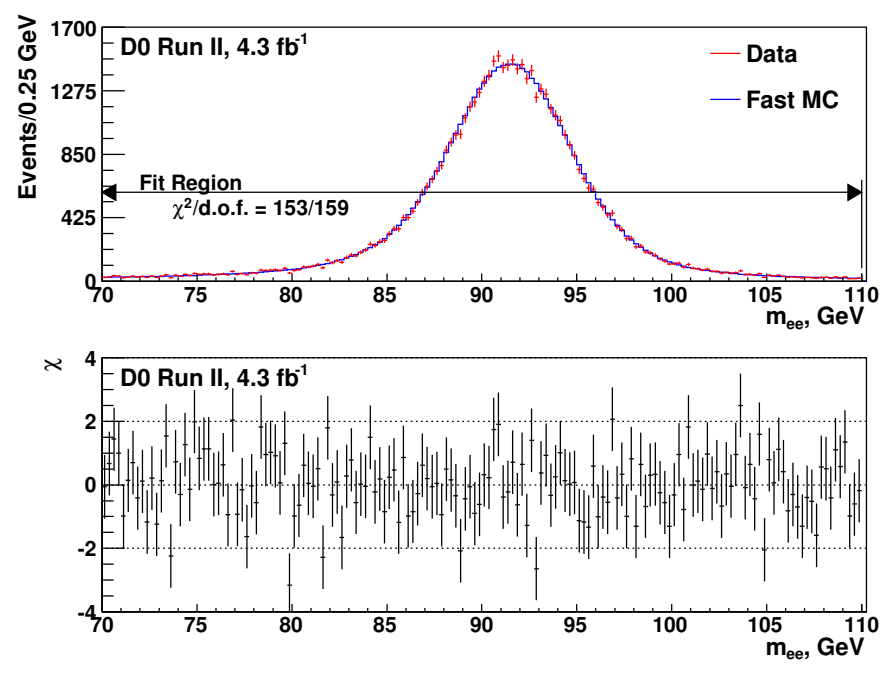

Figure 9.1: The $Z$ mass distribution in data and from the fast $M C$ (top) and the $\chi$ values for each bin (bottom).

The $W$ boson mass is determined by comparing each of the $m_{T}, p_{T}^{e}$ and $\mathbb{E}_{T}$ observable's distribution from collider data to templates generated using the fast MC. The backgrounds are added to the simulated distributions. The $W$ boson mass determined from a given observable is defined as the mass value that minimizes the negative log-likelihood comparing the binned template distribution to the binned collider data distribution. The measurements are performed separately for the $m_{T}, p_{T}^{e}$ and $\mathbb{E}_{T}$ distributions. 

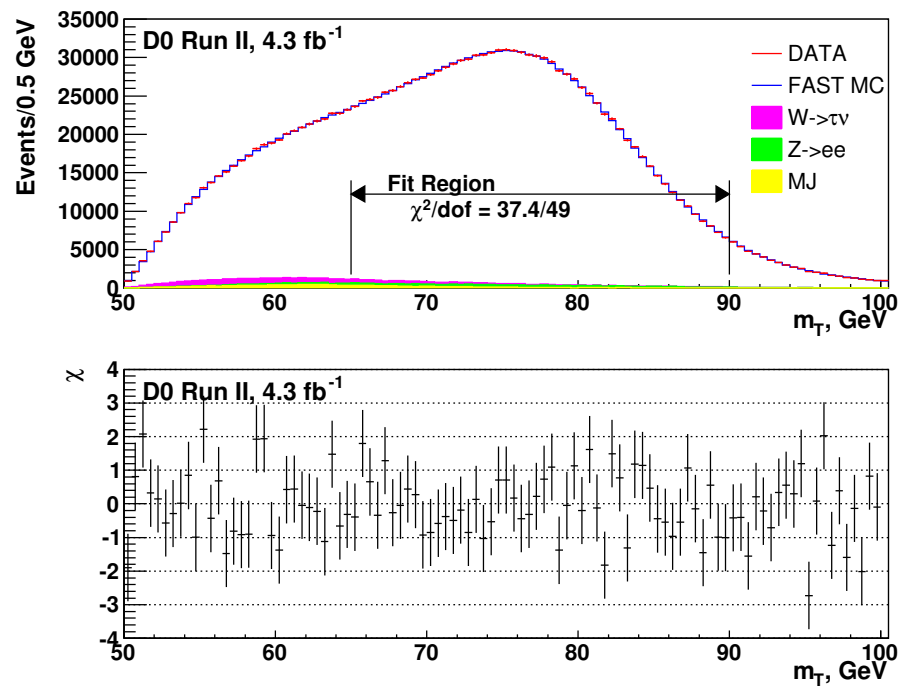

Figure 9.2: The $m_{T}$ distribution for data and fast MC with backgrounds added (top), and the $\chi$ value for each bin (bottom).
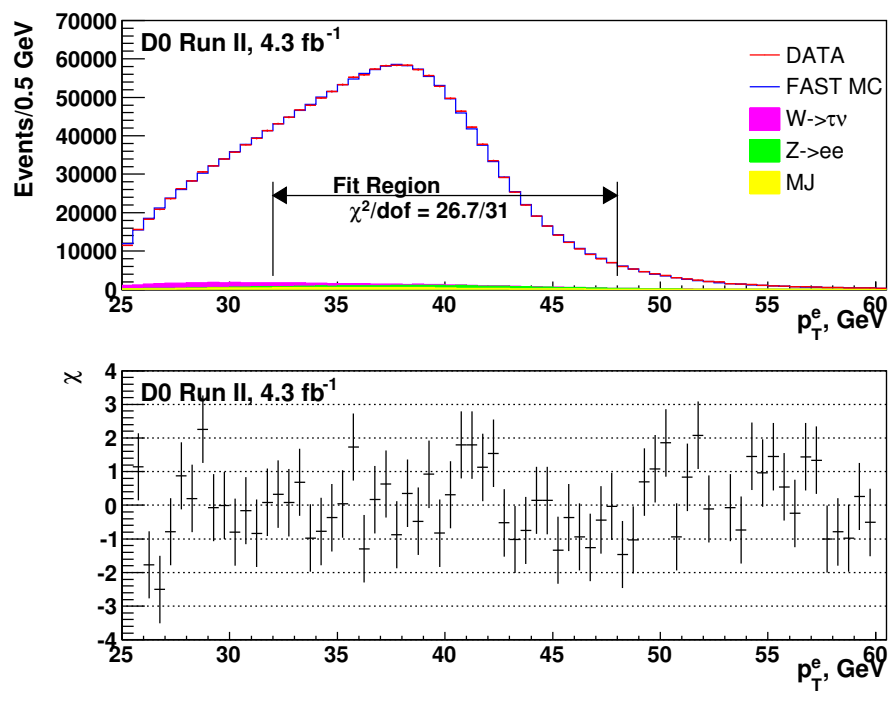

Figure 9.3: The $p_{T}^{e}$ distribution for data and fast MC with backgrounds added (top) and the $\chi$ value for each bin (bottom). 

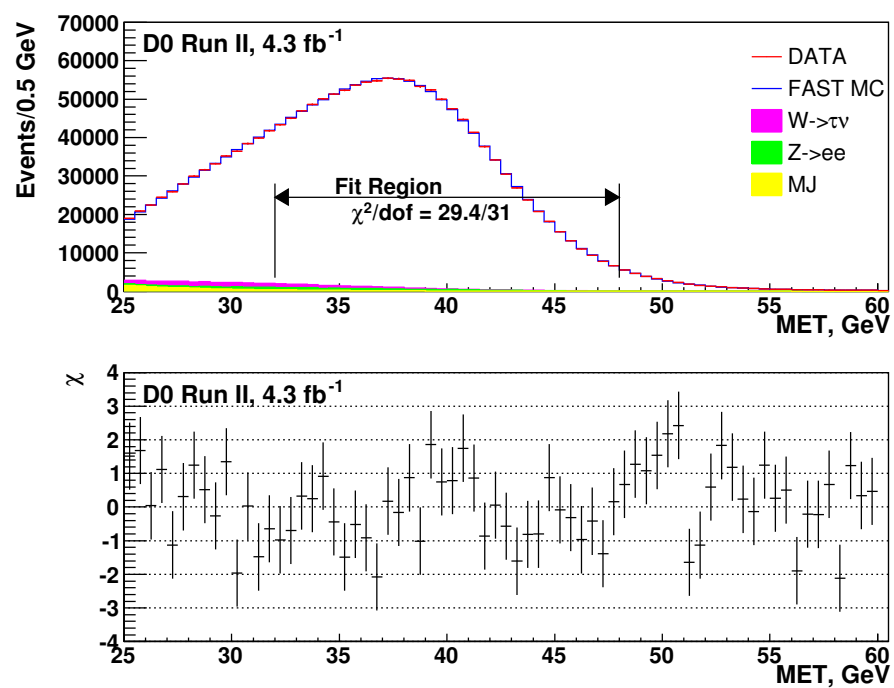

Figure 9.4: The $\mathbb{E}_{T}$ distribution for data and fast MC with backgrounds added (top), and the $\chi$ value for each bin (bottom).

The measurements were performed after we obtained closure in our full MC measurement described in the introduction to Sec.7/and after the measurement was approved by the D0 collaboration following the blinding procedure described in Sec. 3.3 .

After unblinding, the $W$ boson mass fit results from data are given in Table 9.1] [33].

\begin{tabular}{|c|c|cc|}
\hline \hline Variable & Fit Range $(\mathrm{GeV})$ & Result $(\mathrm{GeV})$ & $\chi^{2} /$ d.o.f. \\
\hline$m_{T}$ & $65<m_{T}<90$ & $80.371 \pm 0.013$ & $37 / 49$ \\
$p_{T}^{e}$ & $32<p_{T}^{e}<48$ & $80.343 \pm 0.014$ & $27 / 31$ \\
$\mathbb{E}_{T}$ & $32<\mathbb{E}_{T}<48$ & $80.355 \pm 0.015$ & $29 / 31$ \\
\hline \hline
\end{tabular}

Table 9.1: Results from the fits to data. The uncertainty is only that from the data candidate $W$ boson sample statistics. The $\chi^{2} /$ d.o.f. values are computed over the fit range.

The systematic uncertainties in the $W$ mass boson measurement arise from a variety of sources, but can roughly be categorized as those arising from experimental sources and those arising from the $W$ and $Z$ production models. The methods used to derive the systematic uncertainties and the uncertainties themselves have been described in the corresponding sections above. The systematic uncertainties are summarized in Table 9.2. The largest uncertainty, $16 \mathrm{MeV}$ arises from the precision with which the electron energy scale is known. This is limited by the statistical power of the $Z \rightarrow$ ee sample, and it is expected to improve with more data. 


\begin{tabular}{|l|c|c|c|}
\hline \hline Source & $\sigma\left(m_{W}\right) \mathrm{MeV} m_{T}$ & $\sigma\left(m_{W}\right) \mathrm{MeV} p_{T}^{e}$ & $\sigma\left(m_{W}\right) \mathrm{MeV}_{T}$ \\
\hline \hline Experimental & & & \\
\hline Electron Energy Scale & 16 & 17 & 16 \\
Electron Energy Resolution & 2 & 2 & 3 \\
Electron Shower Model & 4 & 6 & 7 \\
Electron Energy Loss & 4 & 4 & 4 \\
Recoil Model & 5 & 6 & 14 \\
Electron Efficiencies & 1 & 3 & 5 \\
Backgrounds & 2 & 2 & 2 \\
\hline Experimental Total & 18 & 20 & 24 \\
\hline W production and & & & \\
decay model & 11 & 11 & 14 \\
\hline PDF & 7 & 7 & 9 \\
QED & 2 & 5 & 2 \\
Boson $p_{T}$ & 13 & 14 & 17 \\
\hline W model Total & 22 & 24 & 29 \\
\hline Total Systematic Uncertainty & 13 & 14 & 15 \\
\hline W Statistics & 26 & 28 & 33 \\
\hline Total Uncertainty & \multicolumn{2}{|c|}{} \\
\hline \hline
\end{tabular}

Table 9.2: Systematic uncertainties on the $W$ mass results. The dominant systematic uncertainty comes from the electron energy scale, and this is determined by the statistical power of the $Z$ event sample. 


\section{Chapter 10}

\section{Combination}

The measurements from the three observables are correlated. Correlation matrices for the $W$ boson data sample statistical correlation, the electron energy scale, the recoil scale and resolution and parton density functions are determined using ensemble tests and standard uncertainty propagation. The resulting correlation matrices are shown in Table 10.1 . Each of the other uncertainties listed in Table 9.2 is assumed to have a $100 \%$ correlation among the $m_{T}, p_{T}^{e}$ and $\mathbb{E}_{T}$ results. The various sources of uncertainty are assumed to be uncorrelated with each other.

\begin{tabular}{lc}
\hline \hline Source & $4.3 \mathrm{fb}^{-1}$ Correlation Matrices \\
\hline W boson statistics & $\left(\begin{array}{lll}1.000 & 0.658 & 0.744 \\
0.658 & 1.000 & 0.436 \\
0.744 & 0.436 & 1.000\end{array}\right)$ \\
Recoil scale and resolution & $\left(\begin{array}{lll}1.000 & 0.754 & 0.571 \\
0.754 & 1.000 & 0.128 \\
0.571 & 0.128 & 1.000\end{array}\right)$ \\
PDF & $\left(\begin{array}{lll}1.000 & 0.990 & 1.000 \\
0.990 & 1.000 & 0.988 \\
1.000 & 0.988 & 1.000\end{array}\right)$ \\
&
\end{tabular}

Table 10.1: Correlation matrices for the $W$ boson statistical, recoil scale and resolution and the PDF uncertainties. The correlation matrix for each of the other uncertainties is assumed to have a $100 \%$ correlation among the $m_{T}, p_{T}^{e}$ and $\mathbb{E}_{T}$ results. 
The total correlation matrix including all uncertainties is

$$
\rho=\left(\begin{array}{ccc}
\rho_{m_{T} m_{T}} & \rho_{m_{T} p_{T}^{e}} & \rho_{m_{T} \mathbb{Z}_{T}} \\
\rho_{m_{T} p_{T}^{e}} & \rho_{p_{T}^{e} p_{T}^{e}} & \rho_{p_{T}^{e} \mathbb{E}_{T}} \\
\rho_{m_{T} \mathbb{Z}_{T}} & \rho_{p_{T}^{e} \mathbb{E}_{T}} & \rho_{\mathbb{Z}_{T} \mathbb{E}_{T}}
\end{array}\right)=\left(\begin{array}{ccc}
1.0 & 0.89 & 0.86 \\
0.89 & 1.0 & 0.75 \\
0.86 & 0.75 & 1.0
\end{array}\right)
$$

The measurement based on $\mathbb{E}_{T}$ has considerably larger systematic uncertainty than the other two measurements, and is strongly correlated with them. In this situation, the combination using the BLUE method [76, 77] becomes hard to interpret since it results in negative weights which are very sensitive to the exact value of the correlation between the measurements. To better understand the situation, consider the example of a combination of two measurements with uncertainty ratio $\sigma_{2} / \sigma_{1}=\lambda$ and correlation $\rho$. Using the BLUE prescription, the best value for the combination is:

$$
\bar{x}=\frac{(\lambda-\rho) x_{1}+(1 / \lambda-\rho) x_{2}}{(\lambda+1 / \lambda-2 \rho)}
$$

The case in hand is such that $x_{1} \simeq x_{2}$ and $\lambda>\rho>1 / \lambda$. It is clear that the value of $\bar{x}$ depends on the cancellation of two small numbers: the difference $x_{2}-x_{1}$ and $\lambda+1 / \lambda-$ $2 \rho$. The smallness of the weight with which $\mathbb{Z}_{T}$ contributes does not justify introducing this instability.

Therefore, we combine only the $m_{T}$ and $p_{T}^{e}$ measurements, that despite also being strongly correlated, have similar systematic uncertainties. We obtain:

$$
m_{W}=80.367 \pm 0.013 \text { (stat) } \pm 0.022 \text { (syst) } \mathrm{GeV}=80.367 \pm 0.026 \mathrm{GeV}
$$

The $\chi^{2}$ probability of this combination is $2.8 \%$. This result is combined with an earlier D0 measurement [32] to give the new D0 Run II result

$$
m_{W}=80.375 \pm 0.023 \mathrm{GeV} .
$$

For the combination of this new measurement and the measurement in reference [32], the production model uncertainties are treated as 100\% correlated between the two measurements, and all other uncertainties, driven by statistics, are assumed to be uncorrelated.

The combination with the other measurements described in section 1.3 is done assuming no correlations between statistical uncertainties and experimental systematic uncertainties. Model and theoretical uncertainties are treated by an ad hoc procedure. An uncertainty which has a common source in two or more experiments is split $\delta_{u}=\delta_{u}^{\mathrm{min}}+\delta_{u}^{\mathrm{rem}}$, where $\delta_{u}^{\mathrm{min}}$ is the smallest uncertainty in the set of experiments considered. The part $\delta_{u}^{\mathrm{min}}$ is considered totally correlated among all experiments while $\delta_{u}^{\text {rem }}$ is considered totally uncorrelated. Although there is no formal justification for this procedure, any potential bias it introduces is small. Also small is the bias introduced by not combining individual measurements, but combinations of measurements from each experiment. 
All experiments measure the physical $W$ mass assuming the running-width scheme treatment for the $W$ boson width. In this scheme, the propagator is written with a mass dependent imaginary part $1 /\left(\hat{s}+M_{W}^{2}+i \hat{s} \Gamma_{W} / M_{W}\right)$ and the fitted value of $M_{W}$ depends weakly on the $\Gamma_{W}$ [34]. The measurement described in this dissertation assumes $\Gamma_{W}=$ $2100.4 \mathrm{MeV}$ while the world average $M_{W}$ assumes $\Gamma_{W}=2092.2 \pm 1.5 \mathrm{MeV}$.

The correlation between the D0 Run I measurement [78], that uses both CC and EC electrons, with all other hadron collider measurements can be estimated using PYTHIA and the 40 members of the CTEQ6.1 error eigenset. The correlation matrix obtained with this procedure is:

$$
\begin{aligned}
& m_{T}(\mathrm{CC}) \quad p_{T}^{e}(\mathrm{CC}) \quad m_{T}(\mathrm{CC})
\end{aligned}
$$

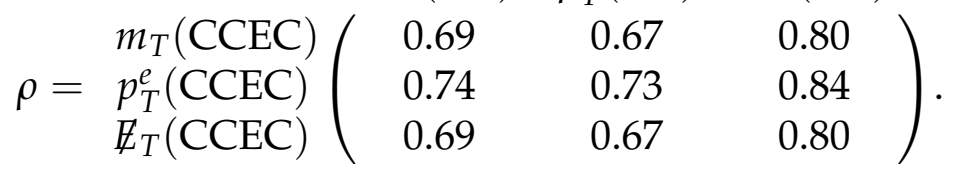

Figure 10.1 shows the D0 Run II combined measurement, the world average top quark mass measurement [79], and the comparison with the SM prediction assuming a Higgs boson mass of $125.7 \mathrm{GeV}$.

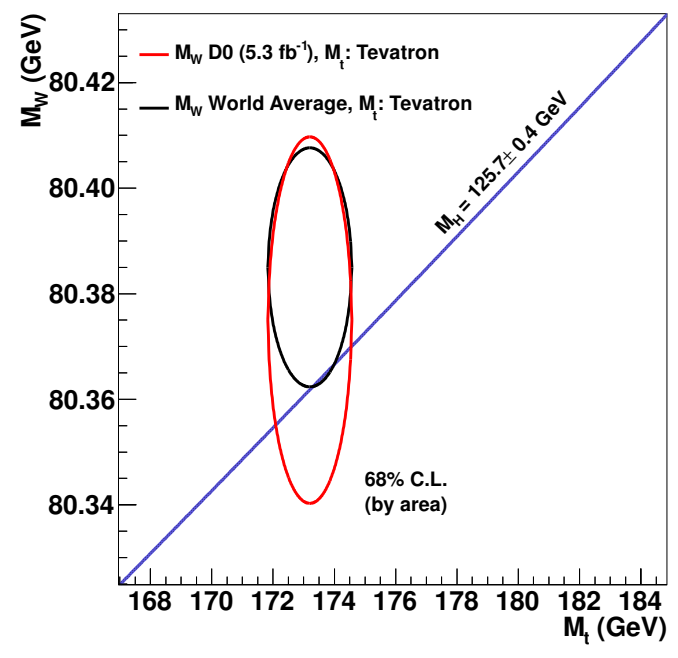

Figure 10.1: The D0 Run II measurement of $M_{W}$ plotted with the world-average mass of the top quark $M_{t}$ at $68 \% \mathrm{CL}$. by area (red ellipse). The new world-average for $M_{W}$ is also plotted (black ellipse). The thin blue band is the prediction of $M_{W}$ in the Standard Model given by equation 1.4 , chapter 1 , assuming $M_{H}=125.7 \pm 0.4 \mathrm{GeV}$ 


\section{Chapter 11}

\section{Consistency Checks}

In this chapter we present consistency checks of the analysis. Two forms of checks are made. For the first, we vary the fit ranges shown in Table 9.1 used in the final $M_{W}$ fits. For the second form, we determine the $W$ and $Z$ mass for many different subsets of the data. We then check that the ratio of the $W$ mass to the $Z$ mass is stable. The subsets are defined using variables that are a priori considered to be difficult to describe or which have critical impact on the result.

To check the impact of the fit ranges shown in Figs. 9.2 - 9.4 and used to determine $M_{W}$, the mass measurements were repeated by changing the range. Figure 11.1 shows the variation resulting from these tests applied to the $m_{T}$ and $p_{T}^{e}$ distribution. The result is stable to within the uncertainty as the fit range is varied. Similar study of the fit ranges for $\mathbb{E}_{T}$ also shows stable results.

We divide the $W$ and $Z$ samples into four subsets of instantaneous luminosity per bunch using the same subsets as for the parametrization of the electron identification efficiencies (Sec.7.2.9) and for the tuning of the absolute EM energy scale (Sec. 7.3.4). The ratio of the $W$ boson mass and $Z$ boson mass measurements are summarized in Fig. 11.2(a),

We also divide the data according to data-taking period. We make four sub-samples. The first pair and the last pair are separated by a long accelerator shutdown. For the divisions within each pair, the integrated luminosities are equal. The ratio of the $W$ boson mass and $Z$ boson mass measurements are summarized in Fig. 11.2(b).

The next division is based on electron $\left|\eta_{\text {det }}\right|$. We divide the data sample into five subsets as defined in Fig. 11.3(a). This is the same categorization that is used in the determination of the $\eta$ dependence of the EM energy scale (Sec. 7.3.3). The $W$ boson mass for each of the five sub-samples is shown in Fig. 11.3(a). We do not show the $W$ boson mass to $Z$ boson mass ratio because we have an explicit $\eta$ dependent calibration and because there are two electrons in each $Z \rightarrow e e$ event.

We now split the candidate $W$ boson sample into a sub-sample of negative $u_{\|}$and a sub-sample of positive $u_{\|}$. There is no direct equivalent of this for the $Z$ sample $e^{1}$, so we show only the $W$ mass fits in Fig. 11.3(b).

\footnotetext{
${ }^{1}$ because there are two electrons from each $\mathrm{Z}$ decay that are reconstructed in roughly opposite directions
} 

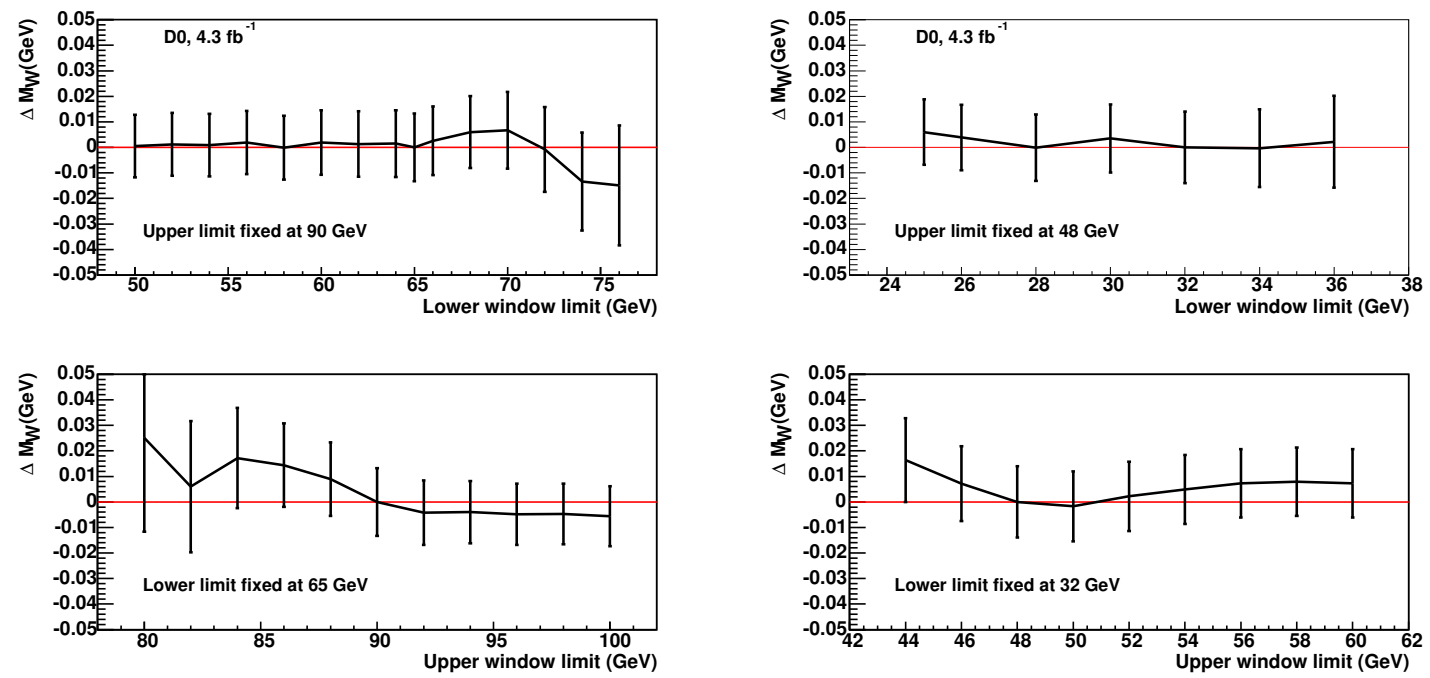

(a) Variation in the mass determined from fits to (b) Variation in the mass determined from fits to the $m_{T}$ spectrum as the fit range was changed. the $p_{T}^{e}$ spectrum as the fit range was changed. The upper plot shows the impact of varying the The upper plot shows the impact of varying the lower edge of the $m_{T}$ fit range, and the bottom lower edge of the $p_{T}^{e}$ fit range, and the bottom plot shows the impact of varying the upper edge. plot shows the impact of varying the upper edge. For each of the variations the difference between For each of the variations the difference between the result from the varied range and the result the result from the varied range and the result from the nominal range is shown. The uncertain- from the nominal range is shown. The uncertainties represent the statistical uncertainties of the ties represent the statistical uncertainties of the varied range fits. varied range fits.

Figure 11.1: Impact of the fitting range on the fitted value of the observables.

We now vary the electron $\phi$ fiducial requirement (Sec. 7.2.5). The nominal requirement, $\phi_{\bmod }(0.80)$, removes $10 \%$ of the phase space at each edge of each CC EM module. We also study four tighter versions of the requirement, namely $\phi_{\bmod }(0.75), \phi_{\bmod }(0.70)$, $\phi_{\text {mod }}(0.60)$ and $\phi_{\bmod }(0.50)$. They remove $12.5 \%, 15 \%, 20 \%$ and $25 \%$ respectively of the phase space at each edge of each CC EM module. The effects of these variations are summarized in Fig. 11.4(a),

The last division is based on recoil $\phi$. We divide the data sample into eight subsets, as defined in Fig. 11.4(b) The results of the ratio of the $W$ mass to the $Z$ mass are shown in the same figure.

in the transverse plane 

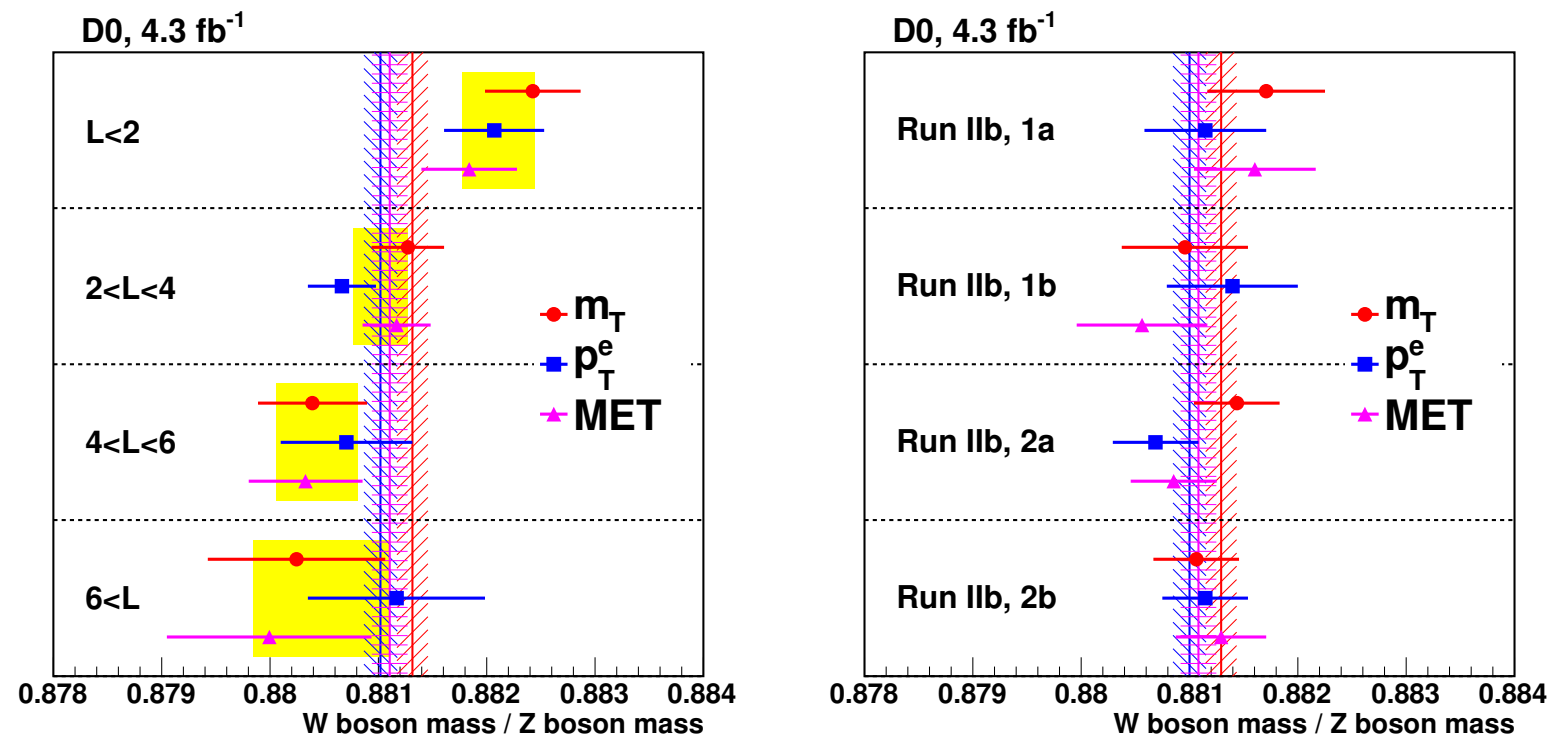

(a) The measured $W$ boson mass to $Z$ boson mass (b) The measured $W$ boson mass to $Z$ boson mass ratios, separately for the $m_{T}, p_{T}^{e}$ and $\mathbb{Z}_{T}$ observables ratios, separately for the $m_{T}, p_{T}^{e}$ and $\mathbb{E}_{T}$ observables and in four bins of instantaneous luminosity. The and for four data taking periods. The uncertainuncertainties for each observable represent the com- ties for each observable represent the combined stabined statistical uncertainty due to limited $W$ statis- tistical uncertainty due to limited $W$ statistics and tics and $Z$ statistics. The yellow bands indicate the $Z$ statistics. The three vertical lines with hashed uncontribution from the $Z$ statistics (which is fully cor- certainties indicate the results from the three observrelated for the three observables). The three verti- ables for the full data sample. The response of the cal lines with hashed uncertainty regions indicate the calorimeter is expected to be time dependent esperesults from the three observables for the full data cially due to the recalibration between Run IIb1 and sample. In the fast MC model, the instantaneous Run IIb2 and due to the anomalous dark currents in luminosity is directly relevant for the model of the the central calorimeter.

relative response loss due to the high-voltage drop,

the hadronic dependent efficiency model and for the

electron window effect model.

Figure 11.2: Consistency checks by splitting the $W$ and $Z$ samples in subsets with different instantaneous luminosity and time. 
D0, $4.3 \mathrm{fb}^{-1}$

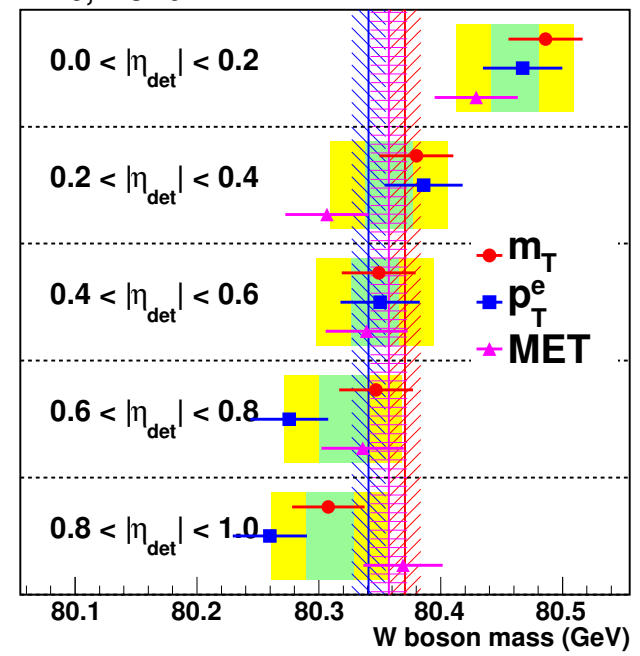

$\mathrm{D} 0,4.3 \mathrm{fb}^{-1}$

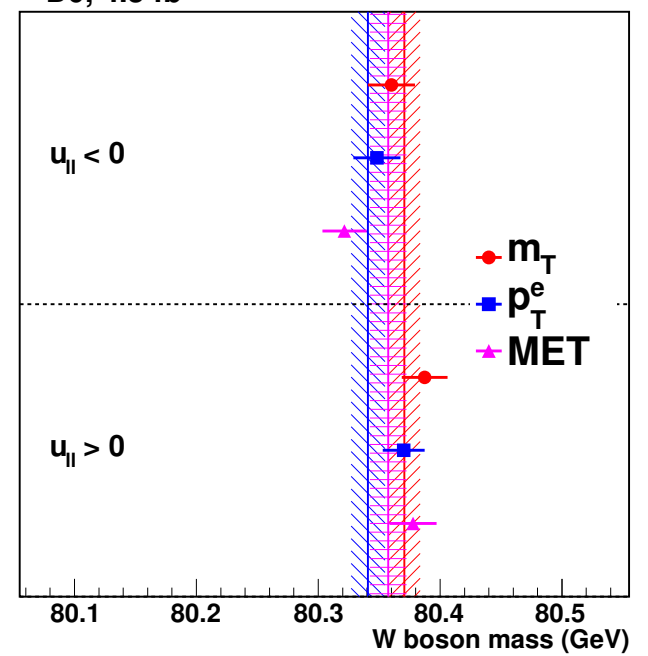

(a) $W$ mass as measured from the $m_{T}, p_{T}^{e}$ and $\mathbb{E}_{T}$ (b) $W$ mass as measured from the $m_{T}, p_{T}^{e}$ and observables, separately for five different regions $\mathbb{E}_{T}$ observables, separately for positive and negin electron $\left|\eta_{\text {det }}\right|$. The uncertainties for each ob- ative $u_{\|} \cdot u_{\|}$is an important observable since it is servable represent the statistical uncertainty due the most relevant component of the recoil transto limited $W$ statistics. The green bands indi- verse vector for the transverse mass when $p_{T}^{e} \gg$ cate the systematic uncertainty arising from PDF $u_{T}$. The sub-samples with $u_{\|}>0$ and $u_{\|}<0$ are variations. The uncertainty is $100 \%$ correlated also different because, on the former, the average among the three observables and highly corre- hadronic energy over the electron cone is larger. lated between neighbor $\eta_{\text {det }}$ bins. The yellow Thus, the detection efficiency is smaller and the bands indicate the quadrature sum of the system- underlying energy represents a larger fraction of atic uncertainties arising from PDF variations and the electron reconstruction efficiency.

from limited $Z$ statistics. The $Z$ statistics uncertainty is $100 \%$ correlated between the different observables but uncorrelated between each $\eta_{\text {det }}$ bin. Almost every electron, recoil and efficiency model is $\eta$ dependence since the detector geometry changes with the polar direction an so does the distribution of underlying energy.

Figure 11.3: Consistency checks by splitting the $W$ and $Z$ samples in subsets with different detector $\eta$ and $u_{\|}$. 

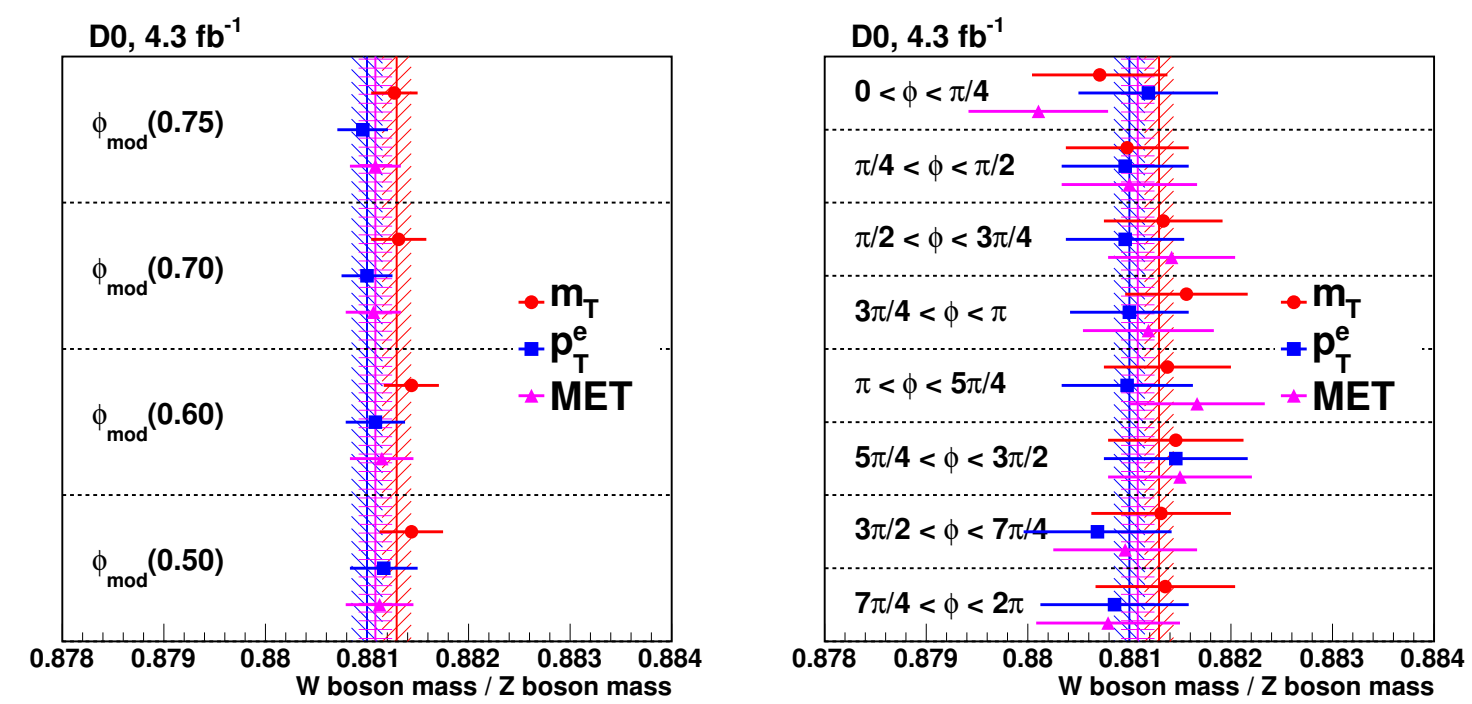

(a) The measured $W$ boson mass to $Z$ boson (b) The $W$ boson mass to $Z$ boson mass ratios, mass ratios, separately for the $m_{T}, p_{T}^{e}$ and $\mathbb{E}_{T}$ ob- separately for the $m_{T}, p_{T}^{e}$ and $\mathbb{Z}_{T}$ observables and servables and for four $\phi_{\text {mod }}$ selection variations. for eight bins in recoil $\phi$. Although there is not Although the response to electrons in the D0 preferred direction in the production of $W$, the D0 calorimeter is made azimuthaly uniform by the detector is not perfectly $\phi$ symmetric. In particu$\phi$-intercalibration [80], the presence of cracks be- lar, the length of the cables of the top part of the tween the $32 \mathrm{EM}$ modules in the central calorime- End Calorimeters is shorter than the cables in the ter changes the energy response and detection ef- bottom part. Due to the different cable lengths, ficiency when the electron is detected close to the the pedestal widths in the top part are smaller boundary relative to the average values in that $\phi$ than in the bottom part. Soft hadronic energy desegment of the calorimeter. The loss in response posits a small amount of energy per cell, close to and efficiency, as well as the bias in the calorime- the zero suppression. Thus, the recoil transverse ter direction pointing, are explicitly modeled in vector distribution is not azimuthaly symmetric. the fast MC.

In the fast MC model, the different recoil response in different directions is present in the zero-bias library.

Figure 11.4: Consistency checks by splitting the $W$ and $Z$ samples in subsets with different electron $\phi_{\text {mod }}$ and recoil $\phi$. 


\section{Part III}

\section{$W$ Boson Mass Measurement with Forward Electrons}




\section{Chapter 12}

\section{Understanding the PDF uncertainty in the $W$ mass measurement}

The parton distribution function (PDF) uncertainty in the $W$ boson measurement with 4.3 $\mathrm{fb}^{-1}$ was determined using PYTHIA with the leading-order (LO) CTEQ6.1 [70] PDF error set. This choice was largely motivated by the impossibility, in RESBOS, to have access to the parton level information on a per-event basis. Recently, the RESBOS authors prepared grid files for all the error sets of the CTEQ6.6 [66], CT10, and CT10w [81] PDFs allowing the propagation of the PDF uncertainty with this generator.

\subsection{Partons and PDF uncertainty}

We compare different predictions for the PDF uncertainty based on PYTHIA [82] and LO CTEQ6.1 with RESBOS and POWHEG [83] showered with PYTHIA for many different PDF sets. The results are summarized in Table 12.1, where the uncertainties have been calculated with the prescription:

$$
\delta A=\sqrt{\frac{1}{2.705} \times \frac{1}{4} \sum_{i=1,3, \ldots}^{N_{\text {eigen }}}\left(A_{i}-A_{i+1}\right)^{2}}
$$

where $A_{i}$ is the value of the observable $A$ when measured using the member $i$ of PDF error set ${ }^{1}$ with $N_{\text {eigen }}$ elements. The factor 2.705 allows the converstion from the $90 \%$ CL. tolerance with which the CTEQ PDF sets are published to the $68 \% \mathrm{CL}$. interval we use to report the uncertainty in this Dissertation.

There are large differences among the results, and while some are expected, others should be better understood. The CT10w set uses the same parametrization as CT10, but considers the $W$ charge asymmetry measured by D0 in the electron channel [84] in

\footnotetext{
${ }^{1}$ For all PDF sets, the member 0 corresponds to the default best fit value.
} 


\begin{tabular}{|c|c|c|c|c|c|}
\hline \hline Generator/PDF & CTEQ61 & CTEQ6.6 & CT10 & CT10(12) & CT10w \\
\hline PYTHIA & 10.9 & - & - & - & - \\
POWHEG & - & 12.7 & 14.7 & - & 9.3 \\
RESBOS & - & 15.5 & 16.8 & 13.3 & 11.4 \\
\hline \hline
\end{tabular}

Table 12.1: Comparison between the PDF uncertainty propagated to the fitted $W$ boson mass using the transverse mass distribution from the fast $\mathrm{MC}$ tuned for the measurement with $4.3 \mathrm{fb}^{-1}$.

the QCD global fit. The $W$ charge asymmetry constrains the $u / d \mathrm{PDH}^{2}$ at large scales $Q \simeq M_{W}$, which is the relevant kinematical regime for $W$ production at the Tevatron. Therefore, the PDF uncertainty is expected to be smaller for CT10w than for others. Indeed, the PDF uncertainties estimated with POWHEG and RESBOS are, respectively, 36\% and 33\% smaller when comparing CT10w to CT10. CT10 has more freedom in the PDF parametrizations than CTEQ6.6 and, therefore, the data in the QCD global fit have less constraining power for each parameter in the PDF parametrization. POWHEG and RESBOS indicate an increase in the uncertainty of $15 \%$ and $8 \%$, respectively, when comparing CT10 to CTEQ6.6.

When we compare PowHeg and ResBos both with CTEQ6.6, POwHEG predicts a PDF uncertainty almost $3 \mathrm{MeV}$ smaller than RESBOS. Both generators calculate the $W$ production at the same fixed order in perturbation theory $\left(\mathcal{O}\left(\alpha_{s}\right)\right)$ but deal with the soft radiation very differently. While RESBOS performs a high-precision NNLL resummation of soft gluon radiation, POWHEG uses PYTHIA for the showering. For making an approximate comparison with PYTHIA, a special RESBOS grid file has been produced[85], in which the CSS resummation of soft-gluons is not performed and only the production $u \bar{d} \rightarrow W$ (an CP conjugate) at LO are considered. The grid file uses the CT10 PDF and, when used to propagate the PDF uncertainty, predicts an uncertainty $21 \%$ smaller compared to the full CT10 grid file. This clearly shows that heavy quarks, higher order correction and soft gluon resummation, collectively, increase the PDF uncertainty. It would be interesting to compare each effect individually.

One interesting observable that can be calculated to further understand the partonic origin of the PDF uncertainty is the so-called PDF-induced correlation [66]:

$$
\rho_{a}=\frac{\sum_{i=1}^{N_{\text {eigen }}}\left(M_{W}^{i}-M_{W}^{0}\right) \times\left(f_{a}^{i}-f_{a}^{0}\right)}{\delta M_{W} \times \delta f_{a}}
$$

where $f_{a}$ is the value of the PDF for the flavor $a$ for a given $(x, Q)$. Because of this, $\rho_{a}$ also depends on $(x, Q)$. We can interpret each member of the PDF error set as a measurement of the PDF and, therefore, this observable measures the correlation between the measured value of the $W$ boson mass and the PDF. Unfortunately, even when calculating

\footnotetext{
${ }^{2} \mathrm{By} u / d$ PDF we mean the ratio between the $u$ and $d$ quarks PDF, both evaluated at the same $(x, Q)$ point.
} 
this observable using $f_{a}$ at a single $(x, Q)$ value, the value of $\rho_{a}$ still depends on all the momentum fractions and scales probed during $W$ production.

Figures 12.1, 12.2, and 12.3 show, on their top right panels, the $u / d$ PDF-induced correlation as a function of $x$ for three values of the scale $Q$. The region $x \simeq 0.1$ has the largest correlation between PDF variations and the fitted value of the $W$ mass. We study of the variation of the error set at this particular value. The bottom panels of the same figures contain the variation of $u / d$ at $\left(x=0.1, Q=M_{W}\right)$ for each member of the error set. Their variations are similar to the ones on the top left panels, which show the variations of $M_{W}$.
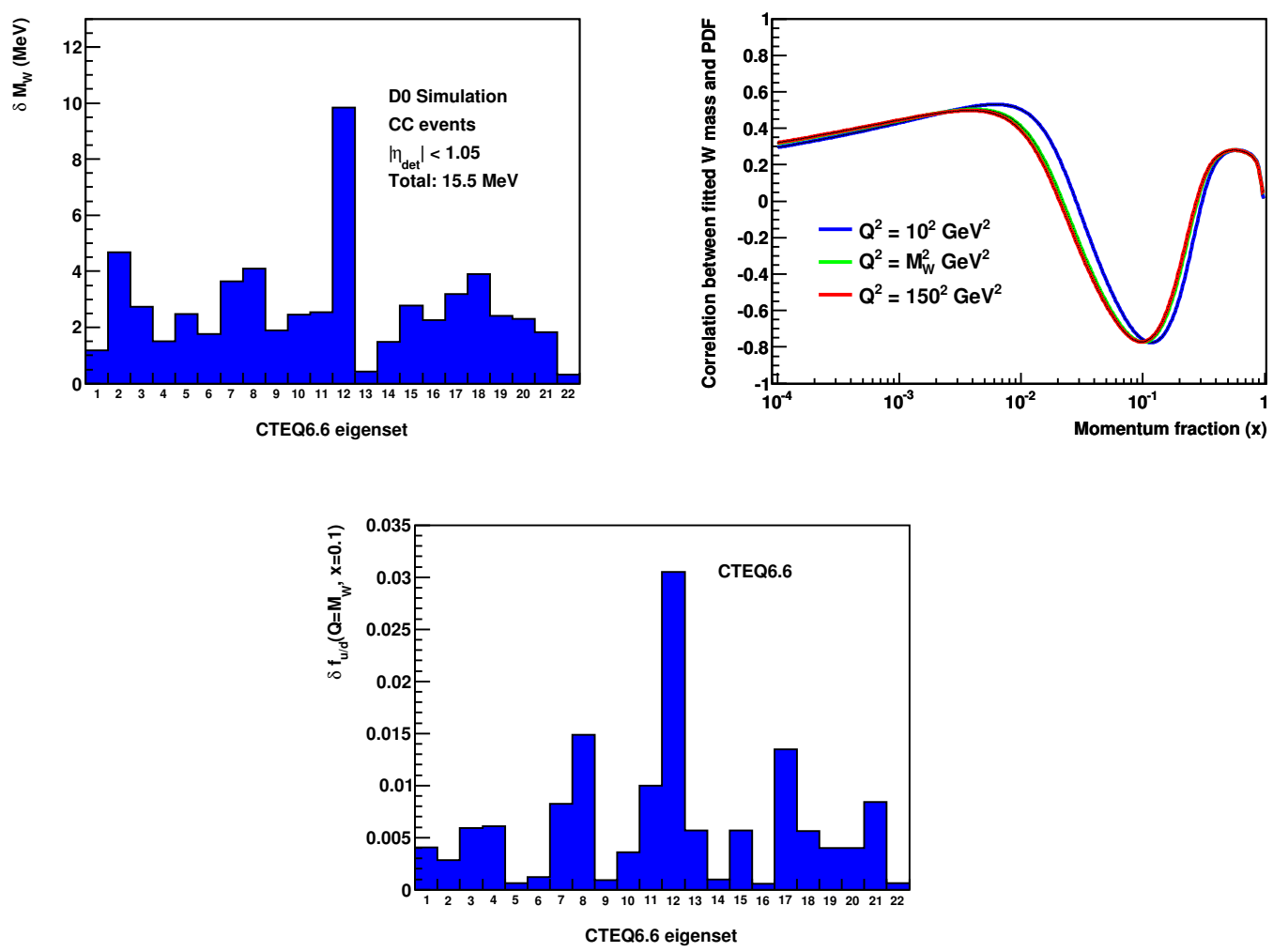

Figure 12.1: (top left) Propagation of the variation of each member of the CTEQ6.6 error eigenset to the fitted value of the $W$ boson mass using the transverse mass distribution of pseudo-experiments simulated with REsBos. (top right) PDF-induced correlation between the $W$ mass and the $u / d$ PDF as a function of the parton momentum fraction for three different mass scales. (bottom) Variation of the $u / d$ PDF in each member of the CTEQ6.6 error eigenset for $x=0.1$ and $Q=M_{W}$.

It is clear from figures 12.1 and 12.3 , that eigenset 12 in CTEQ6.6 and 13 in the CT10 family parametrize most of the $u$ / $d$ variation. It also clearly shows the power of the $W$ charge asymmetry measurement in constraining this variation. 

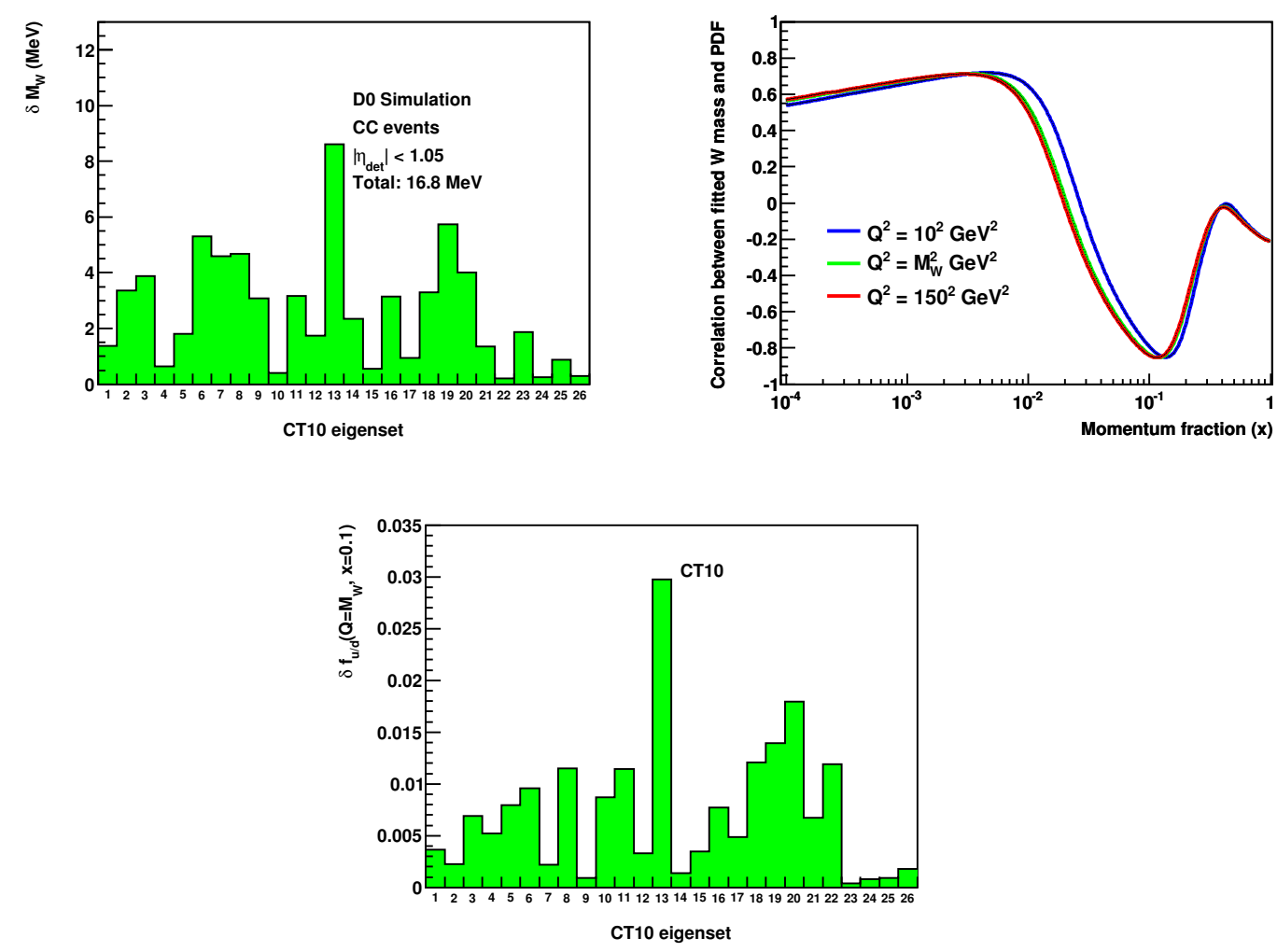

Figure 12.2: (top left) Propagation of the variation of each member of the CT10 error eigenset to the fitted value of the $W$ boson mass using the transverse mass distribution of pseudo-experiments simulated with REsBos. (top right) PDF-induced correlation between the $W$ mass and the $u / d$ PDF as a function of the parton momentum fraction for three different mass scales. (bottom) Variation of the $u / d$ PDF in each member of the CT10 error eigenset for $x=0.1$ and $Q=M_{W}$.

\subsection{Detectors and PDF uncertainties}

The PDF uncertainty is, to a large extent, an acceptance uncertainty. The total integrated PDFs are constrained by the proton quantum numbers. Hence, only when sampling over smaller momentum fraction ranges, will large variations from the uncertainties in the determination of PDFs be present. In the $W$ mass measurement, the most important constraint on the momentum fraction comes from the $\left|\eta_{\text {det }}\right|$ acceptance requirement. By relaxing this requirement, we can reduce the uncertainty by almost $45 \%{ }^{3}$, as shown in

\footnotetext{
${ }^{3}$ Unfortunately, the RESBOS CT10w grid file publicly available returns inconsistent results for forward electrons and, at this moment, it is impossible to test both improvements at the same time. We believe that both theoretical and experimental community would benefit from having another resummed calculation of $W$ boson production so that this type of instability in the overall quality of grid files could be sorted quicker.
} 

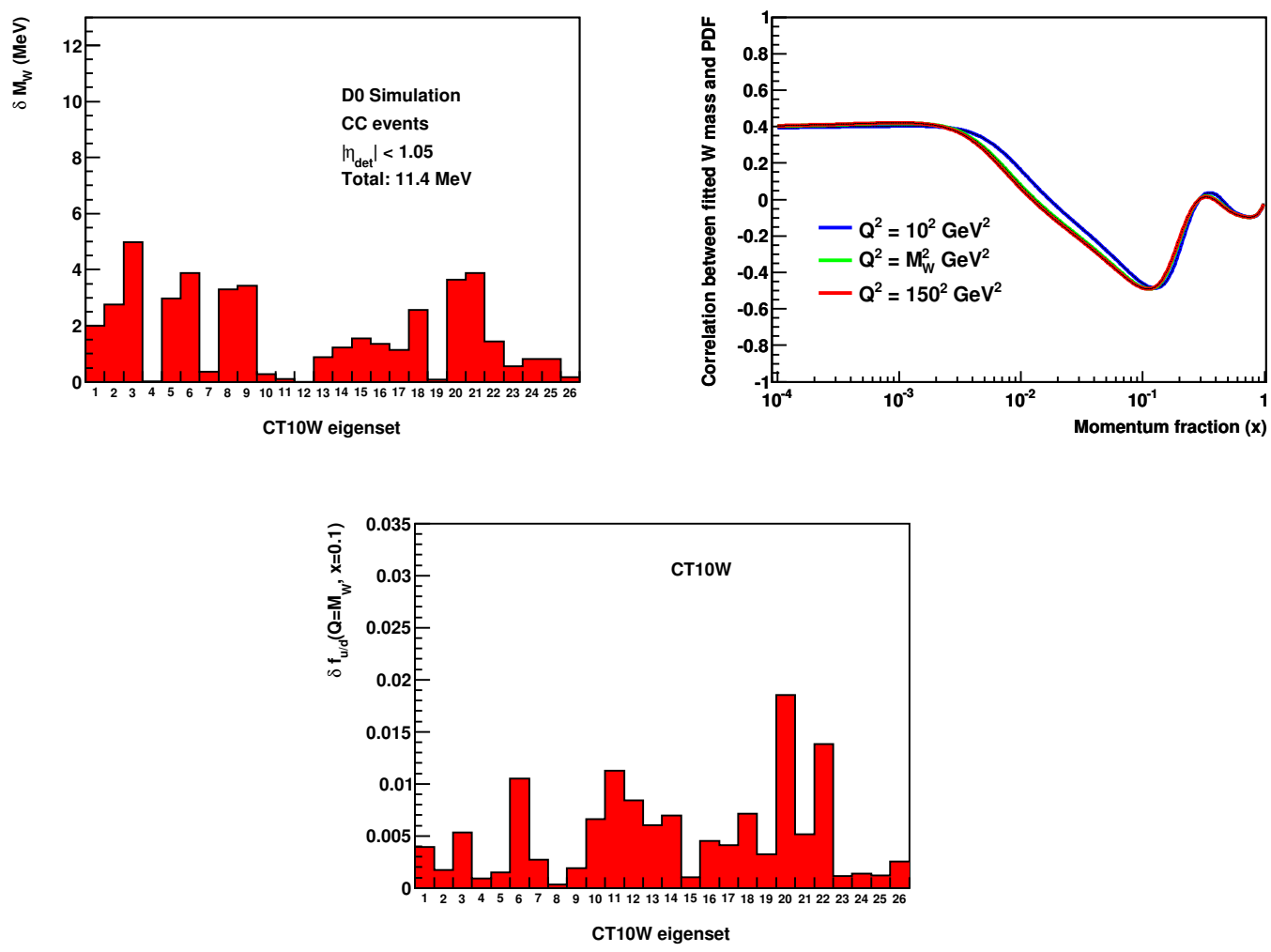

Figure 12.3: (top left) Propagation of the variation of each member of the CT10w error eigenset to the fitted value of the $W$ boson mass using the transverse mass distribution of pseudo-experiments simulated with REsBos. (top right) PDF-induced correlation between the $W$ mass and the $u / d$ PDF as a function of the parton momentum fraction for three different mass scales. (bottom) Variation of the $u / d$ PDF in each member of the CT10w error eigenset for $x=0.1$ and $Q=M_{W}$.

Figs. 12.4, 12.5, 12.6 and summarized in table 12.2

The second selection criteria that imposes constraints on $x$ is the recoil transverse momentum threshold. Figure 12.7 shows the dependence of the PDF uncertainty, calculated with RESBOS and CTEQ6.6 with respect to the recoil relative response and resolution 4

The detector resolution for hadronic particles is fixed by the detector construction and reconstruction algorithms. However, the D0 End Calorimeters can be used to extend the electron $\eta$ acceptance and reduces the PDF uncertainty in the measurement. This project is not without challenges. The uninstrumented material in the forward region of the D0 detector is highly inhomogeneous. The underlying energy flow is larger in the

\footnotetext{
${ }^{4}$ Reference [86] predicts an unrealistically small PDF uncertainty in future $W$ mass measurements exactly because assumes a perfect hadronic recoil response and resolution.
} 


\begin{tabular}{|c|c|c|}
\hline \hline Fitted observable & $\mathrm{CC}\left(\left|\eta_{\operatorname{det}}\right|<1.05\right)$ only $(\mathrm{MeV})$ & $\mathrm{CC}$ and EC $\left(1.5<\left|\eta_{\text {det }}\right|<2.5\right)(\mathrm{MeV})$ \\
\hline$m_{T}$ & 15.5 & 8.4 \\
$p_{T}^{e}$ & 22.0 & 15.8 \\
$\mathbb{Z}_{T}$ & 16.7 & 11.0 \\
\hline \hline
\end{tabular}

Table 12.2: PDF uncertainty in the D0 $W$ boson mass measurement. The uncertainty is propagated from the CTEQ6.6 error set using pseudo-experiments generated with ResBos and simulated with the $W$ mass fast MC.
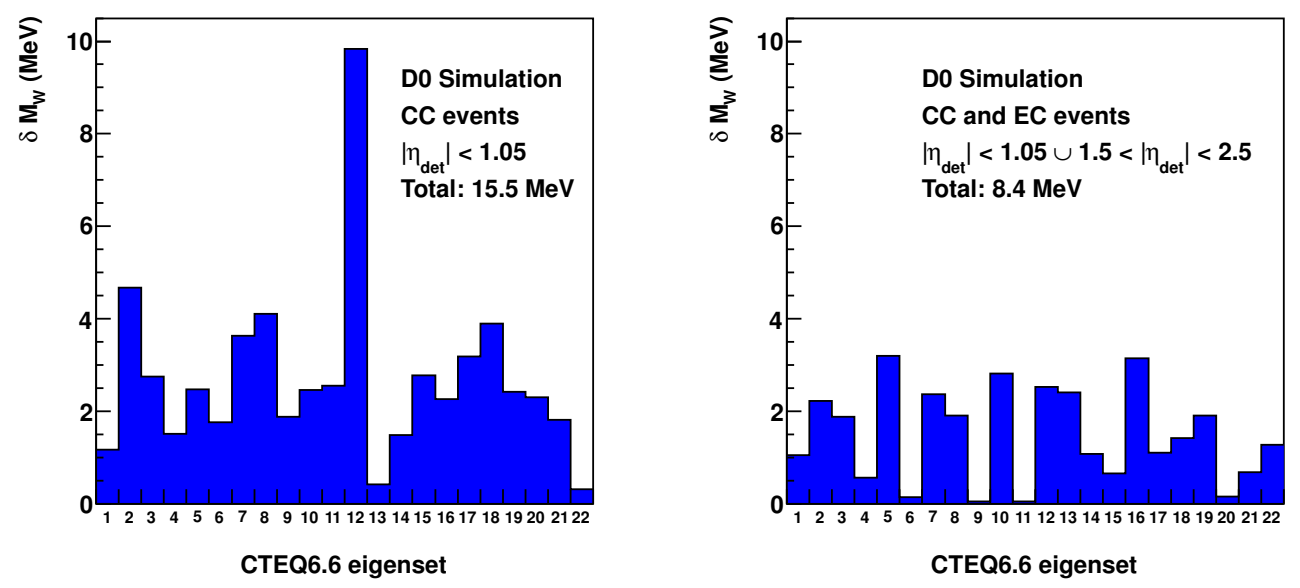

Figure 12.4: Propagation of the variation in each member of the CTEQ6.6 error eigenset to the fitted value of the $W$ boson mass using the transverse mass observable. On the left, the propagation with central electrons $\left(\left|\eta_{\text {det }}\right|<1.05\right)$ only. On the right, the same propagation but including a restricted region of the forward calorimeter $\left(1.5<\left|\eta_{\text {det }}\right|<2.5\right)$. The propagation was done with events generated by RESBOS and simulated with the $W$ mass fast MC. The total uncertainty is the sum in quadrature of each variation. 

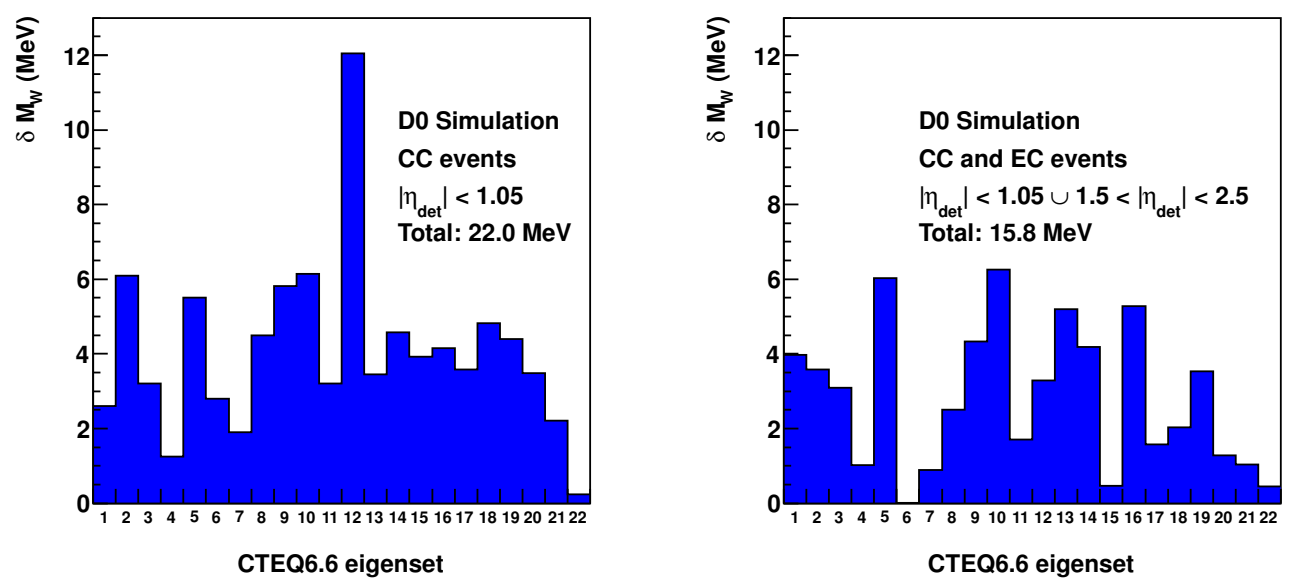

Figure 12.5: Propagation of the variation in each member of the CTEQ6.6 error eigenset to the fitted value of the $W$ boson mass using the electron transverse momentum observable. On the left, the propagation with central electrons $\left(\left|\eta_{\text {det }}\right|<1.05\right)$ only. On the right, the same propagation but including a restricted region of the forward calorimeter $(1.5<$ $\left.\left|\eta_{\text {det }}\right|<2.5\right)$. The propagation was done with events generated by REsBos and simulated with the $W$ mass fast MC. The total uncertainty is the sum in quadrature of each variation.
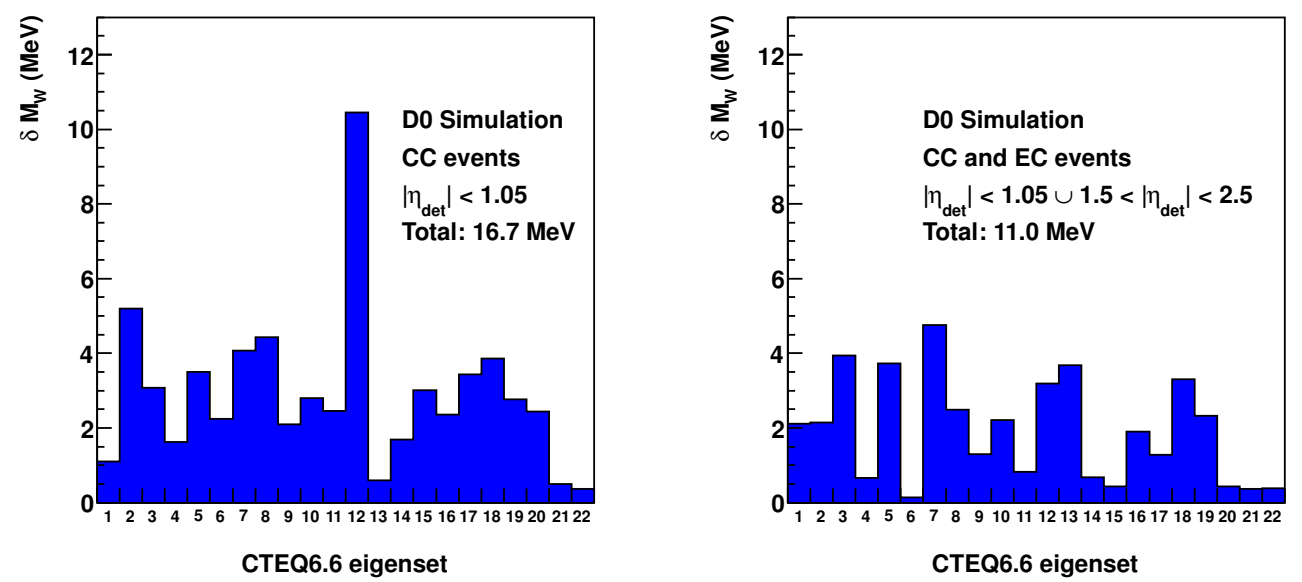

Figure 12.6: Propagation of the variation in each member of the CTEQ6.6 error eigenset to the fitted value of the $W$ boson mass using the missing transverse energy observable. On the left, the propagation with central electrons $\left(\left|\eta_{\text {det }}\right|<1.05\right)$ only. On the right, the same propagation but including a restricted region of the forward calorimeter $\left(1.5<\left|\eta_{\text {det }}\right|<\right.$ 2.5). The propagation was done with events generated by RESBOS and simulated with the $W$ mass fast MC. The total uncertainty is the sum in quadrature of each variation. 


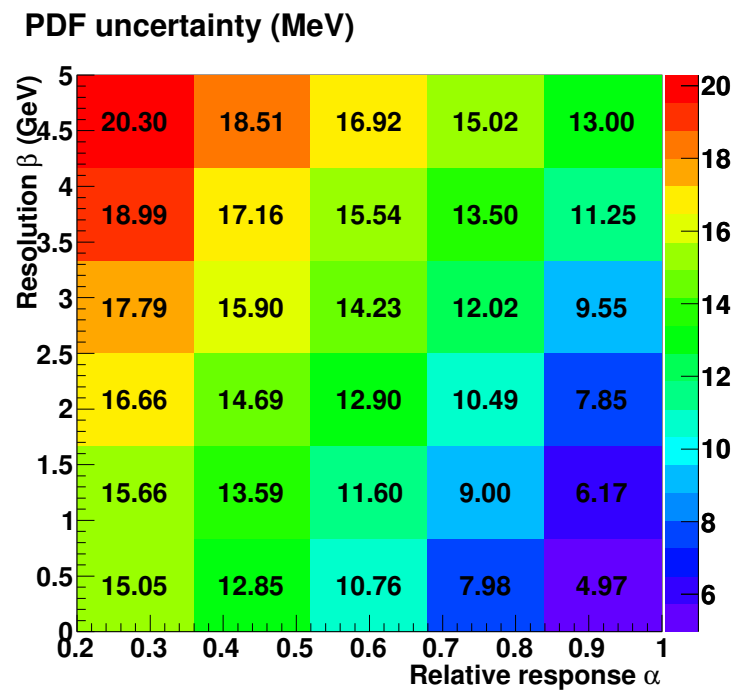

Figure 12.7: Dependence of the PDF uncertainty on the hadronic recoil relative response and resolution. The PDF uncertainty is propagated using $m_{T}$ fits of RESBOS pseudoexperiments simulated with the fast MC and the CTEQ6.6 error set. The recoil transverse momentum vector is simulated as $\vec{u}_{T}=-\alpha \vec{p}_{T}^{W}+\beta^{\prime} \hat{x}+\beta^{\prime \prime} \hat{y}$ where $\beta^{\prime}$ and $\beta^{\prime \prime}$ are independent random variables normally distributed with common width $\beta$.

high $\eta$ region and the number of misreconstructed jets is also potentially larger. While there is not yet a complete fast $\mathrm{MC}$ model for the detector in the forward region, the final part of this Dissertation tries to address these specific challenges. We describe a detailed study of the dead material and calibration of the End Calorimeter, study the effect of the underlying event in many new parametrized models for forward electrons and the multijet fake rate. 


\section{Chapter 13}

\section{Calibration of the D0 End Calorimeters for the $W$ Mass Measurement}

This chapter describes a dedicated calibration of a restricted section of the D0 End Calorimeters performed specifically for the $W$ mass measurement with forward electrons. We review the improvements in the GEANT [64] shower simulation first implemented for the equivalent calibration of the CC (see Sec. 5). We then use $Z \rightarrow e e$ events with CC-EC topology to measure the amount of dead material upstream of the forward calorimeters. With the improved geometry model, we determine new corrections for the energy lost in the dead material before the first active layer of the calorimeter. We then re-derive the calibration constants for each $\eta$ ring in the restricted region of the forward calorimeter. The uncertainties in these procedures are propagated to the $W$ mass measurement and shown to have negligible impact.

\subsection{General Idea of the Calorimeter Calibration}

The D0 Calorimeter is a sampling calorimeter. Therefore, it only detects a fraction, called sampling fraction, of the shower energy that passed through a given cell. The first step in the calibration is to determine accurately the relationship between the energy sampled by the active region of the calorimeter and the number of ADC counts at the end of the electronic read out chain. This part is known as the "electronic calibration". Because of non-linearities in the read out electronics, this calibration is not trivial. However, it is outside the scope of this Dissertation and the details can be found elsewhere [87, 88].

Once an accurate electronic read out is available and the sampling fraction is known, we have an initial estimate of the energy in each cell $E_{i}^{\text {initial }}$. The goal of the calibration described in this chapter is to determine further dimensionless constants $d_{i}$ :

$$
E_{i}^{\text {corrected }}=d_{i} \times E_{i}^{\text {initial }}
$$

such that the $\sum E_{i}^{\text {corrected }}$ over the cells of an electron cluster is a more accurate repre- 
sentation of the energy of the electron that generated the shower. These constants are determined using data control samples which have a precisely known energy reference. For EM calorimeters, the calibration control sample by excellence is $Z \rightarrow e e$. The $Z$ boson mass is very precisely known and, therefore, can be used as a reference to determine the constants $d_{i}$. There are two shortcomings in this idea:

1. The sample size is limited. At D0, we were able to record approximately 10,000 $\mathrm{Z} \rightarrow$ ee candidates per $\mathrm{fb}^{-1}$ with both electrons in the Central Calorimeter. With the inclusion of electrons in the End Calorimeters, this number increases to approximately $14,000 \mathrm{Z} \rightarrow$ ee candidates per $\mathrm{fb}^{-1}$. However, there are approximatelly 30,000 cells in the EM calorimeter and it is impossible to use only this sample to determine the constant $d_{i}$ for each cell.

2. Even if it were possible, the $Z$ is a narrow resonance and the electrons detected at a given direction will have a very small spread in energy. Therefore, the use of $Z$ to describe possible non-linear deviations from the hypothesis in Eq. 13.1 is impossible.

The limitation (1) above forces us to determine the constants $d_{i}$ collectively for large domains of cells. To reduce the number of degrees of freedom in the calibration program, D0 performs a $\phi$ inter-calibration. Since the beams that interact in the Tevatron are not polarized, the energy flow produced in the interactions is, on average, azimuthally isotropic. Therefore, so must be the average deposit of energy in each $\phi$ line. By adjusting the relative value of the constants $d_{i}$ with a common $\phi$ direction, we can equalize the $\phi$ response and leave just one degree of freedom $d_{i}^{\prime}$ undetermined. In practice, the D0 $\phi$ inter-calibration is done independently for each $\eta$ ring, adjusting the energy response of each tower to be equal to the average value of the ring before the inter-calibration:

$$
E_{i}^{\text {corrected }}=d_{i}^{\prime} \times c_{i}^{\phi,(\eta)} \times E_{i}^{\text {initial }}
$$

where the $d_{i}^{\prime}$ do not depend on the direction $\phi$ of the cell. Most of the details in this intercalibration revolve around avoiding bias from another sub-detector or from the trigger system [80].

Limitation (2) above is also present in our calibration, but attacked with another strategy. We recognize that the main source of non-linearity is the energy lost by the particle that initiated the shower before it reached the calorimeter. By comparing the development of EM showers along its axis in the calorimeter between data and a detailed simulation of the D0 detector, we develop an ultra-detailed simulation of the D0 calorimeter (see Sec. 13.2) which is then used to derive an "energy loss" function, which recovers on average the energy lost by the particle and accounts for any residual energy dependency on $d_{i}^{\prime}$. This comparison also allows us to determine the relative calibration constants for each layer of the calorimeter by requiring that the fractional integrated deposit of energy in each layer agrees with the simulation. Therefore,

$$
E_{i}^{\text {corrected }}=d_{i}^{\prime \prime} \times c_{\text {layer }} \times c_{\phi,(\eta)} \times f_{\mathrm{EL}}\left(E_{i}^{\text {initial }}\right)
$$




\begin{tabular}{lc}
\hline \hline $\begin{array}{l}\text { Detector } \\
\text { Element }\end{array}$ & $\begin{array}{c}\text { Material } \\
\text { Thickness }\end{array}$ \\
\hline Inner Detector & $0.2 \mathrm{X}_{0}$ \\
Solenoid & $0.9 \mathrm{X}_{0}$ \\
Preshower (detector) & $0.3 \mathrm{X}_{0}$ \\
Preshower (absorber) & $1.0 \mathrm{X}_{0}$ \\
Cryostat & $1.3 \mathrm{X}_{0}$ \\
\hline \hline
\end{tabular}

Table 13.1: Material in front of the first calorimeter layer as seen by an electron traveling from the interaction point to the $\mathrm{CC}$ at normal incidence.

where the constants $d_{i}^{\prime \prime}$ do not depend on the azimuthal direction, which has been factored out by the $\phi$ constants $c_{\phi,(\eta)}$; do not depend on the depth, which has been factored out by the layer constants $c_{\text {layer }}$; and do not depend on the energy $E_{i}^{\text {initial }}$, since all non-linearities have been described by the energy loss function $f_{\mathrm{EL}}(E)$.

The only degree of freedom left is the $\eta$ direction $d_{i}^{\prime \prime} \equiv c_{\eta}$. We use $Z \rightarrow e e$ candidates in which at least one electron has been detected in a given $\eta$ direction and the known value of the $Z$ mass to determine the calibration constants per $\eta$ ring, which also fixes the absolute calibration:

$$
E_{i}^{\text {corrected }}=c_{\eta} \times c_{\text {layer }} \times c_{\phi,(\eta)} \times f_{\mathrm{EL}}\left(E_{i}^{\text {initial }}\right)
$$

\subsection{A Review of the $W$ Mass Group Modifications to GEANT}

Before the electron reaches the first active layer of the calorimeter, it deposits a sizable part of its energy in other detector components. Independently of whether this is an active material of another D0 sub-detector or not, we refer to this material as dead material. The amount of dead material is not small, as table 13.1 shows.

The energy lost in the dead material can be recovered on average if we know the dead material precisely and if the physics of transport and energy loss in these materials are well simulated. In the standard D0 calorimeter reconstruction, the layer weights are not proportional to the inverse of the sampling fraction of the layer. Table 13.2 shows the weights used in the Run IIb data reconstruction software. The layer weight applied to EM1 is considerably larger. These layers constants were determined by Monte Carlo simulation using single monochromatic electrons of $50 \mathrm{GeV}$ and fitting the constants to obtain the best possible resolution. The best way to achieve this goal is to artificially increase the layer weight of the first EM layer as if it was reading the energy lost before it and to decrease the weight of the subsequent layer. The layer weights of EM3 and EM4 are closer to the inverse of the sampling fractions of the D0 calorimeter. This choice has many shortcomings. It artificially enhances the $\eta$ and energy dependence of the response, since the average EM fractions and fluctuations around this average can change drastically 


\begin{tabular}{cccc}
\hline \hline Calorimeter Layer & Weight & Thickness $\left(X_{0}\right)$ & Weight/Thickness $\left(1 / X_{0}\right)$ \\
\hline \multicolumn{4}{c}{ Central Calorimeter } \\
EM1 & 31.1991 & 1.4 & 22.2851 \\
EM2 & 9.3992 & 2.0 & 4.6996 \\
EM3 & 25.7156 & 6.8 & 3.7817 \\
EM4 & 28.0328 & 9.8 & 2.8605 \\
\hline \multicolumn{4}{c}{ End Calorimeter } \\
EM1 & 32.9612 & 1.6 & 20.6008 \\
EM2 & 17.0792 & 2.6 & 6.5689 \\
EM3 & 33.1245 & 7.9 & 4.1930 \\
\hline \hline
\end{tabular}

Table 13.2: Layer weights applied during data reconstruction in the D0 calorimeter. The values are further multiplied by a global ADC to $\mathrm{GeV}$ conversion factor of $3.086 \times 10^{-4}$ $\mathrm{GeV} /$ count.

depending on the position of the shower maximum and, therefore, on the exact amount of dead material, see Fig. 13.1.

The absolute energy scale of the calorimeter is determined with $Z \rightarrow e e$ events. Since the $\eta$ and $E$ spectrum of electrons from $Z$ boson decays is different from those from $W$ boson decays as shown in Fig. 13.2, it is important to describe these dependencies accurately. With this goal, the Do $W$ mass group engaged in a program to carefully evaluate the transport and energy loss algorithms implemented in GEANT [89]. This section briefly reviews the improvements implemented in these algorithms.

To be able to customize the transport parameters, the simulations performed for the calibration do not use the AUTO mode in GEANT for particle tracking. The tracking parameters are fixed to values such that the approximations assumed, especially those in Molière theory, are comfortably within the validity range. The values chosen are listed below.

Decay electron cut (DCUTE): Ionization electrons ( $\delta$-rays) are tracked down to $10 \mathrm{keV}$ compared to the nominal value of $1 \mathrm{MeV}$ [64, PHYS331].

Maximum fractional energy loss in one step due to continuous ionization (DEEMAX): The automatic value is estimated to be:

$$
\text { DEEMAX }= \begin{cases}0.25 & \text { if non-sensitive detector and } X_{0}<2 \mathrm{~cm} \\ 0.25-\frac{0.2}{\sqrt{X_{0}}} & \text { if non-sensitive detector and } X_{0} \geq 2 \mathrm{~cm} \\ \frac{0.2}{\sqrt{X_{0}}} & \text { if sensitive detector }\end{cases}
$$

For uranium, where $X_{0}=0.32 \mathrm{~cm}$, this value is set to 0.25 , which allows large steps and renders the values of $d E / d x$ at the beginning and end of the tracking steps bad approximations for its average value. In the simulation used for the calibration, the value is set 

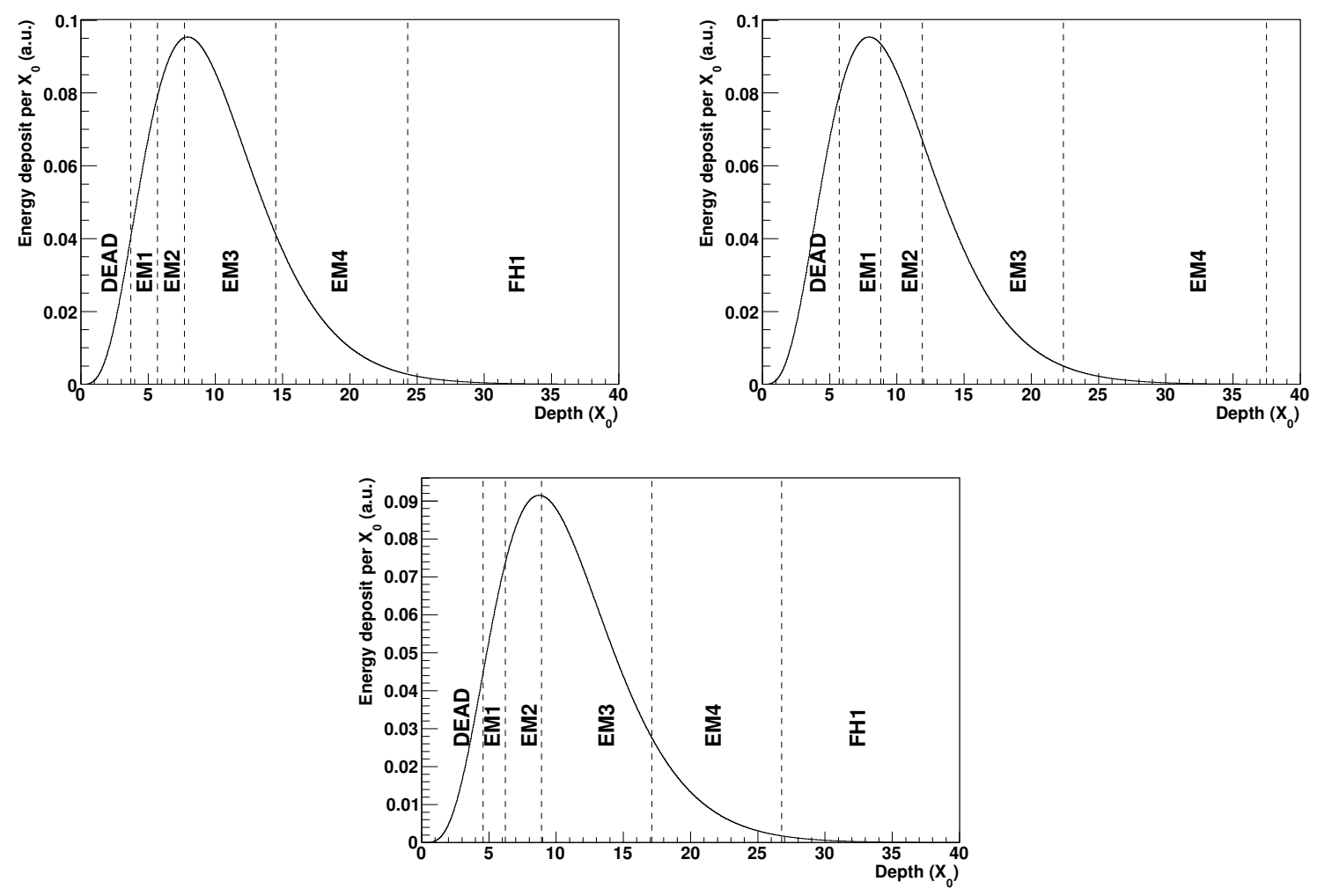

Figure 13.1: (top) Average shower for a $45 \mathrm{GeV}$ electron in the CC with $\eta=0$ and $\eta=1$. (bottom) Average shower for a $100 \mathrm{GeV}$ electron in the $\mathrm{EC}$ with $\eta=2$. The simulations use the GFlash parametrization and represent the average over 1000 simulated EM showers.

to 0.005 [64, PHYS010].

Maximum step taken in multiple scattering simulation (STMAX): The upper bound on the step size from DEEMAX is given by:

$$
\text { step }<E \frac{\text { DEEMAX }}{d E / d x}
$$

With the value of DEEMAX set above, this bound is already a severe constraint. For an electron with momentum $400 \mathrm{keV}$ in uranium, which is close to a minimally ionizing particle, $E /(d E / d x) \simeq 0.02$ and the step will be limited to $\mathcal{O}\left(10^{-4}\right) \mathrm{cm}$. For higher energies, the upper bound loses power since the bremsstrahlung cross section does not vary quickly enough. To avoid large steps at large energies, an energy independent limit is set to 0.1 cm [64, PHYS010].

Minimum step taken in multiple scattering simulation (STMIN): Another limitation imposed on the maximum step comes from Molière theory. To stay within the validity of 


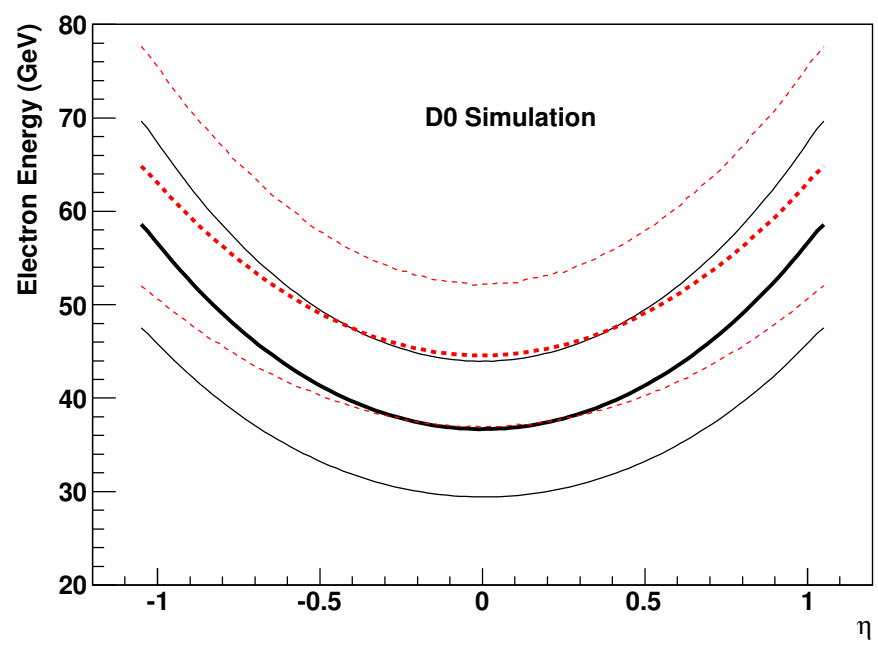

Figure 13.2: The mean electron energy (thick) versus $\eta$ for electrons from $Z$ (red dashes) and $W$ (black solid line) events in data. The thin lines indicate the standard deviations of the energy distributions versus $\eta$.

the approximations made, the maximum allowed step should be [90]:

$$
t_{\text {Bethe }}=\frac{E^{2} \beta^{4}}{\chi_{c c}^{2} Z_{\text {inc }}^{2} \ln \left[b_{c} E^{2} \beta^{2} / \chi_{c c}^{2}\right]},
$$

where $\chi_{c c}$ and $b_{c}$ are constants of the medium for a given incident particle [64, PHYS325] and $Z_{i n c}$ is the charge of the incident particle. As a precaution, GEANT adopts another upper bound for the step given by [64, PHYS010]:

$$
\text { step }<\min \left(t_{\text {Bethe }}, 10 X_{0}\right) .
$$

For a $400 \mathrm{keV}$ electron in uranium, $t_{\mathrm{Bethe}}$ is $\mathcal{O}\left(10^{-4}\right) \mathrm{cm}$ and orders of magnitude smaller than $10 X_{0}$. At this energy, this constraint is similar to the one imposed by DEEMAX. If GEANT obeyed these upper bounds strictly, there would be no major problems in the simulation. However, to save computing time, GEANT also implements a lower bound to the step in the simulation, given in automatic mode by:

$$
\text { STMIN }=\frac{5 R}{\sqrt{X_{0}}}
$$

where $R$ is the range of the particle. If this is lower than $\min \left(t_{\text {Bethe, }}, 10 X_{0}\right)$ this bound is not a problem. For uranium, this lower bound turns out to larger than $t_{\text {Bethe }}$. GEANT chooses to strictly obey the lower bound in detriment of the formal, theoretical upper bound. To avoid using Molière theory outside its validity range, we set the minimum step to the fixed value of $10^{-7} \mathrm{~cm}$. 
Absolute tracking precision (EPSIL): After the parameters described above are set, the size of the step is severely constrained. The precision with which GEANT determines if a track is still inside a given volume must be consistent with the step. A track closer to a boundary than EPSIL is assumed to be leaving the volume. We set this value to $10^{-3} \mathrm{~cm}$ which is ten times smaller than the default value of $10^{-2} \mathrm{~cm}$ in GEANT [64, CONS200]. This is particularly important for a sampling calorimeter in which the value e/mip is always less than unity and whose precise value depends on soft bremsstrahlung photons that cross the boundary between the absorber and active layers.

After setting the tracking parameters, the main limitation of the GEANT shower simulation becomes the precision of the interaction cross sections. Both bremsstrahlung and pair creation total cross sections have been updated using the calculations in references [91, 92] $]^{1}$. Figure 13.3 shows a comparison between the standard GEANT parametrization and the updated one. The red curve shows the bremsstrahlung cross section parametrization used by EGS4[94]. Other parametrizations could be improved, such as the differential distributions for bremsstrahlung and pair creation or the photoelectric cross section, but they have smaller impact in this calibration.
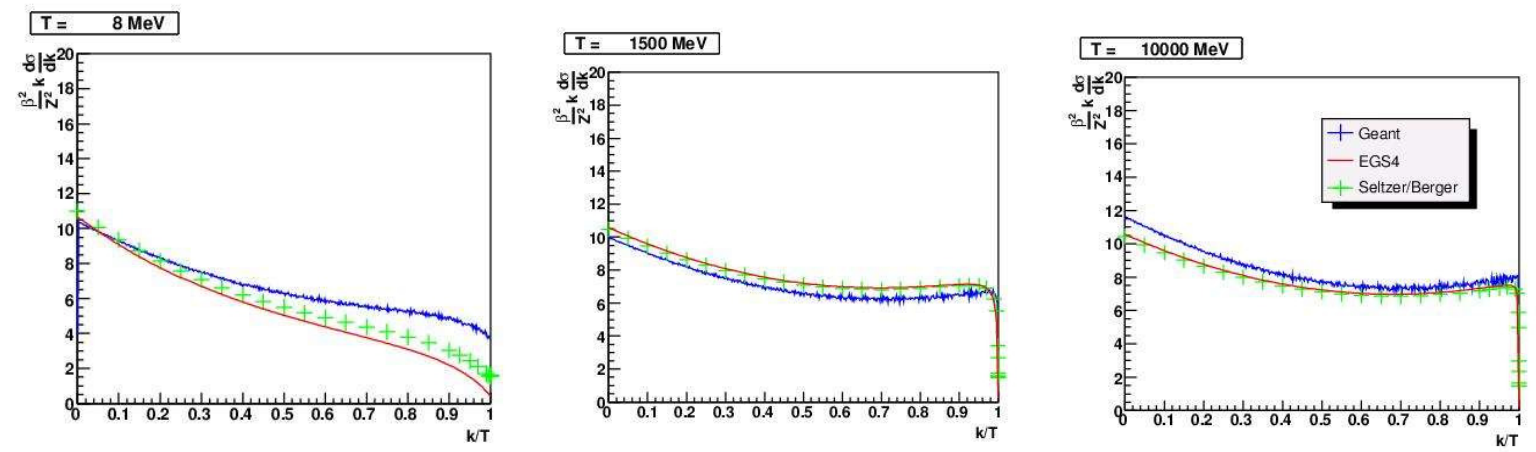

Figure 13.3: Comparison between the parametrization of the bremsstrahlung cross section present in GEANT (blue points) and the first principle calculation by Seltzer and Berger used for this calibration (green points).

\subsection{The D0 Geometry Model in the Forward Region}

In the calibration of the $\mathrm{CC}$ done for the $W$ mass measurement with central electrons, the cylindrical geometry of all elements upstream of the first active layer of the calorimeter was used as a handle to determine any imperfection in the D0 material model. Comparing the shower development in data and Monte Carlo simulations, it was clear that a small amount of dead material was unaccounted for in the GEANT model. A copper cylinder,

\footnotetext{
${ }^{1}$ Since version 9.5, GEANT4 has implemented the same parametrization for bremsstrahlung as used in this Dissertation [93]. The D0 detector simulation is done with GEANT3.
} 
coaxial to the detector, was introduced. Although the radius of the cylinder has an impact in the shower development, it can be almost perfectly compensated for by the thickness.

The copper cylinder was introduced in the GEANT model inside the solenoid mother volume. Since the geometry was taken a priori, all electrons detected in the CC could be used to determine the single parameter left undetermined, namely, the cylinder thickness. In other words, once the geometry is fixed, the amount of extra dead material a particle crosses before reaching the calorimeter depends only on the incident angle, ie. physics $\eta$, and not where in the detector the shower developed, ie. detector $\eta$ [62].

The situation in the ECs is very different. Figure 13.4 has a diagrammatic representation of the elements in the GEANT model of the region around the forward calorimeters. Owing to the curved shapes and many transitions, it is not reasonable to assume a fixed
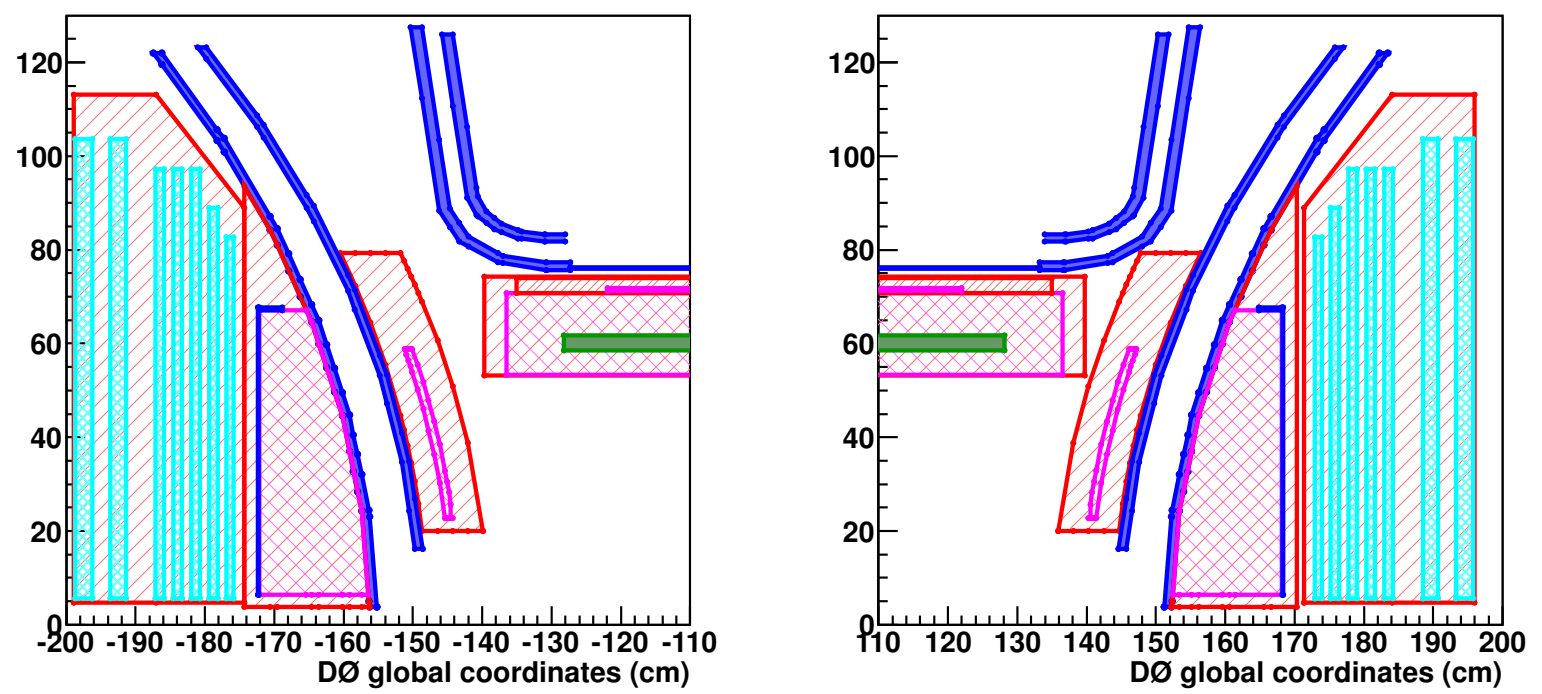

Figure 13.4: Geometry of the D0 GEANT model around the central and End Calorimeter cryostats. The red hatched areas are logical mother volumes. The blue solid areas are the cryostat walls and the internal support structure of the rohacell excluder. The pink hatched areas represent a series of other dead materials that, although important for the overall material budget, will have smaller importance to the strategy of this calibration (solenoid, CPS and FPS absorbers). The green solid area represents the cylinder that was added in the CC calibration. Finally, the cyan hatched areas are the sensitive elements of the forward calorimeter.

shape. Any dead material study has to be done separately for each detector $\eta$ region. Although necessary, this choice is problematic since showers with same detector $\eta$ can be initiated by electrons with different incident angle. This problem is slightly mitigated in the forward region, since the derivative of $\eta_{\text {det }}$ with respect to the $z$ position of the primary vertex is smaller for large values of $\eta$. On the other hand, this choice disentagles 
gain calibration effects from material effects, what is not possible with the strategy chosen for the CC.

Another difficulty in the forward region is the incompleteness of the material model. While all detector elements in the central region are accounted for in the GEANT model, the forward region model notably ignores known detector elements. In particular, there is no description of the effect of the inner tracker readout cables, cryogenic services and the cuts in the detector that accommodate these elements. The inner tracker cables can be well modeled by the $\eta$ dependent correction described here. The cryogenic service pipes and "chimney", on the other hand, create a detector $\phi$ dependence that will not be explicitly modeled for the ECS. Their effects on the energy scale calibration is correctly modeled by the D0 $\phi$ inter-calibration. The same is not true for the non-linearities introduced by the absence of the lead in the dead material budget. However, the effective area they occupy on the south side of the detector is sufficiently small to justify such choice.

\subsection{Improvements in the Material Model}

Fundamentally, the strategy used to measure the effective amount of dead material a particle crosses before being sampled by the EC is the same as in the CC. As it is clear from Fig. 13.1. the mean fraction of energy deposited in each layer of the D0 calorimeter is sensitive to the energy lost before reaching it, since the peak position of the shower shifts accordingly. Because of the relatively large weight given to EM1 during reconstruction, and since it is located in the sharply rising edge of the shower profile (see Fig. 13.1), this layer is especially sensitive to any change in the material. We segment the cryostat wall in straight sections approximately $\Delta \eta_{\text {det }}=0.1$ wide. The region between $1.5<\eta_{\text {det }}<1.7$, where the transitions between the solenoid and the FPS absorber are located, is segmented in smaller bins, $\Delta \eta_{\text {det }}=0.05$ wide, to better describe the rapidly changing amount of dead material.

To determine the effective amount of dead material we use $Z \rightarrow e e$ events with CC-EC topology. The electrons must pass the following selection criteria:

- $p_{T}>25 \mathrm{GeV}$,

- EM fraction >0.9,

- Isolation $<0.15$,

- HMatrix7 $<12$ (central electrons) or HMatrix8 $<20$ (forward electrons),

- $|\eta|<1.05$ (central electrons) of $1.5<|\eta|<2.5$ (forward electrons),

- Spatial track match with $P\left(\chi_{\mathrm{TM}}\right)>0.01, p_{T}^{\text {track }}>10 \mathrm{GeV}$ and at least $1 \mathrm{SMT}$ hit.

The events must pass the following selection criteria: 
- At least 2 good electrons, out of which the two hardest are selected as candidates,

- One central and one forward electron,

- Invariant mass $70<m_{e e}<110 \mathrm{GeV}$.

Those are the same criteria used in the selection for the $W$ mass measurement (see Sec. 4.6), but without a hadronic recoil $u_{T}$ cut, since the EM layer energy fractions (EMLF) are sufficiently insensitive to this variable and we gain a considerable number of events. The raw energy of the electron is defined as the sum of the uncorrected ${ }^{2}$ energies deposited inside the EM cluster defined in the four electromagnetic layers plus the first hadronic layer.

We measure the energy fraction deposited in each layer in both data and full MC in bins of $\eta_{\text {det }}$. Figure 13.5 shows the ratio between the mean of the two distributions with an uniform binning of $\Delta \eta=0.1$.
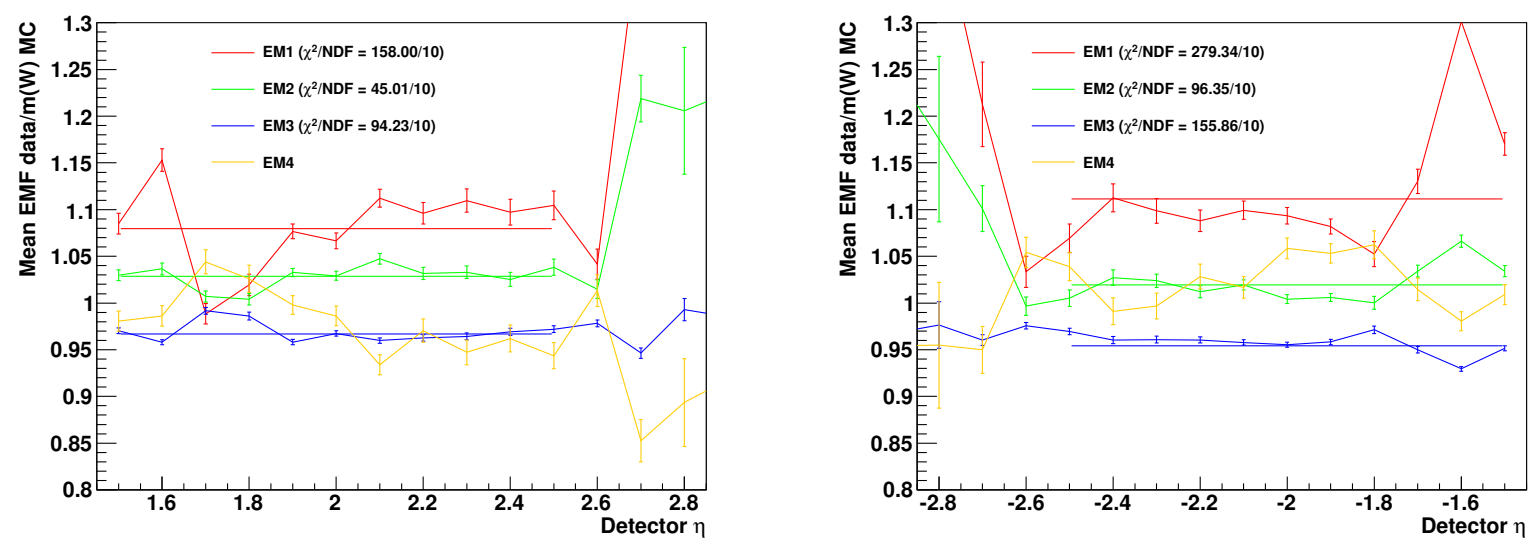

Figure 13.5: Ratio of the mean of the EM energy fraction distribution for data and full MC. The full MC is simulated with the settings described in Sec. 13.2. The average value and $\chi^{2}$ around the mean are calculated in the range $1.5<\eta_{\text {det }}<2.5$.

The energy fraction in EM1 has large derivatives showing the inadequacy of the D0 material model. Two regions are particularly problematic: $1.5<\left|\eta_{\text {det }}\right|<1.6$, in the transition region between the solenoid and FPS, and $\left|\eta_{\text {det }}\right|>2.5$, after the end of the FPS absorber where the face size of the cell becomes small and the method presented here becomes impractical.

After increasing the segmentation of the polycone that models the cryostat wall, there are 20 points in the model of the internal cryostat wall, out of which 10 are in the region $1.5<\left|\eta_{\text {det }}\right|<2.5$. These points that define the cryostat wall can be moved to change the amount of dead material upstream of each $\eta_{\text {det }}$ bin, adding or removing stainless steel in

\footnotetext{
${ }^{2}$ By uncorrected, or raw, we refer to the the energy read out without layer weight corrections and the $\eta$ absolute calibration, but with otherwise other previously applied corrections, such as the $\phi$ inter-calibration.
} 
the dead material budget. In order to study the dependence of the EM energy fractions with respect to the added material, three $a d$ hoc modifications were prepared and used to simulate $750 \mathrm{k} Z \rightarrow$ ee events with CC-EC topology $\mathrm{b}^{3}$. The modified geometries are prepared by thicknening the internal cryostat wall but keeping its profile as described in the standard D0 simulation. Table C.2 in App. C.1 describes these modifications. The events are generated with RESBOS[65, 16, 17] and overlaid with data unsuppressed zerobias events.

Using these modified geometries, together with the nominal D0 geometry model, we build parametrized models for the variations of the EM energy fractions as a function of the variation of the thickness of the internal cryostat wall. The parametrizations are built independently for each layer and for each $\eta_{\text {det }}$ bin. Appendix C.2 has the complete set of parametrizations used for both north and south End Calorimeters. From these parametrizations the data/fullMC EMLF ratio can be calculated for any variation of the dead material. For each End Calorimeter separately, we let the $z$ position of each of the 10 points in the region $1.5<\eta_{\text {det }}<2.5$ vary to minimize the test statistic:

$$
\chi^{2}=\sum_{i=\mathrm{EM} 1, \mathrm{EM} 2, \mathrm{EM} 3} \sum_{j=1}^{12} \frac{\left(x_{i j}^{2}-\bar{x}_{i}\right)^{2}}{\sigma_{i j}^{2}},
$$

where $x_{i j}$ is the data / fullMC EMLF ratio for a given layer $i$ and for a given $\eta_{\operatorname{det}}$ bin $j$ out of the 12 bins we use to gauge the shower development, $\sigma_{i j}$ is the uncertainty in this quantity propagated through the parametrized model described above and $\bar{x}_{i}$ is the average ratio for a given layer $i$ in the range $1.5<\eta_{\text {det }}<2.5$. The minimization is achieved with a $\chi^{2} / \mathrm{NDF}$ of 26/20 for the north End Calorimeter and 34/20 for the south. The result can be seen in Fig. 13.6 and table 13.3 .

The test statistic chosen can only equalize the $\eta_{\text {det }}$ differences in the mean energy fraction, but does not attempt to bring the ratio to one. There are two possible sources for the overall mismatch in the energy fractions. Either there is still an unaccounted for dead material with constant projective thickness or the layer weights chosen in the reconstruction and listed in table 13.2 are sub-optimal. The two choices are not equivalent in the sense that an unaccounted dead material is essentially non-linear in energy response while the reconstruction layer weights are, by definition, linear. The reason is most probably a combination of both. We choose to bring the energy fractions to 1 by changing the reconstruction weights. In the CC calibration, this was the choice made and the overall correction was small, on the order of 1 to $2 \%$. For the forward calorimeters, the correction can be as large as $8 \%$. If such large correction was all due to dead material, the non-linearities could have a large impact in the calibration.

In order to verify whether this large mismatch could arise from some yet unaccounted for dead material with constant projective thickness, we add disks with different thickness in front of the cryostats and use a test statistic with respect to unit $\sum_{j=1}^{12}\left(x_{i j}^{2}-1\right)^{2} / \sigma_{i j}^{2}$

\footnotetext{
${ }^{3}$ At the generator level, we assume $\eta=1.3$ as the boundary between the central and forward calorimeter. This is a good assumption given the wide space between the cryostat.
} 

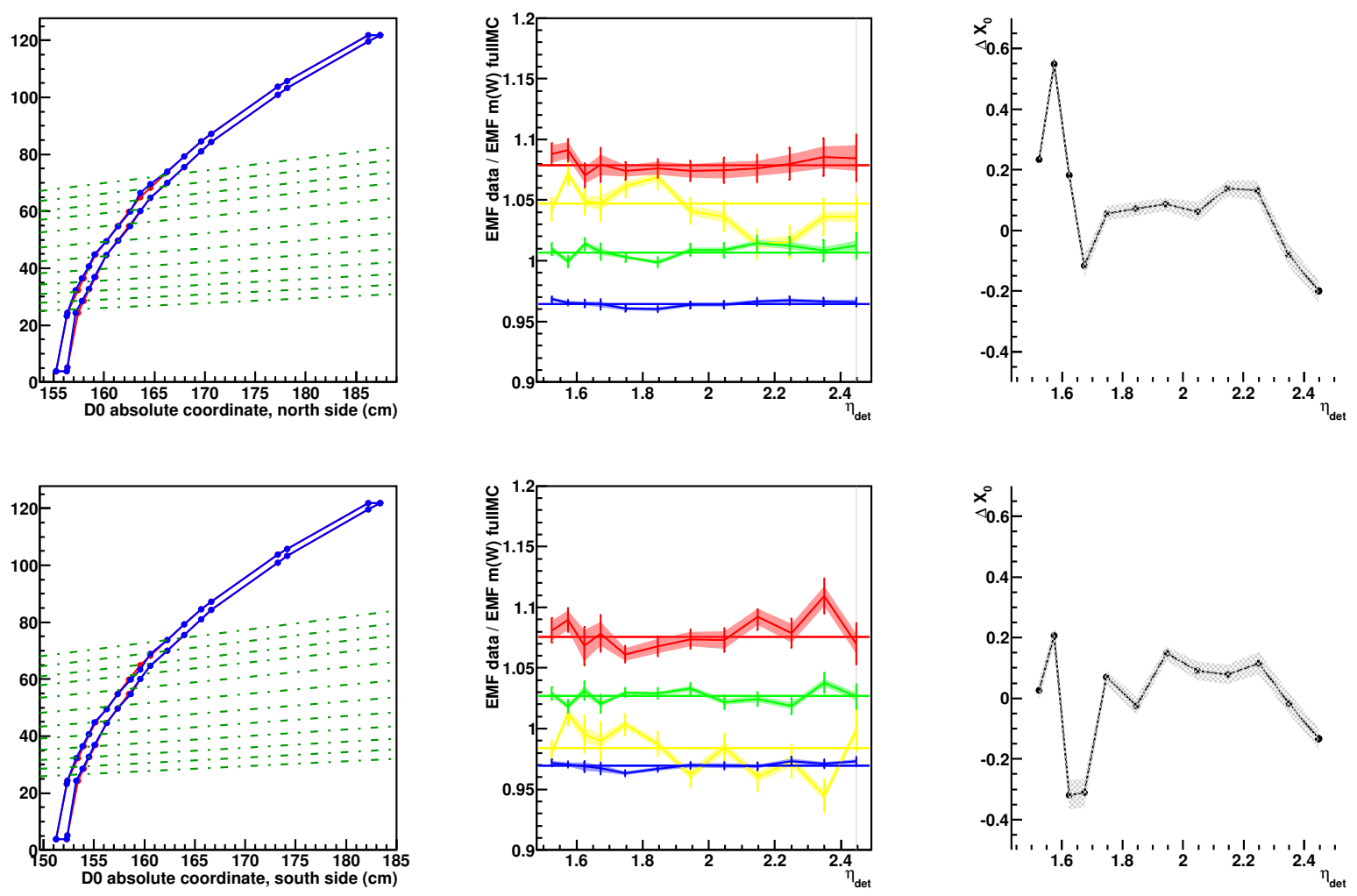

Figure 13.6: Best fit for north (top panels) and south (bottom panels) cryostats that minimize the test statistic 13.10. The dash-dotted green lines represent the average $\eta_{\text {det }}$ from $Z \rightarrow$ ee data events in each of the $12 \eta_{\text {det }}$ bins with boundaries $(1.5,1.55,1.6,1.65,1.7,1.8,1.9,2.0,2.1,2.2,2.3,2.4,2.5)$ used to calculate the value of $\chi^{2}$. The vertical and horizontal axis in the left panels are absolute coordinates of the volume in the D0 geometry model, ie, relative to the center of the tracking system. The central panels show the energy fraction distribution after the minimization for EM1 (red), EM2(green), EM3 (blue) and EM4 (yellow) layers. The right panels show the amount of dead material (in radiation lengths of stainless steel) added or subtracted for each $\eta_{\text {det }}$ bin. The shaded areas depict the uncertainty due to the dead material, whose procedure is explained in 13.4.1. 


\begin{tabular}{|c||c|c|c||c|c|c|}
\hline \hline \multicolumn{1}{|c||}{ Polycone } & \multicolumn{3}{c||}{ North Cryostat } & \multicolumn{3}{c|}{ South Cryostat } \\
point & $z(\mathrm{~cm})$ & $r_{\min }(\mathrm{cm})$ & $r_{\max }(\mathrm{cm})$ & $z(\mathrm{~cm})$ & $r_{\min }(\mathrm{cm})$ & $r_{\max }(\mathrm{cm})$ \\
\hline 1 & 150.6116 & 3.8100 & 3.8100 & 150.6116 & 3.8100 & 3.8100 \\
\hline 2 & 151.6801 & 3.8100 & 23.0944 & 151.6801 & 3.8100 & 23.0944 \\
\hline 3 & 151.7512 & 5.0948 & 24.3792 & 151.7512 & 5.0948 & 24.3792 \\
\hline 4 & 152.5737 & 24.2772 & 32.1395 & 152.6041 & 24.2772 & 32.1395 \\
\hline 5 & 153.1900 & 28.4912 & 36.3535 & 153.2303 & 28.4912 & 36.3535 \\
\hline 6 & 153.8942 & 32.7051 & 40.5675 & 153.8724 & 32.7051 & 40.5675 \\
\hline 7 & 154.4618 & 36.9191 & 44.7815 & 154.4577 & 36.9191 & 44.7815 \\
\hline 8 & 155.5975 & 44.5941 & 49.5521 & 155.6585 & 44.5941 & 49.5521 \\
\hline 9 & 156.7831 & 49.7074 & 54.6654 & 156.7141 & 49.7074 & 54.6654 \\
\hline 10 & 157.9878 & 54.8206 & 59.7787 & 158.0453 & 54.8206 & 59.7787 \\
\hline 11 & 158.9762 & 59.9339 & 66.4551 & 158.9762 & 59.9339 & 63.3519 \\
\hline 12 & 160.0087 & 64.6203 & 69.5465 & 160.0087 & 64.6203 & 68.8328 \\
\hline 13 & 161.6774 & 70.0607 & 73.9335 & 161.6774 & 70.0607 & 73.7529 \\
\hline 14 & 163.3461 & 75.5011 & 79.1390 & 163.3461 & 75.5011 & 79.1390 \\
\hline 15 & 165.0148 & 80.9415 & 84.5794 & 165.0148 & 80.9415 & 84.5794 \\
\hline 16 & 166.0221 & 84.2254 & 87.1132 & 166.0221 & 84.2254 & 87.1132 \\
\hline 17 & 172.6220 & 100.8277 & 103.7156 & 172.6220 & 100.8277 & 103.7156 \\
\hline 18 & 173.5974 & 103.2814 & 105.6887 & 173.5974 & 103.2814 & 105.6887 \\
\hline 19 & 181.6217 & 119.5128 & 121.9200 & 181.6217 & 119.5128 & 121.9200 \\
\hline 20 & 182.8117 & 121.9200 & 121.9200 & 182.8117 & 121.9200 & 121.9200 \\
\hline
\end{tabular}

Table 13.3: Best modification of the internal cryostat wall (CC+1 volume in dogstar). These modifications are implemented in geometry p20_ECdevelB6 in package d0AltGeom. The coordinates are given with respect to the center of the calorimeter coordinate system and additional shifts are necessary to translate them to the center of the tracking system which is used as reference for the D0 global coordinate system. The additional shifts used in this study are those present in the D0 GEANT geomtry model. 
to determine the amount of extra missing material. Figure 13.7 shows the behavior of the test static for each layer. It is clear that any attempt to bring the energy fractions ratio to 1 is bound to fail. Hence, we repeat the same strategy as done in the calibration of the CC and determine corrections to the layer weights, which are summarized in table 13.4 .
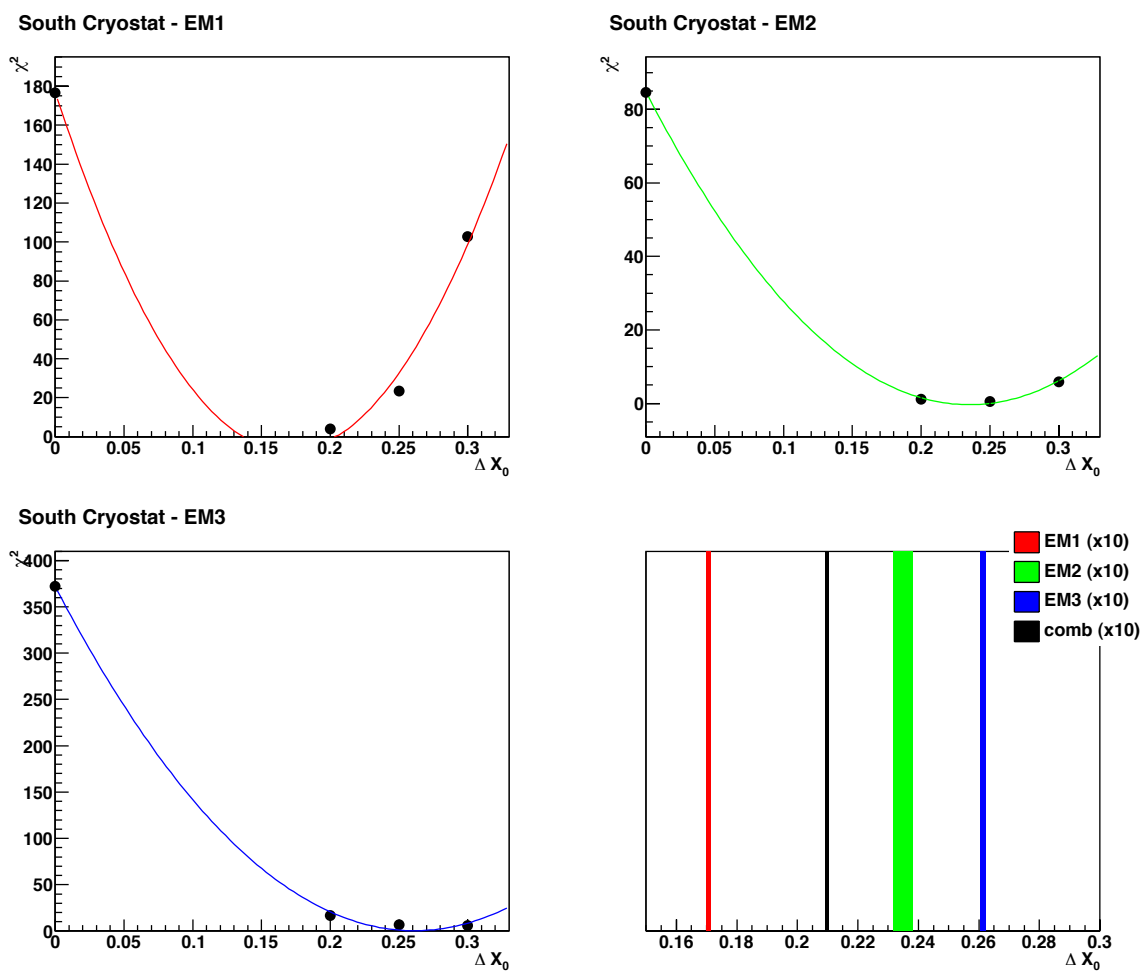

Figure 13.7: EM energy fractions for layers EM1, EM2 and EM3 after adding a disk with constant projective thickness in front of the south calorimeter. The values $\chi^{2}$ in the vertical axis are with respect to unity. The bottom right pad shows a summary of how much extra material would be needed to bring the EMLF ratio to one for each layer. In this panel, the uncertainties have been multiplied by 10 , for easier visualization. Similar results are observed for the north calorimeter.

\subsubsection{Uncertainties}

In order to determine the best correction to D0 geometry model, we allowed 10 points to float simultaneously. The 10-dimensional parameter space makes the study of systematic uncertainties more complicated than in the equivalent calibration by this method done for the CC. To properly study and propagate the uncertainty, we diagonalize the two 10dimensional covariance matrices and create 20 geometries corresponding to a variation 


\begin{tabular}{|c|c|c|}
\hline \hline Layer & ECN & ECS \\
\hline EM1 & $0.9272 \pm 0.0025$ & $0.9296 \pm 0.0026$ \\
EM2 & $0.9933 \pm 0.0016$ & $0.9737 \pm 0.0016$ \\
EM3 & $1.0368 \pm 0.0009$ & $1.0316 \pm 0.0010$ \\
EM4 & $0.9549 \pm 0.0030$ & $1.0165 \pm 0.0034$ \\
FH1 & $0.5580 \pm 0.0067$ & $0.5780 \pm 0.0070$ \\
\hline \hline
\end{tabular}

Table 13.4: Multiplicative correction to the reconstruction default layer weights. They are determined as the inverse of the mean energy fraction across the region $1.5<\eta_{\operatorname{det}}<2.5$ after the improvement in the material model.

of $\pm 1 \sigma$, where $\sigma$ is the square root of the corresponding eigenvalue, in the direction of each eigenvector. Those variations are independent and can be used to propagate the uncertainty in the material determination to any measurement that uses this calibration, with the following prescription:

$$
\sigma_{f}=\frac{1}{2} \sqrt{\sum_{i=1}^{10}\left(f_{2 i-1}-f_{2 i}\right)^{2}} .
$$

The shaded areas in Fig. 13.6 are determined using equation 13.11. Appendix C.3 has the complete set of geometry eigensets.

\subsubsection{Closure Test of the Parametrized Model}

The correction to the D0 material model was determined by using the parametrized model built from the three ad hoc geometries. Although the agreement between the parametrized model and each geometry variation is very good, it only describes the average response in a discrete set of 12 points. To validate the model, we perform a full simulation with the modifications described in table 13.3 and compare it to the prediction from the parametrized model. The results can be seen in Figs. 13.8 and 13.9.

The origin of the large disagreement in the region $1.55<\left|\eta_{\text {det }}\right|<1.6$ for both north and south End Calorimeters is tantalizing and should be object of further investigation.

\subsubsection{Consistency Checks}

As discussed above, the standard D0 geometry model does not attempt to describe many existing elements in the forward region. In particular, the tracking cables and the calorimeter cryogenic services are absent. These volumes create an $\eta$ and $\phi$ dependence in the energy loss. The $\eta$ dependence is properly described in this calibration since it is done in bins of $\eta_{\text {det }}$. The $\phi$ dependence, on the other hand, is averaged out. The differences in the $\phi$ can be seen by doing the fitting to the dead material in separate azimuthal regions. We 

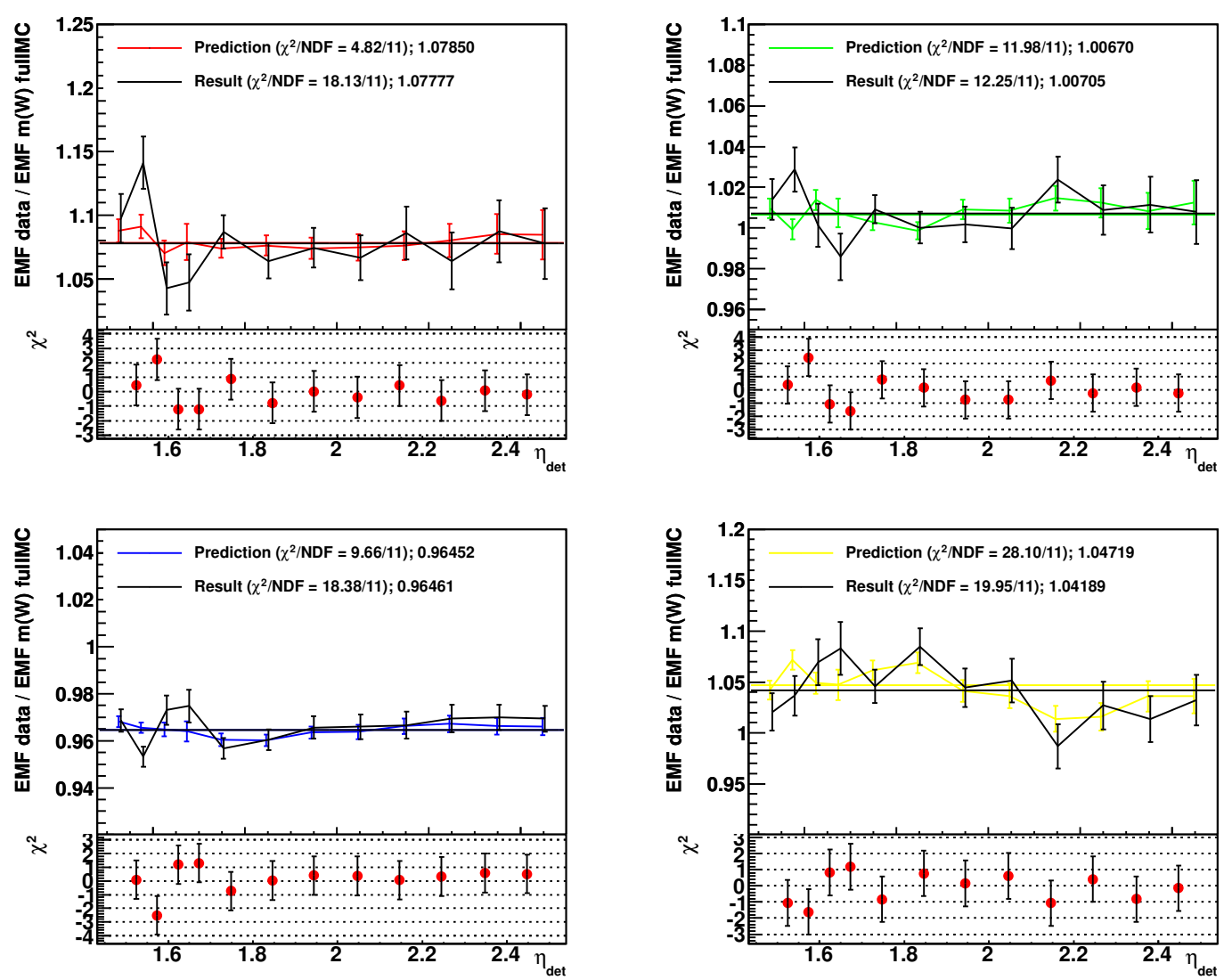

Figure 13.8: Comparison between the full dogstar simulation and the parametrized model prediction in the south EC for EM1 (top left), EM2 (top right), EM3 (bottom left) and EM4 (bottom right) layers. Both simulations are done with the same 750k REsBos CC-EC events at the generator level.

separate each EC in four quadrants $[0, \pi / 2],[\pi / 2, \pi],[\pi, 3 \pi / 2],[3 \pi / 2,2 \pi]$ and repeat the same procedure described above. The result can be seen in Fig. 13.10.

Another source of concern in this calibration is the luminosity dependence. The equivalent calibration for the $\mathrm{CC}$ did not take the dependence of the gains with the instantaneous into account, leaving for the fast $\mathrm{MC}$ to model this uncalibrated dependence. Here, we use a simulation which contains a first principle model for this dependence [95]. However, the parameters of the model were determined using CC data. In principle, there is no fundamental problem with this procedure, since the parameters in the model are measurements of the relationship between the average energy deposited in zero-bias events with the current drawn from the high-voltage sources, which has limited sensitivity on the position of the cell (see Sec. 2.4.5). In practice, the CC has large anomalous currents which have to be subtracted, while this currents are not present in the ECs. We could take advantage of this to determine the luminosity dependence more precisely, but the current 

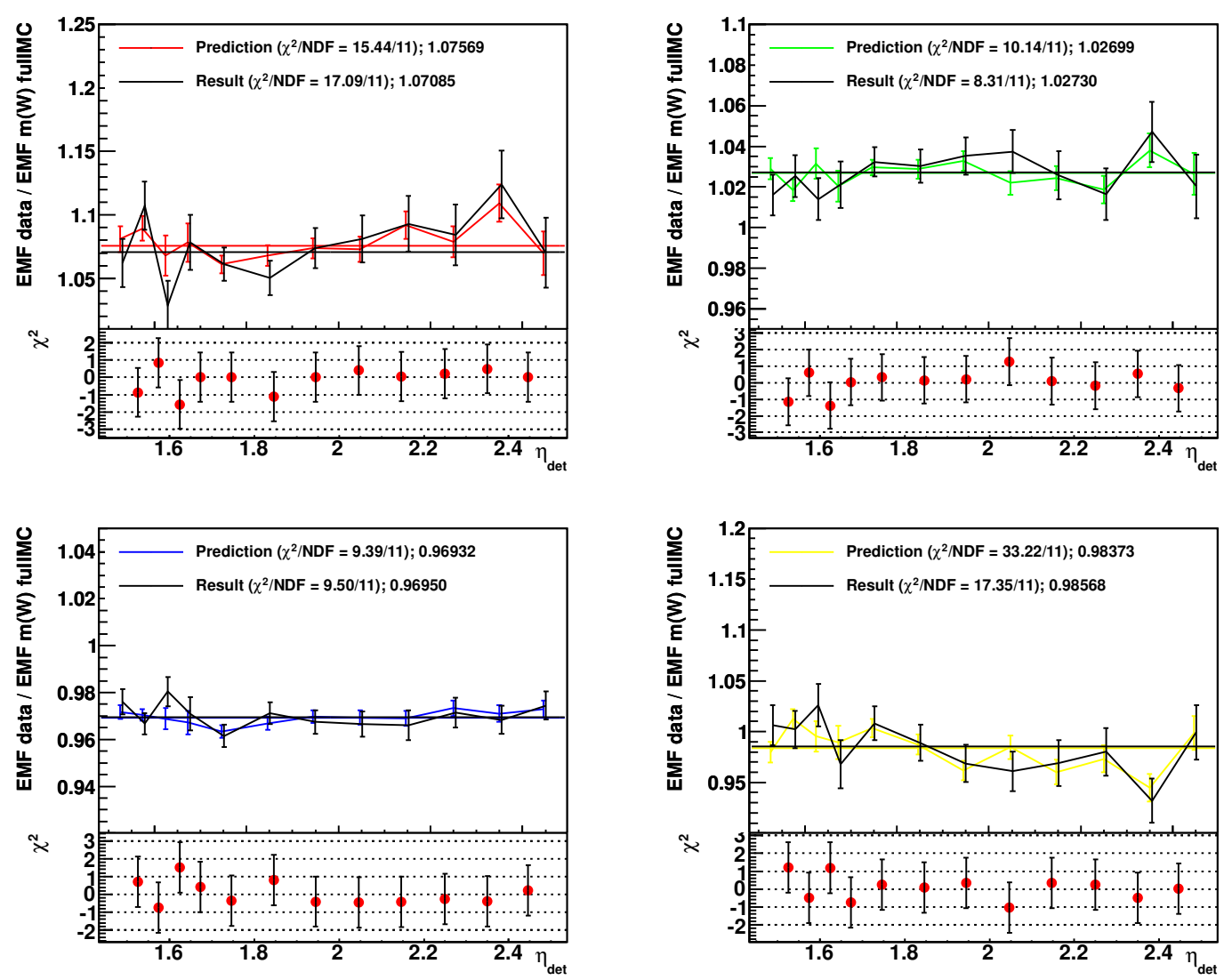

Figure 13.9: Comparison between the full dogstar simulation and the parametrized model prediction in the south EC for EM1 (top left), EM2 (top right), EM3 (bottom left) and EM4 (bottom right) layers. Both simulations are done with the same 750k REsBOS CC-EC events at the generator level.

model is sufficient for our purposes here. We divide our $Z \rightarrow$ ee sample in four bins of instantaneous luminosity per tick with boundaries $2 \times 10^{30} \mathrm{~cm}^{-2} \mathrm{~s}^{-1}, 4 \times 10^{30} \mathrm{~cm}^{-2} \mathrm{~s}^{-1}$, and $6 \times 10^{30} \mathrm{~cm}^{-2} \mathrm{~s}^{-1}$ and measure the correction to the layer weights, which are directly related to the gains, for each sub-sample. Figure 13.11 shows satisfactory agreement among the four luminosity regimes.

\subsection{The Energy Loss Correction Function}

We use the improved material model to re-derive the energy loss correction functions. For a given electron true energy, we determine the average reconstructed energy in bins of $\eta_{\text {det }}$. We use the same binning as the one used for the dead material correction. Using the inverse of this relationship, we determine, for a given average reconstructed energy, a 

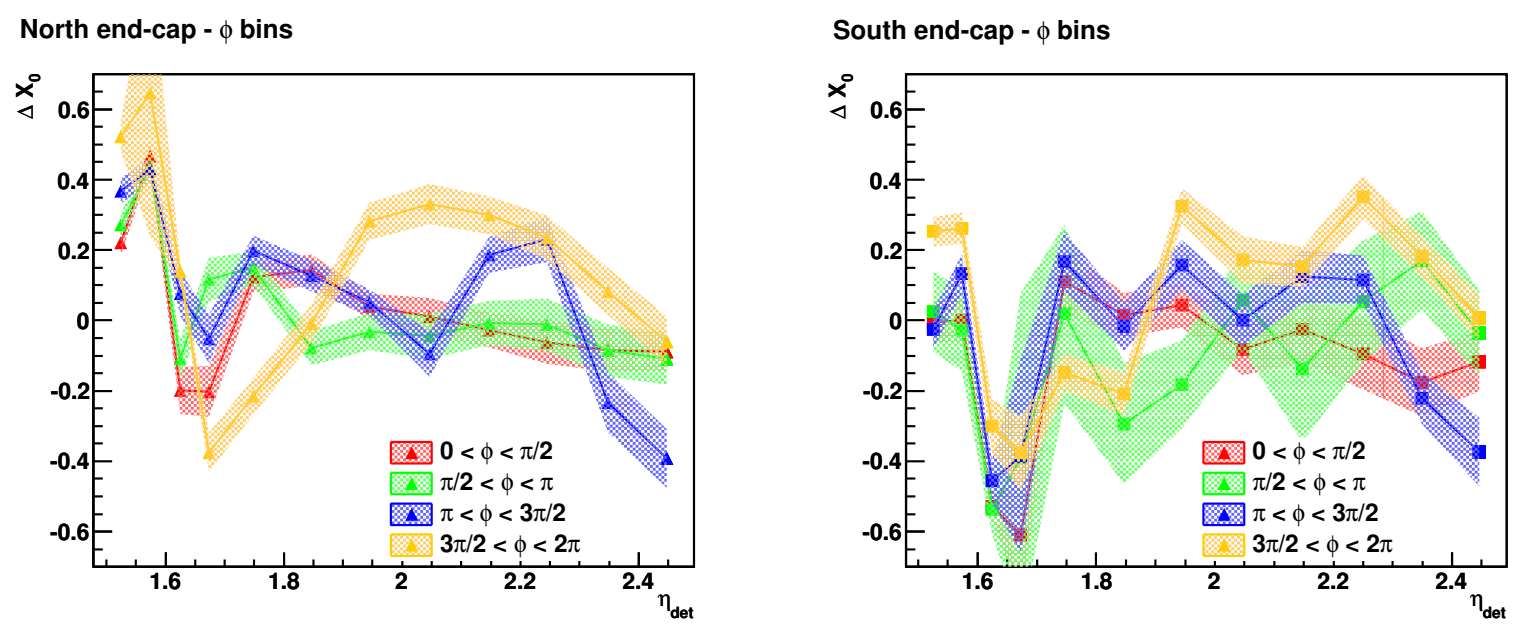

Figure 13.10: Determination of the correction to the material model in different $\phi$ bins for the north (left) and south (right) End Calorimeters.
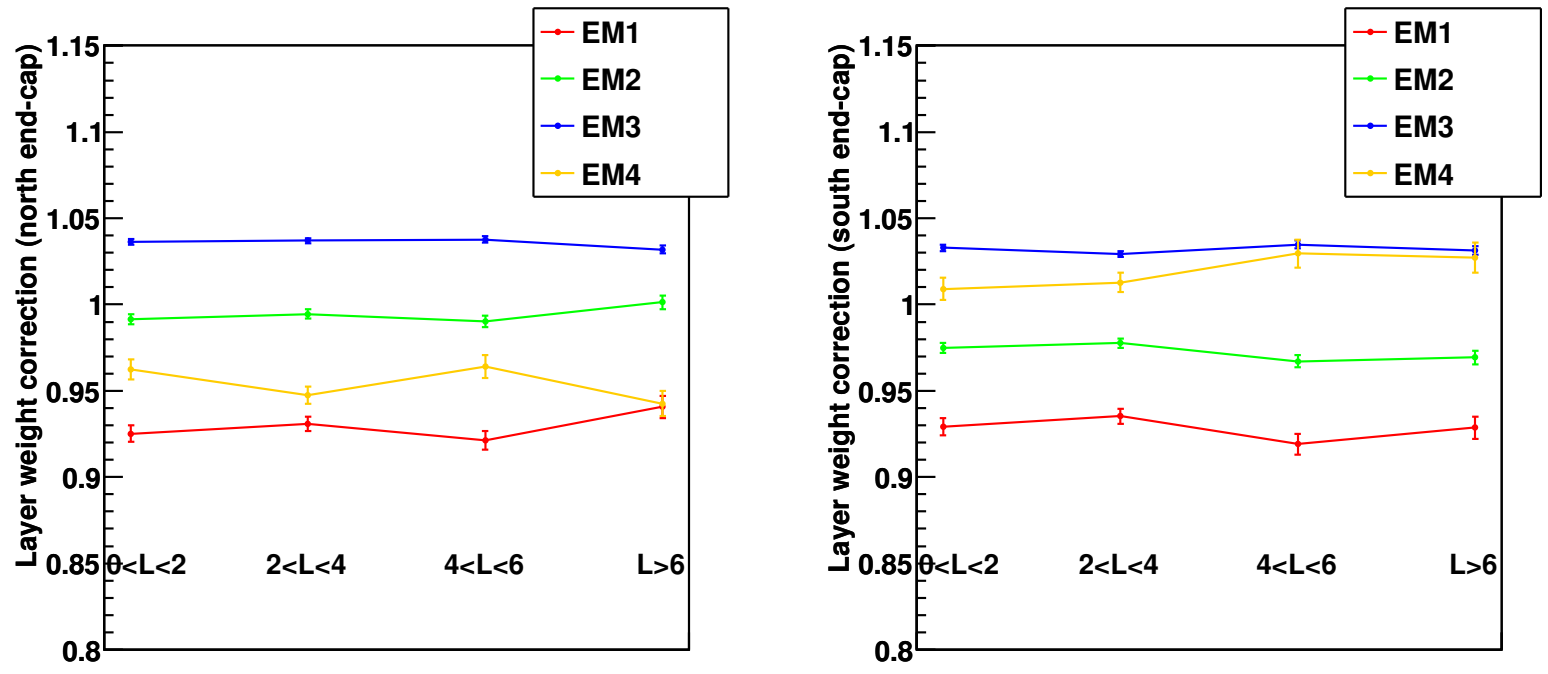

Figure 13.11: Dependence of the layer weights correction with the instantaneous luminosity. The consistency among luminosity bins shows that the model for the luminosity dependence of the gains in d0sim works sufficiently well for this calibration. 
correction factor to recover, on average, the original energy. Since this is a complete simulation of the electron cluster reconstruction, this function not only recovers the energy lost in the dead material but also the energy leaked outside the reconstruction cluster.

Using the same settings for GEANT as described above for the dead material studies, we simulate single monochromatic electrons with energies 10, 15, 20, 35, 45, 55, 65, 75, 85, $95,105,135,165,195,225,285,315,345,375 \mathrm{GeV}$. These samples have a flat distribution in $\eta$ in the region $[-4.5,-1.1] \cup[1.1,4.5]$. We generate 200k events for each of the 20 bins in true energy. The generation is done with the nominal geometry, as well as the ad hoc modifications ECdevelB1, ECdevelB2, and ECdevelB3. Electrons are reconstructed with the same selection criteria as described in Sec. 13.4. except for the electron $p_{T}$ cut.

For each bin in $\eta_{\text {det }}$ we then fit the pair of points $\left(\bar{E}_{\text {reco }}, E_{\text {true }}\right)$ to a third order polynomial in $\log (E)$ in the range $[25,400]$. Appendix C.3 has the result of this fitting for each of the four geometries.

Using the set of energy loss functions derived for the four different geometries, we build parametrized functions for the function coefficients as a function of added material in the internal cryostat wall. Then, we use the result of the material tune to write corresponding energy loss functions for the best fit and corresponding uncertainties eigensets. The results are summarized in table 13.5 and the plots with associated uncertainties in section C.3.

\subsection{Absolute Calibration with Improved Dead Material Model}

With the improved energy loss determination, we can recalibrate the calorimeter using the well known value of the $Z$ boson mass as an absolute reference. We select events using the same criteria as already described but not limited to CC-EC topology $y^{5}$,

As the signal model we use a Voigtian $\sqrt{6}$ with the width of the Breit-Wigner component fixed to the $Z$ boson natural width, but otherwise unconstrained. The complete model is this Voigtian with a constant added as a rudimentary background model. We fit the raw invariant mass distribution to determine the signal fraction. The target distribution function is then defined as the same model but with Voigtian mean fixed at the $Z$ boson mass, the signal normalization as measured with the raw energies, the $Z$ boson natural width as the Breit-Wigner width and gaussian resolution fixed at $2.1 \mathrm{GeV}$, which is undoubtedly better than the resolution of the D0 calorimeter 7

\footnotetext{
${ }^{4}$ The points with true energy 10 and $15 \mathrm{GeV}$ are not well described by such a function. However, since the electron selection in the $W$ boson mass analysis has an energy cut at $58 \mathrm{GeV}$ (corresponding to a $p_{T}$ cut of $25 \mathrm{GeV}$ at $\eta=1.5$ ), whether this energy loss function describes any energy below the $55 \mathrm{GeV}$ is purely academic.

${ }^{5}$ Since there was a long shutdown between Run Ilb1 (D0 runs 221697 to 234914) and Run Ilb2 (D0 runs 237341 to 252919$)$, we calibrate them separately.

${ }^{6} \mathrm{~A}$ Voigtian distribution $V\left(x, \mu, \sigma, \sigma^{\prime}\right)$ is defined as the convolution of a Breit-Wigner distribution $L(x, \mu, \sigma)$ with a Gaussian distribution $\mathcal{N}\left(x, 0, \sigma^{\prime}\right)$, ie., $V\left(x, \mu, \sigma, \sigma^{\prime}\right)=\int_{-\infty}^{\infty} \mathcal{N}\left(x^{\prime}, 0, \sigma^{\prime}\right) \times L\left(x-x^{\prime}, \mu, \sigma\right) d x^{\prime}$.

${ }^{7}$ Historically, $2.1 \mathrm{GeV}$ was the average EM resolution predicted by the Run IIa full MC.
} 


\begin{tabular}{|c|c|c|c|c|}
\hline \multicolumn{5}{|c|}{ North End Calorimeter } \\
\hline$\eta_{\text {det }}$ range & $p_{0}$ & $p_{1}$ & $p_{2}$ & $p_{3}$ \\
\hline $1.5<\eta_{\operatorname{det}}<1.55$ & 1.2644 & $-1.3841 \times 10^{-1}$ & $2.2171 \times 10^{-2}$ & $-1.1885 \times 10^{-3}$ \\
\hline $1.55<\eta_{\operatorname{det}}<1.6$ & 1.2783 & $-1.6511 \times 10^{-1}$ & $2.9123 \times 10^{-2}$ & $-1.7032 \times 10^{-3}$ \\
\hline $1.6<\eta_{\operatorname{det}}<1.65$ & 1.3373 & $-1.7874 \times 10^{-1}$ & $2.8675 \times 10^{-2}$ & $-1.5360 \times 10^{-3}$ \\
\hline $1.65<\eta_{\text {det }}<1.7$ & 1.3427 & $-1.6268 \times 10^{-1}$ & $2.3587 \times 10^{-2}$ & $-1.1415 \times 10^{-3}$ \\
\hline $1.7<\eta_{\mathrm{det}}<1.8$ & 1.4545 & $-2.1502 \times 10^{-1}$ & $3.1567 \times 10^{-2}$ & $-1.5468 \times 10^{-3}$ \\
\hline $1.8<\eta_{\mathrm{det}}<1.9$ & 1.4786 & $-2.2562 \times 10^{-1}$ & $3.3299 \times 10^{-2}$ & $-1.6580 \times 10^{-3}$ \\
\hline $1.9<\eta_{\mathrm{det}}<2.0$ & 1.5039 & $-2.2900 \times 10^{-1}$ & $3.2796 \times 10^{-2}$ & $-1.5871 \times 10^{-3}$ \\
\hline $2.0<\eta_{\mathrm{det}}<2.1$ & 1.5878 & $-2.7190 \times 10^{-1}$ & $4.0822 \times 10^{-2}$ & $-2.1170 \times 10^{-3}$ \\
\hline $2.1<\eta_{\mathrm{det}}<2.2$ & 1.5691 & $-2.4587 \times 10^{-1}$ & $3.4144 \times 10^{-2}$ & $-1.6254 \times 10^{-3}$ \\
\hline $2.2<\eta_{\mathrm{det}}<2.3$ & 1.4656 & $-1.8692 \times 10^{-1}$ & $2.3093 \times 10^{-2}$ & $-9.4124 \times 10^{-4}$ \\
\hline $2.3<\eta_{\mathrm{det}}<2.4$ & 1.4533 & $-1.8603 \times 10^{-1}$ & $2.3261 \times 10^{-2}$ & $-9.4606 \times 10^{-4}$ \\
\hline $2.4<\eta_{\mathrm{det}}<2.5$ & 1.2713 & $-1.0104 \times 10^{-1}$ & $1.0855 \times 10^{-2}$ & $-3.7913 \times 10^{-4}$ \\
\hline \multicolumn{5}{|c|}{ South End Calorimeter } \\
\hline 1.55 & 1.3461 & $-1.7657 \times 10^{-1}$ & $2.8730 \times 10^{-2}$ & -1.591 \\
\hline $1.55<\eta_{\operatorname{det}}<1.6$ & 1.3026 & $-1.7255 \times 10^{-1}$ & $2.9708 \times 10^{-2}$ & $-1.7020 \times 10^{-3}$ \\
\hline $1.6<\eta_{\operatorname{det}}<1.65$ & 1.5767 & $-3.2314 \times 10^{-1}$ & $5.7726 \times 10^{-2}$ & $-3.4777 \times 10^{-3}$ \\
\hline $1.65<\eta_{\operatorname{det}}<1.7$ & 1.4501 & $-2.1237 \times 10^{-1}$ & $3.1638 \times 10^{-2}$ & $-1.5979 \times 10^{-3}$ \\
\hline $1.7<\eta_{\mathrm{det}}<1.8$ & 1.5450 & $-2.5851 \times 10^{-1}$ & $3.9315 \times 10^{-2}$ & $-2.0408 \times 10^{-3}$ \\
\hline $1.8<\eta_{\mathrm{det}}<1.9$ & 1.5621 & $-2.6795 \times 10^{-1}$ & $4.0931 \times 10^{-2}$ & $-2.1372 \times 10^{-3}$ \\
\hline $1.9<\eta_{\mathrm{det}}<2.0$ & 1.6829 & $-3.2401 \times 10^{-1}$ & $5.0544 \times 10^{-2}$ & $-2.7119 \times 10^{-3}$ \\
\hline $2.0<\eta_{\text {det }}<2.1$ & 1.5398 & $-2.2784 \times 10^{-1}$ & $3.0358 \times 10^{-2}$ & $-1.3594 \times 10^{-3}$ \\
\hline $2.1<\eta_{\mathrm{det}}<2.2$ & 1.6297 & $-2.7347 \times 10^{-1}$ & $3.8814 \times 10^{-2}$ & $-1.9113 \times 10^{-3}$ \\
\hline $2.2<\eta_{\text {det }}<2.3$ & 1.5463 & $-2.2180 \times 10^{-1}$ & $2.8565 \times 10^{-2}$ & $-1.2466 \times 10^{-3}$ \\
\hline $2.3<\eta_{\mathrm{det}}<2.4$ & 1.6208 & $-2.7811 \times 10^{-1}$ & $4.0985 \times 10^{-2}$ & $-2.1095 \times 10^{-3}$ \\
\hline $2.4<\eta_{\mathrm{det}}<2.5$ & 1.3060 & $-1.1894 \times 10^{-1}$ & $1.4423 \times 10^{-2}$ & $-6.2950 \times 10^{-4}$ \\
\hline
\end{tabular}

Table 13.5: Energy loss functions using the best fit for the material tune. The function used is $f_{\mathrm{EL}}\left(E_{\text {reco }}\right)=p_{0}+p_{1} \log \left(E_{\text {reco }}\right)+p_{2} \log ^{2}\left(E_{\text {reco }}\right)+p_{3} \log ^{3}\left(E_{\text {reco }}\right)$ with $E_{\text {reco }}$ in GeV. The fit uncertainties are negligible compared to the propagated uncertainties from the dead material tune. 
We allow one calibration constant per i $\eta$ ring 8 . However, since our event selection is limited to $\left|\eta_{\text {det }}\right|<1.05$ and $1.5<\left|\eta_{\text {det }}\right|<2.5$ we fix to unity the calibration constants of $|i \eta|$ rings $12,13,14,15$, as well as all $|i \eta|$ rings larger than 25 . It is true that these rings will contribute to the sampling of the showers close to the acceptance boundary, but these events do not provide enough statistics to determine calibration constants on its own.

We repeat the fit varying the energy loss functions in the error set as well as varying the layer weights corrections by their corresponding uncertainties. Figures 13.12 and 13.13 show the results of the minimization. The minimization uses the energy loss functions for central electrons derived for the $W$ mass measurement with central electrons using 1 $\mathrm{fb}^{-1}$ (see Sec. 5). The calibration constants for $|i \eta|<11$ are statistically consistent with the values obtained with CC-CC events only that were used for the $W$ mass measurement with central electrons using $4.3 \mathrm{fb}^{-1}$. It is equally true and important that the calibrations constants for $16 \leq|i \eta| \leq 25$ are also consistent with the result we would obtain had we selected only events with EC-EC topology. Tables 13.6 and 13.7 list the calibration

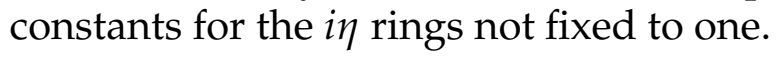

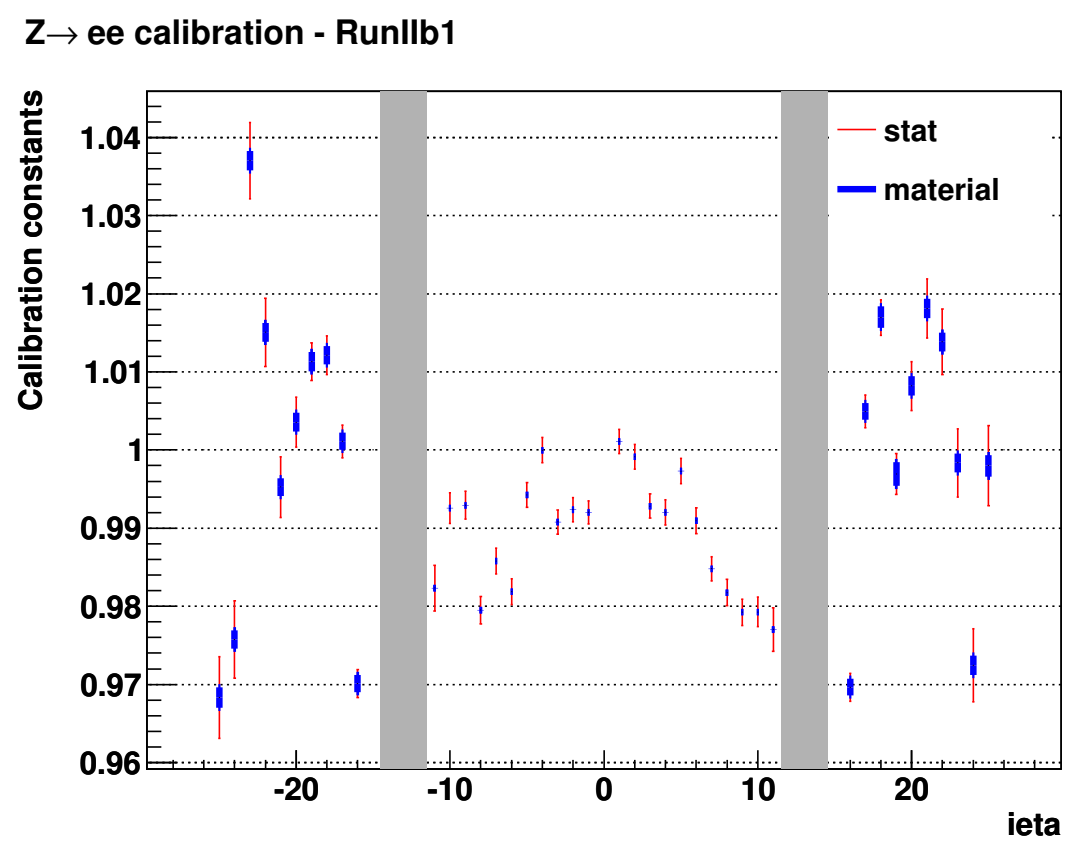

Figure 13.12: Absolute calibration for Run IIb1. The red thin error bars are statistical and the this blue errors bars are from the propagation of the material uncertainty through the energy loss function and through the layer weights corrections.

\footnotetext{
${ }^{8} i \eta$ and $i \phi$ are integers that, together with the layer, determine the position of a cell in the calorimeter. $i \eta$ is defined in Fig. 2.8. i $\phi$ is determined by dividing the transverse plane in 64 equal wedges starting from $\phi=0$.
} 


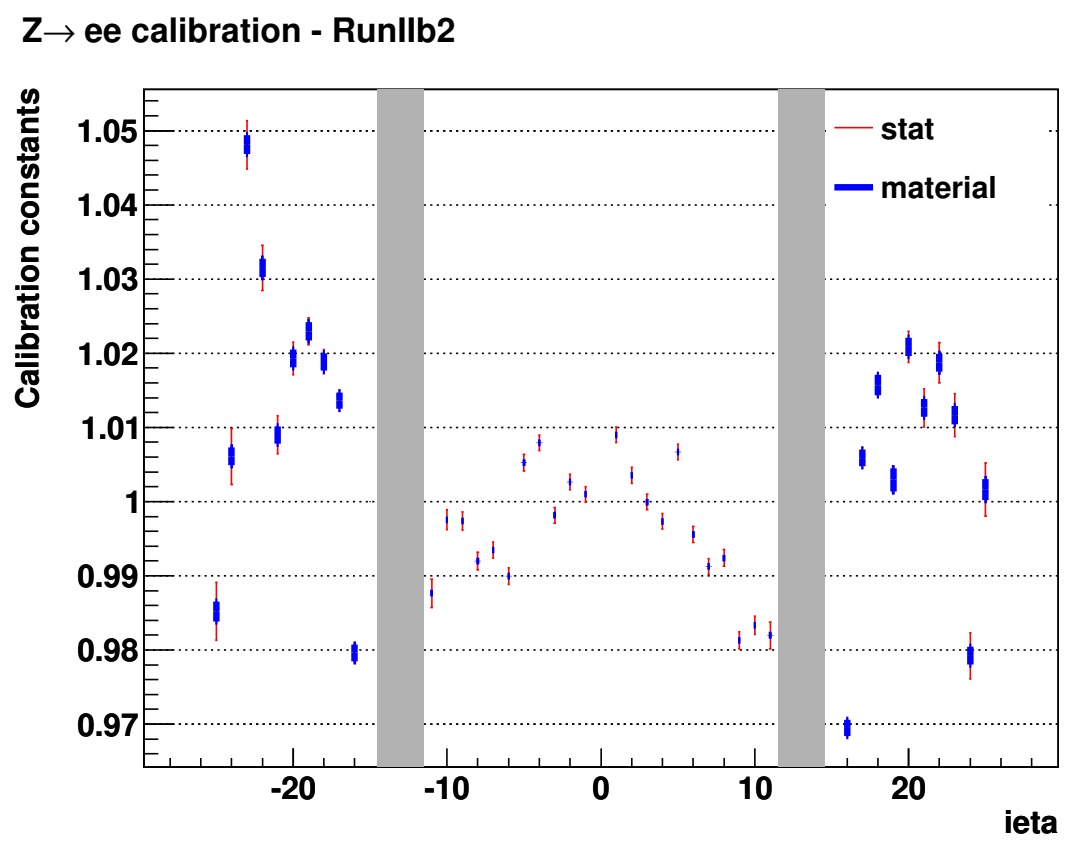

Figure 13.13: Absolute calibration for Run IIb2. The red thin error bars are statistical and the this blue errors bars are from the propagation of the material uncertainty through the energy loss function and through the layer weights corrections.

After setting the calibration constants to their best values, we redo the fit using the same model as initially chosen to determine the signal fraction. The invariant mass distribution for Run IIb1 and Run IIb2 epochs can be found in Fig. 13.14. The returned value of the $Z$ boson from the fit is somewhat larger than the $Z$ boson mass world average. We interpret this as a shortcoming of the oversimplified model for the invariant mass distribution. A detailed description, as simulated with the $W$ mass fast MC, would be necessary to account for all details of the calibration, as described in Sec.7.3.4. Moreover, while it is true that with further effort we could bring the mean of the Voigtian to the $Z$ boson mass world average, we prefer to perform a consistent calibration with the CC.

\subsection{Systematic Uncertainties}

In the previous sections we used CC-EC $Z \rightarrow e e$ events to determine corrections to the D0 material model in the GEANT simulation. The uncertainty on this determination was specified with error sets for the north and south EC with 10 members each. For each member of this eigenset, we derived an energy loss correction with which we could propagate the uncertainty in the material determination to the absolute calibration constant of the calorimeter. We also determined the variation of the calibration constants from the uncertainty in the layer weight corrections, one for each of the five layers used to determine 


\begin{tabular}{|c|c|c|}
\hline \hline i ring & Run Ilb1 constants & Run Ilb2 constants \\
\hline-25 & $0.9683 \pm 0.0052 \pm 0.0013$ & $0.9852 \pm 0.0039 \pm 0.0013$ \\
-24 & $0.9758 \pm 0.0049 \pm 0.0012$ & $1.0061 \pm 0.0038 \pm 0.0012$ \\
-23 & $1.0370 \pm 0.0049 \pm 0.0013$ & $1.0481 \pm 0.0033 \pm 0.0013$ \\
-22 & $1.0151 \pm 0.0044 \pm 0.0012$ & $1.0315 \pm 0.0030 \pm 0.0012$ \\
-21 & $0.9953 \pm 0.0039 \pm 0.0012$ & $1.0090 \pm 0.0026 \pm 0.0012$ \\
-20 & $1.0036 \pm 0.0032 \pm 0.0012$ & $1.0193 \pm 0.0022 \pm 0.0012$ \\
-19 & $1.0113 \pm 0.0024 \pm 0.0012$ & $1.0229 \pm 0.0018 \pm 0.0012$ \\
-18 & $1.0121 \pm 0.0025 \pm 0.0011$ & $1.0188 \pm 0.0016 \pm 0.0012$ \\
-17 & $1.0011 \pm 0.0021 \pm 0.0011$ & $1.0136 \pm 0.0015 \pm 0.0011$ \\
-16 & $0.9701 \pm 0.0018 \pm 0.0011$ & $0.9796 \pm 0.0012 \pm 0.0011$ \\
-11 & $0.9823 \pm 0.0029 \pm 0.0000$ & $0.9877 \pm 0.0019 \pm 0.0000$ \\
-10 & $0.9926 \pm 0.0020 \pm 0.0000$ & $0.9975 \pm 0.0013 \pm 0.0000$ \\
-9 & $0.9929 \pm 0.0018 \pm 0.0000$ & $0.9974 \pm 0.0012 \pm 0.0000$ \\
-8 & $0.9795 \pm 0.0018 \pm 0.0000$ & $0.9920 \pm 0.0012 \pm 0.0000$ \\
-7 & $0.9858 \pm 0.0016 \pm 0.0000$ & $0.9935 \pm 0.0011 \pm 0.0000$ \\
-6 & $0.9819 \pm 0.0017 \pm 0.0000$ & $0.9900 \pm 0.0011 \pm 0.0000$ \\
-5 & $0.9943 \pm 0.0016 \pm 0.0000$ & $1.0053 \pm 0.0011 \pm 0.0000$ \\
-4 & $1.0000 \pm 0.0016 \pm 0.0000$ & $1.0079 \pm 0.0011 \pm 0.0000$ \\
-3 & $0.9908 \pm 0.0015 \pm 0.0000$ & $0.9982 \pm 0.0011 \pm 0.0000$ \\
-2 & $0.9924 \pm 0.0016 \pm 0.0000$ & $1.0026 \pm 0.0011 \pm 0.0000$ \\
-1 & $0.9920 \pm 0.0015 \pm 0.0000$ & $1.0010 \pm 0.0011 \pm 0.0000$ \\
\hline \hline
\end{tabular}

Table 13.6: Calibration constants for the north in ring using the improved energy loss functions and layer weights corrections. The first uncertainty is statistical, the second due to the uncertainty on the material tune in the forward direction. The determination is done separately for Run IIb1 and Run IIb2.

the energy of an EM cluster. In total, we have an error set for the calibration constants with 15 members for each EC, 10 of which must be used consistently with the energy loss function that was used to derived it.

Using equation 13.11, we can propagate the uncertainty in the calibration to any measurement, in particular to the measurement of the $W$ boson mass with forward electrons. We consider two scenarios: a measurement of the $W$ mass with forward electrons only and a measurement that includes both central and forward electrons. It is strongly advisable to work with the latter, since events with $Z \rightarrow e e$ CC-EC topology can improve the determination of the absolute electron energy scale in the fast MC.

For the propagation, we use pseudo-experiments generated with RESBOS and simulated with the $W$ mass fast MC. Electrons are selected as described in section 13.4 and $W$ events must pass the following requirements, which reproduce the criteria used in the $W$ boson mass measurement. 


\begin{tabular}{|c|c|c|}
\hline \hline i ring & Run Ilb1 constants & Run Ilb2 constants \\
\hline 1 & $1.0011 \pm 0.0015 \pm 0.0000$ & $1.0090 \pm 0.0011 \pm 0.0000$ \\
2 & $0.9991 \pm 0.0016 \pm 0.0000$ & $1.0035 \pm 0.0011 \pm 0.0000$ \\
3 & $0.9928 \pm 0.0016 \pm 0.0000$ & $0.9999 \pm 0.0011 \pm 0.0000$ \\
4 & $0.9920 \pm 0.0016 \pm 0.0000$ & $0.9973 \pm 0.0011 \pm 0.0000$ \\
5 & $0.9973 \pm 0.0016 \pm 0.0000$ & $1.0067 \pm 0.0011 \pm 0.0000$ \\
6 & $0.9909 \pm 0.0017 \pm 0.0000$ & $0.9956 \pm 0.0011 \pm 0.0000$ \\
7 & $0.9848 \pm 0.0016 \pm 0.0000$ & $0.9913 \pm 0.0011 \pm 0.0000$ \\
8 & $0.9817 \pm 0.0017 \pm 0.0000$ & $0.9924 \pm 0.0011 \pm 0.0000$ \\
9 & $0.9792 \pm 0.0017 \pm 0.0000$ & $0.9813 \pm 0.0012 \pm 0.0000$ \\
10 & $0.9793 \pm 0.0019 \pm 0.0000$ & $0.9833 \pm 0.0012 \pm 0.0000$ \\
11 & $0.9770 \pm 0.0028 \pm 0.0000$ & $0.9820 \pm 0.0018 \pm 0.0000$ \\
16 & $0.9697 \pm 0.0018 \pm 0.0011$ & $0.9695 \pm 0.0012 \pm 0.0011$ \\
17 & $1.0049 \pm 0.0021 \pm 0.0011$ & $1.0059 \pm 0.0014 \pm 0.0011$ \\
18 & $1.0170 \pm 0.0023 \pm 0.0014$ & $1.0156 \pm 0.0016 \pm 0.0014$ \\
19 & $0.9969 \pm 0.0026 \pm 0.0015$ & $1.0030 \pm 0.0018 \pm 0.0015$ \\
20 & $1.0082 \pm 0.0031 \pm 0.0012$ & $1.0208 \pm 0.0021 \pm 0.0013$ \\
21 & $1.0181 \pm 0.0038 \pm 0.0012$ & $1.0126 \pm 0.0025 \pm 0.0012$ \\
22 & $1.0138 \pm 0.0042 \pm 0.0012$ & $1.0187 \pm 0.0027 \pm 0.0012$ \\
23 & $0.9983 \pm 0.0044 \pm 0.0012$ & $1.0116 \pm 0.0029 \pm 0.0012$ \\
24 & $0.9724 \pm 0.0047 \pm 0.0012$ & $0.9792 \pm 0.0031 \pm 0.0012$ \\
25 & $0.9980 \pm 0.0051 \pm 0.0013$ & $1.0016 \pm 0.0036 \pm 0.0014$ \\
\hline \hline
\end{tabular}

Table 13.7: Calibration constants for south in ring using the improved energy loss functions and layer weights corrections. The first uncertainty is statistical, the second due to the uncertainty on the material tune in the forward direction. The determination is done separately for Run IIb1 and Run IIb2.

- Transverse mass $50<m_{T}<200 \mathrm{GeV}$

- Missing transverse energy $\mathbb{E}_{T}>25 \mathrm{GeV}$

- Recoil transverse momentum $u_{T}<15 \mathrm{GeV}$

To avoid introducing statistical fluctuations between the pseudo-experiments corresponding to each member of the error eigensets, we use the same events but reweight them using the ratio of each energy loss function in the error set by the central one multiplied by the corresponding calibration constant in the error set divided by the central one. With this prescription, we are able to measure sub-MeV differences since the statistical uncertainties in 13.11 will be almost totally correlated. For the variation of the calibration constants propagated from the uncertainty in the layer weight correction we do not vary the energy loss functions. The results can be seen on Fig. 13.15-13.17 for the fitted $W$ 

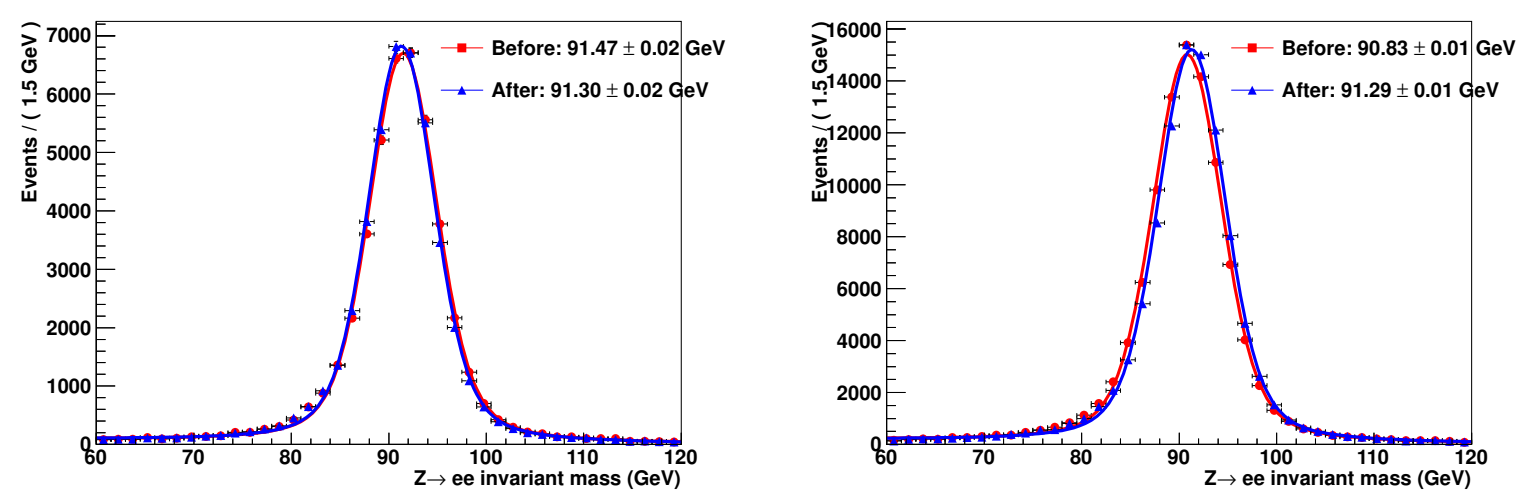

Figure 13.14: Invariant mass distribution of $Z \rightarrow e e$ events before and after the determination of the i $\eta$ calibration constants. The left (right) panel shows the distribution for Run IIb1 (Run IIb2) candidates.

mass from the transverse mass distribution, electron transverse momentum distribution and missing transverse energy distribution, respectively. In all cases, the variation turns out to be negligible.
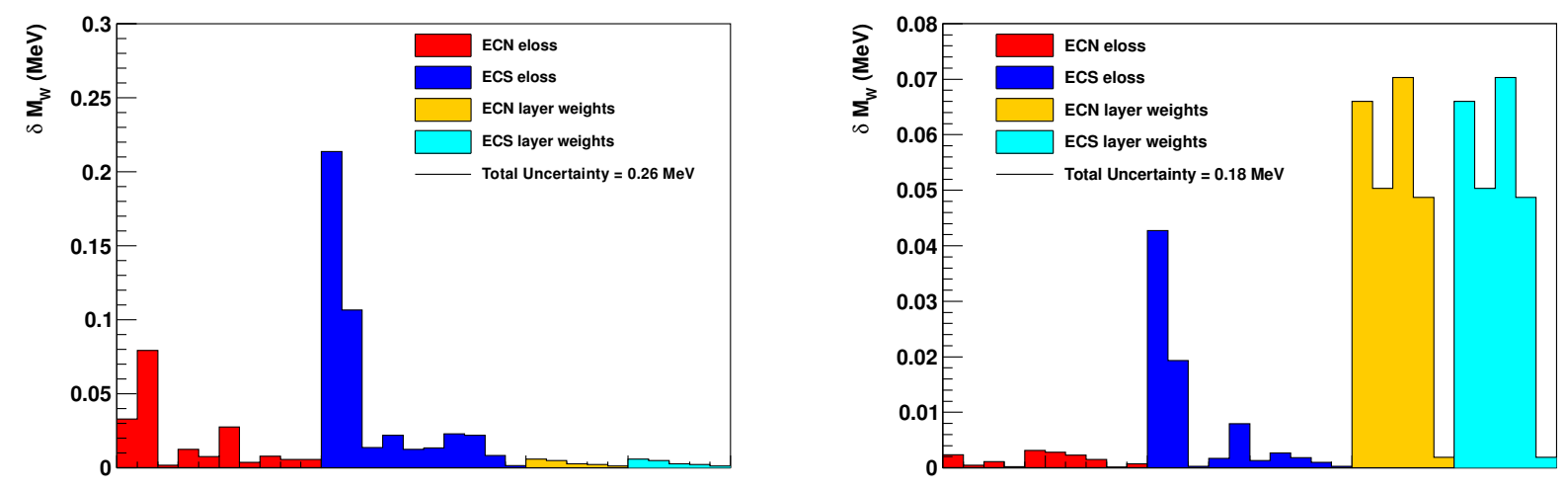

Figure 13.15: Propagation of uncertainty from the dead material determination using $Z \rightarrow e e$ events to the fitted $W$ boson mass using the transverse mass distribution. The left panel considers a measurement with forward electrons $1.5<\left|\eta_{\text {det }}\right|<2.5$ only, while the right panel includes central electrons $\left|\eta_{\text {det }}\right|<1.05$. 

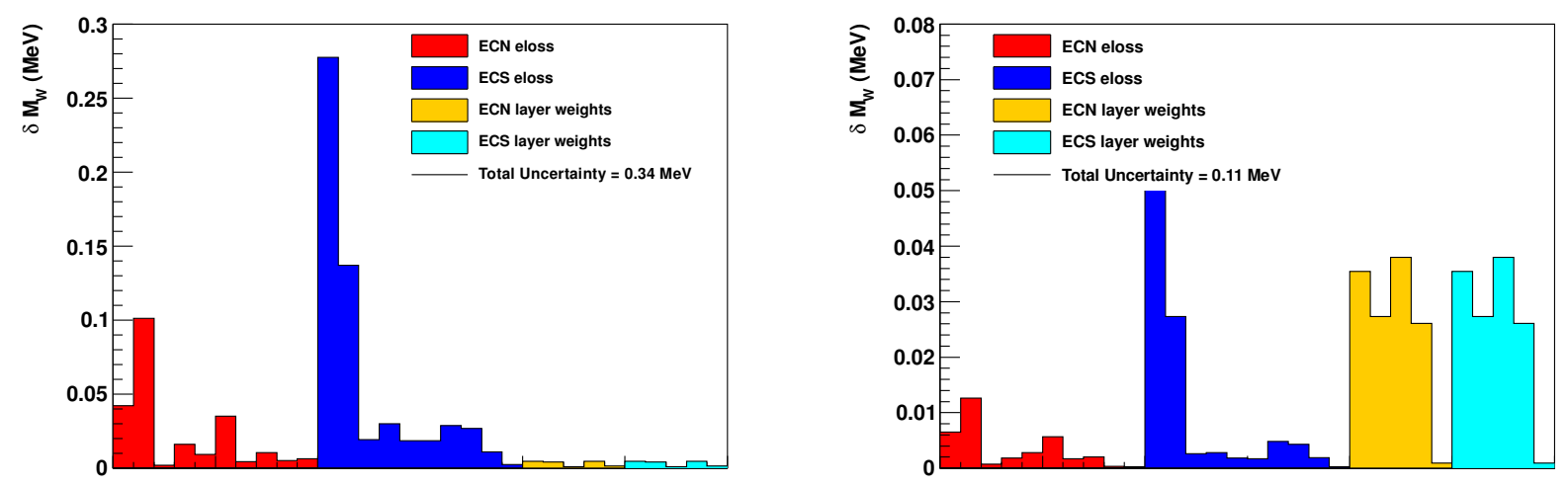

Figure 13.16: Propagation of uncertainty from the dead material determination using $Z \rightarrow e e$ events to the fitted $W$ boson mass using the electron transverse momentum distribution. The left panel considers a measurement with forward electrons $1.5<\left|\eta_{\text {det }}\right|<2.5$ only, while the right panel includes central electrons $\left|\eta_{\text {det }}\right|<1.05$.
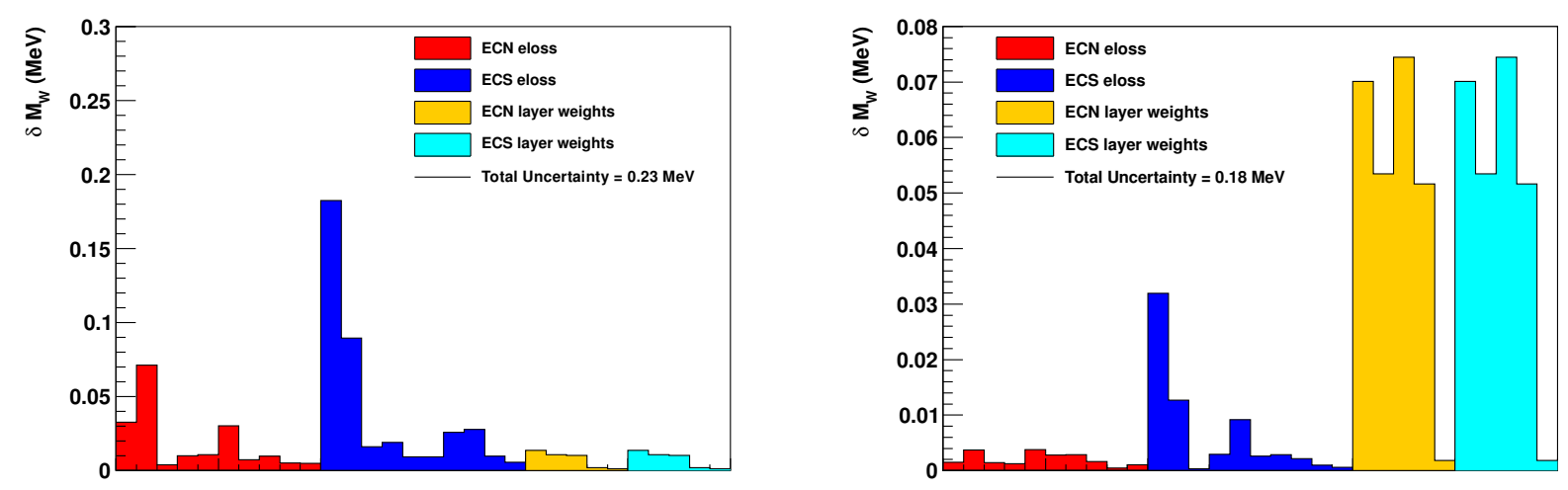

Figure 13.17: Propagation of uncertainty from the dead material determination using $Z \rightarrow e e$ events to the fitted $W$ boson mass using the missing transverse energy distribution. The left panel considers a measurement with forward electrons $1.5<\left|\eta_{\operatorname{det}}\right|<2.5$ only, while the right panel includes central electrons $\left|\eta_{\text {det }}\right|<1.05$. 


\section{Chapter 14}

\section{Parametrized Models for a Forward Electrons fast $\mathrm{MC}$}

The fast Monte Carlo built for the central electron measurement with $4.3 \mathrm{fb}^{-1}$ had a very rudimentary model for the forward electrons, since they only had a secondary role in the calibration. The model in the forward region did not include most of the details necessary for a good description of the $W$ and $Z$ kinematical distributions. Therefore, the initial effort for a measurement with forward electrons is to re-build all the models for the electron response, resolution and efficiency that successfully described the central electron events for the electrons detected in the EC.

A new measurement with central electrons will also strongly benefit from revisiting some of the models used in the fast Monte Carlo. Some of the models have not been updated since the measurement with $1 \mathrm{fb}^{-1}$. Using models calibrated with $1 \mathrm{fb}^{-1}$ had only a modest impact on the uncertainty budget for the measurement with $4.3 \mathrm{fb}^{-1}$, however, with the full data set, it becomes necessary to have all the parameters determined as precisely as $10 \mathrm{fb}^{-1}$ of $W$ and $Z$ boson candidates allow.

\subsection{Final State Radiation Efficiency and Response for Forward Elec- trons}

The Final State Radiation (FSR) model describes the effect of photons in the detection efficiency and energy response of electrons. Since the photon is a massless particle, it is impossible to distinguish an electron from the system of electrons and soft (almost) collinear photons around it. Experimentally, we have yet another scale corresponding to the size of the reconstruction cluster inside which it is physically impossible to distinguish the energy deposited by an electron of that from an almost collinear photon. Therefore, the real object for which the fast MC is built is the electron and photon system, and this is the object that is used to calibrate every other model.

In our simulation, the photons in the final state are simulated by the shower algo- 
rithm implemented in PнотоS. Thus, we use a event-by-event probability to determine whether a photon that carries a momentum fraction $X$ and lies at distance $\Delta R$ of the parent electron will obstruct the identification of the electron with energy $E$ and direction $\eta$. Furthermore, we use an event-by-event observable $\kappa$ to define what fraction of the photon momentum contributes to the electron-photon system. The observable $\kappa$ is defined as:

$$
\kappa=\frac{E_{\text {reco }}-E_{\text {reco }}[\text { not showered }]}{E_{\text {true }}[\text { not showered }]-E_{\text {true }}}
$$

where "not showered" refers to the electron without the final state photon showered. We prepare two samples of single electron events from $W$ decays generated with REsBos. In one of them, all electrons in the final state are forcibly showered with PHOTOS, while the other is kept unshowered. Both are simulated, digitized and reconstructed with the standard D0 full Monte Carlo.

For each corresponding event in the showered and unshowered samples, we measure the number of fully reconstructed electrons using the same selection criteria that are used in the $W$ mass measurement. The ratio between the yield in the showered sample to the unshowered is calculated in bins of $X, \Delta R, \eta$ and $E$. We also calculate the $\kappa$ observable and profile it in bins of the same variables. Figures 14.1 and 14.2 show the FSR efficiency and response integrated over $\eta$ and $E$.

Many of the features are similar to the ones observed in the equivalent parametrization for central electrons. However, the size of the D0 end-cap EM cluster is $\eta$ dependent. In the central calorimeter, a cluster is defined by a fixed number of towers (13) which have a maximum radius of $\Delta \bar{R}=2$. In the $\mathrm{EC}$, the cluster window size is defined by:

$$
\Delta \bar{R}=\left\lfloor 1+\frac{64}{2 \pi} \times \frac{10 \mathrm{~cm}}{r}\right\rfloor \Rightarrow \Delta R \simeq 0.1 \times\left\lfloor 1+\frac{64}{2 \pi} \times \frac{10 \sinh \eta}{172.4}\right\rfloor
$$

where $r$ is the distance of the hottest cell in the cluster to the calorimeter cylindrical axis. The window size at $\eta_{\text {det }}=1.5$ is $\Delta \bar{R}=2$ as in the central calorimeter, but can be as big as $\Delta \bar{R}=4$ for $\eta_{\text {det }}=2.5$. The formula on the right-hand side of Eq. 14.2 is a good approximation that can be used in a fast MC.

This model has an important shortcoming when compared to the equivalent one built for the central electron analysis: it does not takes into account the effect of the underlying energy. In the central calorimeter analysis, we overlaid both showered and unshowered with data unsuppressed $\mathrm{ZB}$ events. However, since we construct observables that require event-by-event comparisons, the same ZB event has to be overlaid in matched events. In the high instantaneous luminosity environment of Run II, the difference is important and the same problem will return in all other models described in this note. But it still needs to be addressed in the FSR model.

\subsubsection{Closure test of the FSR model}

We test the parametrized FSR model by using a toy fast MC, which uses full MC to all simulations but the FSR merging, for which we use the model constructed here. Figure 14.3 

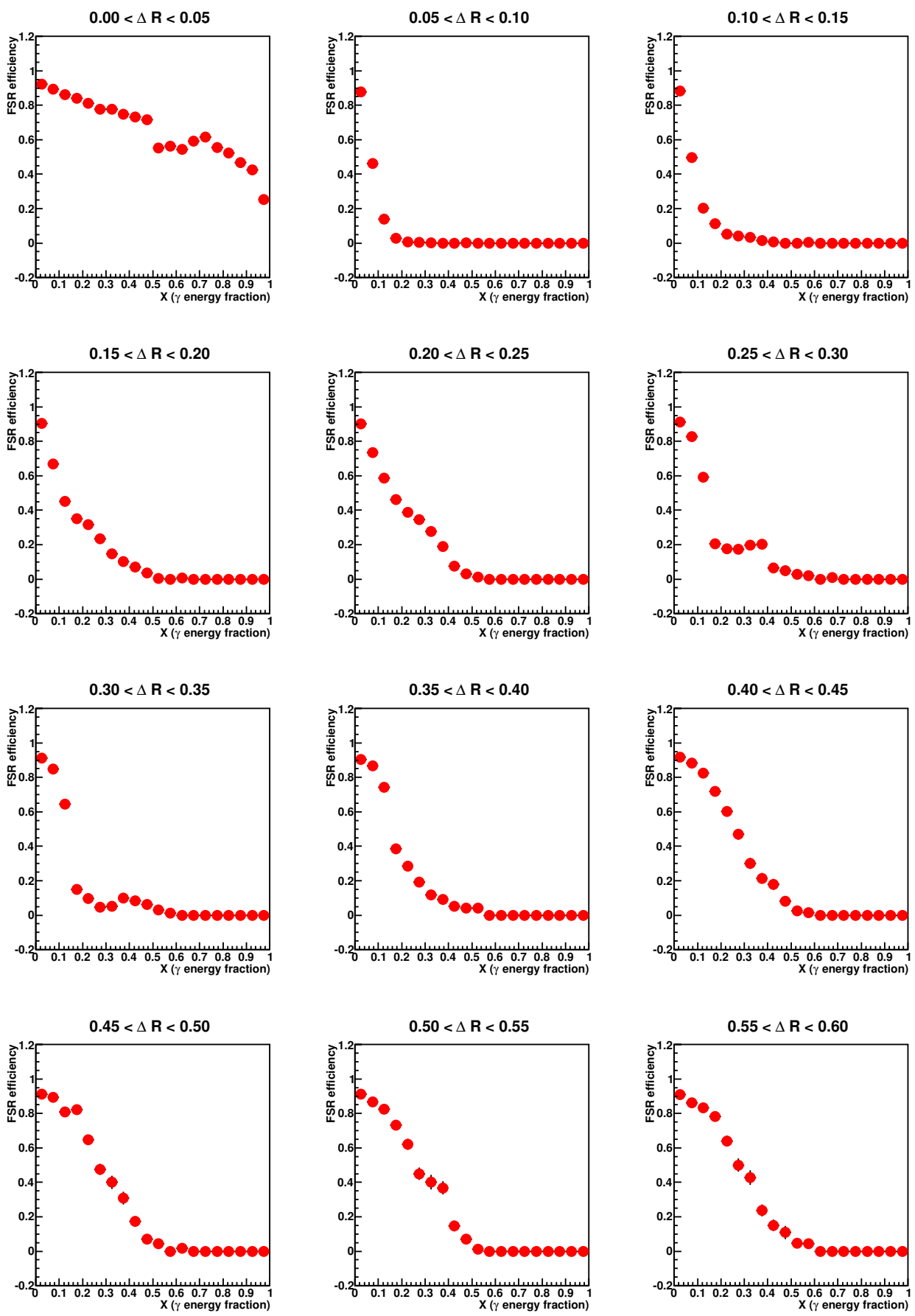

Figure 14.1: Efficiency of detection of an electron in the presence of a showered photon as a function of the fraction of the momentum carried by the photon in bins of $\Delta R$ between the electron and the photon. 

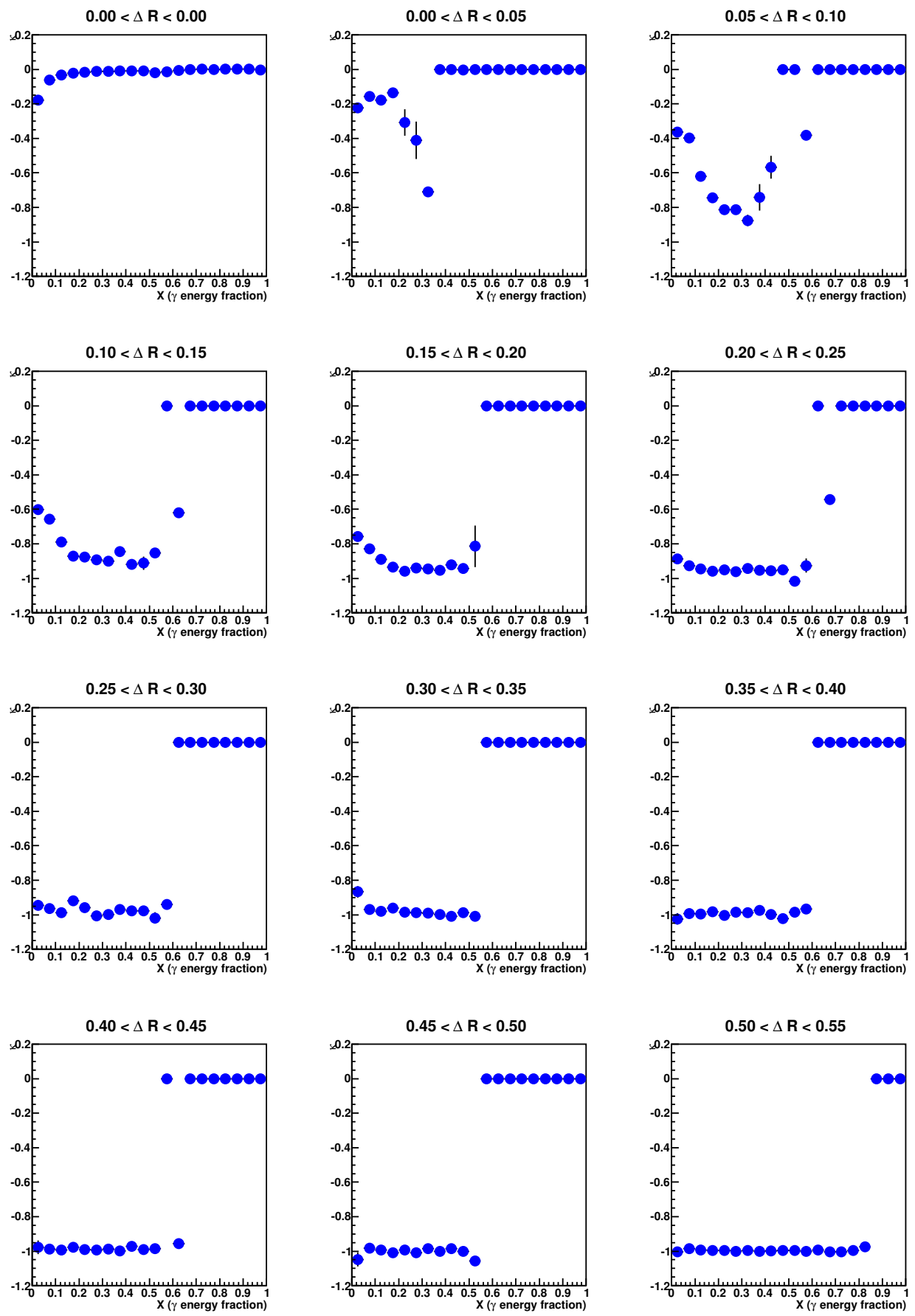

Figure 14.2: Fraction of the photon energy that does not contribute to the electron cluster reconstructed energy as a function of the fraction of the momentum carried by the photon in bins of $\Delta R$ between the electron and the photon. 
compares the distribution of the photon energy fraction and the $\Delta R$ between the electron and the FSR photon for those electron with FSR that are well reconstructed. Similar good agreement is found comparing the other two variables, electron energy and $\eta$, on which the model depends.
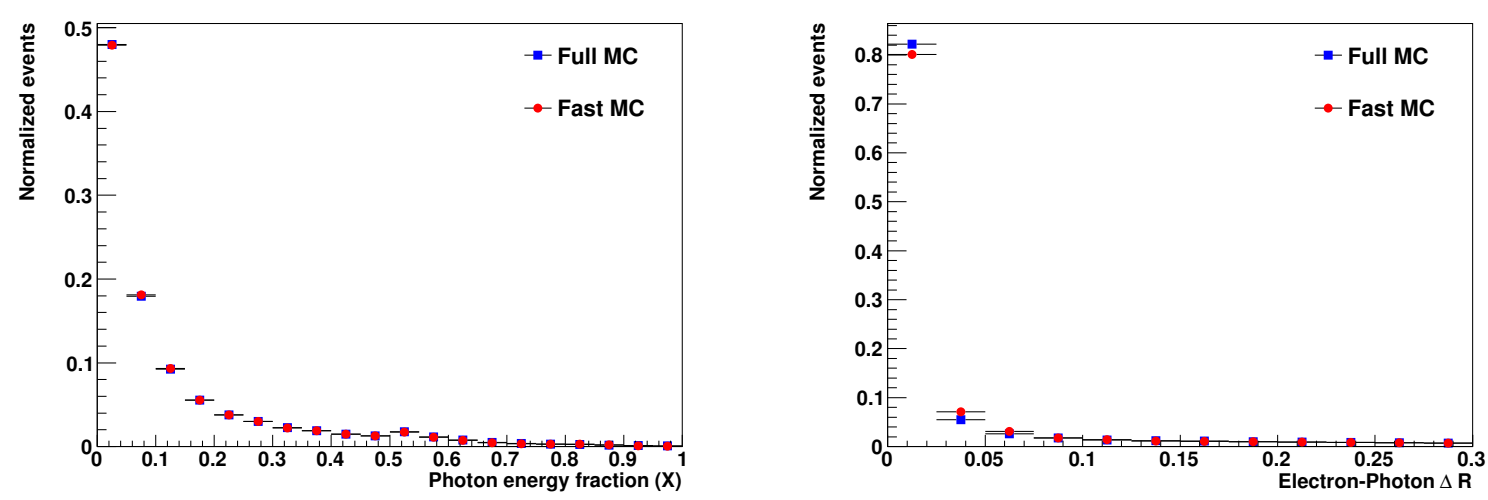

Figure 14.3: Comparison between the photon energy fraction (left) and $\Delta R$ (right) between the electron and photon for reconstructed electrons in full MC and fast $M C$, where the fast MC uses the parametrized model described here.

For those electrons with FSR showers that were identified and passed the selection criteria, we also compare the photon energy loss between fast and full MC using the observable $\kappa$ described above. The result can be seen in Fig. 14.4. Again, the agreement is satisfactory.

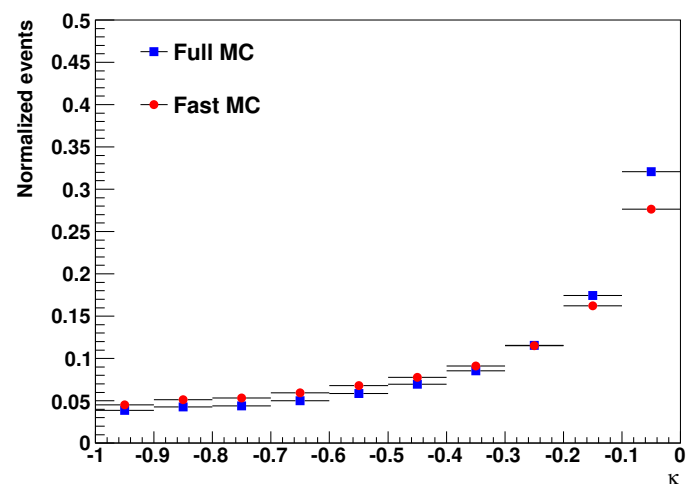

Figure 14.4: Comparison between the photon energy loss in reconstructed electrons with FSR in fast and full MC. 


\subsection{Leakage Model for Forward Electrons}

For wide EM showers, part of the electron energy will not be included in the reconstructed cluster and, therefore, will be added into the recoil. The leakage model describes the probability that an electron, produced at an angle $\eta$ will have some its energy leaked into the recoil system and, when it does, what fraction is lost.

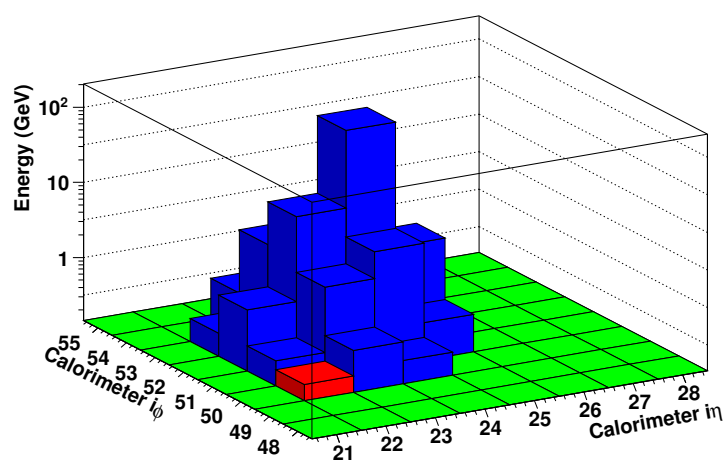

Figure 14.5: Example of a reconstructed full MC electron shower in the EC, after ZB subtraction. The blue bars represent the energy inside the electron cluster. The red is the leaked electron energy into the recoil system. We call attention to how irregular a EC cluster can be.

The leakage will happen through small deposits of energy in the cells around the electron cluster, as shown in Fig. 14.5. Therefore, it is necessary to consider the effect of the hadronic energy flowing through the calorimeter that pushes the baseline energy on the cells close to the zero suppression threshold and makes the leaked energy visible. This is essentially the same issue as briefly described above for the FSR model, but here we treat it fully. The leaked energy is also missing from the reconstructed electron energy, but the difference is recovered by the energy loss function 13.5. since it is derived from reconstructed electron clusters.

We use two full MC samples: a single electron from $W$ decay generated with RESBOS, showered with PНОTOS and overlaid with data ZB events; and one with only the ZB event. Both samples are simulated, digitized and reconstructed with the standard full MC chain. We select only the events in which the reconstructed electron satisfies the same criteria as used in the $W$ boson mass measurement.

We measure the hadronic recoil transverse momentum $u_{T}$, as defined in Sec. 4.3 , in the samples with and without a reconstructed electron and count the fraction of events in which there was a measured increase in $u_{T}$. We also profile the ratio of the leaked transverse momentum, evaluated by the difference in $u_{T}$ on the two samples, by the electron transverse momentum $p_{T}$. Since the calorimeter cells are brought close to zero suppression threshold by the presence of the ZB overlay, we parametrize the probability and the 
leakage fraction also as a function of the ZB scalar $E_{T}$. We build the model separately for the electrons without any photon close to the electron, for events with one photon showered close to the electron and for events with two. Events with photons have a wider shower and tend to have larger leakage than events without (see Fig. 14.6). We define that a photon is close to the electron if their angular separation satisfies $\Delta R=0.3$.
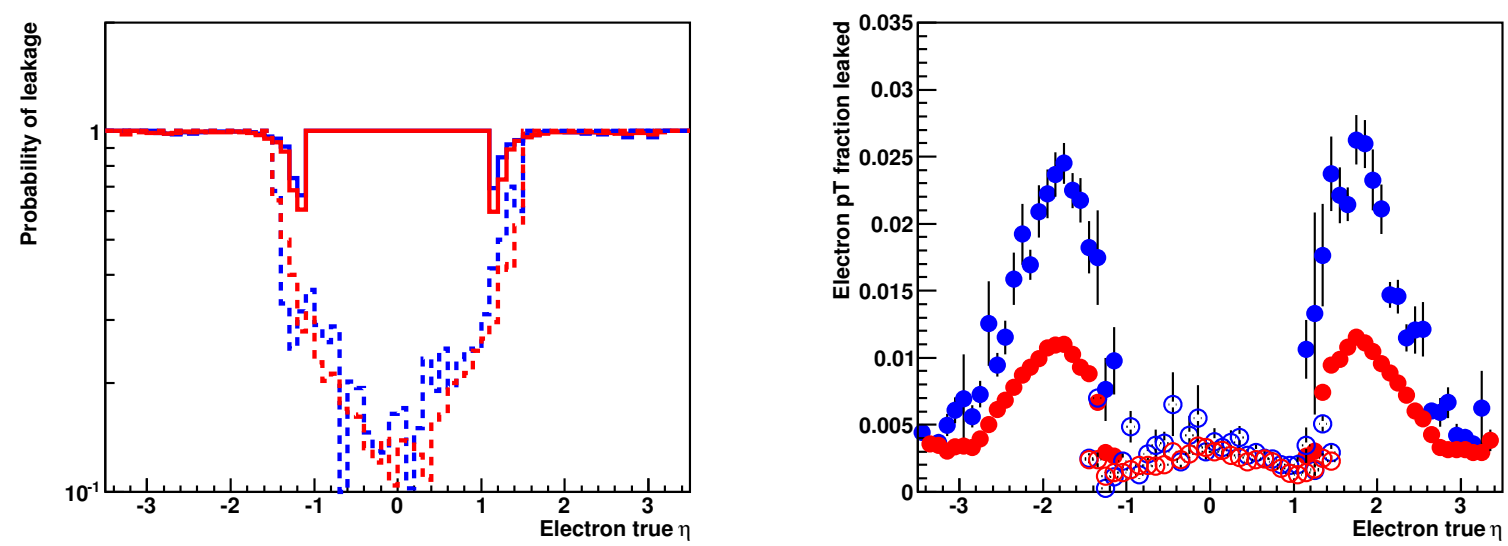

Figure 14.6: Leakage model for both central electrons, dashed line on the left and open makers on the right, and forward electrons, full line on the left and full markers on the right. (left) The probability of an electron produced at angle $\eta$ to leak part of its transverse momentum. In the EC, pratically all EM clusters have some leakage. (right) Average transverse momentum fraction leaked when it happens. The model is shown separately for the case with no showered electron and for the case with one showered electron closer than $\Delta R=0.3$, and integrated over SET.

\subsection{Underlying Energy Model for Forward Electrons}

The underlying event model describes the hadronic energy that is reconstructed as part of the electron cluster. The model has two components: the $\Delta u_{T}$ library, which measures how much of the recoil transverse energy is lost because it is reconstructed inside the EM cluster, and the $\Delta u_{T} \rightarrow \Delta E_{T}$ translation, which describes, for a given recoil transverse momentum inside the electron cluster, how much the electron transverse energy $E_{T}$ changes. Naïvely, this translation would be just the identity function. In practice, because of zero suppression and other noise suppression algorithms (see Sec. 2.4.4), there exists a nontrivial relationship between them. For the simple pedestal zero suppression it is easy to understand the source of the difference. The energies in the cells inside an electron cluster are above the zero suppression threshold, meaning that every hadron that deposits its energy in those cells will have its energy added to the cluster energy. The cells in the 
recoil system, on the other hand, are very close to the zero suppression threshold and often below it. Therefore, on average, some of the hadrons energy deposition will not result in visible reconstructed energy. This simple picture works well at large SET values. For small SET values, that can be a result of negative energies from the BLS system (see Fig. 2.14, the $\Delta E_{T}$ is, on average, negative.

\subsubsection{Rotated clusters and $\Delta u_{T}$ library}

To build the $\Delta u_{T}$ library, we measure, in $W$ candidate events, the average energy flowing in the calorimeter in sections which have the same geometry as the electron cluster, but are away from it. The size of an EC EM cluster is, as described in Sec. 4.1. $\eta_{\text {det }}$ dependent. We look at the 64 possible rotated clusters ${ }^{1}$ around the $\eta_{\text {det }}$ ring in which the electron cluster hottest cell is located.

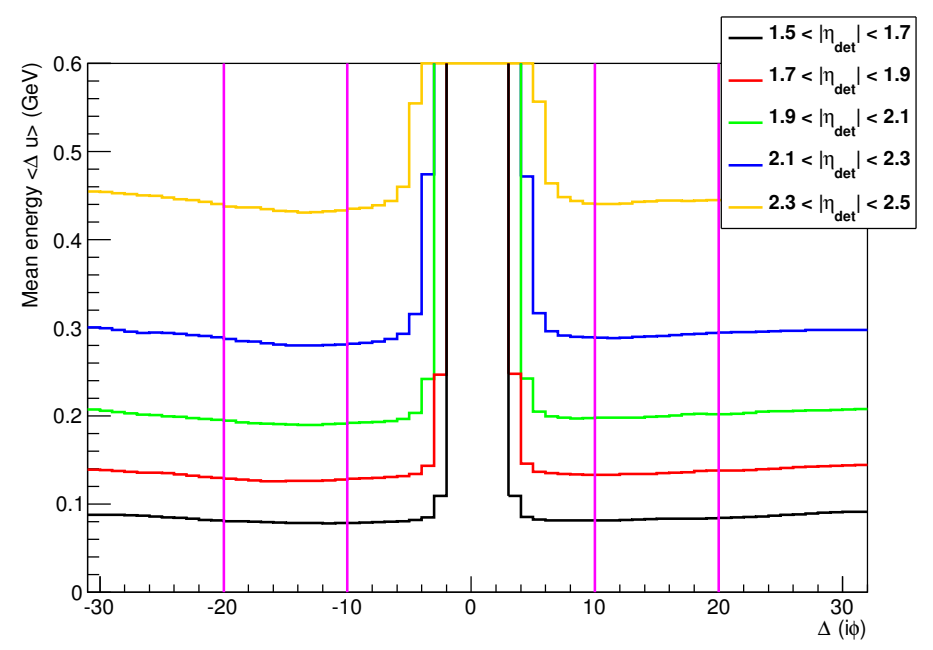

Figure 14.7: Average energy deposited in rotated cluster, in bins of $\left|\eta_{\text {det }}\right|$, measured in a $W \rightarrow e v$ full MC sample. The large tower of energy around $\Delta(i \phi)=0$ is the electron cluster and the small deposit of energy opposite to it is the hard recoil of longitudinally boosted $W$ bosons, which are kinematically correlated to the electron. The region $10<$ $|\Delta(i \phi)|<20$ is free of both contamination and used for the average.

Figure 14.7 shows the average energy in bins of detector $\eta$, each 0.2 wide as measured with a $W \rightarrow e v$ full MC sample. The tower of energy around $\Delta(i \phi)=0$ is the electron

\footnotetext{
${ }^{1}$ Here it is important to make a distinction between the CC and the EC cases. In the CC, the cluster is a well defined cone with 13 towers and there is no ambiguity in the meaning of a rotated cone since we use the central tower as reference. In the EC, the cluster is defined around a cell, not a tower, and, for consistency, we use the term "rotated cluster" instead of "rotated cone". For each cluster, we determine the seeding cell reversing the D0 clustering algorithm and rotate the set of cells clustered around it. However, clusters with identical detector $\eta$ but seeded around different cells will have different rotated clusters and the model here works only on average.
} 
cluster and the small deposit of energy opposite to it is the hard recoil of longitudinally boosted $W$ bosons. Differently from the recoil of central electrons, the contamination from the hard recoil is small because it mostly lies in the opposite EC.

We build the $\Delta u_{T}$ library by counting the fraction of rotated clusters in the region $10<|\Delta(i \phi)|<20$ inside which there is a non-zero deposit of hadronic energy. For the cases with visible energy, the distribution of the cluster uncorrected transverse energies is interpreted as a probability distribution for energy flow in the underlying energy. We measure both the probability of having a non-zero energy and the distribution of nonzero energy separately in bins of $u_{\|}$, SET, instantaneous luminosity $\mathcal{L}$ and detector $\eta_{\text {det }}$. The $u_{\|}$dependence models the hard recoil reconstructed under the electron cluster, while the SET dependence models the soft recoil contribution. Figure 14.8 show the average transverse energy integrated in all variables but one, and 14.9 shows how the fluctuations depend strongly on the SET, both for full MC $W \rightarrow$ ev events.
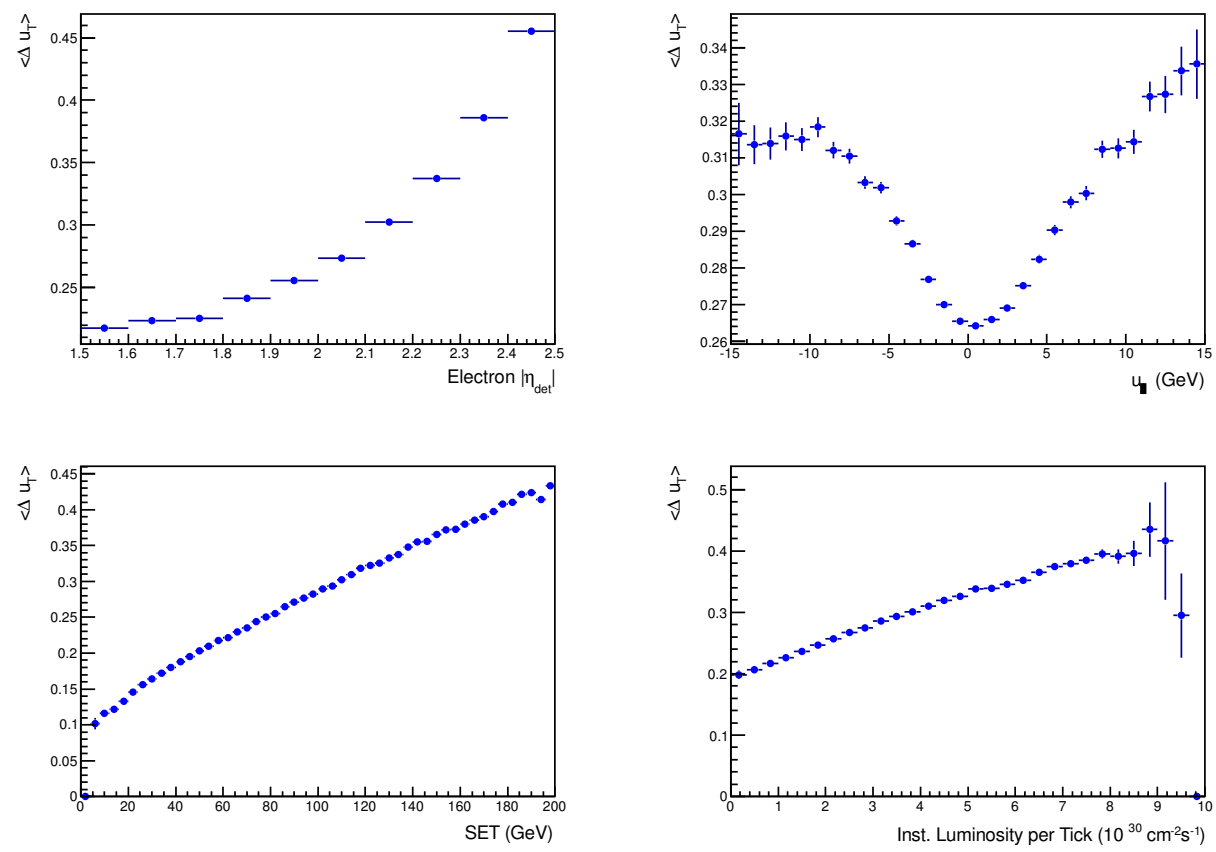

Figure 14.8: Average transverse hadronic energy in rotated clusters as a function of $\left|\eta_{\text {det }}\right|$, SET, instantaneous luminosity $\mathcal{L}$ and $u_{\|}$. We use $W \rightarrow e v$ events, and the average is done on rotated clusters around the same i $\eta$ ring the electron cluster is centered on. We remove, from the average, clusters with electron and hard recoil contamination. The strong SET dependence shows the importance of the soft recoil for the underlying energy flow. We consider both SET and $\mathcal{L}$ dependence simultaneously to describe effects related to the calorimeter readout, such as the BLS system 2.4.4. The complete model is a function of the four variables shown here. 


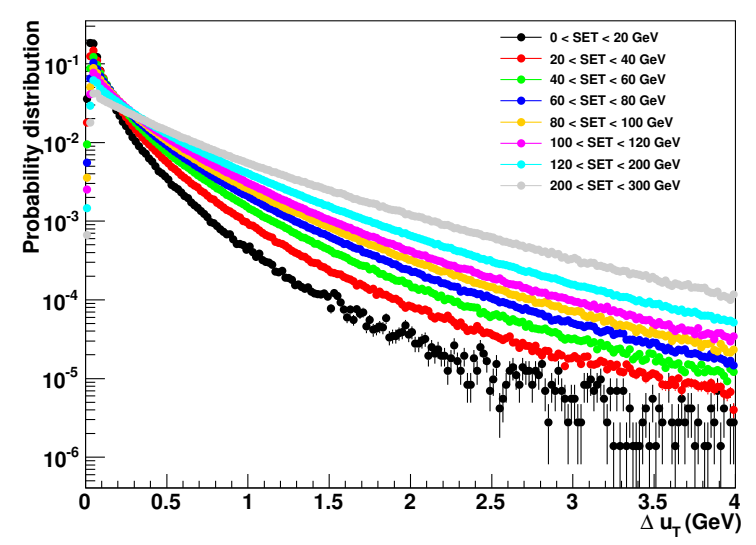

Figure 14.9: Probability distribution for the transverse hadronic energy in rotated clusters as a function of SET, showing the dependence of the noise fluctuations on this variable.

\subsection{2 $\Delta u_{T} \rightarrow \Delta E_{T}$ Mapping and Closure Test of the Underlying Event Model}

The $\Delta u_{T} \rightarrow \Delta E_{T}$ translation is studied using dedicated full MC samples, similar to the one prepared for the analysis with central electrons and described in Sec. 7.3.6. For the model described there, the full MC samples are $W \rightarrow e v$ events generated with PYTHIA where the electron from with a $W$ boson parent are required to have $\left|\eta_{\text {det }}\right|>1$.1. These events are simulated with the standard D0 simulation program. The GEANT hits in the calorimeter are digitized with using three different options:

Everything: The full MC events have a collider data unsuppressed ZB event added and all GEANT tracks ${ }^{2}$ are digitized.

ElecOnly: The events have no ZB events added and only the tracks that descend from the electron or photons that have the $W$ boson as parent are digitized.

RemoveElectron: The events are overlaid with the data unsuppressed $\mathrm{ZB}$ events, using the same pairing between PYTHIA events and ZB events as in the Everything sample, and all the tracks, except the ones that descend from the electron or photons that have the $W$ as parent, are digitized.

The interpretation of each sample is straightforward. The clusters in the Everything sample are the target of the model in the fast MC, while the associated clusters in the ElecOnly correspond to the energy before the electron window correction due to the underlying energy $\Delta u_{T}$ in the RemoveElectron sample. Thus, $\Delta E_{T}$ is the difference in the

\footnotetext{
${ }^{2}$ By tracks, in this context, we mean the segments followed by each shower particle inside the calorimeter during the GEANT simulation. Not to be confused with tracks in the tracking detectors.
} 
reconstructed energy in the Everything sample to the one in the ElecOnly sample.

We parametrize the map $\Delta u_{T} \rightarrow \Delta E_{T}$ as a function of $\Delta u_{T}$, detector $\eta$, SET and instantaneous luminosity. Figure 14.10 shows the dependence of $\Delta E_{T}$ on detector $\eta$, SET and instantaneous luminosity. The figure also shows the result of a closure test using a toy fast MC, which uses full MC for all simulations but the underlying hadronic energy under the electron cluster and the change in the electron reconstructed energy, for which we use the model described here.
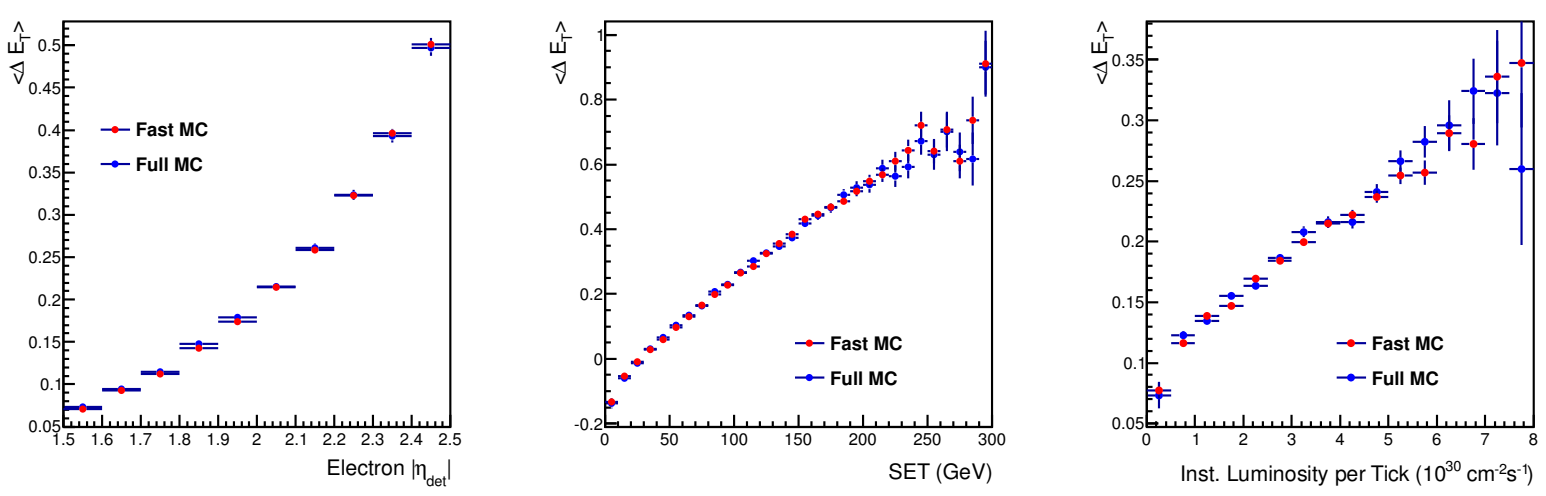

Figure 14.10: The electron cluster $\Delta E_{T}$, in $\mathrm{GeV}$, as a function of detector $\eta$ (left), SET (center) and instantaneous luminosity (right). The red markers shows the closure test with a toy fast MC which uses the parametrized model described in this section.

\subsection{Single Photon Model for Forward Electrons}

The FSR model described in section 14.1 only parametrizes the photons reconstructed as part of an electron cluster. The photon shower may lie outside the electron cluster and, therefore, be reconstructed as part of the recoil system. The recoil system is a global object and the fast $\mathrm{MC}$ for central electrons has a parametrization of the photon response in all the calorimeter acceptance region used for recoil reconstruction. However, since final state photons tend to be close to the electron, the existing model has a very rough description of the EC calorimeter response to soft photons. Moreover, as already mentioned, the effect of the underlying event is important for soft photon energy response, but neglected in the existing model. Despite these shortcomings, the model has been shown to be good enough for the central calorimeter

Here we construct a new model intended primarily for EC electrons. We generate $W \rightarrow e v$ events from RESBOS and simulate the final state photons with PHOTOS. We simulate, digitize and reconstruct only the photon. This sample is initially overlaid with data unsuppressed ZB events. We then use the same ZB only samples described in section 14.2 
to subtract the energy of the underlying event on a per-event basis. This is essentially the same strategy used in the analysis of the leakage model.

We count the fraction of events in which there is a visible energy in the calorimeter. The probability is parametrized as a function of the photon generated energy and $\eta$ as well as the ZB SET. Figure 14.11 shows the importance of these variables for the probability integrated over the other variables. For those events that have visible energy, we parametrize the fraction of the photon energy that contributes to the recoil transverse momentum as a function of $\eta$ and SET, as shown in Fig.14.12.
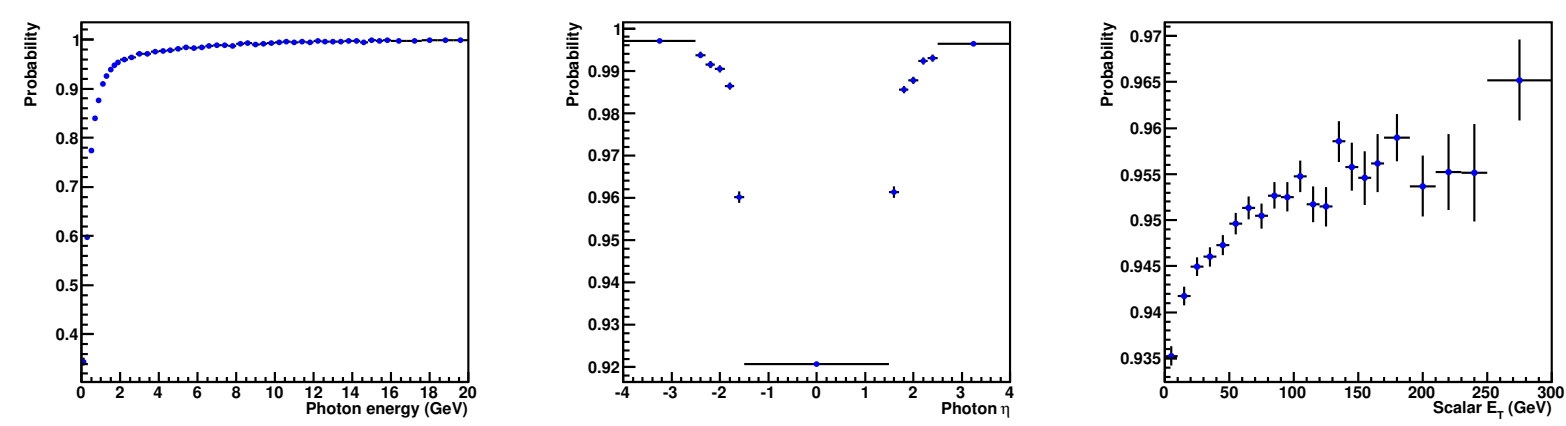

Figure 14.11: Probability of an out-of-cluster FSR photon to reach the calorimeter and leave a visible deposit of energy as a function of the photon energy (left), $\eta$ (center) and event SET (right).
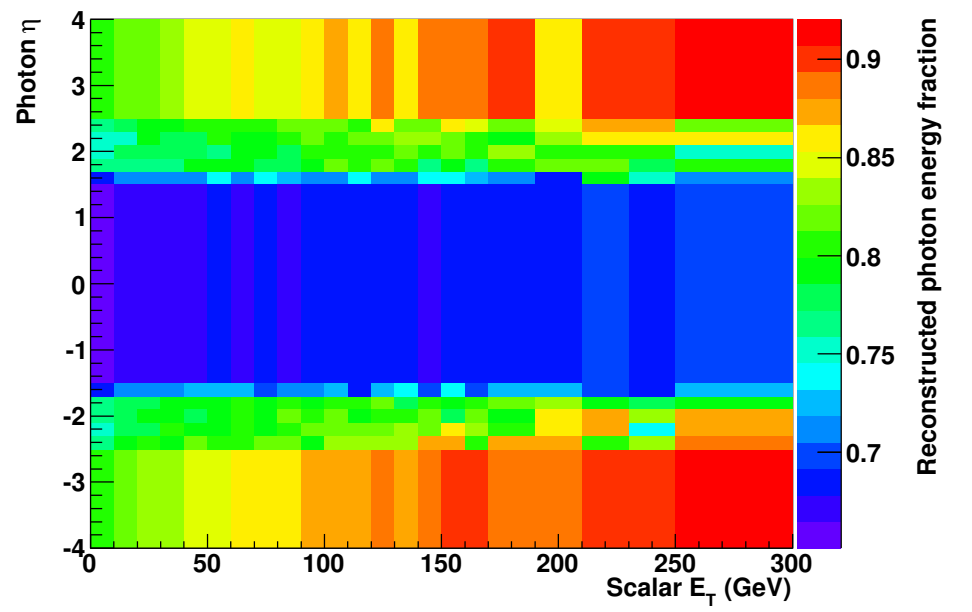

Figure 14.12: The EC calorimeter response to soft photons as a function of the photon pseudo-rapidity $\eta$ and event ZB SET. 


\subsubsection{Closure Test for Out-of-Cone FSR Photons}

We perform a closure test using the same technique already describe for the FSR model and for the underlying event model. We create a toy fast MC in which all the event is simulated in full MC but the contribution of photons to the recoil system. For this specific deposit of energy we use the model constructed here and compare to the prediction from full MC. Figure 14.13 shows good agreement and validate the parametrized model.

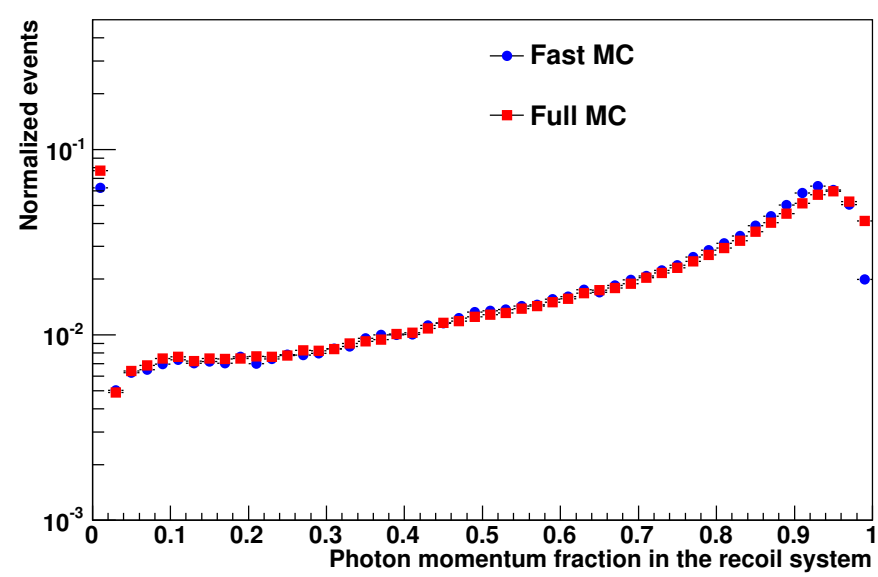

Figure 14.13: Comparision between the fraction of the photon energy deposited in the recoil system as measured in full MC (red dots) and in the toy fast MC (blue dots) using the model described in this Section.

\subsection{The Hard Recoil Model}

The effect of the underlying energy has be shown to be relevant for the modeling of soft energy in the recoil system coming from the electron leakage, from the recoil under the electron cluster and from out-of-cluster FSR photons. Here, we study its effect in the hard recoil, ie. hadrons that balance the transverse momentum of the vector boson. In the $W$ mass fast MC, the hard component of the recoil is modeled from a full MC $Z \rightarrow v v$ sample (see Sec. 7.4). We parametrize probability distributions for the relative response $u_{T} / q_{T}$ and relative direction $\Delta \phi$ between the recoil transverse vector and the vector boson $V$ transverse momentum. The model used for the $W$ mass measurement with central electrons was constructed from a sample digitized without $\mathrm{ZB}$ overlay. Thus, a significant part of the recoil energy was zero suppressed and not visible.

Here we adopt the same strategy as already used twice above: we use a sample with data ZB overlay and then subtract, on a per-event basis, the energy from the ZB event. We select events in which both neutrinos have $|\eta|<2.5$ to reproduce, as closely as possible, the electron fiducial cut in the End Calorimeter. Figure 14.14 shows the effect of the 
underlying energy in the hard recoil relative response and SET. Figure 14.15 shows the probability distribution of the parametrized model in two different $Z q_{T}$ ranges.
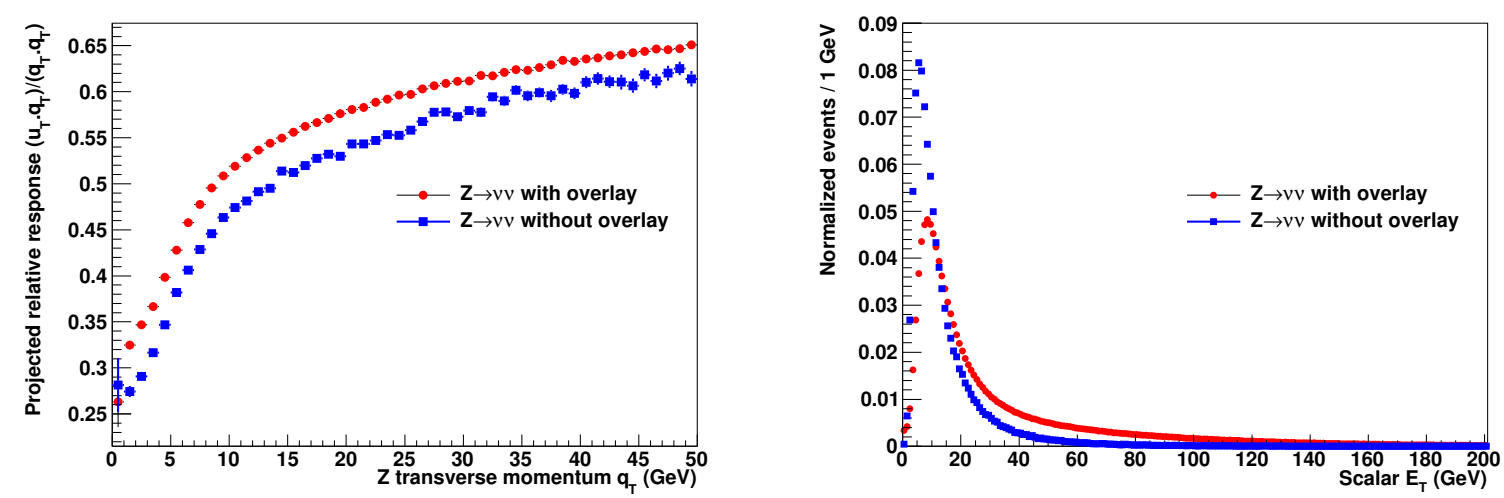

Figure 14.14: (left) Projected relative response $\left(u_{T} \cdot q_{T}\right) /\left(q_{T} \cdot q_{T}\right)$ of the recoil transverse vector $u_{T}$ with respect to the boson generator transverse momentum $q_{T}$ as a function of $q_{T}$. (right) SET distribution from the hard recoil only. The blue histograms were generated from full MC samples without overlay while the red histograms are generated form histograms with ZB overlay in which the underlying energy was removed event by event. Once again, the importance of the underlying energy in bringing the cells closer to the noise suppression threshold is clearly seen in the amount of visible soft energy in the detector.

\subsection{Resolution Model for Forward Electrons}

The complete electron resolution model is divided in three parts: the sampling resolution, the noise resolution and the constant term. The constant term is due to channel-to-channel variations and is determined directly from data. The noise contribution is mainly due to the fluctuations in the underlying hadronic system and is properly described by the $\Delta u_{T}$ library (see section 14.3.1 and figure 14.9). The sampling contribution is due to Poisson fluctuations in the ionization charge readout by the calorimeter. It is strongly dependent on the shower development and, since the electron showering depends on the material upstream of calorimeter, it is dependent on a detailed knowledge of the dead material.

The amount of dead material was precisely determined comparing $Z \rightarrow$ ee data to a detailed simulation of electron showers with four different ad hoc geometries (App. C.1). Here, we use the ad hoc geometry (ECdevelB2) closest to the fitted amount of dead material to study the sampling resolution. We use a sample of single monochromatic electrons generated with energies $10,15,20,35,45,55,65,75,85,95,105,135,165,195,225,285,315$, $345,375 \mathrm{GeV}$ in the $\eta$ region $[-4.5,-1.1] \cup[1.1,4.5]$. We apply the energy loss correction derived to this sample C.1 and study the fluctuations around the mean energy. 

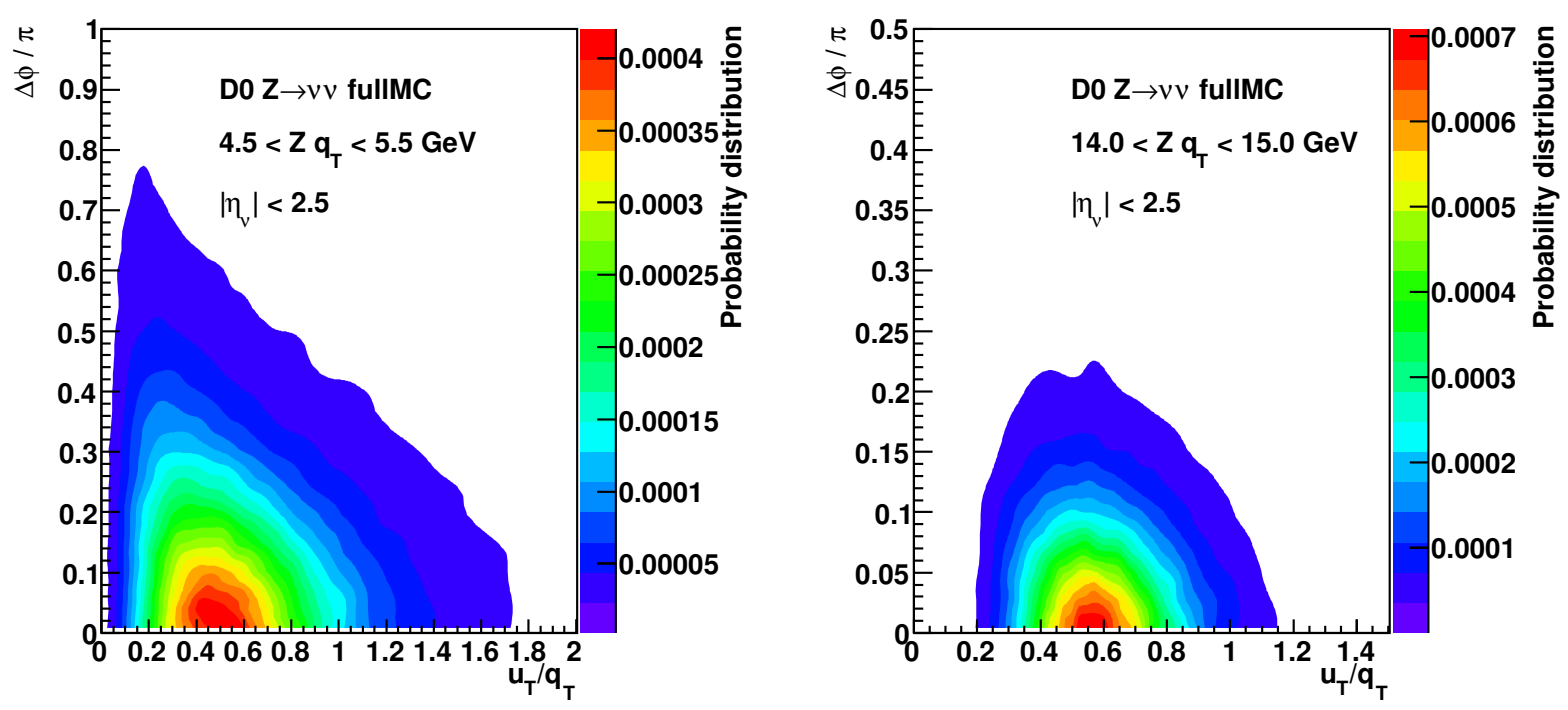

Figure 14.15: Hard recoil parametrization with new $Z \rightarrow v v$ full MC. The left panel shows the relative response and direction for relatively low $Z q_{T}$ when the recoil is dominated by soft gluon emission. In this case the angular correlation is very soft.

We study the sampling resolution using the same $\eta_{\text {det }}$ binning as used for the energy loss function. The large amount of dead material in front of the calorimeter creates a low energy tail for the reconstructed energy, as can be seen in Fig. 14.16, and complicates the choice of response function. We separate the fluctuations in the radiative loss and the fluctuations in the detected shower by fitting a landau distribution convoluted with a gaussian. We interpret the width of the gaussian as a measure of the detector resolution.

We observe a strong dependence of the resolution on the angle of incidence, as can be seen in Fig. 14.17, which supports our binning in $\eta_{\text {det }}$. The energy dependence of the parameters in the models is fitted to a polynomial in $1 / \sqrt{E}$.

\subsubsection{Closure Test for the Resolution Model}

We test the parametrization described here for the electron energy resolution by comparing the energy fluctation in single electron samples to the parametrized model built for the fast MC. Since these samples do not have noise fluctuations, their resolution must be well described by our model. We do the test in two steps: first, we compare the distribution of energy sampling fluctation in both full MC and fast MC. The result can be seen on Fig. 14.18. Then, we compare the energy resolution as a function of the electron energy and $\eta$, again in full MC and fast MC. Both comparisons can be seen on Fig. 14.19. Both tests show good closure of the model. 

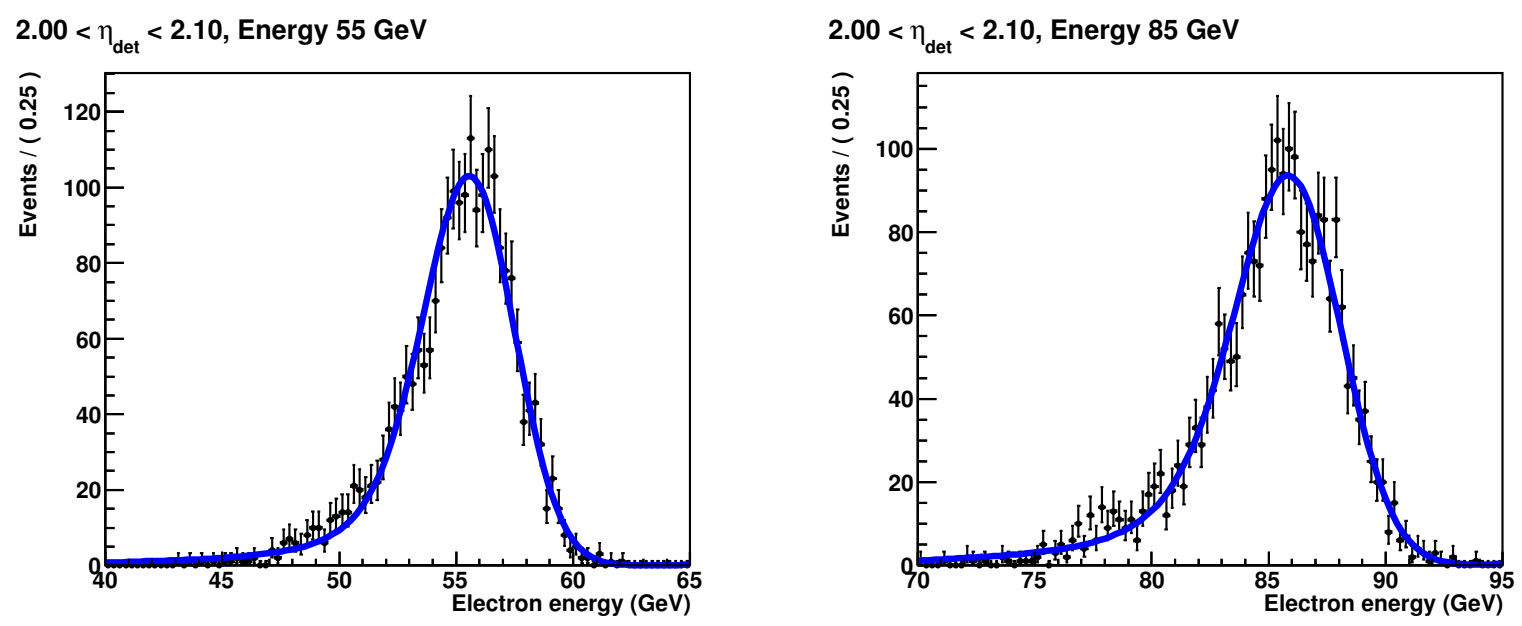

Figure 14.16: Distribution of the reconstructed energy, after energy loss correction, for monochromatic electrons with true energy $55 \mathrm{GeV}$ (left) and $85 \mathrm{GeV}$ (right). The model used to describe the electron sampling resolution is the convolution of a Landau distribution with a gaussian. The Landau describes the fluctuations in the radiative energy loss, while the gaussian describes the sampling fluctuations.
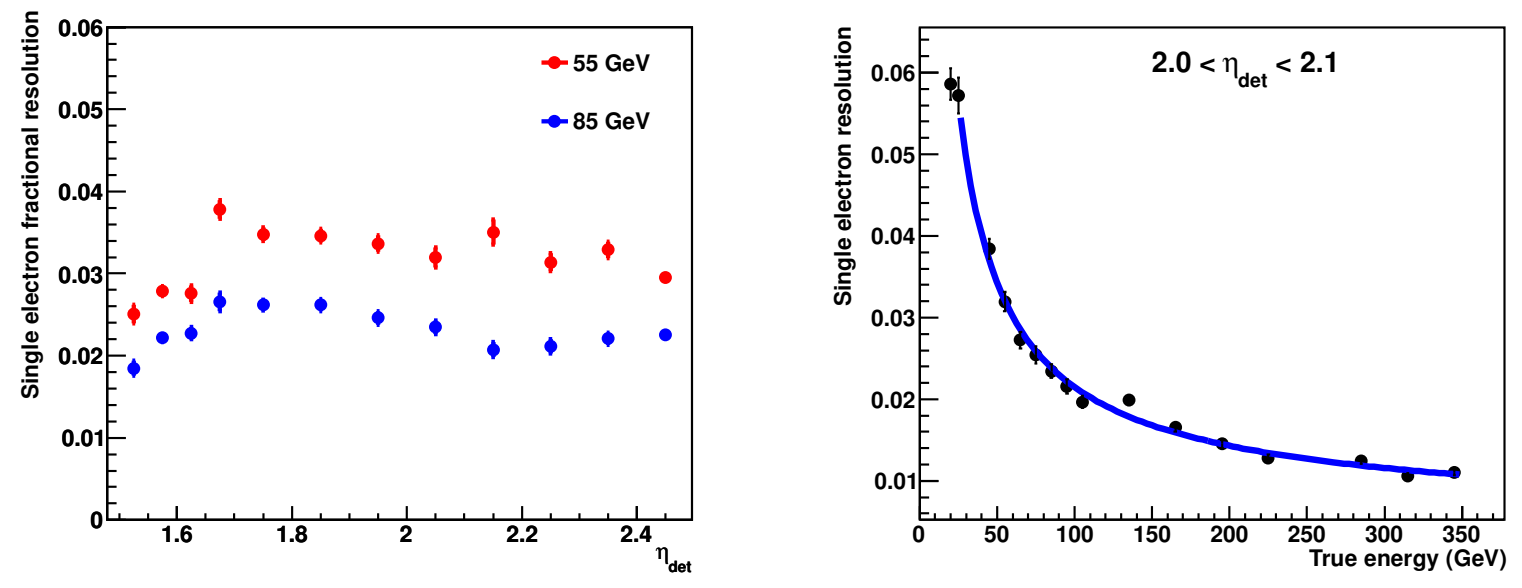

Figure 14.17: (left) Gaussian width in the energy distribution for single monochromatic electrons with energy $55 \mathrm{GeV}$ (red markers) and $85 \mathrm{GeV}$ (blue markers). As expected, the large amount of dead material introduces strong angular dependence. Due to the nonuniformity of the material budget in the forward region, the distribution is not smooth as for the CC. (right) Energy dependence for electrons reconstructed with $2.0<\eta_{\text {det }}<2.1$. The dependence is fitted to a polynomial in $1 / \sqrt{E}$ with an important contribution from the $1 / E$ term. 


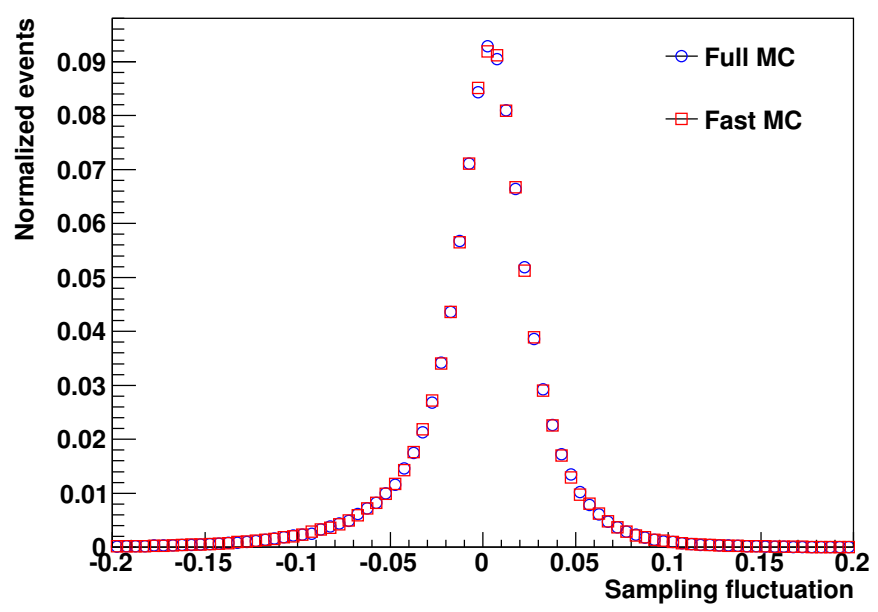

Figure 14.18: Distribution of the electron fractional energy fluctuation for both full MC (blue markers) and fast MC (red markers). This comparison uses a sample of single electrons in which all the energy fluctuation is due to the sampling resolution and, therefore, represents a good test for our model.
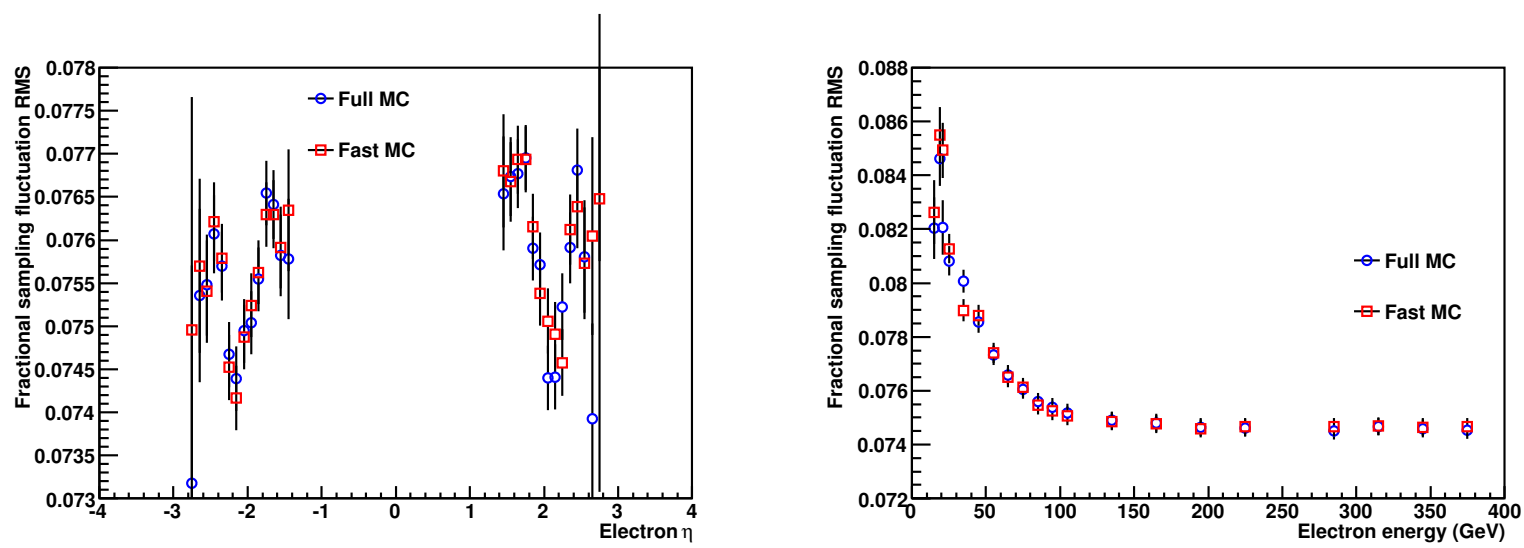

Figure 14.19: We compare the RMS of the electron fractional energy sampling fluctuation in both full MC (blue markers) and fast MC (red markers) for events with different $\eta$ (left) and energy (right) regimes. 


\subsection{EC Jet Misidentification Rate}

We have not yet discussed backgrounds for the measurement using EC electrons. The background from hadronic events in which a jet is misidentified as electron (called a "fake") may be quite different for events with EC electrons than for events with CC electrons used in the previous analysis. Although an assessment of the absolute multijet background events would require a complete study of the track matching probably, in the restricted region of the EC we are interested, this efficiency is similar to the CC. If there is a similar increase in the instrumental fake rate, the multijet background could conceivably be larger. In this section we study the probability of a highly electromagnetic jet to fake an electron.

In the central electron analysis, the tracking match selection criteria removes most of the MJ background. In the efficiency studies for central electrons, forward electrons were selected with a much simpler track matching condition, only requiring the track match probability (see Sec. 4.1) to be larger than 0.01 , but without any requirement on the track $p_{T}$ or number of SMT hits, which were included for the full CC electron selection requirements. Thus, before measuring the fake rate, we study the possibility of keeping this simpler criterion for the $W$ mass analysis with forward electrons.

We select dijet events using the same single electron trigger as used in the analysis, but require the presence of an additional reconstructed jet object. The goal is to select dijet events in which one of the jets has a large EM fraction and was reconstructed as an electron. The electron object is required to satisfy the same selection criteria described in section 4.6 for forward electrons, with a matched track, but without any quality criteria on the matching. The jet object is required to satisfy:

- The jet should be away from the ICD region which has poor energy resolution: $\left|\eta_{\text {det }}\right|<0.8$ or $1.5<\left|\eta_{\text {det }}\right|<2.5$

- High transverse momentum: $p_{T}>20 \mathrm{GeV}$

- EM fraction: $0.05<$ EMF $<0.95$

- At least one track matched to the jet.

- Fraction of energy in the coarse hadronic calorimeter: CHF $<0.4$

- Minimum number of towers comprising at least $90 \%$ of jet scalar $E_{T}:$ N90 $>1$

- Ratio of the energy in the hottest cell to the next-to-hottest cell: HCR $<10$

Additionally, we require electron-jet system to have $\Delta \phi_{\mathrm{e}-\mathrm{jet}}>2.8$. A sample selected with this criteria will have a small, but non negligible, contamination from $Z \rightarrow e e$ and $W \rightarrow e v$ events. Thus, to veto these events, we further require:

- Missing transverse energy: $\mathbb{Z}_{T}<10 \mathrm{GeV}$ 
- Electron-jet invariant mass: $m_{\mathrm{e}-\text { jet }}<70 \mathrm{GeV}$ or $m_{\mathrm{e}-\text { jet }}>110 \mathrm{GeV}$.

We measure the fraction of events in which the associated track to the electron object satisfies an additional quality criteria. Figure 14.20 shows the fake rate as a function of the track matching probability criterion. With this single criterion, the relative fake rates are in the $20-25 \%$ range, larger than the $10-15 \%$ in the CC. It is clear that, by itself, this condition would not be enough to control the multijet background. It is interesting to note the north-south asymmetry in the fake rate, for which we do not have an understanding.
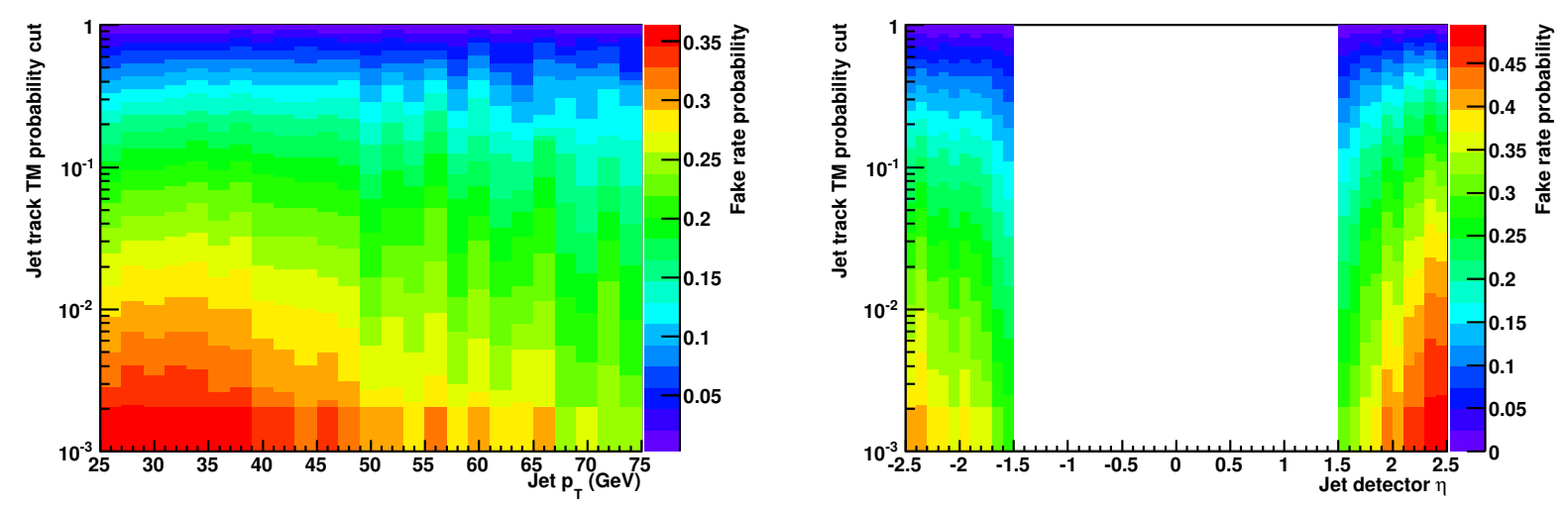

Figure 14.20: Probability of a highly electromagnetic jet in the EC to fake an electron with a associated track as a function of the spatial track matching probability and as a function of the jet $p_{T}$ (left) and jet detector $\eta$ (right).

Figure 14.21 shows the fake rate as a function of the minimum track $p_{T}$ selection criteria. Once again, by itself, the track $p_{T}$ criterion cannot reduce the fake rate to levels similar as in the central electron analysis.

Since neither single criterion is strong enough to control the multijet background, we measure the fake rate adopting the same track selection as done for the $\mathrm{CC}$ analysis ${ }^{3}$,

- Track matching probability $>0.01$

- Track $p_{T}>10 \mathrm{GeV}$

- At least one SMT hit

Figure 14.22 shows the fake rate, separately for the north and south EC, as a function of the jet $p_{T}$ and detector $\eta$ when the three criteria as applied. The fake rate is back to the range $10-15 \%$, similar to the CC. However, differently from the CC, it is not possible to assume a $\eta_{\text {det }}$-independent fake rate parametrization. We parametrize the fake rate in the north and south ECs individually using a simple quadratic polynomial:

\footnotetext{
${ }^{3}$ Although we could test the number of SMT hits criterion the same way as we tested the other criteria, there are additional reasons to require at least one hit in the silicon tracker.
} 

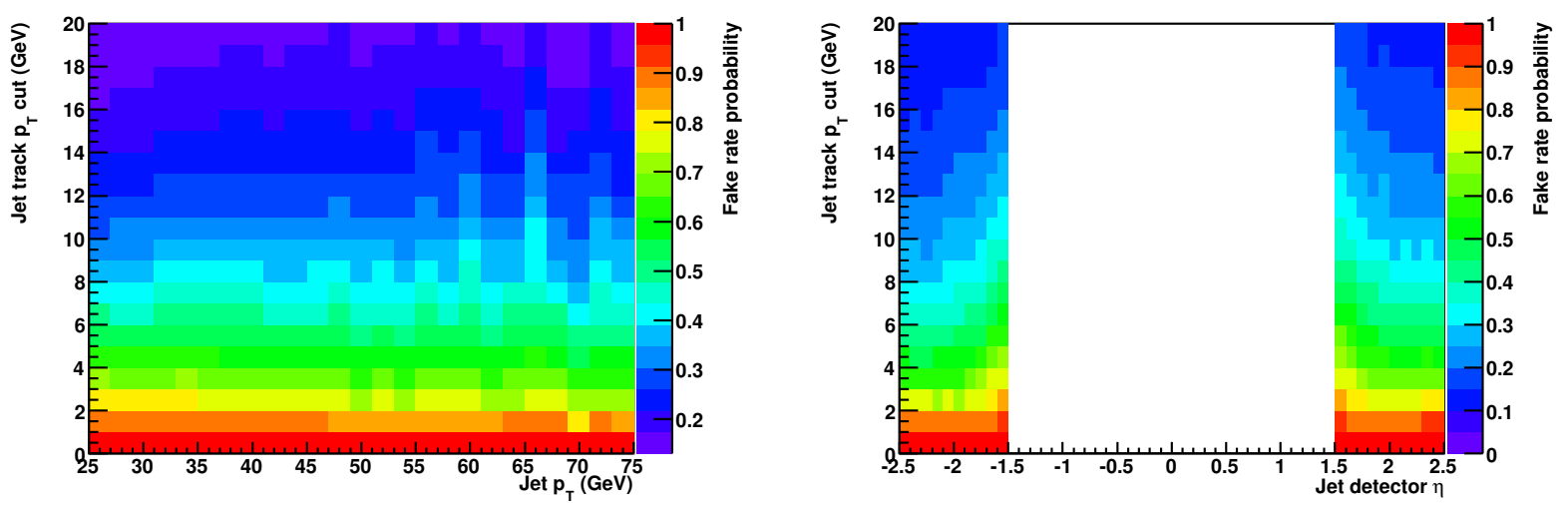

Figure 14.21: Relative probability of a highly electromagnetic jet in the EC to fake an electron with a associated track as a function of the minimum track $p_{T}$ criterion and as a function of the jet $p_{T}$ (left) and jet detector $\eta$ (right).
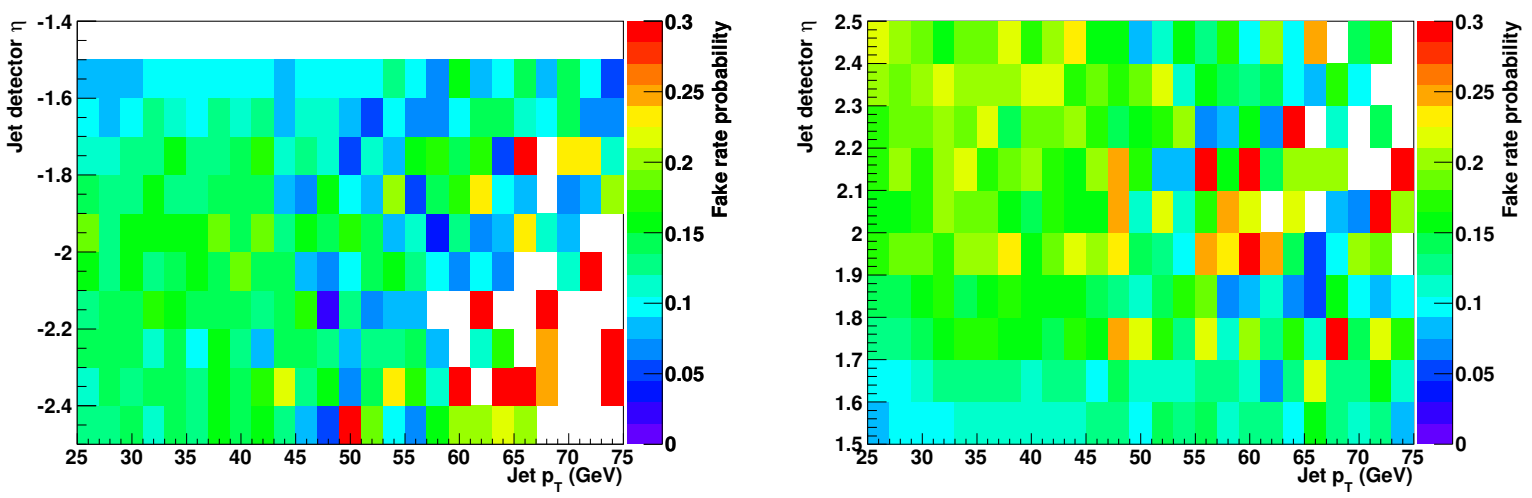

Figure 14.22: Relative probability of a highly electromagnetic jet in the north (left) and south (right) ECs to fake an electron with the same track criteria as in the central calorimeter analysis, as a function of the jet $p_{T}$ and jet detector $\eta$. 


$$
\text { fake rate }=\left(p_{0}+p_{1} \times \eta_{\mathrm{det}}+p_{2} \times \eta_{\mathrm{det}}^{2}\right)+\left(p_{3}+p_{4} \times \eta_{\mathrm{det}}+p_{5} \times \eta_{\mathrm{det}}^{2}\right) \times p_{T}
$$

The values for the parameters are listed in table 14.1. Figures 14.23 and 14.24 show the function with the fitted parameters and the residuals distribution. Both functions fit well the distribution in the whole acceptance range.

\begin{tabular}{|c|c|c|}
\hline \hline Parameter & North end-cap & South end-cap \\
\hline$p_{0}$ & $-1.7529 \pm 0.0088$ & $-0.8215 \pm 0.0076$ \\
$p_{1}$ & $-1.8725 \pm 0.0065$ & $0.7812 \pm 0.0055$ \\
$p_{2}$ & $-0.4525 \pm 0.0027$ & $-0.1392 \pm 0.0023$ \\
$p_{3}$ & $0.03446 \pm 0.00025$ & $0.00694 \pm 0.00022$ \\
$p_{4}$ & $0.03513 \pm 0.00019$ & $-0.00371 \pm 0.00016$ \\
$p_{5}$ & $0.00863 \pm 0.00008$ & $0.00003 \pm 0.00007$ \\
\hline \hline
\end{tabular}

Table 14.1: Parametrization of the fake rate probability in the north and south ECs.
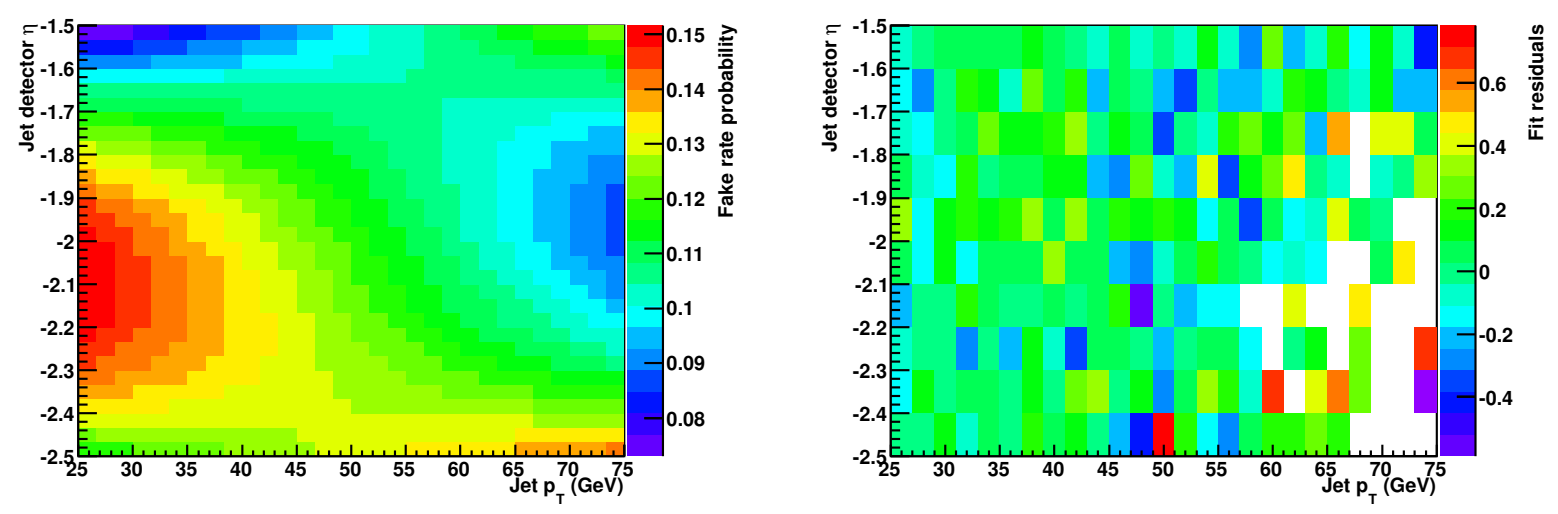

Figure 14.23: Parametrization of the fake rate probability in the north EC as a function of the jet $p_{T}$ and $\eta_{\text {det }}$. The left plot shows a graph of the parametrized function. The right plot shows the signed $\chi$ distribution with respect to the parent distribution.

\subsection{Full MC Efficiency Model for Forward Electrons}

The efficiency model used in the fast MC was thoroughly described in Sec. 7.2. It has multiple components that describe either the impact of some particular source of inefficiency or the effect of all sources on a particular set of kinematical variables. Here, we 

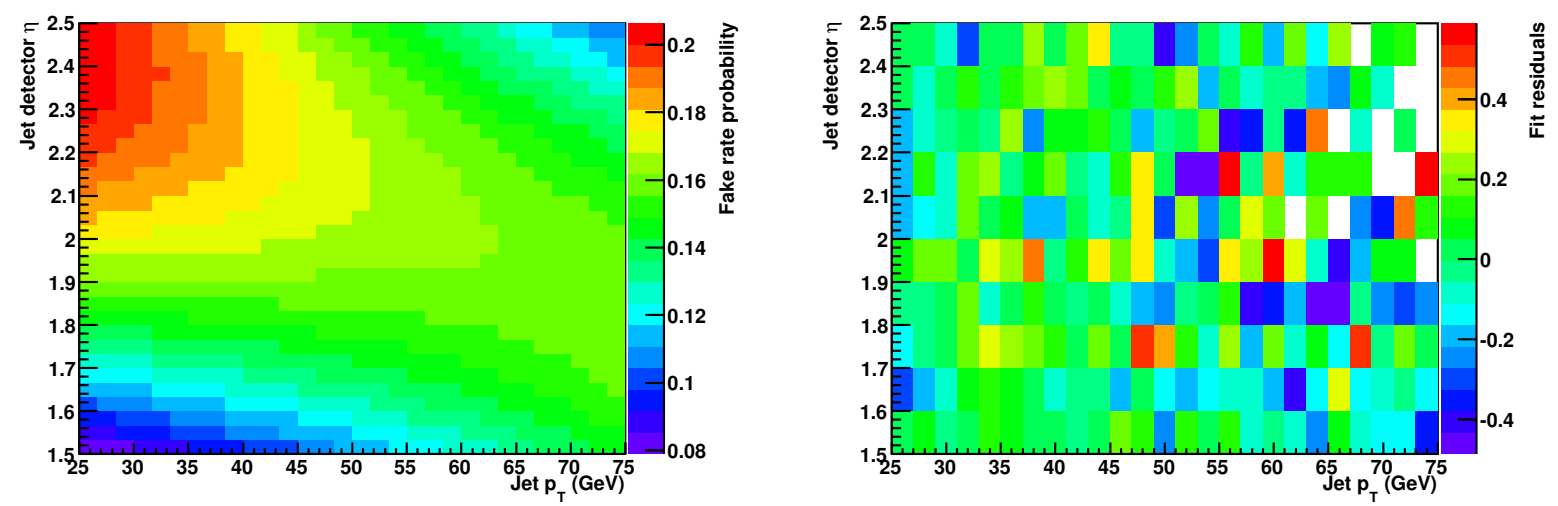

Figure 14.24: Parametrization of the fake rate probability in the south EC as a function of the jet $p_{T}$ and $\eta_{\text {det }}$. The left plot shows a graph of the parametrized function. The right plot shows the signed $\chi$ distribution with respect to the parent distribution.

will focus on the former category since the latter depends on the existence of an already $Z \rightarrow e e$ tuned version of the fast MC. We have already described the FSR efficiency parameterization (see Sec.14.1) and there is no trigger simulation in our full MC simulation, so the trigger efficiency is not a worry for the full MC test. Thus, we will consider here the track matching efficiency and the EMID criteria efficiency. The track matching efficiency is further divided into two parts: the loose track matching efficiency, defined by requiring that the cluster and associated track directions are within $\Delta \eta<0.05$ and $\Delta \phi<0.05$ of each other, and the tight track matching efficiency, defined by requiring a good probability for the spatial matching as well as a hit in the SMT and a mininum track $p_{T}$ (as motivated in Sec. 14.7). In the EC there is no equivalent of a $\phi_{\text {mod }}$ efficiency, since the EM End Calorimeter was built as a monolithic ring and there were no cracks, as described in Sec. 2.4.1.

To measure these three efficiencies we use $Z \rightarrow e e$ full MC events. They are measured using both the tag and probe method as well as directly counting the number of generated electrons that are reconstructed and satisfy the criteria for each efficiency (truth level efficiency). By comparing both methods, we can study biases introduced by the requirement of a tagging electron. The selection of $Z \rightarrow e e$ events is exactly the same as used in the $W$ mass analysis. The EMID efficiency is measured relatively to reconstructed energy clusters, the loose track matching efficiency is measured relatively to reconstructed clusters that satisfy the EMID requirements and, finally, the tight track matching efficiency is measured realtively to reconstructed clusters that satisfy both the EMID requirement and the loose track matching criterion. 


\subsubsection{EMID Efficiency}

The EMID efficiency is determined as a function of the electron $\eta_{\operatorname{det}}$ and $p_{T}$. Figure 14.25 shows the efficiency for the tag and probe and truth methods. Figure 14.26 compares the projection of the two methods over $\eta_{\mathrm{det}}$ and $p_{T}$. It is clear that a bias exists at large $p_{T}$. Since we select $Z$ boson with low $p_{T}$, when the probe electron has high $p_{T}$, compared to half the $Z$ boson mass, the tag electron will have low $p_{T}$. Electrons at low momentum have smaller detection efficiency which biases the tagging towards events in which, as a whole, is easier to detect electrons (for instance, low hadronic activity). The tag and probe bias is only relevant for electrons with $p_{T}>50 \mathrm{GeV}$. These events are not in the fit region for the $p_{T}^{e}$ observable and contribute marginally to the other two observable. We can use the measured ratio between the two methods to correct the determination of this efficiency for collider data events, when only the tag and probe method is possible.
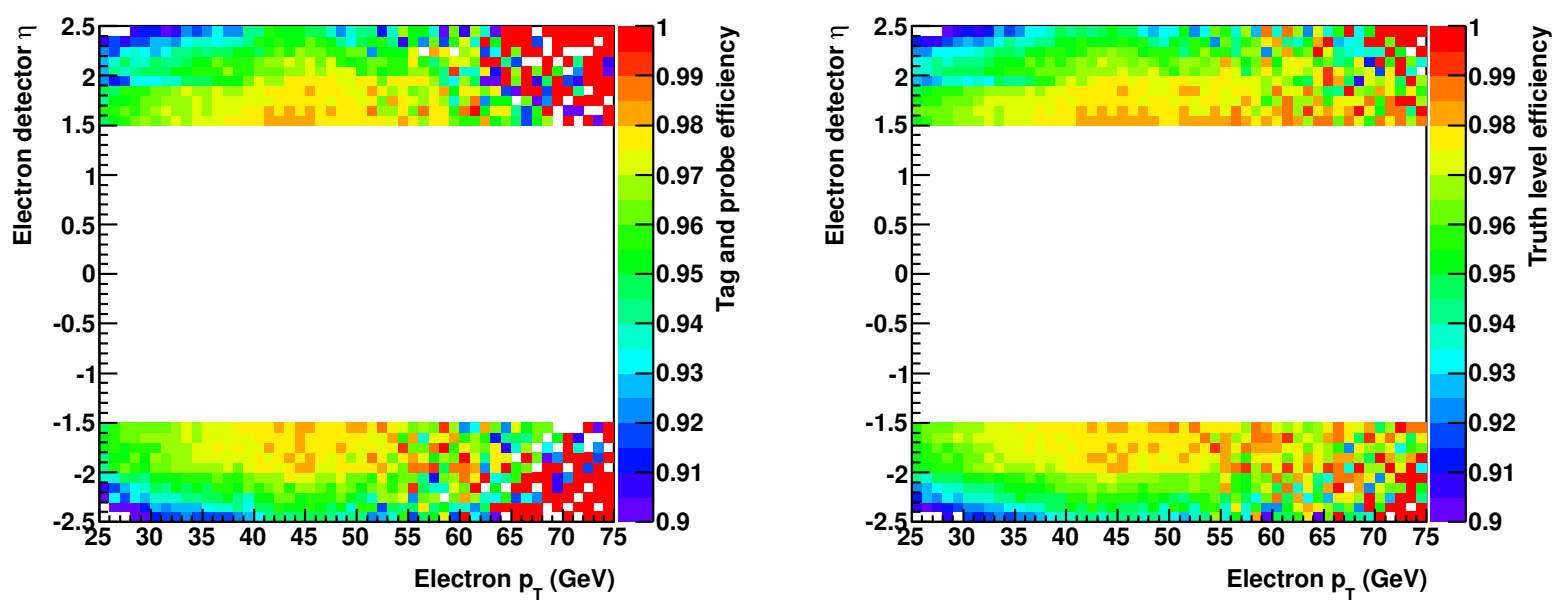

Figure 14.25: EMID efficiency as a function of the electron $p_{T}$ and $\eta_{\text {det }}$. The efficiency is derived using the tag and probe method (left) and the truth method (right).

\subsubsection{Track Matching Efficiencies}

The loose and tight track matching efficiencies are determined as a function of the track $\eta$ and primary vertex $z$ coordinate. Figure 14.27 shows the loose track matching efficiency determined with both tag and probe method and truth method. The loose track matching criteria is the most important source of inefficiency in the forward direction, since a large part of the calorimeter acceptance is only covered by the SMT for particles originating from the center of the detector. Figure 14.28 shows the tag and probe bias in both variables.

There is a very large bias in the determination of the loose track matching efficiency for events with $\left|z_{V}\right|>30 \mathrm{~cm}$. By inspecting the plots on Fig. 14.27, it is clear that the the 

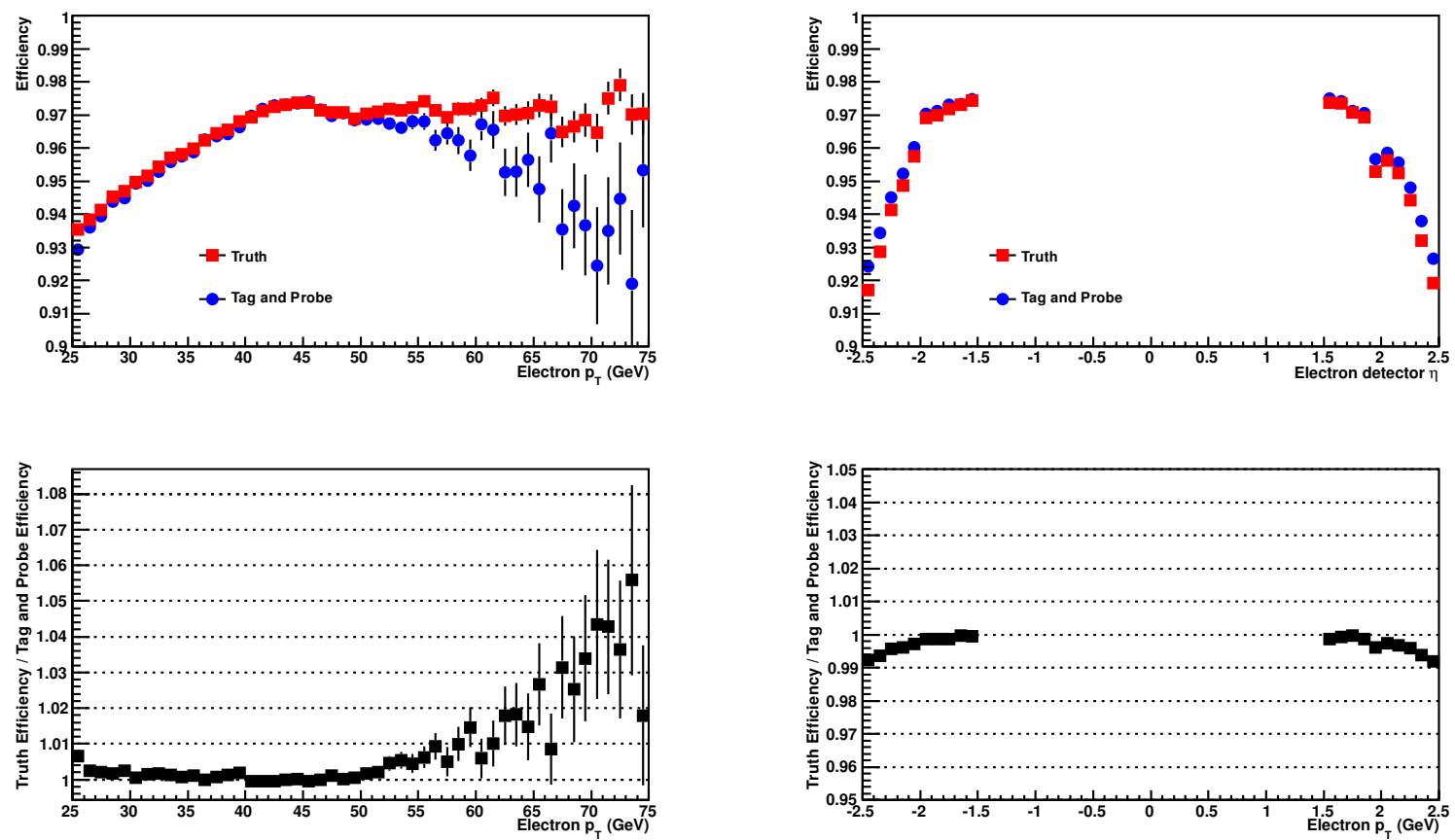

Figure 14.26: Projection of the EMID efficiency over electron $p_{T}$ (left) and $\eta_{\text {det }}$ (right). The bottom plots show the ratio between the efficiency derived by the truth method to the tag and probe method.
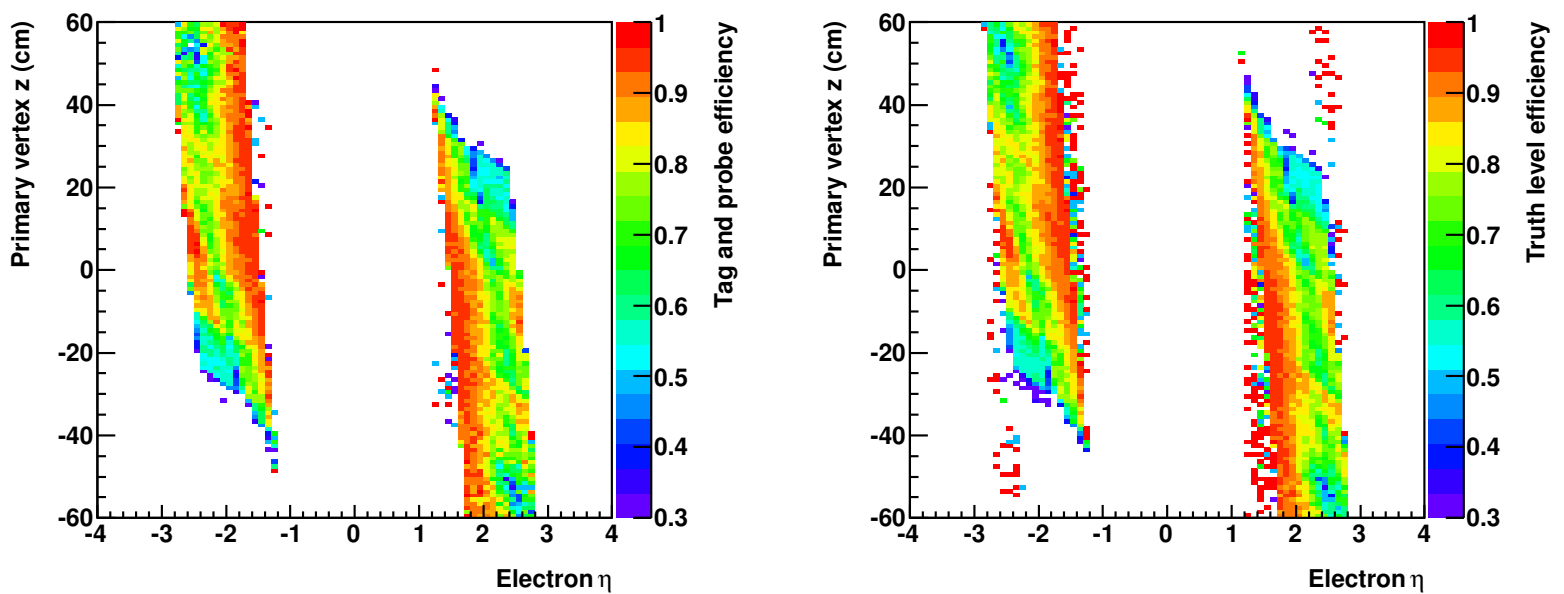

Figure 14.27: Loose track match efficiency as a function of the electron $p_{T}$ and $\eta_{\text {det }}$. The efficiency is derived using the tag and probe method (left) and the truth method (right). 

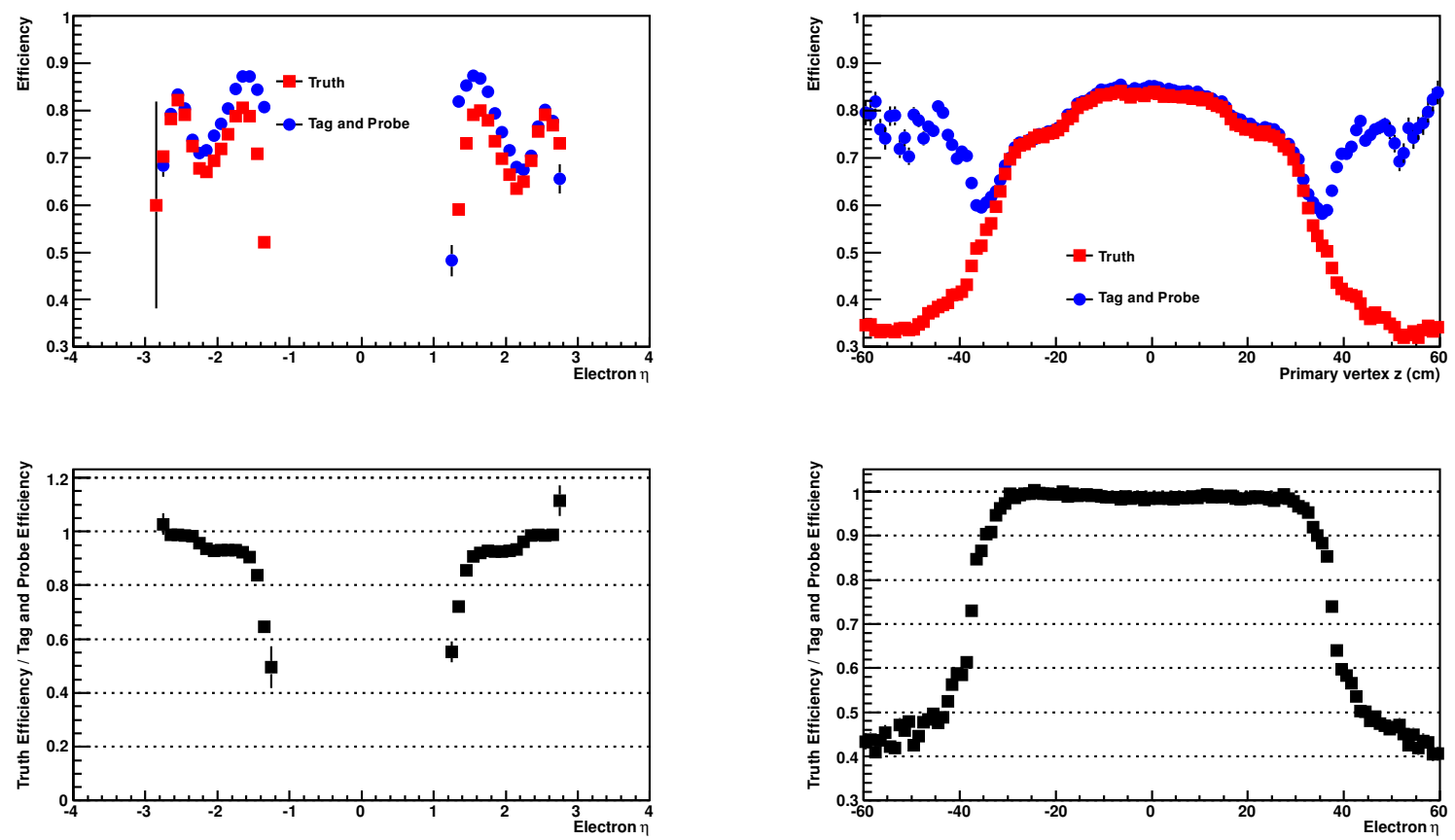

Figure 14.28: Projection of the loose efficiency on electron $\eta$ (left) and $z_{V}$ (right). The bottom plots show the ratio between the efficiency derived by the truth method to the tag and probe method.

bias arises mostly from events with $\left|z_{V}\right|>30 \mathrm{~cm}$ and $z_{V} \times \eta>0$. Those are electrons whose tracks depend solely on the three outer F disks and on the single functional H disk after the installation of the Layer 0 . To avoid correcting a bias larger than $50 \%$, we propose an additional fiducial criteria in the EC electron selection:

- If $\left|z_{V}\right|>30 \mathrm{~cm}$, then an EC electron must satisfy $z_{V} \times \eta<0$.

With this additional requirement, we can rederive the loose track matching efficiency and find a smaller bias in the tag and probe determination, as seen on Figs. 14.29 and 14.30 Using the same additional fiducial requirement, we determine the tight track matching efficiency relative to clusters with a loose track match, by requiring:

- Probability of the track matching $\mathrm{P}\left(\chi_{\mathrm{TM}}^{2}\right)>0.01$, based on $\chi_{\mathrm{TM}}^{2}$ defined on Sec. 4.1 ,

- Track momentum $p_{T}^{\text {track }}>10 \mathrm{GeV}$,

- At least one SMT hit.

The result for both tag and probe and truth methods can be seen on Fig. 14.31 . 

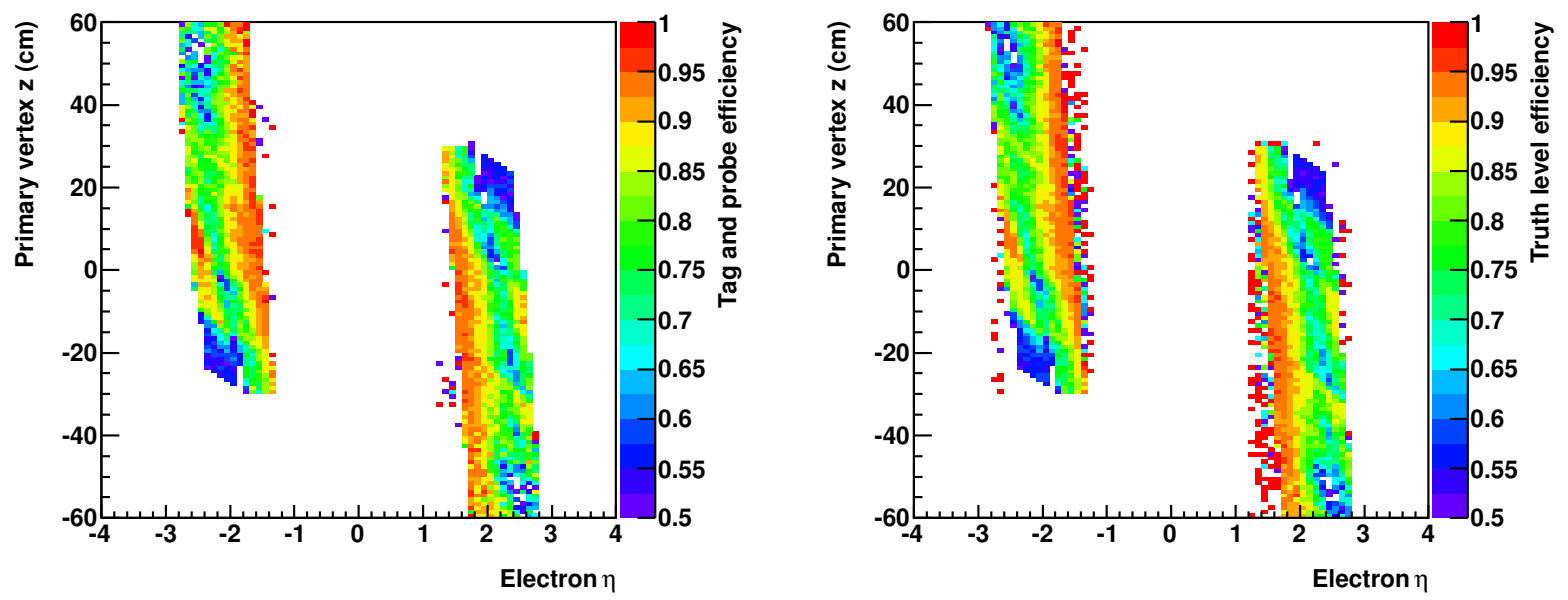

Figure 14.29: Loose track match efficiency as a function of the electron $\eta$ and $z_{V}$. The efficiency is derived using the tag and probe method (left) and the truth method (right). The EC electrons are required to satisfy the fiducial criterion $z_{V} \times \eta<0$ when $\left|z_{V}\right|>$ $30 \mathrm{~cm}$.
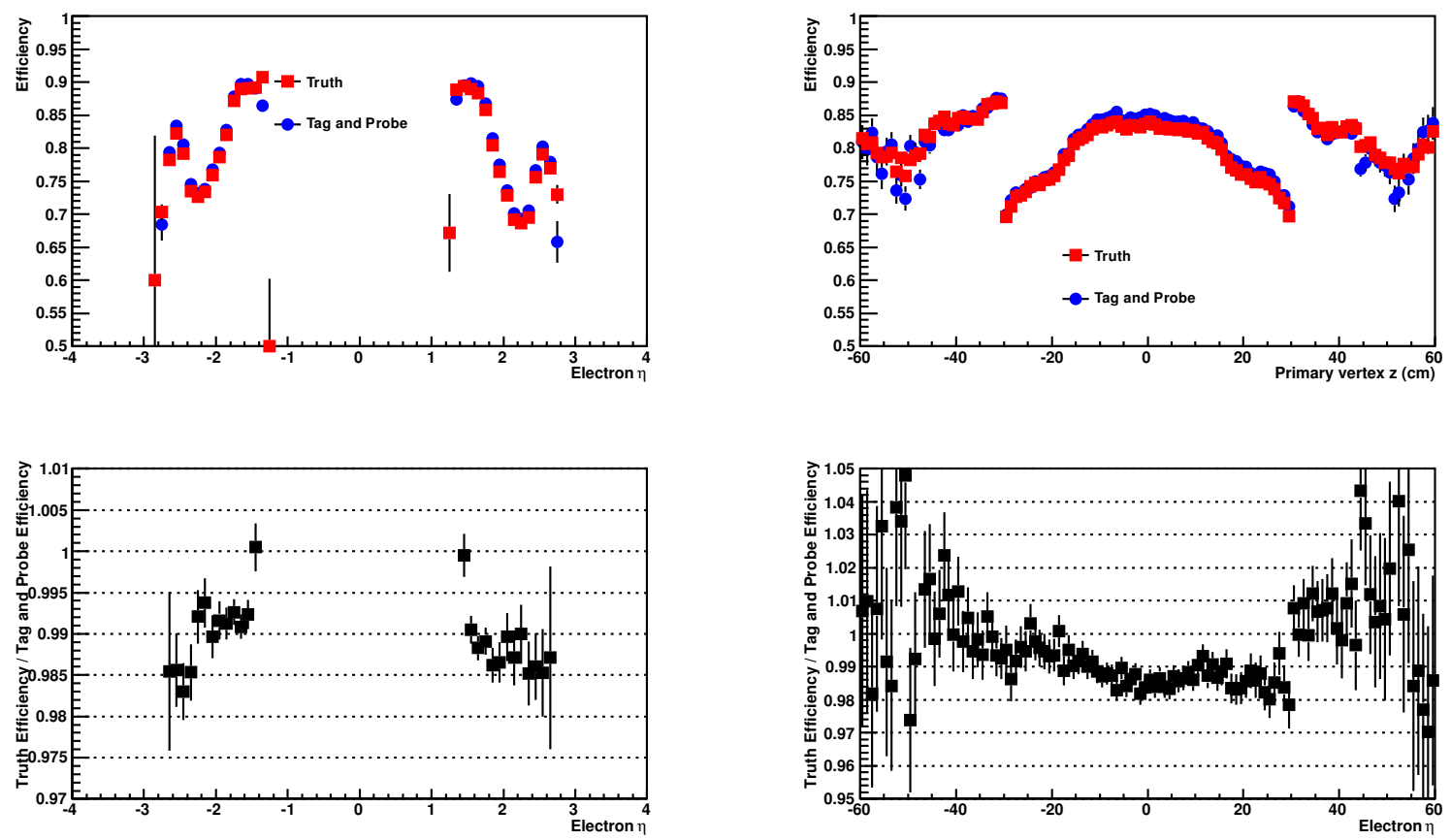

Figure 14.30: Projection of the loose efficiency on electron $\eta$ (left) and $z_{V}$ (right). The bottom plots show the ratio between the efficiency derived by the truth method to the tag and probe method. The EC electrons are required to satisfy the fiducial criterion $z_{V} \times \eta<0$ when $\left|z_{V}\right|>30 \mathrm{~cm}$. 

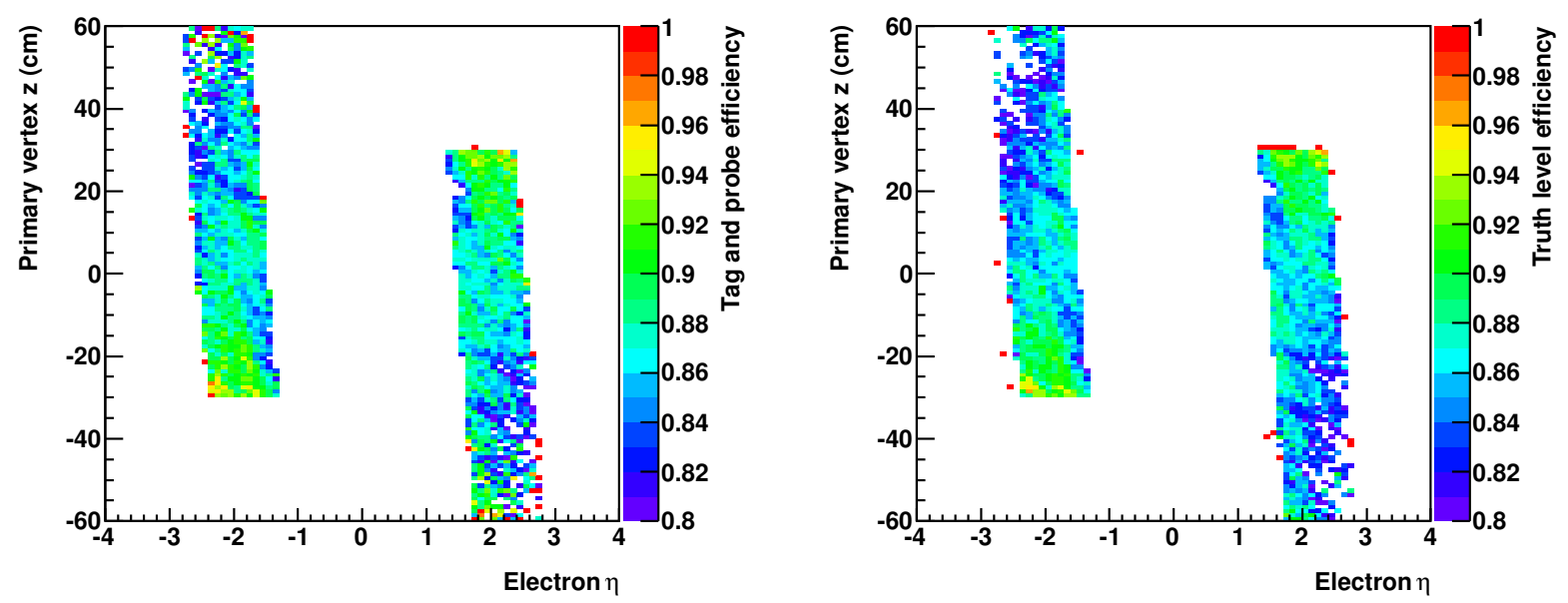

Figure 14.31: Tight track match efficiency as a function of the electron $\eta$ and $z_{V}$. The efficiency is derived using the tag and probe method (left) and the truth method (right). The EC electrons are required to satisfy the fiducial criterion $z_{V} \times \eta<0$ when $\left|z_{V}\right|>$ $30 \mathrm{~cm}$.

\subsection{Basic Comparison Plots for Forward Electrons}

We close this section comparing a fast MC for forward electrons which has all the models presented here with a $W \rightarrow e v$ full MC sample. The size of the full MC sample used is equivalent to $24 \mathrm{fb}^{-1}$ of integrated luminosity. It is a crude comparison since it does not have any fine tune using $Z \rightarrow e e$ events for the electron response and resolution models, for the electron efficiency model nor for the hadronic recoil response and resolution models. Moreover, it uses the very incomplete efficiency model described above which does not fully describe the complicated kinematical correlations a complete model will eventually know about. It is, thus, an exercise to see the reach of the models presented here by themselves and a starting points to a complete tuning of the parametrizations using $Z \rightarrow e e$ events. Figure 14.32 shows the comparison between fast and full MC for the basic transverse mass $m_{T}$, electron transverse momentum $p_{T}^{e}$ and missing transverse energy $\mathbb{E}_{T}$ distributions. The incompleteness of the efficiency model is evident as well as the need for the response and resolution tune. 

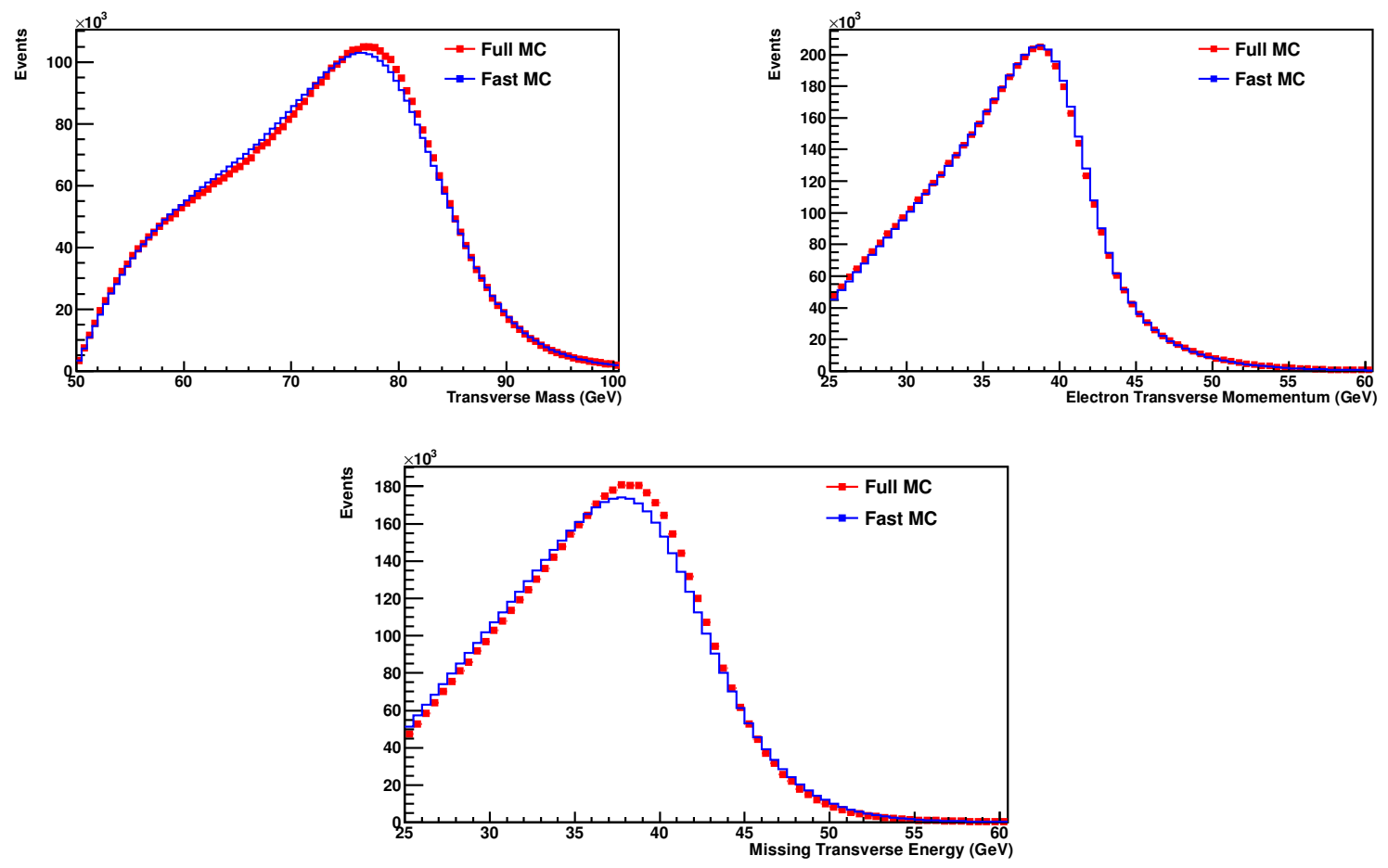

Figure 14.32: Comparison between fast and full MC transverse mass $m_{T}$ (top left), electron transverse momentum $p_{T}^{e}$ (top right), and missing transverse energy $\mathbb{E}_{T}$ (bottom) distributions from $W \rightarrow e v$ events. The fast MC has all the models described in this section but lacks any tune of the electron response and resolution, electron efficiency and hadronic response and resolution using $Z \rightarrow e e$ events. The efficiency model is incomplete, as can be clearly seen in low energy part of the histograms. 


\section{Part IV}

\section{Conclusions}




\section{Chapter 15}

\section{Conclusions}

The $W$ boson mass is, today, the most important Electroweak Precision Observable that can be measured with the experimental tools available. It provides a strong consistency test of the electroweak sector of the Standard Model and complementary to the Higgs observables that are being measured at the LHC experiments.

We presented a measurement of the $W$ boson mass using $4.3 \mathrm{fb}^{-1}$ of integrated luminosity of central electrons, collected with the D0 detector between 2006 and 2009. The result, when combined with the previous $1.0 \mathrm{fb}^{-1}$ measurement, gives:

$$
M_{W}=80.375 \pm 0.023 \mathrm{GeV},
$$

which is as precise as the world average was before this and the most recent CDF collaboration measurement. When these two measurements are combined with the measurements done by the LEP experiments, the new world average value of the $W$ boson mass is:

$$
M_{W}=80.385 \pm 0.015 \mathrm{GeV} .
$$

Both measurement have been published in Physical Review Letters journal in April 2012.

The uncertainty in the measurement presented in this Dissertation is dominated by the electron energy scale determination and by the knowledge of the parton distribution functions. We proposed methods of reducing the systematic uncertainties in future measurements, in particular the one related to the parton distribution function. We showed that including forward electrons is necessary to reach the planned final uncertainty of $\delta M_{W}=10 \mathrm{MeV}$ at the Tevatron [96].

We presented a detailed calibration of the D0 End Calorimeter towards a future measurement with forward electrons. Many parametrized models for electron response have been constructed, building on the experience of the central electron fast $\mathrm{MC}$, but facing important challenges of the high $\eta$ region. A complete fast MC for forward electron would still require a thorough understanding and modeling of the electron detection efficiency in this region. But this work sets important milestones in this direction. 


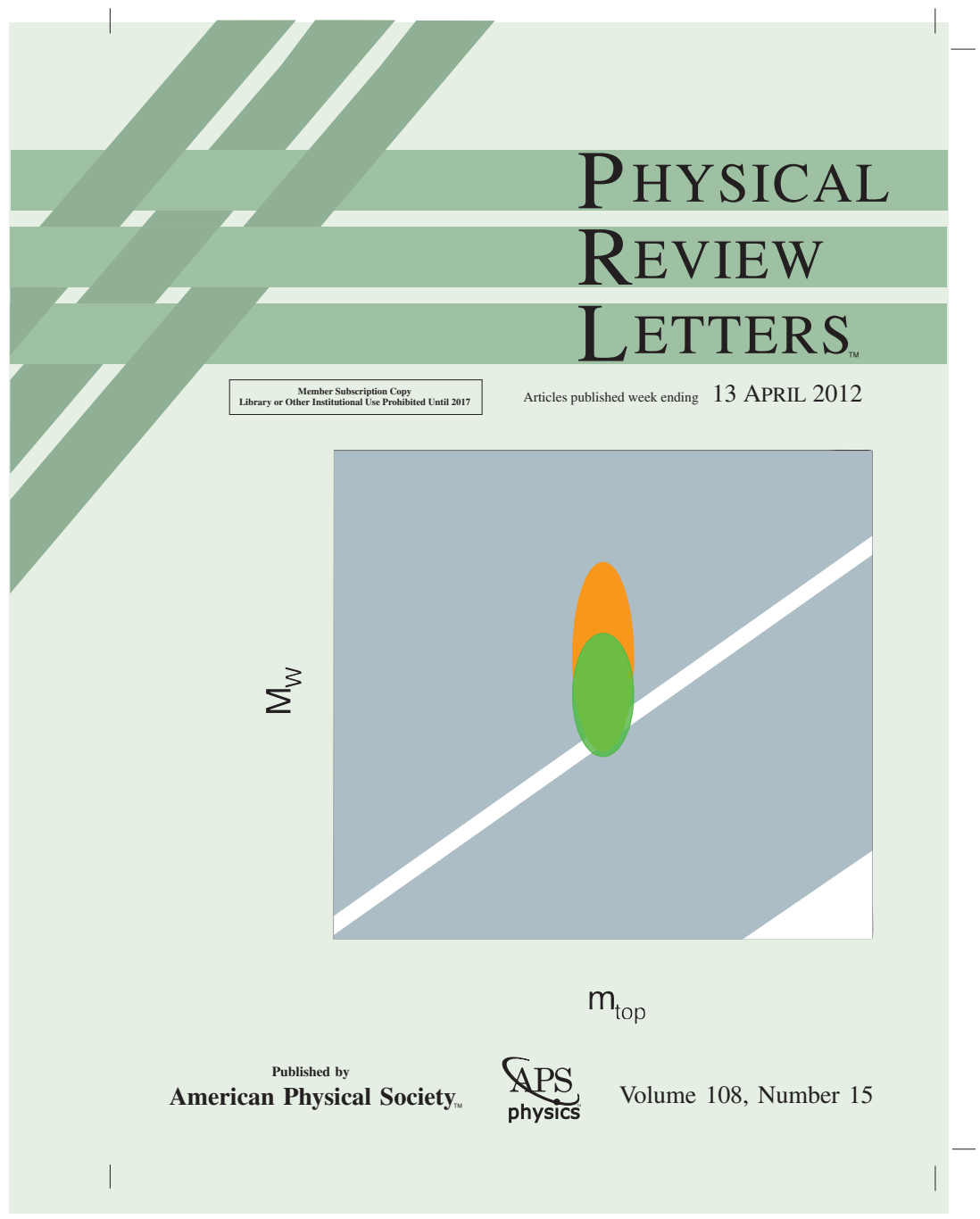

Figure 15.1: Cover of the Physical Review Letters issue in which the latest D0 and CDF measurements of the $W$ boson mass have been published. 


\section{Bibliography}

[1] R. Wigmans, Calorimetry: Energy Measurements in Particle Physics. Oxford Science Publications. Clarendon Press, 2000. http://books . google.com/books?id=vD9RFluMD5sC

[2] ATLAS Collaboration , G. Aad et al., "Observation of a new particle in the search for the Standard Model Higgs boson with the ATLAS detector at the LHC," Phys.Lett. B716 (2012) 1-29, arXiv: 1207.7214 [hep-ex].

[3] CMS Collaboration, S. Chatrchyan et al., "Observation of a new boson at a mass of $125 \mathrm{GeV}$ with the CMS experiment at the LHC," Phys.Lett. B716 (2012) 30-61, arXiv:1207.7235 [hep-ex].

[4] M. Baak, M. Goebel, J. Haller, A. Hoecker, D. Kennedy, et al., "The Electroweak Fit of the Standard Model after the Discovery of a New Boson at the LHC," Eur.Phys.J. C72 (2012) 2205, arXiv: 1209.2716 [hep-ph].

[5] MuLan Collaboration, V. Tishchenko et al., "Detailed Report of the MuLan Measurement of the Positive Muon Lifetime and Determination of the Fermi Constant," arXiv:1211.0960 [hep-ex].

[6] M. Awramik, M. Czakon, A. Freitas, and G. Weiglein, "Precise prediction for the W boson mass in the standard model," Phys.Rev. D69 (2004) 053006. arXiv:hep-ph/0311148 [hep-ph].

[7] A. Freitas, W. Hollik, W. Walter, and G. Weiglein, "Electroweak two loop corrections to the $M(W)-M(Z)$ mass correlation in the standard model," Nucl.Phys. B632 (2002) 189-218, arXiv: hep-ph/0202131 [hep-ph].

[8] ALEPH Collaboration, DELPHI Collaboration, L3 Collaboration, OPAL Collaboration, SLD Collaboration, LEP Electroweak Working Group, SLD Electroweak Group, SLD Heavy Flavour Group , S. Schael et al., "Precision electroweak measurements on the $Z$ resonance," Phys.Rept. 427 (2006) 257-454, arXiv:hep-ex/0509008 [hep-ex].

[9] ALEPH Collaboration, CDF Collaboration, D0 Collaboration, DELPHI Collaboration, L3 Collaboration, OPAL Collaboration, SLD Collaboration, LEP 
Electroweak Working Group, Tevatron Electroweak Working Group, SLD Electroweak and Heavy Flavour Groups, "Precision Electroweak Measurements and Constraints on the Standard Model," arXiv:1012.2367 [hep-ex].

[10] CDF Collaboration, T. Aaltonen et al., "Precise measurement of the $W$-boson mass with the CDF II detector," Phys.Rev.Lett. 108 (2012) 151803, arXiv:1203. 0275 [hep-ex].

[11] M. Davier, A. Hoecker, B. Malaescu, and Z. Zhang, "Reevaluation of the Hadronic Contributions to the Muon $g-2$ and to $\alpha\left(M_{Z}\right)$," Eur.Phys.J. C71 (2011) 1515, arXiv:1010.4180 [hep-ph].

[12] T. Blum, M. Bucho, N. Christ, A. Kronfeld, P. Mackenzie, S. Sharpe, R. Sugar, and R. Van de Water, "Lattice QCD at the Intensity Frontier." Feb., 2012.

[13] R. Ellis, W. Stirling, and B. Webber, QCD and Collider Physics. Cambridge Monographs on Particle Physics, Nuclear Physics and Cosmology. Cambridge University Press, 2003. http://books . google.com/books?id=TqrPVoS6s0UC

[14] J. Collins, Foundations of Perturbative QCD. Cambridge Monographs on Particle Physics, Nuclear Physics and Cosmology. Cambridge University Press, 2011. http://books. google.com/books?id=0xGi1KW9vykC

[15] J. Collins, D. E. Soper, and G. Sterman, "Transverse momentum distribution in Drell-Yan pair and $W$ and $Z$ boson production," Nuclear Physics B 250 no. 14, (1985) $199-224$ http://www.sciencedirect.com/science/article/pii/0550321385904791.

[16] C. Balazs and C.-P. Yuan, "Soft gluon effects on lepton pairs at hadron colliders," Phys.Rev. D56 (1997) 5558-5583, arXiv: hep-ph/9704258 [hep-ph].

[17] F. Landry, R. Brock, P. M. Nadolsky, and C.-P. Yuan, "Tevatron Run-1 Z boson data and Collins-Soper-Sterman resummation formalism," Phys.Rev. D67 (2003) 073016 , arXiv:hep-ph/0212159 [hep-ph].

[18] G. Bozzi, S. Catani, G. Ferrera, D. de Florian, and M. Grazzini, "Production of Drell-Yan lepton pairs in hadron collisions: Transverse-momentum resummation at next-to-next-to-leading logarithmic accuracy," Phys.Lett. B696 (2011) 207-213, arXiv:1007.2351 [hep-ph].

[19] W. Marciano and A. Sirlin, "Radiative Corrections to Neutrino Induced Neutral Current Phenomena in the $S U(2)_{L} \times U(1)$ Theory," Phys.Rev. D22 (1980) 2695.

[20] A. Sirlin and A. Ferroglia, "Radiative corrections in precision electroweak physics: A historical perspective," Rev. Mod. Phys. 85 (Feb, 2013) 263-297. http://link.aps.org/doi/10.1103/RevModPhys.85.263 
[21] A. Sirlin, "Radiative Corrections in the $S U(2)_{L} \times U(1)$ Theory: A Simple Renormalization Framework," Phys.Rev. D22 (1980) 971-981.

[22] F. Jegerlehner, M. Y. Kalmykov, and O. Veretin, " $\overline{\mathrm{MS}}$ versus pole masses of gauge bosons: Electroweak bosonic two loop corrections," Nucl.Phys. B641 (2002) 285-326, arXiv:hep-ph/0105304 [hep-ph].

[23] F. Jegerlehner, M. Y. Kalmykov, and O. Veretin, "MS versus pole masses of gauge bosons. 2. Two loop electroweak fermion corrections," Nucl.Phys. B658 (2003) 49-112, arXiv:hep-ph/0212319 [hep-ph].

[24] UA2 Collaboration, J. Alitti et al., "An Improved determination of the ratio of $W$ and $Z$ masses at the CERN $\bar{p} p$ collider," Phys.Lett. B276 (1992) 354-364.

[25] UA1 Collaboration , C. Albajar et al., "Studies of Intermediate Vector Boson Production and Decay in UA1 at the CERN Proton - Antiproton Collider," Z.Phys. C44 (1989) 15-61.

[26] H1 Collaboration , Z.-Q. Zhang, "A determination of electroweak parameters at HERA," POS HEP2005 (2006) 293.

[27] ZEUS Collaboration, S. Chekanov et al., "Measurement of high $Q^{2}$ charged current cross-sections in e- $\mathrm{p}$ deep inelastic scattering at HERA," Phys.Lett. B539 (2002) 197-217, arXiv: hep-ex/0205091 [hep-ex].

[28] ALEPH Collaboration, S. Schael et al., "Measurement of the $W$ boson mass and width in $e^{+} e^{-}$collisions at LEP," Eur.Phys.J. C47 (2006) 309-335, arXiv:hep-ex/0605011 [hep-ex].

[29] DELPHI Collaboration, J. Abdallah et al., "Measurement of the Mass and Width of the $W$ Boson in $e^{+} e^{-}$Collisions at $\sqrt{s}=161-\mathrm{GeV}-209-\mathrm{GeV}$," Eur.Phys.J. C55 (2008) 1-38, arXiv:0803.2534 [hep-ex].

[30] L3 Collaboration, P. Achard et al., "Measurement of the mass and the width of the W boson at LEP," Eur.Phys.J. C45 (2006) 569-587, arXiv: hep-ex/0511049 [hep-ex].

[31] OPAL Collaboration , G. Abbiendi et al., "Measurement of the mass and width of the $W$ boson," Eur.Phys.J. C45 (2006) 307-335, arXiv: hep-ex/0508060 [hep-ex]

[32] D0 Collaboration, V. M. Abazov et al., "Measurement of the W boson mass," Phys.Rev.Lett. 103 (2009) 141801, arXiv:0908.0766 [hep-ex].

[33] D0 Collaboration , V. M. Abazov et al., "Measurement of the $W$ Boson Mass with the D0 Detector," Phys.Rev.Lett. 108 (2012) 151804, arXiv:1203.0293 [hep-ex]. 
[34] CDF Collaboration, D0 Collaboration, T. E. W. Group, "2012 Update of the Combination of CDF and D0 Results for the Mass of the $W$ Boson," arXiv:1204.0042 [hep-ex].

[35] V. Papadimitriou, "Luminosity determination at the Tevatron," arXiv:1106.5182 [physics.ins-det].

[36] M. Begel, D. Edmunds, P. Laurens, and R. Partridge, “D0 Luminosity in Run II: Delivered." D0 Note 3970, May, 2003.

[37] B. Casey, M. Corcoran, K. DeVaughan, Y. Enari, E. Gallas, et al., "The D0 Run IIb Luminosity Measurement," Nucl.Instrum.Meth. A698 (2013) 208-223, arXiv:1204.0461 [hep-ex].

[38] D0 Collaboration, V. Abazov et al., "The Upgraded D0 detector," Nucl.Instrum.Meth. A565 (2006) 463-537, arXiv : physics/0507191 [physics.ins-det].

[39] D0 Collaboration , G. A. Alves, "The Forward proton detector at D0," arXiv:hep-ex/9804005 [hep-ex].

[40] D0 Collaboration, R. Angstadt et al., "The Layer 0 Inner Silicon Detector of the D0 Experiment," Nucl.Instrum.Meth. A622 (2010) 298-310, arXiv: 0911.2522 [physics.ins-det].

[41] D0 Collaboration, V. Abazov et al., "Measurement of the forward-backward charge asymmetry and extraction of $\sin ^{2} \theta_{W}^{\text {eff }}$ in $p \bar{p} \rightarrow Z / \gamma^{*}+X \rightarrow e^{+} e^{-}+X$ events produced at $\sqrt{s}=1.96 \mathrm{TeV}, "$ Phys.Rev.Lett. 101 (2008) 191801. arXiv:0804.3220 [hep-ex].

[42] D0 Collaboration , V. M. Abazov et al., "Measurement of the anomalous like-sign dimuon charge asymmetry with $9 \mathrm{fb}^{-1}$ of $p \bar{p}$ collisions," Phys.Rev. D84 (2011) 052007, arXiv: 1106.6308 [hep-ex].

[43] T. Andeen, Measurement of the W Boson Mass with the D0 Run II Detector using the Electron $p_{T}$ Spectrum. PhD thesis, Northwestern University, June, 2009. http: //www-d0.fnal.gov/results/publications_talks/thesis/andeen/thesis.pdf.

[44] J.-R. Vlimant, U. Bassler, G. Bernardi, and S. Trincaz-Duvoid, "Technical description of the T42 algorithm for the calorimeter noise suppression." D0 Note 4146, June, 2003.

[45] H1 Collaboration, I. Abt et al., "The H1 detector at HERA," Nucl.Instrum.Meth. A386 (1997) 310-347.

[46] A. Güntherschulze and H. Betz, "Die bewegung der ionengitter von isolatoren bei extremen elektrischen feldstärken," Zeitschrift für Physik 92 no. 5-6, (1934) 367-374. 
[47] L. Malter, "Anomalous secondary electron emission a new phenomenon," Phys. Rev. 49 (Mar, 1936) 478-478, http://link.aps.org/doi/10.1103/PhysRev.49.478.

[48] L. Malter, "Thin film field emission," Phys. Rev. 50 (Jul, 1936) 48-58. http://link.aps.org/doi/10.1103/PhysRev.50.48

[49] A. Stevenson, "How McCarthy's Malter Theory of DC Currents in Uranium Modules fits the EC data." D0 Note 1154, July, 1991.

[50] R. McCarthy, "Malter Theory of D0 Currents in Uranium Modules." D0 Note 780, Feb., 1988.

[51] J. Stark, "Review of Literature on Hysteresis Effects in thin Semiconductor Layers in the Context of Rising Guentherschulze Currents in the D0 Calorimeter." D0 Note 6361, Sept., 2012.

[52] S. Palestini, G. Barr, A. Ceccucci, J. Cogan, D. Cundy, et al., "Space charge in ionization detectors and the NA48 electromagnetic calorimeter," Nucl.Instrum.Meth. A421 (1999) 75-89.

[53] C. Biscarat, "MET at High Luminosity, T42, Negative Energy and all that." Talk presentend at the D0 Calorimeter Algorithms internal meeting on 09/06/2007, Sept., 2007.

[54] W. Walkowiak, "Drift velocity of free electrons in liquid argon," Nucl.Instrum.Meth. A449 (2000) 288-294.

[55] J. Bystricky, D. Calvet, P. Le Du, E. Perez, G. Tarte, et al., "Algorithms and architecture for the L1 calorimeter trigger at D0 Run IIb," IEEE Trans.Nucl.Sci. 51 (2004) 351-355.

[56] CERN and ATLAS Collaboration, ATLAS, Level-1 Trigger: Technical Design Report. ATLAS technical design report. CERN, 1998. http://cds.cern.ch/record/381429?In=en. Report Number: ATLAS-TDR-12; CERN-LHCC-98-014.

[57] A. Rujula and A. Galindo, "Measuring the W-Boson mass at a hadron collider: a study of phase-space singularity methods," JHEP 1108 (2011) 023, arXiv:1106.0396 [hep-ph].

[58] F. James and M. Roos, "Minuit: A System for Function Minimization and Analysis of the Parameter Errors and Correlations," Comput.Phys.Commun. 10 (1975) 343-367.

[59] T. Andeen et al., "Modeling the Hadronic Recoil for a precision measurement of the W mass in the Run II (Method I)." D0 Note 5668, Feb., 2009. 
[60] R. Engelmann et al., "Response of a Highly Segmented Extruded Lead Glass Calorimeter fo Electrons and Pions between $15-\mathrm{GeV} / \mathrm{c}$ and $45 \mathrm{GeV} / \mathrm{c}^{\prime \prime}$ Nucl.Instrum.Meth. 216 (1983) 45.

[61] A. Schwartzman, Medición de la vida media del mesón $B^{ \pm}$e identificación de quarks top a partir de la reconstrucción de decaimientos de quarks $b$. PhD thesis, 2004. http://www-d0.fnal.gov/results/publications_talks/thesis/schwartzman/ thesis.pdf.

[62] J. Stark, Mise au point de la calorimétrie au Run II de l'expríence D0 et mesure de la masse du boson $W$. Habilitation thesis, Université Joseph Fourier - Grenoble I, February, 2013.

[63] G. Grindhammer, M. Rudowicz, and S. Peters, "The fast simulation of electromagnetic and hadronic showers," Nucl.Instrum.Meth. A290 (1990) 469

[64] R. Brun, F. Carminati, and S. Giani, “GEANT Detector Description and Simulation Tool,".

[65] G. Ladinsky and C.-P. Yuan, "The Nonperturbative regime in QCD resummation for gauge boson production at hadron colliders," Phys.Rev. D50 (1994) 4239. arXiv:hep-ph/9311341 [hep-ph].

[66] P. M. Nadolsky, H.-L. Lai, Q.-H. Cao, J. Huston, J. Pumplin, et al., "Implications of CTEQ global analysis for collider observables," Phys.Rev. D78 (2008) 013004, arXiv:0802.0007 [hep-ph].

[67] P. Golonka and Z. Was, "PHOTOS Monte Carlo: A Precision tool for QED corrections in $Z$ and $W$ decays," Eur.Phys.J. C45 (2006) 97-107, arXiv:hep-ph/0506026 [hep-ph].

[68] U. Baur, S. Keller, and D. Wackeroth, "Electroweak radiative corrections to $W$ boson production in hadronic collisions," Phys.Rev. D59 (1999) 013002, arXiv:hep-ph/9807417 [hep-ph].

[69] U. Baur, O. Brein, W. Hollik, C. Schappacher, and D. Wackeroth, "Electroweak radiative corrections to neutral current Drell-Yan processes at hadron colliders," Phys.Rev. D65 (2002) 033007, arXiv: hep-ph/0108274 [hep-ph].

[70] D. Stump, J. Huston, J. Pumplin, W.-K. Tung, H. L. Lai, et al., “Inclusive jet production, parton distributions, and the search for new physics," JHEP 0310 (2003) 046, arXiv: hep-ph/0303013 [hep-ph].

[71] Particle Data Group , J. Beringer et al., "Review of Particle Physics (RPP)," Phys.Rev. D86 (2012) 010001. 
[72] C. Edwards, R. Partridge, C. Peck, F. Porter, D. Antreasyan, et al., "Study of the decay $J / \psi \rightarrow \gamma \eta \pi \pi, "$ Phys.Rev.Lett. 51 (1983) 859.

[73] S. Jadach, J. H. Kuhn, and Z. Was, “TAUOLA: A Library of Monte Carlo programs to simulate decays of polarized tau leptons," Comput.Phys.Commun. 64 (1990) 275-299.

[74] M. Jezabek, Z. Was, S. Jadach, and J. H. Kuhn, "The tau decay library TAUOLA, update with exact $\mathcal{O}(\alpha)$ QED corrections in $\tau \rightarrow \mu(e) v \bar{v}$," Comput.Phys.Commun. 70 (1992) 69-76.

[75] R. Lopes de Sá, "PDF Uncertainties in the W Mass Measurement." D0 Note 6147, Feb., 2012.

[76] L. Lyons, D. Gibaut, and P. Clifford, "How to combine correlated estimates od a single physical quantity," Nucl.Instrum.Meth. A270 (1988) 110.

[77] A. Valassi, "Combining correlated measurements of several different physical quantities," Nucl.Instrum.Meth. A500 (2003) 391-405.

[78] D0 Collaboration, B. Abbott et al., "A measurement of the $W$ boson mass using electrons at large rapidities," Phys.Rev.Lett. 84 (2000) 222-227, arXiv:hep-ex/9909030 [hep-ex].

[79] CDF Collaboration, D0 Collaboration, T. Aaltonen et al., "Combination of the top-quark mass measurements from the Tevatron collider," Phys.Rev. D86 (2012) 092003, arXiv:1207.1069 [hep-ex].

[80] Q. Zhu, Measurement of the $W$ boson mass in pp collisions at $\sqrt{s}=1.8 \mathrm{TeV}$. PhD thesis, Apr., 1994. http://www-d0.fnal.gov/results/publications_talks/thesis/zhu/ thesis_1side.ps.

[81] H.-L. Lai, M. Guzzi, J. Huston, Z. Li, P. M. Nadolsky, et al., “New parton distributions for collider physics," Phys.Rev. D82 (2010) 074024, arXiv:1007.2241 [hep-ph].

[82] T. Sjostrand, S. Mrenna, and P. Z. Skands, "PYTHIA 6.4 Physics and Manual," JHEP 0605 (2006) 026, arXiv: hep-ph/0603175 [hep-ph].

[83] S. Alioli, P. Nason, C. Oleari, and E. Re, "NLO vector-boson production matched with shower in POWHEG," JHEP 0807 (2008) 060, arXiv:0805.4802 [hep-ph]

[84] Do Collaboration, V. M. Abazov et al., "Measurement of the electron charge asymmetry in $p \bar{p} \rightarrow W+X \rightarrow e v+X$ events at $\sqrt{s}=1.96-\mathrm{TeV}, "$ Phys.Rev.Lett. 101 (2008) 211801, arXiv:0807.3367 [hep-ex]. 
[85] http://hep.pa.msu.edu/resum/grids/resbos_p/w_z/tev2/general_purpose/w+ /2012/nlo_pdfs/lo_vkm/. We thank P. Nadolsky and M. Guzzi for providing this specially prepared grid file.

[86] G. Bozzi, J. Rojo, and A. Vicini, "The Impact of PDF uncertainties on the measurement of the $W$ boson mass at the Tevatron and the LHC," Phys.Rev. D83 (2011) 113008, arXiv: 1104.2056 [hep-ph].

[87] R. Zitoun, "Study of the non-linearity of the D0 calorimeter readout chain." D0 Note 3997, June, 2003.

[88] U. Bassler, "Calorimeter Calibration: Online Calibration Procedures and Online/Offline Database Design." D0 Note 5316, 2007.

[89] J. Stark, "Modifications to the Calorimeter Simulation." Talk presentend at the D0 MC Summit internal meeting on 06/20/2006, June, 2006.

[90] H. Bethe, "Moliere's theory of multiple scattering," Phys.Rev. 89 (1953) 1256-1266.

[91] S. M. Seltzer and M. J. Berger, "Bremsstrahlung Spectra from Electron Interactions with Screened Atomic Nuclei and Orbital Electrons," Nuclear Instruments and Methods in Physics Research Section B: Beam Interactions with Materials and Atoms 12 no. 1, (1985) $95-134$ http://www.sciencedirect.com/science/article/pii/0168583X85907074.

[92] J. H. Hubbell, H. A. Gimm, and I. O. verbø, "Pair, triplet, and total atomic cross sections (and mass attenuation coefficients) for 1 mev- 100 gev photons in elements $\mathrm{z}=1$ to 100," Journal of Physical and Chemical Reference Data 9 no. 4, (1980) 1023-1148. http://link. aip.org/link/?JPR/9/1023/1.

[93] A. Dotti, "Simulation of Showers with Geant4." https://indico.in2p3.fr/getFile.py/access?contribId=71\&sessionId= 13\&resId=0\&materialId=slides\&conf Id=7691. Accessed: 2013-04-30.

[94] W. R. Nelson and Y. Namito, "THE EGS4 CODE SYSTEM: SOLUTION OF gamma-ray AND ELECTRON TRANSPORT PROBLEMS,".

[95] J. Stark, "On the Dependence of the Calorimeter Gains on the Instantaneous Luminosity." Talk presentend at the D0 Calorimeter Algorithms internal meeting on 04/08/2008, Apr., 2009.

[96] A. V. Kotwal and J. Stark, "Measurement of the W Boson Mass at the Tevatron," Ann.Rev.Nucl.Part.Sci. 58 (2008) 147-175.

[97] M. Peskin and D. Sschroeder, An introduction to quantum field theory. Advanced book classics. Addison-Wesley Publishing Company, 1995. http://books.google.com/books?id=i35LALNOGosC 
[98] Tevatron Electroweak Working Group, D0 and CDF Collaborations , "Combination of CDF and DO results on the mass of the top quark using up to $8.7^{-1}$ at the Tevatron," arXiv:1305.3929 [hep-ex].

[99] D. Hanneke, S. Fogwell, and G. Gabrielse, "New Measurement of the Electron Magnetic Moment and the Fine Structure Constant," Phys.Rev.Lett. 100 (2008) 120801, arXiv:0801.1134 [physics.atom-ph].

[100] D. Y. Bardin, A. Leike, T. Riemann, and M. Sachwitz, "Energy Dependent Width Effects in e+ e- Annihilation Near the Z Boson Pole," Phys.Lett. B206 (1988) 539-542.

[101] R. Tenchini and C. Verzegnassi, The physics of the Z and W bosons. World Scientific Publishing Company Incorporated, 2008. http://books.google.com/books?id=apW5PYVm4k0C 
Appendices 


\section{Appendix A}

\section{Renormalization of the Electroweak Sector of the Standard Model}

The goal of this appendix is to study in more details the definition of the $W$ boson mass in the Standard Model. The full Standard Model lagrangian can be found in standard quantum field theory textbooks [97]. Here, we focus on the electroweak sector, which is written as (without CKM mixing):

$$
\begin{aligned}
\mathcal{L}_{\mathrm{EW}}= & {\overline{\Psi_{L}}}^{\alpha} i \gamma^{\mu}\left(D_{\mu} \Psi\right)_{\alpha}+\overline{\Psi_{R}} i \gamma^{\mu}\left(D_{\mu} \Psi_{R}\right)-\frac{1}{4} F_{\mu v}^{a} F_{a}^{\mu \nu}-\frac{1}{4} B_{\mu v} B^{\mu v} \\
& +\left(D_{\mu} \Phi\right)^{\dagger \alpha}\left(D^{\mu} \Phi\right)_{\alpha}-V(\Phi)-G_{f}\left[\overline{\Psi_{R}} \Phi^{\dagger \alpha} \Psi_{L \alpha}+\text { h.c. }\right]
\end{aligned}
$$

where, only this time, we wrote explicitly the $\mathfrak{s u}(2)$ representation indices, using greek indices from the beggining of the alphabet for the fundamental representation and latin indices for the adjoint representation. In the lagragian above, $\Psi_{L}$ and $\Psi_{R}$ are the chiral and anti-chiral components of the matter fields, $F_{\mu v}$ is the $\mathrm{SU}(2)$ field strength, $B_{\mu v}$ is the $\mathrm{U}(1)$ field strength, and $\Phi$ is the Higgs field. For completeness, we define:

$$
\begin{aligned}
F_{\mu \nu} & =\partial_{\mu} W_{v}^{a}-\partial_{\nu} W_{\mu}^{a}-g_{W} f_{b c}^{a} W_{\mu}^{b} W_{v}^{c} \\
B_{\mu v} & =\partial_{\mu} B_{v}-\partial_{\nu} B_{\mu} \\
D_{\mu} & =\partial_{\mu}+i g_{W} W_{\mu}^{a} t_{a}+i \frac{g_{B}}{2} \Upsilon B_{\mu} \\
V(\Phi) & =\lambda\left(|\Phi|^{2}-v^{2}\right)^{2}
\end{aligned}
$$

At tree level, it is easy to show that:

$$
\begin{aligned}
M_{W}^{2} & =\frac{g_{W}^{2} v^{2}}{4} \\
M_{Z}^{2} & =\frac{g_{Z}^{2} v^{2}}{4}
\end{aligned}
$$


where $g_{Z}^{2}=g_{W}^{2}+g_{B}^{2} \cdot g_{Z}$ is the gauge coupling constant of the $Z$ boson. We can also read out the gauge coupling constant of the $\gamma$ as:

$$
e^{2}=\frac{\alpha}{4 \pi}=\left(\frac{g_{B} g_{W}}{\sqrt{g_{B}^{2}+g_{W}^{2}}}\right)^{2}
$$

Besides the masses and the gauge coupling constants of the $Z$ and $\gamma$ boson, any other observable can be expressed using the independent variables in the EW lagragian, namely $\left(g_{W}, g_{B}, G_{f}, v, \lambda\right)$. Experimentally, it is convenient to choose another set of variables, namely $\left(G_{F}, M_{H}, m_{t}, M_{Z}, \alpha\right)$, where $M_{Z}$ and $\alpha$ have been defined above, $m_{t}$ is the top quark mass which substitutes the most relevant Yukawa coupling for electroweak physics, $M_{H}$ is the Higgs boson mass and $G_{F}=g_{Z}^{2} / 8 M_{Z}^{2}$, at tree level.

These variables are convenient because they can be measured with very high precision [5, 2, 3, 98, 8, 99]. Other observables, measured with relatively less precision, are interpreted as derived and the comparisons of their predicted value, using the five quantities above as input, to the directly measured value are stringent tests of the internal consistency of the Standard Model. Before studying the renormalization of the EW theory, we define some of these observables and discuss their interpretation.

\section{A.1 The Weak Angle}

Originally, the Weak (or Weinberg) angle was defined as:

$$
\sin ^{2} \theta_{W}=s_{W}^{2}=1-\frac{M_{W}^{2}}{M_{Z}^{2}}
$$

This definition is renormalization group invariant and constains no additional information than what is contained in the $W$ boson mass. However, this is not what is commonly measured. A second definition, which agrees at tree level to the definition above, but is not equivalent to $M_{W}$ is:

$$
J_{N}^{\mu}=J_{3}^{\mu}-Q_{F} \sin ^{2} \theta_{\text {eff }}^{f} J_{Q}^{\mu}
$$

where $J_{N}$ is the neutral weak current, $J_{3}$ is the third isospin component of the weak current and $J_{Q}$ is the electromagnetic current. This Weak angle is a function of the renormalization scale (tipically taken as the momentum transfer scale) and it is the one measured at collider experiments, at the $Z$ peak, and from deep inelastic neutrino scattering, a low momentum transfer scales. A third definition $\hat{s}^{2}=g_{B}^{2}(\mu) /\left(g_{B}^{2}(\mu)+g_{W}^{2}(\mu)\right)$ using the modified minimum subtraction scheme $(\overline{\mathrm{MS}})$ is theoretically interesting but almost never used experimentally. 


\section{A.2 W Boson Mass}

Using the five variables singled out as independent, the $W$ boson mass is written as:

$$
M_{W}^{2}\left(1-\frac{M_{W}^{2}}{M_{Z}^{2}}\right)=\frac{\pi \alpha}{\sqrt{2} G_{F}}(1+\Delta r)
$$

As a function of the renormalization scale, it is natural to consider $G_{F} \equiv G_{F}\left(\mu=m_{\mu}\right)$ and $\alpha \equiv \alpha\left(\mu=m_{e} \simeq 0\right)$. On the other hand, the natural scale for $\sin ^{2} \theta_{W}$ is $M_{Z}$. The $\Delta r$ can be thought as a connection between the two scales. $M_{W}^{2}$ :

In order to have a feeling of the importance of $\Delta r$, we can expand the expression for

$$
\begin{aligned}
M_{W}^{2} & =\frac{M_{Z}^{2}}{2}\left[1+\sqrt{1-\frac{4 \pi \alpha}{\sqrt{2} G_{F} M_{Z}^{2}}}\right]+M_{W}^{2} \frac{1-M_{W}^{2} / M_{Z}^{2}}{1-2 M_{W}^{2} / M_{Z}^{2}} \times \Delta r+\mathcal{O}\left(\Delta r^{2}\right) \\
\Rightarrow M_{W} & =80.939 \times[1-0.185 \times \Delta r+\cdots] \mathrm{GeV}
\end{aligned}
$$

where the numerical estimation uses the values in the references listed above. Since the experimental precision on $M_{W}$ is $15 \mathrm{MeV}$, we are sensitive to $\Delta r \gtrsim 0.001$.

\section{A.3 Renormalization of the Gauge Boson Propagator}

In this section, we will refer to any SM massive vector boson as $V=W, Z$, as already done in some parts of this Dissertation. The free field propagator of a vector boson is written as:

$$
D_{V 0}^{\mu v}=-i \frac{\left(g^{\mu \nu}-q^{\mu} q^{v} / M_{V}^{2}\right)+B\left(q^{2}\right) q^{\mu} q^{v}}{q^{2}-M_{V}^{2}+i \epsilon}
$$

where $B\left(q^{2}\right)$ is a gauge dependent part that, in the $R_{\xi}$ gauge, is written as $\left(\xi / q^{2}\right) /\left(q^{2}-\right.$ $\left.\xi M_{V}^{2}\right)$. Our goal here is not to give a general proof of the renormalization of spontanously broken non-abelian gauge theories, but rather to study the specific case relevant for the definition of the $W$ boson mass. Therefore, we ignore the second term and work only with 1 .

$$
D_{V 0}^{\mu \nu}=\left(g^{\mu \nu}-q^{\mu} q^{v} / M_{V}^{2}\right) D_{V 0}=\left(g^{\mu \nu}-q^{\mu} q^{v} / M_{V}^{2}\right) \times \frac{-i}{q^{2}-M_{V}^{2}}
$$

\footnotetext{
${ }^{1}$ Formally, what we are doing is choosing $\xi=0$. The reader should also note that, for conserved vector currents, any term proportional to $q^{\mu}$ vanishes and for axial currents it is proportional to the mass in the loop, which is negligible compared to the $W$ and $Z$ boson masses in most cases.
} 
The one-loop correction to the propagator from a loop of fermions with mass $m_{1}$ and $m_{2}$ can be written as [97]:

$$
\begin{aligned}
-i \sum_{V_{1} V_{2}}^{\mu v}\left(q^{2}\right)= & -g_{V_{1} V_{2}} \int \frac{d^{4} k}{(2 \pi)^{4}} \operatorname{Tr}\left[\frac{i\left(k+m_{1}\right)}{k^{2}+m_{1}^{2}} i \gamma^{v}\left(v_{2}-a_{2} \gamma_{5}\right)\right. \\
& \left.\times \frac{i\left(q+k+m_{2}\right)}{(k+q)^{2}+m_{1}^{2}} i \gamma^{v}\left(v_{1}-a_{1} \gamma_{5}\right)\right]
\end{aligned}
$$

We factor out the gauge invariant part:

$$
\Sigma_{V_{1} V_{2}}^{\mu v} \equiv\left(g^{\mu \nu}-q^{\mu} q^{v} / M_{V}^{2}\right) \Sigma_{V_{1} V_{2}}\left(q^{2}\right)
$$

The expression above is UV divergent, but the divergencies can be absorbed in the mass and wave function counterterms:

$$
\widehat{\Sigma}_{V_{1} V_{2}}\left(q^{2}\right)=\Sigma_{V_{1} V_{2}}\left(q^{2}\right)-\delta M_{V}^{2}+\delta Z_{V}\left(q^{2}-M_{V}^{2}\right)
$$

Now we can properly define the on-shell scheme as:

Definition 1 (On-Shell Renormalization Scheme (part 1: mass counterterms)). In this scheme, the mass counterterm satisfies:

$$
\delta M_{V}^{2}=\operatorname{Re} \Sigma_{V V}\left(M_{V}^{2}\right)
$$

And the real part of the pole of the propagator will be at the physical mass of the gauge boson.

The Dyson resummed propagator will be:

$$
D_{V}=\frac{-i}{q^{2}-M_{V}^{2}+\widehat{\Sigma}_{V V}\left(q^{2}\right)}
$$

and we define the finite self-energy function:

Definition 2 (Finite Self-Energy Function). The finite self-energy function $\widehat{\Pi}_{V}\left(q^{2}\right)$ is defined as:

$$
\begin{aligned}
\operatorname{Re} \widehat{\Sigma}_{V V}\left(q^{2}\right) & =\left(q^{2}-M_{V}^{2}\right) \widehat{\Pi}_{V}\left(q^{2}\right) \\
\widehat{\Pi}_{V}\left(q^{2}\right) & =\operatorname{Re} \frac{\Sigma_{V V}\left(q^{2}\right)-\Sigma_{V V}(0)}{q^{2}-M_{V}^{2}}+\delta Z_{V}=\Pi_{V}\left(q^{2}\right)+\delta Z_{V}
\end{aligned}
$$

We see that the propagator in the interacting theory has the form:

$$
D_{V}\left(q^{2}\right)=-i \times \frac{1}{1+\widehat{\Pi}_{V}\left(q^{2}\right)} \times \frac{1}{q^{2}-M_{V}^{2}+i M_{V} \Gamma_{V}} ; \quad M_{V} \Gamma_{V}=\operatorname{Im} \frac{\widehat{\Sigma}_{V V}\left(q^{2}\right)}{1+\widehat{\Pi}_{V}\left(q^{2}\right)}
$$

corresponding to a non-zero decay width $\Gamma_{V}{ }^{2}$ and a renormalized gauge coupling constant:

$$
g_{V}^{2}\left(q^{2}\right)=\frac{g_{V}^{2}}{1+\widehat{\Pi}_{V}\left(q^{2}\right)}
$$

\footnotetext{
${ }^{2}$ This propagator form is not in the so-called running width scheme, in which $D_{V}\left(q^{2}\right) \propto \frac{-i}{q^{2}-\bar{M}_{V}^{2}+i q^{2} \Gamma_{V} / \bar{M}_{V}}$. The two parameters can be related by $M_{W, Z}=\bar{M}_{W, Z}-\Gamma_{W, Z}^{2} /\left(2 \bar{M}_{W, Z}\right)$ [100].
} 


\section{A.4 Photon-Z Mixing}

The situation in the EW sector of the Standard Model is slightly more complicated because of the mixing introduced by the Higgs mechanism. Then, we have a total of four selfenergy functions to renormalize: $\Sigma_{W W}, \Sigma_{Z Z}, \Sigma_{Z \gamma}$, and $\Sigma_{\gamma \gamma}$.

The $\gamma$ and $Z$ fields are mixtures written as:

$$
\left[\begin{array}{l}
Z_{\mu} \\
A_{\mu}
\end{array}\right]=\left[\begin{array}{cc}
c_{W} & -s_{W} \\
s_{W} & c_{W}
\end{array}\right]\left[\begin{array}{c}
W_{3 \mu} \\
B_{\mu}
\end{array}\right]
$$

If we define the wave function counterterms $W_{\mu}^{(0)}=\left(1+\delta Z_{W} / 2\right) W_{\mu}, B_{\mu}^{(0)}=\left(1+\delta Z_{B} / 2\right) B_{\mu}$, and $s_{W}^{2}=s_{W}^{(0) 2}+\delta s_{W}^{2}$ as well as:

$$
\left[\begin{array}{l}
Z_{0 \mu} \\
A_{0 \mu}
\end{array}\right]=\left[\begin{array}{cc}
1+\frac{1}{2} \delta Z_{Z} & \frac{1}{2} \delta Z_{Z A} \\
\frac{1}{2} \delta Z_{A Z} & 1+\frac{1}{2} \delta Z_{A}
\end{array}\right]\left[\begin{array}{l}
Z_{\mu} \\
A_{\mu}
\end{array}\right]
$$

Then, we have the following relationships:

$$
\begin{gathered}
{\left[\begin{array}{l}
\delta Z_{Z} \\
\delta Z_{A}
\end{array}\right]=\left[\begin{array}{ll}
c_{W}^{2} & s_{W}^{2} \\
s_{W}^{2} & c_{W}^{2}
\end{array}\right]\left[\begin{array}{l}
Z_{\mu} \\
A_{\mu}
\end{array}\right]} \\
\delta Z_{Z A}=s_{W} c_{W}\left(\delta Z_{W}-\delta Z_{B}\right)-\frac{\delta s_{W}^{2}}{c_{W} s_{W}} \\
\delta Z_{A Z}=s_{W} c_{W}\left(\delta Z_{W}-\delta Z_{B}\right)+\frac{\delta s_{W}^{2}}{c_{W} s_{W}} \\
\frac{\delta s_{W}^{2}}{c_{W} s_{W}}=\frac{c_{W}}{s_{W}}\left(\frac{\delta M_{Z}^{2}}{M_{Z}^{2}}-\frac{\delta M_{W}^{2}}{M_{W}^{2}}\right)
\end{gathered}
$$

The physics of $\delta Z_{Z A}$ and $\delta Z_{A Z}$ is really straightforward: the former is a direct coupling of the $\gamma$ with the weak neutral current and the latter a direct coupling of the $Z$ with the electromagnetic current. With these definitions, we can write the renormalized selfenergies:

$$
\begin{aligned}
\widehat{\Sigma}_{\gamma \gamma}\left(q^{2}\right) & =\Sigma_{\gamma \gamma}\left(q^{2}\right)+\delta Z_{A} q^{2} \equiv q^{2}\left[\Pi_{\gamma}\left(q^{2}\right)+\delta Z_{A}\right] \\
\widehat{\Sigma}_{\gamma Z}\left(q^{2}\right) & =\Sigma_{\gamma Z}\left(q^{2}\right)+\frac{1}{2}\left[\delta Z_{A Z} q^{2}+\delta Z_{Z A}\left(q^{2}-M_{Z}^{2}\right)\right] \\
\widehat{\Sigma}_{Z Z}\left(q^{2}\right) & =\Sigma_{Z Z}\left(q^{2}\right)-\delta M_{Z}^{2}+\delta Z_{z}\left(q^{2}-M_{Z}^{2}\right) \\
\widehat{\Sigma}_{W W}\left(q^{2}\right) & =\Sigma_{W W}\left(q^{2}\right)-\delta M_{W}^{2}+\delta W_{z}\left(q^{2}-M_{W}^{2}\right)
\end{aligned}
$$


Definition 3 (On-Shell Renormalization Scheme (part 2: wave function counterterms)). In the on-shell scheme, the wave function counterterms are defined such that the poles have residue one and that the $r$ field has no $\mathrm{Z}$ component, ie.,

$$
\begin{aligned}
\delta Z_{A} & =-\Pi_{\gamma}(0) \\
\delta Z_{Z A} & =\frac{2 \Sigma_{\gamma Z}}{M_{Z}^{2}}
\end{aligned}
$$

Below we summarize the expression for the renormalized self energy in the on-shell scheme:

$$
\begin{aligned}
\widehat{\Sigma}_{\gamma \gamma}\left(q^{2}\right) & =q^{2} \widehat{\Pi}_{\gamma}\left(q^{2}\right)=\Sigma_{\gamma \gamma}\left(q^{2}\right)-q^{2} \Pi_{\gamma}(0)=q^{2}\left(\Pi_{\gamma}\left(q^{2}\right)-\Pi_{\gamma}(0)\right) \\
\widehat{\Sigma}_{\gamma Z}\left(q^{2}\right) & =q^{2} \widehat{\Pi}_{\gamma Z}\left(q^{2}\right)=\Sigma_{\gamma Z}\left(q^{2}\right)-\Sigma_{\gamma Z}(0)+q^{2} \frac{\delta^{\prime} s_{W}^{2}}{c_{W} s_{W}} \\
\widehat{\Sigma}_{\gamma Z}\left(q^{2}\right) & =\left(q^{2}-M_{Z}^{2}\right) \widehat{\Pi}_{Z}\left(q^{2}\right)=\Sigma_{Z Z}-\operatorname{Re} \Sigma_{Z Z}\left(M_{Z}^{2}\right)+\left(q^{2}-M_{Z}^{2}\right) \delta Z_{Z} \\
\widehat{\Sigma}_{\gamma W}\left(q^{2}\right) & =\left(q^{2}-M_{W}^{2}\right) \widehat{\Pi}_{W}\left(q^{2}\right)=\Sigma_{W W}-\operatorname{Re} \Sigma_{W W}\left(M_{W}^{2}\right)+\left(q^{2}-M_{W}^{2}\right) \delta Z_{W} \\
\delta Z_{Z} & =-\Pi_{\gamma}+\frac{c_{W}^{2}-s_{W}^{2}}{c_{W}^{2} s_{W}^{2}} \delta^{\prime} s_{W}^{2} \\
\delta Z_{W} & =-\Pi_{\gamma}+\frac{1}{s_{W}^{2}} \delta^{\prime} s_{W}^{2} \\
\delta^{\prime} s_{W}^{2} & =c_{W}^{2}\left[\frac{\Sigma_{Z Z}\left(M_{Z}^{2}\right)}{M_{Z}^{2}}-\frac{\Sigma_{W W}\left(M_{W}^{2}\right)}{M_{W}^{2}}+2 \frac{s_{W}}{c_{W}} \frac{\Sigma_{\gamma Z}(0)}{M_{Z}^{2}}\right]
\end{aligned}
$$

where the explicit expression for the one-loop Feynman diagrams $\Sigma_{V V}$ can be found in textbooks [101]. The charge renormalization could be calculated independently, but it is related to the wave-function renormalizaton by a Slavnov-Taylor identity, as seen in Eq. A.18 (here, with an extra term from the $\delta Z_{Z A}$ coupling):

$$
\frac{\delta e}{e}=\frac{1}{2} \Pi_{\gamma}(0)+\frac{s_{W}}{c_{W}} \frac{\Sigma_{\gamma Z}(0)}{M_{Z}^{2}}
$$

\section{A.5 The muon decay and $G_{F}$}

The last piece still undefined in the $W$ mass prediction is the Fermi coupling constant $G_{F}$. It has been defined before 1.3 using the muon decay time as derived from an nonrenormalizable invariant amplitude:

$$
\mathcal{M}=\frac{G_{F}}{\sqrt{2}}\left[\bar{u}\left(p_{e}\right) \gamma_{\mu}\left(1-\gamma_{5}\right) v\left(p_{v_{e}}\right)\right]\left[\bar{u}\left(p_{v_{\mu}}\right) \gamma^{\mu}\left(1-\gamma_{5}\right) v\left(p_{\mu}\right)\right]
$$



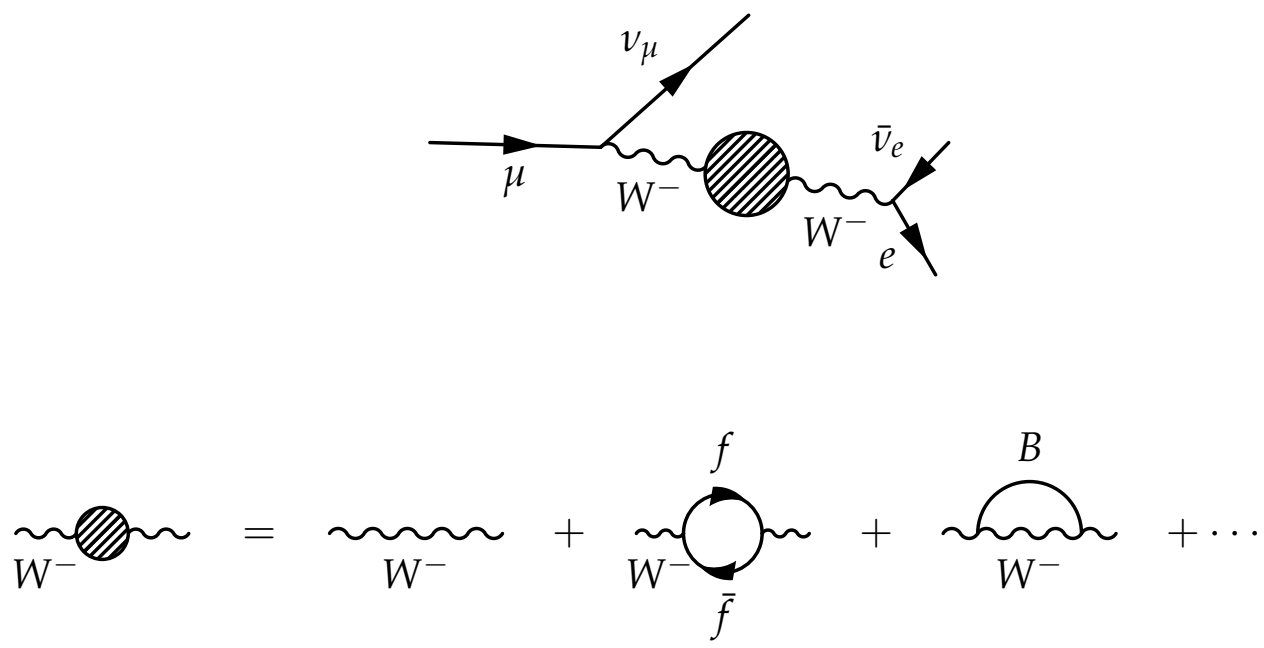

Figure A.1: Feynman diagram for the muon decay in the Standard Model. We consider only loop corrections to the propagator, ignoring sub-leading corrections from the vertex renormalization as well as box-like diagrams. On the last diagram, $B$ represents either a vector boson $Z, \gamma$ or the Higgs boson $H$.

We can compare this expression with the equivalent one calculated from the full electroweak lagragian. However, we will only consider the dominant one-loop correction namely, the renormalization of the $W$ propagator that have been worked out in details above (see Fig. A.1). The invariant amplitude is:

$$
\begin{aligned}
\mathcal{M} & =-\frac{g_{W}^{2}}{M_{W}^{2}\left[1+\widehat{\Pi}_{W}\left(q^{2}\right)\right]} \frac{\left[\bar{u}\left(p_{e}\right) \gamma_{\mu}\left(1-\gamma_{5}\right) v\left(p_{v_{e}}\right)\right]\left[\bar{u}\left(p_{v_{\mu}}\right) \gamma^{\mu}\left(1-\gamma_{5}\right) v\left(p_{\mu}\right)\right]}{q^{2}-M_{W}^{2}+i M_{W} \Gamma_{W}} \ldots \\
& \simeq \frac{e^{2}}{8 M_{W}^{2} s_{W}^{2}} \frac{\left[\bar{u}\left(p_{e}\right) \gamma_{\mu}\left(1-\gamma_{5}\right) v\left(p_{v_{e}}\right)\right]\left[\bar{u}\left(p_{v_{\mu}}\right) \gamma^{\mu}\left(1-\gamma_{5}\right) v\left(p_{\mu}\right)\right]}{1+\widehat{\Pi}_{W}\left(q^{2}\right)}+\cdots
\end{aligned}
$$

Comparing Eqs. A.29 and A.30 with definition A.7, it is clear that the leading contribution to $\Delta r$ is:

$$
\Delta r=-\widehat{\Pi}_{W}(0)+\cdots=\operatorname{Re} \frac{\Sigma_{W W}(0)-\Sigma_{W W}\left(M_{W}^{2}\right)}{M_{W}^{2}}+\Pi_{\gamma}(0)-\frac{\delta^{\prime} s_{W}^{2}}{s_{W}^{2}}+\cdots
$$


and, using the expression for the renormalized self-energy in the on-shell scheme A.27.

$$
\begin{aligned}
\Delta r=\Pi_{\gamma}(0) & -\frac{c_{W}^{2}}{s_{W}^{2}} \operatorname{Re}\left[\frac{\Sigma_{Z Z}\left(M_{Z}^{2}\right)-\Sigma_{Z Z}(0)}{M_{Z}^{2}}\right] \\
& +\frac{c_{W}^{2}-s_{W}^{2}}{s_{W}^{2}} \operatorname{Re}\left[\frac{\Sigma_{W W}\left(M_{W}^{2}\right)-\Sigma_{W W}(0)}{M_{W}^{2}}\right] \\
& +\frac{c_{W}^{2}}{s_{W}^{2}} \operatorname{Re}\left[\frac{\Sigma_{Z Z}(0)}{M_{Z}^{2}}-\frac{\Sigma_{W W}(0)}{M_{W}^{2}}+2 \frac{s_{W}}{c_{W}} \frac{\Sigma_{\gamma Z}(0)}{M_{Z}^{2}}\right]
\end{aligned}
$$

The first three terms are the contributions from the $\gamma, Z$, and $W$ self-energies to the value of the $W$ boson mass. The last term is interpreted as the deviation of the Standard Model $\rho$ parameter:

Definition 4 (Standard Model $\rho$ parameter). We define the Standard Model $\rho$ parameter as:

$$
\rho\left(q^{2}\right)=\frac{1+\widehat{\Pi}_{W}\left(q^{2}\right)}{1+\widehat{\Pi}_{Z}\left(q^{2}\right)} \simeq 1+\widehat{\Pi}_{W}\left(q^{2}\right)-\widehat{\Pi}_{Z}\left(q^{2}\right)
$$

And we interpret the last term of $E q$. A.33 as the renormalization $\Delta \rho\left(q^{2}\right)$.

It is easier to interpret $\Delta \rho$ if we separate the isospin components (see Eq. A.6):

$$
\begin{aligned}
\Sigma_{\gamma \gamma} & =e^{2} Q^{2} \Sigma_{Q Q} \\
\Sigma_{\gamma Z} & =e g_{Z}\left(Q \Sigma_{3 Q}-Q^{2} s_{W}^{2} \Sigma_{Q Q}\right) \\
\Sigma_{Z Z} & =g_{Z}^{2}\left(\Sigma_{33}-2 Q s_{W}^{2} \Sigma_{3 Q}+Q^{2} s_{W}^{4} \Sigma_{Q Q}\right) \\
\Sigma_{W W} & =g_{W}^{2} \Sigma_{11}
\end{aligned}
$$

where the index $Q$ is isospin invariant. Substituting:

$$
\Delta \rho(0)=\frac{e^{2}}{M_{W}^{2} s_{W}^{2}}\left(\Sigma_{33}(0)-\Sigma_{11}(0)\right)
$$

It is clear that only the loops that break isospin invariance will contribute to $\Delta \rho$. The most important contribution is the one form the top-bottom loop, whose result is [101]:

$$
\Delta \rho_{\mathrm{tb}}=\frac{3 e^{2}}{64 \pi^{2} M_{W}^{2} s_{W}^{2}}\left(m_{t}^{2}+m_{b}^{2}-2 \frac{m_{t}^{2} m_{b}^{2}}{m_{t}^{2}-m_{b}^{2}} \ln \frac{m_{t}^{2}}{m_{b}^{2}}\right) \simeq \frac{3 G_{F} m_{t}^{2}}{8 \sqrt{2} \pi^{2}}
$$

Using the value of the quark top mass from the Tevatron [98], the numerical value is $\Delta \rho_{\mathrm{tb}}=0.00941 \Rightarrow \Delta r_{t b}=-0.0328$. We close this appendix by rewritting the expression for $\Delta r$ as it is usually found in the literature:

$$
\Delta r=\Delta \alpha\left(M_{Z}^{2}\right)-\frac{c_{W}^{2}}{s_{W}^{2}} \Delta \rho(0)+r_{1}
$$


where $\Delta \alpha\left(M_{Z}^{2}\right)=-\widehat{\Pi}_{\gamma}\left(M_{Z}^{2}\right)$ from the $Z$ boson self-energy contribution and $r_{1}$ is a subleading remainder with all other contributions. The calculation of $\Delta \alpha$ is both important for the numerical value of $\Delta r$ and delicate, since the contribution from light quarks cannot be calculated perturbatively and it is estimated from dispersion relations and the measurement of total cross section $\sigma\left(e^{-} e^{+} \rightarrow\right.$ hadrons $)$. Using the value from reference [11], we obtain $\Delta \alpha=0.0594$. The interest in measuring the $W$ boson and the quark top masses is to test the strong relationship that comes form this large value of $\Delta \alpha$ and $\Delta \rho_{\mathrm{tb}}$. The two-loop value for $\Delta r$, with the uncertainty from the two sources discussed above, is [71]:

$$
\Delta r=0.0358 \mp 0.0004_{m_{t}} \pm 0.00011_{\alpha\left(M_{Z}\right)}
$$




\section{Appendix B}

\section{Central Calorimeter fast MC Comparison Plots}

\section{B.1 Z $\rightarrow$ ee Comparison Plots}

Since the $\mathrm{Z}$ boson is used as the main control sample to parametrize the fast $\mathrm{MC}$ it is important to check the agreement between the fast $\mathrm{MC}$ and the data sample on which the tuning was performed. Figures B.1. B.2 and B.3 show the $p_{T}^{e}, p_{T}^{Z}, u_{T}$, comparisons between data and fast MC. Figures B.4. B.5 and B.6 show the comparisons between data and fast MC for the $u_{\|}$and $u_{\perp}$ distributions of electrons from $Z \rightarrow e e$ events and the $\mathbb{Z}_{T}$ in $Z$ events respectively.
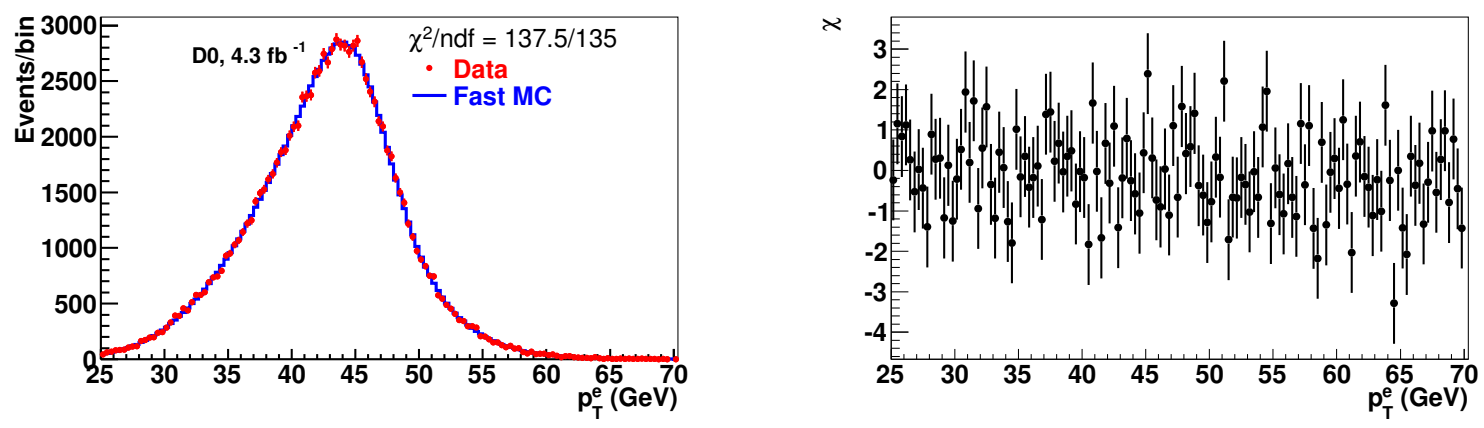

Figure B.1: (left) Comparison between data and fast $\mathrm{MC}$ for the $p_{T}^{e}$ distribution of the electrons from $Z \rightarrow e e$. (right) the corresponding $\chi$ value per bin. 

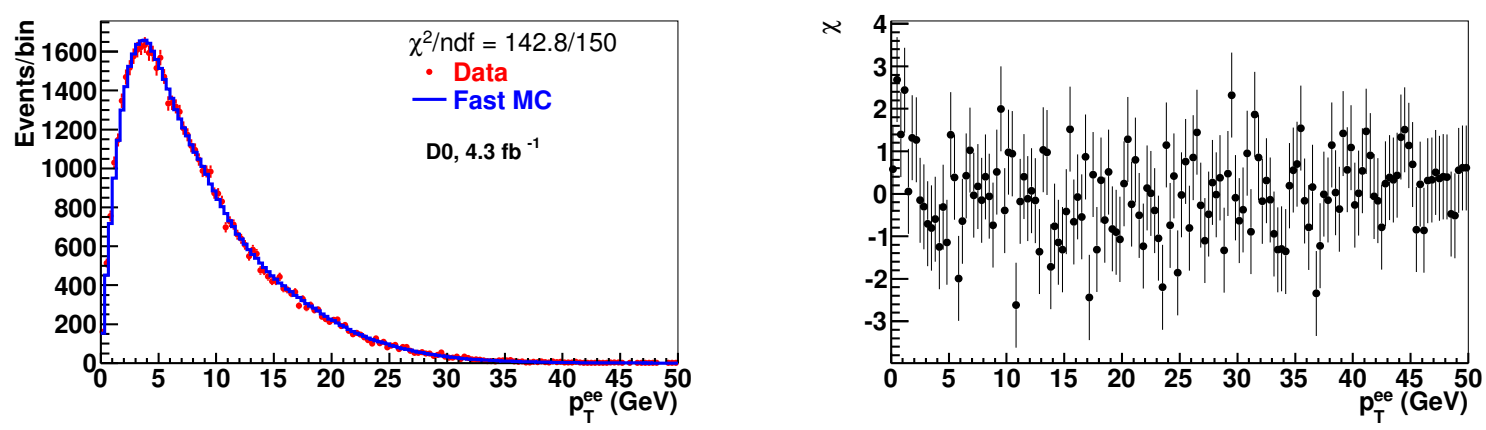

Figure B.2: (left) Comparison between data and fast $\mathrm{MC}$ for the $p_{T}^{Z}$ distribution of $Z \rightarrow e e$ events. (right) the corresponding $\chi$ value per bin.
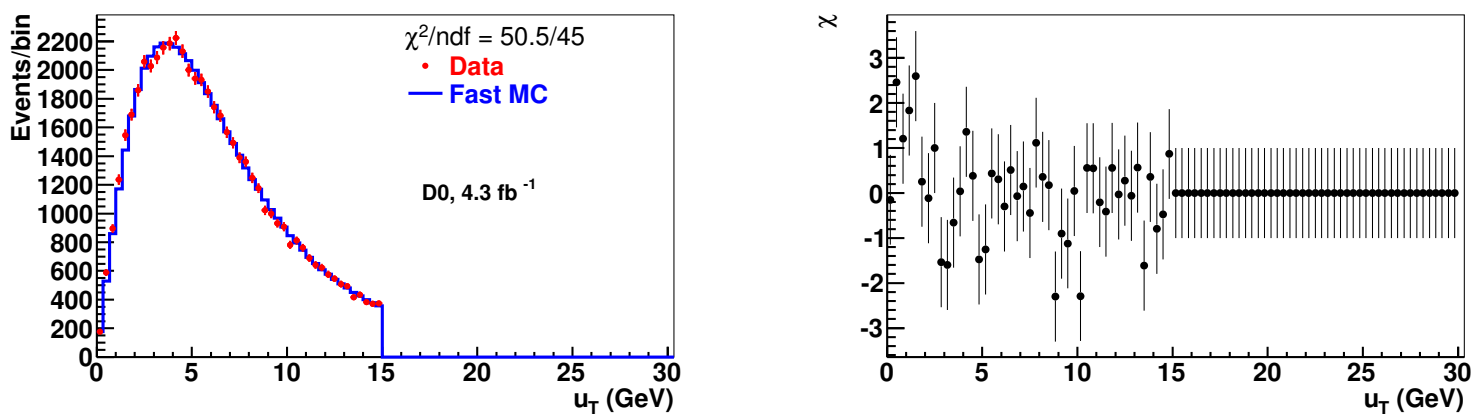

Figure B.3: (left) Comparison between data and fast $\mathrm{MC}$ for the $u_{T}$ distribution of $Z \rightarrow e e$ events. (right) the corresponding $\chi$ value per bin.
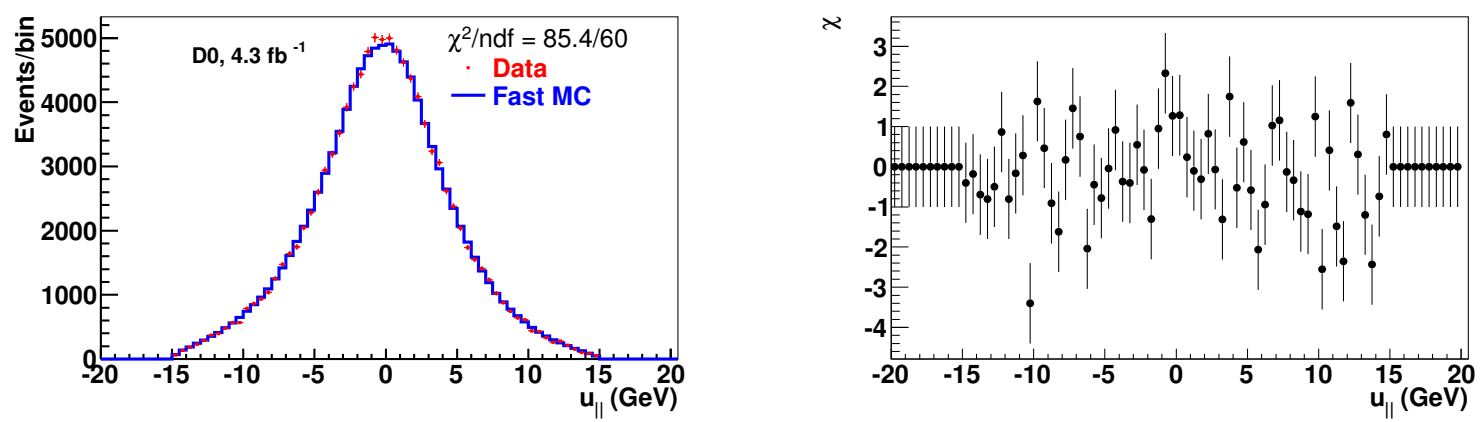

Figure B.4: (left) Comparison between data and fast MC for the $u_{\|}$distribution of electrons in $Z \rightarrow e e$ events. (right) the corresponding $\chi$ value per bin. 

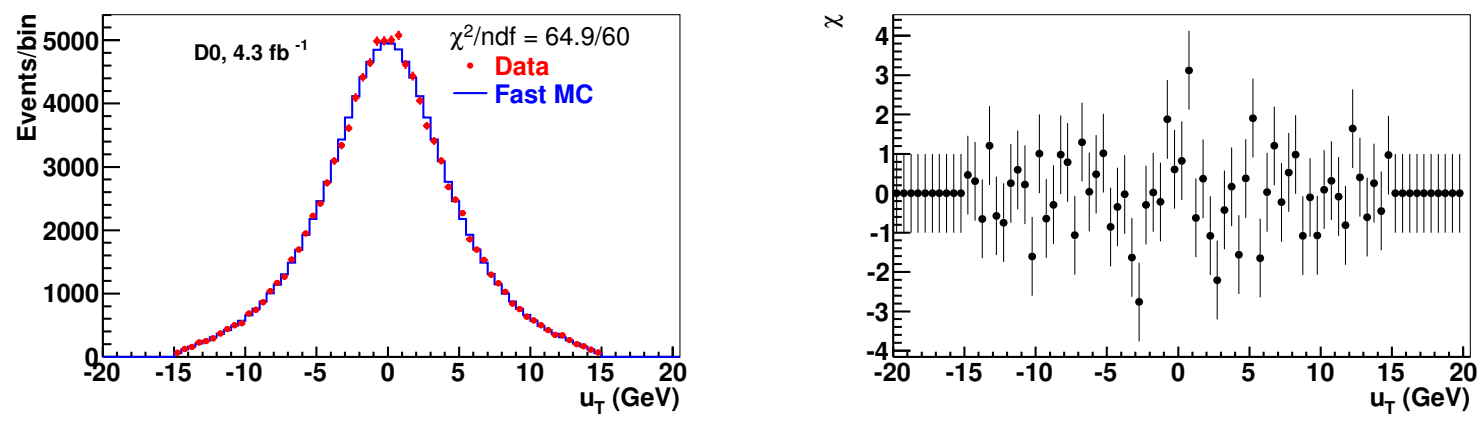

Figure B.5: (left) Comparison between data and fast $\mathrm{MC}$ for the $u_{\perp}$ distribution of electrons in $Z \rightarrow e e$ events. (right) the corresponding $\chi$ value per bin.
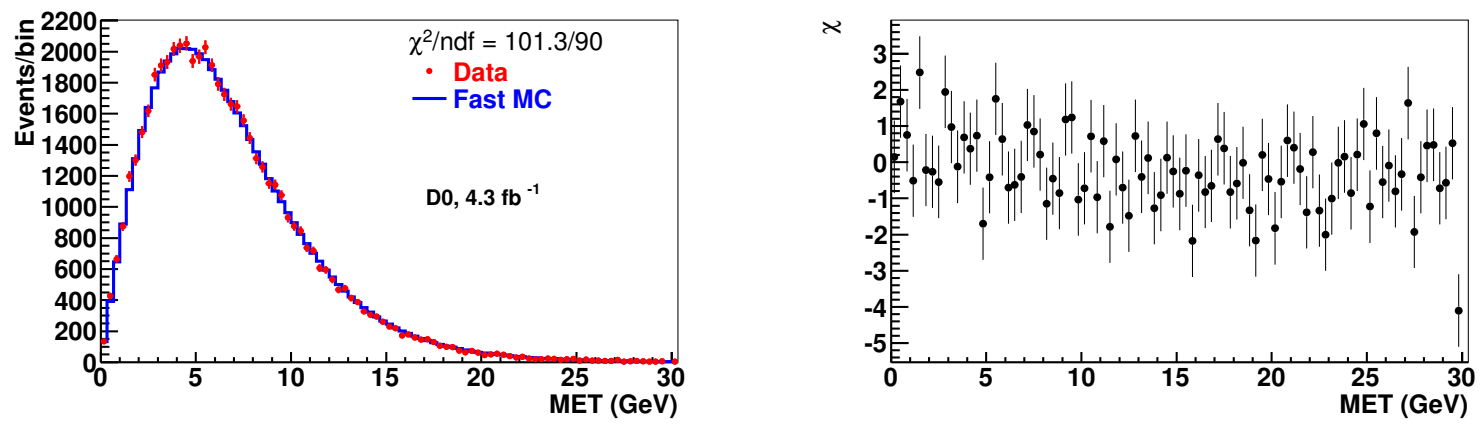

Figure B.6: (left) Comparison between data and fast $\mathrm{MC}$ for the $\mathbb{E}_{T}$ distribution of in $Z \rightarrow e e$ events. (right) the corresponding $\chi$ value per bin. 


\section{B.2 $W \rightarrow$ ev Comparison Plots}

We have shown the excellent agreement between data and fast $\mathrm{MC}$ for the $\left(m_{T}, p_{T}^{e}, \mathbb{E}_{T}\right)$ distributions which are used in Sec. 9 to measure $M_{W}$. In these distributions there are typically 50,000 events per $0.5 \mathrm{GeV}$ bin so the statistical error is about $0.5 \%$ in a typical bin. The accuracy of our model in fast MC is good. We present further comparison plots of the $u_{T}, u_{\|}, u_{\perp}, \eta, \eta_{\text {det }}, L$ and SET distributions in $W$ events in Figs. B.7, B.8, B.9. B.10. and B.11. The agreement is worse for some of these variables (particularly $u_{T}, L$, and SET) but still good considering the statistical precision of our data and the fact that our fast MC was tuned with $Z$ and not $W$ events. The agreement is good enough between data and fast $\mathrm{MC}$ for our purposes, since we do not use these distributions to directly measure the $W$ mass.
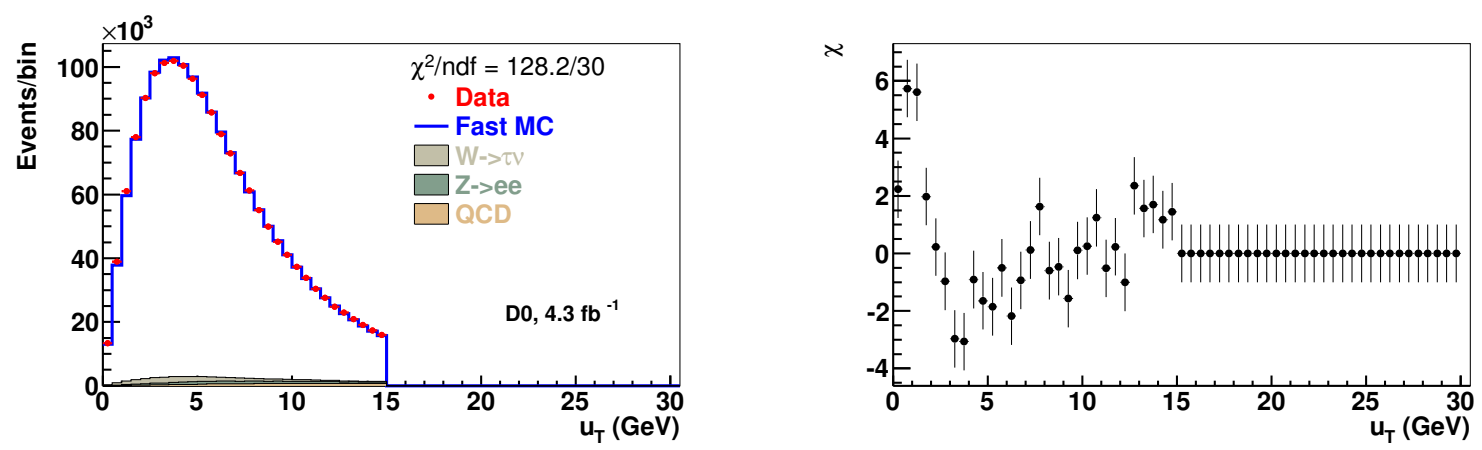

Figure B.7: (left) Comparison between data and fast MC for the $u_{T}$ distribution in $\mathrm{W}^{ \pm} \rightarrow$ $\mathrm{e}^{ \pm} v$ data. (right) the corresponding $\chi$ value per bin.
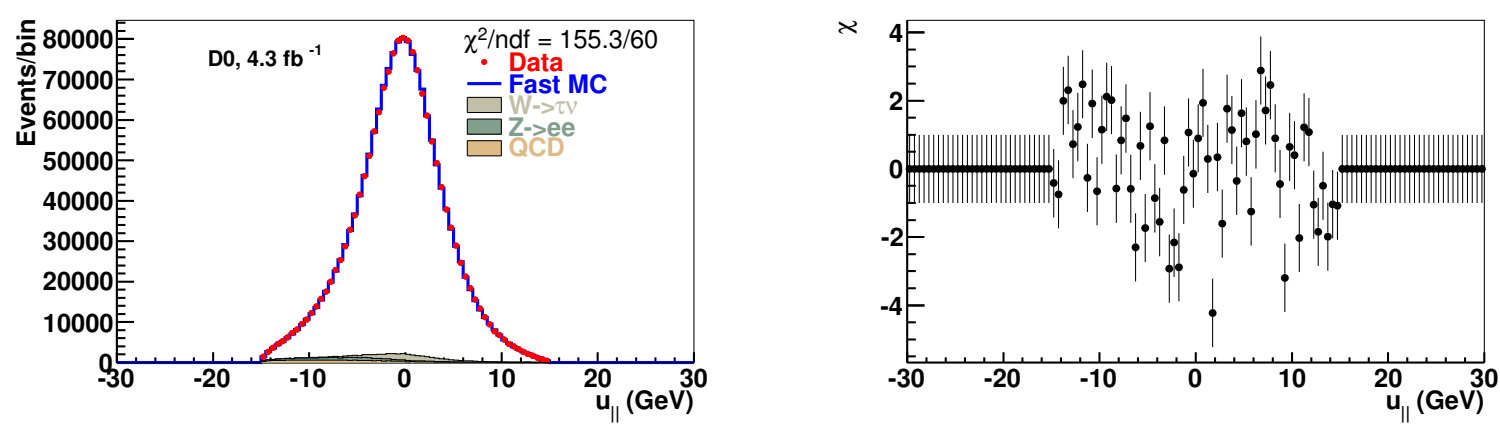

Figure B.8: (left) Comparison between data and fast $\mathrm{MC}$ for the $u_{\|}$distribution in $\mathrm{W}^{ \pm} \rightarrow$ $\mathrm{e}^{ \pm} v$ data. (right) the corresponding $\chi$ value per bin. 

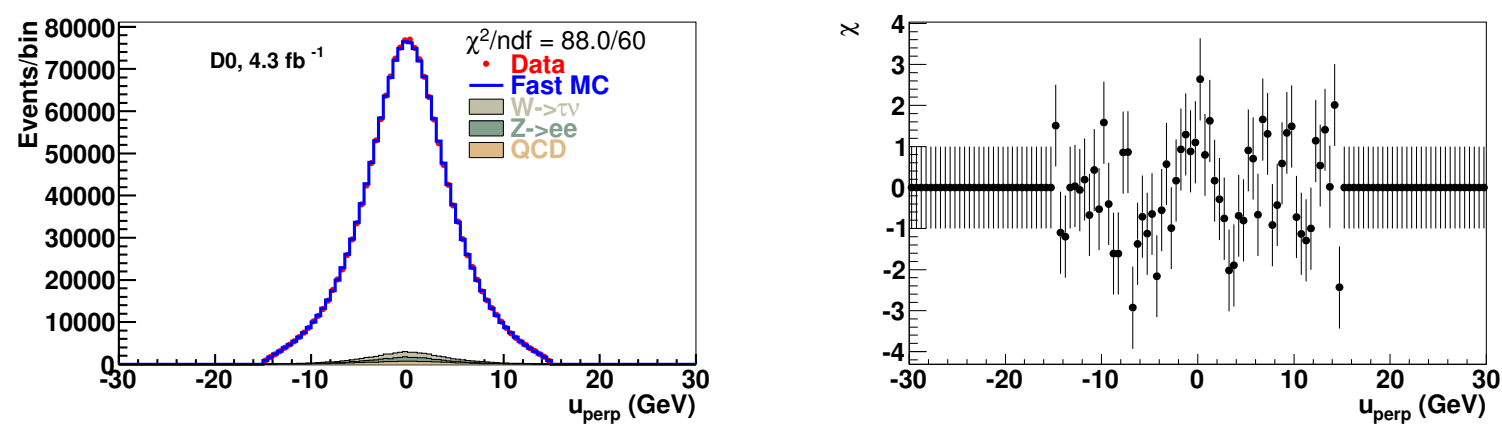

Figure B.9: (left) Comparison between data and fast $\mathrm{MC}$ for the $u_{\perp}$ distribution in $\mathrm{W}^{ \pm} \rightarrow$ $\mathrm{e}^{ \pm} v$ data. (right) the corresponding $\chi$ value per bin.
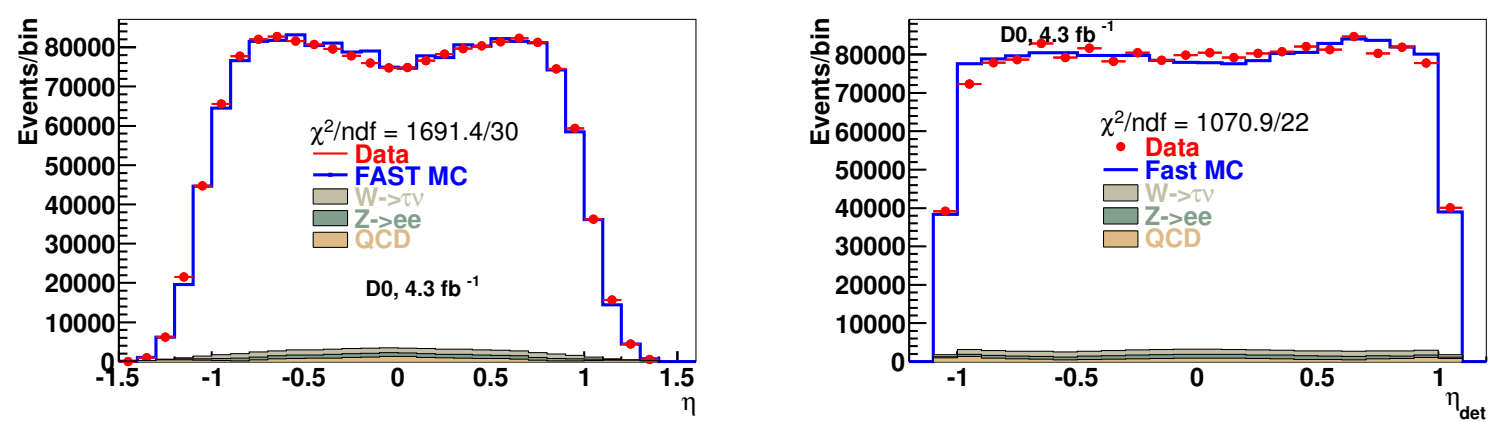

Figure B.10: (left) Comparison between data and fast $\mathrm{MC}$ for the physics $\eta$ distribution of electrons from $\mathrm{W}^{ \pm} \rightarrow \mathrm{e}^{ \pm} v$. (right) Comparison between data and fast MC for the detector $\eta$ distribution of electrons from $\mathrm{W}^{ \pm} \rightarrow \mathrm{e}^{ \pm} \nu$.
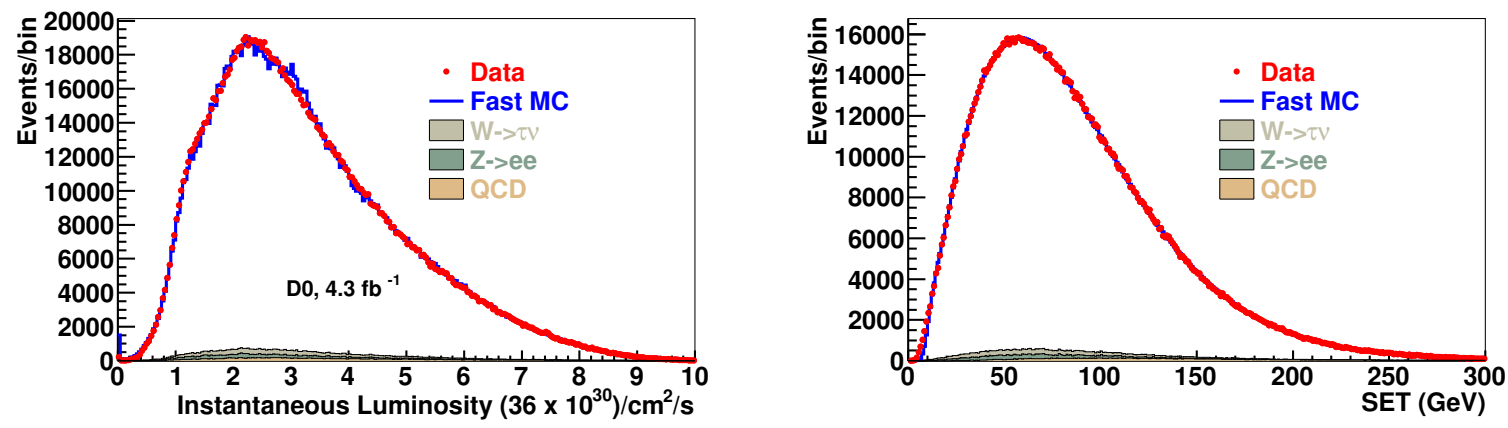

Figure B.11: (left) Comparison between data and fast MC for the instantaneous luminosity distribution of $\mathrm{W}^{ \pm} \rightarrow \mathrm{e}^{ \pm} v$ events. (right) Comparison between data and fast MC for the SET distribution of $\mathrm{W}^{ \pm} \rightarrow \mathrm{e}^{ \pm} v$ events. 


\section{Appendix C}

\section{Parametrized Model for the End-Cap Calibration}

\section{C.1 Ad hoc Geometries}

We prepared a number of different geometry models, changing detector elements in the forward region. The cryostat wall, due to its large number of points, is especially adequate to perform a $\eta_{\text {det }}$ dependent study of the dead material. The model of the cryostat wall in dogstar is represented by a GEANT polycone with 14 points. To increase the flexibility, we add extra points interpolating the larger straight segments. The goal was to have, as best as possible, one point in each region 0.1 wide in $\eta$. The interpolated geometry of the CC +1 element in dogstar, the D0 GEANT interface, is described in table C.1. The ad hoc modifications used in this calibration were prepared by varying the $z$ and $r_{\max }$ coordinate of the polycone points. By varying $r_{\max }$, we delay having to deal with overlapping of volumes until the final fit. The variations for ECdevelB1, ECdevelB2 and ECdevelB3 are described in table C.2. These modified geometries are properly implemented in the package d0AtlGeom under the directories p20_ECdevelB1, p20_ECdevelB2 and p20_ECdevelB3, respectively. 


\begin{tabular}{|c|c|c|c|c|c|c|c|}
\hline \hline $\begin{array}{c}\text { Polycone } \\
\text { point }\end{array}$ & $z(\mathrm{~cm})$ & $r_{\min }(\mathrm{cm})$ & $r_{\max }(\mathrm{cm})$ & $\begin{array}{c}\text { Polycone } \\
\text { point }\end{array}$ & $z(\mathrm{~cm})$ & $r_{\min }(\mathrm{cm})$ & $r_{\max }(\mathrm{cm})$ \\
\hline 1 & 150.6116 & 3.8100 & 3.8100 & 11 & 158.9762 & 59.9339 & 64.8920 \\
\hline 2 & 151.6801 & 3.8100 & 23.0944 & 12 & 160.0087 & 64.6203 & 68.2582 \\
\hline 3 & 151.7512 & 5.0948 & 24.3792 & 13 & 161.6774 & 70.0607 & 73.6986 \\
\hline 4 & 152.8140 & 24.2772 & 32.1395 & 14 & 163.3461 & 75.5011 & 79.1390 \\
\hline 5 & 153.3911 & 28.4912 & 36.3535 & 15 & 165.0148 & 80.9415 & 84.5794 \\
\hline 6 & 153.9682 & 32.7051 & 40.5675 & 16 & 166.0221 & 84.2254 & 87.1132 \\
\hline 7 & 154.5453 & 36.9191 & 44.7815 & 17 & 172.6220 & 100.8277 & 103.7156 \\
\hline 8 & 155.5964 & 44.5941 & 49.5521 & 18 & 173.5974 & 103.2814 & 105.6887 \\
\hline 9 & 156.7230 & 49.7074 & 54.6654 & 19 & 181.6217 & 119.5128 & 121.9200 \\
\hline 10 & 157.8496 & 54.8206 & 59.7787 & 20 & 182.8117 & 121.9200 & 121.9200 \\
\hline \hline
\end{tabular}

Table C.1: Polycone model for the D0 end-cap internal cryostat wall (volume CC +1 in dogstar). The number of points was increased by simple interpolation to provide additional flexibility in the fit. The coordinates are given with respect to the center of the calorimetry system and, in dogstar, additional shifts are applied to translate them to the center of tracker system, which is used as origin of the D0 global coordinates. 


\begin{tabular}{|c|c|c|c|c|c|c|}
\hline \hline Polycone & \multicolumn{2}{|c|}{ ECdevelB1 } & \multicolumn{2}{c|}{ ECdevelB2 } & \multicolumn{2}{c|}{ ECdevelB3 } \\
\hline point & $\delta z(\mathrm{~cm})$ & $\delta r_{\max }(\mathrm{cm})$ & $\delta z(\mathrm{~cm})$ & $\delta r_{\max }(\mathrm{cm})$ & $\delta z(\mathrm{~cm})$ & $\delta r_{\max }(\mathrm{cm})$ \\
\hline \hline 1 & -0.6576 & 0.0000 & -0.4076 & 0.0000 & -0.2506 & 0.0000 \\
\hline 2 & -0.4001 & 2.8497 & -0.2471 & 1.7639 & -0.1521 & 1.0854 \\
\hline 3 & -0.0002 & 4.8024 & -0.0002 & 2.9725 & -0.0002 & 1.8291 \\
\hline 4 & 0.0000 & 4.8025 & 0.0000 & 2.9727 & 0.0000 & 1.8292 \\
\hline 5 & -0.0001 & 4.1967 & -0.0001 & 2.5977 & -0.0001 & 1.5984 \\
\hline 6 & -0.0002 & 3.5909 & -0.0002 & 2.2227 & -0.0002 & 1.3677 \\
\hline 7 & -0.0003 & 2.9851 & -0.0003 & 1.8477 & -0.0003 & 1.1369 \\
\hline 8 & -0.0004 & 2.9851 & -0.0004 & 1.8477 & -0.0004 & 1.1369 \\
\hline 9 & -0.0003 & 2.7048 & -0.0003 & 1.6742 & -0.0003 & 1.0302 \\
\hline 10 & -0.0003 & 2.4246 & -0.0003 & 1.5007 & -0.0003 & 0.9234 \\
\hline 11 & -0.0002 & 2.1443 & -0.0002 & 1.3272 & -0.0002 & 0.8167 \\
\hline 12 & 0.0003 & 2.1443 & 0.0003 & 1.3273 & 0.0003 & 0.8167 \\
\hline 13 & 0.0003 & 1.9810 & 0.0003 & 1.2262 & 0.0003 & 0.7545 \\
\hline 14 & 0.0002 & 1.8177 & 0.0002 & 1.1251 & 0.0002 & 0.6923 \\
\hline 15 & 0.0002 & 1.6544 & 0.0002 & 1.0240 & 0.0002 & 0.6301 \\
\hline 16 & -0.0001 & 1.6545 & -0.0001 & 1.0241 & -0.0001 & 0.6301 \\
\hline 17 & 0.0000 & 1.3304 & 0.0000 & 0.8234 & 0.0000 & 0.5064 \\
\hline 18 & -0.0004 & 1.3303 & -0.0004 & 0.8233 & -0.0004 & 0.5063 \\
\hline 19 & -0.6577 & 0.0000 & -0.4067 & 0.0000 & -0.2507 & 0.0000 \\
\hline 20 & 0.0003 & 0.0000 & 0.0003 & 0.0000 & 0.0003 & 0.0000 \\
\hline \hline
\end{tabular}

Table C.2: The three ad hoc variations built by varying $z$ and $r_{\max }$ in the polycone model of the internal cryostat wall. 


\section{C.2 EM Energy Fraction Model}

We build parametrized models for the EM energy fraction variation in full MC as a function of the variation in thickness of the internal cryostat wall. Since the variation is small, a linear parametrization works perfectly well, as can be seen in figures C.2 to C.5 for the south end-cap and figures C.6 to C.9 for the north one.

Although we do not parametrize directly the data/fullMC EMF ratio, this choice has many advantages. Firstly, since the fullMC are statistically independent, there is no correlation between the different points. Had we divided by the data EMF, the points would be very correlated making the determination of the parameters in the model and tests of goodness of fit more complicated. Secondly, working only with variations avoid large correlations between the parameters of the fit, improving their numerical determination and simplifying the propagation of uncertainties. Figure C.1 depicts the EMF for data and for fullMC with nominal geometry which, together with the model, allow the reconstruction of the observable used to fit the amount of dead material.

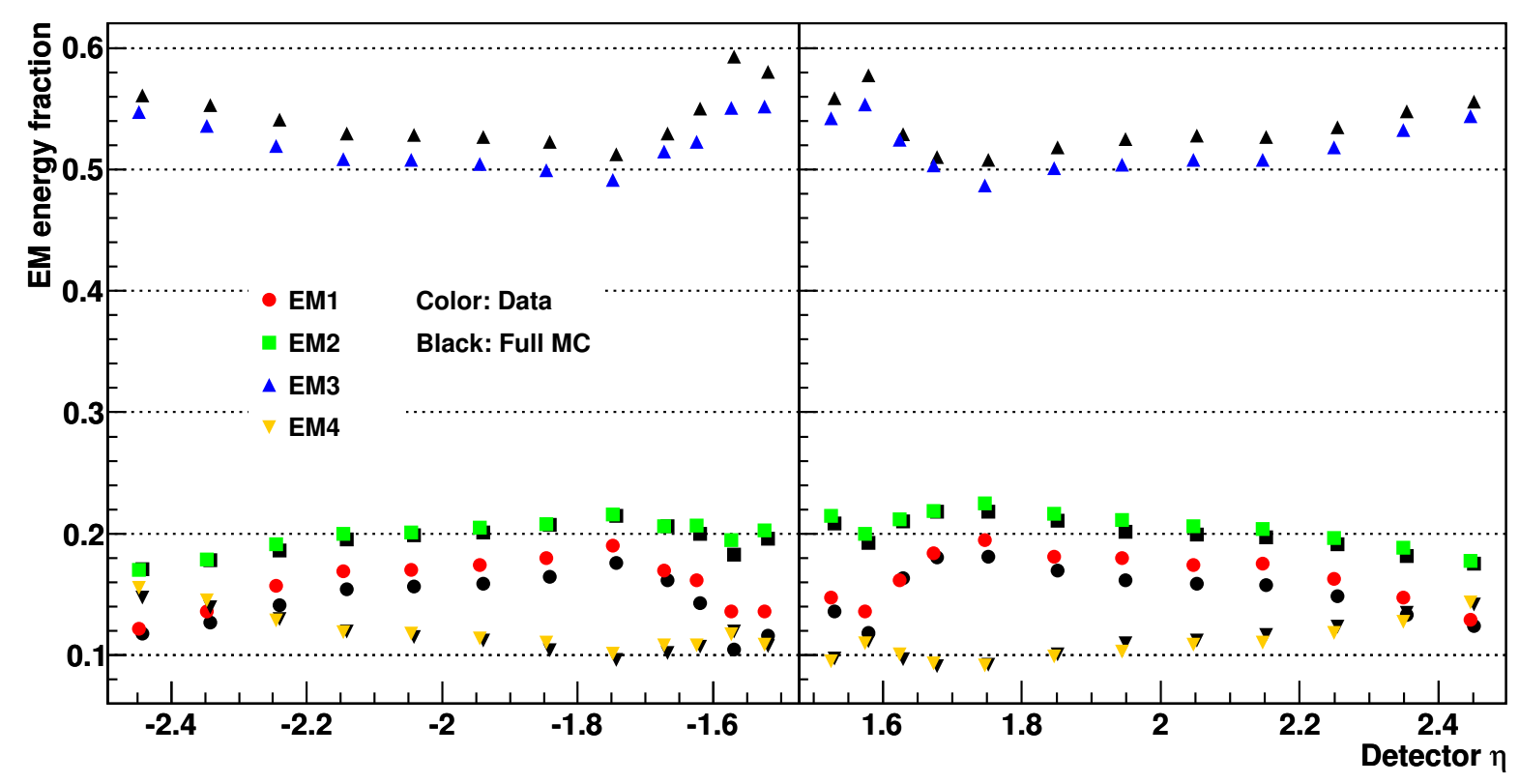

Figure C.1: Data and fullMC mean of the EMF distributions in bins of $\eta_{\text {det }}$ using the same binning as used for the fitting of dead material. 

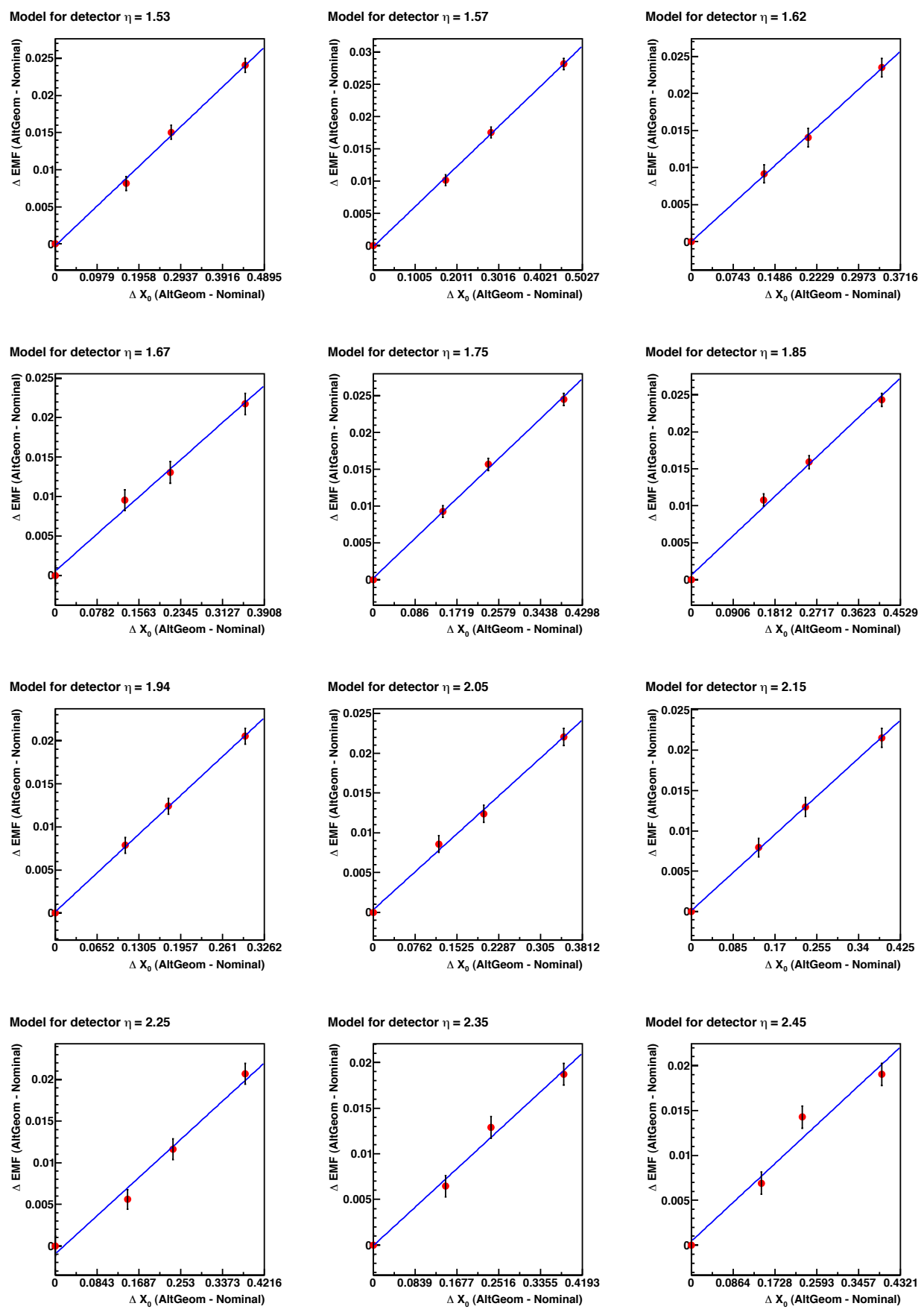

Figure C.2: Parametrized model for the variation of the mean EM1 energy fraction as a function of the variation of the dead material. Since the model is built with independent full MC samples, the bins are statistically independent. 

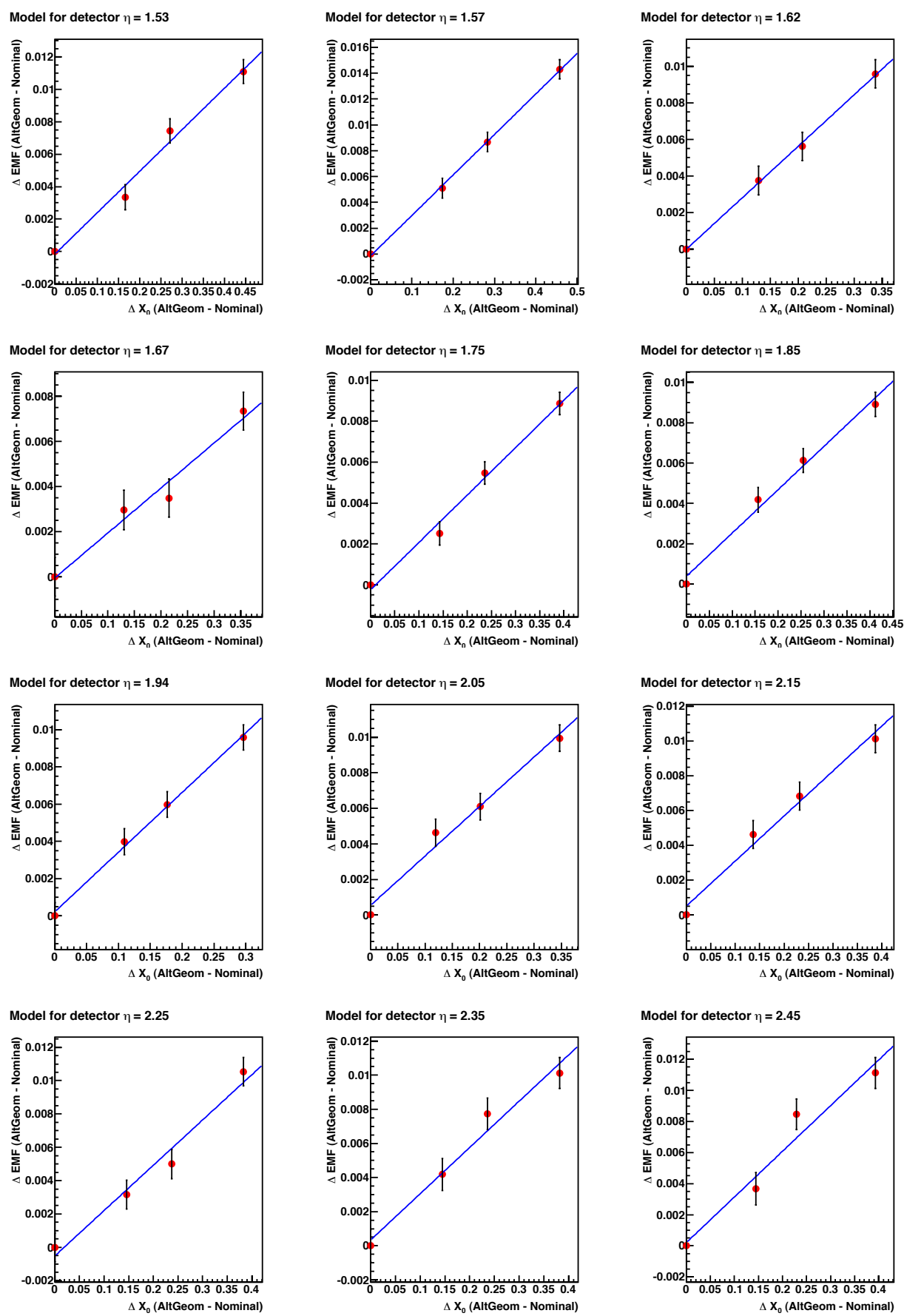

Figure C.3: Parametrized model for the variation of the mean EM2 energy fraction as a function of the variation of the dead material. Since the model is built with independent full MC samples, the bins are statistically independent. 

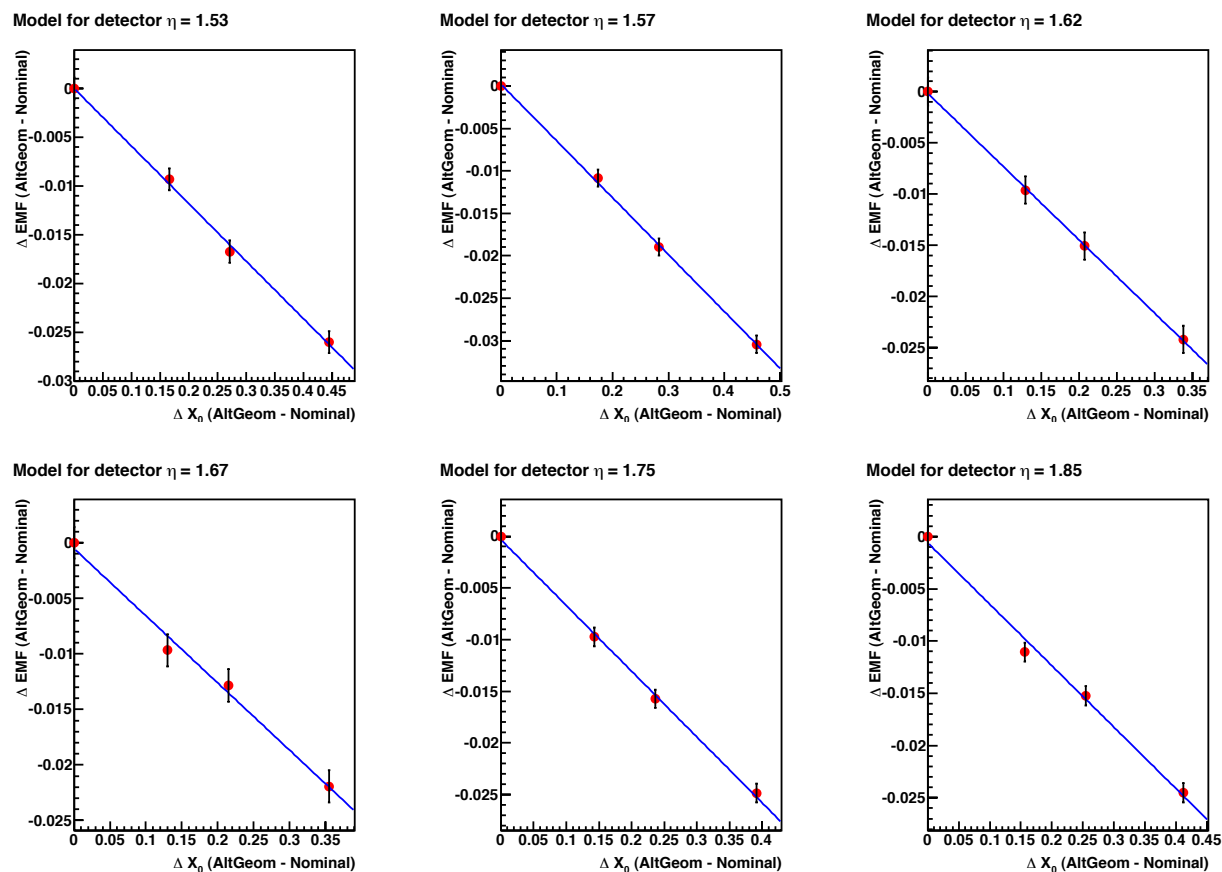

Model for detector $\eta=1.94$
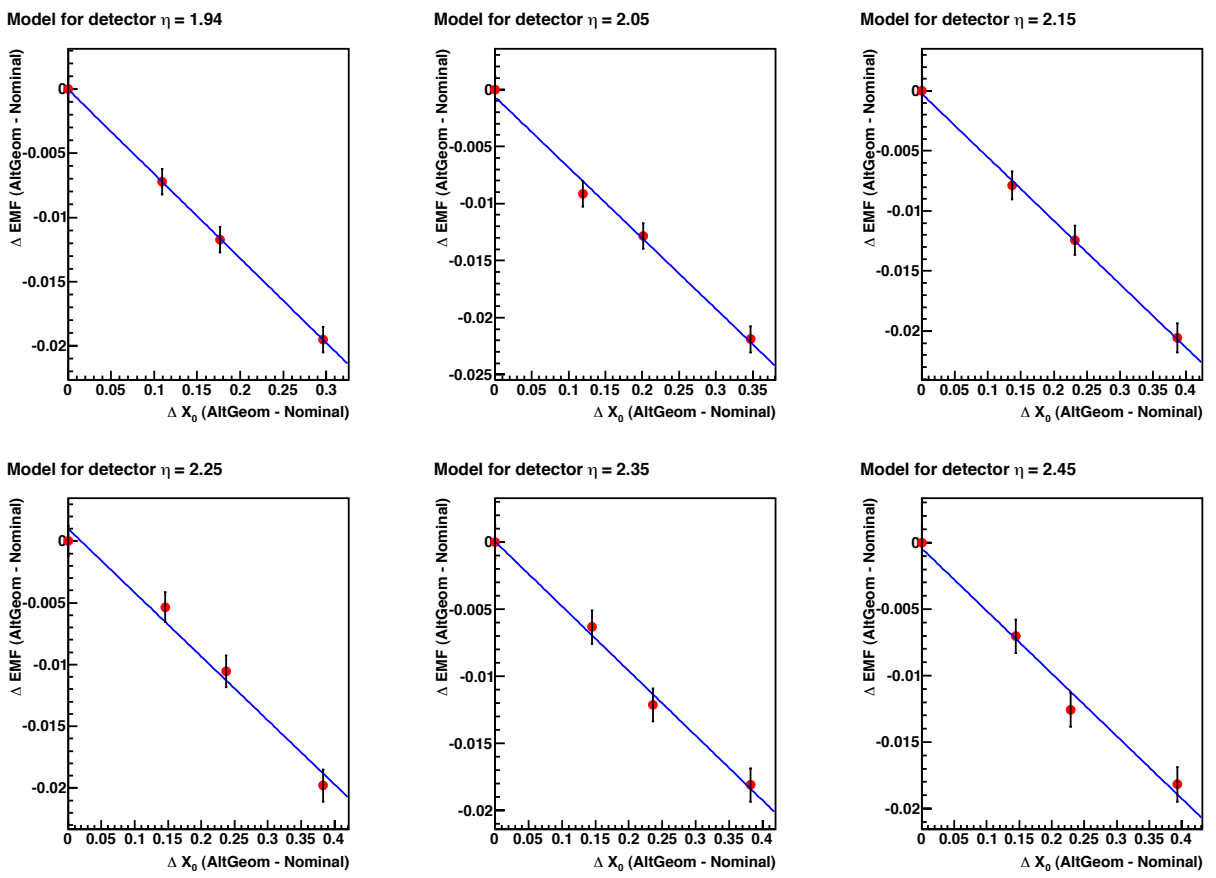

Figure C.4: Parametrized model for the variation of the mean EM3 energy fraction as a function of the variation of the dead material. Since the model is built with independent full MC samples, the bins are statistically independent. 

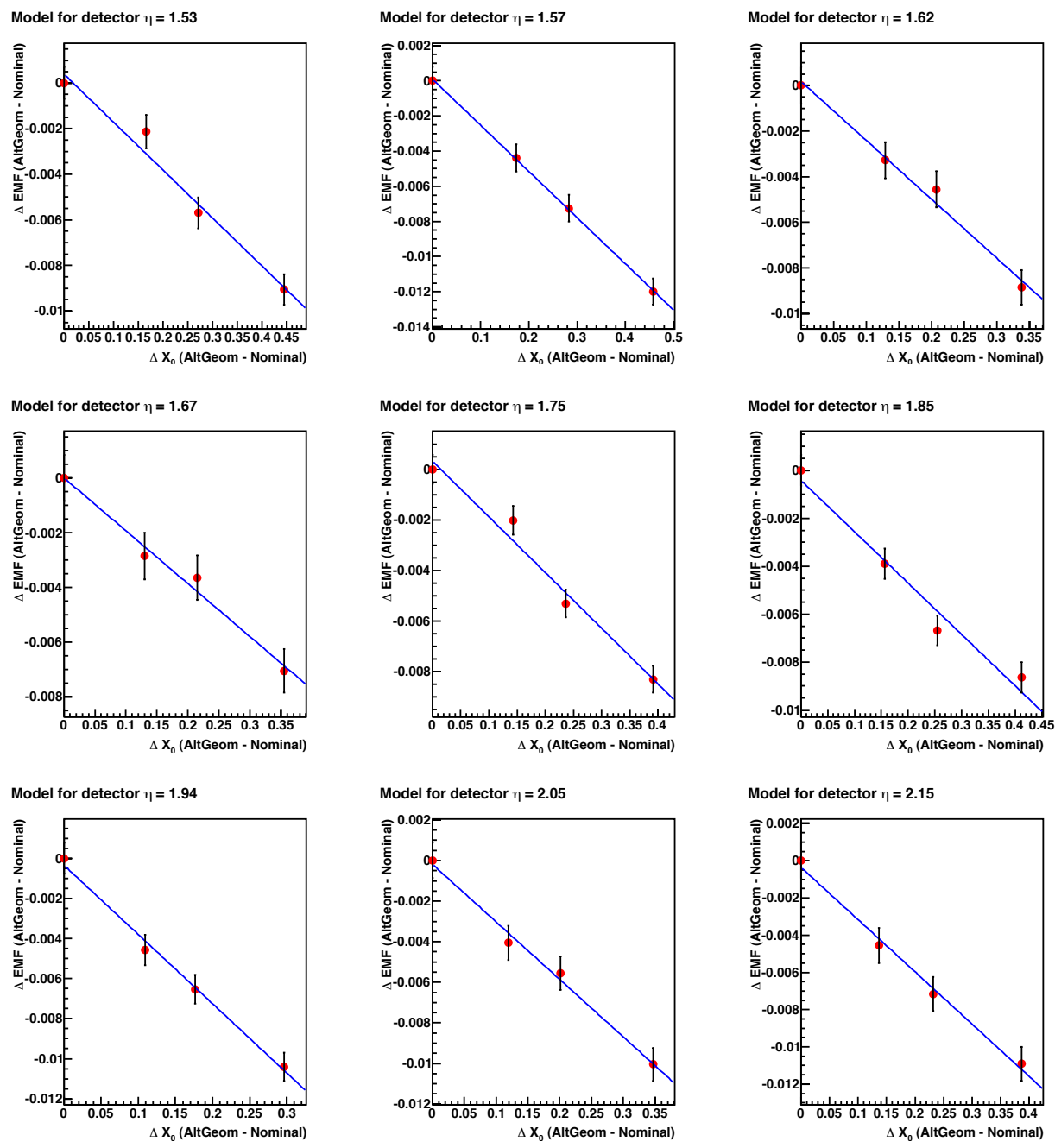

Model for detector $\eta=\mathbf{2} .15$
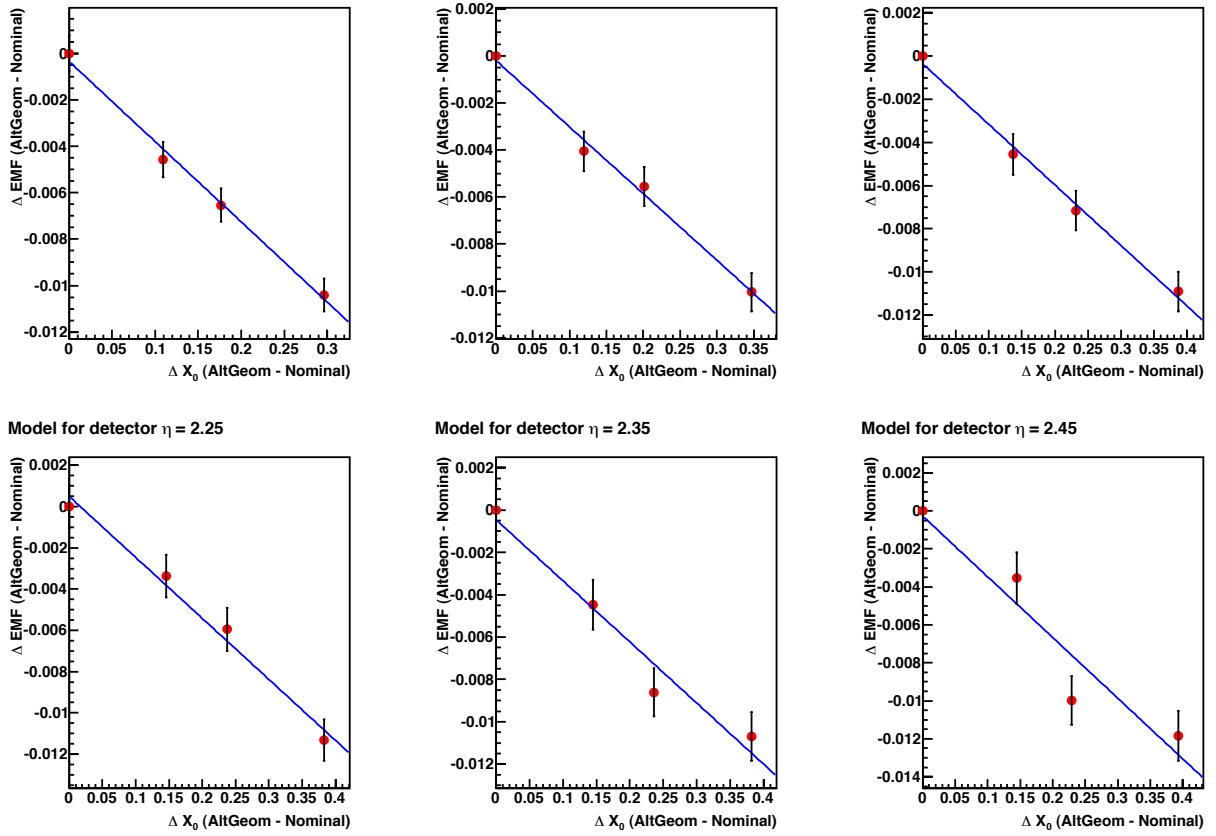

Figure C.5: Parametrized model for the variation of the mean EM4 energy fraction as a function of the variation of the dead material. Since the model is built with independent full MC samples, the bins are statistically independent. 

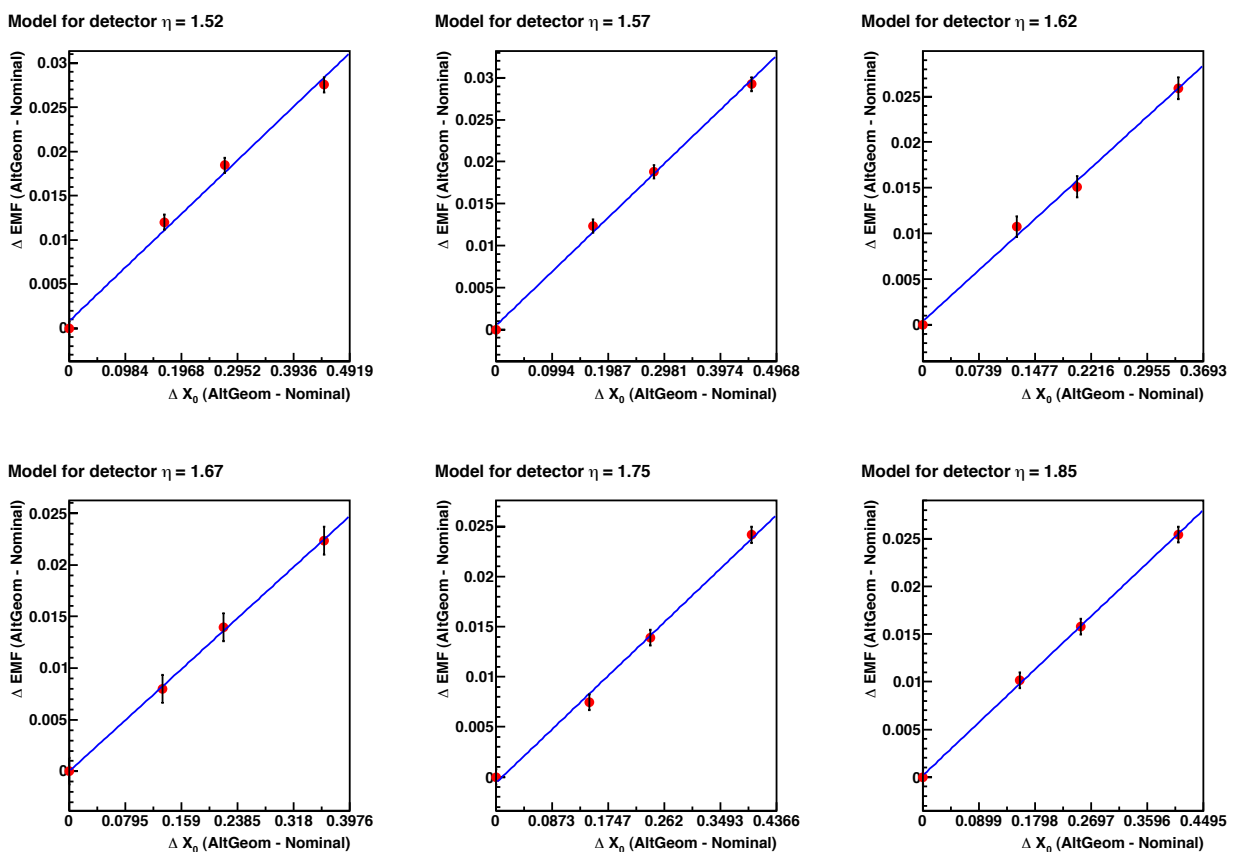

Model for detector $\eta=1.94$
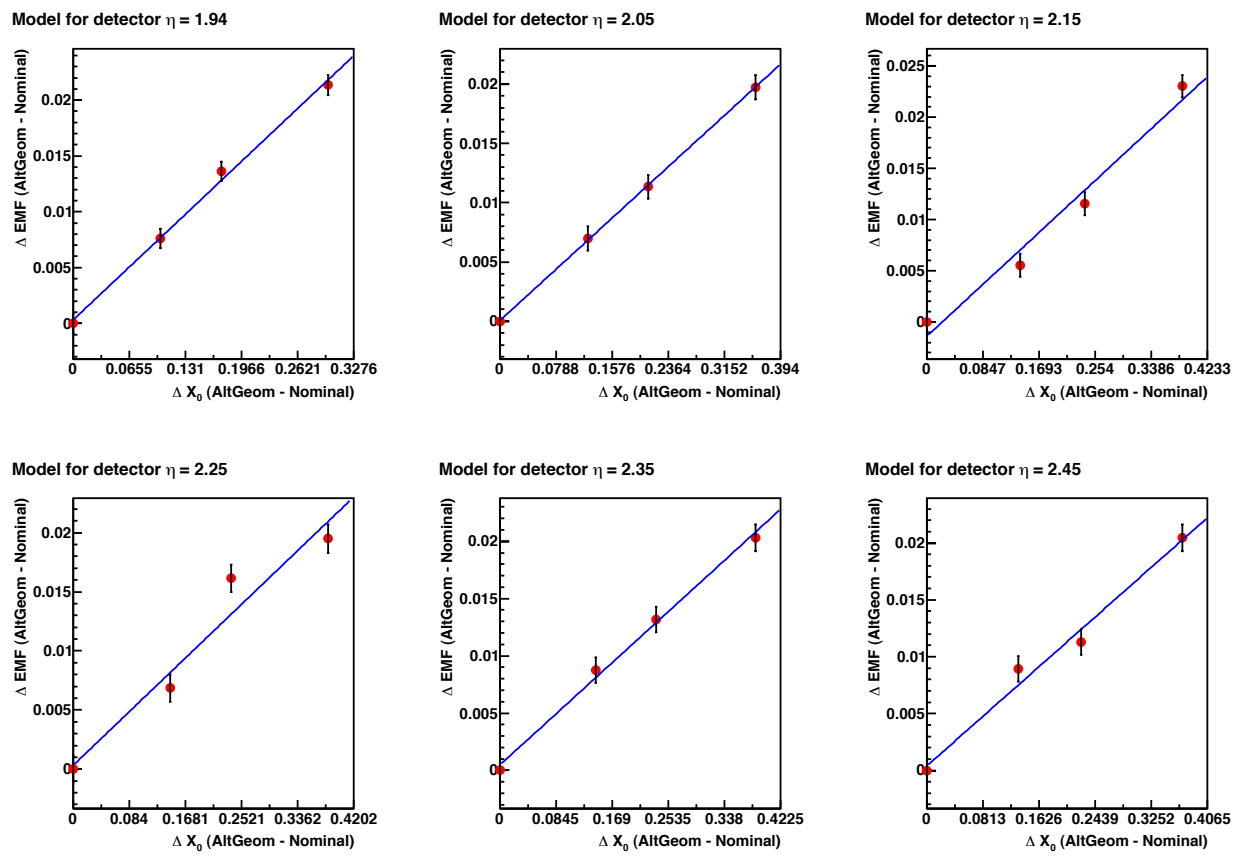

Figure C.6: Parametrized model for the variation of the mean EM1 energy fraction as a function of the variation of the dead material. Since the model is built with independent full MC samples, the bins are statistically independent. 

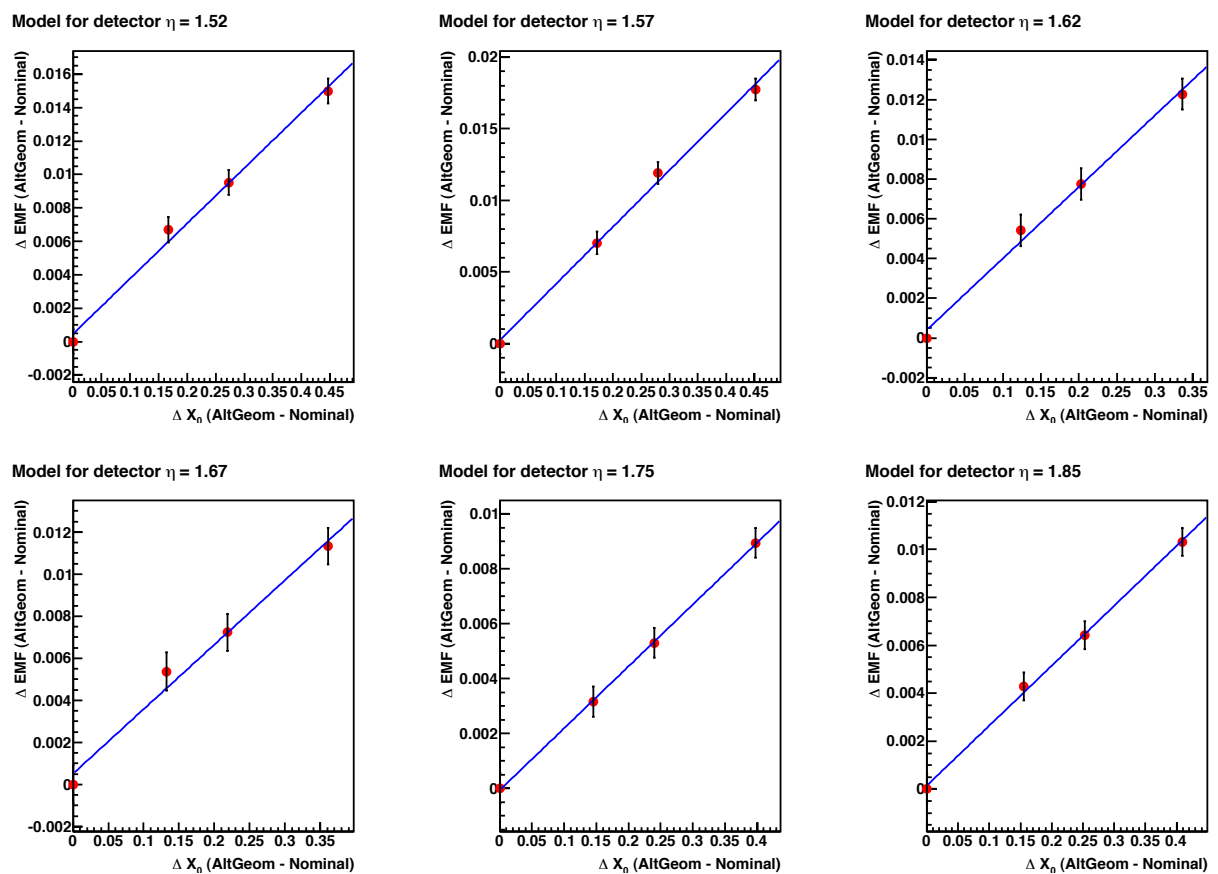

Model for detector $\eta=1.94$
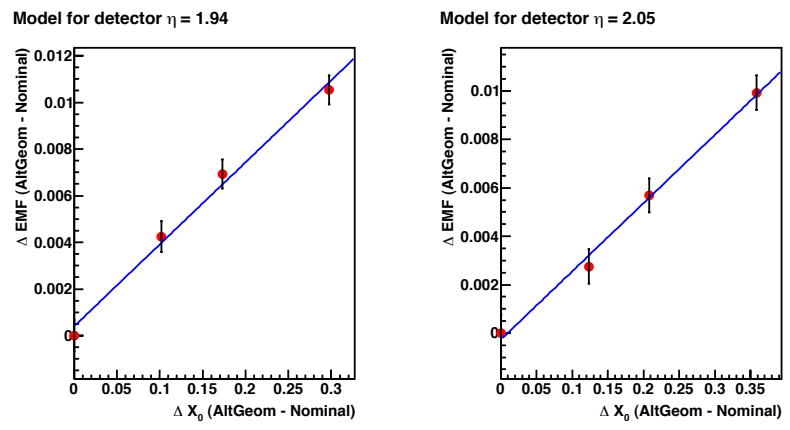

Model for detector $\eta=2.15$
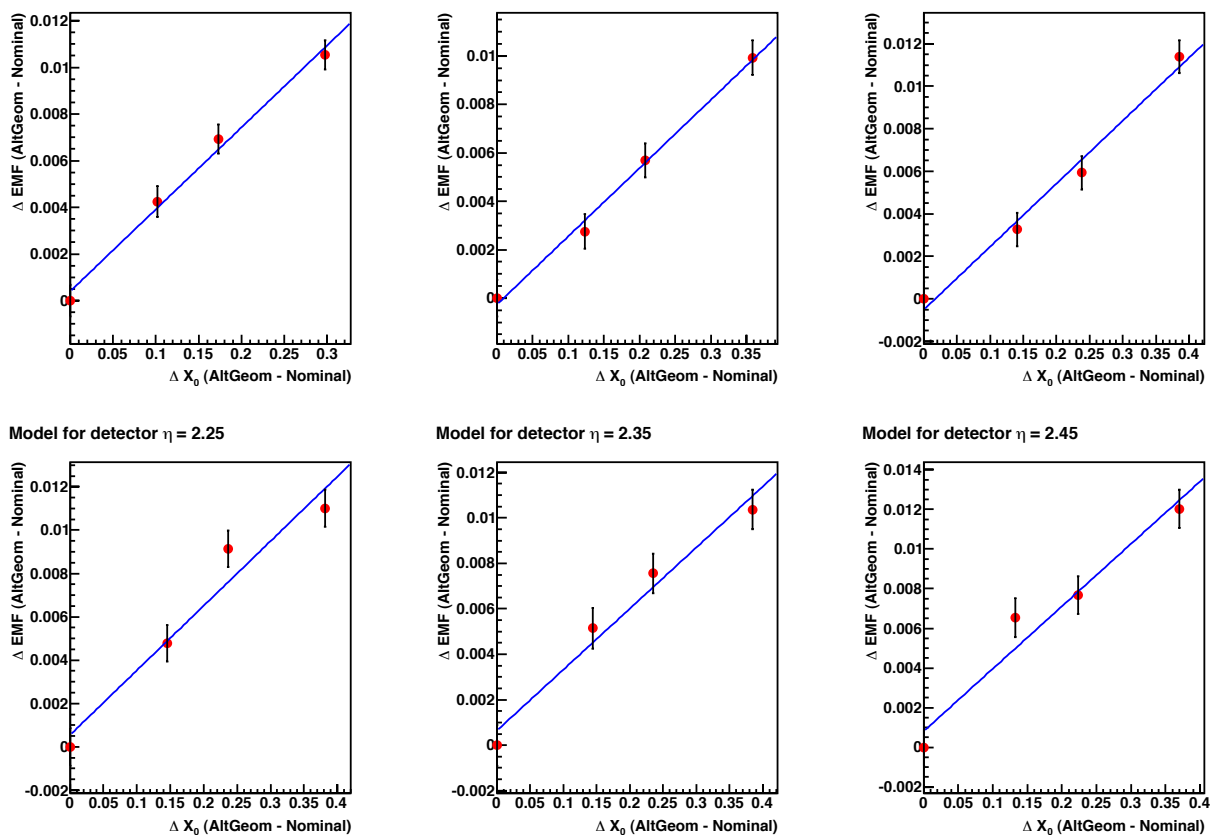

Figure C.7: Parametrized model for the variation of the mean EM2 energy fraction as a function of the variation of the dead material. Since the model is built with independent full MC samples, the bins are statistically independent. 

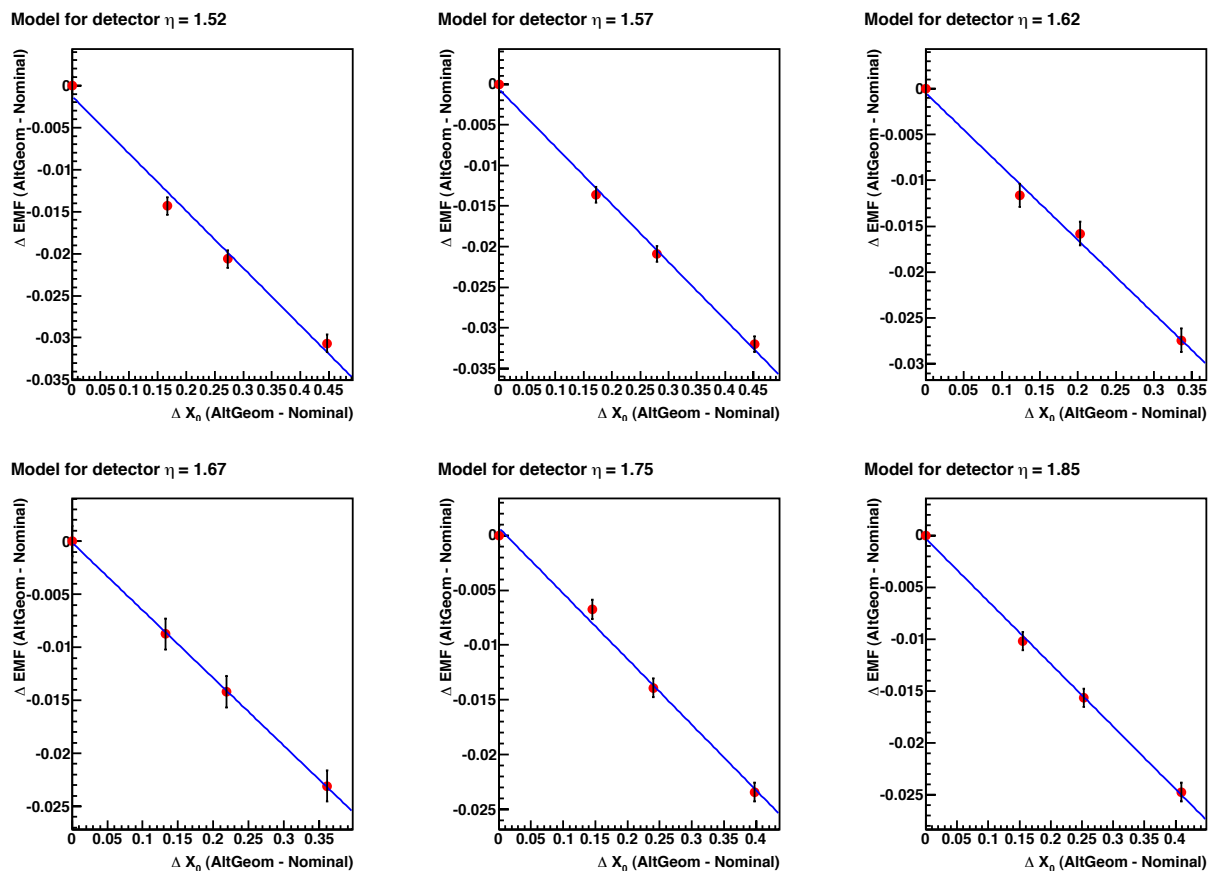

Model for detector $\eta=1.94$
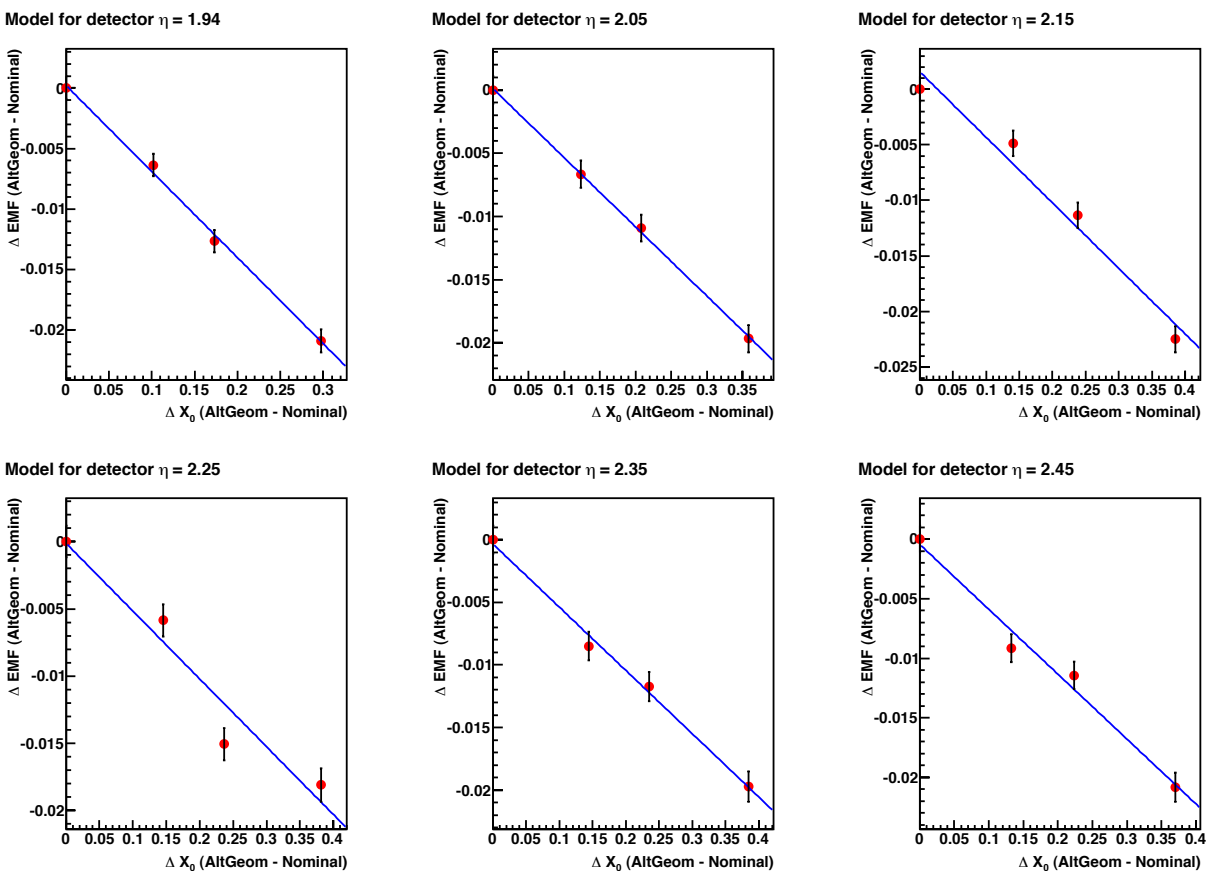

Figure C.8: Parametrized model for the variation of the mean EM3 energy fraction as a function of the variation of the dead material. Since the model is built with independent full MC samples, the bins are statistically independent. 

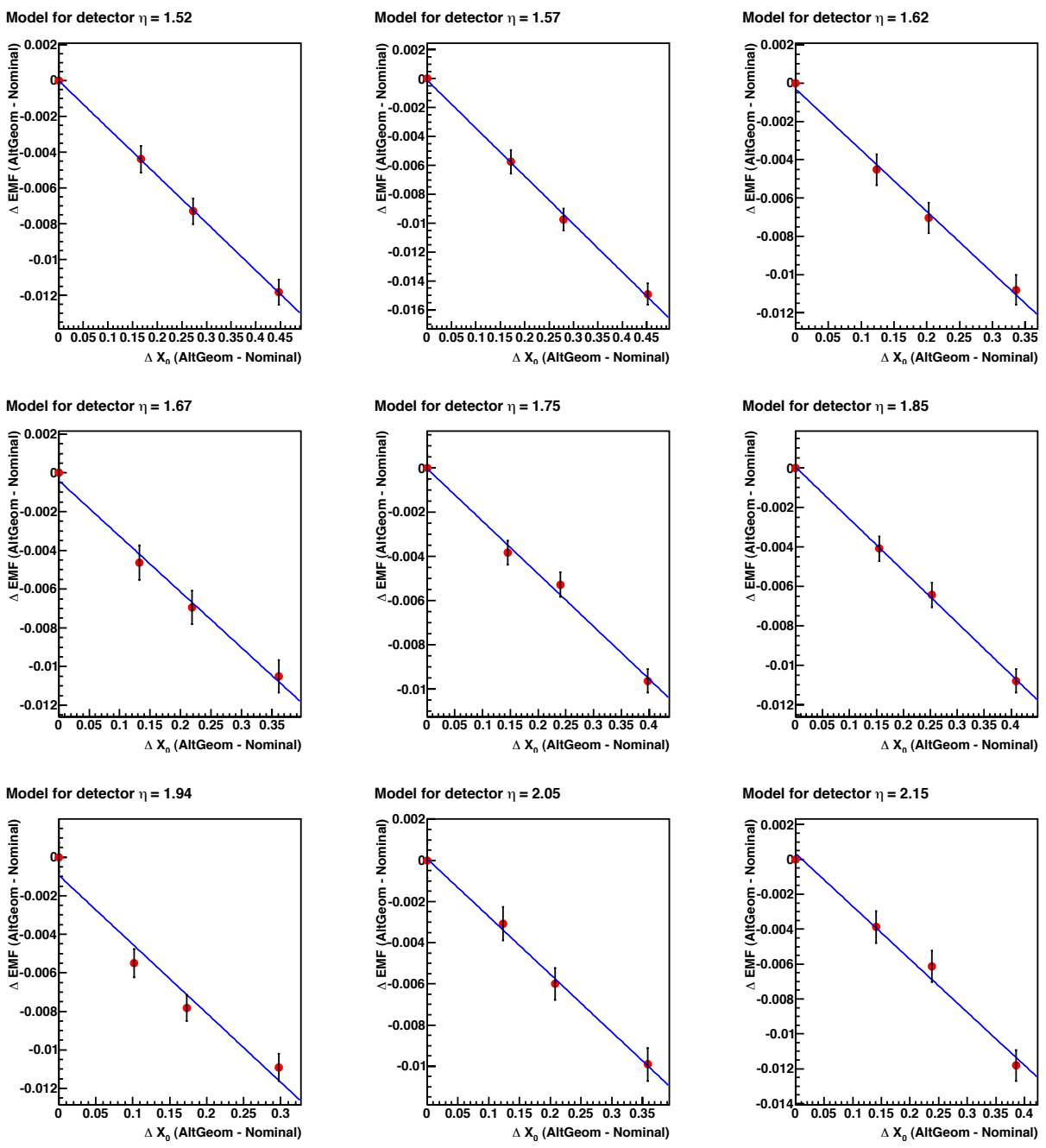

Model for detector $\eta=2.15$
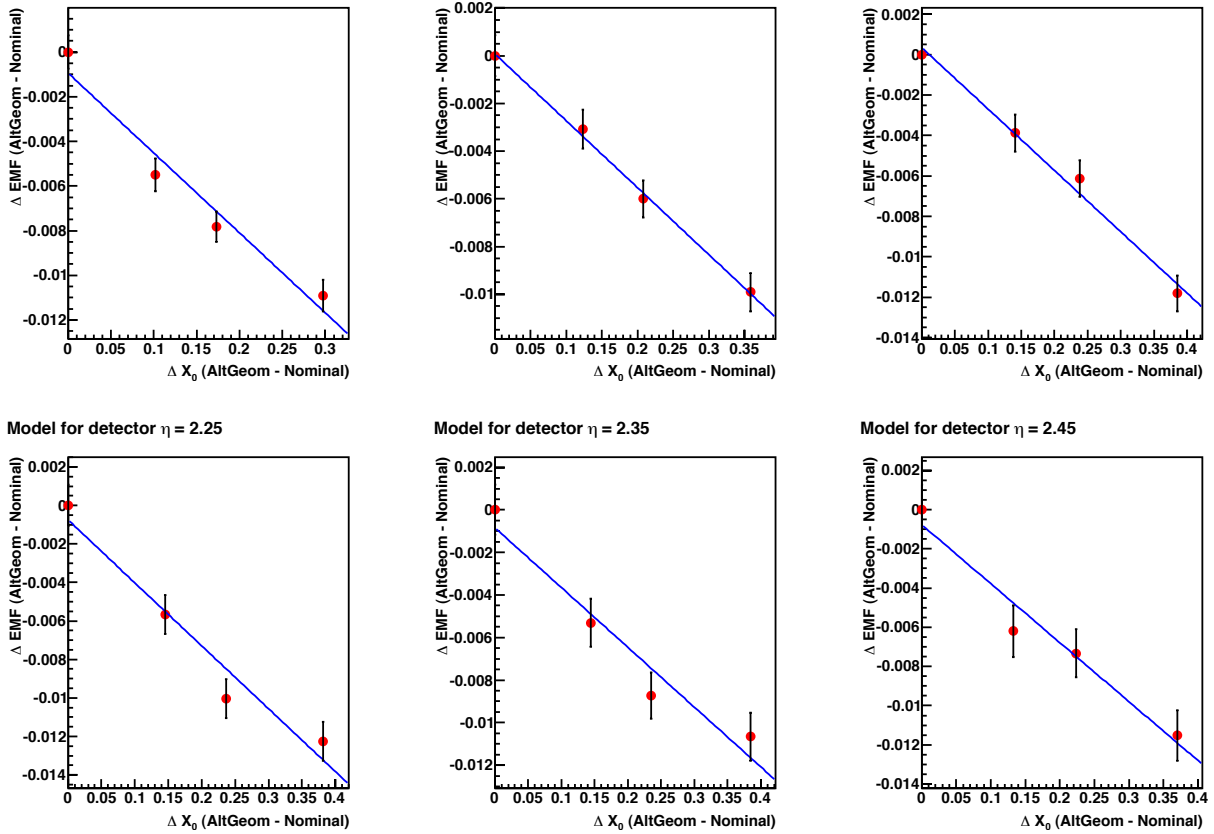

Figure C.9: Parametrized model for the variation of the mean EM4 energy fraction as a function of the variation of the dead material. Since the model is built with independent full MC samples, the bins are statistically independent. 


\section{C.3 Geometry Eigensets and Energy Loss Function}

The error eigensets are derived from the covariance matrix returned from MINUIT during the minimization of the test statistic (eq. 13.10). We diagonalize the covariance matrix and shift each normalized eigenvector by $\pm 1 \sigma$, where $\sigma$ is the square root of the associated eigenvalue. It is expected that the correlations for distant bins in $\eta_{\text {det }}$ is negligible. Therefore, the error sets will have variations concentrated around one $\eta_{\text {det }}$ region, up to unavoidable statistical fluctuations in the covariance matrix.

Figures C.10 to C.14 show the variations associated to each member of the error set for the south end-cap. They are ordered by decreasing value of the associated eigenvalue. In each figure, the left panel shows the variation in the EM energy fraction distribution while the right panel shows the variation in the amount of dead material added to the internal cryostat wall. Figures C.15 to C.19 show the equivalent distributions for the north End Calorimeter.

The energy loss functions are determined in bins of $\eta_{\text {det }}$. We bins with boundaries 1.5, $1.55,1.6,1.65,1.7,1.8,1.9,2.0,2.1,2.2,2.3,2.4$, and 2.5. The derivation is done separately for the north and south cryostat. The many transitions present in the forward direction thwarts any attempt to smooth the energy function in $\eta_{\text {det }}$, similarly as what was done for the central calorimeter calibration. The energy dependence of the energy loss functions is parametrized with the following function:

$$
\operatorname{eloss}(E)=p_{0}+p_{1} \log (E)+p_{2} \log ^{2}(E)+p_{3} \log ^{3}(E),
$$

where $E$ is the uncorrected (raw) energy read out by the calorimeter.

The parameters are derived for the ad hoc geometries ECdevelB1, ECdevelB2, ECdevelB3. We use a single monochromatic electron sample, flat in $\eta$ to study the dependence of the average reconstructed energy for a given true energy and define eloss $(E)$ as the ratio between the two. The fit to the parametric form is done in the range $E \in[25,400]$ and shown in figures C.20 to C.27.

The intrinsic non-linear dependence of the parameters in the fit also prevents the determination of a linear flow with the respect to the modification of the material in each $\eta_{\text {det }}$ bin. The image of the energy loss function, on the other hand, does not suffer from this problem. Therefore, we used the fitted functions for each ad hoc functions to build a model of the energy loss binned in both uncorrected energy and modification in the dead material eloss $\left(E_{\text {raw }}, \Delta X_{0}\right)$ and, then, used the measured values of $\Delta X_{0}$ to determine the best energy loss function. Then, the energy points are fitted to the same functional form as in equation C.1.

The results are shown in figures C.28 to C.31. The shaded areas show the sum in quadrature of the variation in the energy loss function determined from the error eigenset in the material tune. For visibility, the variations shown correspond to a $3 \sigma$ shift, where $\sigma$ is the eigenvalue of the corresponding eigenvalue. 

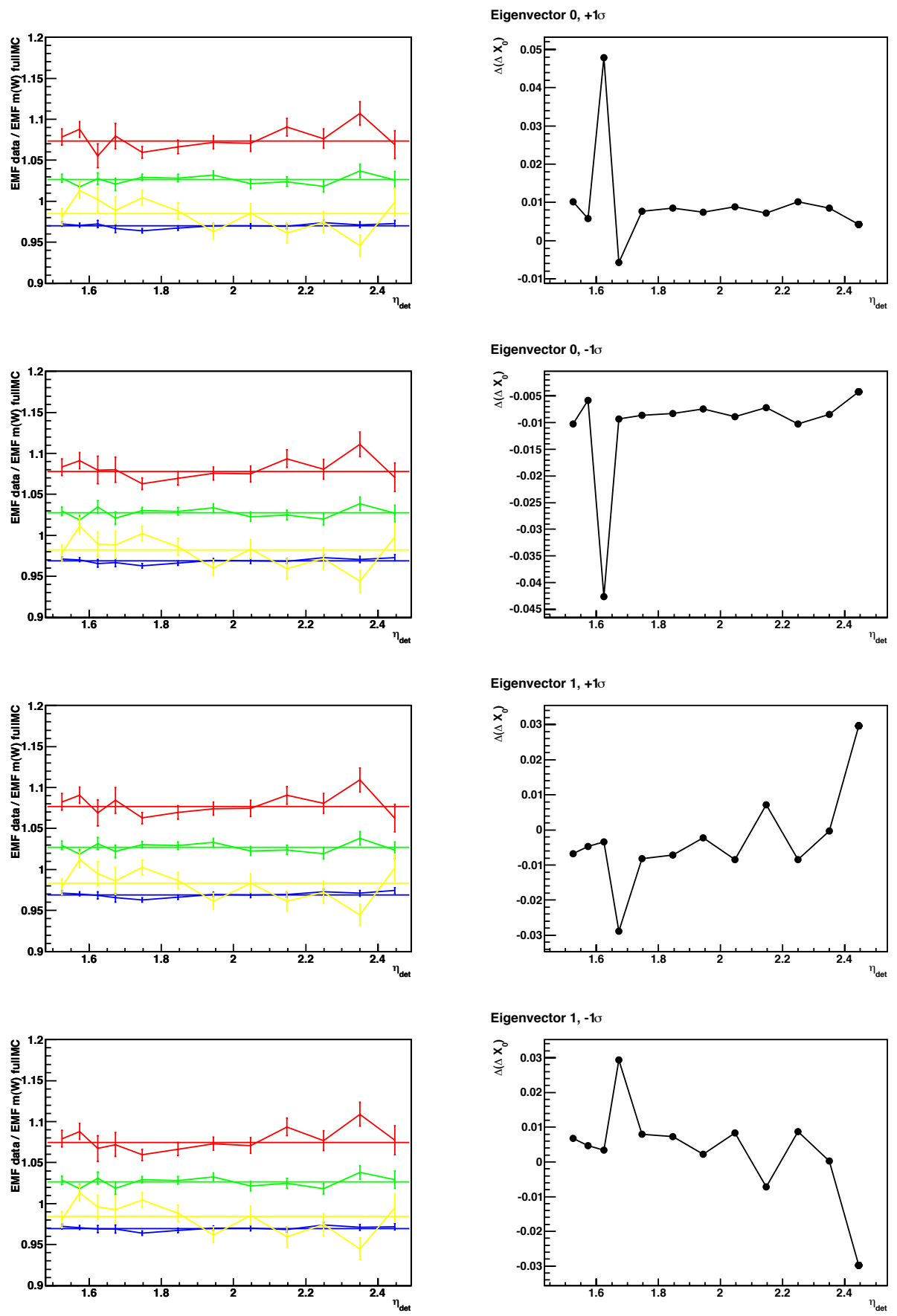

Figure C.10: South end-cap, eigenset 1 (two upper panels) and 2 (two bottom panels) with variations corresponding to $\pm 1 \sigma$ where $\sigma$ is the square root of the corresponding eigenvalue. The eigenvectors are ordered by decreasing eigenvalue. 

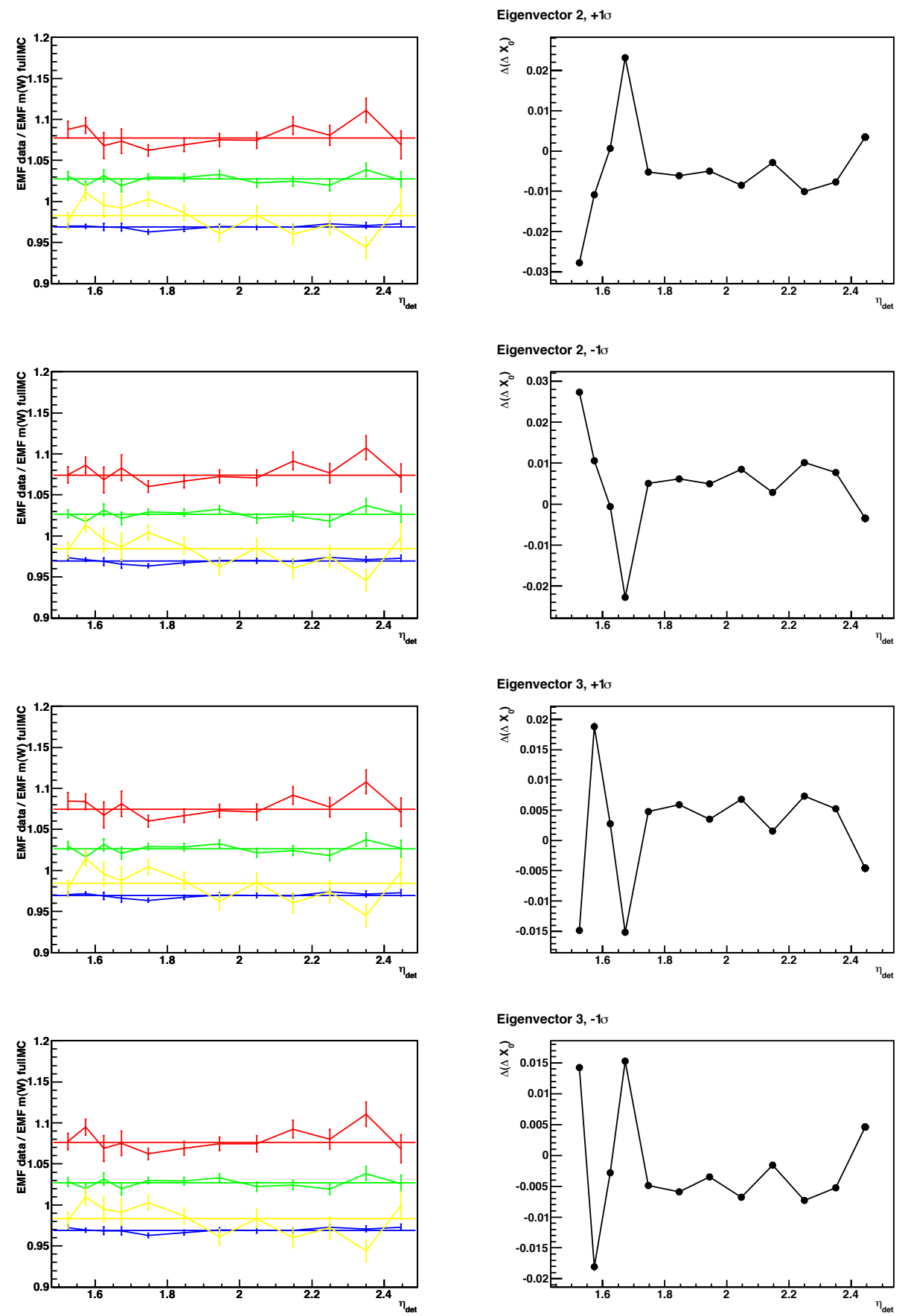

Figure C.11: South end-cap, eigenset 3 (two upper panels) and 4 (two bottom panels) with variations corresponding to $\pm 1 \sigma$ where $\sigma$ is the square root of the corresponding eigenvalue. The eigenvectors are ordered by decreasing eigenvalue. 

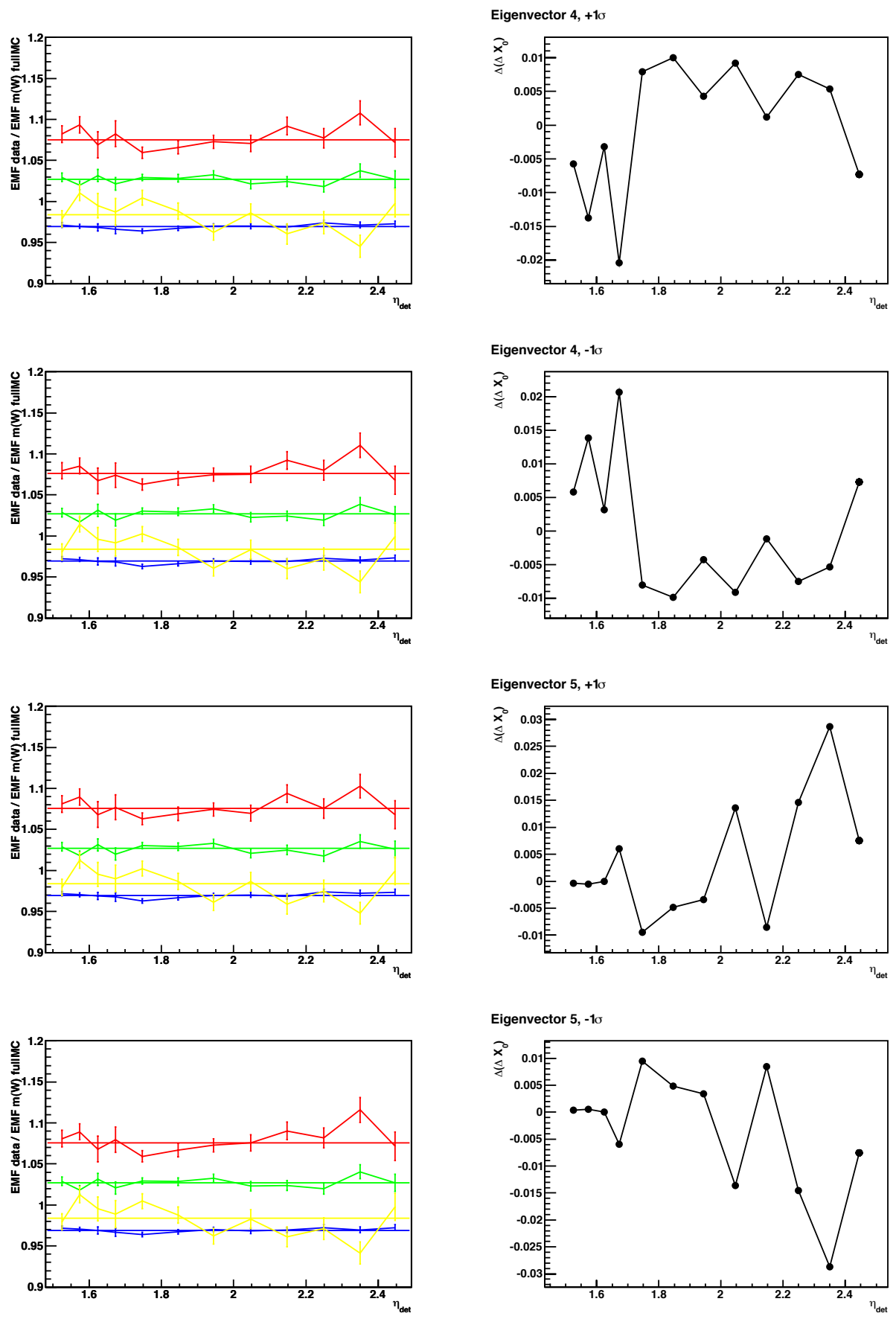

Figure C.12: South end-cap, eigenset 5 (two upper panels) and 6 (two bottom panels) with variations corresponding to $\pm 1 \sigma$ where $\sigma$ is the square root of the corresponding eigenvalue. The eigenvectors are ordered by decreasing eigenvalue. 

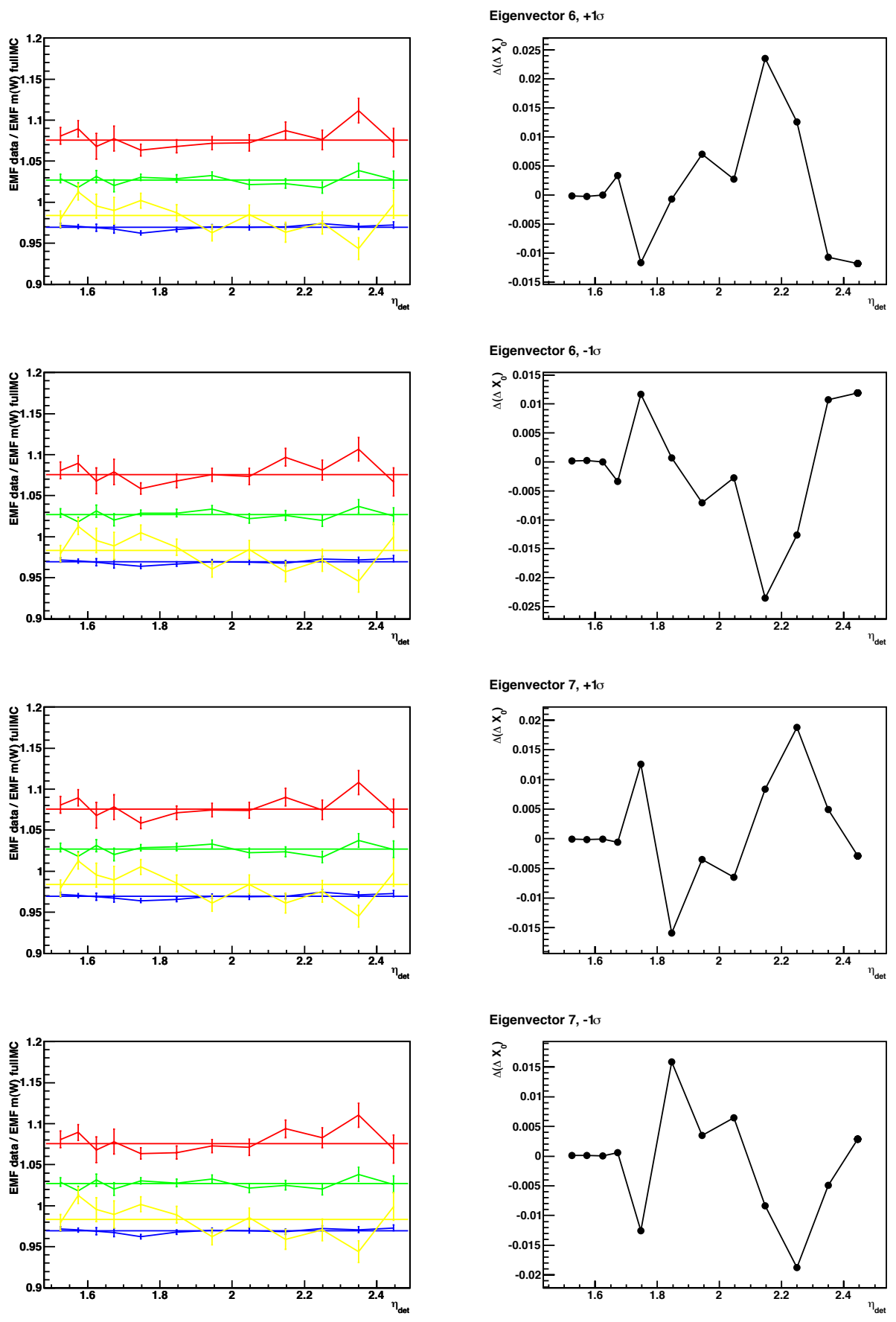

Figure C.13: South end-cap, eigenset 7 (two upper panels) and 8 (two bottom panels) with variations corresponding to $\pm 1 \sigma$ where $\sigma$ is the square root of the corresponding eigenvalue. The eigenvectors are ordered by decreasing eigenvalue. 

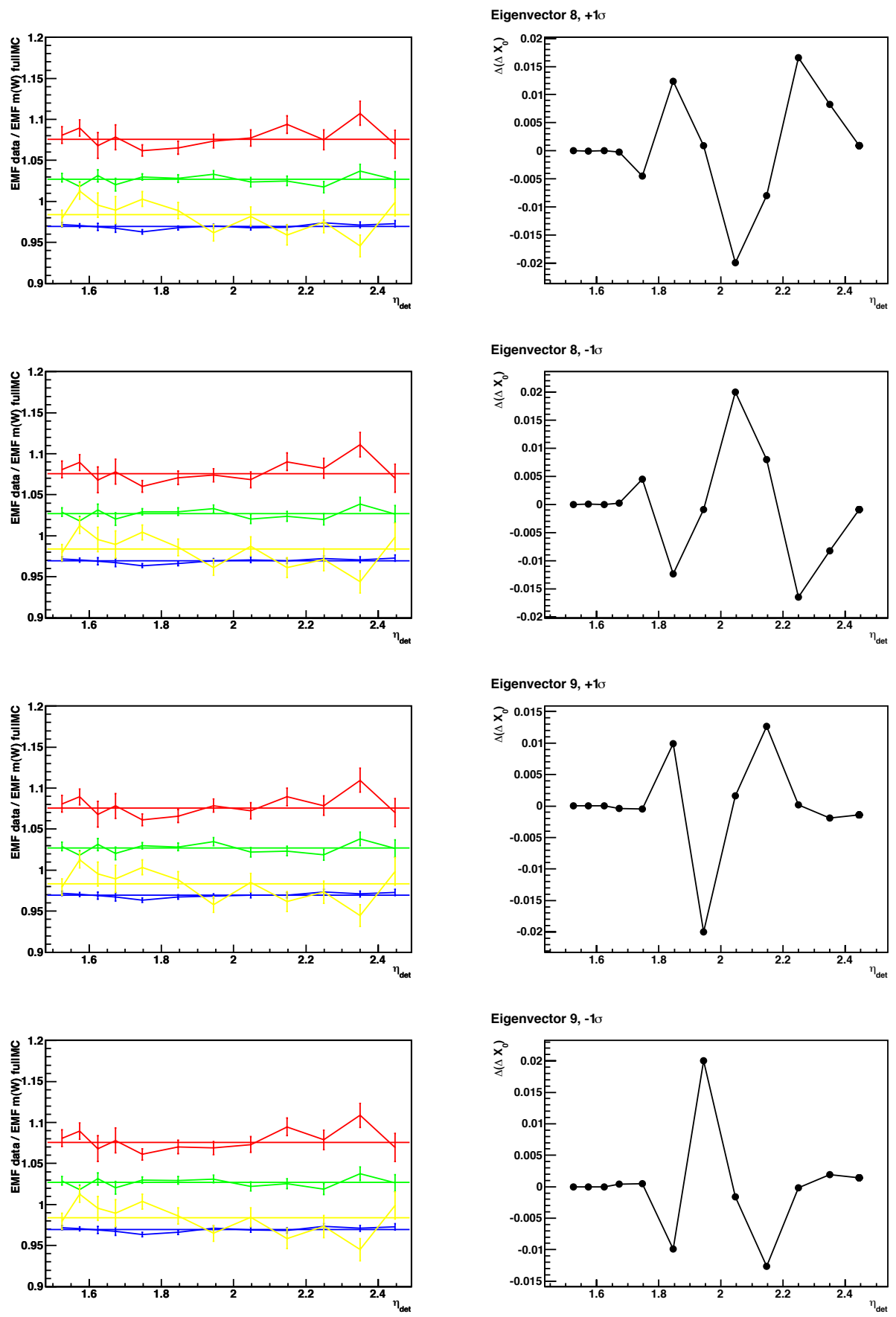

Figure C.14: South end-cap, eigenset 9 (two upper panels) and 10 (two bottom panels) with variations corresponding to $\pm 1 \sigma$ where $\sigma$ is the square root of the corresponding eigenvalue. The eigenvectors are ordered by decreasing eigenvalue. 

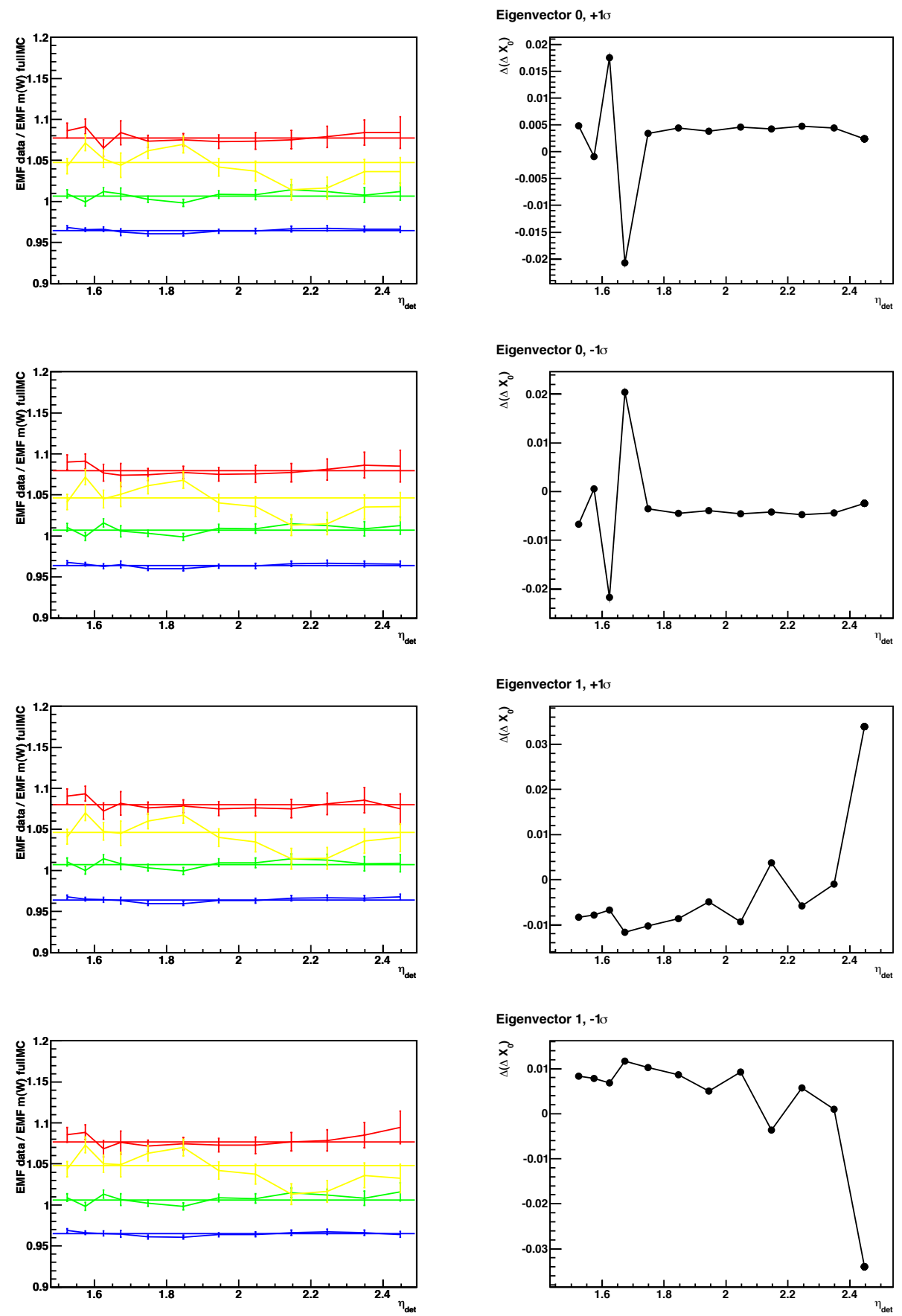

Figure C.15: North end-cap, eigenset 1 (two upper panels) and 2 (two bottom panels) with variations corresponding to $\pm 1 \sigma$ where $\sigma$ is the square root of the corresponding eigenvalue. The eigenvectors are ordered by decreasing eigenvalue. 

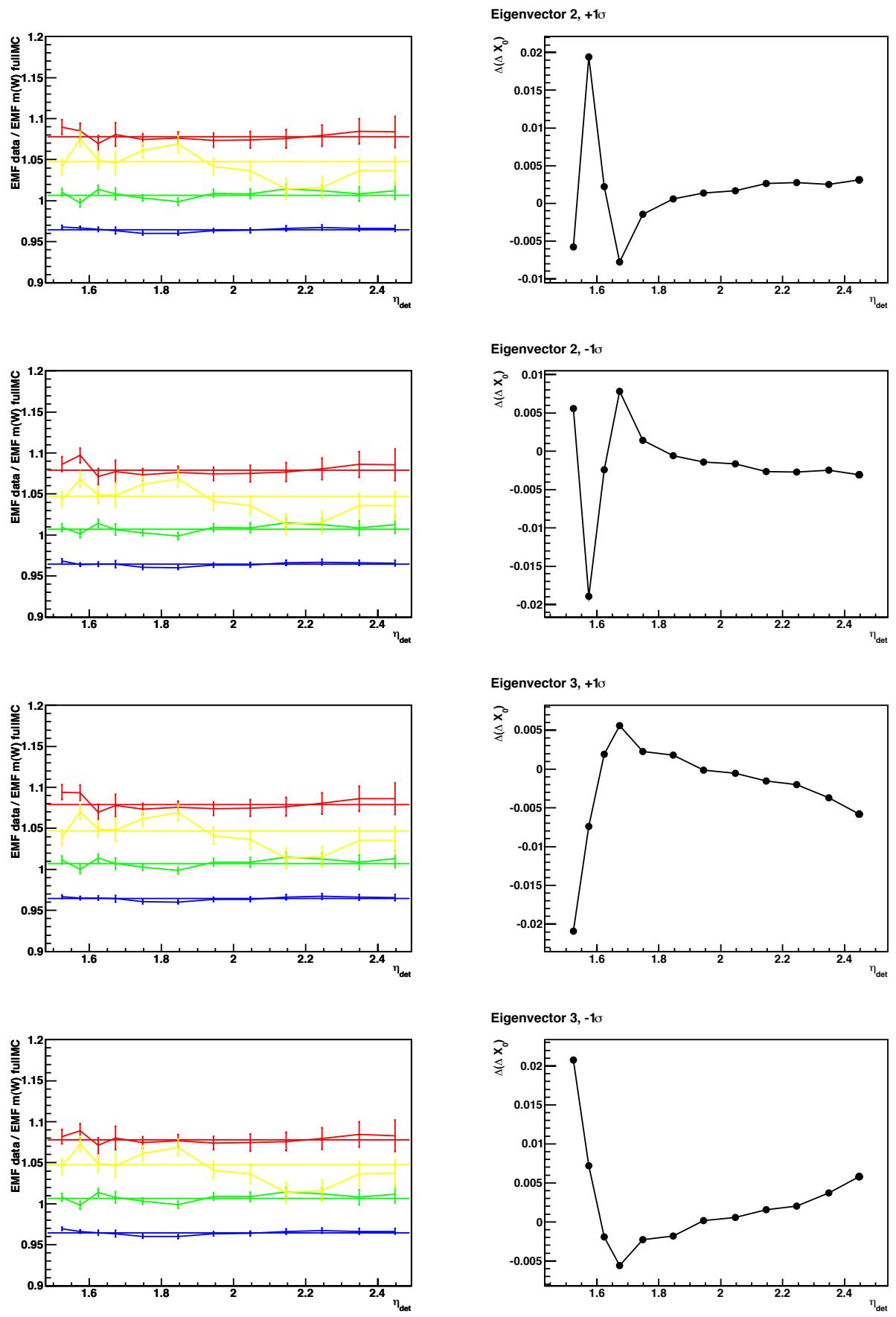

Figure C.16: North end-cap, eigenset 3 (two upper panels) and 4 (two bottom panels) with variations corresponding to $\pm 1 \sigma$ where $\sigma$ is the square root of the corresponding eigenvalue. The eigenvectors are ordered by decreasing eigenvalue. 

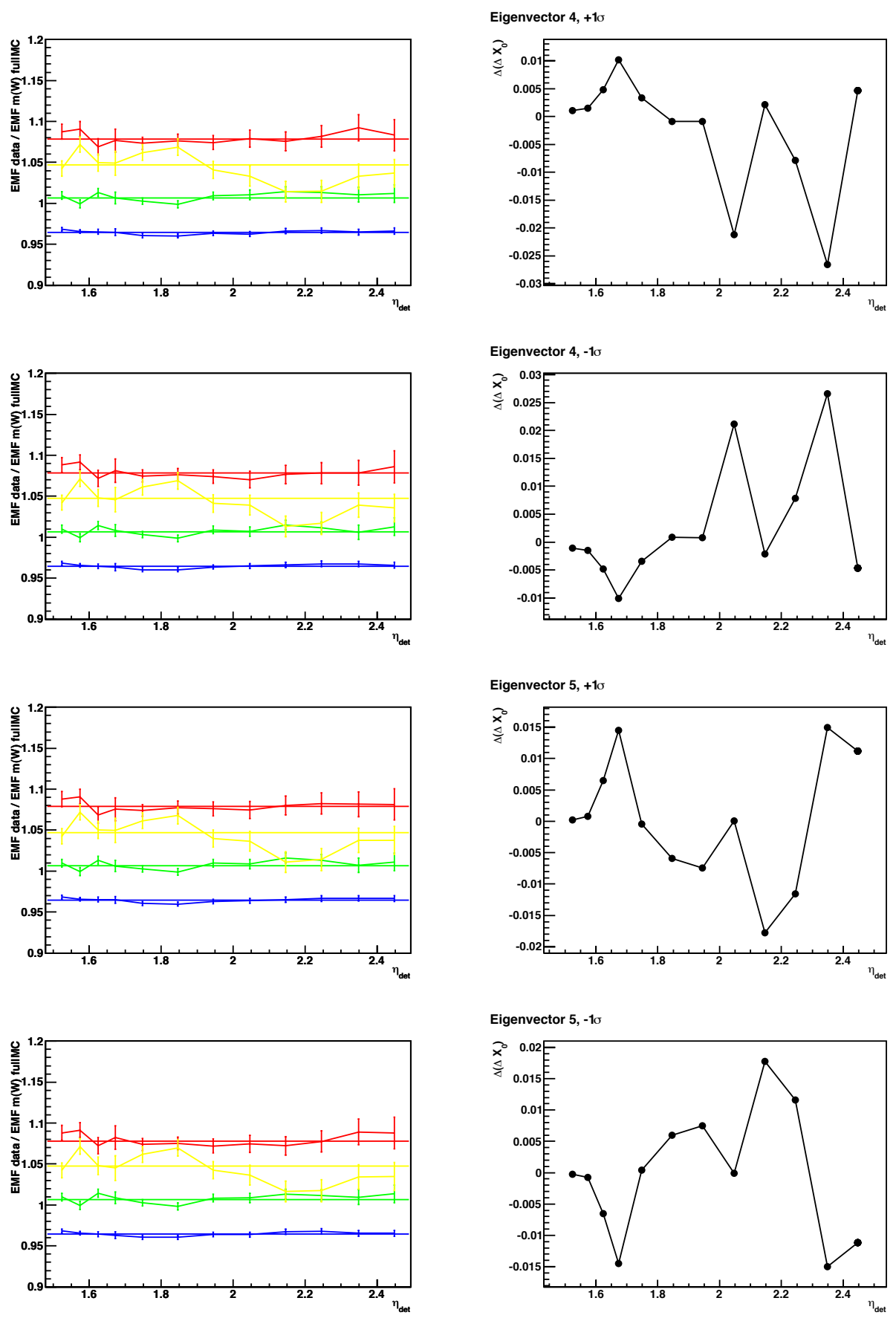

Figure C.17: North end-cap, eigenset 5 (two upper panels) and 6 (two bottom panels) with variations corresponding to $\pm 1 \sigma$ where $\sigma$ is the square root of the corresponding eigenvalue. The eigenvectors are ordered by decreasing eigenvalue. 

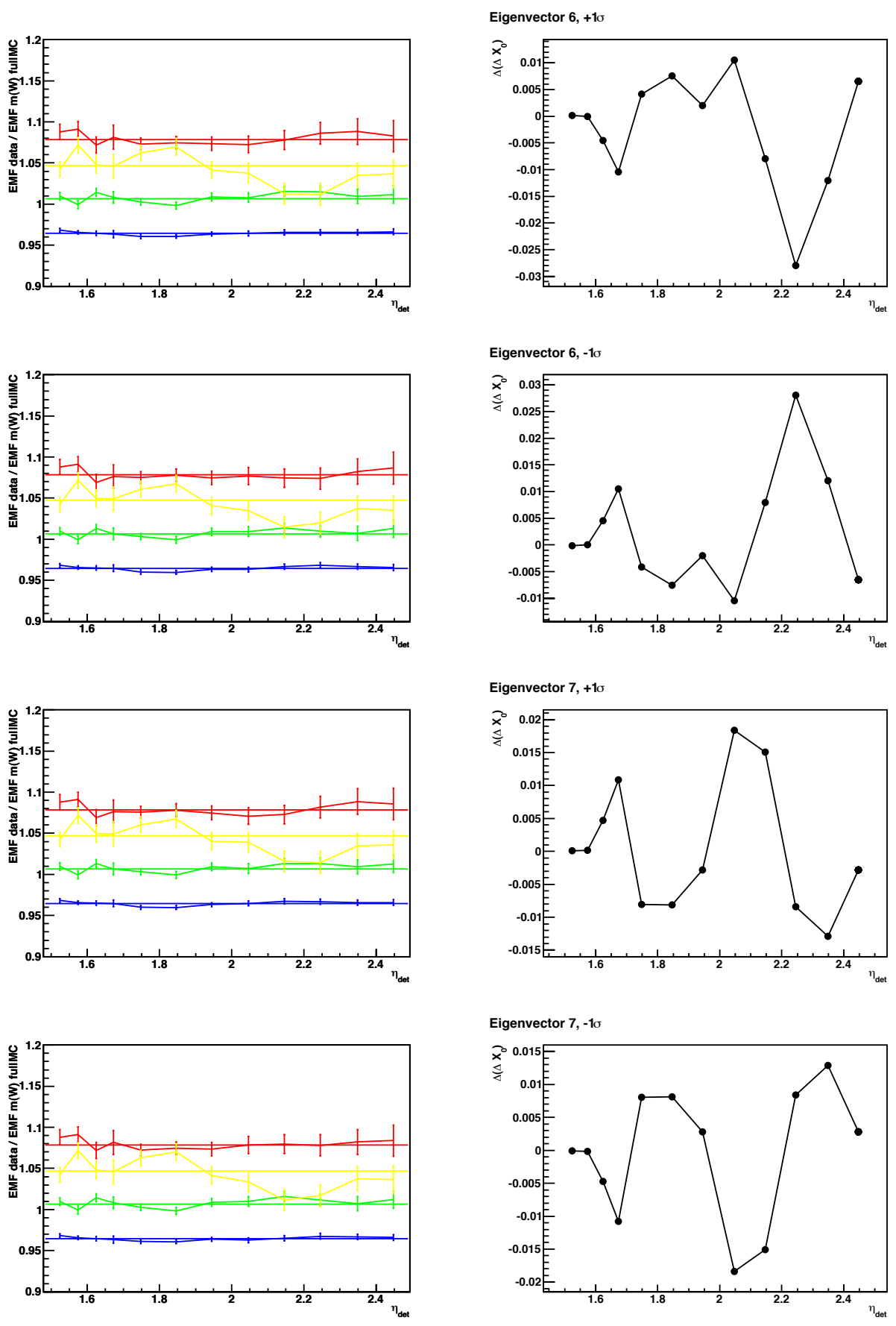

Figure C.18: North end-cap, eigenset 7 (two upper panels) and 8 (two bottom panels) with variations corresponding to $\pm 1 \sigma$ where $\sigma$ is the square root of the corresponding eigenvalue. The eigenvectors are ordered by decreasing eigenvalue. 

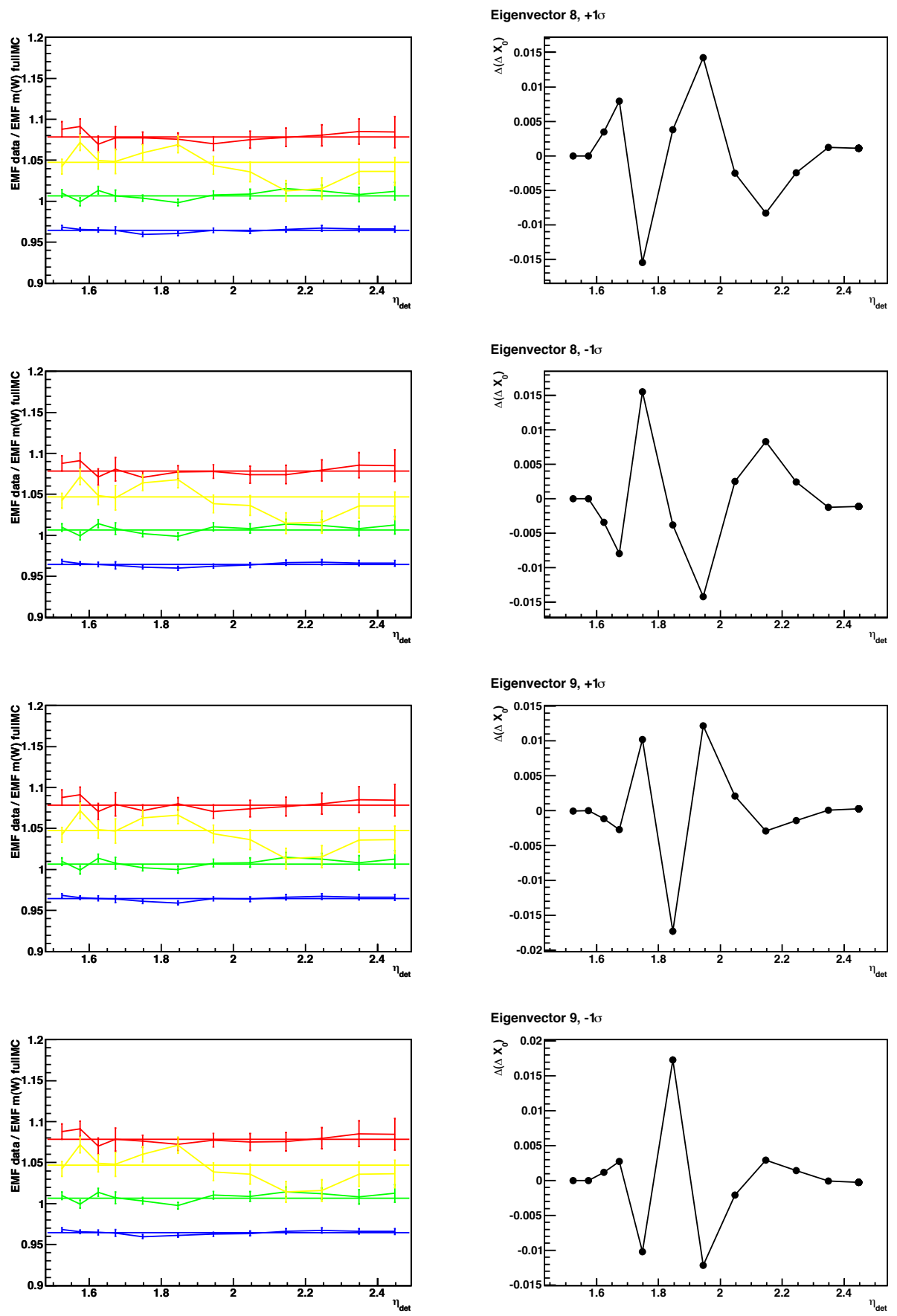

Figure C.19: North end-cap, eigenset 9 (two upper panels) and 10 (two bottom panels) with variations corresponding to $\pm 1 \sigma$ where $\sigma$ is the square root of the corresponding eigenvalue. The eigenvectors are ordered by decreasing eigenvalue. 

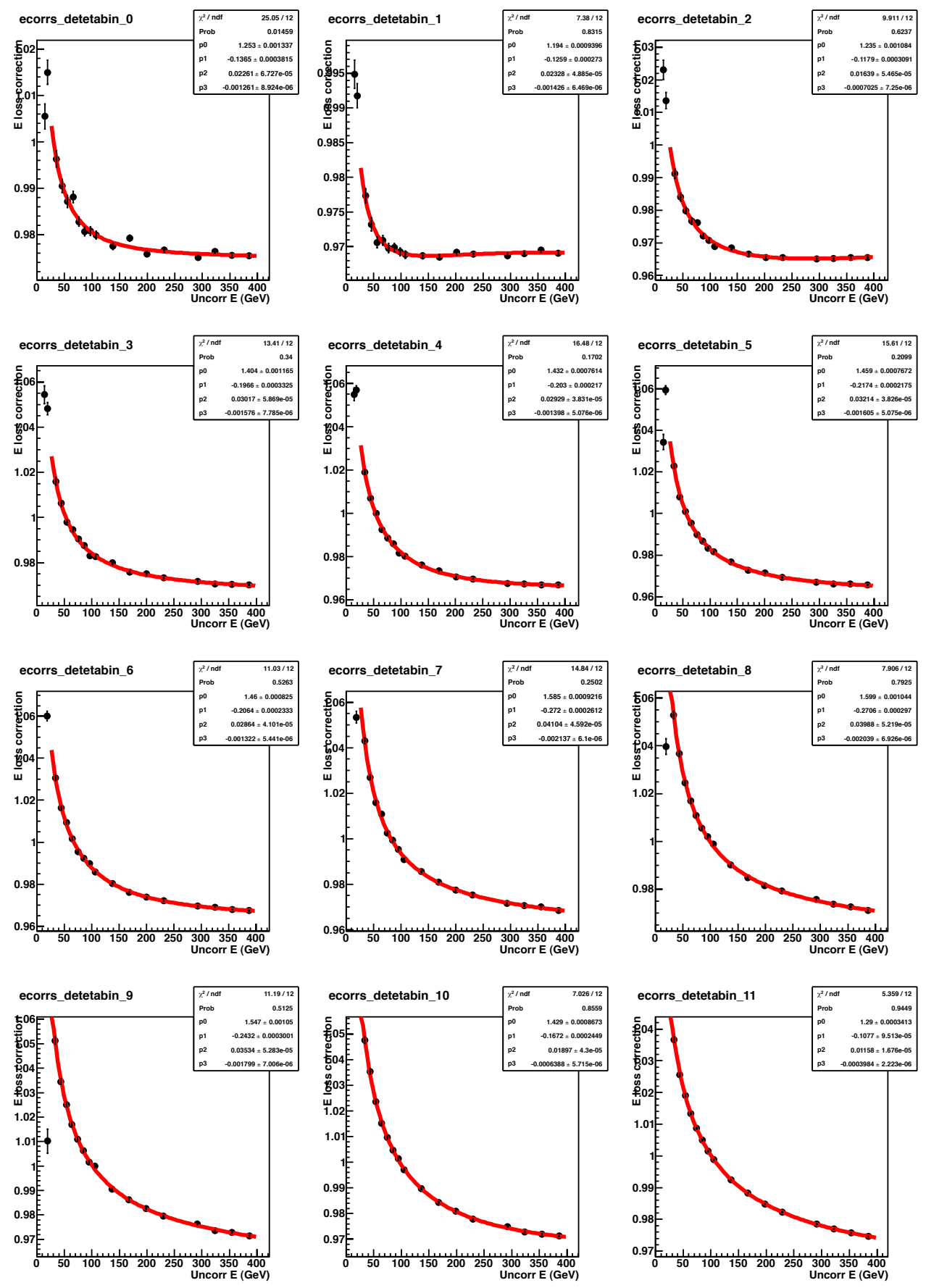

Figure C.20: North End Calorimeter energy loss function fit for using the D0 nominal geometry model. 

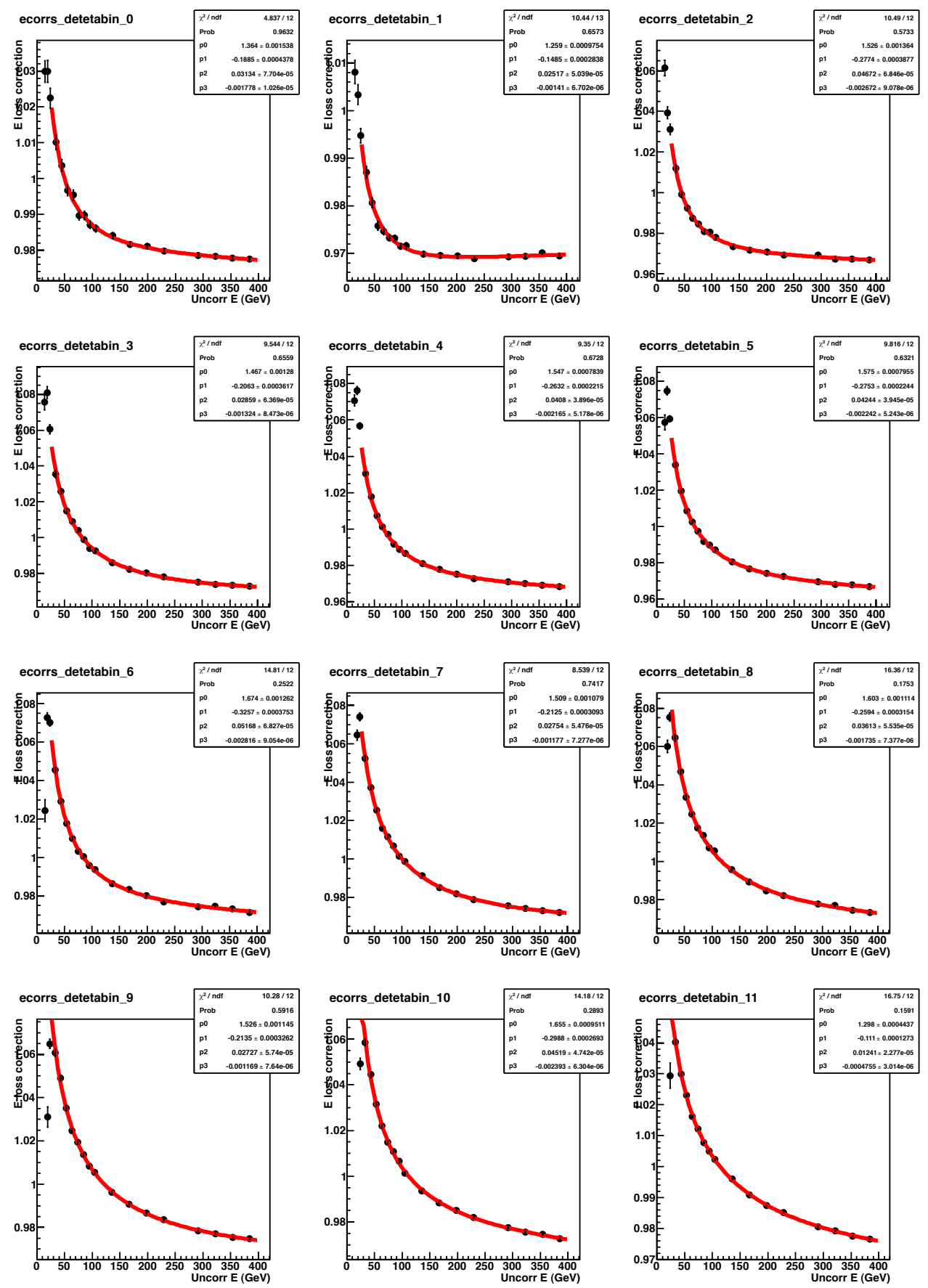

Figure C.21: South End Calorimeter energy loss function fit for using the D0 nominal geometry model. 

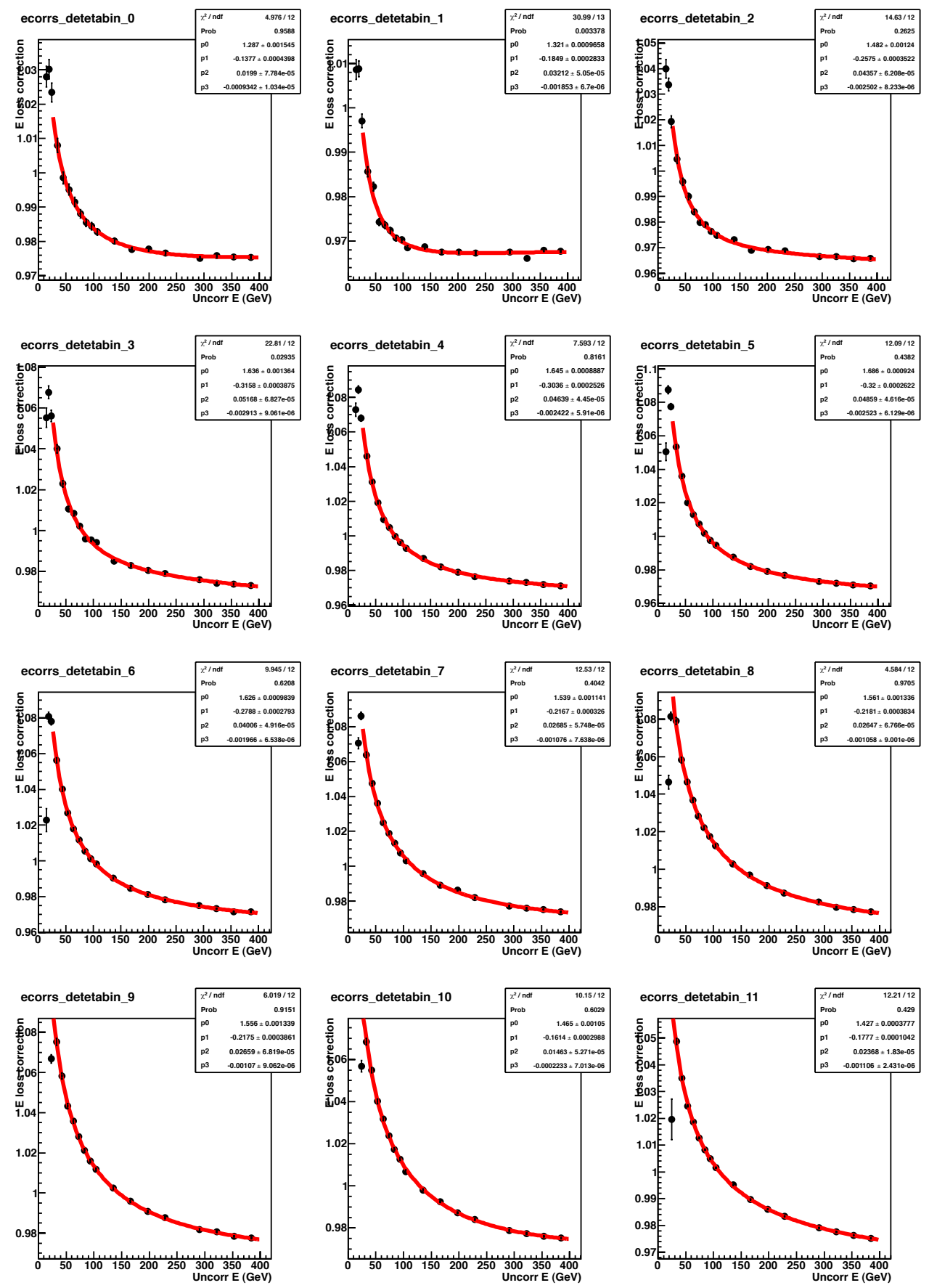

Figure C.22: North End Calorimeter energy loss function fit for using the ad hoc ECdevelB1 geometry. 

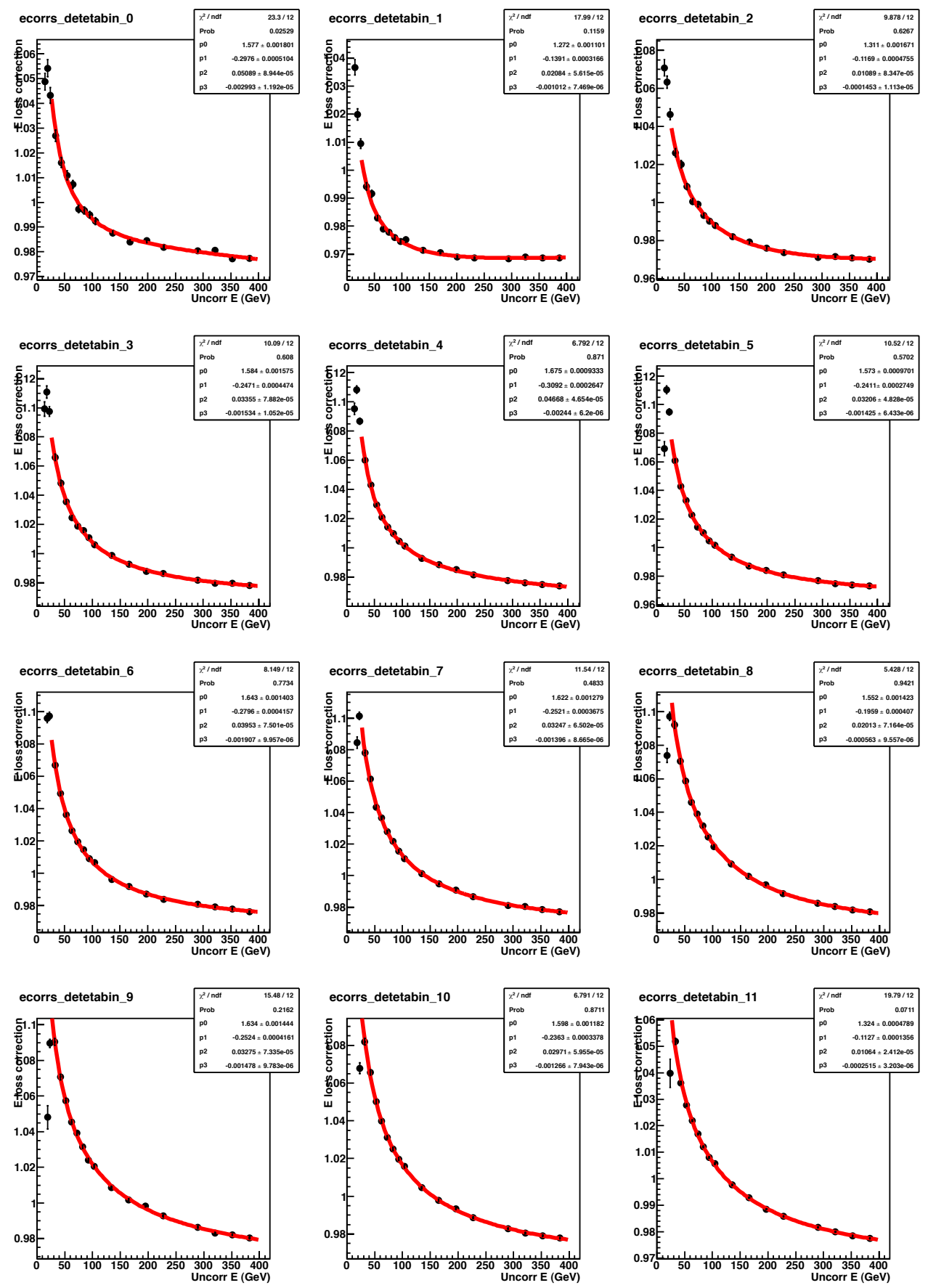

Figure C.23: South End Calorimeter energy loss function fit for using the ad hoc ECdevelB1 geometry. 

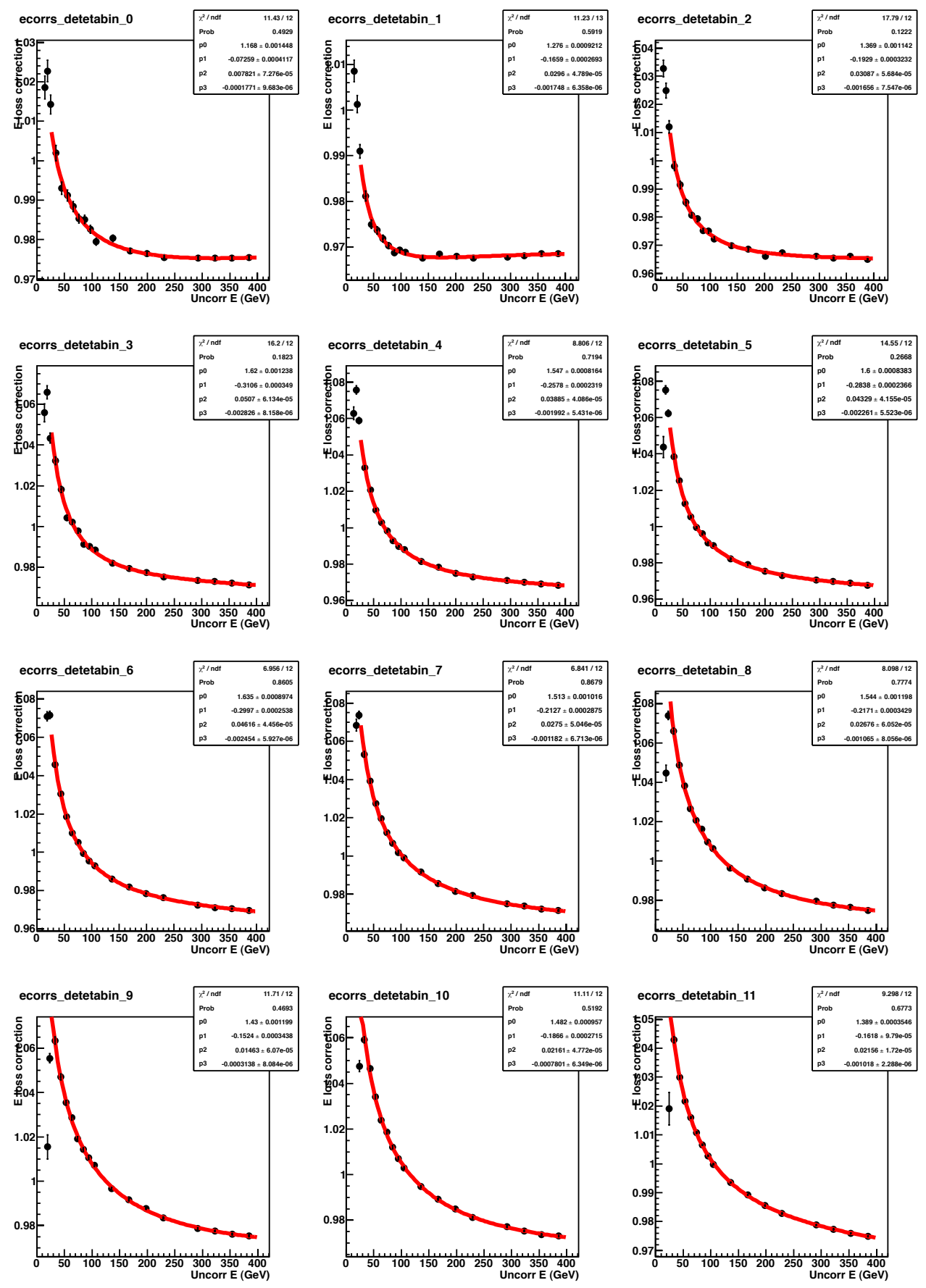

Figure C.24: North End Calorimeter energy loss function fit for using the ad hoc ECdevelB2 geometry. 

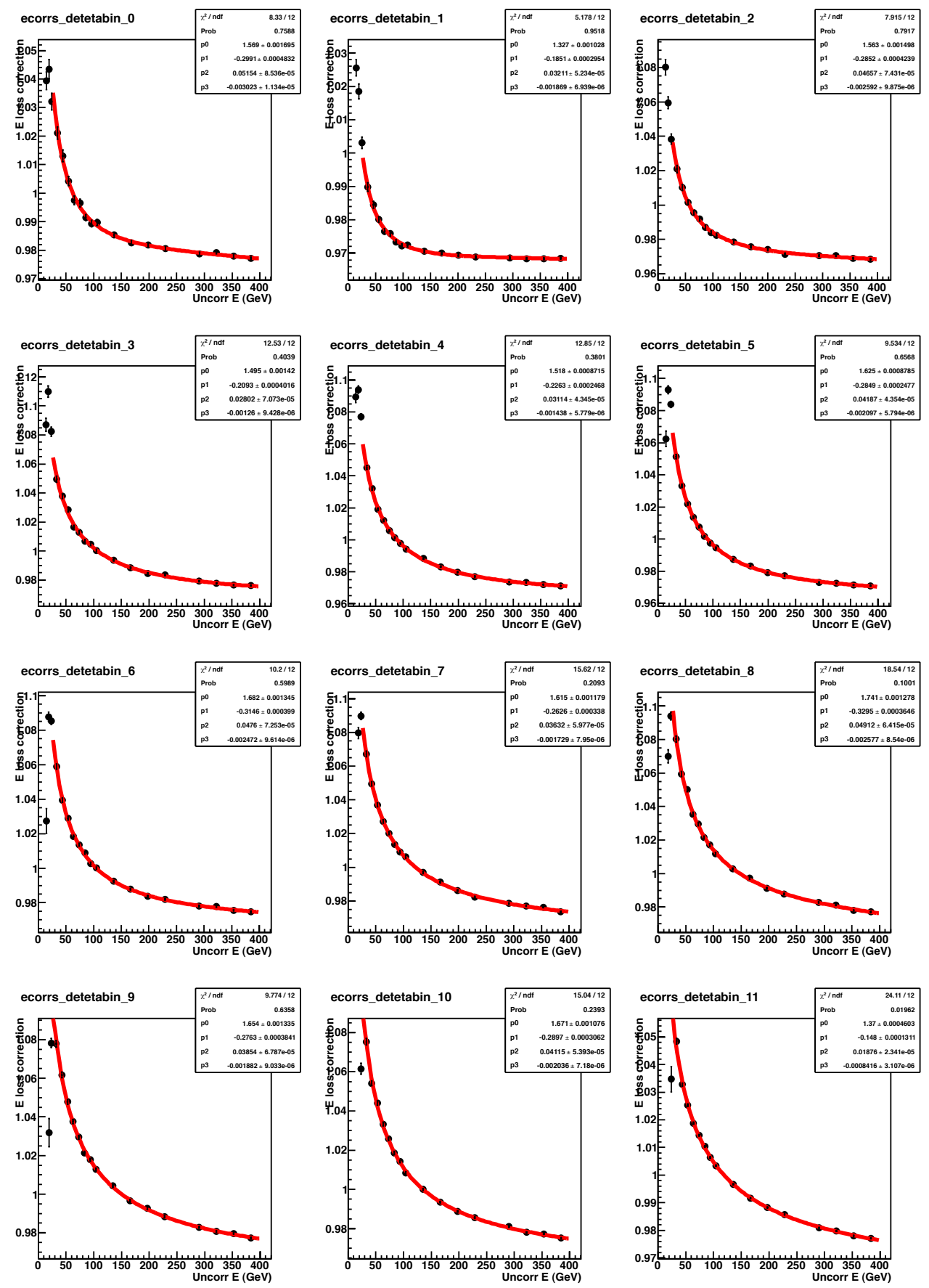

Figure C.25: South End Calorimeter energy loss function fit for using the ad hoc ECdevelB2 geometry. 

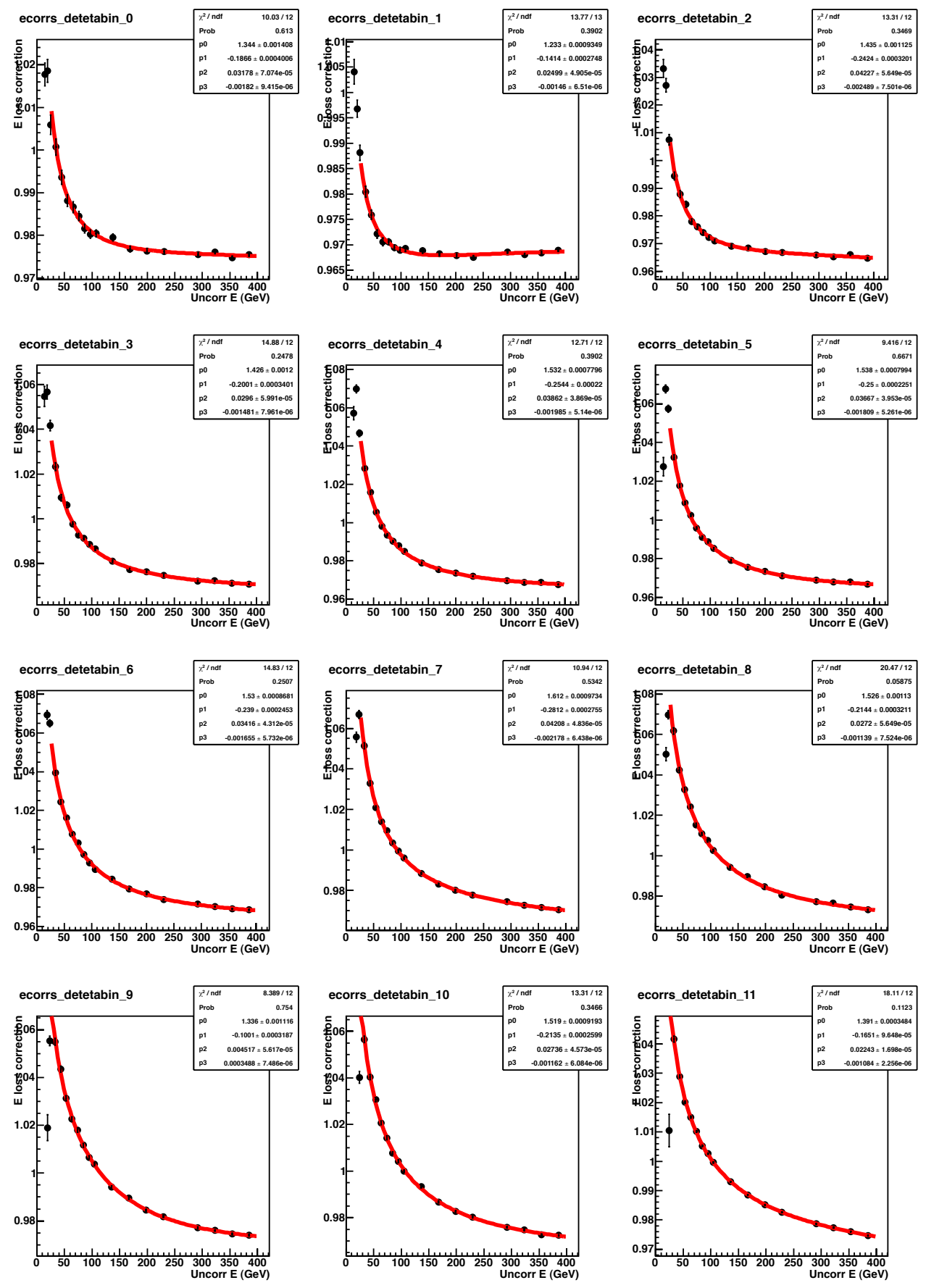

Figure C.26: North End Calorimeter energy loss function fit for using the ad hoc ECdevelB3 geometry. 

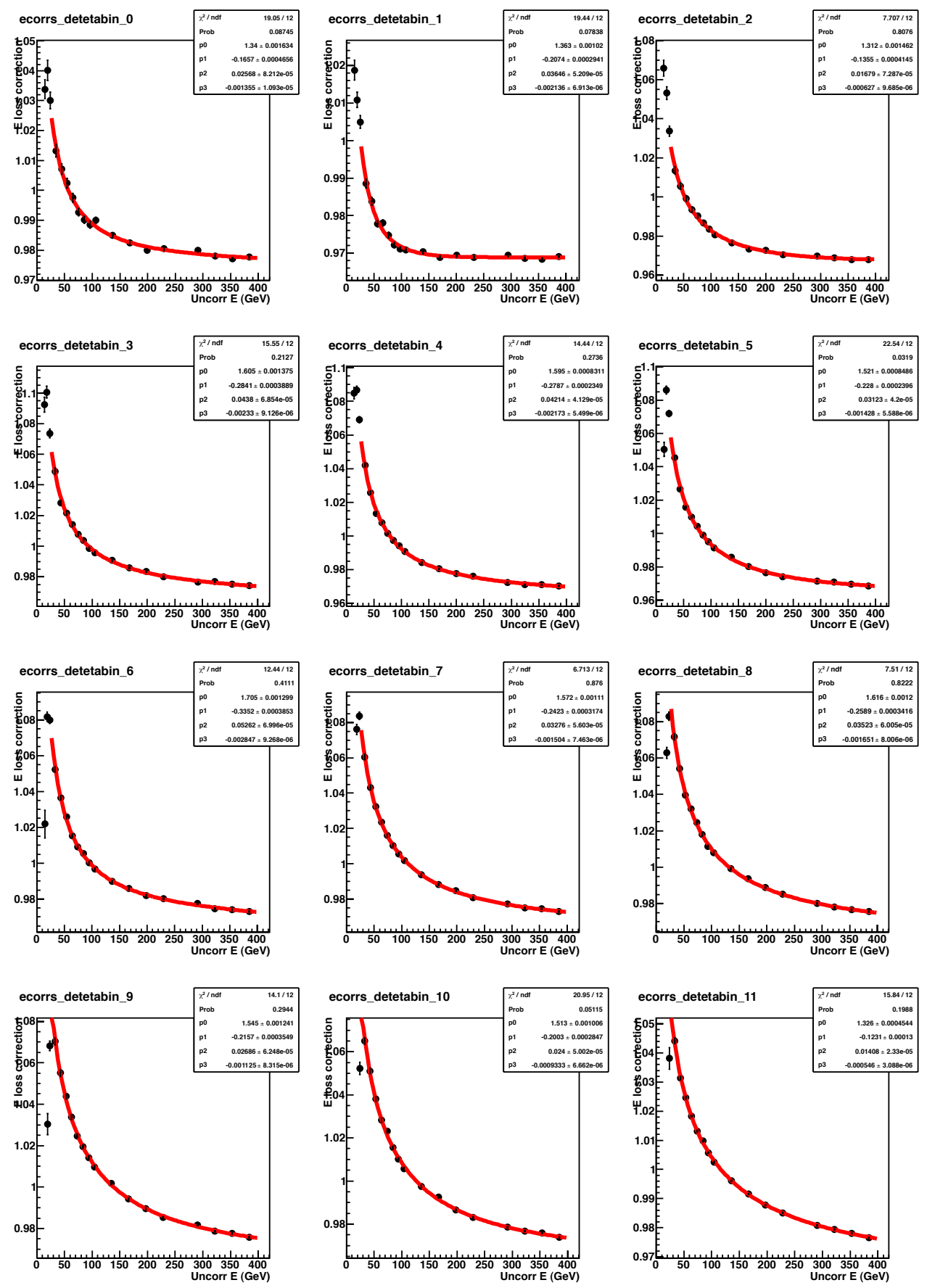

Figure C.27: South End Calorimeter energy loss function fit for using the ad hoc ECdevelB3 geometry. 
Energy loss correction for $\eta_{\text {det }}=1.52$

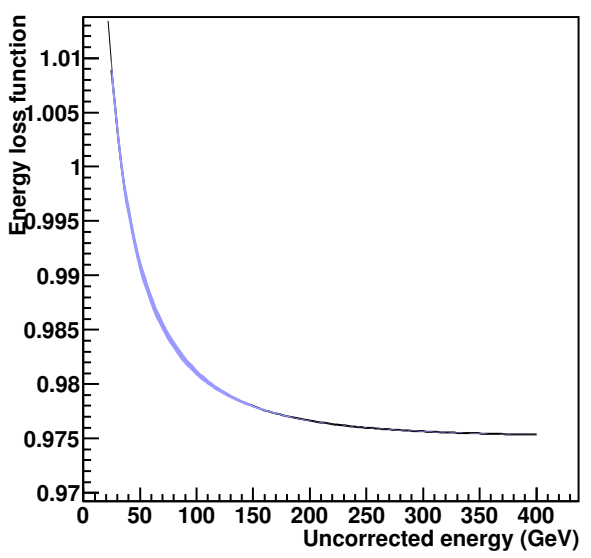

Energy loss correction for $\eta_{\text {det }}=1.62$

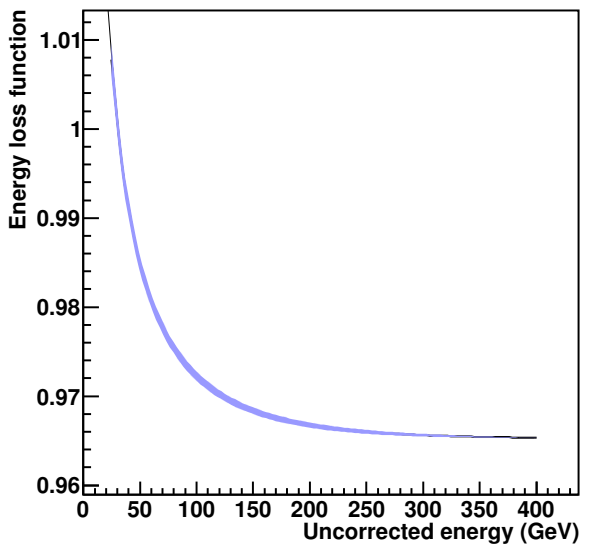

Energy loss correction for $\eta_{\text {det }}=1.75$

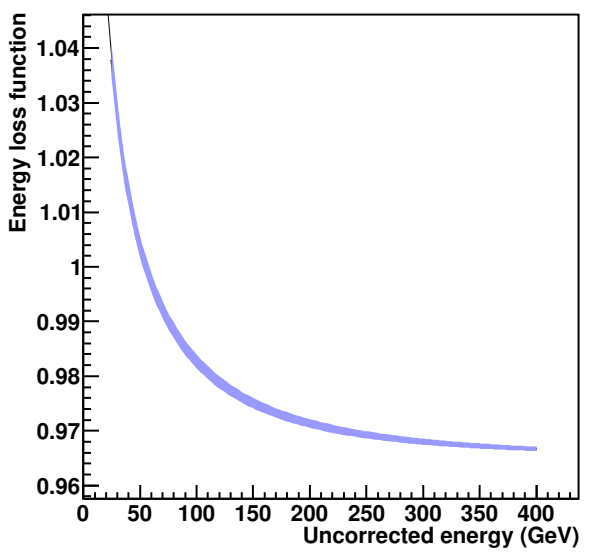

Energy loss correction for $\eta_{\text {det }}=1.57$

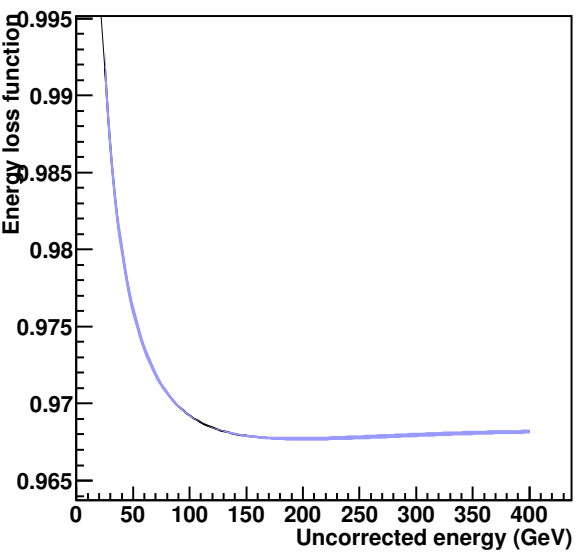

Energy loss correction for $\eta_{\text {det }}=1.67$

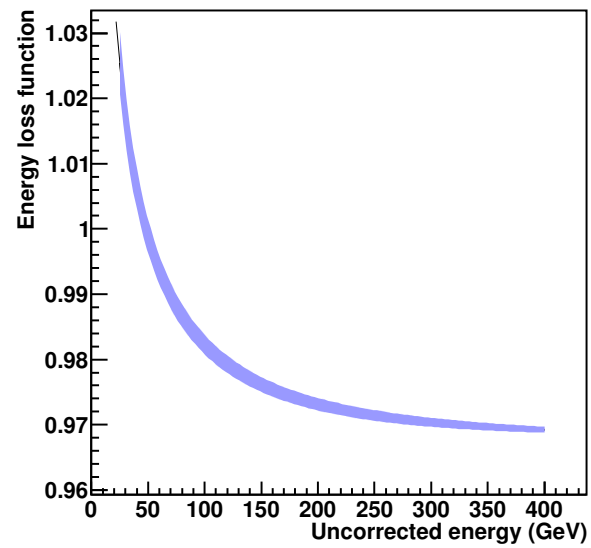

Energy loss correction for $\eta_{\text {det }}=1.85$

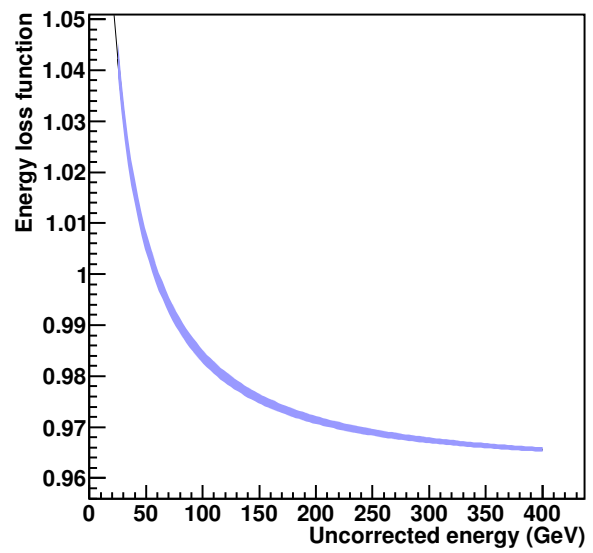

Figure C.28: North end-cap, energy loss functions for the first six $\eta_{\text {det }}$ bins $\left(1.5<\eta_{\text {det }}<\right.$ 1.9). The shaded area represents the 3 standard deviations propagated from the material tune. 

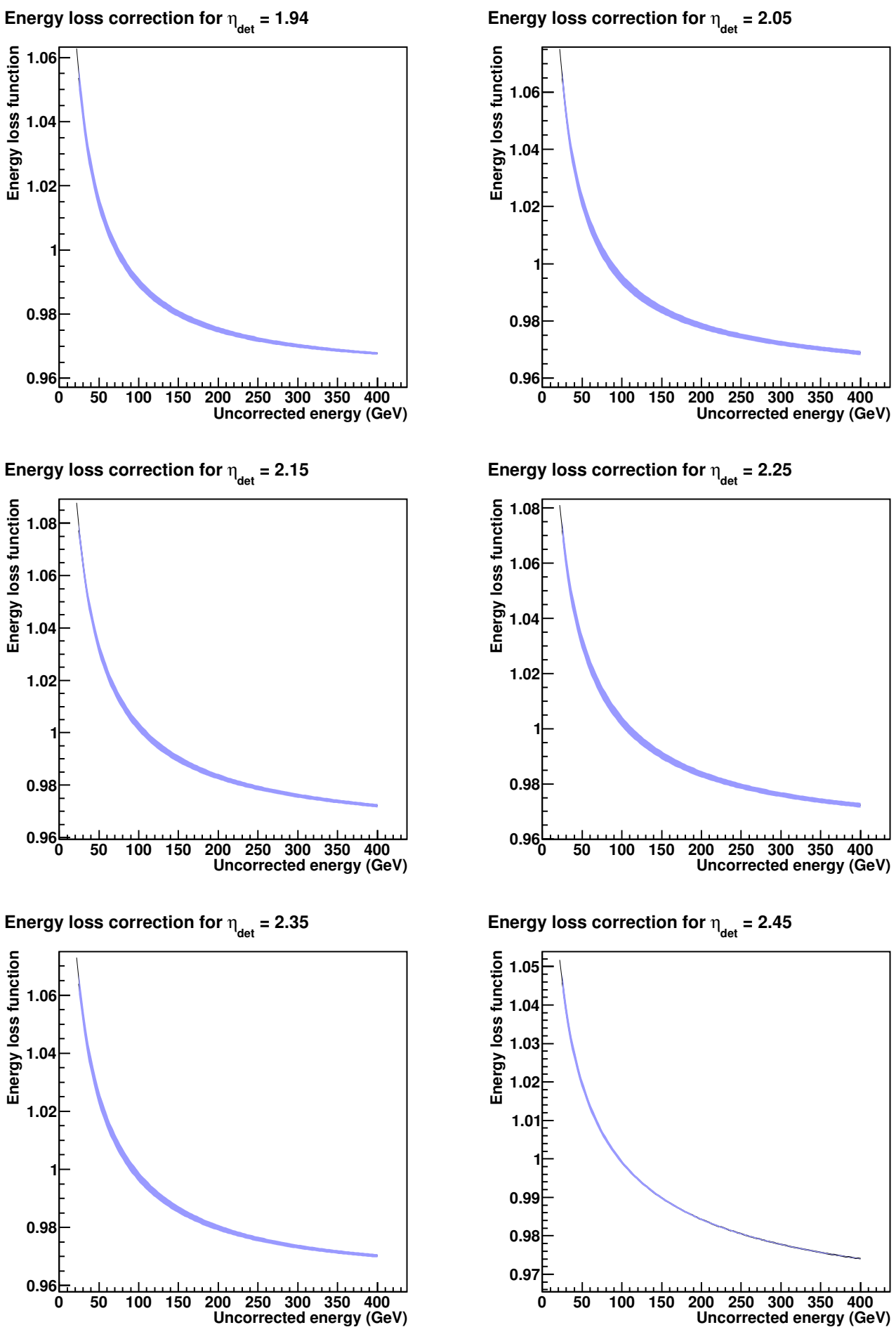

Figure C.29: North end-cap, energy loss functions for the last six $\eta_{\text {det }}$ bins $\left(1.9<\eta_{\text {det }}<\right.$ 2.5). The shaded area represents the 3 standard deviations propagated from the material tune. 
Energy loss correction for $\eta_{\text {det }}=1.53$

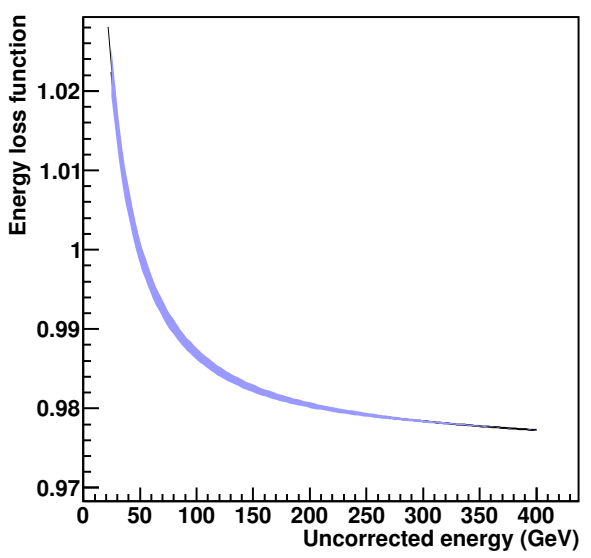

Energy loss correction for $\eta_{\operatorname{det}}=1.62$

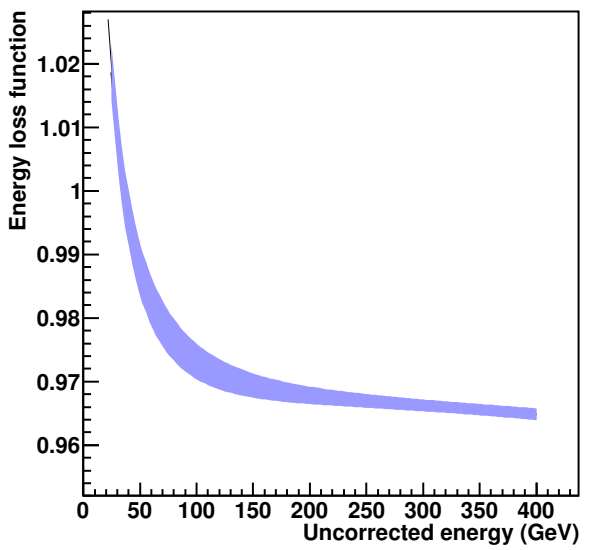

Energy loss correction for $\eta_{\operatorname{det}}=1.75$

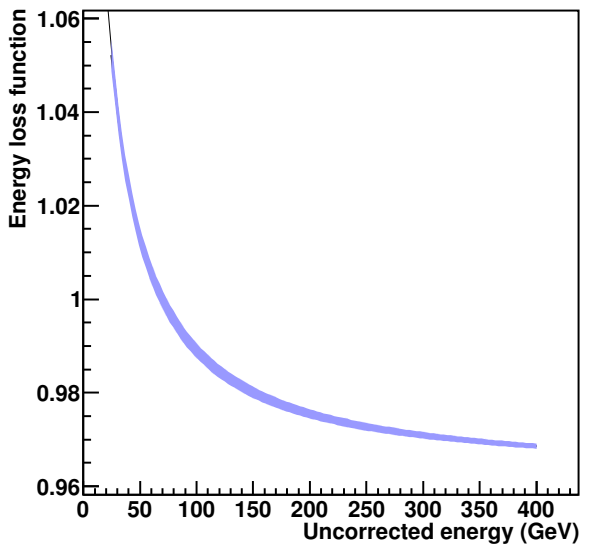

Energy loss correction for $\eta_{\text {det }}=1.57$

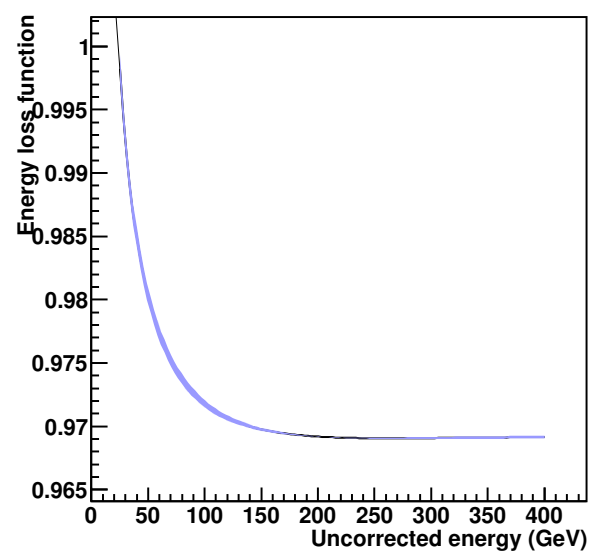

Energy loss correction for $\eta_{\operatorname{det}}=1.67$

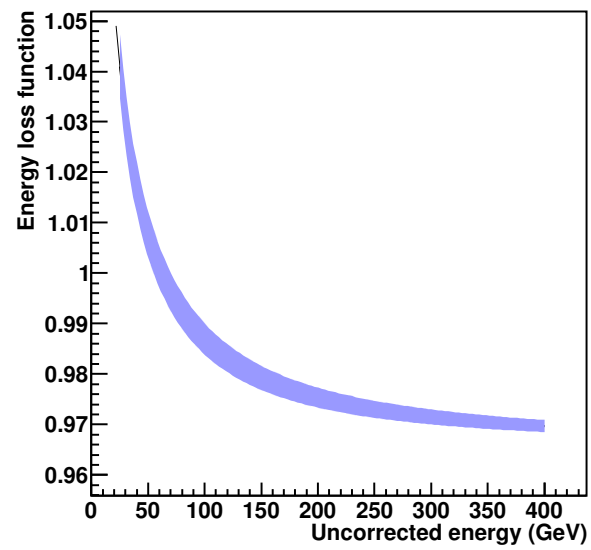

Energy loss correction for $\eta_{\text {det }}=1.85$

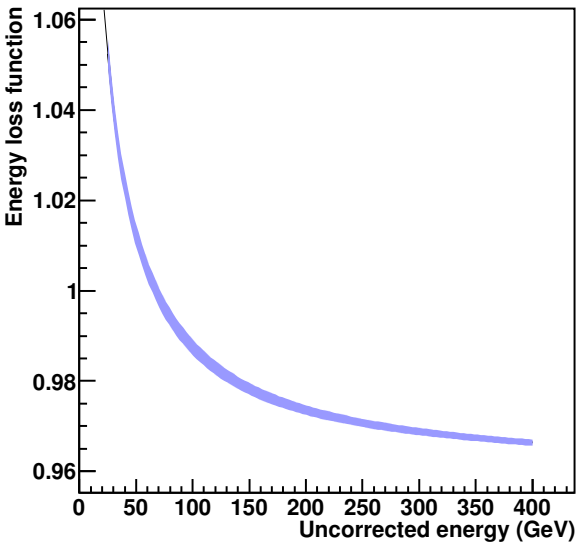

Figure C.30: South end-cap, energy loss functions for the last six $\eta_{\text {det }}$ bins $\left(1.9<\eta_{\text {det }}<\right.$ 2.5). The shaded area represents the 3 standard deviations propagated from the material tune. 

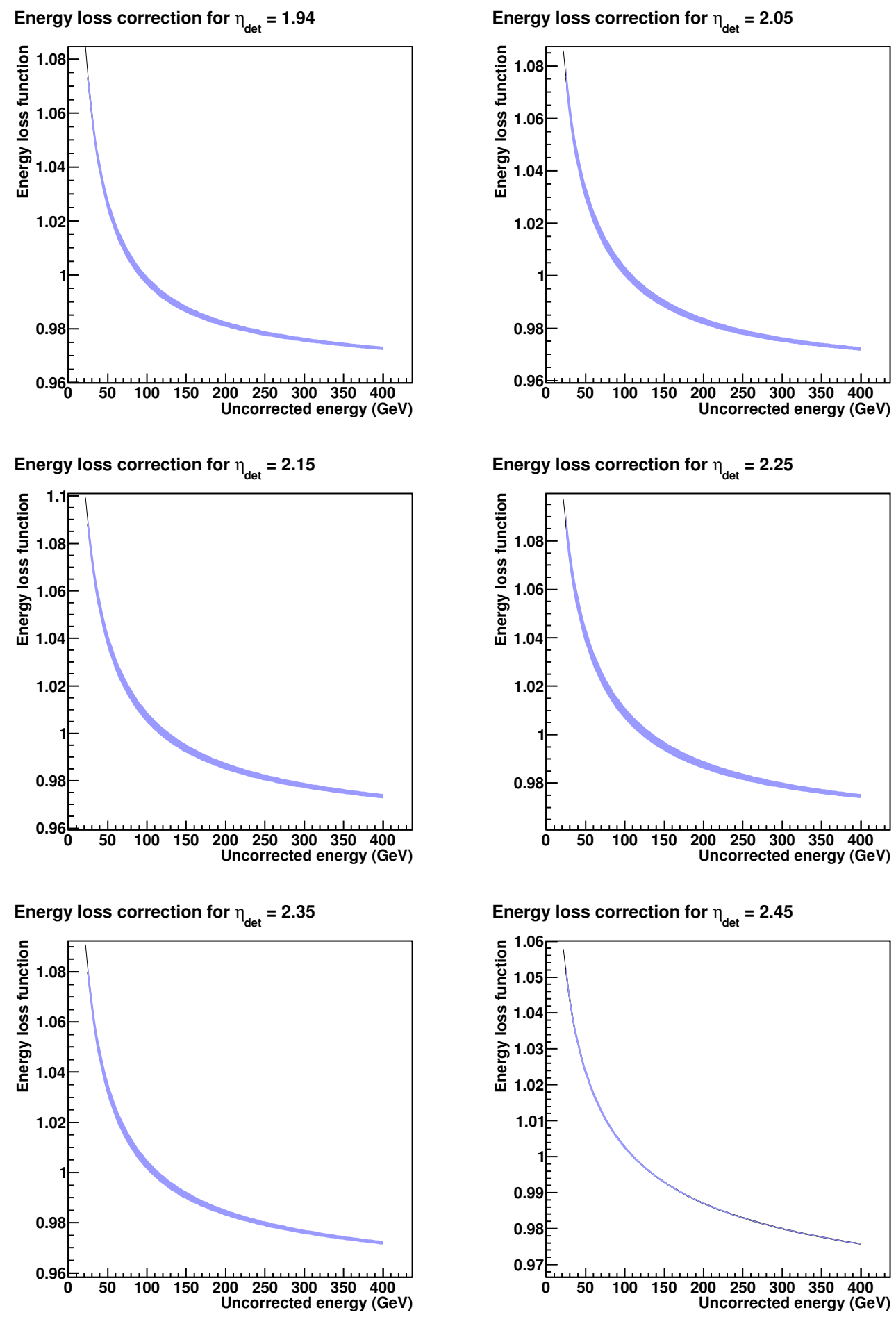

Figure C.31: South end-cap, energy loss functions for the last six $\eta_{\text {det }}$ bins $\left(1.9<\eta_{\text {det }}<\right.$ 2.5). The shaded area represents the 3 standard deviations propagated from the material tune. 